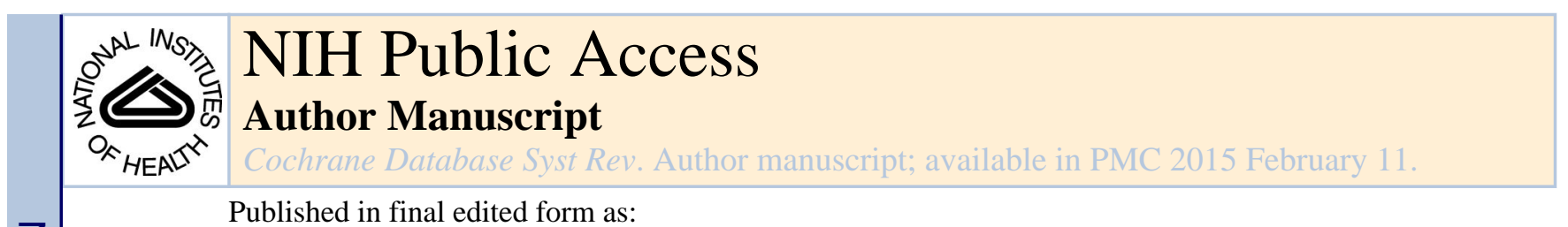

Published in final edited form as:

Cochrane Database Syst Rev. ; (3): CD002759. doi:10.1002/14651858.CD002759.pub2.

\title{
Progressive resistance strength training for improving physical function in older adults
}

\author{
Chiung-ju Liu ${ }^{1}$ and Nancy K Latham ${ }^{2}$ \\ ${ }^{1}$ Department of Occupational Therapy, Indiana University at Indianapolis, Indianpolis, Indiana, \\ USA \\ ${ }^{2}$ Health and Disabilty Research Institute, School of Public Health, Boston University, Boston, MA, \\ USA
}

\section{Abstract}

Background-Muscle weakness in old age is associated with physical function decline. Progressive resistance strength training (PRT) exercises are designed to increase strength.

Objectives-To assess the effects of PRT on older people and identify adverse events.

Search methods-We searched the Cochrane Bone, Joint and Muscle Trauma Group Specialized Register (to March 2007), the Cochrane Central Register of Controlled Trials (The Cochrane Library 2007, Issue 2), MEDLINE (1966 to May 01, 2008), EMBASE (1980 to February 06 2007), CINAHL (1982 to July 012007 ) and two other electronic databases. We also searched reference lists of articles, reviewed conference abstracts and contacted authors.

Copyright (C) 2009 The Cochrane Collaboration. Published by John Wiley \& Sons, Ltd.

Contact address: Chiung-ju Liu, Department of Occupational Therapy, Indiana University at Indianapolis, 1140 W Michigan ST CF 303, Indianpolis, Indiana, 46202, USA. liu41@iupui.edu.

*Indicates the major publication for the study

Contributions of Authors: For the first version of the review (completed 2002), Dr Nancy Latham, Dr Craig Anderson, Dr Derrick Bennett and Dr Caroline Stretton contributed to the development of the protocol, the analysis and interpretation of the data and the write-up of the review. Dr Nancy Latham took the lead in conducting the analyses and writing the protocol and review. In addition, Dr Latham and Dr Stretton conducted the searches, identified the trials, conducted the quality assessments and extracted the data. Dr Bennett provided methodological and statistical guidance for the review. Dr Anderson served as the adjudicator when a consensus about data issues could not be reached between the two reviewers, and provided guidance about the methods and interpretation of the review.

The review was substantially updated in 2009 by Dr Chiung-ju Liu and Dr Nancy Latham. Dr Liu took the lead in conducting the update, which included undertaking the searches, screening search results, organizing retrieval of papers, screening retrieved papers against inclusion criteria, appraising quality of papers, extracting data, contacting authors for additional information, entering data into RevMan, doing the analyses and writing up. The project was completed when Dr Liu was a post-doctoral research fellow at the Health and Disability Research Institute at Boston University. Dr Latham assisted in identifying the trials, conducting the quality assessments, extracting the data, interpreting the results and writing the review.

Both Dr Chiung-ju Liu and Dr Nancy Latham are guarantors for the review.

Declarations of Interest: Dr. Latham is an author for two trials. The trials were rated independently by other reviewers in the first review.

Notes: Substantial updates of reviews such as this one often take a considerable time to prepare and then take through the editorial process. They can therefore seem 'out of date' before publication, particularly in research active areas. However, although an updated search made in May 2008 revealed nine more potentially eligible trials (which await assessment, pending the next update), it is unlikely that the review's main findings will be substantively changed by these. [Comment by Helen Handoll, Co-ordinating Editor, May 2009] 
Selection criteria-Randomised controlled trials reporting physical outcomes of PRT for older people were included.

Data collection and analysis-Two review authors independently selected trials, assessed trial quality and extracted data. Data were pooled where appropriate.

Main results-One hundred and twenty one trials with 6700 participants were included. In most trials, PRT was performed two to three times per week and at a high intensity. PRT resulted in a small but significant improvement in physical ability (33 trials, 2172 participants; SMD $0.14,95 \%$ CI 0.05 to 0.22 ). Functional limitation measures also showed improvements: e.g. there was a modest improvement in gait speed (24 trials, 1179 participants, MD $0.08 \mathrm{~m} / \mathrm{s}, 95 \%$ CI 0.04 to 0.12 ); and a moderate to large effect for getting out of a chair (11 trials, 384 participants, SMD $-0.94,95 \%$ CI -1.49 to -0.38 ). PRT had a large positive effect on muscle strength (73 trials, 3059 participants, SMD $0.84,95 \%$ CI 0.67 to 1.00 ). Participants with osteoarthritis reported a reduction in pain following PRT (6 trials, 503 participants, SMD -0.30, 95\% CI -0.48 to -0.13). There was no evidence from 10 other trials (587 participants) that PRT had an effect on bodily pain. Adverse events were poorly recorded but adverse events related to musculoskeletal complaints, such as joint pain and muscle soreness, were reported in many of the studies that prospectively defined and monitored these events. Serious adverse events were rare, and no serious events were reported to be directly related to the exercise programme.

Authors' conclusions-This review provides evidence that PRT is an effective intervention for improving physical functioning in older people, including improving strength and the performance of some simple and complex activities. However, some caution is needed with transferring these exercises for use with clinical populations because adverse events are not adequately reported.

\section{Medical Subject Headings (MeSH)}

Activities of Daily Living; Muscle Weakness [*rehabilitation]; Randomized Controlled Trials as Topic; Recovery of Function [physiology]; Resistance Training [adverse effects; *methods]

\section{MeSH check words}

Aged; Humans

\section{Background}

\section{Description of the condition}

Muscle strength is the amount of force produced by a muscle. The loss of muscle strength in old age is a prevalent condition. Muscle strength declines with age such that, on average, the strength of people in their $80 \mathrm{~s}$ is about $40 \%$ less than that of people in their 20s (Doherty 1993). Muscle weakness, particularly of the lower limbs, is associated with reduced walking speed (Buchner 1996), increased risk of disability (Guralnik 1995) and falls in older people (Tinetti 1986). 


\section{Description of the intervention}

Progressive resistance training (PRT) is often used to increase muscle strength. During the exercise, participants exercise their muscles against some type of resistance that is progressively increased as strength improves. Common equipment used for PRT includes exercise machines, free weights, and elastic bands.

\section{How the intervention might work}

Contrary to long held beliefs, the muscles of older people (i.e. people aged 60 years and older) continue to be adaptable, even into the extremes of old age (Frontera 1988). Trials have revealed that older people can experience large improvements in their muscle strength, particularly if their muscles are significantly overloaded during training (Brown 1990; Charette 1991; Fiatarone 1994).

\section{Why it is important to do this review}

Despite evidence of benefit from PRT in terms of improving muscle strength, there is still uncertainty about how these effects translate into changes in substantive outcomes such a reduction in physical disability (Chandler 1998). Most studies have been under-powered to determine the effects of PRT on these outcomes or have included PRT as part of a complex intervention. In addition, there is uncertainty about the effects of PRT when more pragmatic, home or hospital-based programmes are used, and the safety and effectiveness of this intervention in older adults who have health problems and/or functional limitations. Finally, there is uncertainty about the relative benefits of PRT compared with other exercise programmes, or the effectiveness of varying doses of PRT (i.e. programmes of varying intensity and duration). This update of our review (Latham 2003a) has continued to assess and summarise the evidence for PRT.

\section{Objectives}

To determine the effects of progressive resistance strength training (PRT) on physical function in older adults through comparing PRT with no exercise, or another type of care or exercise (e.g. aerobic training). Comparisons of different types (e.g. intensities, frequencies, or speed) of PRT were included also. We considered these effects primarily in terms of measures of physical (dis)ability and adverse effects, and secondary measures of functional impairment (muscle strength \& aerobic capacity) and limitation (e.g. gait speed).

\section{Methods}

\section{Criteria for considering studies for this review}

Types of studies-Any randomised clinical trials meeting the specifications below were included. All non-randomised controlled trials (e.g. controlled before and after studies) were excluded. Also excluded were trials for which details were provided that indicated these used quasi-randomised methods, such as allocation based on date of birth.

Types of participants-Older people, resident in institutions or at home in the community. Trials were included if the mean age of participants was 60 or over, but 
excluded if participants aged less than 50 were enrolled. The participants could include frail or disabled older people, people with identified diseases or health problems, or fit and healthy people.

Types of interventions-Any trial that had one group of participants who received PRT as a primary intervention was considered for inclusion. PRT was defined as a strength training programme in which the participants exercised their muscles against an external force that was set at specific intensity for each participant, and this resistance was adjusted throughout the training programme. The type of resistance used included elastic bands or tubing (i.e. therabands), cuff weights, free weights, isokinetic machines or other weight machines. This type of training could take place in individual or group exercise programmes, and in a home-based or gymnasium/clinic setting. Studies that utilised only isometric exercises were excluded. Studies that included balance, aerobic or other training as part of the exercise intervention (and not simply part of the warm-up or cool-down) were also excluded.

We found the following comparisons between groups in the trials:

- $\quad$ PRT versus no exercise (greatest difference between groups was expected)

- Different types of PRT: high intensity versus low intensity, high frequency versus low frequency, or higher speed (power training) versus regular speed (greatest effect expected in the higher intensity groups). Power training refers to the type of PRT that emphasizes speed.

- PRT versus regular care (including regular therapy or exercise)

- PRT versus another type of exercise (smaller difference between groups expected)

\section{Types of outcome measures}

Primary outcomes: This review assessed physical function in older adults at the level of impairment, functional limitation and disability. The primary outcome of this review was physical disability. This was assessed as a continuous variable. The outcomes were categorized based on the Nagi model of health states (Nagi 1991). In this model, disability is considered to be a limitation in performance of socially defined roles and tasks that can relate to self-care, work, family etc. In this review, the primary assessment of physical disability included the evaluation of self-reported measures of activities of daily living (ADL, i.e. the Barthel Index) and the physical domains of health-related quality of life (HRQOL, i.e. the physical function domain of the SF-36). Data from these measures were pooled for the main analysis of physical disability. However, because these two types of measures (ADL and physical domains of HRQOL) evaluate different health concepts, they were also evaluated in separate analyses. The Nagi model also includes firstly, the domain of 'functional limitations' which are limitations in performance at the level of the whole person and includes activities such as walking, climbing or reaching, and secondly, 'impairments' that are defined as anatomical or physiological abnormalities.

Since the protocol of this review was written, the International Classification of Functioning, Disability and Handicap (ICF) has been released (WHO 2001). Under this system, disability 
is an umbrella term for impairments, activity limitations and participation restrictions. Using the ICF, the outcome measures evaluated in this review fall under the domains of impairments, limitations in simple activities (similar to 'functional limitations' in Nagi's system) and limitations in complex activities (similar to some aspects of disability in Nagi's model).

\section{Secondary outcomes}

Measures of impairment (outcome comparisons 2 and 3): The following secondary outcomes were assessed as continuous variables:

- muscle strength (e.g. 1 repetition maximum test, isokinetic and isometric dynamometry)

- aerobic capacity (e.g. 6 minute walk test, $\mathrm{VO} 2$ max: maximal oxygen uptake during exercise)

Measures of functional limitation (simple physical activities): The following secondary outcomes were assessed as continuous variables:

- balance (e.g. Berg Balance Scale, Functional Reach Test)

- $\quad$ gait speed, timed walk

- timed 'up-and-go' test

- chair rise (sit to stand)

- $\quad$ stair climbing (added in 2008)

The balance outcome is also reviewed in a separate Cochrane review (Howe 2007).

Other outcomes: The dichotomous secondary outcomes assessed were adverse events, admission to hospital and death. The effect of PRT on falls was also evaluated, although these outcomes are considered in a separate Cochrane review (Gillespie 2003). Pain and vitality measures were evaluated as continuous outcomes, and were used to provide additional information about the potential adverse effects or benefits of PRT.

Outcomes removed after the protocol: In the original protocol for this review, measures of fear of falling and participation in social activities were also included as outcomes.

However, when the size and complexity of this review became apparent, the authors decided to limit this review to assessments of physical disability as this was the prespecified primary aim of the review. Therefore, these outcomes are not included in the current review. In addition, the protocol also stated that assessments of disability using the Barthel Index and Functional Independence Measure (FIM) would be dichotomised. However as no trials included the FIM as an outcome and only three trials used the Barthel Index, the decision was made to report these data as continuous outcomes only.

\section{Search methods for identification of studies}

Electronic searches-We searched the Cochrane Bone, Joint and Muscle Trauma Group Specialised Register (March 2007), the Cochrane Central Register of Controlled Trials (The 
Cochrane Library 2002, Issue 2; February 2007), MEDLINE (1966 to May 01, 2008), EMBASE (1980 t February 06, 2007), CINAHL (1982 to July 01, 2007), SPORTDiscus (1948 to February 07, 2007), PEDro - The Physiotherapy Evidence Database (accessed February 07, 2007) and Digital Dissertations (accessed February 01, 2007). No language restrictions were applied.

In MEDLINE (OVID Web) the subject specific search strategy was combined with the first two phases of the Cochrane optimal search strategy (Higgins 2006). This search strategy, along with those for EMBASE (OVID Web), The Cochrane Library (Wiley InterScience), CINAHL (OVID Web), SPORTDiscus (OVID Web) and PEDro, can be found in Appendix 1 .

Searching other resources-We contacted authors and searched reference lists of identified studies, and reviews (Anonymous 2001; Buchner 1993; Chandler 1996; Fiatarone 1993; Keysor 2001; King 1998; King 2001; Mazzeo 1998; Singh 2002).

We also handsearched the following conference proceedings:

- 16th International Association of Gerontology World Congress; 1997; Adelaide (Australia).

- 17th International Association of Gerontology World Congress; 2001; Vancouver (Canada).

- Proceedings of the 13th World Congress of Physical Therapy; 1995; Washington (DC).

- Proceedings of the 14th World Congress of Physical Therapy; 1999; Japan.

- New Zealand Association of Gerontology Conferences -1996 Dunedin, 1999 Wellington and 2002 Auckland (New Zealand).

- The 60th annual scientific meeting of the Gerontological Society of America; 2007, San Francisco, CA.

- The American Congress of Rehabilitation Medicine -American Society of Neurorehabilitation Joint Conference; 2006, Boston, MA.

\section{Data collection and analysis}

Selection of studies-For this update (Issue 3, 2009), one author (CJL) conducted the searches. Both listed authors (CJL, NL) reviewed the titles, descriptors or abstracts identified from all literature searches to identify potentially relevant trials for full review. A copy of the full text of all trials that appeared to be potentially suitable for the review was obtained. Both authors independently used previously defined inclusion criteria to select the trials. In all cases, the reviewers reached a consensus when they initially disagreed about the inclusion of a trial. Before this update, the same method of identifying and assessing studies was used, although other members of the previous review team assisted (Latham 2003a).

Data extraction and management-Two authors independently extracted the data and recorded information on a standardised paper form. They considered all primary and 
secondary outcomes. If the data were not reported in a form that enabled quantitative pooling, the authors were contacted for additional information. If the authors could not be contacted or if the information was no longer available, the trial was not included in the pooling for that specific outcome.

Assessment of risk of bias in included studies-The methodological quality of each trial was independently assessed by two authors (NL, CS in the first review; CJL, NL in the update) using a scoring system that was based on the Cochrane Bone, Joint and Muscle Trauma Group's former evaluation tool. The review authors were blinded to the trial authors' institution, journal that the trial was published in and the results of the trial. A third review author (CA) was consulted in the first review if a consensus about the trial quality could not be reached. No third review author was involved in the review update. The criteria for assessing internal and external validity can be found in Table 1 .

Assessment of heterogeneity-The chi $^{2}$ test was used to assess heterogeneity. In future updates, we will also assess heterogeneity by visual inspection of the forest plots and consideration of the $\mathrm{I}^{2}$ statistic.

Data synthesis-Where it was thought appropriate, the results from the studies were combined. Data synthesis was carried out using MetaView in Review Manager version 5.0. For continuous outcomes, mean differences (MD) and 95\% confidence intervals (CI) were calculated when similar measurement units were used. To pool outcomes using different units, standardised units (i.e. standardised mean differences, SMD) were created as appropriate. We calculated risk ratios and $95 \% \mathrm{CI}$ for dichotomous outcomes, where possible. If minimal statistical heterogeneity $(\mathrm{P}<0.1)$ existed, fixed-effect meta-analysis was performed.

For trials that compared two or more different dosages of PRT versus a control group, data from the higher or highest intensity group were used in the analyses of PRT versus control.

Subgroup analysis and investigation of heterogeneity-If substantial statistical heterogeneity existed, the review authors looked for possible explanations. Specifically, we considered differences in age and baseline disability of the study participants, the methodological quality of the trials and the intensity and duration of the interventions. If the statistical heterogeneity could be explained, we considered the possibility of presenting the results as subgroup analyses. If the statistical heterogeneity could not be explained, we considered not combining the studies at all, using a random-effects model with cautious interpretation or using both fixed-effect and random-effects models to assist in explaining the uncertainty around an analysis with heterogeneous studies.

Sensitivity analysis-Sensitivity analyses were conducted to assess the effect of differences in methodological quality. These included allocation concealment, blinding of outcome assessors, statements of intention-to-treat analysis and use of attention control. 


\section{Results}

\section{Description of studies}

See: Characteristics of included studies; Characteristics of excluded studies.

Results of the search—Please see the 'Characteristics of included studies'.

One hundred and twenty-one trials with 6700 participants at entry were included in this review. Four studies were published only as abstracts and/or theses (Collier 1997; Fiatarone 1997; Moreland 2001; Newnham 1995).

Included studies-There was variation across the trials in the characteristics of the participants, the design of the PRT programmes, the interventions provided for the comparison group and the outcomes assessed. More detailed information is provided in the 'Characteristics of included studies'; however, a brief summary is provided here.

Language: All reviewed trials were published in English.

Location: Sixty-eight trials were conducted in the USA, 13 in Canada, 9 in Australia or New Zealand, and 31 in various European countries.

Study size: Most of these studies were small, with less than 40 participants in total, but 14 studies had 100 or more participants in total in a PRT group and a control group (Buchner 1997; Chandler 1998; Chin A Paw 2006; de Vos 2005; Ettinger 1997; Jette 1996; Jette 1999; Judge 1994; Latham 2003; Maurer 1999; McCartney 1995; Mikesky 2006; Moreland 2001; Segal 2003).

\section{Participants}

Health status: The participants in 59 trials were healthy older adults. In the remaining 62 trials, the participants had a health problem, functional limitation and/or were residing in a hospital or residential care. Thirty-two trials included older people with a specific medical condition, including diabetes (Brandon 2003), prostate cancer (Segal 2003), osteoarthritis (Baker 2001; Ettinger 1997; Foley 2003; Maurer 1999; Mikesky 2006; Schilke 1996; Topp 2002), osteoporosis/osteopenia (Liu-Ambrose 2005), peripheral arterial disease (Hiatt 1994; McGuigan 2001), recent stroke (Moreland 2001; Ouellette 2004), congestive heart failure (Brochu 2002; Pu 2001; Selig 2004; Tyni-Lenne 2001), chronic airflow limitation (Casaburi 2004; Kongsgaard 2004; Simpson 1992), clinical depression (Sims 2006; Singh 1997; Singh 2005), low bonemineral density (Parkhouse 2000), hip replacement due to osteoarthritis (Suetta 2004), hip/lower limb fracture (Mangione 2005; Miller 2006), obesity (Ballor 1996), chronic renal insufficiency (Castaneda 2001; Castaneda 2004) and coronary artery bypass graft surgery three or more months before exercise training (Maiorana 1997). Nineteen other trials recruited participants who did not have a specific health problem, but were considered frail and/or to have a functional limitation (Bean 2004; Boshuizen 2005; Chandler 1998; Fiatarone 1994; Fiatarone 1997; Fielding 2002; Hennessey 2001; Jette 1999; Krebs 2007; Latham 2003; Manini 2005; McMurdo 1995; Mihalko 1996; Miszko 2003; Newnham 1995; Skelton 1996; Sullivan 2005; Topp 2005; Westhoff 2000). In nine trials, the participants 
resided in a resthome or nursing home (Baum 2003; Bruunsgaard 2004; Chin A Paw 2006; Fiatarone 1994; Hruda 2003; McMurdo 1995; Mihalko 1996; Newnham 1995; Seynnes 2004). In addition, two trials included participants who were in hospital at the time the exercise programme was carried out (Donald 2000; Latham 2001). In the other trials, most or all of the participants lived in the community.

Gender: Most studies included both men and women, although 10 trials included men only (Fatouros 2002; Hagerman 2000; Haykowsky 2000; Hepple 1997; Izquierdo 2004;

Katznelson 2006; Kongsgaard 2004; Maiorana 1997; Segal 2003; Sousa 2005) and 22 trials included women only (Bean 2004; Brochu 2002; Charette 1991; Damush 1999; Fahlman 2002; Flynn 1999; Frontera 2003; Haykowsky 2005; Jones 1994; Kallinen 2002; LiuAmbrose 2005; Macaluso 2003; Madden 2006; Nelson 1994; Nichols 1993; Parkhouse 2000; Pu 2001; Rhodes 2000; Sipila 1996; Skelton 1995; Skelton 1996; Taaffe 1996).

Age: In 49 studies the mean or median age of the participants was between 60 and 69 years old; in 57 studies, the mean/median age was between 70 and 79 years old; and in 20 studies, it was 80 years old or over.

Lifestyle: Fifteen studies specifically recruited participants with a sedentary lifestyle (Ades 1996; Beneka 2005; Charette 1991; Fatouros 2002; Fatouros 2005; Frontera 2003; Kalapotharakos 2005; Katznelson 2006; Malliou 2003; Mihalko 1996; Parkhouse 2000; Pollock 1991; Rhodes 2000; Topp 1996; Tsutsumi 1997).

\section{PRT Programmes}

Settings: Most training programmes took place in gym or clinic settings with all sessions fully supervised. Ten studies were entirely home-based (Baker 2001; Chandler 1998; Fiatarone 1997; Jette 1996; Jette 1999; Katznelson 2006; Krebs 2007; Latham 2003; Mangione 2005; McMurdo 1995), while 12 additional studies carried out some of the training at home and some in gym/clinic settings (Boshuizen 2005; Ettinger 1997; Jones 1994; Mikesky 2006; Simoneau 2006; Skelton 1995; Skelton 1996; Topp 1993; Topp 1996; Topp 2002; Topp 2005; Westhoff 2000).

Intensity: The resistance training programmes in most trials (i.e. 83 trials) involved high intensity training. Most of these trials used specialized exercise machines for training. Thirty-six trials used low-intensity to moderate-intensity training, with most using elastic tubing or bands. All of the high-intensity training was carried out at least in part in gym or clinic based settings, with the exception of two published trials (Baker 2001; Latham 2003) and a trial published as an abstract (Fiatarone 1997).

Frequency and duration: The frequency of training was consistent across studies, with the exercise programme carried out two to three times a week in almost all trials. Two exceptions to this were the two trials conducted in hospital which carried out the exercises on a daily basis (Donald 2000; Latham 2001). In contrast, there was large variation in the duration of the exercise programmes and the number of exercises performed in each programme. Although most of the programmes (i.e. 71 trials) were eight to 12 weeks long, 
the duration ranged from two to 104 weeks. In 54 trials the exercise programme was longer than 12 weeks. The number of exercises performed also varied, from one to more than 14 .

Adherence: Data about adherence to the PRT programme are reported in the 'Characteristics of included studies'. These data are difficult to interpret because different definitions for adherence or compliance were used across the trials. In most trials, adherence referred to the percentage of exercise sessions attended compared with the total number of prescribed sessions and in this case the reported adherence rate is high (i.e. greater than 75\%). Many trials only included participants that completed the entire trial (i.e. excluded drop-outs), while some trials reported these data with drop-outs included.

Comparison interventions: Comparisons were conducted between a PRT group and a control group and between a PRT group and a group that received other type of intervention. In addition, comparisons between high intensity or frequency and low intensity or frequency, different sets, and different types of contraction training were also conducted. Multiple comparisons within a trial were possible when the trial included more than two groups that were relevant to the review. Twenty-eight trials had three groups. Among these trials, 14 included an aerobic training group in addition to a PRT group and a control group (Ettinger 1997; Fahlman 2002; Fatouros 2002; Haykowsky 2005; Hiatt 1994; Jubrias 2001; Kallinen 2002; Madden 2006; Malliou 2003; Mangione 2005; Pollock 1991; Sipila 1996; Topp 2005; Wood 2001), and seven included two PRT groups that exercised at different intensities in addition to a control group (de Vos 2005; Fatouros 2005; Hortobagyi 2001; Hunter 2001; Kalapotharakos 2005; Seynnes 2004; Singh 2005). One trial had a PRT group, a functional training group, and a PRT with functional training group (Manini 2005). The other six trials either had a balance training group (Judge 1994), functional training group (Chin A Paw 2006; de Vreede 2007), an endurance training group (Sipila 1996), a mobility training group (McMurdo 1995), or a power training group (Miszko 2003) in addition to a PRT group and a control group. One trial had three groups that exercised at three different frequencies in addition to a control group (Taaffe 1999).

PRT versus controls: One hundred and four trials compared PRT with a control group. The control group might receive no exercise, regular care, or attention control (i.e. the control group receives matching attention as the intervention group).

\section{Comparisons of PRT dosage}

High intensity versus low intensity: Ten studies compared PRT programmes at high intensity versus low intensity (Beneka 2005; Fatouros 2005; Harris 2004; Hortobagyi 2001; Seynnes 2004; Singh 2005; Sullivan 2005; Taaffe 1996; Tsutsumi 1997; Vincent 2002).

Different frequencies of PRT: Two trials (DiFrancisco 2007; Taaffe 1999) compared PRT performed at different frequencies (i.e. once, twice, or three times per week).

Different sets: One study compared PRT at different sets, i.e. 3-sets versus 1-set (Galvao 2005). One set of exercise means several continuous repeated movements. 
Concentric versus eccentric training: One study (Symons 2005) compared PRT at two types of contraction training: concentric versus eccentric training. During concentric training, speed was added at concentric contraction phase and vise versa for eccentric training.

PRT versus aerobic training: PRT was compared with aerobic (endurance) training in 17 trials (Ballor 1996; Buchner 1997; Earles 2001; Ettinger 1997; Fatouros 2002; Hepple 1997; Hiatt 1994; Izquierdo 2004; Jubrias 2001; Kallinen 2002; Madden 2006; Malliou 2003; Mangione 2005; Pollock 1991; Sipila 1996; Topp 2005; Wood 2001).

PRT versus balance training: One study compared PRT with balance training (Judge 1994). Balance training included training on a computerized balance platform and non-platform training (i.e. balancing on different surfaces, with varying bases of support, with different perturbations). Both exercise programmes were performed in a research center three times per week for three months.

PRT versus functional training: Three studies compared PRT to functional training (Chin A Paw 2006; de Vreede 2007; Manini 2005). Functional training involves game-like activities or exercise movements in various directions. In Chin A Paw 2006, functional training involved game-like or cooperative activities; and in de Vreede 2007, functional training involved moving with a vertical or horizontal component, carrying an object, and changing position between lying, sitting, and standing.

PRT versus flexibility training: One study compared PRT with flexibility training (Barrett 2002).

Power training: Power training refers to the type of PRT that emphasizes speed. Three studies applied this type of training (de Vos 2005; Macaluso 2003; Miszko 2003).

Outcomes: A variety of outcomes were assessed in these studies: the primary outcomes of physical function and secondary outcomes of measures of impairment and functional limitation.

Excluded studies-The excluded studies and their reasons for exclusion are listed in the 'Characteristics of excluded studies'. The main reasons for exclusion were that the study was not a randomised controlled trial or that the study design caused serious threats to its internal validity ( 57 trials); the studies used a combination of exercise interventions (i.e. not resistance training alone) (51 trials); the strength training programme did not use a progressive resistance approach (32 trials); and some participants were not elderly (i.e. did not have a mean age of at least 60 years and/or included some participants below 50 years of age) (25 trials).

Studies awaiting assessment: Nine trials were identified on a search update to May 2008, and a further trial was added after a referee's comment. 


\section{Risk of bias in included studies}

Methodological quality scores of each item for all included studies are given in Table 2. A summary of the findings of key indicators of internal validity are listed below.

Allocation concealment-Eleven studies provided some information about the method of randomisation that suggested that randomisation was probably concealed (i.e. the use of concealed envelopes or the randomisation was generated by an independent person) (Baker 2001; Chin A Paw 2006; Donald 2000; Foley 2003; Jette 1999; Latham 2001; Latham 2003; McMurdo 1995; Moreland 2001; Sims 2006; Sullivan 2005). Nineteen studies used randomisation list/table but allocation concealment was unclear (Barrett 2002; Baum 2003; Buchner 1997; de Vos 2005; de Vreede 2007; DiFrancisco 2007; Ettinger 1997; Krebs 2007; Liu-Ambrose 2005; Maurer 1999; Miller 2006; Schilke 1996; Segal 2003; Singh 1997; Singh 2005; Skelton 1995; Suetta 2004; Vincent 2002; Wieser 2007).

Loss to follow-up-Some trials had high drop-out rates, with several studies reporting more than 20\% of their participants were lost to follow-up (Bruunsgaard 2004; Chin A Paw 2006; DeBeliso 2005; Donald 2000; Katznelson 2006; Kongsgaard 2004; Mangione 2005; Mikesky 2006; Topp 1996). In some studies there was clear evidence of bias associated with the deliberate exclusion of patients such as those who failed to adhere to the exercise programme (Izquierdo 2004; Madden 2006; Topp 1996; Vincent 2002) or those who had adverse responses (Hagerman 2000).

Intention-to-treat analysis-Twenty-two studies stated that they used intention-to-treat analysis (Baker 2001; Barrett 2002; Baum 2003; Buchner 1997; Chin A Paw 2006; Ettinger 1997; Fiatarone 1994; Foley 2003; Judge 1994; Katznelson 2006; Latham 2003; LiuAmbrose 2005; Macaluso 2003; Mikesky 2006; Miller 2006; Moreland 2001; Nelson 1994; Ouellette 2004; Pu 2001; Segal 2003; Sims 2006; Sullivan 2005).

Blinded outcome assessment-Thirty-three studies stated that they used a blinded assessor for all outcome measures (Barrett 2002; Baum 2003; Bean 2004; Boshuizen 2005; Buchner 1997; Casaburi 2004; Castaneda 2004; Chin A Paw 2006; de Vreede 2007; Ettinger 1997; Foley 2003; Haykowsky 2005; Jette 1996; Jette 1999; Jones 1994; Judge 1994; Kalapotharakos 2005; Katznelson 2006; Krebs 2007; Latham 2003; Liu-Ambrose 2005; Mangione 2005; Maurer 1999; McMurdo 1995; Mikesky 2006; Miller 2006; Moreland 2001; Newnham 1995; Segal 2003; Sims 2006; Singh 2005; Sullivan 2005; Westhoff 2000).

Eight additional studies used a blinded outcome assessor for some, but not all outcome assessments (Baker 2001; Castaneda 2001; de Vos 2005; Fiatarone 1994; Ouellette 2004; Pu 2001; Singh 1997; Suetta 2004).

Blinding of participants-Blinding of participants is difficult in studies of exercise interventions. However, the use of attention control groups can help to minimise bias. Thirty-six studies used some type of attention programme for the control group (Baker 2001; Baum 2003; Bean 2004; Brochu 2002; Bruunsgaard 2004; Castaneda 2001; Castaneda 2004; Chin A Paw 2006; Damush 1999; Ettinger 1997; Fiatarone 1994; Fiatarone 1997; Foley 2003; Judge 1994; Kongsgaard 2004; Latham 2003; Liu-Ambrose 2005; Mangione 2005; 
Maurer 1999; McCartney 1995; McMurdo 1995; Mihalko 1996; Mikesky 2006; Miller 2006; Miszko 2003; Moreland 2001; Newnham 1995; Ouellette 2004; Pu 2001; Seynnes 2004; Simons 2006; Sims 2006; Singh 1997; Suetta 2004; Topp 1993; Topp 1996). In 10 of these studies, the control group received 'sham' exercise programmes (Bean 2004; Brochu 2002; Castaneda 2001; Castaneda 2004; Kongsgaard 2004; Liu-Ambrose 2005; Mikesky 2006; Ouellette 2004; Pu 2001; Seynnes 2004).

Duration of follow-up-Five studies continued to follow up the participants after intervention had ended (Buchner 1997; Fiatarone 1994; Moreland 2001; Newnham 1995; Sims 2006). Two of these followed up falls for more than one year (Buchner 1997; Fiatarone 1994).

\section{Effects of interventions}

Eleven studies did not report final means and standard deviations for some or all of their outcome measures but instead reported baseline mean scores and mean change in scores from baseline (Baum 2003; Bean 2004; Buchner 1997; Chandler 1998; Fiatarone 1994; Hiatt 1994; Jette 1996; Lamoureux 2003; Madden 2006; Sullivan 2005; Topp 1996). If additional data could not be obtained from the investigators, the final mean score was estimated by adding the change in score to the baseline score, and the standard deviation of the baseline score was used for the final score.

Four studies did not report standard deviations for some or all of their outcome measures but instead reported standardized errors (Ouellette 2004; Seynnes 2004; Suetta 2004; Topp 2002). The standard deviations were estimated based on reported standardized errors and sample sizes.

Eight studies did not report numerical results of outcomes of interest for the purpose of this review and additional data were not provided by the investigators (Castaneda 2004; Fielding 2002; Harris 2004; Haykowsky 2005; Krebs 2007; Miller 2006; Topp 2005; Wieser 2007).

\section{PRT versus control}

Measures of physical (dis)ability/HRQOL (complex physical activities): The main function (disability) measures from trials that had appropriate data were pooled using the standardised mean difference (SMD) and a fixed-effect model. Because studies measured function in scales with different directions, a higher score indicates either less disability/ better function or more disability/poor function, a transformation was conducted to make all the scales point in the same direction. Mean values from trials in which a higher score indicates more disability/poor function were multiplied by -1 . There is a significant effect of PRT in decreasing disability (see Figure 1; Analysis 1.1: 33 trials, 2172 participants; SMD $0.14,95 \%$ CI 0.05 to 0.22 ). When the physical function domain of SF-36 or SF-12 was pooled from 14 studies $(\mathrm{n}=778)$ using a fixed-effect model, no difference was found (Analysis 1.2: SMD 0.07, 95\% CI -0.08 to 0.21). No difference was found from the pooled results of three trials for activity of daily living measures (Analysis 1.3). A number of studies had function measures (i.e. measures of activity, function or HRQOL) that could not be pooled. The available data from these measures are reported in Table 3. 


\section{Measures of impairment}

Strength: Many different muscle groups were tested and a number of methods were used to evaluate muscle strength in these trials. To minimise clinical heterogeneity, data were pooled from one muscle group. The leg extensor group of muscles was selected since this group was the most frequently evaluated. The effect size was calculated using standardised mean difference (SMD) to allow the pooling of data that used different units of measurement. Seventy-three studies involving 3059 participants reported the effect of resistance training on a lower-limb extensor muscle group and provided data that allowed pooling. A moderate-to-large beneficial effect was found (Analysis 1.5: SMD 0.84, 95\% CI 0.67 to 1.00 , random-effects model; fixed-effect model: SMD $0.53,95 \%$ CI 0.46 to 0.61 ).

Supplementary analyses: Significant statistical heterogeneity was apparent in these data ( $\mathrm{P}$ $<0.0001)$. Since a large number of studies assessed this outcome, it was possible to explore this heterogeneity by stratifying the data. Differences in treatment effects due to the quality of the trials were investigated. We also explored subgroups of trials that were based on the design of the treatment programmes and the characteristics of the participants.

To explore the effect of data quality on treatment effects, data were stratified by four design features that are associated with internal validity. These are allocation concealment; blinded assessors; intention-to-treat analysis (ITT); and attention control groups. The fixed-effect model was used throughout in order to obtain the results for the test for subgroup differences. The effect was smaller in the few studies with clear allocation concealment (6 trials, 607 participants) compared with studies with unknown concealment of allocation (67 trials, 2452 participants): Analysis 10.1: test for subgroup differences: $\mathrm{Chi}^{2}=32.69, \mathrm{df}=1$ ( $\mathrm{P}<0.00001)$. The effect was also smaller in studies that used blinded assessors (19 trials, 1523 participants) compared with studies that did not use blinded assessors (54 trials, 1536 participants): Analysis 10.2: test for subgroup differences: $\mathrm{Chi}^{2}=70.56, \mathrm{df}=1(\mathrm{P}<$ 0.00001). This was also true for studies that used intention-to-treat analysis (ITT) (12 trials, 1041 participants) versus no ITT (61 trials, 2018 participants): Analysis 10.3: test for subgroup differences: $\mathrm{Chi}^{2}=49.74, \mathrm{df}=1(\mathrm{P}<0.00001)$. It is noticable that trials that applied better design features tend to be the larger trials. The effect was smaller when attention control groups were used (attention control: 24 studies, 1408 participants, no attention control: 49 studies, 1651 participants): Analysis 10.4: test for subgroup differences: $\mathrm{Chi}^{2}=25.04, \mathrm{df}=1(\mathrm{P}<0.00001)$.

Subgroup analyses were conducted to explore the effect of PRT when the design of the exercise programme and the characteristics of the participants differed. The effect of differences in the exercise programme was explored by examining effect estimates in studies that used different intensity and duration. High intensity strength training was compared with low to moderate intensity training. This analysis suggests that while both training approaches are probably effective in improving strength, higher intensity training (54 trials, 2026 participants) has a larger effect on strength than low to moderate intensity training (19 trials, 1033 participants): Analysis 10.5: test for subgroup differences: $\mathrm{Chi}^{2}=7.24, \mathrm{df}=1(\mathrm{P}$ $=0.007)$. Longer duration programmes (i.e. greater than 12 weeks) were also compared with shorter duration programmes (less than 12 weeks). The duration of the trial appeared to have 
minimal effect on the strength outcome ( $<12$ weeks: 20 trials, 828 participants; $>12$ weeks: 36 trials, 1736 participants): Analysis 10.6: test for subgroup differences: $\mathrm{Chi}^{2}=0.04, \mathrm{df}=1$ $(\mathrm{P}=0.85)$.

Treatment effects in older people with and without a chronic disease (or functional limitation) were also assessed. Again, resistance training appeared to be effective in improving strength in both groups of older people, but there was statistical heterogeneity in the effects. Studies that included participants who had specific health problems and/or functional limitations were compared with studies that included only healthy older people. The effect in older adults who were healthy has a larger effect size than older adults with specific health problems (healthy older adults: 46 trials, 1502 participants; older adults with specific health problems: 19 trials, 926 participants): Analysis 10.7: test for subgroup differences: $\mathrm{Chi}^{2}=19.85, \mathrm{df}=1(\mathrm{P}<0.00001)$. In addition, PRT in studies that included older adults who had a physical disability or functional limitation appeared to be less effective than in those that included older adults who did not have functional limitations (people with functional limitations: 13 studies, 784 participants; people with no functional limitations: 41 studies, 1349 participants): Analysis 10.8: test for subgroup differences: $\mathrm{Chi}^{2}$ $=29.33, \mathrm{df}=1(\mathrm{P}<0.00001)$. However, this result could be confounded by the intensity of the PRT programmes, as almost all programmes that included people with functional limitations were carried out at a low to moderate intensity. There were insufficient data available to compare the results by gender (men only: 5 trials with 107 participants; women only: 15 trials with 486 participants).

Aerobic capacity: The main measure of aerobic capacity was pooled from 29 studies ( $\mathrm{n}=$ 1138) using a random-effects model. These results suggest that PRT has a significant effect on aerobic capacity (Analysis 1.6: SMD $0.31,95 \%$ CI 0.09 to 0.53 ). Further analyses were performed for three specific measures of aerobic capacity: $\mathrm{VO} 2 \mathrm{max}(\mathrm{ml} / \mathrm{kg} / \mathrm{min})$, peak oxygen uptake (L/min) and the six-minute walk test (meters). A consistent significant effect was found for VO2 max (Analysis 1.7: 18 trials, $\mathrm{n}=710$, MD $1.5 \mathrm{ml} / \mathrm{kg} / \mathrm{min}, 95 \%$ CI 0.49 to 2.51). Similarly, a significant positive effect was found for the six-minute walk test (Analysis 1.8: 11 trials, $\mathrm{n}=325$, MD 52.37 meters, $95 \%$ CI 17.38 to 87.37 ).

\section{Measures of functional limitations (simple physical activities)}

Balance/postural control: Results from all balance performance measures were pooled using SMD and a fixed-effect model. Data pooled from 17 studies with 996 participants showed a small but non-significant benefit (higher score indicates better balance) for balance (Analysis 1.9: SMD 0.12 (95\% CI 0.00 to 0.25 ).

Gait speed: Two different measures of walking speed were used: gait speed (measured in meters per second) and timed walk (i.e. time to walk a set distance, measured in seconds). A higher gait speed score indicates faster mobility, while a higher timed walk score indicates slower mobility. Because of this difference, these data were analyzed separately. Data for gait speed were available from 24 studies that included 1179 participants (Analysis 1.11: MD $0.08 \mathrm{~m} / \mathrm{s}, 95 \%$ CI 0.04 to 0.12 , random-effects). This indicated that PRT has a modest but significant beneficial effect on gait speed. Only eight trials measured the timed walk 
(seconds) as an outcome measure and no evidence of an effect was found (Analysis 1.12; 204 participants, MD -0.23 seconds, $95 \%$ CI -1.07 to 0.62 , fixed-effect).

Timed up-and-go: Timed up-and-go (i.e. time to stand from a chair, walk three meters, turn, and return to sitting, measured in seconds) was analysed using a fixed-effect model. Data, available from 12 trials and a total of 691 participants, showed the PRT group took significantly less time to complete this mobility task (Analysis 1.13: MD -0.69 seconds, $95 \% \mathrm{CI}-1.11$ to -0.27$)$.

Timed chair rise: Time to stand up from a sitting position data were available in 11 studies $(\mathrm{n}=384)$. Because different numbers of sit-to-stand were counted, SMD and a randomeffects model was used to pool these results. These showed a significant, moderate to large effect on this task in favour of the PRT group (Analysis 1.14: SMD -0.94, 95\% CI -1.49 to $-0.38)$.

Stair climbing: Time to climb stairs data, which were available from eight trials, also favoured PRT (Analysis 1.15). However, these results were highly heterogenous.

Falls: Thirteen studies collected data about the effect of resistance training on falls or reported the incident of falls, but the outcomes reported did not allow pooling of the data. The available data is reported in Table 4. Three of these studies (Buchner 1997; Fiatarone 1994; Judge 1994) were part of the FICSIT trial, a prospective preplanned meta-analysis to determine the effectiveness of exercise to prevent falls in older people (Province 1995). The data were extracted from the main FICSIT paper, because papers published about the individual exercise programmes did not provide useful data about the effect of resistance training alone on falls. One additional trial investigated the effect of resistance training on falls in older people while they were in hospital (Donald 2000). Another trial also assessed the effect of PRT on frail older people following discharge from hospital (Latham 2003). There is a more comprehensive review of the effect of exercise on falls in a separate Cochrane review (Gillespie 2003).

With the exception of Latham 2003, all of these trials were small (i.e. less than 80 participants in the resistance training and control groups). Only Donald 2000 found a significant reduction in falls, but there were few fall events in this trial.

Adverse events: Adverse events are reported for all trials in the review at the end of the results section.

Vitality: The vitality (VT) domain of the SF-36 health status measure was assessed in 10 studies involving 611 participants. For this measure, a higher score indicates better health (i.e. more vitality): there was no evidence of an effect of PRT from the pooled data (Analysis 1.17: MD 1.33 95\% CI -0.89 to 3.55).

Pain: Data of bodily pain (BP) domain of the SF-36 health status measure were provided by 10 studies involving 587 participants. For this measure, a higher score indicates better health (i.e. less pain), there was no evidence that PRT had an effect on bodily pain (Analysis 1.18: 
MD 0.34, 95\% CI -3.44 to 4.12). In contrast, six studies with 503 participants included pain measures where a higher score indicates more pain, and found evidence to support a modest reduction in pain following PRT (Analysis 1.19: SMD -0.30, 95\% CI -0.48 to -0.13). These six studies all included participants with osteoarthritis and used pain measures designed specifically for this population, which could have increased their sensitivity to change.

Health service use, hospitalization and death: Five studies provided data about hospitalization rates, length of stay and/or outpatient visits. Donald 2000 reported that people who received PRT in addition to regular in-hospital physiotherapy had a length of stay of 27 days compared with 32 days for the control group. Latham 2003 found that 42/120 people in the PRT group were admitted to hospital over six months compared to 35/123 in the control group. The third trial by Singh 1997 reported that, over a 10 week period, people in the PRT group had mean 2.1 (SD 0.4) visits to a health professional and mean 0.24 (SD 0.2) hospital days compared to controls mean of 2.0 (SD 0.5) visits and mean 0.53 (0.4) hospital days. The fourth study by Singh 2005 reported visits to a health professional over the study (average numbers per person): high intensity group, 2 (2); low intensity group, 2 (1.8); controls, 5 (1.8). The fifth study by Miller 2006 reported participants' discharge destinations but did not specify the group: 52 participants were discharged to a rehabilitation programme, 12 were transferred to a community hospital, 16 were discharged to higher level care, and 20 returned directly to their pre-injury admission accommodation. An additional study, Buchner 1997, provided data about health service use, but only reported data that were pooled to include participants in aerobic training, combined aerobic training and PRT and PRT alone. This study found no change in hospital admissions between those in the exercise and control groups, but an increased number of outpatient visits by those in the control group. Finally, two studies stated that there was no difference in health care visits (Fiatarone 1997) or hospitalization (Pu 2001) but no specific data were provided.

Thirteen studies provided data about participant deaths that allowed pooling (Baum 2003; Boshuizen 2005; Chin A Paw 2006; Donald 2000; Ettinger 1997; Fiatarone 1994; Kallinen 2002; Latham 2003; Mangione 2005; Miller 2006; Moreland 2001; Newnham 1995; Selig 2004). The risk ratio of death in the PRT group was not significantly different from the control group (Analysis 1.20: 20 deaths versus 21 deaths; $R R=0.89,95 \%$ CI 0.52 to 1.54 ).

Comparisons of PRT dosage-Thirteen trials investigated the effects of different doses of PRT. Note that data from medium intensity were not examined in the following.

\section{High versus low intensity PRT}

Physical function, pain and vitality: Of the 10 studies comparing high versus low intensity PRT, only two (Singh 2005; Tsutsumi 1997), evaluated physical function, pain and vitality using the domains of the SF-36. No significant difference was found for physical function (Analysis 2.1) or pain (Analysis 2.4), but vitality scores were statistically significantly higher for high intensity (Analysis 2.5 : $\mathrm{MD}=6.54,95 \% \mathrm{CI} 0.69$ to 12.39 ). 
Strength: Data from all nine studies $(\mathrm{n}=219)$ were available to examine the effect of high versus low intensity PRT on lower limb strength (Beneka 2005; Fatouros 2005; Harris 2004; Hortobagyi 2001; Seynnes 2004; Sullivan 2005; Taaffe 1996; Tsutsumi 1997; Vincent 2002). The results indicate that high intensity training results in greater lower limb strength, as a moderate effect was seen (Analysis 2.2: $\mathrm{SMD}=0.48,95 \% \mathrm{CI} 0.03$ to 0.93 ; random-effects model).

Aerobic capacity: Three studies compared the effect of high versus low intensity PRT on aerobic capacity (Fatouros 2005; Tsutsumi 1997; Vincent 2002). These studies $(\mathrm{n}=101)$ did not show greater benefit from high intensity compared with low intensity training (Analysis 2.3: MD $1.82 \mathrm{ml} / \mathrm{kg} / \mathrm{min}, 95 \% \mathrm{CI}-0.79$ to 4.43 ; higher score favours high-intensity group).

High intensity versus variable intensity PRT: One trial (Hunter 2001) comparing high intensity PRT with variable intensity PRT showed no statistically significant differences for strength (Analysis 3.1: $\mathrm{n}=24, \mathrm{MD}=0.61,95 \% \mathrm{CI}-0.21$ to 1.44 ) and aerobic capacity (Analysis 3.2).

Frequency: Taaffe 1999 and DiFrancisco 2007 compared PRT at different frequencies, respectively three times a week versus once a week, and twice a week versus once a week. Both studies recruited few participants and applied high intensity intervention. There were no significant differences between the two exercise frequencies in muscle strength (Analysis 4.1: $\mathrm{MD}=0.40,95 \% \mathrm{CI}-0.44$ to $1.25 ; \mathrm{MD}=-0.46,95 \% \mathrm{CI}-1.40$ to 0.48 ).

Sets: Galvao 2005 compared PRT at 3-sets versus 1-set in 28 participants. No significant differences between the two groups were found for muscle strength (Analysis 5.1), six minute walk test (Analysis 5.2), sit-to-stand (Analysis 5.4) and stair climbing (Analysis 5.5). However, participants who exercised at 3-sets walked significantly faster than those who exercised at 1-set (Analysis 5.3: $\mathrm{MD}=-29.6$ seconds, $95 \%-54.23$ to -4.97 ).

\section{PRT versus aerobic training}

Physical function: Five studies evaluated the effect of PRT compared with aerobic training on physical function. Four studies (Buchner 1997; Earles 2001; Hiatt 1994; Mangione 2005) used outcomes in which a higher score indicates less disability $(n=125)$, and found no significant difference (see Analysis 6.1: SMD -0.21, 95\% CI -0.56 to 0.15; lower score favours the aerobic training group). The other study (Ettinger 1997) $(n=237)$ also found no significant difference between the groups for function (see Analysis 6.2: SMD 0.05, 95\% CI -0.21 to 0.30 ; higher score favours aerobic group).

Strength: Data on lower extremity strength were available from 10 studies $(\mathrm{n}=487)$ (Ballor 1996; Buchner 1997; Earles 2001; Ettinger 1997; Fatouros 2002; Izquierdo 2004; Malliou 2003; Pollock 1991; Sipila 1996; Wood 2001). These data when pooled using a randomeffects model showed that PRT had a significant benefit compared with aerobic training on strength (see Analysis 6.3: SMD 0.44, 95\% CI 0.08 to 0.80; higher score favours PRT).

Aerobic capacity: Aerobic capacity was evaluated in eight studies involving 423 participants (Ballor 1996; Buchner 1997; Ettinger 1997; Hepple 1997; Hiatt 1994; Kallinen 
2002; Madden 2006; Pollock 1991). This was measured using VO2 max in $\mathrm{ml} / \mathrm{kg} / \mathrm{min}$. Using the random-effects model, aerobic training had a non-significant benefit compared to PRT for this outcome (Analysis 6.4: MD $-1.13 \mathrm{ml} / \mathrm{kg} / \mathrm{min}, 95 \% \mathrm{CI}-2.63$ to 0.38 ; higher values favours PRT).

Gait speed: Mangione 2005 reported on gait speed $(\mathrm{m} / \mathrm{s})$ and found no significant difference between groups (Analysis 6.6 : MD $-0.08 \mathrm{~m} / \mathrm{s}, 95 \%$ CI -0.30 to 0.14 ; higher speed favours PRT group)

Pain: Ettinger 1997 found no significant difference between groups in pain (Analysis 6.7: MD 0.12; $95 \%$ CI -0.14 to 0.37 ; lower score favours PRT).

PRT versus balance: One study (Judge 1994) compared PRT with balance retraining ( $\mathrm{n}=$ 55). This study found that strength improved in the PRT group, but not in the balance training group. Chair rise time and gait speed did not improve in any group, with gait speed actually declining in the balance training group. However, balance improved in the balance training group compared with the PRT group.

PRT versus functional training-Three studies compared PRT with functional training (Chin A Paw 2006; de Vreede 2007; Manini 2005). No significant differences between the two interventions were found for the reported outcomes (see: Analysis 7.1 physical function; Analysis 7.2 strength; Analysis 7.3 timed up and go; Analysis 7.4 vitality; Analysis 7.5 pain).

PRT versus flexibility training-Barrett $2002(n=40)$ compared a group of older adults who undertook PRT with a control group who did mainly stretching for the major muscle groups (flexibility training). No statistically significant differences were found for any of the reported outcomes (see: Analysis 8.1: SF-36 physical function; Analysis 8.2: strength; Analysis 8.3: timed walk; Analysis 8.4: chair stand; Analysis 8.5: vitality; Analysis 8.6: pain).

Power training-Two studies (de Vos 2005; Miszko 2003) ( $\mathrm{n}=76$ ) compared power training with a control group. de Vos 2005 and another study (Macaluso 2003) also compared different intensities of power training. While the data for muscle strength for de Vos 2005 favoured high intensity power training, data pooling was inappropriate given the substantial and significant heterogeneity (see Analysis 9.1).

Adverse events-Among 121 studies that were reviewed, 53 studies provided no comment at all about adverse events associated with the training programme. Of the remaining 68 studies, 25 reported no adverse events and 43 reported some adverse reaction to the exercise programme. An additional eight studies did not report adverse events as such, but it is possible that an event occurred since these studies reported drop-outs from the exercise group secondary to increasing pain or specific injuries (Chandler 1998; Charette 1991; Fiatarone 1997; Hagerman 2000; Hortobagyi 2001; Jette 1996; Maurer 1999; Topp 1993). Given that there were considerably more drop-outs from the PRT group than from the 
control group (see methodological quality section above), it is possible that the number of cases of adverse events reported here are an underestimate.

Only nine studies provided an a priori definition of an adverse event in the study methods or objectives (Earles 2001; Ettinger 1997; Judge 1994; Kallinen 2002; Latham 2003; LiuAmbrose 2005; Moreland 2001; Pollock 1991; Singh 1997). Eight of these nine studies detected adverse events (Earles 2001; Ettinger 1997; Judge 1994; Kallinen 2002; Latham 2003; Liu-Ambrose 2005; Moreland 2001; Pollock 1991). However, there was little consistency in the definition that was used, with some studies only reporting serious events that the investigators thought were possibly related to the exercise programme (i.e. Ettinger 1997) while other studies reported all adverse events that occurred in each group. Most adverse events were musculoskeletal problems. Serious adverse events were rare, and none appeared to be directly related to the exercise programme. One study reported one death of myocardial information in the PRT group (Kallinen 2002). Another two studies reported one death in the PRT group but the reason of death was not reported (Baum 2003; Chin A Paw 2006). Further details about all adverse events reported in these trials can be found in Table 5.

\section{Discussion}

\section{Summary of main results}

This review identified, graded and synthesized the available literature regarding the effect of a specific exercise intervention, PRT, on a particular population, older people. To increase the generalisability of these data, the trials included participants with a range of health problems, and the dose and delivery of the PRT programmes varied. This made it possible to assess overall effects of the intervention on older people, with a potential for exploring the effects on subgroups (i.e. in different groups of older people or with different doses of PRT). Overall, this review suggests that PRT has a small but significant effect on improving physical function (complex activities), a small to moderate effect on decreasing some impairments and functional limitations, and a large effect on increasing strength. Adverse events were poorly reported in most studies, which limits the ability of this review to assess the risks associated with this intervention. Additionally, there is some preliminary evidence that suggests that PRT might reduce pain in older people with osteoarthritis. The effect of exercise on reducing pain in people with osteoarthritis is reported in another Cochrane review (Brosseau 2003). The sparse data did not allow an adequate assessment of the effect of PRT on fall risk. However, a separate Cochrane review (Gillespie 2003) has reviewed fall prevention.

\section{Overall completeness and applicability of evidence}

This review update highlights the fact that exercise training in older adults continues to be a dynamic area of research, with the number of included studies doubling in the five years since the previous review. A quick update extending the MEDLINE search to May 2008 identified nine further studies. However, the majority of the trials continue to be studies with small sample sizes. 
This review deliberately used broad inclusion criteria and multiple strategies to try to identify as many studies as possible that used PRT training with older adults. Despite these efforts, given the broad coverage of our review it is inevitable that we have missed some trials. It is particularly challenging to identify unpublished trials in this area because the studies could have been presented at many different types of conferences (stoke, OA, CHD etc). We acknowledge that it was not possible to hand search all of the potential conferences where studies in this area could be presented, and it is therefore possible that we missed some studies that had negative or neutral results and are more difficult to get published. Although we attempted to contact authors when there was any uncertainty about data, it is also likely that data could also have been missed both from the excluded trials (i.e. the outcomes may have been recorded but not reported) and the included trials (i.e. data not reported and/or data not available for pooling).

\section{Quality of the evidence}

The 121 studies in this review were generally of poor methodological quality, as most of the studies did not use design features that are known to increase internal validity, such as concealed randomisation; intention-to-treat analysis, blinded outcome assessors, or attention control groups. Only 11 studies used concealed randomisation; 22 studies used intention-totreat analysis; and 33 studies used blinded outcome assessors for all outcomes. Therefore, caution is required when drawing conclusions from these data. When data were stratified by indicators of study quality for the outcome muscle strength, results from the high quality trials continued to support the positive effect of resistance training on strength. However, these data also indicate that low quality trials, usually small studies, that comprise the majority of the studies in the review probably overestimate the effect of resistance training because of random chance effects from small studies. The long-term outcome of PRT is unclear because the majority of studies stopped following up participants once the intervention had ended.

PRT versus control-PRT shows small positive effect on measures of physical function (disability). PRT also appears to have a positive effect on aerobic capacity and most measures of functional limitations, including gait speed, timed "Up-and-Go" and, the time to stand up from a chair. All of these effects were statistically significant, although the effect sizes tended to be small to moderate, and the clinical significance of these effects is unclear. PRT appears to have a large positive effect on strength and aerobic capacity in older people. However, there was a large amount of statistical heterogeneity associated with the estimate in strength. This variation was reduced, but not eliminated, by investigating differences in outcome in different groups of participants, types of intervention and in trials that used different quality indicators. Please note that results from such exploratory analysis are tentative. In exploratory subgroup analyses, it appeared that training intensity has the greatest effect on strength (i.e. high intensity training has a greater effect on strength than lower intensity training), while the duration of the training appears to have a reduced effect. The magnitude of the effect was influenced by participants' health status or functional status. PRT in healthy participants had a greater effect than in those with a chronic disease or functional limitation. In other words, it appeared that people with a pre-existing health condition or with functional limitations had smaller gains in strength. Additionally, men had 
larger gains in strength than women; although there were fewer trials in men. These subgroup analyses must be interpreted with caution as the number of participants is reduced which decreases the precision of these estimates. In addition, it is possible that study size is a source of heterogeneity, as several of the largest and highest quality trials included people with function limitations and/or lower intensity training programmes, and study quality appears to reduce the effect estimates. Overall, the effect of PRT on function is positive for older adults; although the effect seems diminished when it transfers from muscle strength to functional limitations and disability.

It was not possible to pool fall data because falls were reported differently in the five studies that measured this outcome. These data might suggest a trend towards PRT reducing falls, since four of the five studies found that participants in the PRT group had fewer falls than those in the control group. However, the effect of PRT alone on falls is still not clear.

Adverse events were poorly monitored and reported in most of these trials. This makes it difficult to assess the risk of injury or other adverse events associated with resistance training. The finding that several studies reported drop-outs from the exercise programme due to pain or injury, yet failed to report any adverse events, suggests that adverse events might have been under-reported in some trials. This hypothesis is further supported by the finding that the studies with a clear definition of adverse events in their study methods were more likely to detect these events than those with no definition. The large number of dropouts from the PRT group compared to controls also raises the possibility that people are experiencing adverse effects from PRT that are not identified in these trials. However, it is reassuring that participant's pain and vitality were not affected by PRT, and in fact PRT appeared to decrease pain in people with osteoarthritis. Furthermore, there was no evidence of increased risk of hospitalization. A few studies reported decreased use of health care services in the PRT group. Finally, there were a few reports of serious adverse events (i.e. myocardial infarction or death) in the PRT group but there was no evidence that these events were directly associated with the intervention. There was also no evidence of increased risk of death in the PRT group when compared with the control group.

Comparison of PRT dosage-There are currently few randomized data available to guide the dose and prescription of PRT. Trials investigated different aspects of this issue were all small studies and most were of poor quality. When high intensity training was compared with low intensity training, data from 10 trials show that high intensity training has a greater effect on strength than lower intensity training. Among these 10 trials, three show that high intensity training has a greater effect on aerobic capacity. Eight of the 10 trials were healthy older people who participated in highly supervised, gym-based programmes. Therefore, it is not clear if high intensity PRT is more beneficial than low intensity training in less fit or healthy older people and/or in home or hospital based programmes. Limited evidence are available for exercise frequencies and sets.

PRT versus other training-Overall, no significant differences were found between the different types of training. When PRT is compared to aerobic training, PRT tended to produced larger gains in strength than aerobic training. However, these two types of training are not different in aerobic capacity. This finding is to be expected, given that the strength 
outcome is more specific to PRT. There are fewer data available to determine the comparative effect of these types of training on physical disability, but the available data suggest that the two training programmes have a similar effect on this outcome. There are too few data to draw conclusions about other forms of training such as balance or mobility training compared to PRT.

\section{Authors' Conclusions}

\section{Implications for practice}

Doing PRT two to three times a week can improve physical function in older adults, including reducing physical disability, some functional limitations (i.e. balance, gait speed, timed walk, timed 'up-and-go', chair rise; and climbing stairs) and muscle weakness in older people. Therefore, it would appear to be an appropriate intervention for many older people to improve performance of some simple physical tasks. The training also shows a reduction in pain in people with osteoarthritis. However, some caution is warranted with this intervention as in many studies adverse effects have been poorly monitored. Nonetheless, serious adverse events appear to be rare. When used in clinical practice, clinicians should monitor for adverse effects, particularly when older people who might be at higher risk of injury (i.e. frail or recently ill older people) are undertaking PRT. Additionally, there is no information regarding how long these effects can be maintained because the majority of the studies did not follow up the effect after the training had ended.

\section{Implications for research}

We recommend that future trials investigating the effect of PRT in older people should:

- minimise bias by using concealed randomisation, blinded outcome assessors, intention-to-treat analysis and attention control groups;

- recruit an adequate number of participants so that a precise estimate of the effect of the intervention can be determined (should have a priori power calculations);

- $\quad$ include a careful assessment of adverse events in both treatment groups, so that both the benefits and risks of PRT are fully evaluated;

- follow up participants after the programmes have completed to examine the longterm effects of PRT.

Future trials should include participants and interventions that are similar to those in health care settings (i.e. frail or recently ill older people), so that, if proven to be effective, resistance training can be incorporated into routine health care services. Well-designed trials are also required to determine the most appropriate dose of PRT to use with different participants and in different settings.

\section{Acknowledgments}

The authors would like to thank the editorial team of the Cochrane Bone, Joint and Muscle Trauma Group, particularly Lindsey Elstub, Lesley Gillespie, Joanne Elliott and Leeann Morton, for their assistance throughout the review process. In particular, thanks to Lesley and Joanne for searching the Cochrane registers and assistance with developing the search strategies. Thank you to Leeann and Lindsey for their advice and guidance about the 
procedures and content. We would also like to thank the Review Group's editors and the external referees, Prof John Campbell, Dr Keith Hill and Professor David Stott for their helpful comments on earlier drafts of this review.

The reviewers would like to thank Craig Anderson, Derrick Bennett, and Caroline Stretton for their contribution for the first review. The reviewers also would like to thank the National Institute of Disability and Rehabilitation Research (NIDDR) for a post-doctoral fellowship to the first author (H133P001) through Boston University and Switzer research fellowship (H133F060030) and National Institute of Aging (P30 AG031679) to the second author for supporting the review update. In addition, the second author received support for this review through a Pepper Center Trainee award from Boston Pepper Center funded by the National Institute of Aging (1P30Ag031679-01).

\section{Sources of Support}

Internal sources

- Health and Disability Research Institute, School of Public Health, Boston University, USA.

- Department of Occupational Therapy, School of Health and Rehabilitation Sciences, Inidana University at Indianapolis, USA.

External sources

- $\quad$ NIDRR Post-doctoral Fellowship, grant \# H133P001, USA.

- $\quad$ NIDRR Switzer Research Fellowship, grant \#H133F060030, USA.

- $\quad$ National Institute of Aging, grant \# P30 AG031679, USA.

\section{Characteristics of Studies}

\section{Characteristics of included studies [ordered by study ID]}

\section{Ades 1996}

\begin{tabular}{|c|c|}
\hline Methods & $\begin{array}{l}\text { RCT (randomised controlled trial) } \\
\text { Method of randomisation: unclear } \\
\text { Assessor blinding: no } \\
\text { Participant blinding: no } \\
\text { Loss to follow-up: not reported } \\
\text { Intention-to-treat analysis: no } \\
\text { Post-program follow up: no }\end{array}$ \\
\hline Participants & $\begin{array}{l}\text { Location: USA } \\
\mathrm{N}=24 \\
\text { Sample: healthy, sedentary } \\
\text { Age: mean } 70.4 \text { years (SD 4) } \\
\text { Inclusion criteria: healthy, sedentary older people } \\
\text { Exclusion criteria: angina or electrocardiographic ischaemia during exercise test, resting BP } \\
\text { >160/90, non-cardiopulmonary limitation of exercise capacity (i.e. claudication, arthritis, } \\
\text { cerebrovascular disease) }\end{array}$ \\
\hline Interventions & $\begin{array}{l}\text { PRT (progressive resistance strength training) versus control } \\
\text { 1. PRT } \\
\text { Type of exercises: } 4 \text { UL (upper limb), 3LL (lower limb) } \\
\text { Equipment: machines (Universal Gym) } \\
\text { Intensity: high (50-80\% of 1RM) } \\
\text { Frequency: Ex3 } \\
\text { Reps/ sets: } 8 / 3 \\
\text { Duration: } 12 \text { weeks } \\
\text { Setting: gym } \\
\text { Supervision: not reported } \\
\text { Adherence: not reported } \\
\text { 2. Control Group: instructed not to alter their home activity habits }\end{array}$ \\
\hline Outcomes & $\begin{array}{l}\text { Strength ( } 1 \text { repetition maximum) } \\
\text { Peak aerobic capacityComments on adverse events: no }\end{array}$ \\
\hline \multicolumn{2}{|l|}{ Notes } \\
\hline \multicolumn{2}{|l|}{ Risk of bias } \\
\hline Item & Authors' judgement \\
\hline
\end{tabular}




\begin{tabular}{l|l|l} 
Allocation concealment? & Unclear & B - Unclear \\
\hline
\end{tabular}

\section{Baker 2001}

\begin{tabular}{|c|c|c|}
\hline Methods & \multicolumn{2}{|c|}{$\begin{array}{l}\text { RCT } \\
\text { Method of randomisation: generated by statistician, concealed from investigators } \\
\text { Assessor blinding: blinded for primary measures, not for secondary (including strength) } \\
\text { Participant blinding: yes } \\
\text { Loss to follow-up: } 2 / 46 \\
\text { Intention-to-treat analysis: yes for primary, no for secondary measures } \\
\text { Post-program follow up: no }\end{array}$} \\
\hline Participants & \multicolumn{2}{|c|}{$\begin{array}{l}\text { Location: USA } \\
\mathrm{N}=46 \\
\text { Sample: older people with osteoarthritis. Recruited through community advertising } \\
\text { Age: mean } 68 \text { years (SD 6) in the treatment group } \\
\text { Inclusion criteria: age } 55 \text { or older, body mass index less than } 40 \mathrm{~kg} / \mathrm{m} 2 \text {, pain on more than } \\
\text { half the days of the past month and during activities and radiographic evidence of OA } \\
\text { Exclusion criteria: medical condition that precluded safe participation in an exercise } \\
\text { program or was more limiting than OA, inflammatory OA, or had participated in any } \\
\text { regular exercise program in the last } 6 \text { months }\end{array}$} \\
\hline Interventions & \multicolumn{2}{|c|}{$\begin{array}{l}\text { PRT versus control } \\
\text { 1. PRT } \\
\text { Type of Ex: } 2 \text { functional exercises (squats and step-ups), } 5 \text { LL isotonic exercises } \\
\text { Equipment: velcro ankle weights (isotonic ex only) } \\
\text { Intensity: initially low ( } 3-5 \text { on Borg scale), progressed to } 8 \text { ("hard" on Borg scale) } \\
\text { Frequency: Ex3 } \\
\text { Reps/ sets: } 12 / 2 \\
\text { Program duration: } 16 \text { weeks } \\
\text { Setting: home-based } \\
\text { Supervision: low ( } 12 \text { visits over } 16 \text { weeks) } \\
\text { Adherence: } 84 \% \text { (SD } 27 \text { ) of sessions } \\
\text { 2. Control: given nutrition info, } 7 \text { home visits over } 16 \text { weeks, kept food logs } 3 / 14 \text { days }\end{array}$} \\
\hline Outcomes & \multicolumn{2}{|c|}{$\begin{array}{l}\text { Primary: WOMAC pain and physical function subscales, SF- } 36 \\
\text { Secondary: Strength (1RM), clinical knee exam, nutrition, physical performance (stair } \\
\text { climb, chair stand time) } \\
\text { Comments on adverse events: yes }\end{array}$} \\
\hline \multicolumn{3}{|l|}{ Notes } \\
\hline \multicolumn{3}{|l|}{ Risk of bias } \\
\hline Item & Authors' judgement & Description \\
\hline Allocation concealment? & Yes & A - Adequate \\
\hline
\end{tabular}

\section{Balagopal 2001}

\begin{tabular}{|c|c|}
\hline Methods & $\begin{array}{l}\text { RCT } \\
\text { Method of randomisation: not reported } \\
\text { Assessor blinding: no } \\
\text { Participant blinding: no } \\
\text { Loss to follow-up: not reported } \\
\text { Intention-to-treat analysis: no } \\
\text { Post-program follow up: no }\end{array}$ \\
\hline Participants & $\begin{array}{l}\text { Location: USA } \\
\mathrm{N}=20 \\
\text { Sample: healthy older people } \\
\text { Age: mean } 71 \text { years (SD 1) } \\
\text { Inclusion criteria: older people aged } 65-79 \text {, healthy (based on physical exam and blood } \\
\text { tests) } \\
\text { Exclusion criteria: subjects who exercised regularly for }>\text { or }=2 \text { days per week, women } \\
\text { taking hormone replacement }\end{array}$ \\
\hline Interventions & PRT versus control \\
\hline
\end{tabular}




\begin{tabular}{l|l|l}
\multicolumn{1}{l|}{} & $\begin{array}{l}\text { 1. PRT } \\
\text { Type of Ex: 4UL, 3LL } \\
\text { Equipment: resistance training machines } \\
\text { Intensity: 50-80\% 1RM } \\
\text { Frequency: Ex3 } \\
\text { Reps/ sets: } 8 / 3 \\
\text { Duration: 3 months } \\
\text { Setting: gym } \\
\text { Supervision: full } \\
\text { Adherence: not reported } \\
\text { 2. Control Group: not reported }\end{array}$ \\
\hline Outcomes & $\begin{array}{l}\text { Muscle strength (1RM) } \\
\text { Comments on adverse events: no }\end{array}$ \\
\hline Notes & \multicolumn{2}{|l}{} \\
\hline Risk of bias & Authors' judgement & Description \\
\hline Item & Unclear & B - Unclear \\
\hline Allocation concealment? &
\end{tabular}

Ballor 1996

\begin{tabular}{|c|c|c|}
\hline Methods & \multicolumn{2}{|c|}{$\begin{array}{l}\text { RCT } \\
\text { Method of randomisation: not reported } \\
\text { Assessor blinding: no } \\
\text { Participant blinding: no } \\
\text { Loss to follow-up: not reported } \\
\text { Intention-to-treat analysis: no } \\
\text { Post-program follow up: no }\end{array}$} \\
\hline Participants & \multicolumn{2}{|c|}{$\begin{array}{l}\text { Location: USA } \\
\mathrm{N}=18 \\
\text { Sample: obese, recently completed dietary program } \\
\text { Age: mean } 61 \text { years (SE 1) } \\
\text { Inclusion criteria: aged } 55-70 \text { years, a BMI before weight loss of }>32 \mathrm{~kg} / \mathrm{m} \text { squared, no } \\
\text { signs, symptoms or history of heart disease, non-diabetic, non-smoker, resting blood } \\
\text { pressure < } 160 / 90 \mathrm{~mm} \mathrm{Hg} \text {, no symptoms that would preclude safe participation in an } \\
\text { exercise program } \\
\text { Exclusion criteria: not reported }\end{array}$} \\
\hline Interventions & \multicolumn{2}{|c|}{$\begin{array}{l}\text { PRT versus aerobic } \\
\text { 1. PRT } \\
\text { Type of Ex: 4UL, 3LL } \\
\text { Equipment: machines (Universal Gym) } \\
\text { Intensity: } 50-80 \% \text { of } 1 \text { RM } \\
\text { Frequency: Ex3 } \\
\text { Reps/ sets: } 8 / 3 \\
\text { Program duration: } 12 \text { weeks } \\
\text { Setting: gym } \\
\text { Supervision: full } \\
\text { Adherence: not reported } \\
\text { 2. Aerobic Training Group: exercised } 3 \text { times per week on a motorised treadmill at } \\
\text { approximately } 50 \% \text { of maximum aerobic uptake for } 20-60 \text { minutes per session }\end{array}$} \\
\hline Outcomes & \multicolumn{2}{|c|}{$\begin{array}{l}\text { Strength (1RM) } \\
\text { Aerobic capacity } \\
\text { Comments on adverse events: no }\end{array}$} \\
\hline Notes & \multicolumn{2}{|c|}{ Data from PRT and aerobic training group were compared } \\
\hline \multicolumn{3}{|l|}{ Risk of bias } \\
\hline Item & Authors' judgement & Description \\
\hline Allocation concealment? & Unclear & B - Unclear \\
\hline
\end{tabular}




\section{Barrett 2002}

\begin{tabular}{l|l}
\hline Methods & RCT \\
Method of randomisation: a computer generalized list \\
Assessor blinding: yes \\
Participant blinding: yes \\
Loss to follow-up: 4/44 \\
Intention-to-treat analysis: yes for primary, no for secondary measures \\
Post-program follow up: no
\end{tabular}

\section{Baum 2003}

\begin{tabular}{l|l}
\hline Methods & RCT \\
Method of randomisation: a computer generated algorithm stratified by the location of the \\
facility \\
Assessor blinding: yes \\
Participant blinding: not reported \\
Loss to follow-up: $1 / 11$ in PRT group \\
Intention-to-treat analysis: yes \\
Post-program follow up: no
\end{tabular}




\begin{tabular}{l|l|l} 
& $\begin{array}{l}\text { Reps/ sets: increased from 5/1 to 10/2 } \\
\text { Duration: } 1 \text { year (after 6 months the two groups switched program. the results extracted at } \\
\text { the end of the first } 6 \text { months) }\end{array}$ \\
& $\begin{array}{l}\text { Setting: not reported, (Gym in the facility?) } \\
\text { Supervision: full by an exercise physiologist } \\
\text { Adherence: (80\%-Ex group; 56\%-control) } \\
\text { 2. Control group: did activities such as painting, drawing, or puzzles with an art therapist or } \\
\text { social worker,3 times a week }\end{array}$ \\
\hline Outcomes & $\begin{array}{l}\text { Primary: FIM, physical performance test } \\
\text { Secondary: TUAG, Berg balance scale } \\
\text { Comments on adverse events: yes }\end{array}$ \\
\hline Notes & $\begin{array}{l}\text { Means and SDs at } 12 \text { months were not reported. Portion results at 6 months could be } \\
\text { estimated from baseline score and change score. Because of small sample size, the } \\
\text { precision is questionable }\end{array}$ \\
\hline Risk of bias & Authors' judgement & Description \\
\hline Item & Unclear & B - Unclear \\
\hline Allocation concealment?
\end{tabular}

\section{Bean 2004}

\begin{tabular}{|c|c|c|}
\hline Methods & \multicolumn{2}{|c|}{$\begin{array}{l}\text { RCT } \\
\text { Method of randomisation: not reported } \\
\text { Assessor blinding: yes } \\
\text { Participant blinding: not reported } \\
\text { Loss to follow-up: } 1 / 10 \text { in the control group } \\
\text { Intention-to-treat analysis: no } \\
\text { Post-program follow up: no }\end{array}$} \\
\hline Participants & \multicolumn{2}{|c|}{$\begin{array}{l}\text { Location: USA } \\
\mathrm{N}=21 \text { ( } 11 \text { in PRT) } \\
\text { Sample: community dwelling older females (with physical performance limitations??) } \\
\text { Age: mean } 77.1 \text { years ( } \mathrm{SD}=5.7 \text { ) } \\
\text { Inclusion criteria: female sex, age of } 70 \text { and older, and a score between four and } \\
\text { 10ontheShort Physical Performance Battery } \\
\text { Exclusion criteria: unstable acute or chronic medical conditions, a score less than } 23 \text { on the } \\
\text { MMSE, or a neuromusculoskeletal condition interfering with exercise participation }\end{array}$} \\
\hline Interventions & \multicolumn{2}{|c|}{$\begin{array}{l}\text { PRT versus control } \\
1 \text { 1. PRT } \\
\text { Type of Ex: } 2 \text { UL/4LL with fast concentric phase } \\
\text { Equipment: weighted vest } \\
\text { Intensity: increased to the next level (increase } 2 \% \text { of the subject's baseline body mass) after } \\
10 \text { reps/ } 3 \text { sets } \\
\text { Frequency: Ex } 3 \\
\text { Reps/Sets: } 8 / 3 \\
\text { Duration: } 12 \text { weeks } \\
\text { Setting: research center (Gym?) } \\
\text { Supervision: full } \\
\text { Adherence: } 88 \text { to } 90 \% \\
\text { 2. Control group: slow velocity and low resistance exercise with body or limb weight, } 3 \\
\text { times a week }\end{array}$} \\
\hline Outcomes & \multicolumn{2}{|c|}{$\begin{array}{l}\text { Primary: Short Physical Performance Battery (including chair rise) } \\
\text { Secondary: Muscle strength } \\
\text { Comments on adverse events: yes }\end{array}$} \\
\hline Notes & \multicolumn{2}{|c|}{ Post mean $=$ baseline + change score; baseline SD was used } \\
\hline \multicolumn{3}{|l|}{ Risk of bias } \\
\hline Item & Authors' judgement & Description \\
\hline Allocation concealment? & Unclear & B - Unclear \\
\hline
\end{tabular}




\section{Beneka 2005}

\begin{tabular}{|c|c|c|}
\hline Methods & \multicolumn{2}{|c|}{$\begin{array}{l}\text { RCT with } 4 \text { groups: low intensity, medium intensity and high intensity and control group } \\
\text { Method of randomisation: not reported, stratified by gender } \\
\text { Assessor blinding: not reported } \\
\text { Participant blinding: not reported } \\
\text { Loss to follow-up: no } \\
\text { Intention-to-treat analysis: no } \\
\text { Post-program follow up: no }\end{array}$} \\
\hline Participants & \multicolumn{2}{|c|}{$\begin{array}{l}\text { Location: Greece } \\
\mathrm{N}=16 \text { for each group (Control, LI, MI, \& HI) } \\
\text { Sample: healthy but inactive elderly } \\
\text { Age: male-mean } 70 \text { years; female-mean } 67 \text { years } \\
\text { Inclusion criteria: inactive prior to the study, no anaemia, hepatic complications, thyroid } \\
\text { disorders, and kidney problems } \\
\text { Exclusion criteria: hypertension or taking anti-hypertensive medication, didn't pass } \\
\text { diagnostic treadmill test, didn't pass physician's screen }\end{array}$} \\
\hline Interventions & \multicolumn{2}{|c|}{$\begin{array}{l}\text { PRT (low intensity, medium intensity, and high intensity) versus control } \\
\text { 1. PRT } \\
\text { Type of Ex: } 3 \text { LL } \\
\text { Equipment: Universal machines } \\
\text { Intensity: LI-50\% of } 1 \text { RM; MI-70\% of } 1 \text { RM; HI-90\% of } 1 \text { RM } \\
\text { Frequency: Ex3 } \\
\text { Reps/ sets: LI -12 to } 14 / 3 \text {; MI-8 to } 10 / 3 \text {; HI-4 to } 6 / 3 \\
\text { Duration: } 16 \text { weeks } \\
\text { Setting: not reported (Gym?) } \\
\text { Supervision: not reported } \\
\text { Adherence: not reported } \\
\text { 2. Control group: no training }\end{array}$} \\
\hline Outcomes & \multicolumn{2}{|c|}{$\begin{array}{l}\text { Muscle strength } \\
\text { Comments on adverse events: no }\end{array}$} \\
\hline Notes & \multicolumn{2}{|c|}{$\begin{array}{l}\text { Results from males were extracted } \\
\text { Comparisons: low intensity versus high intensity, and high intensity versus control }\end{array}$} \\
\hline \multicolumn{3}{|l|}{ Risk of bias } \\
\hline Item & Authors' judgement & Description \\
\hline Allocation concealment? & Unclear & B - Unclear \\
\hline
\end{tabular}

\section{Bermon 1999}

\begin{tabular}{l|l}
\hline Methods & RCT \\
& Method of randomisation: not reported \\
Assessor blinding: no \\
Participant blinding: no \\
Loss to follow-up: 1 \\
Intention-to-treat analysis: no \\
Post-program follow up: no \\
\hline \multirow{2}{*}{ Participants } & Location: France \\
& N=32 \\
& Sample: healthy older people \\
& Age: mean 70 years \\
& Inclusion criteria: elderly adults, free of cardiorespiratory and neurological diseases, \\
& sedentary to moderately active, passed screening procedure including medical history and \\
physical examination & Exclusion criteria: not reported \\
\hline \multirow{2}{*}{ Interventions } & PRT versus control \\
& 1. PRT \\
& Type of Ex: 1 UL, 2LL \\
& Equipment: weight machine (Marcy Vertex II) \\
& Intensity: (80\% of 1RM) \\
& Frequency: Ex3 \\
& Reps/ sets: $8 / 3$ \\
& Program duration: 8 weeks
\end{tabular}




\begin{tabular}{l|l|l}
\multicolumn{1}{l|}{} & $\begin{array}{l}\text { Setting: gym } \\
\text { Supervision: full } \\
\text { Adherence: not reported } \\
\text { 2. Control Group: asked to maintain customary activities and dietary patterns }\end{array}$ \\
\hline Outcomes & $\begin{array}{l}\text { Strength (1RM) } \\
\text { Anthropometry } \\
\text { Hormones } \\
\text { Comments on adverse events: no }\end{array}$ \\
\hline Notes & \multicolumn{2}{l}{} \\
\hline Risk of bias & Authors' judgement & Description \\
\hline Item & Unclear & B - Unclear \\
\hline Allocation concealment?
\end{tabular}

\section{Boshuizen 2005}

\begin{tabular}{|c|c|c|}
\hline Methods & \multicolumn{2}{|c|}{$\begin{array}{l}\text { RCT } \\
\text { Method of randomisation: not reported } \\
\text { Assessor blinding: yes } \\
\text { Participant blinding: not reported } \\
\text { Loss to follow-up: } 18 \text { in total ( } 2 \text { in high-guidance group, } 10 \text { in medium-guidance group, } \\
\text { and } 5 \text { in controls, } 1 \text { was not mentioned) } \\
\text { Intention-to-treat analysis: no } \\
\text { Post-program follow up: no }\end{array}$} \\
\hline Participants & \multicolumn{2}{|c|}{$\begin{array}{l}\text { Location: Netherlands } \\
\mathrm{N}=46 \text { ( } 24 \text { in high-guidance group; } 22 \text { in control) } \\
\text { Sample: experiencing difficulty in chair rising } \\
\text { Age: mean = } 80 \text { years ( } \mathrm{SD}=6.7) \\
\text { Inclusion criteria: experiencing difficulty in chair rising } \\
\text { Exclusion criteria: with a maximum knee-extensor torque of both legs exceeding } 25 \mathrm{~kg} \\
\text { force; self-reported diseases that would be adversely affected by the exercises }\end{array}$} \\
\hline Interventions & \multicolumn{2}{|c|}{$\begin{array}{l}\text { PRT Group (high-guidance) versus control } \\
\text { 1. PRT } \\
\text { Type of Ex : LLs } \\
\text { Equipment: elastic bands } \\
\text { Intensity: increased to the next level after } 8 \text { reps/3sets } \\
\text { Frequency: Ex3 } \\
\text { Reps/ sets: } 8 / 3 \\
\text { Duration: } 10 \text { weeks } \\
\text { Setting: welfare centers (Gym?) } \\
\text { Supervision: two supervised sessions/week by two physical therapists and one unsupervised } \\
\text { home session/week } \\
\text { Adherence: } 73 \% \text { at group sessions and } 90 \% \text { at home sessions } \\
\text { 2.Control group: no exercise training }\end{array}$} \\
\hline Outcomes & \multicolumn{2}{|c|}{$\begin{array}{l}\text { Primary: disability measure (Groningen Activity Restriction Scale) } \\
\text { Secondary: muscle strength, timed walk, TUAG, balance test } \\
\text { Comments on adverse events: yes }\end{array}$} \\
\hline \multicolumn{3}{|l|}{ Notes } \\
\hline \multicolumn{3}{|l|}{ Risk of bias } \\
\hline Item & Authors' judgement & Description \\
\hline Allocation concealment? & Unclear & B - Unclear \\
\hline
\end{tabular}

\section{Brandon 2000}

\begin{tabular}{l|l}
\hline Methods & RCT \\
BUT some changing of groups allowed before intervention began (husband/wives or people \\
sharing rides changed groups) \\
Method of randomisation: not reported
\end{tabular}




\begin{tabular}{l|l|l} 
& $\begin{array}{l}\text { Assessor blinding: no } \\
\text { Participant blinding: no } \\
\text { Loss to follow-up: not reported } \\
\text { Intention-to-treat analysis: no } \\
\text { Post-program follow up: no }\end{array}$ \\
\hline Participants & $\begin{array}{l}\text { Location: USA } \\
\text { N = 85 } \\
\text { Sample: healthy older adults, participants in community activities } \\
\text { Age: mean 72 years } \\
\text { Inclusion criteria: “community-dwelling older adults", no symptoms of cardiovascular } \\
\text { disease, consent from physician, } \\
\text { Exclusion criteria: depression (according to Beck Inventory), MMSE > 19, } \\
\text { contraindications on sub maximal aerobic test }\end{array}$ \\
\hline Interventions & $\begin{array}{l}\text { PRT versus control } \\
\text { 1. PRT } \\
\text { Type of Ex: 3LL } \\
\text { Equipment: Nautilaus machines } \\
\text { Intensity: moderate-high (50-70\% of 1RM) }\end{array}$ \\
\hline Frequency: Ex3 \\
Reps/ sets: 8-12/3 \\
Duration: 4 months \\
Setting: gym-based \\
Supervision: full \\
Adherence: 95\% \\
2. Control Group: no intervention
\end{tabular}

\section{Brandon 2003}

\begin{tabular}{|c|c|}
\hline Methods & $\begin{array}{l}\text { RCT } \\
\text { Method of randomisation: not reported } \\
\text { Assessor blinding: not reported } \\
\text { Participant blinding: not reported } \\
\text { Loss to follow-up: } 13 / 29 \text { in the PRT group; } 8 / 23 \text { in the control group } \\
\text { Intention-to-treat analysis: no } \\
\text { Post-program follow up: no }\end{array}$ \\
\hline Participants & $\begin{array}{l}\text { Location: USA } \\
\mathrm{N}=52(29 \text { in PRT) } \\
\text { Sample: community dwelling, diabetes } \\
\text { Age: mean } 65.8 \text { years }(\mathrm{SD}=7.6) \\
\text { Inclusion criteria: not reported } \\
\text { Exclusion criteria: elevated blood glucose, depression, altered cognitive function, } \\
\text { cardiovascular diseases, strokes, and hypertension }\end{array}$ \\
\hline Interventions & $\begin{array}{l}\text { PRT versus control } \\
\text { 1. PRT } \\
\text { Type of Ex: } 5 \mathrm{LL} \\
\text { Equipment: Nautilus machine } \\
\text { Intensity: ( } 50 \%, 60 \% \text {, and } 70 \% \text { for set } 1,2 \text {, and } 3 \text { separately) } \\
\text { Frequency: Ex3 during the first } 6 \text { months, and Ex } 2 \text { from month } 7 \text { to } 24 \\
\text { Reps/Sets: } 8-12 \text { /3 } \\
\text { Duration: } 24 \text { months } \\
\text { Setting: not reported, (Gym?) } \\
\text { Supervision: full } \\
\text { Adherence: }>85 \% \\
\text { 2. Control group: no training }\end{array}$ \\
\hline Outcomes & $\begin{array}{l}\text { Muscle strength (1RM/body weight) } \\
\text { TUAG }\end{array}$ \\
\hline
\end{tabular}


50-foot walk

Walk up and down stairs

Comments on adverse events: yes

\begin{tabular}{l|l|l}
\hline \multicolumn{2}{l}{ Notes } \\
\hline Risk of bias \\
\hline Item & Authors' judgement & Description \\
\hline Allocation concealment? & Unclear & B - Unclear \\
\hline
\end{tabular}

Brochu 2002

\begin{tabular}{|c|c|c|}
\hline Methods & \multicolumn{2}{|c|}{$\begin{array}{l}\text { RCT } \\
\text { Method of randomisation: stratified by physical function scores of SF-36 } \\
\text { Assessor blinding: no } \\
\text { Participant blinding: no } \\
\text { Loss to follow-up: } 5 / 30 \\
\text { Intention-to-treat analysis: no } \\
\text { Post-program follow up: no }\end{array}$} \\
\hline Participants & \multicolumn{2}{|c|}{$\begin{array}{l}\text { Location: USA } \\
\mathrm{N}=30 \text { ( } 15 \text { in each group) } \\
\text { Sample: disabled women with } \mathrm{CHD} \\
\text { Age: mean } 70.5 \text { years }(\mathrm{SD}=4) \\
\text { Inclusion criteria: age }>65 \text { years SF- } 36 \text { physical function }<85 \text { Had definite CHD } \\
\text { Exclusion criteria: hospitalization for an acute coronary syndrome within } 6 \text { months, very } \\
\text { low threshold angina, exercise-test limiting noncardiac comorbility, uncontrolled BP, } \\
\text { sternal nonunion after coronary surgery, recent participation in a cardiac rehabilitation } \\
\text { program, inflammatory arthritis, and dementia }\end{array}$} \\
\hline Interventions & \multicolumn{2}{|c|}{$\begin{array}{l}\text { PRT versus control } \\
\text { 1. PRT } \\
\text { Type of Ex: 5UL, 3LL } \\
\text { Equipment: Universal weights and dumbbells } \\
\text { Intensity: high ( } 80 \% \text { of } 1 \mathrm{RM}) \\
\text { Frequency: Ex3 } \\
\text { Reps/Sets: } 10 / 2 \\
\text { Duration: } 24 \text { weeks } \\
\text { Setting: gym } \\
\text { Supervision: not reported } \\
\text { Adherence: required to be } 75 \% \\
\text { 2. Control Group: } 30 \text { to } 40 \text { minutes of stretching, calisthenics, light yoga, and deep- } \\
\text { breathing progressiverelaxation exercise }\end{array}$} \\
\hline Outcomes & \multicolumn{2}{|c|}{$\begin{array}{l}\text { Primary: CS physical performance test, SF-36 } \\
\text { Secondary: strength (1 RM), peak V02, 6-minute walk } \\
\text { Comments on adverse events: yes }\end{array}$} \\
\hline \multicolumn{3}{|l|}{ Notes } \\
\hline \multicolumn{3}{|l|}{ Risk of bias } \\
\hline Item & Authors' judgement & Description \\
\hline Allocation concealment? & Unclear & B - Unclear \\
\hline
\end{tabular}

\section{Bruunsgaard 2004}

\begin{tabular}{l|l}
\hline Methods & RCT \\
& Method of randomisation: not reported \\
& Assessor blinding: not reported \\
& Participant blinding: not reported \\
& Loss to follow-up: 18 (39 enrolled) \\
& Intention-to-treat analysis: no \\
& Post-program follow up: no \\
\hline Participants & Location: Demark
\end{tabular}




\begin{tabular}{l|l|l} 
& $\begin{array}{l}\text { N }=21 \text { (10 in PRT) } \\
\text { Sample: frail nursing home residents } \\
\text { Age: mean 88.6 years-PRT, 90.6 years-control } \\
\text { Inclusion criteria: not reported } \\
\text { Exclusion criteria: acute illness, hypertension, severe cardiovascular disease, moderate/ } \\
\text { severe cognitiveimpairment, severe impairment of motor function, and neurological } \\
\text { disorder }\end{array}$ \\
\hline Interventions & $\begin{array}{l}\text { PRT versus control } \\
\text { 1. PRT } \\
\text { Type of Ex: } 2 \text { LL } \\
\text { Equipment: training chair (Quadriceps Exercise Table) } \\
\text { Intensity: 50\% to 80\% of 1 RM } \\
\text { Frequency: Ex3 } \\
\text { Reps/Sets: } 8 / 3 \\
\text { Duration: 12 weeks } \\
\text { Setting: nursing home facility (Gym?) } \\
\text { Supervision: full by a physiotherapist } \\
\text { Adherence: 84\% for the PRT group, 97\% for the control group } \\
\text { 2. Control group: social activities, twice a week by an occupational therapist }\end{array}$ \\
\hline Outcomes & $\begin{array}{l}\text { Muscle strength (1 RM) } \\
\text { Comments on adverse events: no }\end{array}$ \\
\hline Notes & \multicolumn{2}{|l}{} \\
\hline Risk of bias & Authors' judgement & Description \\
\hline Item & Unclear & B - Unclear \\
\hline Allocation concealment? &
\end{tabular}

\section{Buchner 1997}

\begin{tabular}{l|l}
\hline Methods & RCT: with four groups: strength training alone, endurance training alone, strength and \\
endurance training and control group \\
Method of randomisation: variation of randomly permuted blocks \\
Assessor blinding: yes \\
Participant blinding: no \\
Loss to follow-up: 4 (from PRT/control) \\
Intention to-treat analysis: yes \\
Post-program follow up: exercisers assessed at 9 months, all participants monitored for falls \\
for median 1.42 years (max 2.35 years)
\end{tabular}




\begin{tabular}{l|l|l}
\multicolumn{1}{l|}{} & Strength (isokinetic) \\
& Balance \\
& Gait \\
& SF-36 \\
& Sickness Impact Profile & Lawton IADL scale \\
& Stair climbing & Falls \\
& Health care use \\
& Comments on adverse events: yes \\
\hline Notes & Data from PRT and control group were compared \\
& Data from PRT and aerobic training group were compared \\
\hline Risk of bias & \multicolumn{2}{|l}{} \\
\hline Item & Authors' judgement & Description \\
\hline Allocation concealment? & Unclear & B - Unclear \\
\hline
\end{tabular}

\section{Casaburi 2004}

\begin{tabular}{|c|c|c|}
\hline Methods & \multicolumn{2}{|c|}{$\begin{array}{l}\text { RCT } \\
\text { Method of randomisation: not reported } \\
\text { Assessor blinding: yes } \\
\text { Participant blinding: not reported } \\
\text { Loss to follow-up: 1/12-Tx, 1/12-Control } \\
\text { Intention-to-treat analysis: no } \\
\text { Post-program follow up: no }\end{array}$} \\
\hline Participants & \multicolumn{2}{|c|}{$\begin{array}{l}\text { Location: USA } \\
\mathrm{N}=24 \text { ( } 12 \text { for each group) } \\
\text { Sample: people with COPD } \\
\text { Age: mean } 68.9 \text { years (SD=9.8) } \\
\text { Inclusion criteria: age } 55 \text { to } 80 \text { years, FEV1 of } 60 \% \text { predicted or less, and FEV1 to vital } \\
\text { capacity ratio of } 60 \% \text { or less. Screening serum testosterone was } 400 \mathrm{ng} / \mathrm{dl} \text { or less } \\
\text { Exclusion criteria: significant cardiovascular or orthopedic impairments, body weight of } \\
\text { less than } 75 \% \text { or more than } 130 \% \text { of ideal, symptomatic benign prostatic hypertrophy, } \\
\text { prostate cancer history, serum prostate specific antigen of more than } 4 \text { ?g/L, or hemoglobin } \\
\text { of more than } 16 \text { ug/dl }\end{array}$} \\
\hline Interventions & \multicolumn{2}{|c|}{$\begin{array}{l}\text { PRT versus control } \\
1 \text {. PRT } \\
\text { Type of Ex: } 5 \text { LL with eumetabolic diet } \\
\text { Equipment: not reported } \\
\text { Intensity: first } 4 \text { weeks, } 60 \% \text { of } 1 \mathrm{RM} \text { then increased to } 80 \% \text { of } 1 \mathrm{RM} \\
\text { Frequency: Ex } 3 \\
\text { Reps/Sets: first } 4 \text { weeks, } 12 / 3 \text { then increased to } 8-10 / 4 \\
\text { Duration: } 10 \text { weeks } \\
\text { Setting: not reported } \\
\text { Supervision: full by an exercise trainer } \\
\text { Adherence: at least } 25 \text { of } 30 \text { scheduled sessions } \\
\text { 2. Control Group: no training }\end{array}$} \\
\hline Outcomes & \multicolumn{2}{|c|}{$\begin{array}{l}\text { Muscle strength } \\
\text { VO2max } \\
\text { Comments on adverse events: yes }\end{array}$} \\
\hline \multicolumn{3}{|l|}{ Notes } \\
\hline \multicolumn{3}{|l|}{ Risk of bias } \\
\hline Item & Authors' judgement & Description \\
\hline Allocation concealment? & Unclear & B - Unclear \\
\hline
\end{tabular}




\section{Castaneda 2001}

\begin{tabular}{|c|c|c|}
\hline Methods & \multicolumn{2}{|c|}{$\begin{array}{l}\text { RCT both groups were also on a low-protein diet (run-in period for } 6 \text { weeks to evaluate } \\
\text { this); comparison was between low-protein diet alone or low-protein diet plus resistance } \\
\text { training } \\
\text { Method of randomisation: not reported } \\
\text { Assessor blinding: blind for all assessments except strength } \\
\text { Participant blinding: yes, sham-exercises } \\
\text { Loss to follow-up: no } \\
\text { Intention-to-treat analysis: not stated } \\
\text { Post-program follow up: no }\end{array}$} \\
\hline Participants & \multicolumn{2}{|c|}{$\begin{array}{l}\text { Location: USA N }=26 \\
\text { Sample: patients with moderate chronic renal insufficiency, recruited from nephrology } \\
\text { clinics } \\
\text { Age: mean } 65 \text { years (SD 9) } \\
\text { Inclusion criteria: older than } 50 \text { years of age; serum creatinine concentrations between } \\
133-422 \text { umol/L }(1.5 \text { and } 5.0 \mathrm{mg} / \mathrm{dL}) \text {; physician approval to follow a low protein diet; } \\
\text { nephrologist confirmed diagnosis of chronic renal insufficiency } \\
\text { Exclusion criteria: myocardial infarction within the last } 6 \text { months; any unstable chronic } \\
\text { condition; dementia; alcoholism; dialysis or previous renal; current resistance training; } \\
\text { recent involuntary weight change }(+/-2 \mathrm{~kg}) \text {; albumin level less than } 30 \mathrm{~g} / \mathrm{L} ; \text { proteinuria } \\
\text { greater than } 10 \mathrm{~g} / \mathrm{d} \text {; abnormal stress test on screening }\end{array}$} \\
\hline Interventions & \multicolumn{2}{|c|}{$\begin{array}{l}\text { PRT versus control } \\
\text { 1. PRT plus low-protein diet } \\
\text { Type of Ex: 2UL, 3LL } \\
\text { Equipment: machines (Keiser) } \\
\text { Intensity: } 80 \% \text { of } 1 \text { RM } \\
\text { Frequency: Ex3 } \\
\text { Reps/Sets: } 8 / 3 \\
\text { Duration: } 12 \text { weeks } \\
\text { Setting: gym at research centre } \\
\text { Supervision: full } \\
\text { Adherence: } 91 \% \\
\text { 2. Control Group: on low-protein diet; performed 5-8 sham exercises (gentle movements } \\
\text { while standingsitting and bending) for upper and lower body }\end{array}$} \\
\hline Outcomes & \multicolumn{2}{|c|}{$\begin{array}{l}\text { Strength }(1 \mathrm{RM}) \text {, } \\
\text { Peak oxygen consumption } \\
\text { Comments on adverse events: yes }\end{array}$} \\
\hline \multicolumn{3}{|l|}{ Notes } \\
\hline \multicolumn{3}{|l|}{ Risk of bias } \\
\hline Item & Authors' judgement & Description \\
\hline Allocation concealment? & Unclear & B - Unclear \\
\hline
\end{tabular}

\section{Castaneda 2004}

\begin{tabular}{l|l}
\hline Methods & RCT \\
& Method of randomisation: not reported \\
Assessor blinding: yes & Participant blinding: yes \\
& Loss to follow-up: 0 \\
& Intention-to-treat analysis: no \\
& Post-program follow up: no
\end{tabular}




\begin{tabular}{|c|c|c|}
\hline & \multicolumn{2}{|c|}{$\begin{array}{l}\text { Type of Ex : } 2 \mathrm{UL} / 3 \mathrm{LL} \\
\text { Equipment: Keiser Sports Health Equipments } \\
\text { Intensity: } 80 \% \text { of } 1 \mathrm{RM} \\
\text { Frequency: Ex3 } \\
\text { Reps/Sets: } 8 / 3 \\
\text { Duration: } 12 \text { weeks } \\
\text { Setting: research center (Gym?) } \\
\text { Supervision: full } \\
\text { Adherence: not reported } \\
\text { 2.Control group: stretching and flexibility exercise }\end{array}$} \\
\hline Outcomes & \multicolumn{2}{|c|}{$\begin{array}{l}\text { Muscle strength ( } 1 \mathrm{RM}) \\
\text { Comments on adverse events: yes }\end{array}$} \\
\hline Notes & \multicolumn{2}{|c|}{ Reported whole body muscle strength (data were not pooled) } \\
\hline \multicolumn{3}{|l|}{ Risk of bias } \\
\hline Item & Authors' judgement & Description \\
\hline Allocation concealment? & Unclear & B - Unclear \\
\hline
\end{tabular}

\section{Chandler 1998}

\begin{tabular}{|c|c|c|}
\hline Methods & \multicolumn{2}{|c|}{$\begin{array}{l}\text { RCT } \\
\text { Method of randomisation: block randomised and stratified by } 2 \text { levels of functioning } \\
\text { Assessor blinding: some measures } \\
\text { Participant blinding: no } \\
\text { Loss to follow-up: } 13 \\
\text { Intention-to-treat analysis: no } \\
\text { Post-program follow-up: no }\end{array}$} \\
\hline Participants & \multicolumn{2}{|c|}{$\begin{array}{l}\text { Location: USA } \\
\mathrm{N}=100 \\
\text { Sample: community-dwelling older people with functional limitations } \\
\text { Age: mean } 77.6 \text { years } \\
\text { Inclusion criteria: community-dwelling; aged } 64 \text { or above; unable to descend stairs step } \\
\text { over step without holding onto the railing } \\
\text { Exclusion criteria: }>\text { or }=3 \text { on Reuben's Advanced Activities of Daily Living; terminal } \\
\text { illness (i.e. not expected to survive } 6 \text { months); severe unstable cardiac disease including MI } \\
\text { in the past } 6 \text { months; severe fixed or progressive neurologic disease; complete blindness; } \\
\text { lower extremity amputation; score below } 18 \text { on MM SE and unable to follow a 3-step } \\
\text { command }\end{array}$} \\
\hline Interventions & \multicolumn{2}{|c|}{$\begin{array}{l}\text { PRT versus control } \\
\text { 1. PRT } \\
\text { Type of Ex: } 8 \mathrm{LL} \\
\text { Equipment: Theraband } \\
\text { Intensity: progressively increased ( } 8 \mathrm{RM} \text { to } 2 \text { sets of } 10 \mathrm{RM}) \\
\text { Frequency: Ex3 } \\
\text { Reps/Sets: } 10 / 2 \\
\text { Duration: } 10 \text { weeks } \\
\text { Setting: home-based } \\
\text { Supervision: not reported } \\
\text { Adherence: not reported } \\
\text { 2. Control Group: could begin exercise after } 10 \text { weeks, one friendly phone call at } 5 \text { weeks }\end{array}$} \\
\hline Outcomes & \multicolumn{2}{|l|}{$\begin{array}{l}\text { HRQoL }(\mathrm{SF}-36) \\
\text { Lower limb strength (Cybex) } \\
\text { 6-minute walk test } \\
\text { Chair rise } \\
\text { Functional reach } \\
\text { Falls Self-Efficacy (/100) } \\
\text { Comments on adverse events: no }\end{array}$} \\
\hline \multicolumn{3}{|l|}{ Notes } \\
\hline \multicolumn{3}{|l|}{ Risk of bias } \\
\hline Item & Authors' judgement & Description \\
\hline Allocation concealment? & Unclear & B- Unclear \\
\hline
\end{tabular}




\section{Charette 1991}

\begin{tabular}{|c|c|c|}
\hline Methods & \multicolumn{2}{|c|}{$\begin{array}{l}\text { RCT } \\
\text { Method of randomisation: not reported } \\
\text { Assessor blinding: no } \\
\text { Participant blinding : no } \\
\text { Loss to follow-up: } 8 \\
\text { Intention-to-treat analysis: no } \\
\text { Post-program follow-up: no }\end{array}$} \\
\hline Participants & \multicolumn{2}{|c|}{$\begin{array}{l}\text { Location: USA } \\
\mathrm{N}=27 \\
\text { Sample: healthy, sedentary women } \\
\text { Age: mean } 69 \text { years } \\
\text { Inclusion criteria: aged } 64-86 ; \text { healthy; female, Palo Alto community } \\
\text { Exclusion criteria: pre-existing disability or illness that would preclude participation in a } \\
\text { weight training program of moderate intensity }\end{array}$} \\
\hline Interventions & \multicolumn{2}{|c|}{$\begin{array}{l}\text { PRT versus control } \\
\text { 1. PRT } \\
\text { Type of Ex: 7LL } \\
\text { Equipment: weight training machines } \\
\text { Intensity: } 65-75 \% \text { of } 1 \text { RM } \\
\text { Frequency: Ex3 } \\
\text { Reps/Sets: } 6 / 3 \text {, increased to } 6 \text { sets for leg extension and press after } 2 \text { weeks } \\
\text { Program Duration: } 12 \text { weeks } \\
\text { Setting: gym } \\
\text { Supervision: full } \\
\text { Adherence: } 90 \% \text { completed all sessions } \\
\text { 2. Control Group: maintain normal activities, asked not to start an exercise program. Could } \\
\text { undertaketraining at the end of the program. Contacted to make appointments/ maintain } \\
\text { interest }\end{array}$} \\
\hline Outcomes & \multicolumn{2}{|c|}{$\begin{array}{l}\text { Strength (1RM) } \\
\text { Comments on adverse events: no }\end{array}$} \\
\hline \multicolumn{3}{|l|}{ Notes } \\
\hline \multicolumn{3}{|l|}{ Risk of bias } \\
\hline Item & Authors' judgement & Description \\
\hline Allocation concealment? & Unclear & B - Unclear \\
\hline
\end{tabular}

\section{Chin A Paw 2006}

\begin{tabular}{|c|c|}
\hline Methods & $\begin{array}{l}\text { RCT with } 4 \text { groups: PRT, control, functional training, and combined training } \\
\text { Method of randomisation: the random allocation sequence was generated by computer by } \\
\text { two independent students } \\
\text { Assessor blinding: yes } \\
\text { Participant blinding: not reported } \\
\text { Loss to follow-up: } 21 / 57 \text { in PRT; } 22 / 60 \text { in function-skills; } 17 / 56 \text { in combined training; } \\
\text { 23/51 in controls } \\
\text { Intention-to-treat analysis: yes. Data analysed: } 40 \text { in PRT, } 44 \text { in function-skills, } 44 \text { in } \\
\text { combined training, } 31 \text { in controls } \\
\text { Post-program follow up: no }\end{array}$ \\
\hline Participants & $\begin{array}{l}\text { Location: Netherlands } \\
\mathrm{N}=108 \text { ( } 57 \text { in PRT) } \\
\text { Sample: elders lived in long-term care facilities } \\
\text { Age: mean } 81.3 \text { (SD }=4.4 \text { ) } \\
\text { Inclusion criteria: } 1 \text { ) aged } 65 \text { or older; } 2 \text { ) living in a nursing home or residential care } \\
\text { facility; } 3 \text { ) able to walk } 6 \text { m or more (with or without a walking aid); } 4 \text { ) able to comprehend } \\
\text { the study procedures; } 5 \text { ) no medical contraindication for study participation; } 6 \text { ) no rapidly } \\
\text { progressive or terminal illness; } 7 \text { ) and not moving away from the home within the 6-months } \\
\text { intervention period } \\
\text { Exclusion criteria: not reported }\end{array}$ \\
\hline Interventions & $\begin{array}{l}\text { PRT versus control, versus functional training, and versus combined training } \\
\text { 1. PRT } \\
\text { Type of Ex: } 3 \mathrm{UL} / 2 \mathrm{LL}\end{array}$ \\
\hline
\end{tabular}




\begin{tabular}{l|l|l} 
& $\begin{array}{l}\text { Equipment: TechnoGym equipment, dump bells and ankle/wrist weights } \\
\text { Intensity: high (60-80\% of 1 RM) } \\
\text { Frequency: Ex2 } \\
\text { Reps/Sets: 8-12/2 } \\
\text { Duration: } 24 \text { weeks } \\
\text { Setting: long-term care facility (Gym?) } \\
\text { Supervision: full by a physical therapist and an assistant } \\
\text { Adherence: } 78 \% \\
\text { 2.Control group: mean age }=81 \text {, educational program (group discussion about topics of } \\
\text { interest) } \\
\text { 3. Functional training group: N=60, mean age = 82 years, game-like or cooperative } \\
\text { activities } \\
\text { 4. Combined training group: N=56, mean age = 81 years, one strength training and one } \\
\text { functional trainingper week }\end{array}$ \\
\hline Outcomes & $\begin{array}{l}\text { Primary: physical activities/ADL disability } \\
\text { Secondary: muscle strength, vitality plus scales, balance, gait speed, chair rise } \\
\text { Comments on adverse events: yes }\end{array}$ \\
\hline Notes & Comparisons: PRT versus control, PRT versus functional training \\
\hline Risk of bias & \multicolumn{2}{|l}{} \\
\hline Item & Authors' judgement & Description \\
\hline Allocation concealment? & Yes & A - Adequate \\
\hline
\end{tabular}

\section{Collier 1997}

\begin{tabular}{|c|c|c|}
\hline Methods & \multicolumn{2}{|c|}{$\begin{array}{l}\text { RCT } \\
\text { Method of randomisation: not reported } \\
\text { Assessor blinding: no } \\
\text { Participant blinding: no } \\
\text { Loss to follow-up: } 1 \\
\text { Intention-to-treat analysis: no } \\
\text { Post-program follow up: no }\end{array}$} \\
\hline Participants & \multicolumn{2}{|c|}{$\begin{array}{l}\text { Location: USA } \\
\mathrm{N}=39 \\
\text { Sample: healthy, community-dwelling } \\
\text { Age: range } 65-85 \text { years } \\
\text { Inclusion criteria: aged } 65-85 \text {, approval of physician, community residents } \\
\text { Exclusion criteria: not reported }\end{array}$} \\
\hline Interventions & \multicolumn{2}{|c|}{$\begin{array}{l}\text { PRT versus control } \\
\text { 1. PRT } \\
\text { Type of Ex: 5UL, 2LL } \\
\text { Equipment: Universal Hercules Gym Machine } \\
\text { Intensity: not specified, but progressed throughout } \\
\text { Frequency: Ex3 } \\
\text { Reps/Sets: } 10 / 2 \\
\text { Program Duration: } 10 \text { weeks } \\
\text { Setting: gym } \\
\text { Supervision: full } \\
\text { Adherence: not reported } \\
\text { 2. Control Group: no active intervention }\end{array}$} \\
\hline Outcomes & \multicolumn{2}{|c|}{$\begin{array}{l}\text { Strength (number of reps at } \% \text { of body weight) } \\
\text { Functional Fitness Assessment for adults }>60 \\
\text { Agility Assessment (walking between cones) } \\
\text { Hand-eye co-ordination ("soda pop" test) } \\
\text { Grip strength } \\
\text { Physical Self-Efficacy Scale (PSE) } \\
\text { Comments on adverse events: no }\end{array}$} \\
\hline \multicolumn{3}{|l|}{ Notes } \\
\hline \multicolumn{3}{|l|}{ Risk of bias } \\
\hline Item & Authors' judgement & Description \\
\hline Allocation concealment? & Unclear & B - Unclear \\
\hline
\end{tabular}


Damush 1999

\begin{tabular}{|c|c|c|}
\hline Methods & \multicolumn{2}{|c|}{$\begin{array}{l}\text { RCT } \\
\text { Method of randomisation: not reported } \\
\text { Assessor blinding: no } \\
\text { Participant blinding: no, attention control group used } \\
\text { Loss to follow-up: } 9 \\
\text { Intention-to-treat analysis: no } \\
\text { Post-program follow up: no }\end{array}$} \\
\hline Participants & \multicolumn{2}{|c|}{$\begin{array}{l}\text { Location: USA } \\
\mathrm{N}=71 \\
\text { Sample: community-dwelling women, recruited through media-based promotion } \\
\text { Age: mean } 68 \text { years (SD 5.6) } \\
\text { Inclusion criteria: age 55+, living in retirement residential community, clearance from GP } \\
\text { Exclusion criteria: GP-identified contraindications to exercise }\end{array}$} \\
\hline Interventions & \multicolumn{2}{|c|}{$\begin{array}{l}\text { PRT versus control } \\
\text { 1.PRT } \\
\text { Type of Ex: 4UL, 3LL } \\
\text { Equipment: Theraband } \\
\text { Intensity: low to moderate ( } 4 / 10 \text { on Borg scale) } \\
\text { Frequency: Ex3 } \\
\text { Reps/Sets: } 1 \text { set, as many reps to reach } 4 / 10 \text { on Borg } \\
\text { Program Duration: } 8 \text { weeks } \\
\text { Setting: gym, group-based } \\
\text { Supervision: full } \\
\text { Adherence: } 88 \% \\
\text { 2. Control Group: attended all of the exercise sessions to allow social contact }\end{array}$} \\
\hline Outcomes & \multicolumn{2}{|c|}{$\begin{array}{l}\text { HRQoL (SF-36) } \\
\text { Strength (3RM) } \\
\text { Grip strength } \\
\text { Comments on adverse events: no }\end{array}$} \\
\hline \multicolumn{3}{|l|}{ Notes } \\
\hline \multicolumn{3}{|l|}{ Risk of bias } \\
\hline Item & Authors' judgement & Description \\
\hline Allocation concealment? & Unclear & B - Unclear \\
\hline
\end{tabular}

\section{de Vos 2005}

\begin{tabular}{l|l}
\hline Methods & RCT with 4 groups: high intensity, medium intensity, and low intensity, and control \\
Method of randomisation: computerized randomisation program, stratified by gender \\
Assessor blinding: only for tests at baseline \\
Participant blinding: blinded to the research hypothesis \\
Loss to follow-up: 12 (4-high intensity, 3-medium intensity, 3-low intensity, 2-control) \\
Intention-to-treat analysis: no \\
Post-program follow up: no
\end{tabular}




\begin{tabular}{l|l|l} 
& $\begin{array}{l}\text { Duration: 8-12 weeks (M = 10 weeks) } \\
\text { Setting: not reported } \\
\text { Supervision: Experienced exercise trainers } \\
\text { Adherence: }>90 \% \text { for each training group } \\
\text { 2. Control Group: maintain current level of activities }\end{array}$ \\
\hline Outcomes & $\begin{array}{l}\text { Dynamic muscle strength } \\
\text { Muscle power } \\
\text { Muscle endurance } \\
\text { Balance } \\
\text { Comments on adverse events: yes }\end{array}$ \\
\hline Notes & Involved power training & \multicolumn{2}{|l}{} \\
\hline Risk of bias & \multicolumn{2}{|l}{} \\
\hline Item & Authors' judgement & Description \\
\hline Allocation concealment? & Unclear & B - Unclear \\
\hline
\end{tabular}

de Vreede 2007

\begin{tabular}{|c|c|c|}
\hline Methods & \multicolumn{2}{|c|}{$\begin{array}{l}\text { RCT with } 3 \text { groups: PRT, control, and functional task exercise group } \\
\text { Method of randomisation: by computer using a random numbers table } \\
\text { Assessor blinding: yes } \\
\text { Participant blinding: not reported } \\
\text { Loss to follow-up: 6/34 in PRT group } \\
\text { Intention-to-treat analysis: no } \\
\text { Post-program follow up: no }\end{array}$} \\
\hline Participants & \multicolumn{2}{|c|}{$\begin{array}{l}\text { Location: Netherlands } \\
\mathrm{N}=65 \text { ( } 34 \text { in PRT) } \\
\text { Sample: community-dwelling older adults } \\
\text { Age: mean } 74.8 \text { years (SD = 4) } \\
\text { Inclusion Criteria: age over } 70 \text { years } \\
\text { Exclusion Criteria: recent fractures, unstable cardiovascular or metabolic disease, } \\
\text { musculoskeletal condition or chronic illness, severe airflow obstruction, recent depression } \\
\text { or emotional distress, loss of mobility for more than one week in the previous months, } \\
\text { exercised at a sports club more than } 3 \text { times a week }\end{array}$} \\
\hline Interventions & \multicolumn{2}{|c|}{$\begin{array}{l}\text { PRT versus control and versus functional task exercise } \\
\text { 1. PRT } \\
\text { Type of Ex: } 5 \mathrm{UL}, 9 \mathrm{LL} \\
\text { Equipment: weights, elastic tub } \\
\text { Intensity: } 7-8 \text { on a } 10 \text {-point rated perceived exertion scale } \\
\text { Frequency: Ex3 } \\
\text { Reps/Sets: } 10 / 3 \\
\text { Duration: } 12 \text { weeks } \\
\text { Setting: a local leisure center } \\
\text { Supervision: at least two experienced instructors } \\
\text { Adherence: } 74 \% \text { (SD }=34.6 \% \text { ) } \\
\text { 2. Control Group: to keep normal activity level } \\
\text { 3. Functional task exercise group: } \mathrm{N}=33 \text {, moving with a vertical component, moving with } \\
\text { a horizontal component, carrying an object, and changing position between lying, sitting, } \\
\text { and standing. Practice phase for } 2 \text { weeks, variation phase for } 4 \text { weeks, and daily tasks for } 6 \\
\text { weeks }\end{array}$} \\
\hline Outcomes & \multicolumn{2}{|c|}{$\begin{array}{l}\text { Primary: SF-36 } \\
\text { Secondary: TUAG } \\
\text { Comments on adverse events: yes }\end{array}$} \\
\hline Notes & \multicolumn{2}{|c|}{$\begin{array}{l}\text { Data of SF- } 36 \text { were provided by the trial authors } \\
\text { Data from PRT and functional training group were compared }\end{array}$} \\
\hline \multicolumn{3}{|l|}{ Risk of bias } \\
\hline Item & Authors' judgement & Description \\
\hline Allocation concealment? & Unclear & B - Unclear \\
\hline
\end{tabular}




\section{DeBeliso 2005}

\begin{tabular}{|c|c|c|}
\hline Methods & \multicolumn{2}{|c|}{$\begin{array}{l}\text { RCT } \\
\text { Method of randomisation: not reported-stratified by gender and strength } \\
\text { Assessor blinding: not reported } \\
\text { Participant blinding: not reported } \\
\text { Loss to follow-up: } 8 / 21 \text { in control; } 5 / 18 \text { in fixed repetition group; } 4 / 21 \text { in periodised group } \\
\text { Intention-to-treat analysis: no } \\
\text { Post-program follow up: no }\end{array}$} \\
\hline Participants & \multicolumn{2}{|c|}{$\begin{array}{l}\text { Location: USA } \\
\mathrm{N}=18 \text {-fixed repetition; } \mathrm{N}=21 \text {-periodised repetition; } \mathrm{N}=21 \text {-control } \\
\text { Sample: independent and community dwelling older adults } \\
\text { Age: fixed repetition, mean } 71.4 \text { years }(\mathrm{SD}=5.4) \text {; periodised, mean }=70.6 \text { years }(\mathrm{SD}= \\
4.7) \\
\text { Inclusion Criteria: no previous background in resistance training } \\
\text { Exclusion Criteria: not reported }\end{array}$} \\
\hline Interventions & \multicolumn{2}{|c|}{$\begin{array}{l}\text { PRT (fixed repetition and periodised repetition) versus control } \\
1 \text {. PRT } \\
\text { Type of Ex: } 5 \mathrm{UL} / 3 \mathrm{LL} \\
\text { Equipment: Flex machines } \\
\text { Intensity: fixed repetition-9 RM; periodised-week } 1 \text { to } 6,15 \mathrm{RM} \text {; week } 7 \text { to } 12,9 \mathrm{RM} \text {; } \\
\text { week } 13 \text { to } 18,6 \mathrm{RM} \\
\text { Frequency: Ex2 } \\
\text { Reps/Sets: fixed repetition-9/3; periodised-week } 1 \text { to } 6,15 / 2 \text {; week } 7 \text { to } 12,9 / 3 \text {; week } 13 \text { to } \\
\text { 18, } 6 / 4 \\
\text { Duration: } 18 \text { weeks } \\
\text { Setting: training facility (Gym?) } \\
\text { Supervision: full by trainers } \\
\text { Adherence: fixed repetition group } 77 \% \text {; periodised group } 62 \% \\
\text { 2. Control group: maintain current recreational activities }\end{array}$} \\
\hline Outcomes & \multicolumn{2}{|l|}{$\begin{array}{l}\text { Muscle strength (1 RM) } \\
\text { Comments on adverse events: yes }\end{array}$} \\
\hline \multicolumn{3}{|l|}{ Notes } \\
\hline \multicolumn{3}{|l|}{ Risk of bias } \\
\hline Item & Authors' judgement & Description \\
\hline Allocation concealment? & Unclear & B - Unclear \\
\hline
\end{tabular}

\section{DiFrancisco 2007}

\begin{tabular}{l|l}
\hline Methods & RCT \\
& Method of randomisation: a table of random numbers \\
Assessor blinding: not reported \\
Participant blinding: not reported \\
Loss to follow-up: 0 \\
Intention-to-treat analysis: no \\
Post-program follow up: no
\end{tabular}




\begin{tabular}{l|l|l}
\hline Outcomes & Adherence: not reported \\
\hline Notes & $\begin{array}{l}\text { Strength (1RM) } \\
\text { Comments on adverse events: yes }\end{array}$ \\
\hline Risk of bias & Date from 2 times a week and one time a week were compared \\
\hline Item & Authors' judgement & Description \\
\hline Allocation concealment? & Unclear & B - Unclear \\
\hline
\end{tabular}

\section{Donald 2000}

\begin{tabular}{|c|c|}
\hline Methods & $\begin{array}{l}\text { RCT, factorial design (comparison of floor surface types not included here) } \\
\text { Method of randomisation: randomised envelopes } \\
\text { Assessor blinding: no } \\
\text { Participant blinding: no } \\
\text { Loss to follow-up: } 22 \\
\text { Intention-to-treat analysis: no } \\
\text { Post-program follow up: no }\end{array}$ \\
\hline Participants & $\begin{array}{l}\text { Location: UK } \\
\mathrm{N}=58 \\
\text { Sample: hospitalised older people } \\
\text { Age: mean } 81 \text { years } \\
\text { Inclusion criteria: admitted to elderly care rehabilitation ward from Feb to Sept 1996, } \\
\text { consent from patient and carers } \\
\text { Exclusion criteria: not reported }\end{array}$ \\
\hline Interventions & $\begin{array}{l}\text { PRT versus control } \\
\text { 1. PRT } \\
\text { Type of Ex: } 2 \text { LL } \\
\text { Equipment: not reported } \\
\text { Intensity: high (maximum weight the patient could manage) } \\
\text { Frequency: twice daily } \\
\text { Reps/Sets: } 10 / 3 \\
\text { Program duration: not reported (length of hospital stay) } \\
\text { Setting: hospital } \\
\text { Supervision: full } \\
\text { Adherence: not reported } \\
\text { 2. Control Group: regular in-hospital daily physiotherapy }\end{array}$ \\
\hline Outcomes & $\begin{array}{l}\text { Falls (during hospital stay) } \\
\text { Barthel Index (ADL measure) } \\
\text { Strength (hand-held dynamometer, hand-grip strength) } \\
\text { Comments on adverse events: no }\end{array}$ \\
\hline \multicolumn{2}{|l|}{ Notes } \\
\hline \multicolumn{2}{|l|}{ Risk of bias } \\
\hline Item & Authors' judgement \\
\hline Allocation concealment? & A - Adequate \\
\hline
\end{tabular}

Earles 2001

\begin{tabular}{l|l}
\hline Methods & RCT, PRT vs moderate aerobic exercise \\
& Method of randomisation: randomised, with subjects blocked for gender and residence \\
& Assessor blinding: no \\
& Participant blinding: no \\
& Loss to Follow-up: 3 \\
& Intention-to-treat analyses: no \\
& Post-program follow up: no \\
\hline Participants & Location: USA \\
& $\mathrm{N}=43$
\end{tabular}

Cochrane Database Syst Rev. Author manuscript; available in PMC 2015 February 11. 
Sample: independent community volunteers

Age: mean 77 years (SD 5) in PRT group

Inclusion criteria: age greater than 70 years; score of 8 or higher on the Short Physical

Performance Battery; ability to travel (by using public or private transportation) to the

retirement community where exercise sessions were held; willingness to attend exercise sessions for 12 weeks

Exclusion criteria: myocardial infarction in the past 6 months; heart failure (New York

Heart Association classification <1); angina with moderate activity; chronic obstructive pulmonary disease or shortness of breath while walking at a normal pace; stroke with residual motor deficits; poorly controlled hypertension $(>174 \mathrm{mmHg}$ systolic, $>100 \mathrm{mmHg}$ diastolic); cancer with chemotherapy or radiation in the past year; physical performance

limited by arthritis; on any of the following medications: neuroleptics, oral steroids, testosterone or growth hormones

\begin{tabular}{|c|c|c|}
\hline Interventions & \multicolumn{2}{|c|}{$\begin{array}{l}\text { PRT versus aerobic } \\
\text { 1. PRT } \\
\text { Type of Ex: } 2 \text { LL; also did step-ups, chair rises and plantar flexion exercises in standing } \\
\text { Equipment: Pneumatic resistance machines } \\
\text { Intensity: high for leg press- started at } 50 \% \text { of } 1 \text { RM, increased by } 10 \% \text { during each week of } \\
\text { training; moderate for other exercises } \\
\text { Frequency: Ex3 } \\
\text { Reps/Sets: } 10 / 3 \\
\text { Duration: } 12 \text { weeks } \\
\text { Setting: gym at retirement center } \\
\text { Supervision: full } \\
\text { Adherence: } 90 \% \\
\text { 2. Aerobic training group: moderate intensity exercise } 30 \text { minutes daily, } 6 \text { days weekly }\end{array}$} \\
\hline Outcomes & \multicolumn{2}{|c|}{$\begin{array}{l}\text { Short physical performance battery (SPPB) } \\
\text { Balance (semi-tandem stance, single leg stance) } \\
\text { Chair rise (5) } \\
\text { 8-foot walk } \\
\text { Aerobic capacity (6-minute walk) } \\
\text { Muscle strength } \\
\text { Comments on adverse events: yes }\end{array}$} \\
\hline Notes & \multicolumn{2}{|c|}{ Data from PRT and aerobic training group were compared } \\
\hline \multicolumn{3}{|l|}{ Risk of bias } \\
\hline Item & Authors' judgement & Description \\
\hline Allocation concealment? & Unclear & B- Unclear \\
\hline
\end{tabular}

\section{Ettinger 1997}

\begin{tabular}{|c|c|}
\hline Methods & $\begin{array}{l}\text { RCT with } 3 \text { groups: PRT, aerobic training and health education (attention control) } \\
\text { Method of randomisation: stratified, variable block randomisation, computer generated } \\
\text { Assessor blinding: yes } \\
\text { Participant blinding: attention control group used } \\
\text { Loss to follow-up: } 75 \text { total ( } 48 \text { from PRT and control group) at } 18 \text { months } \\
\text { Intention-to-treat analysis: yes } \\
\text { Post-program follow up: participants followed after initial supervised sessions ( } 3 \text { months) } \\
\text { to home-based sessions ( } 3-18 \text { months) }\end{array}$ \\
\hline Participants & $\begin{array}{l}\text { Location: USA } \\
\mathrm{N}=439 \text { total ( } 295 \text { in PRT versus control) } \\
\text { Sample: community-dwelling people with osteoarthritis resulting in functional limitation } \\
\text { Age: mean } 68 \text { years (SD 6) in PRT group } \\
\text { Inclusion criteria: age } 60 \text { years or more, pain on most days in } 1 \text { or more knees, difficulty } \\
\text { with at least } 1 \text { of the following due to knee pain: walking a quarter mile, climbing stairs, } \\
\text { getting in and out of a car, lifting and carrying groceries, getting out of bed, getting out of } \\
\text { the bathtub or performing shopping, cleaning or self-care activities; radiographic evidence } \\
\text { of knee osteoarthritis in the tibial-femoral compartment. } \\
\text { Exclusion criteria: person has a medical condition that precluded safe participation in the } \\
\text { exercise program or prevented completion of the study (myocardial infarction or stroke in } \\
\text { the past } 3 \text { months, evidence of ischemia during the exercise treadmill test, congestive heart } \\
\text { failure, severe chronic obstructive pulmonary disease, active treatment for cancer, insulin } \\
\text { dependent diabetes mellitus, hemoglobin less than } 110 \mathrm{~L} / \mathrm{L} \text {, creatinine greater than } 176.8 \\
\text { umol/L, severe systemic disease or major psychiatric disease), inflammatory arthritis (i.e., } \\
\text { rheumatoid or psoriatic), exercised regularly (defined as aerobic activity or resistance } \\
\text { training more than } 1 \text { time per week for } 20 \text { minutes or longer), planned to move from the } \\
\text { area or be admitted to a long-term care facility in the next } 2 \text { years; unable to walk at least }\end{array}$ \\
\hline
\end{tabular}




\begin{tabular}{|c|c|c|}
\hline & \multicolumn{2}{|c|}{$\begin{array}{l}420 \text { feet in } 6 \text { minutes without a cane or assistive device; unable to walk on a treadmill } \\
\text { without an assistive device; participating in another research study; resided in a long-term } \\
\text { care facility }\end{array}$} \\
\hline Interventions & \multicolumn{2}{|c|}{$\begin{array}{l}\text { PRT versus control and versus aerobic } \\
\text { 1. PRT } \\
\text { Type of Ex: 4UL, 4LL, } 1 \mathrm{Tr} \\
\text { Equipment: cuff-weights, dumb bells } \\
\text { Intensity: moderate to high ( } 2 \text { sets of } 12 \text { reps max) } \\
\text { Frequency: Ex3 } \\
\text { Reps/Sets: } 12 / 2 \\
\text { Duration: } 78 \text { weeks } \\
\text { Setting: facility-based group for } 3 \text { months, then home- based for } 15 \text { months } \\
\text { Supervision: high for gym-based, telephone contact and visits during home based phase } \\
\text { (diminishing contact over time) } \\
\text { Adherence: } 70 \% \text { at } 18 \text { months } \\
\text { 2. Control Group: health education program (meetings and telephone contact) } \\
\text { 3. Aerobic Training Group: walking program for } 40 \text { minutes } 3 \text { times per week at } 50-70 \% \text { of } \\
\text { HR reservegroup facility based for } 3 \text { months then home-based for } 15 \text { months (same contact } \\
\text { as PRT) }\end{array}$} \\
\hline Outcomes & \multicolumn{2}{|c|}{$\begin{array}{l}\text { Primary: self-report physical disability ( } 23 \text { item scale developed for use in this trial) } \\
\text { Secondary: } 6 \text { minute walk test, stair climbing, lifting object, timed task in and out of car, } \\
\text { graded sub maximal aerobic treadmill test, strength (isokinetic dynamometer), knee x-rays, } \\
\text { knee pain } \\
\text { Comments on adverse events: yes }\end{array}$} \\
\hline Notes & \multicolumn{2}{|c|}{ Data from PRT and aerobic training group were compared } \\
\hline \multicolumn{3}{|l|}{ Risk of bias } \\
\hline Item & Authors' judgement & Description \\
\hline Allocation concealment? & Unclear & B - Unclear \\
\hline
\end{tabular}

Fahlman 2002

\begin{tabular}{|c|c|}
\hline Methods & $\begin{array}{l}\text { RCT with } 3 \text { groups: PRT, control, and aerobic group } \\
\text { Method of randomisation: not reported } \\
\text { Assessor blinding: not reported } \\
\text { Participant blinding: not reported } \\
\text { Loss to follow-up: } 0 \\
\text { Intention-to-treat analysis: N/A } \\
\text { Post-program follow-up: no }\end{array}$ \\
\hline Participants & $\begin{array}{l}\text { Location: USA } \\
\mathrm{N}=30 \text { ( } 15 \text { in each group) } \\
\text { Sample: highly active and functioning women } \\
\text { Age: mean } 73 \text { years (SD = 3) } \\
\text { Inclusion criteria: not reported } \\
\text { Exclusion criteria: dementia screened by MMSE, did not meet the criteria of the American } \\
\text { College of Sports Medicine, the presence of activity-limiting arthritis; being bedridden } \\
\text { within } 3 \text { months of the study; the presence of central or peripheral nervous system } \\
\text { disorders, stroke, acute or chronic infection, major affective disorder, human } \\
\text { immunodeficiency virus infection or autoimmune disorders, or metabolic disorders (type I } \\
\text { diabetes mellitus); being a smoker or smokeless tobacco user; participating in regular } \\
\text { aerobic or resistance training within the previous } 3 \text { months; using oral steroids or } \\
\text { medications known to have an effect on blood lipids except hormone replacement therapy; } \\
\text { having surgery within the previous } 3 \text { months; and consuming caffeine in excess of the } \\
\text { equivalent of } 4 \text { cups of coffee per day }\end{array}$ \\
\hline Interventions & $\begin{array}{l}\text { PRT versus control and aerobic } \\
\text { 1. PRT } \\
\text { Type of Ex: } 7 \mathrm{LL} \\
\text { Equipment: not reported } \\
\text { Intensity: } 8 \mathrm{RM} \\
\text { Frequency: Ex3 } \\
\text { Reps/Sets: } 8 / 3 \\
\text { Duration: } 10 \text { weeks } \\
\text { Setting: not reported } \\
\text { Supervision: not reported } \\
\text { Adherence: > 95 \% } \\
\text { 2. Control Group: maintain normal activity level }\end{array}$ \\
\hline
\end{tabular}




\begin{tabular}{l|l|l} 
& $\begin{array}{l}\text { 3. Aerobic training group: stretching and walking exercise at 70\% heart rate reserve, } \\
\text { duration increasedfrom 20 minutes to } 30 \text { minutes through out the program }\end{array}$ \\
\hline Outcomes & $\begin{array}{l}\text { Muscle strength (1RM) } \\
\text { 1-minet walk (no data available for the PRT group) } \\
\text { VO2 max } \\
\text { Comments on adverse events: no }\end{array}$ \\
\hline Notes & Comparisions: PRT versus control, and PRT versus aerobic \\
\hline Risk of bias & Authors' judgement & Description \\
\hline Item & Unclear & B - Unclear \\
\hline Allocation concealment? &
\end{tabular}

Fatouros 2002

\begin{tabular}{|c|c|c|}
\hline Methods & \multicolumn{2}{|c|}{$\begin{array}{l}\text { RCT with } 3 \text { groups: PRT, control, and aerobic group } \\
\text { Method of randomisation: not reported } \\
\text { Assessor blinding: not reported } \\
\text { Participant blinding: not reported } \\
\text { Loss to follow-up: } 0 \\
\text { Intention-to-treat analysis: no } \\
\text { Post-program follow-up: no }\end{array}$} \\
\hline Participants & \multicolumn{2}{|c|}{$\begin{array}{l}\text { Location: Greece } \\
\mathrm{N}=8 \text { in each group } \\
\text { Sample: inactive elder men } \\
\text { Age: mean } 71.8 \text { years }(\mathrm{SD}=2.5) \\
\text { Inclusion criteria: completely inactive prior to the study, VO2 max below } 25 \mathrm{ml} / \mathrm{kg} / \mathrm{min} \text {, no } \\
\text { anemia, hepatic complications, thyroid disorders or kidney problems, normal blood } \\
\text { pressure } \\
\text { Exclusion criteria: respiratory complications or } \mathrm{BP}>240 / 110 \mathrm{mmHg} \text { during the exercise } \\
\text { test }\end{array}$} \\
\hline Interventions & \multicolumn{2}{|c|}{$\begin{array}{l}\text { PRT versus control and versus aerobic (cardiovascular training) } \\
\text { 1. PRT } \\
\text { Type of Ex: } 5 \text { UL/3 LL } \\
\text { Equipment: Universal resistance exercise machines } \\
\text { Intensity: Week 1-4 (55\%-60\% of } 1 \mathrm{RM}) \text {; Week } 5-8(60 \%-70 \% \text { of } 1 \mathrm{RM}) \text {; week } 9-12 \\
\text { (70\%-80\% of } 1 \mathrm{RM}) \text {; week 13-16 (80\% of } 1 \mathrm{RM}) \\
\text { Frequency: Ex3 } \\
\text { Reps/Sets: Week 1-4 (12-14/2); Week 5-8 (10-12/3); week 9-12 (8-10/3); week 13-16 (8/3) } \\
\text { Duration: } 16 \text { weeks } \\
\text { Setting: not reported } \\
\text { Supervision: not reported } \\
\text { Adherence: required the participants not miss more than } 4 \text { training sessions, } \\
\text { 2. Control Group: no exercise } \\
\text { 3. Cardiovascular training group: walking, jogging on a treadmill, the intensity was } \\
\text { increased through out the training }\end{array}$} \\
\hline Outcomes & \multicolumn{2}{|c|}{$\begin{array}{l}\text { Muscle strength ( } 1 \mathrm{RM}) \\
\text { Comments on adverse events: no }\end{array}$} \\
\hline Notes & \multicolumn{2}{|c|}{ Data from PRT and aerobic training group were compared } \\
\hline \multicolumn{3}{|l|}{ Risk of bias } \\
\hline Item & Authors' judgement & Description \\
\hline Allocation concealment? & Unclear & B - Unclear \\
\hline
\end{tabular}

Fatouros 2005

Methods

RCT with 3 groups: high intensity PRT, low intensity PRT, and control

Method of randomisation: not reported

Assessor blinding: not reported

Participant blinding: not reported 


\begin{tabular}{|c|c|c|}
\hline & \multicolumn{2}{|c|}{$\begin{array}{l}\text { Loss to follow-up: not reported } \\
\text { Intention-to-treat analysis: N/A } \\
\text { Post-program follow-up: yes }\end{array}$} \\
\hline Participants & \multicolumn{2}{|c|}{$\begin{array}{l}\text { Location: Greece } \\
\mathrm{N}=18(\mathrm{LI}) ; \mathrm{N}=20(\mathrm{HI}) ; \mathrm{N}=14 \text { (control) } \\
\text { Sample: inactive older adults } \\
\text { Age: } \mathrm{HI}-\text { mean } 72.4 \text { years }(\mathrm{SD}=3.5) \text {; LI-mean } 70.3 \text { years }(\mathrm{SD}=4.4) \\
\text { Inclusion criteria: at least } 65 \text { years of age, inactive before the study, free from health } \\
\text { problems and potentially damaging orthopedic, neuromuscular, metabolic, and } \\
\text { cardiovascular limitations } \\
\text { Exclusion criteria: not reported }\end{array}$} \\
\hline Interventions & \multicolumn{2}{|c|}{$\begin{array}{l}\text { PRT (high intensity and low intensity) versus control } \\
\text { 1. PRT } \\
\text { Type of Ex: } 5 \text { UL/3LL } \\
\text { Equipment: Universal machines } \\
\text { Intensity: low- } 55 \% \text { of 1RM; high- } 82 \% \text { of } 1 \text { RM } \\
\text { Frequency: Ex3 } \\
\text { Reps/Sets: low intensity: } 14-16 / 2 \text { (after week } 8,3 \text { sets), high intensity: } 6-8 / 2 \text { (after week } 8 \\
3 \text { sets) } \\
\text { Duration: } 24 \text { weeks } \\
\text { Setting: not reported } \\
\text { Supervision: Full } \\
\text { Adherence: } 98 \% \\
\text { 2. Control Group: not reported }\end{array}$} \\
\hline Outcomes & \multicolumn{2}{|c|}{$\begin{array}{l}\text { Muscle strength } \\
\text { VO2max } \\
\text { TUAG } \\
\text { Step climbing } \\
\text { 50-feet walk } \\
\text { Comments on adverse comments: yes }\end{array}$} \\
\hline Notes & \multicolumn{2}{|c|}{ Data from high intensity and low intensity PRT group were compared } \\
\hline \multicolumn{3}{|l|}{ Risk of bias } \\
\hline Item & Authors' judgement & Description \\
\hline Allocation concealment? & Unclear & B - Unclear \\
\hline
\end{tabular}

\section{Fiatarone 1994}

\begin{tabular}{|c|c|}
\hline Methods & $\begin{array}{l}\text { RCT, factorial design (comparison of nutritional supplements versus placebo not } \\
\text { considered here) } \\
\text { Method of randomisation: not reported } \\
\text { Assessor blinding: for some assessments, not for all } \\
\text { Participant blinding: no, but recreational activities offered to control group (? quantity) } \\
\text { Loss to follow-up: } 6 \text { total ( } 4 \text { in PRT and control groups) } \\
\text { Intention-to-treat analysis: yes } \\
\text { Post-program follow up: falls monitored median } 1.53 \text { years, max } 4.11 \text { years }\end{array}$ \\
\hline Participants & $\begin{array}{l}\text { Location: USA } \\
\mathrm{N}=51 \text { in PRT vs control } \\
\text { Sample: residents of a long term care facility for older people } \\
\text { Age: mean } 87.1 \text { years (SE 0.6) } \\
\text { Inclusion criteria: residential status, age over } 70 \text { years, ability to walk } 6 \mathrm{~m} \\
\text { Exclusion criteria: severe cognitive impairment; rapidly progressive or terminal illness, } \\
\text { acute illness or unstable chronic illness; myocardial infarction; fracture of a lower extremity } \\
\text { within the six months before the study; insulin dependent diabetes mellitus; on a weight- } \\
\text { loss diet or undergoing resistance training at the time of enrolment; tests of muscle strength } \\
\text { revealed a musculoskeletal or cardiovascular abnormality }\end{array}$ \\
\hline Interventions & $\begin{array}{l}\text { PRT versus control } \\
\text { 1. PRT } \\
\text { Type of Ex: } 2 \mathrm{LL} \\
\text { Equipment: weight training machines } \\
\text { Intensity: high }(80 \% \text { of } 1 \mathrm{RM}) \\
\text { Frequency: Ex } 3 \\
\text { Reps/sets: } 8 / 3 \\
\text { Program duration: } 10 \text { weeks } \\
\text { Setting: nursing home }\end{array}$ \\
\hline
\end{tabular}




\begin{tabular}{l|l|l}
\multicolumn{1}{l|}{} & $\begin{array}{l}\text { Supervision: full } \\
\text { Adherence: } 97 \% \\
\text { 2. Control Group: engaged in 3 activities of their choice offered by recreational therapy }\end{array}$ \\
\hline Outcomes & $\begin{array}{l}\text { Strength (1RM) } \\
\text { Gait speed } \\
\text { Stair climbing power } \\
\text { Anthropometric measurements } \\
\text { Physical activity (leg monitors) } \\
\text { Comments on adverse events: yes }\end{array}$ \\
\hline Notes & \multicolumn{2}{l}{} \\
\hline Risk of bias & Authors' judgement & Description \\
\hline Item & Unclear & B - Unclear \\
\hline Allocation concealment?
\end{tabular}

Fiatarone 1997

\begin{tabular}{|c|c|c|}
\hline Methods & \multicolumn{2}{|c|}{$\begin{array}{l}\text { RCT } \\
\text { Method of randomisation: not reported } \\
\text { Assessor blinding: no } \\
\text { Participant blinding: no, but control group received weekly phone calls } \\
\text { Loss to follow-up: } 4 \\
\text { Intention-to-treat analysis: no } \\
\text { Post-program follow up: no }\end{array}$} \\
\hline Participants & \multicolumn{2}{|c|}{$\begin{array}{l}\text { Location: USA } \\
\mathrm{N}=34 \\
\text { Sample: frail older people } \\
\text { Age: mean } 82 \text { years } \\
\text { Inclusion criteria: community dwelling older people, moderate to severe functional } \\
\text { impairment } \\
\text { Exclusion criteria: not reported }\end{array}$} \\
\hline Interventions & \multicolumn{2}{|c|}{$\begin{array}{l}\text { PRT versus control } \\
\text { 1. PRT } \\
\text { Type of Ex: } 11 \text { total to UL and LL } \\
\text { Equipment: arm and leg weights } \\
\text { Intensity: high } \\
\text { Frequency: Ex3 } \\
\text { Reps/Sets: not reported } \\
\text { Program Duration: } 16 \text { weeks } \\
\text { Setting: home-based } \\
\text { Supervision: low - } 2 \text { weeks of home instruction, then phone calls } \\
\text { Adherence: } 90 \% \\
\text { 2. Control Group: weekly phone calls }\end{array}$} \\
\hline Outcomes & \multicolumn{2}{|c|}{$\begin{array}{l}\text { Strength } \\
\text { Gait velocity } \\
\text { Self-reported activity level } \\
\text { Attitude towards Ageing on the PGC Morale Scale } \\
\text { Bed days } \\
\text { Falls } \\
\text { Health care visits } \\
\text { Comments on adverse events: no }\end{array}$} \\
\hline \multicolumn{3}{|l|}{ Notes } \\
\hline \multicolumn{3}{|l|}{ Risk of bias } \\
\hline Item & Authors' judgement & Description \\
\hline Allocation concealment? & Unclear & B - Unclear \\
\hline
\end{tabular}


Fielding 2002

\begin{tabular}{|c|c|c|}
\hline Methods & \multicolumn{2}{|c|}{$\begin{array}{l}\text { RCT } \\
\text { Method of randomisation: not reported } \\
\text { Assessor blinding: not reported } \\
\text { Participant blinding: not reported } \\
\text { Loss to follow-up: } 3 / 15 \text { in high velocity group, } 2 / 15 \text { in low velocity group } \\
\text { Intention-to-treat analysis: no } \\
\text { Post-program follow up: no }\end{array}$} \\
\hline Participants & \multicolumn{2}{|c|}{$\begin{array}{l}\text { Location: USA } \\
\mathrm{N}=30 \text { ( } 15 \text { in high velocity, } 15 \text { in low velocity) } \\
\text { Sample: community dwelling elderly with self reported disability } \\
\text { Age: high velocity-mean } 73.2 \text { years }(\mathrm{SD}=1.2) \text {; low velocity-mean } 72.1 \text { years }(\mathrm{SD}=1.3) \\
\text { Inclusion criteria: at least of } 65 \text { years of age, community dwelling, could walk with or } \\
\text { without an assistive device, reported } 2 \text { or more deficits on the physical function subscale of } \\
\text { SF-36 } \\
\text { Exclusion criteria: acute or terminal illness, myocardial infarction in the past } 6 \text { months, } \\
\text { unstable cardiovascular disease or other medical condition, upper extremities or lower } \\
\text { extremities fractures in the past } 6 \text { months, amputations, cognitive impairments, current } \\
\text { participations in regular exercise sessions, and unwilling to be randomised }\end{array}$} \\
\hline Interventions & \multicolumn{2}{|c|}{$\begin{array}{l}\text { PRT (high velocity versus low velocity) } \\
\text { Type of Ex: } 2 \text { LL, leg press \& knee extension } \\
\text { Equipment: machines (Keiser pneumatic resistance training equipment) } \\
\text { Intensity: high velocity group- } 70 \% \text { of } 1 \mathrm{RM} \text {, extension as fast as possible during concentric } \\
\text { phase, then maintain full extension for } 1 \text { second, and eccentric phase of each repetition over } \\
2 \text { seconds; low velocity group- extension concentric phase, maintain full extension, and } \\
\text { eccentric phase of each repetition } 2,1,2 \text { seconds } \\
\text { Frequency: Ex3 } \\
\text { Reps/Sets: } 8 / 3 \\
\text { Duration: } 16 \text { weeks } \\
\text { Setting: human physiology lab } \\
\text { Supervision: exercise trainers } \\
\text { Adherence: } 95 \% \text { for high velocity group, } 94 \% \text { for low velocity group }\end{array}$} \\
\hline Outcomes & \multicolumn{2}{|c|}{$\begin{array}{l}\text { Muscle strength } \\
\text { Chair rise } \\
\text { Stair climbing } \\
\text { Comments on adverse events: yes }\end{array}$} \\
\hline Notes & \multicolumn{2}{|c|}{ No reported results can be pooled (missing $\mathrm{M}$ and SD for each group) } \\
\hline \multicolumn{3}{|l|}{ Risk of bias } \\
\hline Item & Authors' judgement & Description \\
\hline Allocation concealment? & Unclear & B - Unclear \\
\hline
\end{tabular}

Flynn 1999

\begin{tabular}{l|l}
\hline Methods & RCT \\
Method of randomisation: not reported \\
Assessor blinding: no \\
Participant blinding: no \\
Loss to follow-up: not reported \\
Intention-to-treat analysis: no \\
Post-program follow up: no
\end{tabular}

Cochrane Database Syst Rev. Author manuscript; available in PMC 2015 February 11. 


\begin{tabular}{|c|c|c|}
\hline & \multicolumn{2}{|c|}{$\begin{array}{l}\text { surgery within the previous } 3 \text { months, caffeine consumption in excess of four cups of } \\
\text { coffee per day, adequate flexibility and mobility (screened with performance tests) }\end{array}$} \\
\hline Interventions & \multicolumn{2}{|c|}{$\begin{array}{l}\text { PRT versus control } \\
\text { 1. PRT } \\
\text { Type of Ex: } 8 \text { LLEquipment: not reportedIntensity: high ( } 70-80 \% \text { of } 1 \text { RM) } \\
\text { Frequency: Ex3 } \\
\text { Reps/sets: } 8 / 3 \\
\text { Duration: } 10 \text { weeks } \\
\text { Setting: gym } \\
\text { Supervision: not reported } \\
\text { Adherence: not reported } \\
\text { 2. Control Group: asked to maintain their normal activity level }\end{array}$} \\
\hline Outcomes & \multicolumn{2}{|c|}{$\begin{array}{l}\text { Strength (1RM - ? data collected for controls) } \\
\text { Comments on adverse events: no }\end{array}$} \\
\hline \multicolumn{3}{|l|}{ Notes } \\
\hline \multicolumn{3}{|l|}{ Risk of bias } \\
\hline Item & Authors' judgement & Description \\
\hline Allocation concealment? & Unclear & B - Unclear \\
\hline
\end{tabular}

Foley 2003

\begin{tabular}{|c|c|c|}
\hline Methods & \multicolumn{2}{|c|}{$\begin{array}{l}\text { RCT } \\
\text { Method of randomisation: a computer generated randomisation list generated by person } \\
\text { external to the study as was managed by an external department } \\
\text { Assessor blinding: yes } \\
\text { Participant blinding: not reported } \\
\text { Loss to follow-up: } 3 / 35 \text { in the gym group, } 3 / 35 \text { in the control group } \\
\text { Intention-to-treat analysis: yes } \\
\text { Post-program follow up: no }\end{array}$} \\
\hline Participants & \multicolumn{2}{|c|}{$\begin{array}{l}\text { Location: Australia } \\
\mathrm{N}=70 \text { ( } 35 \text { in each group) } \\
\text { Sample: community living adults with OA of the hip or knee } \\
\text { Age: mean } 69.8 \text { years ( } \mathrm{SD}=9.2) \\
\text { Inclusion criteria: read, write, and speak English, could give informed consent, and provide } \\
\text { transport to attend the training sessions } \\
\text { Exclusion criteria: had received physiotherapy or hydrotherapy in the past } 6 \text { weeks, } \\
\text { attending community exercise classes; joint replacement surgery within the past } 12 \text { months } \\
\text { or the next } 12 \text { weeks; and cognitive impairment }\end{array}$} \\
\hline Interventions & \multicolumn{2}{|c|}{$\begin{array}{l}\text { PRT versus control } \\
\text { 1. PRT } \\
\text { Type of Ex: } 1 \mathrm{UE} / 4 \mathrm{LL} \\
\text { Equipment: weighted gaiters } \\
\text { Intensity: } 10 \mathrm{RM} \\
\text { Frequency: Ex3 } \\
\text { Reps/Sets: not reported } \\
\text { Duration: } 6 \text { weeks } \\
\text { Setting: gym } \\
\text { Supervision: not reported } \\
\text { Adherence: } 75 \% \\
\text { 2. Control Group: telephone calls to record any changes in their condition drug use or } \\
\text { injuries }\end{array}$} \\
\hline Outcomes & \multicolumn{2}{|c|}{$\begin{array}{l}\text { Primary: SF-12, Adelaide Activities profile, WOMAC } \\
\text { Secondary: muscle strength, Arthritis Self-Efficacy Questionnaire } \\
\text { Comments on adverse events: yes }\end{array}$} \\
\hline \multicolumn{3}{|l|}{ Notes } \\
\hline \multicolumn{3}{|l|}{ Risk of bias } \\
\hline Item & Authors' judgement & Description \\
\hline Allocation concealment? & Yes & A - Adequate \\
\hline
\end{tabular}


Frontera 2003

\begin{tabular}{|c|c|c|}
\hline Methods & \multicolumn{2}{|c|}{$\begin{array}{l}\text { RCT } \\
\text { Method of randomisation: not reported } \\
\text { Assessor blinding: not reported } \\
\text { Participant blinding: not reported } \\
\text { Loss to follow-up: } 0 \\
\text { Intention-to-treat analysis: N/A } \\
\text { Post-program follow up: no }\end{array}$} \\
\hline Participants & \multicolumn{2}{|c|}{$\begin{array}{l}\text { Location: USA } \\
\mathrm{N}=14 \text { ( } 7 \text { in each group) } \\
\text { Sample: community-dwelling healthy women } \\
\text { Age: mean } 73.7 \text { years }(\mathrm{SD}=3.4) \\
\text { Inclusion criteria: not involved in regular exercise } \\
\text { Exclusion criteria: had conditions that could interfere with neuromuscular function }\end{array}$} \\
\hline Interventions & \multicolumn{2}{|c|}{$\begin{array}{l}\text { PRT versus control } \\
\text { 1. PRT } \\
\text { Type of Ex: knee extensors/flexors, each leg was trained separately } \\
\text { Equipment: Keiser Sports Health Equipment } \\
\text { Intensity: ( } 80 \% \text { of } 1 \text { RM) } \\
\text { Frequency: Ex3 } \\
\text { Reps/Sets: } 8 / 4 \\
\text { Duration: } 12 \text { weeks } \\
\text { Setting: not reported } \\
\text { Supervision: not reported } \\
\text { Adherence: } 98 \% \\
\text { 2. Control Group: not reported }\end{array}$} \\
\hline Outcomes & \multicolumn{2}{|c|}{$\begin{array}{l}\text { Muscle strength (1RM, isokinetic strength of knee extension) } \\
\text { Comments on adverse events: no }\end{array}$} \\
\hline \multicolumn{3}{|l|}{ Notes } \\
\hline \multicolumn{3}{|l|}{ Risk of bias } \\
\hline Items & Authors' judgement & Description \\
\hline Allocation concealment? & Unclear & B - Unclear \\
\hline
\end{tabular}

Galvao 2005

\begin{tabular}{l|l}
\hline Methods & RCT \\
& Method of randomisation: not reported \\
& Assessor blinding: not reported \\
& Participant blinding: not reported \\
& Loss to follow-up: 4/16 in 1-set PRT group \\
& Intention-to-treat analysis: no \\
& Post-program follow up: no \\
\hline Participants & Location: Australia \\
& $\mathrm{N}=16$ for each group \\
& Sample: community dwelling elderly \\
Age: 1-set PRT group-mean 68.9 years (SD=4.8); 3-set PRT group-mean 69.7 years \\
(SD=4.4) \\
Inclusion criteria: not reported \\
Exclusion criteria: musculoskeletal, cardiovascular, or neurological disorder; PRT in the \\
& previous 12 months, inability to undertake upper and lower limb ex. or walk less than 100 \\
& meters; unwilling to undertake 20 weeks of training \\
\hline
\end{tabular}




\begin{tabular}{|c|c|c|}
\hline Interventions & \multicolumn{2}{|c|}{$\begin{array}{l}\text { PRT (3-set versus 1-set) } \\
\text { Type of Ex: 4UL/3LL } \\
\text { Equipment: Strength Fitness Equipment } \\
\text { Intensity: } 8 \text { RM } \\
\text { Frequency: Ex2 } \\
\text { Reps/Sets: } 8 / 3 \text { versus } 8 / 1 \\
\text { Duration: } 20 \text { weeks } \\
\text { Setting: not reported (Gym?) } \\
\text { Supervision: full } \\
\text { Adherence: All completed } 40 \text { training sessions (make-up sessions were provided) }\end{array}$} \\
\hline Outcomes & \multicolumn{2}{|l|}{$\begin{array}{l}\text { Muscle strength (1 RM) } \\
\text { Chair rise } \\
\text { 6-minute walk } \\
\text { Stair climbing } \\
\text { 400-m-walk } \\
\text { Comments on adverse events: yes }\end{array}$} \\
\hline Notes & \multicolumn{2}{|l|}{ 3-set PRT versus 1-set PRT } \\
\hline \multicolumn{3}{|l|}{ Risk of bias } \\
\hline Item & Authors' judgement & Description \\
\hline Allocation concealment? & Unclear & B - Unclear \\
\hline
\end{tabular}

\section{Hagerman 2000}

\begin{tabular}{|c|c|c|}
\hline Methods & \multicolumn{2}{|c|}{$\begin{array}{l}\text { RCT } \\
\text { Method of randomisation: not reported } \\
\text { Assessor blinding: no } \\
\text { Participant blinding: no } \\
\text { Loss to follow-up: } 4 \\
\text { Intention-to-treat analysis: no } \\
\text { Post-program follow up: no }\end{array}$} \\
\hline Participants & \multicolumn{2}{|c|}{$\begin{array}{l}\text { Location: USA } \\
\mathrm{N}=22 \\
\text { Sample: untrained but physically active older men } \\
\text { Age: mean } 63.7 \text { years } \\
\text { Inclusion criteria: male, aged } 60-75 \text {, physically active but not engaged in resistance training } \\
\text { Exclusion criteria: not reported }\end{array}$} \\
\hline Interventions & \multicolumn{2}{|l|}{$\begin{array}{l}\text { PRT versus control } \\
\text { 1. PRT } \\
\text { Type of Ex: } 3 \text { LL } \\
\text { Equipment: machines } \\
\text { Intensity: high ( } 85-90 \% \text { of 1RM) } \\
\text { Frequency: Ex2 } \\
\text { Reps/Sets: } 6-8 / 3 \\
\text { Program Duration: } 16 \text { weeks } \\
\text { Setting: gym } \\
\text { Supervision: full } \\
\text { Adherence: } 100 \% \\
\text { 2. Control Group: not reported }\end{array}$} \\
\hline Outcomes & \multicolumn{2}{|l|}{$\begin{array}{l}\text { Strength (1RM) } \\
\text { Peak aerobic capacity } \\
\text { Comments on adverse events: no }\end{array}$} \\
\hline \multicolumn{3}{|l|}{ Notes } \\
\hline \multicolumn{3}{|l|}{ Risk of bias } \\
\hline Item & Authors' judgement & Description \\
\hline Allocation concealment? & Unclear & B - Unclear \\
\hline
\end{tabular}


Harris 2004

\begin{tabular}{l|l|l}
\hline Methods & \multicolumn{1}{l}{ RCT } \\
Method of randomisation: not reported \\
Assessor blinding: not reported \\
Participant blinding: not reported \\
Loss to follow-up: 2/19 in LI (2 sets of 15 RM); 1/18 in HI (4 sets of 6 RM) \\
Intention-to-treat analysis: no \\
Post-program follow up: no
\end{tabular}

Haykowsky 2000

\begin{tabular}{l|l}
\hline Methods & RCT \\
Method of randomisation: matched according to combined leg press and bench press \\
strength scores, then randomly assigned \\
Assessor blinding: no \\
Participant blinding: no \\
Loss to follow-up: 4 \\
Intention-to-treat analysis: no \\
Post-program follow up: no \\
\hline Participants & Location: Canada \\
& No 22 \\
& Sample: healthy older men \\
& Age: mean 68 years (SD 3) \\
& Inclusion criteria: aged 61 -76; no clinical evidence of cardiovascular disease or \\
& hypertension; normal resting electrocardiograms; normal electrocardiographic response to \\
& graded treadmill exercise; not requiring or using cardiovascular medications; no regular \\
& participation in endurance or RT; absence of cerebrovascular or orthopaedic disability that \\
& would limit RT \\
& Exclusion criteria: not reported \\
\hline Interventions & PRT versus control \\
& 1. PRT \\
& Type of Ex: 5 UL, 3LL \\
& Equipment: machines \\
& Intensity: $60-80 \%$ of 1 RM \\
& Frequency: Ex3 \\
& Reps/Sets: $3 / 10$ \\
& Duration: 16 weeks Setting: gym \\
\hline
\end{tabular}




\begin{tabular}{l|l|l}
\multicolumn{2}{l}{} & $\begin{array}{l}\text { Supervision: not reported } \\
\text { Adherence: } 97 \% \text { attended } \\
\text { 2. Control Group: continued normal activities }\end{array}$ \\
\hline Outcomes & $\begin{array}{l}\text { Strength (1RM) } \\
\text { Comments on adverse events: yes }\end{array}$ \\
\hline Notes & Authors' judgement & Description \\
\hline Risk of bias & B - Unclear \\
\hline Item & Unclear &
\end{tabular}

\section{Haykowsky 2005}

\begin{tabular}{|c|c|c|}
\hline Methods & \multicolumn{2}{|c|}{$\begin{array}{l}\text { RCT with } 3 \text { groups: PRT, control, and aerobic group } \\
\text { Method of randomisation: not reported } \\
\text { Assessor blinding: not reported } \\
\text { Participant blinding: yes for echocadiograms } \\
\text { Loss to follow-up: no } \\
\text { Intention-to-treat analysis: no } \\
\text { Post-program follow up: no }\end{array}$} \\
\hline Participants & \multicolumn{2}{|c|}{$\begin{array}{l}\text { Location: Canada } \\
\mathrm{N}=\text { ? (did not report sample size for each group) } \\
\text { Sample: women } \\
\text { Age: mean = 70 years (SD }=4 \text { ) } \\
\text { Inclusion criteria: a) no clinical evidence of cardiovascular disease; b) normal resting } \\
\text { electrocardiogram (ECG); c) normal ECG response to graded exercise; d) no requirement } \\
\text { or use of cardiovascular medications; e) no regular participation in AT and/or ST; and f) } \\
\text { absence of any cerebrovascular or orthopedic disability that would limit exercise training. } \\
\text { Exclusion criteria: not reported }\end{array}$} \\
\hline Interventions & \multicolumn{2}{|c|}{$\begin{array}{l}\text { PRT versus control and versus aerobic } \\
\text { 1. PRT } \\
\text { Type of Ex: } 3 \mathrm{LL} / 5 \mathrm{UL} \\
\text { Equipment: not reported } \\
\text { Intensity: } 50 \% \text { of } 1 \mathrm{RM} \text { and increased } 2.5 \% \text { per week until } 75 \% \text { of } 1 \mathrm{RM} \\
\text { Frequency: Ex3 } \\
\text { Reps/Sets: } 10 / 2 \\
\text { Duration: } 12 \text { weeks } \\
\text { Setting: not reported (Gym?) } \\
\text { Supervision: full } \\
\text { Adherence: not reported } \\
\text { 2. Control group: continue normal daily activities } \\
\text { 3. Aerobic training: cycle exercise at } 60-80 \% \text { of heart rate reserve }\end{array}$} \\
\hline Outcomes & \multicolumn{2}{|c|}{$\begin{array}{l}\text { Muscle strength } \\
\text { Absolute VO2peak } \\
\text { Comments on adverse events: yes }\end{array}$} \\
\hline Notes & \multicolumn{2}{|c|}{$\begin{array}{l}\text { sample size for each group was not reported. } \\
12 \text { weeks of strength training is as effective as } 12 \text { weeks of aerobic training for increasing } \\
\text { relative VO2peak, however, strength training is more effective than aerobic training for } \\
\text { improving overall muscle strength }\end{array}$} \\
\hline \multicolumn{3}{|l|}{ Risk of bias } \\
\hline Item & Authors' judgement & Description \\
\hline Allocation concealment? & Unclear & B - Unclear \\
\hline
\end{tabular}

\section{Hennessey 2001} \begin{tabular}{l|l} 
Methods & $\begin{array}{l}\text { RCT trial with } 4 \text { groups: PRT alone, growth hormone treatment alone, PRT and growth } \\
\text { hormone treatment and control. Only PRT alone and control are included in this review }\end{array}$
\end{tabular} Method of randomisation: not reported 


\begin{tabular}{|c|c|c|}
\hline & \multicolumn{2}{|c|}{$\begin{array}{l}\text { Assessor blinding: no } \\
\text { Participant blinding: no } \\
\text { Loss to follow-up: not reported } \\
\text { Intention-to-treat analysis: no } \\
\text { Post-program follow up: no }\end{array}$} \\
\hline Participants & \multicolumn{2}{|c|}{$\begin{array}{l}\text { Location: USA } \\
\text { N = } 16 \text { in PRT and control } \\
\text { Sample: frail older people } \\
\text { Age: mean } 71.3 \text { years (SD 4.5) } \\
\text { Inclusion criteria: frail which was defined as scoring between } 12 \text { and } 28 \text { on Reuben's } \\
\text { Physical Performance Test; } \\
\text { Exclusion criteria: medical conditions (cancer, heart disease, diabetes, recent fracture, } \\
\text { carpal tunnel syndrome) that would interfere with administration of growth hormone or the } \\
\text { performance of regular exercise } 3 \text { times per week; did not expect to spend a year in Rhode } \\
\text { Island; their doctor convinced them not to participate for medical reasons or otherwise; } \\
\text { unwilling to inject the drug and be randomised to exercise or no exercise }\end{array}$} \\
\hline Interventions & \multicolumn{2}{|c|}{$\begin{array}{l}\text { PRT versus control } \\
\text { 1. PRT } \\
\text { Type of Ex: } 11 \text { exercises (UL \& LL) } \\
\text { Equipment: ankle and wrist weights and exercise equipment } \\
\text { Intensity: increased from } 20 \% \text { to } 95 \% \text { of } 1 \text { RM-most training was at high intensity } \\
\text { Frequency: Ex } 3 \\
\text { Reps/Sets: } 8 / 3 \\
\text { Duration: } 25 \text { weeks } \\
\text { Setting: gym (in study facilities or local community centers) } \\
\text { Supervision: Full } \\
\text { Adherence: not reported } \\
\text { 2. Control Group: not reported }\end{array}$} \\
\hline Outcomes & \multicolumn{2}{|c|}{$\begin{array}{l}\text { Strength (isokinetic dynamometry) } \\
\text { Physical Performance Test (PPT) } \\
\text { Comments on adverse events: no }\end{array}$} \\
\hline \multicolumn{3}{|l|}{ Notes } \\
\hline \multicolumn{3}{|l|}{ Risk of bias } \\
\hline Item & Authors' judgement & Description \\
\hline Allocation concealment? & Unclear & B - Unclear \\
\hline
\end{tabular}

Hepple 1997

\begin{tabular}{l|l}
\hline Methods & RCT \\
& Method of randomisation: not reported \\
Assessor blinding: no \\
Participant blinding: no \\
Loss to Follow-up: 1 \\
Intention-to-treat analysis: no \\
Post-program follow up: no
\end{tabular}


3. Aerobic Training Group: intermittent walking on treadmill until pain subsided, 3 times per week

\begin{tabular}{l|l|l} 
& $\begin{array}{l}\text { 3. Aerobic Training Group: intermittent walking on treadmill until pain subsided, } 3 \text { times } \\
\text { per week }\end{array}$ \\
\hline Outcomes & $\begin{array}{l}\text { Peak VO2 } \\
\text { Comments on adverse events: no }\end{array}$ \\
\hline Notes & Data from PRT and aerobic training group were compared \\
\hline Risk of bias & Authors' judgement & Description \\
\hline Item & Unclear & B - Unclear \\
\hline Allocation concealment? & &
\end{tabular}

\section{Hiatt 1994}

\begin{tabular}{|c|c|}
\hline Methods & $\begin{array}{l}\text { RCT with } 3 \text { groups: PRT, walking (aerobic training) and control } \\
\text { Method of randomisation: not reported } \\
\text { Assessor blinding: no } \\
\text { Participant blinding: no } \\
\text { Loss to follow-up: } 2 \\
\text { Intention-to-treat analysis: no } \\
\text { Post-program follow up: no }\end{array}$ \\
\hline Participants & $\begin{array}{l}\text { Location: USA } \\
\mathrm{N}=29 \text { (19 in PRT versus control) } \\
\text { Sample: people who have peripheral arterial disease and intermittent claudication Age: } \\
\text { mean } 67 \text { years } \\
\text { Inclusion criteria: intermittent claudication (disabling but stable for } 3 \text { months prior to } \\
\text { enrolment); peripheral arterial disease } \\
\text { Exclusion criteria: leg pain at rest, ischemic ulceration, gangrene, unable to walk on the } \\
\text { treadmill at a speed of at least } 2 \mathrm{mph} \text {; exercise capacity limited by symptoms of angina, } \\
\text { congestive heart failure, chronic obstructive pulmonary disease, arthritis; diabetes; vascular } \\
\text { surgery or angioplasty in the past year }\end{array}$ \\
\hline Interventions & $\begin{array}{l}\text { PRT versus control and versus aerobic } \\
\text { 1. PRT } \\
\text { Type of Ex: 5LL } \\
\text { Equipment: cuff weights } \\
\text { Intensity: high (6RM) } \\
\text { Frequency: Ex3 } \\
\text { Reps/Sets: } 6 / 3 \\
\text { Duration: } 12 \text { weeks } \\
\text { Setting: gym } \\
\text { Supervision: full } \\
\text { Adherence: not reported } \\
\text { 2. Control Group: usual level of activity } \\
\text { 3. Aerobic Training Group: intermittent walking on treadmill until pain subsided, } 3 \text { times } \\
\text { per week }\end{array}$ \\
\hline Outcomes & $\begin{array}{l}\text { Strength (Cybex dynamometer) } \\
\text { Peak Vo2 } \\
\text { Comments on adverse events: no }\end{array}$ \\
\hline Notes & Data from PRT and aerobic training group were compared \\
\hline \multicolumn{2}{|l|}{ Risk of bias } \\
\hline Item & Authors' judgement \\
\hline Allocation concealment? & B - Unclear \\
\hline
\end{tabular}

\section{Hortobagyi 2001}

\begin{tabular}{l|l}
\hline Methods & RCT with 3 groups: High-intensity PRT, Low-intensity PRT and Control \\
& Method of randomisation: not reported \\
& Assessor blinding: no \\
& Participant blinding: no \\
Loss to follow-up: 3
\end{tabular}

Cochrane Database Syst Rev. Author manuscript; available in PMC 2015 February 11. 


\begin{tabular}{|c|c|c|}
\hline & \multicolumn{2}{|c|}{$\begin{array}{l}\text { Intention-to-treat analysis: no } \\
\text { Post-program follow up: no }\end{array}$} \\
\hline Participants & \multicolumn{2}{|c|}{$\begin{array}{l}\text { Location: USA } \\
\mathrm{N}=30 \text { total ( } 20 \text { in high-intensity PRT versus control) } \\
\text { Sample: healthy older people } \\
\text { Age: mean } 72 \text { years (SD } 4.7) \\
\text { Inclusion criteria: older men and women, healthy, had not exercised more than once a week } \\
\text { in the previous } 3 \text { years, approval of GP } \\
\text { Exclusion criteria: more than two risk factors for coronary artery disease; a history of falls, } \\
\text { osteoporosis, osteoarthritis, or orthopaedic or neurological conditions (i.e. stroke); took } \\
\text { medications that cause dizziness or slow movement; smoked; had a BMI greater than } 28 \\
\mathrm{~kg} / \mathrm{m} \text { squared; blood pressure greater than } 140 / 90 \mathrm{mmHg} \text { or a heart condition }\end{array}$} \\
\hline Interventions & \multicolumn{2}{|c|}{$\begin{array}{l}\text { PRT (high intensity and low intensity) versus control } \\
\text { 1. PRT } \\
\text { Type of Ex: 1 LL } \\
\text { Equipment: machine } \\
\text { Intensity: HI - } 80 \% \text { 1RM; LI - 40\% 1RM } \\
\text { Frequency: Ex3 } \\
\text { Reps/Sets: HI: 4-6/5; LI: 8-12/5 } \\
\text { Duration: } 10 \text { weeks } \\
\text { Setting: gym } \\
\text { Supervision: not reported } \\
\text { Adherence: } 98 \% \\
\text { 2. Control Group: not reported }\end{array}$} \\
\hline Outcomes & \multicolumn{2}{|c|}{$\begin{array}{l}\text { Force accuracy and steadiness } \\
\text { Maximal strength (Cybex) } \\
\text { Comments on adverse events: no (not identified as such) }\end{array}$} \\
\hline Notes & \multicolumn{2}{|c|}{ Date from high intensity PRT and low intensity PRT were compared } \\
\hline \multicolumn{3}{|l|}{ Risk of bias } \\
\hline Item & Authors' judgement & Description \\
\hline Allocation concealment? & Unclear & B - Unclear \\
\hline
\end{tabular}

Hruda 2003

\begin{tabular}{|c|c|}
\hline Methods & $\begin{array}{l}\text { RCT } \\
\text { Method of randomisation: in a lottery format, } 2: 1 \text { ratio } \\
\text { Assessor blinding: not reported } \\
\text { Participant blinding: not reported } \\
\text { Loss to follow-up: } 2 / 20 \text { in PRT group, } 3 / 10 \text { in control group } \\
\text { Intention-to-treat analysis: no } \\
\text { Program-post follow up: no }\end{array}$ \\
\hline Participants & $\begin{array}{l}\text { Location: Canada } \\
\mathrm{N}=30 \text { ( } 20 \text { in PRT) } \\
\text { Sample: frail older adults (residents of a long-term care facility) } \\
\text { Age: mean } 84.9 \text { years }(\mathrm{SD}=4.8) \\
\text { Inclusion criteria: able to follow directions and walk across the room; no recent history of } \\
\text { cardiovascular, cerebrovascular, respiratory, systemic, muscular, or uncontrolled metabolic } \\
\text { disease } \\
\text { Exclusion criteria: not reported }\end{array}$ \\
\hline Interventions & $\begin{array}{l}\text { PRT versus control } \\
\text { 1. PRT } \\
\text { Type of Ex : LLs } \\
\text { Equipment: Therabands } \\
\text { Intensity: Increasing repetitions, sets, and speed, } 20 \text { minutes class progressed to an hour } \\
\text { Frequency: Ex3 } \\
\text { Reps/Sets: } 4-8 / 1 \\
\text { Duration: } 10 \text { weeks } \\
\text { Setting: long-term care facility } \\
\text { Supervision: not reported } \\
\text { Adherence: } 71 \% \\
\text { 2. Control Group: maintain usual daily activities }\end{array}$ \\
\hline Outcomes & $\begin{array}{l}\text { TUAG } \\
\text { Chair stand }\end{array}$ \\
\hline
\end{tabular}




\begin{tabular}{l|l|l}
\multicolumn{2}{l|}{} & $\begin{array}{l}\text { 6-meter walk } \\
\text { Muscle strength } \\
\text { Comments on adverse events: yes }\end{array}$ \\
\hline Notes & \multicolumn{2}{l}{} \\
\hline Risk of bias & Authors' judgement & Description \\
\hline Item & B - Unclear \\
\hline Allocation concealment? & Unclear &
\end{tabular}

Hunter 2001

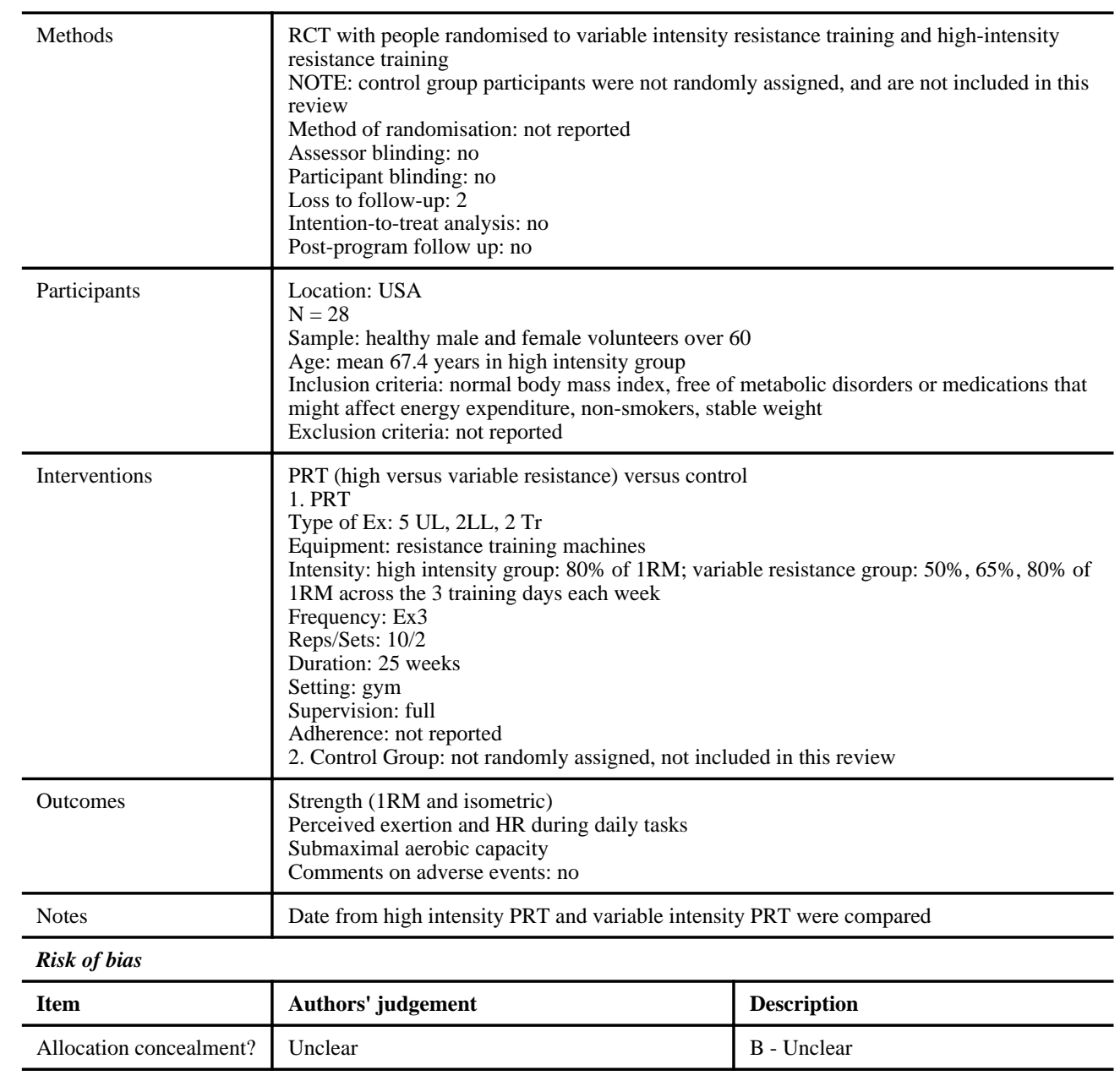

Izquierdo 2004

\begin{tabular}{l|l}
\hline Methods & RCT \\
& Method of randomisation: not reported \\
& Assessor blinding: not reported \\
& Participant blinding: not reported \\
& Loss to follow-up: 0 \\
& Intention-to-treat analysis: N/A \\
& Post-program follow-up: no \\
\hline
\end{tabular}




\begin{tabular}{l|l} 
Participants & Location: Spain \\
N $=10$ in PRT, $\mathrm{N}=11$ in endurance training \\
Sample: healthy men \\
Age: mean 64.8 years (SD = 2.6) \\
Inclusion criteria: had not participated in regular resistance/endurance training or \\
competitive sports for the last 5 years \\
Exclusion criteria: cardiovascular, neuromuscular, arthritic, pulmonary, other debilitating \\
diseases
\end{tabular}

Jette 1996

\begin{tabular}{|c|c|}
\hline Methods & $\begin{array}{l}\text { RCT } \\
\text { Method of randomisation: not reported } \\
\text { Assessor blinding: yes } \\
\text { Participant blinding: no } \\
\text { Loss to follow-up: } 9 \\
\text { Intention-to-treat analysis: no } \\
\text { Post-program follow up: no }\end{array}$ \\
\hline Participants & $\begin{array}{l}\text { Location: USA } \\
\mathrm{N}=102 \\
\text { Sample: non-disabled community-dwelling older people } \\
\text { Age: mean } 72 \text { years } \\
\text { Inclusion criteria: non-disabled, community dwelling, aged } 65 \text { and over; clearance from GP } \\
\text { Exclusion criteria: significant coronary artery disease, angina, congestive heart failure, } \\
\text { myocardial infarction, cardiac surgery, or significant or new onset rhythm disturbance; } \\
\text { neurological disorders with residual deficit; renal failure requiring dialysis; recent cancer } \\
\text { with active chemotherapy or radiation treatment; uncontrolled hypertension, diabetes or } \\
\text { seizure disorders; recent fracture; legal blindness; major mobility limitations; failed } \\
\text { exercise safety evaluation (i.e. resting heart rate greater than } 120 \text { bpm, resting systolic/ } \\
\text { diastolic great than } 165 / 100 \text { or less than } 80 / 50 \text {, or failed treadmill test; English speaking; } \\
\text { have access to a VCR or willing and able to use one provided by the study }\end{array}$ \\
\hline Interventions & $\begin{array}{l}\text { PRT versus control } \\
\text { 1.PRT } \\
\text { Type of Ex: } 10 \text { exercises to the UL, LL and Tr } \\
\text { Equipment: Theraband } \\
\text { Intensity: low to moderate } \\
\text { Frequency: Ex } 3 \\
\text { Reps/Sets: } 10 / 1 \\
\text { Duration: } 12-15 \text { weeks } \\
\text { Setting: home-based } \\
\text { Supervision: low } \\
\text { Adherence: mean } 58 \% \text {, median } 71 \% \\
\text { 2. Control Group: continued with normal activities, on a waiting list for exercises }\end{array}$ \\
\hline Outcomes & Strength (Cybex isokinetic dynamometer) \\
\hline
\end{tabular}




\begin{tabular}{l|l|l}
\multicolumn{2}{l|}{} & $\begin{array}{l}\text { Psychological well-being (Profile of Mood States battery) } \\
\text { SF-36 } \\
\text { Comments on adverse events: no (not identified as such) }\end{array}$ \\
\hline Notes & Authors' judgement & Description \\
\hline Risk of bias & B - Unclear \\
\hline Item & Unclear &
\end{tabular}

Jette 1999

\begin{tabular}{|c|c|c|}
\hline Methods & \multicolumn{2}{|c|}{$\begin{array}{l}\text { RCT } \\
\text { Method of randomisation: randomly permuted blocks by size } 4 \text {, assigned by a staff member } \\
\text { not involved in data collection } \\
\text { Assessor blinding: yes } \\
\text { Participant blinding: no } \\
\text { Loss to follow-up: } 15 \text { at } 6 \text { months } \\
\text { Intention-to-treat analysis: no } \\
\text { Post-program follow up: no, but } 6 \text { months of exercise }\end{array}$} \\
\hline Participants & \multicolumn{2}{|c|}{$\begin{array}{l}\text { Location: USA } \\
\mathrm{N}=215 \\
\text { Sample: older adults with disabilities } \\
\text { Age: PRT group mean } 75.4 \text { years (SD 7.4) } \\
\text { Inclusion criteria: aged } 60 \text { years or over; limitations in at least one of } 9 \text { functional areas } \\
\text { Exclusion criteria: medical history that contained current treatment for cancer, kidney } \\
\text { disease requiring dialysis, recent fracture, uncontrolled diabetes or seizures, regular use of a } \\
\text { wheelchair, current rehabilitation care, current fainting or dizzy spells, sudden loss of } \\
\text { coordination or legal blindness or physician identified contraindications to exercise }\end{array}$} \\
\hline Interventions & \multicolumn{2}{|c|}{$\begin{array}{l}\text { PRT versus control } \\
\text { 1. PRT } \\
\text { Type of Ex: } 11 \text { exercises to UL, LL and Tr } \\
\text { Equipment: Theraband } \\
\text { Intensity: low-moderate } \\
\text { Frequency: Ex3 } \\
\text { Reps/Sets: } 10 \text { reps } \\
\text { Duration: } 6 \text { months } \\
\text { Setting: home-based } \\
\text { Supervision: low } \\
\text { Adherence: } 89 \% \\
\text { 2. Control Group: on a waiting list }\end{array}$} \\
\hline Outcomes & \multicolumn{2}{|c|}{$\begin{array}{l}\text { Strength (hand-held dynamometer) } \\
\text { Balance (functional reach, unilateral stance, tandem stance) } \\
\text { TUAG } \\
\text { Profile of Mood States } \\
\text { Sickness Impact Profile } 68 \\
\text { Comments on adverse events: yes }\end{array}$} \\
\hline \multicolumn{3}{|l|}{ Notes } \\
\hline \multicolumn{3}{|l|}{ Risk of bias } \\
\hline Item & Authors' judgement & Description \\
\hline Allocation concealment? & Yes & A - Adequate \\
\hline
\end{tabular}

\section{Jones 1994}

Methods

RCT: (note: data reported by dominant and non-dominant leg. Data for dominant leg used in analyses)

Method of randomisation: not stated

Assessor blinding: yes

Participant blinding: no

Loss to follow-up: 4 


\begin{tabular}{|c|c|c|}
\hline & \multicolumn{2}{|c|}{$\begin{array}{l}\text { Intention-to-treat analysis: no } \\
\text { Post-program follow up: no }\end{array}$} \\
\hline Participants & \multicolumn{2}{|c|}{$\begin{array}{l}\text { Location: USA } \\
\mathrm{N}=46 \\
\text { Sample: women from a community senior center } \\
\text { Age: mean } 67.4 \text { years } \\
\text { Inclusion criteria: female, from a community senior centre, age }>60 \text {, independently } \\
\text { ambulatory } \\
\text { Exclusion criteria: unstable cardiovascular disease, orthopaedic or neurological } \\
\text { dysfunction, any other uncontrolled chronic conditions that would interfere with the safety } \\
\text { and conduct of the training protocol }\end{array}$} \\
\hline Interventions & \multicolumn{2}{|c|}{$\begin{array}{l}\text { PRT versus control } \\
1 . P R T \\
\text { Type of Ex: } 7 \mathrm{LL} \\
\text { Equipment: velcro leg weights } \\
\text { Intensity: started low, progressed to moderate } \\
\text { Frequency: Ex3 } \\
\text { Reps/Sets: } 3 \text { of } 14 \text { by end of program } \\
\text { Duration: } 16 \text { weeks } \\
\text { Setting: group at local community centre ( } 2 \text { days/week) and home (1 day/week) } \\
\text { Supervision: full in group, none at home } \\
\text { Adherence: } 86-93 \% \\
\text { 2. Control Group: no intervention - contacted to monitor health and activity level }\end{array}$} \\
\hline Outcomes & \multicolumn{2}{|c|}{$\begin{array}{l}\text { Strength and muscular endurance (isokinetic dynamometer) } \\
\text { Comments on adverse events: yes }\end{array}$} \\
\hline \multicolumn{3}{|l|}{ Notes } \\
\hline \multicolumn{3}{|l|}{ Risk of bias } \\
\hline Item & Authors' judgement & Description \\
\hline Allocation concealment? & Unclear & B - Unclear \\
\hline
\end{tabular}

Jubrias 2001

\begin{tabular}{|c|c|}
\hline Methods & $\begin{array}{l}\text { RCT with } 3 \text { groups: PRT, aerobic training and control } \\
\text { Method of randomisation: not reported } \\
\text { Assessor blinding: no } \\
\text { Participant blinding: no } \\
\text { Loss to follow-up: no } \\
\text { Intention-to-treat analysis: no } \\
\text { Post-program follow up: no }\end{array}$ \\
\hline Participants & $\begin{array}{l}\text { Location: USA } \\
\mathrm{N}=40 \text { total }(\mathrm{n}=26 \text { in PRT and control) } \\
\text { Sample: healthy, active older people } \\
\text { Age: } 69.2 \text { years ( } \mathrm{SD}=0.6) \\
\text { Inclusion criteria: healthy (screened with physical exam, exercise testing), physically } \\
\text { active, not engaged in PRT or aerobic training before this study } \\
\text { Exclusion criteria: not reported }\end{array}$ \\
\hline Interventions & $\begin{array}{l}\text { PRT versus control and versus aerobic } \\
\text { 1. PRT } \\
\text { Type of Ex: 1LL, } 2 \text { UL } \\
\text { Equipment: resistance training machines } \\
\text { Intensity: phase } 1-60-70 \% \text { of } 1 \text { RM; phase } 2-70-85 \% \text { of } 1 \text { RM } \\
\text { Frequency: Ex3 } \\
\text { Reps/Sets: phase 1: } 10-15 / 3 \text {; phase } 2: 4-8 / 3-5 \\
\text { Duration: } 24 \text { weeks } \\
\text { Setting: gym } \\
\text { Supervision: not reported } \\
\text { Adherence: } 94.2 \% \text { attendance } \\
\text { 2. Control Group: continued normal activities, asked not to begin PRT or aerobic training } \\
\text { during thetrial } \\
\text { 3. Aerobic Training Group: training began at } 60 \% \text { heart rate reserve for } 10-20 \text { minutes, } \\
\text { progressed to } 80-85 \% \text { HR reserve for a total of } 40 \text { minutes, three times per week }\end{array}$ \\
\hline Outcomes & $\begin{array}{l}\text { Muscle size } \\
\text { Energy and fibre properties }\end{array}$ \\
\hline
\end{tabular}


Comments on adverse events: yes

\begin{tabular}{l|l|l}
\hline \multicolumn{2}{l}{ Notes } & \multicolumn{2}{l}{} \\
\hline Risk of bias & Authors' judgement & Description \\
\hline Item & B - Unclear \\
\hline Allocation concealment? & Unclear &
\end{tabular}

Judge 1994

\begin{tabular}{|c|c|}
\hline Methods & $\begin{array}{l}\text { RCT with factorial design: PRT alone, balance training alone, PRT and balance, control } \\
\text { Method of randomisation: balance block design (blocks of } 4 \text { subjects) } \\
\text { Assessor blinding: yes } \\
\text { Patient blinding: no, but control group received educational sessions } \\
\text { Loss to follow-up: } 3 \text { from PRT and control group } \\
\text { Intention-to-treat analysis: yes } \\
\text { Post-program follow up: yes, monitored for } 6 \text { months after intensive program while } \\
\text { participants undertook tai chi. Falls monitored for median } 0.88 \text { years, max } 1.86 \text { years }\end{array}$ \\
\hline Participants & $\begin{array}{l}\text { Location: USA } \\
\mathrm{N}=110 \text { total ( } 55 \text { in PRT vs control) } \\
\text { Sample: ambulatory older people from voter registration list } \\
\text { Age: mean } 80 \text { years } \\
\text { Inclusion criteria: age } 75 \text { years or greater, the ability to walk without an assistive device for } \\
8 \text { meters, MMSE > } 24 \\
\text { Exclusion criteria: symptomatic cardiovascular disease, poorly controlled hypertension } \\
\text { (>160/96), history or physical findings of focal neurological deficit, Parkinson disease, } \\
\text { peripheral neuropathy of the legs, hip or knee joint replacement, hip fracture, cancer } \\
\text { (metastatic or under active treatment), taking neuroleptic, prednisolone }>5 \mathrm{mg} / \mathrm{day} \text {, } \\
\text { benzodiazepines, significant hip or knee arthritis that requires a cane for ambulation }\end{array}$ \\
\hline Interventions & $\begin{array}{l}\text { PRT versus control } \\
\text { 1. PRT } \\
\text { Type of Ex: } 6 \text { LL } \\
\text { Equipment: cuff-weights and exercise machines } \\
\text { Intensity: } 60-75 \% \text { for exercises with machines; low to moderate for other } \\
\text { Frequency: } 3 \text { times per week } \\
\text { Reps/Sets: } 3 \text { sets to failure with machines; } 13 / 2 \text { with sandbags; } 10 / 2 \text { with body weight } \\
\text { Duration: } 12 \text { weeks } \\
\text { Setting: group exercise } \\
\text { Supervision: full } \\
\text { Adherence: } 82 \% \\
\text { 2. Control group: } 5 \text { education sessions } \\
\text { 3. Balance training: } 3 \text { times per week, } 45 \text { minute sessions, one-on-one with exercise leader } \\
\text { includingbalance platform and floor-based exercises (eyes open and closed on different } \\
\text { surfaces, with pertubationsand base of support changes) }\end{array}$ \\
\hline Outcomes & $\begin{array}{l}\text { Strength (isokinetic dynamometer) } \\
\text { Side effects of training (musculoskeletal or neurologic complaints) } \\
\text { Gait velocity } \\
\text { Chair rise } \\
\text { Balance } \\
\text { Comments on adverse events: yes (a priority outcome of study) }\end{array}$ \\
\hline \multicolumn{2}{|l|}{ Notes } \\
\hline \multicolumn{2}{|l|}{ Risk of bias } \\
\hline Item & Authors' judgement \\
\hline Allocation concealment? & B - Unclear \\
\hline
\end{tabular}

Kalapotharakos 2005

\begin{tabular}{l|l}
\hline Methods & RCT \\
Method of randomisation: not reported \\
Assessor blinding: yes
\end{tabular}

Cochrane Database Syst Rev. Author manuscript; available in PMC 2015 February 11. 


\begin{tabular}{|c|c|c|}
\hline & \multicolumn{2}{|c|}{$\begin{array}{l}\text { Participant blinding: not reported } \\
\text { Loss to follow-up: } 1 \text { in the high resistance group, } 1 \text { in the control group } \\
\text { Intention-to-treat analysis: no } \\
\text { Post-program follow up: no }\end{array}$} \\
\hline Participants & \multicolumn{2}{|c|}{$\begin{array}{l}\text { Location: Greece } \\
\mathrm{N}=12-\mathrm{HI} ; \mathrm{N}=12-\mathrm{MI} ; \mathrm{N}=11 \text {-control } \\
\text { Sample: healthy, inactive but independent living older adults } \\
\text { Age: } \mathrm{HI}-\mathrm{mean} 64.6 \text { years }(\mathrm{SD}=5.1) \text {; MI-mean } 65.7 \text { years }(\mathrm{SD}=4.2) \\
\text { Inclusion criteria: non smokers, free of medication, no symptoms of cardiovascular, } \\
\text { orthopedic, or neuromuscular disease, and physically inactive before } \\
\text { Exclusion criteria: MMSE }<24 \text {, depression }(\mathrm{GDS}>5 \text { ) }\end{array}$} \\
\hline Interventions & \multicolumn{2}{|c|}{$\begin{array}{l}\text { PRT (high intensity and moderate intensity) versus control } \\
\text { 1. PRT } \\
\text { Type of Ex: } 4 \mathrm{UL} / 2 \mathrm{LL} \\
\text { Equipment: Universal gym machines } \\
\text { Intensity: high intensity group: } 80 \% \text { of } 1 \mathrm{RM} \text {; moderate intensity group: } 60 \% \text { of } 1 \mathrm{RM} \\
\text { Frequency: Ex } 3 \\
\text { Reps/Sets: } 8 / 3 \text { for high intensity group; } 15 / 3 \text { for moderate intensity group } \\
\text { Duration: } 12 \text { weeks } \\
\text { Setting: not reported } \\
\text { Supervision: not reported } \\
\text { Adherence: } 98.5 \% \\
\text { 2. Control Group: no exercise }\end{array}$} \\
\hline Outcomes & \multicolumn{2}{|c|}{$\begin{array}{l}\text { Muscle strength (1-RM) } \\
\text { 6-min walk } \\
\text { Chair rise } \\
\text { Vertical jump } \\
\text { 1-leg standing time, } \\
\text { Walking speed } \\
\text { Stair climb } \\
\text { Comments on adverse events: no }\end{array}$} \\
\hline \multicolumn{3}{|l|}{ Notes } \\
\hline \multicolumn{3}{|l|}{ Risk of bias } \\
\hline Item & Authors' judgement & Description \\
\hline Allocation concealment? & Unclear & B - Unclear \\
\hline
\end{tabular}

Kallinen 2002

\begin{tabular}{|c|c|}
\hline Methods & $\begin{array}{l}\text { RCT with } 3 \text { groups: PRT, control, and aerobic group } \\
\text { Method of randomisation: manually perform by drawing lots } \\
\text { Assessor blinding: No } \\
\text { Participant blinding: not reported } \\
\text { Loss to follow-up: } 4 \text { in PRT group, } 3 \text { in endurance group (aerobic) } \\
\text { Intention-to-treat analysis: yes done at the } 30 \text { th month } \\
\text { Post-program follow up: no }\end{array}$ \\
\hline Participants & $\begin{array}{l}\text { Location: Finland } \\
\mathrm{N}=27 \text { (16 in PRT) } \\
\text { Sample: elder women } \\
\text { Age: range } 76-78 \text { years } \\
\text { Inclusion criteria: no severe diseases or functional impairments } \\
\text { Exclusion criteria: not reported }\end{array}$ \\
\hline Interventions & $\begin{array}{l}\text { PRT versus control and versus aerobic (Note: participants in all groups were given } 600 \mathrm{mg} \\
\text { calcium per day) } \\
\text { 1. PRT } \\
\text { Type of Ex: } 4 \mathrm{UL}, 4 \mathrm{LL} \\
\text { Equipment: resistance training machines } \\
\text { Intensity: high - completed } 8 \mathrm{RM} \\
\text { Frequency: Ex3 } \\
\text { Reps/Sets: } 8 / 3 \\
\text { Program Duration: } 2 \text { years } \\
\text { Setting: gym } \\
\text { Supervision: full } \\
\text { Adherence: } 74 \% \\
\text { 2. Control Group: non-exercise group }\end{array}$ \\
\hline
\end{tabular}




\begin{tabular}{l|l|l}
\multicolumn{2}{l}{} & $\begin{array}{l}\text { 3. Aerobic Fitness Group: } \mathrm{N}=15 ; 3 \text { sessions per week, performed same exercises as PRT } \\
\text { group but withno resistance, plus added stationary cycling for 40 second stations }\end{array}$ \\
\hline Outcomes & $\begin{array}{l}\text { PeakVO2 } \\
\text { Peak Power } \\
\text { Comments on adverse events: yes }\end{array}$ \\
\hline Notes & Data from PRT and aerobic training group were compared \\
\hline Risk of bias & Authors' judgement & Description \\
\hline Item & Unclear & B - Unclear \\
\hline Allocation concealment?
\end{tabular}

Katznelson 2006

\begin{tabular}{|c|c|c|}
\hline Methods & \multicolumn{2}{|c|}{$\begin{array}{l}\text { RCT } \\
\text { Method of randomisation: by a block design } \\
\text { Assessor blinding: yes } \\
\text { Participant blinding: yes } \\
\text { Loss to follow-up: } 4 / 19 \text { - placebo + ex; } 1 / 17 \text { - placebo only } \\
\text { Intention-to-treat analysis: yes } \\
\text { Post-program follow up: no }\end{array}$} \\
\hline Participants & \multicolumn{2}{|c|}{$\begin{array}{l}\text { Location: USA } \\
\mathrm{N}=26(19 \text { in PRT) } \\
\text { Sample: men with relative testosterone insufficiency, sedentary and community dwelling } \\
\text { Age: mean } 72 \text { years ( } \mathrm{SD}=5.4) \\
\text { Inclusion criteria: a single fasting serum free-testosterone value < } 14.5 \mathrm{pg} / \mathrm{ml} \text { and BMI is } \\
\text { between } 18-32 \text {; sedentary status } \\
\text { Exclusion criteria: clinically unstable coronary artery or cerebrovascular disease, } \\
\text { osteoarthritis of the lower extremity that could limit ambulation, clinically significant } \\
\text { benign prostatic hypertropy (BPH), prostate cancer, an elevated prostate-specific antigen } \\
\text { (PSA) value, hematocrit }>52 \% \text {, disorders known to affect body composition including } \\
\text { hypokalemia, renal insufficiency, liver dysfunction, diabetes mellitus, hypothyroidism, } \\
\text { alcoholism, thromoboembolic disease or coagulopathy, supraphysiologic glucocorticoid } \\
\text { medication during the previous } 12 \text { months, androgen medications including supplements } \\
\text { during the past } 5 \text { years, clinically significant psychiatric disease, or known pituitary } \\
\text { disease, or radiation of the hypothalamus or pituitary gland }\end{array}$} \\
\hline Interventions & \multicolumn{2}{|c|}{$\begin{array}{l}\text { PRT versus control } \\
\text { 1. PRT } \\
\text { Type of Ex: } 11 \text { resistance exercises adapted from the Strong for Life video } \\
\text { Equipment: elastic bands } \\
\text { Intensity: used the next level of elastic band when the perceived exertion was less than } \\
\text { moderate } \\
\text { Frequency: } 3 \text { to } 4 \text { times a week } \\
\text { Reps/Sets: } 10 / 1 \\
\text { Duration: } 12 \text { weeks } \\
\text { Setting: home } \\
\text { Supervision: returned for an out-patient visit every two weeks and phone calls } \\
\text { Adherence: } 90 \% \\
\text { 2. Control Group: non exercise intervention }\end{array}$} \\
\hline Outcomes & \multicolumn{2}{|c|}{$\begin{array}{l}\text { SF-36 } \\
\text { Comments on adverse events: yes }\end{array}$} \\
\hline \multicolumn{3}{|l|}{ Notes } \\
\hline \multicolumn{3}{|l|}{ Risk of bias } \\
\hline Item & Authors' judgement & Description \\
\hline Allocation concealment? & Unclear & B - Unclear \\
\hline
\end{tabular}

\section{Kongsgaard 2004}

\begin{tabular}{l|l}
\hline Methods & RCT
\end{tabular}




\begin{tabular}{|c|c|c|}
\hline & \multicolumn{2}{|c|}{$\begin{array}{l}\text { Method of randomisation: not reported } \\
\text { Assessor blinding: not reported } \\
\text { Participant blinding: not reported } \\
\text { Loss to follow-up: } 3 / 9 \text { in the ex group; } 2 / 9 \text { in the control group } \\
\text { Intention-to-treat analysis: no } \\
\text { Post-program follow up: no }\end{array}$} \\
\hline Participants & \multicolumn{2}{|c|}{$\begin{array}{l}\text { Location: Denmark } \\
\mathrm{N}=18 \text { ( } 9 \text { in each group) } \\
\text { Sample: home-dwelling elder men with COPD } \\
\text { Age: mean } 71 \text { years ( } \mathrm{SD}=1.3 \text { ) } \\
\text { Inclusion criteria: can transport to the hospital } \\
\text { Exclusion criteria: fractures of the lower extremities within the last } 6 \text { months, neurological } \\
\text { disease, cardiovascular diseases, dependence on more than one walking device and } \\
\text { cognitive dysfunction }\end{array}$} \\
\hline Interventions & \multicolumn{2}{|c|}{$\begin{array}{l}\text { PRT versus control } \\
\text { 1. PRT } \\
\text { Type of Ex: } 3 \text { LL } \\
\text { Equipment: Technogym } \\
\text { Intensity: } 80 \% \text { of } 1 \text { RM } \\
\text { Frequency: Ex2 } \\
\text { Reps/Sets: } 8 / 4 \\
\text { Duration: } 12 \text { weeks } \\
\text { Setting: not reported (Gym?) } \\
\text { Supervision: full } \\
\text { Adherence: extending the training period until a total of } 24 \text { training sessions were finished } \\
\text { 2. Control Group: daily non-supervised breathing ex }\end{array}$} \\
\hline Outcomes & \multicolumn{2}{|c|}{$\begin{array}{l}\text { Primary: simple ADL (interview) } \\
\text { Secondary: forced expiratory volume, muscle strength ( } 5 \mathrm{RM}) \text {, gait speed, timed stair } \\
\text { climbing } \\
\text { Comments on adverse events: no }\end{array}$} \\
\hline \multicolumn{3}{|l|}{ Notes } \\
\hline \multicolumn{3}{|l|}{ Risk of bias } \\
\hline Item & Authors' judgement & Description \\
\hline Allocation concealment? & Unclear & B - Unclear \\
\hline
\end{tabular}

Krebs 2007

\begin{tabular}{|c|c|}
\hline Methods & $\begin{array}{l}\text { RCT } \\
\text { Method of randomisation: a computer-generated table } \\
\text { Assessor blinding: yes } \\
\text { Participant blinding: not reported } \\
\text { Loss to follow-up: } 0 \\
\text { Intention-to-treat analysis: NA } \\
\text { Post-program follow up: no }\end{array}$ \\
\hline Participants & $\begin{array}{l}\text { Location: USA } \\
\mathrm{N}=15 \text { ( } 6 \text { in PRT) } \\
\text { Sample: community dwelling elders with disability } \\
\text { Age: mean } 70.4 \text { years }(\mathrm{SD}=6.5) \\
\text { Inclusion criteria: at least } 60 \text { years of age, cognitive intactness, ambulate independently } \\
\text { more than } 15 \text { feet, had more than one lower-limb impairment, and have more than one } \\
\text { functional limitation on SF-36 } \\
\text { Exclusion criteria: terminal illness, progressive neurological disease, major loss of vision, } \\
\text { and acute pain and non-ambulatory status }\end{array}$ \\
\hline Interventions & $\begin{array}{l}\text { PRT versus functional training } \\
\text { 1. PRT } \\
\text { Type of Ex: resisted proprioceptive neuromuscular facilitation exercise, } 9 \text { LL/2UL } \\
\text { Equipment: elastic bands } \\
\text { Intensity: } 10 \text { RM increased to } 6 \mathrm{RM} \\
\text { Frequency: } 3 \text { to } 5 \text { times a week } \\
\text { Reps/Sets: } 4 \text {-level of normal progression and 4-level of advanced levels } \\
\text { Duration: } 6 \text { weeks } \\
\text { Setting: home? } \\
\text { Supervision: two physical therapists taught the exercises and checked the exercise log at } \\
\text { out-patient visits }\end{array}$ \\
\hline
\end{tabular}




\begin{tabular}{l|l|l} 
& $\begin{array}{l}\text { Adherence: PRT group- exercised average 5 days per week; functional training group- } \\
\text { exercise average 5. 39 days per week }\end{array}$ \\
& $\begin{array}{l}\text { 2. Functional training group: N=6, average age =78.1 years, simulating locomotion } \\
\text { activities at 3 differentspeeds, 3-5 days a week }\end{array}$ \\
\hline Outcomes & $\begin{array}{l}\text { Primary: SF-36 } \\
\text { Secondary: muscle strength, paced gait, chair rise, standing balance } \\
\text { Comment on adverse events: yes }\end{array}$ \\
\hline Notes & Numerical results of means and SDs were not reported. Reported/figured \% difference \\
\hline Risk of bias & Authors' judgement & Description \\
\hline Item & Unclear & B - Unclear \\
\hline Allocation concealment?
\end{tabular}

\section{Lamoureux 2003}

\begin{tabular}{|c|c|c|}
\hline Methods & \multicolumn{2}{|c|}{$\begin{array}{l}\text { RCT } \\
\text { Method of randomisation: not reported } \\
\text { Assessor blinding: not reported } \\
\text { Participant blinding: not reported } \\
\text { Loss to follow-up: } 1 / 29 \text { in the experimental group, } 1 / 16 \text { in the control group } \\
\text { Intention-to-treat analysis: no } \\
\text { Post-program follow up: no }\end{array}$} \\
\hline Participants & \multicolumn{2}{|c|}{$\begin{array}{l}\text { Location: Australia } \\
\mathrm{N}=45 \text { ( } 29 \text { in PRT) } \\
\text { Sample: community dwelling elderly } \\
\text { Age: mean } 68.5 \text { years ( } \mathrm{SD}=1.2) \\
\text { Inclusion criteria: no resistance-training background, no cardiovascular disease, } \\
\text { musculoskeletal disorders, neurological dysfunction and uncontrolled chronic conditions } \\
\text { Exclusion criteria: not reported }\end{array}$} \\
\hline Interventions & \multicolumn{2}{|c|}{$\begin{array}{l}\text { PRT versus control } \\
\text { 1. PRT } \\
\text { Type of Ex: 5 LL } \\
\text { Equipment: Pin-loaded weigh machines } \\
\text { Intensity: from } 60 \% 1 \mathrm{RM} \text { in week } 1 \text { to } 85 \% 1 \mathrm{RM} \text { in week } 14 \\
\text { Frequency: Ex3 -first } 3 \text { months; then Ex2-last } 3 \text { months } \\
\text { Reps/Sets: } 5-8 / 2-5 \\
\text { Duration: } 24 \text { weeks } \\
\text { Setting: not reported } \\
\text { Supervision: not reported } \\
\text { Adherence: } 95.5 \% \\
\text { 2. Control Group: maintain normal activities }\end{array}$} \\
\hline Outcomes & \multicolumn{2}{|c|}{$\begin{array}{l}\text { Muscle strength } \\
\text { Gait velocity } \\
\text { Comments on adverse events: no }\end{array}$} \\
\hline Notes & \multicolumn{2}{|c|}{$\begin{array}{l}\text { Final muscle strength outcome was not available } \\
\text { Data at week } 12 \text { were extracted }=\text { baseline }+ \text { change score. Final } S D=\text { baseline SD }\end{array}$} \\
\hline \multicolumn{3}{|l|}{ Risk of bias } \\
\hline Item & Authors' judgement & Description \\
\hline Allocation concealment? & Unclear & B - Unclear \\
\hline
\end{tabular}

Latham 2001

\begin{tabular}{l|l}
\hline Methods & RCT \\
& Method of randomisation: concealed envelopes \\
& Assessor blinding: no \\
& Participant blinding: no \\
& Loss to follow-up: 3 \\
& Intention-to-treat analysis: no
\end{tabular}

Cochrane Database Syst Rev. Author manuscript; available in PMC 2015 February 11. 


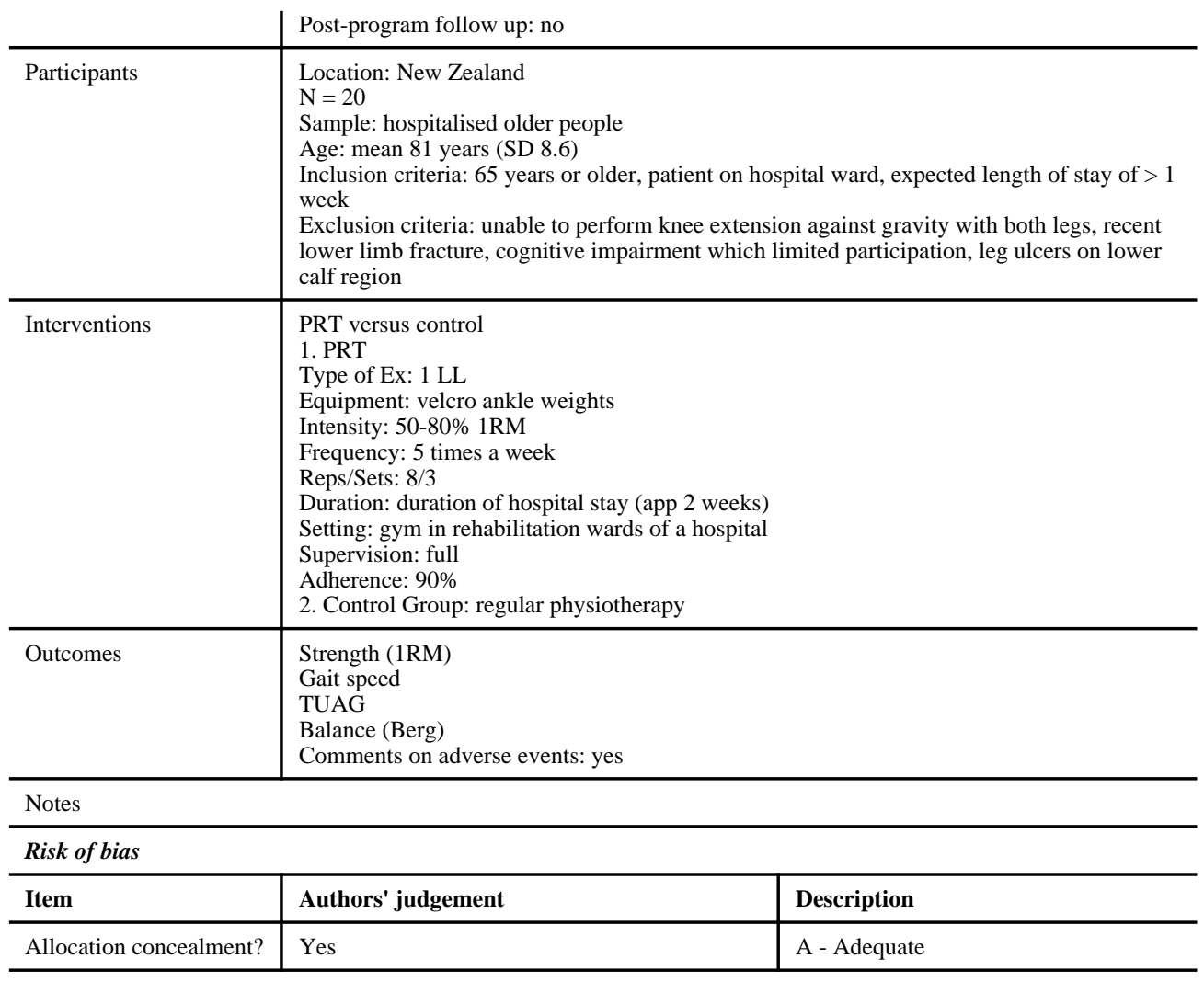

\section{Latham 2003}

\begin{tabular}{|c|c|}
\hline Methods & $\begin{array}{l}\text { RCT with a factorial design (only information about PRT vs control reported, } 3 \text { - month } \\
\text { outcomes reported) } \\
\text { Method of randomisation: central computerised randomisation, blocks of } 6 \text { by centre } \\
\text { Assessor blinding: yes } \\
\text { Participant blinding: no, but attention control } \\
\text { Loss to follow-up: } 21 \\
\text { Intention-to-treat analysis: yes } \\
\text { Post-program follow up: yes, at the } 6 \text { th month }\end{array}$ \\
\hline Participants & $\begin{array}{l}\text { Location: New Zealand and Australia } \\
\mathrm{N}=243 \\
\text { Sample: frail older adults recruited from hospital geriatric services } \\
\text { Age: mean } 79.1 \text { years (SD 6.9) } \\
\text { Inclusion criteria: age } 65 \text { years or more, receiving hospital care from geriatric services, } \\
\text { considered to be frail, not clear indication or contraindication to study treatments } \\
\text { Exclusion criteria: responsible physician considered the interventions definitely hazardous } \\
\text { or required, patients unlikely to survive } 6 \text { months, severe cognitive impairment which could } \\
\text { compromise adherence to the exercise programme, not fluent in the English language }\end{array}$ \\
\hline Interventions & $\begin{array}{l}\text { PRT versus control } \\
\text { 1. PRT } \\
\text { Type of Ex: } 1 \mathrm{LL} \\
\text { Equipment: velcro ankle weights } \\
\text { Intensity: aimed for } 50-80 \% \text { for most of the programme } \\
\text { Frequency: Ex3 } \\
\text { Reps/Sets: } 8 / 3 \\
\text { Duration: } 10 \text { weeks } \\
\text { Setting: home-based } \\
\text { Supervision: limited - fortnightly home visits alternating with phone calls } \\
\text { Adherence: } 82 \% \text { (including drop-outs) } \\
\text { 2. Control Group: frequency-matched phone calls and home visits }\end{array}$ \\
\hline
\end{tabular}




\begin{tabular}{l|l|l} 
Outcomes & $\begin{array}{l}\text { Primary: falls over 6 months, HRQoL (SF-36) } \\
\text { Secondary: balance (Berg), strength (hand-held dynamometer), gait speed, TUAG, Barthel } \\
\text { Index, Adelaide Activities Profile, Falls Self-Efficacy Index, adverse events (limitation in } \\
\text { ADL for 2+ days and/or attention sought from health care professional) } \\
\text { Comments on adverse events: yes }\end{array}$ \\
\hline Notes & Authors' judgement & Description \\
\hline Risk of bias & A - Adequate \\
\hline Item & Yes &
\end{tabular}

Liu-Ambrose 2005

\begin{tabular}{|c|c|c|}
\hline Methods & \multicolumn{2}{|c|}{$\begin{array}{l}\text { RCT } \\
\text { Method of randomisation: computer-generalized list } \\
\text { Assessor blinding: yes } \\
\text { Participant blinding: not reported } \\
\text { Loss to follow-up: } 2 / 34 \text { in PRT, } 2 / 34 \text { in stretching } \\
\text { Intention-to-treat analysis: yes } \\
\text { Post-program follow up: no }\end{array}$} \\
\hline Participants & \multicolumn{2}{|c|}{$\begin{array}{l}\text { Location: Canada } \\
\mathrm{N}=68 \text { ( } 34 \text { in each group) } \\
\text { Sample: elder women with osteoporosis or osteopenia } \\
\text { Age: mean } 79.6 \text { years }(\mathrm{SD}=2.1 \text { ) } \\
\text { Inclusion criteria: age between } 75-85 \text { years with low bone mass and diagnosed with } \\
\text { osteoporosis/osteopenia } \\
\text { Exclusion criteria: living in care facilities, non-Caucasian, exercise regularly more than } 2 \\
\text { times a week, illness or condition that would affect balance, MMSE score lowers than } 23\end{array}$} \\
\hline Interventions & \multicolumn{2}{|c|}{$\begin{array}{l}\text { PRT versus control } \\
\text { 1. PRT } \\
\text { Type of Ex: } 4 \text { UL, } 5 \mathrm{LL} \\
\text { Equipment: machines (Keiser Pressurized Air system) or free weights } \\
\text { Intensity: progressed from } 50-60 \% \text { of } 1 \text { RM to } 75-85 \% \text { of } 1 \text { RM in } 4 \text { weeks } \\
\text { Frequency: Ex2 } \\
\text { Reps/Sets: } 10-15 / 2 \text { (first } 3 \text { weeks); 6-8/2 (after week } 3 \text { ) } \\
\text { Duration: } 25 \text { weeks } \\
\text { Setting: community center } \\
\text { Supervision: certified fitness instructors } \\
\text { Adherence: } 85 \% \text { for PRT, } 79 \% \text { for stretching (control) } \\
\text { 2. Control Group: general stretching, deep breathing and relaxation }\end{array}$} \\
\hline Outcomes & \multicolumn{2}{|c|}{$\begin{array}{l}\text { Primary: health related quality of life, general physical function } \\
\text { Secondary: muscle strength, gait speed, fall risk assessment } \\
\text { Comments on adverse events: yes }\end{array}$} \\
\hline \multicolumn{3}{|l|}{ Notes } \\
\hline \multicolumn{3}{|l|}{ Risk of bias } \\
\hline Item & Authors' judgement & Description \\
\hline Allocation concealment? & Unclear & B - Unclear \\
\hline
\end{tabular}

Macaluso 2003

\begin{tabular}{l|l}
\hline Methods & RCT \\
& Method of randomisation: the principal investigator drew numbers from a bowl that had \\
been thoroughly mixed \\
Assessor blinding: not reported \\
Participant blinding: not reported \\
Loss to follow-up: 7 \\
Intention-to-treat analysis: yes \\
Post-program follow up: no \\
\hline
\end{tabular}




\begin{tabular}{|c|c|c|}
\hline Participants & \multicolumn{2}{|c|}{$\begin{array}{l}\text { Location: } \mathrm{UK} \\
\mathrm{N}=10 \text {-speed group (LI), } \mathrm{N}=10 \text {-Strength group }(\mathrm{HI}) \\
\text { Sample: healthy elder women } \\
\text { Age: mean }=69 \text { years }(\mathrm{SD}=2.7) \\
\text { Inclusion criteria: not reported } \\
\text { Exclusion criteria: not "medical stable" for exercise studies }\end{array}$} \\
\hline Interventions & \multicolumn{2}{|c|}{$\begin{array}{l}\text { PRT (speed versus strength) } \\
\text { Type of Ex: pedal } \\
\text { Equipment: mechanically braked cycle ergometer } \\
\text { Intensity: speed group (LI)-40\% of } 2 \text { max resistance to complete } 2 \text { revolutions (2RM); } \\
\text { strength group (HI)-80\% of } 2 \text { RM } \\
\text { Frequency: Ex3 } \\
\text { Reps/Sets: Speed group (LI)-16 pedal revolutions/8 sets; Strength group (HI)-8 pedals } \\
\text { revolutions/8sets } \\
\text { Duration: } 16 \text { weeks } \\
\text { Setting: not reported (gym?) } \\
\text { Supervision: not reported } \\
\text { Adherence: speed group (LI)-93\%; strength group (HI)-89\% }\end{array}$} \\
\hline Outcomes & \multicolumn{2}{|c|}{$\begin{array}{l}\text { Strength measure } \\
\text { Max treadmill walking speed } \\
\text { Box-stepping test } \\
\text { Vertical jump } \\
\text { Comments on adverse events: yes }\end{array}$} \\
\hline Notes & \multicolumn{2}{|c|}{ Involved power training, no control group } \\
\hline \multicolumn{3}{|l|}{ Risk of bias } \\
\hline Item & Authors' judgement & Description \\
\hline Allocation concealment? & Unclear & B - Unclear \\
\hline
\end{tabular}

\section{Madden 2006}

\begin{tabular}{|c|c|}
\hline Methods & $\begin{array}{l}\text { RCT with } 3 \text { groups: PRT, control, and endurance (aerobic) group } \\
\text { Method of randomisation: not reported } \\
\text { Assessor blinding: not reported } \\
\text { Participant blinding: not reported } \\
\text { Loss to follow-up: } 5 \text { in the endurance training group } \\
\text { Intention-to-treat analysis: no } \\
\text { Post-program follow up: no }\end{array}$ \\
\hline Participants & $\begin{array}{l}\text { Location: USA } \\
\mathrm{N}=30 \text { ( } 15 \text { in each group) } \\
\text { Sample: healthy elder women } \\
\text { Age: mean } 69.8 \text { years }(\mathrm{SD}=1.5) \\
\text { Inclusion criteria: a normal blood pressure, a normal physical exam, normal resting ECG, } \\
\text { normal M-mode and two-dimensional echocardiograms showing no more than mild } \\
\text { valvular regurgitation, a normal Bruce protocol treadmill maximal exercise stress test, and a } \\
\text { normal hematocrit, fasting blood glucose, total cholesterol, and creatinine. } \\
\text { Exclusion criteria: any history of angina, myocardial infarction, stroke, hypertension, } \\
\text { chronic pulmonary disease, diabetes, current medication use (prescription or over the } \\
\text { counter), current smoking, or exercise-limiting orthopedic impairment }\end{array}$ \\
\hline Interventions & $\begin{array}{l}\text { PRT versus control and versus endurance (aerobic) } \\
\text { 1. PRT } \\
\text { Type of Ex: } 10 \text { UL and LL } \\
\text { Equipment: not reported (weight?) } \\
\text { Intensity: } 85 \% 1 \mathrm{RM} \\
\text { Frequency: } 5 \text { times/week } \\
\text { Reps/Sets: } 8-12 / 3 \\
\text { Duration: } 24 \text { weeks } \\
\text { Setting: not reported (Gym?) } \\
\text { Supervision: full-certified trainer } \\
\text { Adherence: required participants to attend } 90 \% \text { of all training sessions to remain enrolled in } \\
\text { the study } \\
\text { 2. Control Group: no training } \\
\text { 3. Endurance Ex (aerobic) Group: } \mathrm{N}=15 \text {, mean age }=70 \text { years }(\mathrm{SD}=2.6) \text {, using cycle } \\
\text { ergometer, } 50-60 \% \text { Max HR to } 80-85 \% \text { Max HR, } 5 \text { times a week }\end{array}$ \\
\hline Outcomes & VO2max \\
\hline
\end{tabular}




\begin{tabular}{l|l|l}
\hline Notes & Comments on adverse events: no \\
\hline & $\begin{array}{l}\text { Baseline + relative change score } \\
\text { Data from PRT and aerobic training group were compared }\end{array}$ \\
\hline Risk of bias & Authors' judgement & Description \\
\hline Item & Unclear & B - Unclear \\
\hline Allocation concealment? & &
\end{tabular}

Maiorana 1997

\begin{tabular}{|c|c|c|}
\hline Methods & \multicolumn{2}{|c|}{$\begin{array}{l}\text { RCT } \\
\text { Method of randomisation: not reported } \\
\text { Assessor blinding: no } \\
\text { Participant blinding: no } \\
\text { Loss to follow-up: } 5 \\
\text { Intention-to-treat analysis: no } \\
\text { Post-program follow up: no }\end{array}$} \\
\hline Participants & \multicolumn{2}{|c|}{$\begin{array}{l}\text { Location: Australia } \\
\mathrm{N}=31 \\
\text { Sample: men at least } 3 \text { months after coronary bypass } \\
\text { Age: mean } 61.2 \text { years (SD } 8.4 \text { ) in training group } \\
\text { Inclusion criteria: male, at least } 3 \text { months after coronary artery bypass surgery, low risk for } \\
\text { recurrent cardiac events (normal left ventricular function, no residual ischemia, and an } \\
\text { exercise capacity exceeding } \\
4 \text { metabolic equivalents during graded exercise testing) } \\
\text { Exclusion criteria: not in an exercise rehabilitation programme at time of recruitment, } \\
\text { moderate/severe left ventricular function, valve replacement/repair, history of CHF, on } \\
\text { beta-blocking medication, significant resting hypertension (systolic BP > 160mmHg or } \\
\text { diastolic } 100 \mathrm{mmHg} \text { ) angina or significant ST depression during graded exercise testing }\end{array}$} \\
\hline Interventions & \multicolumn{2}{|c|}{$\begin{array}{l}\text { PRT versus control } \\
\text { 1. PRT } \\
\text { Type of Ex: 7UL, 4LL, } 1 \mathrm{Tr} \\
\text { Equipment: machines, dumb-bells } \\
\text { Intensity: } 40 \% \text { of MVC at beginning or program, } 60 \% \text { by end } \\
\text { Frequency: Ex3 } \\
\text { Reps/Sets: } 10-15 / 3 \\
\text { Duration: } 10 \text { weeks } \\
\text { Setting: gym } \\
\text { Supervision: full } \\
\text { Adherence: all subjects completed at least } 80 \% \text { of sessions (excluding drop-outs) } \\
\text { 2. Control Group: maintain current physical activity habits }\end{array}$} \\
\hline Outcomes & \multicolumn{2}{|c|}{$\begin{array}{l}\text { Strength (1RM) } \\
\text { Aerobic capacity (Peak VO2 on treadmill test) } \\
\text { Self-efficacy } \\
\text { Comments on adverse events: yes (safety an aim of study) }\end{array}$} \\
\hline \multicolumn{3}{|l|}{ Notes } \\
\hline \multicolumn{3}{|l|}{ Risk of bias } \\
\hline Item & Authors' judgement & Description \\
\hline Allocation concealment? & Unclear & B - Unclear \\
\hline
\end{tabular}

Malliou 2003

\begin{tabular}{l|l}
\hline Methods & RCT with 3 groups: PRT, functional training, and PRT with functional training group \\
& Method of randomisation: not reported \\
& Assessor blinding: not reported \\
& Participant blinding: not reported \\
& Loss to follow-up: 0 \\
& Intention-to-treat analysis: not reported \\
& Post-program follow up: no \\
\hline
\end{tabular}




\begin{tabular}{|c|c|c|}
\hline Participants & \multicolumn{2}{|c|}{$\begin{array}{l}\text { Location: Greece } \\
\mathrm{N}=25 \text { ( } 15 \text { in multi-joint resistance training group) } \\
\text { Sample: healthy inactive elderly } \\
\text { Age: mean } 68 \text { years } \\
\text { Inclusion criteria: inactive prior to the study, not exhibited anemia, hepatic complications, } \\
\text { thyroid disorders or kidney problems, no hypertension, no potential damaging orthopedic } \\
\text { and neuromuscular problems. } \\
\text { Exclusion criteria: not reported }\end{array}$} \\
\hline Interventions & \multicolumn{2}{|c|}{$\begin{array}{l}\text { PRT versus control and versus aerobic } \\
1 . \text { PRT } \\
\text { Type of Ex: } 3 \text { LL- multi-joint resistance training group } \\
\text { Equipment: Universal exercise machines } \\
\text { Intensity: } 90 \% \text { of } 1 \mathrm{RM} \\
\text { Frequency: Ex3 } \\
\text { Reps/Sets: } 12 / 3 \\
\text { Duration: } 10 \text { weeks } \\
\text { Setting: not reported (gym?) } \\
\text { Supervision: full } \\
\text { Adherence: not reported } \\
\text { 2. Control group: no training } \\
\text { 3. Aerobic ex. group: } \mathrm{N}=15 \text {, mean age }=69 \text { years, aerobic exercise with light leg weight }\end{array}$} \\
\hline Outcomes & \multicolumn{2}{|c|}{$\begin{array}{l}\text { Strength measure } \\
\text { Comments on adverse events: no }\end{array}$} \\
\hline Notes & \multicolumn{2}{|c|}{ Data from PRT and aerobic training group were compared } \\
\hline \multicolumn{3}{|l|}{ Risk of bias } \\
\hline Item & Authors' judgement & Description \\
\hline Allocation concealment? & Unclear & B - Unclear \\
\hline
\end{tabular}

\section{Mangione 2005}

\begin{tabular}{|c|c|}
\hline Methods & $\begin{array}{l}\text { RCT with } 3 \text { groups: PRT, control, and aerobic group } \\
\text { Method of randomisation: not reported } \\
\text { Assessor blinding: yes } \\
\text { Participant blinding: not reported } \\
\text { Loss to follow-up: 1/11-control group, 1/13-aerobic training group, 6/17-resistance training } \\
\text { group } \\
\text { Intention-to-treat analysis: no } \\
\text { Post-program follow up: no }\end{array}$ \\
\hline Participants & $\begin{array}{l}\text { Location: USA } \\
\mathrm{N}=28 \text { ( } 17 \text { in PRT) } \\
\text { Sample: post hip fracture } \\
\text { Age: mean } 77.9 \text { years }(\mathrm{SD}=7.9) \\
\text { Inclusion criteria: successful fixation of a hip fracture, at least } 65 \text { years old, living at home, } \\
\text { and willing to come to the study site } \\
\text { Exclusion criteria: history of unstable angina, uncompensated congestive heart failure, } \\
\text { metabolic conditions (i.e., renal dialysis), stroke, Parkinson's disease, life expectancy of } \\
\text { less than } 6 \text { months, MMSE score is less than } 20 \text {, and living in a nursing home }\end{array}$ \\
\hline Interventions & $\begin{array}{l}\text { PRT versus control and versus aerobic } \\
\text { 1.PRT } \\
\text { Type of Ex: } 4 \mathrm{LL} \\
\text { Equipment: portable progressive-resistance ex. machine and body weight } \\
\text { Intensity: } 8 \text { RM } \\
\text { Frequency: first } 2 \text { months-Ex } 2 \text {, the } 3 \text { rd month-Ex } 1 \\
\text { Reps/Sets: } 8 / 3 \\
\text { Duration: } 12 \text { weeks } \\
\text { Setting: participant's home } \\
\text { Supervision: full-6 physical therapists } \\
\text { Adherence: } 98 \% \\
\text { 2. Control group: received biweekly mailing of non-ex health topics } \\
\text { 3. Aerobic group: } \mathrm{N}=13 \text {, mean age }=79.8 \text { years, walking or stepping, LEs/UEs active ROM } \\
\text { ex, } 65-75 \% \text { max heart rate }\end{array}$ \\
\hline
\end{tabular}




\begin{tabular}{l|l|l} 
Outcomes & $\begin{array}{l}\text { Primary: SF-36 } \\
\text { Secondary: strength measure, 6-minute walking test, walking endurance, gait speed } \\
\text { Comments on adverse events: yes }\end{array}$ \\
\hline Notes & Data from PRT and aerobic training group were compared \\
\hline Risk of bias & Authors' judgement & Description \\
\hline Item & B - Unclear \\
\hline Allocation concealment? & Unclear &
\end{tabular}

\section{Manini 2005}

\begin{tabular}{|c|c|c|}
\hline Methods & \multicolumn{2}{|c|}{$\begin{array}{l}\text { RCT with } 3 \text { groups: PRT, functional training, and PRT with functional training } \\
\text { Method of randomisation: not reported } \\
\text { Assessor blinding: not reported } \\
\text { Participant blinding: not reported } \\
\text { Loss to follow-up: } 25 \\
\text { Intention-to-treat analysis: no } \\
\text { Post-program follow up: no }\end{array}$} \\
\hline Participants & \multicolumn{2}{|c|}{$\begin{array}{l}\text { Location: USA } \\
\mathrm{N}=9 \text {-PRT } \\
\text { Sample: functional limited older adults (low isometric knee extension strength) } \\
\text { Age: mean } 72 \text { years }(\mathrm{SD}=10 \text { ) } \\
\text { Inclusion criteria: bilateral isometric knee extension strength test }<3 \mathrm{Nm} / \mathrm{Kg} \text {; pass } \\
\text { physician's clearance } \\
\text { Exclusion criteria: had cardiac or pulmonary difficulty }\end{array}$} \\
\hline Interventions & \multicolumn{2}{|c|}{$\begin{array}{l}\text { PRT versus functional training and versus PRT with functional training } \\
\text { 1.PRT } \\
\text { Type of Ex: } 3 \mathrm{LL} \\
\text { Equipment: Life-Fitness Inc. } \\
\text { Intensity: } 10 \mathrm{RM} \\
\text { Frequency: Ex } 2 \\
\text { Reps/Sets: } 8 / 2 \\
\text { Duration: } 10 \text { weeks ( } 8-10 \text { weeks of control period before intervention) } \\
\text { Setting: not reported (Gym?) } \\
\text { Supervision: not reported } \\
\text { Adherence: not reported } \\
\text { 2. Functional training group: } \mathrm{N}=7 \text {, rising from a chair, rising from kneeling, stair } \\
\text { ascending/descending } \\
\text { 3. PRT and functional training group: } \mathrm{N}=8,1 / \text { week PRT training and } 1 / \text { week of functional } \\
\text { training }\end{array}$} \\
\hline Outcomes & \multicolumn{2}{|c|}{$\begin{array}{l}\text { Muscle strength } \\
\text { Max. knee isometrics } \\
\text { Comments on adverse events: yes }\end{array}$} \\
\hline Notes & \multicolumn{2}{|c|}{ Data from PRT and functional training group were compared } \\
\hline \multicolumn{3}{|l|}{ Risk of bias } \\
\hline Item & Authors' judgement & Description \\
\hline Allocation concealment? & Unclear & B - Unclear \\
\hline
\end{tabular}

Maurer 1999

\begin{tabular}{l|l}
\hline Methods & RCT \\
& Method of randomisation: random number generator, stratified by disease severity \\
& Assessor blinding: yes \\
& Participant blinding: no, but attention control group \\
& Loss to Follow-up: 15 \\
& Intention-to-treat analysis: no \\
& Post-program follow up: yes - at 12 weeks (after 8 weeks of training) \\
\hline Participants & Location: USA
\end{tabular}




\begin{tabular}{|c|c|c|}
\hline & \multicolumn{2}{|c|}{$\begin{array}{l}\mathrm{N}=113 \\
\text { Sample: people with diagnosed OA of the knee } \\
\text { Age: mean } 66.3 \text { years (SD } 8.8 \text { ) in treatment group } \\
\text { Inclusion criteria: met current American College of Rheumatology criteria for OA, between } \\
50-80 \text { years, receiving no drugs for their arthritis other than stable doses of analgesics or } \\
\text { NSAIDs, had mild to moderate knee pain for at least the previous } 3 \text { months, scored 1-3 on } \\
\text { the Kellgren radiographic scale } \\
\text { Exclusion criteria: concurrently receiving physical therapy, actively involved in any other } \\
\text { pharmaceutical or exercise study or had undergone isokinetic strength training within the } \\
\text { previous } 3 \text { years, had significant cardiovascular disease, more than mild knee swelling, } \\
\text { large popliteal cysts, knee instability, major hip or knee surgery on the side to be treated, } \\
\text { systemic disease other than OA that might affect muscle function, severe osteopenia, } \\
\text { history of fracture in the area of the joint to be treated, paresis of the lower extremity }\end{array}$} \\
\hline Interventions & \multicolumn{2}{|c|}{$\begin{array}{l}\text { PRT versus control } \\
\text { 1. PRT } \\
\text { Type of Ex: } 1 \text { LL } \\
\text { Equipment: isokinetic dynamometer } \\
\text { Intensity: appears high } \\
\text { Frequency: Ex3 } \\
\text { Reps/Sets: } 3 \text { reps at } 3 \text { speeds (total } 9 \text { reps) in } 3 \text { sets } \\
\text { Program Duration: } 8 \text { weeks } \\
\text { Setting: gym } \\
\text { Supervision: not reported } \\
\text { Adherence: not reported } \\
\text { 2. Control Group: four classes on OA education and self-management }\end{array}$} \\
\hline Outcomes & \multicolumn{2}{|c|}{$\begin{array}{l}\text { Primary: WOMAC, SF-36 } \\
\text { Secondary: strength (isokinetic dynamometer), AIMS index } \\
\text { Comments on adverse events: yes }\end{array}$} \\
\hline \multicolumn{3}{|l|}{ Notes } \\
\hline \multicolumn{3}{|l|}{ Risk of bias } \\
\hline Item & Authors' judgement & Description \\
\hline Allocation concealment? & Unclear & B - Unclear \\
\hline
\end{tabular}

\section{McCartney 1995}

\begin{tabular}{|c|c|}
\hline Methods & $\begin{array}{l}\text { RCT. All results broken down into four groups by sex and age ( } 60-70 \text { or } 70-80 \text {, only results } \\
\text { for women aged } 70-80 \text { - the largest group - used for pooled comparisons in review) } \\
\text { Method of randomisation: not reported } \\
\text { Assessor blinding: no } \\
\text { Participant blinding: no, but attention/exercise control group } \\
\text { Loss to follow-up: } 23 \\
\text { Intention-to-treat analysis: no } \\
\text { Post-program follow up: no, but exercise program had } 2 \text { year duration }\end{array}$ \\
\hline Participants & $\begin{array}{l}\text { Location: Canada } \\
\mathrm{N}=142 \\
\text { Sample: healthy volunteers } \\
\text { Age: mean } 64 \text { years (SD 2.4) for exercise group } \\
\text { Inclusion criteria: approval of family physician, successful completion of cycle ergometer } \\
\text { test, aged } 60-80 \text { years, no prior resistance training experience } \\
\text { Exclusion criteria: evidence of coronary artery disease, chronic obstructive or restrictive } \\
\text { lung disease, osteoporosis, major orthopaedic disability, smoking, body weight greater than } \\
130 \% \text { of ideal }\end{array}$ \\
\hline Interventions & $\begin{array}{l}\text { PRT versus control } \\
\text { 1. PRT } \\
\text { Type of Ex: 3UL, 3LL, 1Tr } \\
\text { Equipment: weight-lifting machines } \\
\text { Intensity: 50-80\% 1RM } \\
\text { Frequency: Ex2 } \\
\text { Reps/Sets: } 10-12 / 3 \\
\text { Program Duration: } 42 \text { weeks } \\
\text { Setting: gym } \\
\text { Supervision: not reported } \\
\text { Adherence: } 88 \% \text { (at } 1 \text { year) } \\
\text { 2. Control Group: } 2 \text { times per week low-intensity walking }\end{array}$ \\
\hline
\end{tabular}




\begin{tabular}{l|l|l} 
Outcomes & $\begin{array}{l}\text { Strength (1RM) } \\
\text { Maximum cycle ergometry } \\
\text { Treadmill testing } \\
\text { Stair climbing ergometric muscle cross-sectional area } \\
\text { Comments on adverse events: yes }\end{array}$ \\
\hline Notes & Authors' judgement & Description \\
\hline Risk of bias & B - Unclear \\
\hline Item & &
\end{tabular}

\section{McGuigan 2001}

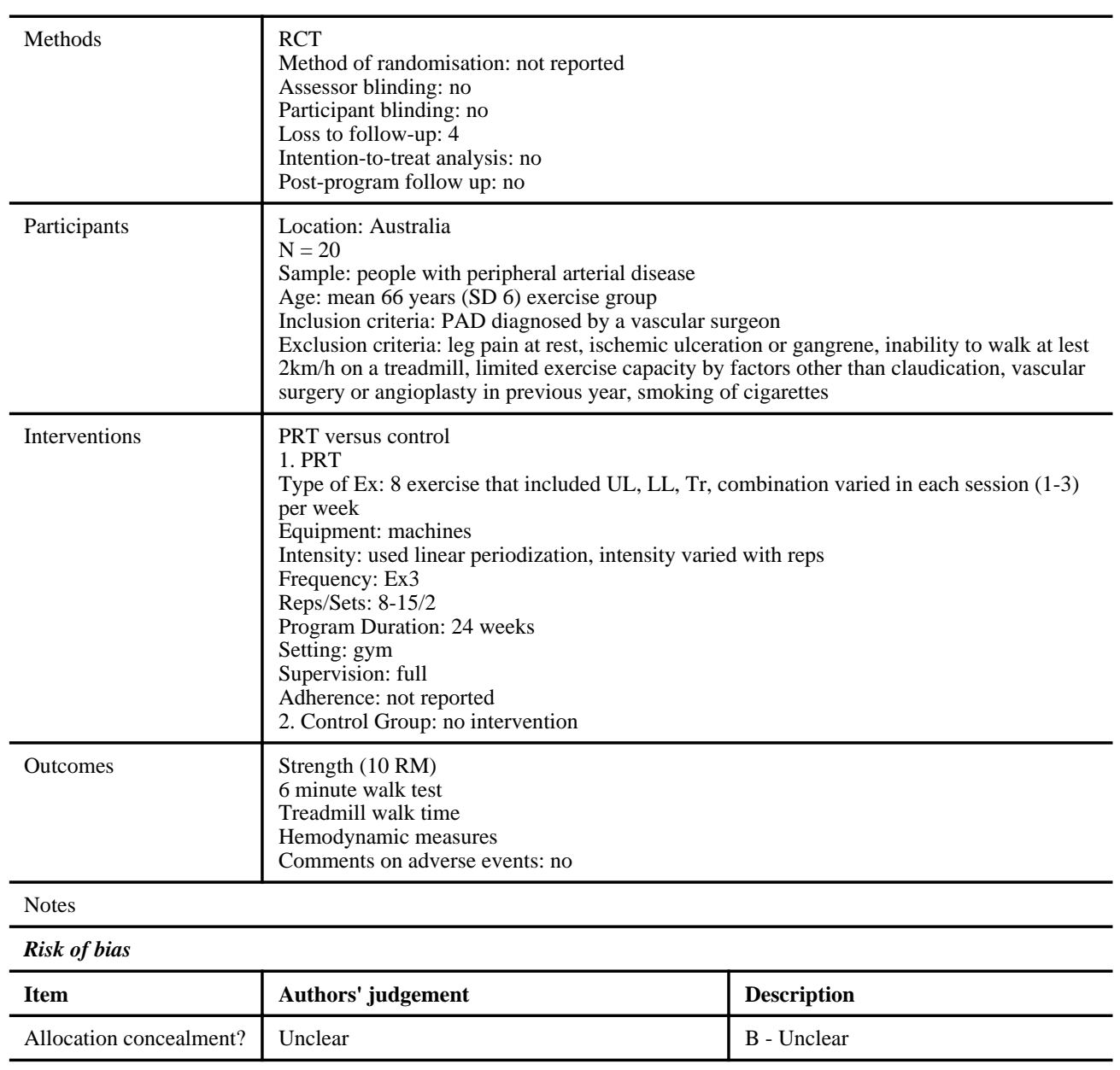

\section{McMurdo 1995}

Methods

RCT with three groups, PRT, mobility exercise programme and attention control

Method of randomisation: sealed envelopes in sequence, computer generated random

number tables generated the sequence

Assessor blinding: yes

Participant blinding: no, but attention control used

Loss to follow-up: 7 from PRT and control group 


\begin{tabular}{|c|c|c|}
\hline & \multicolumn{2}{|c|}{$\begin{array}{l}\text { Intention-to-treat analysis: no } \\
\text { Post-program follow up: no, but program } 6 \text { months long }\end{array}$} \\
\hline Participants & \multicolumn{2}{|c|}{$\begin{array}{l}\text { Location: UK } \\
\mathrm{N}=86 \text { total ( } 55 \text { in PRT vs control) } \\
\text { Sample: residents of sheltered housing complexes } \\
\text { Age: mean } 82 \text { years } \\
\text { Inclusion criteria: age } 75 \text { years and over, limited mobility requiring the use of a walking } \\
\text { aid, dependence in functional activities of daily living requiring the assistance of home help } \\
\text { at least once per week } \\
\text { Exclusion criteria: major neurological disease, unstable cardiovascular disease, severe } \\
\text { cognitive impairment }\end{array}$} \\
\hline Interventions & \multicolumn{2}{|c|}{$\begin{array}{l}\text { PRT versus control and versus mobility } \\
\text { 1. PRT } \\
\text { Type of Ex: } 24 \text { (UL, LL, trunk) } \\
\text { Equipment: theraband, progressive thickness } \\
\text { Intensity: low-moderate } \\
\text { Frequency: daily } \\
\text { Reps/Sets: } 5-10 / 1 \\
\text { Program Duration: } 26 \text { weeks } \\
\text { Setting: home } \\
\text { Supervision: low - visited at home every } 3-4 \text { weeks } \\
\text { Adherence: not reported } \\
\text { 2. Control Group: health education visits every } 3-4 \text { weeks } \\
\text { 3. Mobility Group: same } 24 \text { exercises, but with no resistance }\end{array}$} \\
\hline Outcomes & \multicolumn{2}{|c|}{$\begin{array}{l}\text { TUAG } \\
\text { Sit to stand test (time to complete } 10 \text { full stands) } \\
\text { Grip strength } \\
\text { Functional reach } \\
\text { ADL (Barthel Index) } \\
\text { Comments on adverse events: yes }\end{array}$} \\
\hline \multicolumn{3}{|l|}{ Notes } \\
\hline \multicolumn{3}{|l|}{ Risk of bias } \\
\hline Item & Authors' judgement & Description \\
\hline Allocation concealment? & Yes & A - Adequate \\
\hline
\end{tabular}

\section{Mihalko 1996}

\begin{tabular}{|c|c|}
\hline Methods & $\begin{array}{l}\text { RCT cluster randomised by residence } \\
\text { Method of randomisation: not reported } \\
\text { Assessor blinding: no } \\
\text { Participant blinding: no, but attention control group } \\
\text { Loss to follow-up: not reported } \\
\text { Intention-to-treat analysis: no } \\
\text { Post-program follow up: no }\end{array}$ \\
\hline Participants & $\begin{array}{l}\text { Location: USA } \\
\mathrm{N}=58 \\
\text { Sample: sedentary residents of senior citizen or residential nursing homes } \\
\text { Age: mean } 82.7 \text { years (SD 7.7) } \\
\text { Inclusion criteria: residents of senior citizen and residential nursing home facilities, } \\
\text { sedentary, clearance form personal physician } \\
\text { Exclusion criteria: not reported }\end{array}$ \\
\hline Interventions & $\begin{array}{l}\text { PRT versus control } \\
\text { 1. PRT } \\
\text { Type of Ex: } 5 \text { UL } \\
\text { Equipment: dumb bells } \\
\text { Intensity: high - worked until failure } \\
\text { Frequency: Ex3 } \\
\text { Reps/Sets: } 10-12 \text { reps } \\
\text { Program Duration: } 8 \text { weeks } \\
\text { Setting: gym } \\
\text { Supervision: not reported } \\
\text { Adherence: not reported } \\
\text { 2. Control Group: fluid movement program }\end{array}$ \\
\hline
\end{tabular}




\begin{tabular}{l|l|l} 
Outcomes & $\begin{array}{l}\text { ADL performance (modified version of Lawton and Brody's IADL scale) } \\
\text { Strength (1RM) } \\
\text { Satiffaction with Life Scale } \\
\text { Positive and negative affect } \\
\text { Comments on adverse events: no }\end{array}$ \\
\hline Notes & Authors' judgement & Description \\
\hline Risk of bias & B - Unclear \\
\hline Item & &
\end{tabular}

Mikesky 2006

\begin{tabular}{|c|c|c|}
\hline Methods & \multicolumn{2}{|c|}{$\begin{array}{l}\text { RCT } \\
\text { Method of randomisation: not reported-stratified } \\
\text { Assessor blinding: yes } \\
\text { Participant blinding: not reported } \\
\text { Loss to follow-up: } 36 \% \text { in PRT, } 24 \% \text { in Control (range of motion) } \\
\text { Intention-to-treat analysis: yes, done at the 30th month } \\
\text { Post-program follow up: no }\end{array}$} \\
\hline Participants & \multicolumn{2}{|c|}{$\begin{array}{l}\text { Location: USA } \\
\mathrm{N}=221(113 \text { in PRT) } \\
\text { Sample: knee OA } \\
\text { Age: mean }=69.4 \text { years }(\mathrm{SD}=8) \\
\text { Inclusion criteria: not clearly described } \\
\text { Exclusion criteria: cannot walk without assistance, amputation of either lower extremity, } \\
\text { knee or hip replacement, history of stroke, myocardial infarction, CHF, uncontrolled } \\
\text { hypertension, fibromyalgia... }\end{array}$} \\
\hline Interventions & \multicolumn{2}{|c|}{$\begin{array}{l}\text { PRT versus flexibility (control) } \\
\text { 1.PRT } \\
\text { Type of Ex: 2UL/2LL } \\
\text { Equipment: CYBEX machines at gym; Elastic bands at home, } \\
\text { Intensity: } 8 \text {-10 RM } \\
\text { Frequency: Ex3; first } 3 \text { months ( } 2 / \text { week in the gym, 1/week at home), month } 4-6 \text { (1/week in } \\
\text { the gym, } 2 / \text { week at home), month } 7-9 \text { (2/month in the gym, 3/week at home); month } 10-12 \\
\text { (1/month in the gym, 3/week at home) } \\
\text { Reps/Sets: from 8-10/ } 3 \text { to } 12 / 2 \\
\text { Duration: } 1 \text { year } \\
\text { Setting: gym and home } \\
\text { Supervision: full-1 fitness trainer in the gym } \\
\text { Adherence: attending gym (PRT-59\%, control/ROM-64\%); home ex (PRT-56\%, control/ } \\
\text { ROM-62\%) } \\
\text { 2. Flexibility exercise group: N=108, mean age = } 68.6 \text { years (SD = 7.5), flexibility ex, } 3 \\
\text { times/week }\end{array}$} \\
\hline Outcomes & \multicolumn{2}{|l|}{$\begin{array}{l}\text { Primary: SF- } 36 \text { (at the } 30 \text { month), WOMAC } \\
\text { Secondary: Strength measure (1RM) } \\
\text { Comments on adverse events: yes }\end{array}$} \\
\hline Notes & \multicolumn{2}{|c|}{ SF-36 was not pooled because it was not measured right after the training } \\
\hline \multicolumn{3}{|l|}{ Risk of bias } \\
\hline Item & Authors' judgement & Description \\
\hline Allocation concealment? & Unclear & B - Unclear \\
\hline
\end{tabular}

Miller 2006

\begin{tabular}{l|l}
\hline Methods & RCT \\
& Method of randomisation: computer generated sequence, stratified and block randomization \\
& Assessor blinding: yes \\
& Participant blinding: not reported \\
Loss to follow-up: 3 withdrawn (1 in control), 4 death (2 in PRT)
\end{tabular}

Cochrane Database Syst Rev. Author manuscript; available in PMC 2015 February 11. 


\begin{tabular}{|c|c|c|}
\hline & \multicolumn{2}{|c|}{$\begin{array}{l}\text { Intention-to-teat analysis: yes } \\
\text { Post-program follow up: no }\end{array}$} \\
\hline Participants & \multicolumn{2}{|c|}{$\begin{array}{l}\text { Location: Australia } \\
\mathrm{N}=51 \text { ( } 25 \text { in PRT) } \\
\text { Sample: fall-related lower limb fracture } \\
\text { Age: mean } 84.8 \text { years } \\
\text { Inclusion criteria: at least } 70 \text { years old, fall-related lower limb fracture } \\
\text { Exclusion criteria: (1) did not reside within southern Adelaide, (2) were unable to } \\
\text { comprehend instructions relating to positioning of the upper arm for eligibility assessment, } \\
\text { (3) were unable to fully weight bear on the side of the injury for more than seven days post } \\
\text { admission, (4) were not independently mobile prefracture, (5) were medically unstable } \\
\text { more than } 7 \text { days post admission, (6) were suffering from cancer, chronic renal failure, } \\
\text { unstable angina or unstable diabetes or (7) were not classified as malnourished }\end{array}$} \\
\hline Interventions & \multicolumn{2}{|c|}{$\begin{array}{l}\text { PRT versus control } \\
\text { 1. PRT } \\
\text { Type of Ex: } 5 \mathrm{LL} \\
\text { Equipment: elastic band } \\
\text { Intensity: was appropriate to baseline strength, pain level and range of motion } \\
\text { Frequency: Ex3 } \\
\text { Reps/Sets: increased to } 8 / 2 \text { if exercise could be completed in good form } \\
\text { Duration: } 12 \text { weeks } \\
\text { Setting: a teaching hospital } \\
\text { Supervision: full-pysiotherapist } \\
\text { Adherence: > } 86 \% \\
\text { 2. Control group: attention control, week 1-6: tri-weekly home visits, week 7-12: weekly } \\
\text { home visit;discussion of general information during the visit }\end{array}$} \\
\hline Outcomes & \multicolumn{2}{|c|}{$\begin{array}{l}\text { Primary: SF-12 } \\
\text { Secondary: strength measure, gait speed } \\
\text { Comments on adverse events: no }\end{array}$} \\
\hline Notes & \multicolumn{2}{|c|}{$\begin{array}{l}\text { Reported Median \& 95\%CI. Data from participants who took nutrition supplementation } \\
\text { were not extracted }\end{array}$} \\
\hline \multicolumn{3}{|l|}{ Risk of bias } \\
\hline Item & Authors' judgement & Description \\
\hline Allocation concealment? & Unclear & B - Unclear \\
\hline
\end{tabular}

\section{Miszko 2003}

\begin{tabular}{|c|c|}
\hline Methods & $\begin{array}{l}\text { RCT with } 3 \text { groups: PRE, control, and power exercise } \\
\text { Method of randomisation: stratified by sex } \\
\text { Assessor blinding: not reported } \\
\text { Participant blinding: not reported } \\
\text { Loss to follow-up: } 4 / 17 \text { in PRT, } 7 / 18 \text { in power } \\
\text { Intention-to-treat analysis: no } \\
\text { Post-program follow up: no }\end{array}$ \\
\hline Participants & $\begin{array}{l}\text { Location: USA } \\
\mathrm{N}=28(13 \text { in PRT) } \\
\text { Sample: older adults with below average leg extensor power } \\
\text { Age: mean } 72.8 \text { years (SD }=5.4) \\
\text { Inclusion criteria: below-average leg extensor power } \\
\text { Exclusion criteria: poorly controlled or unstable cardiovascular disease or diabetes, recent } \\
\text { unhealed bone fracture (within the past } 12 \text { months), severe hypertension while resting } \\
\text { quietly in the supine position, leg or arm amputation, excessive alcohol intake (more than } \\
\text { three drinks per day), a classic anterior compression fracture, neuromuscular disorders, } \\
\text { being nonambulatory, or having recent (within } 6 \text { months) involvement in a strength-training } \\
\text { or running or jogging program }\end{array}$ \\
\hline Interventions & $\begin{array}{l}\text { PRT versus control and versus power exercise } \\
\text { 1.PRT } \\
\text { Type of Ex: } 4 \text { UL/4LL \& squats } \\
\text { Equipment: Keiser Inc. } \\
\text { Intensity: } 50 \% \text {-> } 70 \% \text { of } 1 \text { RM by week } 8,80 \% \text { of } 1 \mathrm{RM} \text { the last } 8 \text { weeks } \\
\text { Frequency: Ex3 } \\
\text { Reps/Sets: } 6-8 / 3 \\
\text { Duration: } 16 \text { weeks } \\
\text { Setting: not reported (Gym?) }\end{array}$ \\
\hline
\end{tabular}




\begin{tabular}{l|l|l} 
& $\begin{array}{l}\text { Supervision: not reported } \\
\text { Adherence: not reported } \\
\text { 2. Control Group: maintain usual activity and attend 3 educational presentations over the } \\
\text { study period } \\
\text { 3. Power Ex Group: N=11, mean age =72.3 years (SD = 6.7), the same exercise as the PRT } \\
\text { group butdid jump squats instead of squats, 6-8 repetition at 40\% of 1RM, move as fast as } \\
\text { possible }\end{array}$ \\
\hline Outcomes & $\begin{array}{l}\text { Primary: Continuous Scale Physical Functional Performance } \\
\text { Secondary: strength measure (1 RM) } \\
\text { Comments on adverse events: yes }\end{array}$ \\
\hline Notes & Involved power training & Description \\
\hline Risk of bias & Authors' judgement & B - Unclear \\
\hline Item & Unclear & \\
\hline Allocation concealment? & &
\end{tabular}

Moreland 2001

\begin{tabular}{|c|c|c|}
\hline Methods & \multicolumn{2}{|c|}{$\begin{array}{l}\text { RCT } \\
\text { Method of randomisation: concealed, phoned central office } \\
\text { Assessor blinding: yes } \\
\text { Participant blinding: not reported } \\
\text { Loss to follow-up: } 10 \\
\text { Intention-to-treat analysis: yes } \\
\text { Post-program follow up: yes, at the 6th month }\end{array}$} \\
\hline Participants & \multicolumn{2}{|c|}{$\begin{array}{l}\text { Location: Canada } \\
\mathrm{N}=133 \text { ( } 68 \text { in PRT) } \\
\text { Sample: people post-stroke } \\
\text { Age: mean } 69 \text { years } \\
\text { Inclusion criteria: not reported } \\
\text { Exclusion criteria: not reported }\end{array}$} \\
\hline Interventions & \multicolumn{2}{|c|}{$\begin{array}{l}\text { PRT versus control } \\
\text { 1.PRT } \\
\text { Type of Ex: UL, LL } \\
\text { Equipment: not reported } \\
\text { Intensity: not reported } \\
\text { Frequency: not reported } \\
\text { Reps/Sets: not reported } \\
\text { Program Duration: until hospital discharge } \\
\text { Setting: hospital } \\
\text { Supervision: full } \\
\text { Adherence: not reported } \\
\text { 2. Control Group: regular therapy }\end{array}$} \\
\hline Outcomes & \multicolumn{2}{|c|}{$\begin{array}{l}\text { Primary: Chedoke-McMaster Stroke Assessment } \\
\text { Secondary: 2-minute walk test } \\
\text { Comments on adverse events: yes }\end{array}$} \\
\hline \multicolumn{3}{|l|}{ Notes } \\
\hline \multicolumn{3}{|l|}{ Risk of bias } \\
\hline Item & Authors' judgement & Description \\
\hline Allocation concealment? & Yes & A - Adequate \\
\hline
\end{tabular}

Nelson 1994

\begin{tabular}{l|l}
\hline Methods & RCT \\
& Method of randomisation: not reported \\
& Assessor blinding: no \\
& Participant blinding: no \\
& Loss to follow-up: 1
\end{tabular}




\begin{tabular}{|c|c|c|}
\hline & \multicolumn{2}{|c|}{$\begin{array}{l}\text { Intention-to-treat analysis: yes } \\
\text { Post-program follow up: no, but program had 1-year duration }\end{array}$} \\
\hline Participants & \multicolumn{2}{|c|}{$\begin{array}{l}\text { Location: USA } \\
\text { N=40 } \\
\text { Sample: healthy females post-menopause } \\
\text { Age:mean } 61.1 \text { years (SD 3.7) } \\
\text { Inclusion criteria: at least } 5 \text { years post-menopausal but not older than 70, do not engage in } \\
\text { any regular physical training, weigh less than } 130 \% \text { of ideal body weight, currently non- } \\
\text { smoking, do not have more than one crush fracture of the spine, no history of other } \\
\text { osteoporotic fractures, have not taken estrogen or other medications known to affect bone } \\
\text { for } 12 \text { months, passed physical screening (including ECG during strength training session) } \\
\text { Exclusion criteria: not reported }\end{array}$} \\
\hline Interventions & \multicolumn{2}{|c|}{$\begin{array}{l}\text { PRT versus control } \\
1 . P R T \\
\text { Type of Ex: } 2 \text { LL, } 1 \text { UL, } 2 \mathrm{Tr} \\
\text { Equipment: pneumatic resistance machines (Keiser) } \\
\text { Intensity: } 80 \% \text { of } 1 \mathrm{RM} \\
\text { Frequency: Ex2 } \\
\text { Reps/Sets: } 8 / 3 \\
\text { Program Duration: } 52 \text { weeks } \\
\text { Adherence: } 87.5 \% \\
\text { Setting: gym } \\
\text { Supervision: full } \\
\text { 2. Control Group: asked to maintain normal level of activity, could receive the exercise } \\
\text { program at the end of the trial }\end{array}$} \\
\hline Outcomes & \multicolumn{2}{|c|}{$\begin{array}{l}\text { Strength (1RM) } \\
\text { Balance (backward walking) } \\
\text { Physical activity (Harvard Alumini Questionnaire, kJ/week) } \\
\text { Comments on adverse events: yes }\end{array}$} \\
\hline \multicolumn{3}{|l|}{ Notes } \\
\hline \multicolumn{3}{|l|}{ Risk of bias } \\
\hline Item & Authors' judgement & Description \\
\hline Allocation concealment? & Unclear & B - Unclear \\
\hline
\end{tabular}

\section{Newnham 1995}

\begin{tabular}{l|l}
\hline Methods & RCT \\
& Method of randomisation: not reported \\
& Assessor blinding: yes \\
& Participant blinding: no, but attention control \\
Loss to follow-up: 6 & Intention-to-treat analysis: no \\
& Post-program follow up: yes, at the 24 week \\
\hline \multirow{2}{*}{ Participants } & Location: Canada \\
& N = 30 \\
& Sample: residents of long-term care facility \\
& Age: mean 81.7 years (SD 5.6) \\
& Inclusion criteria: age 70+, independent in ambulation (with or without walking aid) over \\
& 40m at <0.9m/s, 20+ on TUAG; at least 90 degrees of available ROM at knee, can follow a \\
& 3-step command \\
& Exclusion criteria: have Parkinsons Disease or CVA; participation in strength training in \\
& the past year; unstable medical conditions \\
\hline
\end{tabular}




\begin{tabular}{l|l|l} 
& PRT versus control \\
& 1.PRT & Type of Ex: UL, LL \\
& Equipment: pullies \\
& Intensity: 80\% of 1RM & Frequency: Ex3 \\
& Reps/Sets: 10/3 & \\
& Program Duration: 12 weeks & \\
& Setting: gym in nursing home & \\
& Supervision: full & \\
& Adherence: 86\% & \\
& 2. Control Group: attention control & \\
\hline Outcomes & Strength (1RM) & \\
& Gait velocity & \\
& TUAG & \\
& Balance (Berg) & \\
\hline Notes & Comments on adverse events: no & Description \\
\hline Risk of bias & Authors' judgement & B - Unclear \\
\hline Item & Unclear & \\
\hline Allocation concealment? &
\end{tabular}

\section{Nichols 1993}

\begin{tabular}{|c|c|c|}
\hline Methods & \multicolumn{2}{|c|}{$\begin{array}{l}\text { RCT } \\
\text { Method of randomisation: stratified into rank-ordered pairs and randomised } \\
\text { Assessor blinding: no } \\
\text { Participant blinding: no } \\
\text { Loss to follow-up: } 6 \\
\text { Intention-to-treat analysis: no } \\
\text { Post-program follow up: no, but } 6 \text { month duration of program }\end{array}$} \\
\hline Participants & \multicolumn{2}{|c|}{$\begin{array}{l}\text { Location: USA } \\
\mathrm{N}=36 \\
\text { Sample: active healthy women } \\
\text { Age: mean } 67.8 \text { years (SE } 1.6) \\
\text { Inclusion criteria: greater than } 60 \text { years, active for at least } 6 \text { months prior to the trial with } \\
\text { exercise at least } 3 \text { times per week, physician's consent } \\
\text { Exclusion criteria: previous weight training, history of cardiovascular disease, taking } \\
\text { thyroid or cardiac medications, nonestrogen repleted }\end{array}$} \\
\hline Interventions & \multicolumn{2}{|c|}{$\begin{array}{l}\text { PRT versus control } \\
\text { 1. PRT } \\
\text { Type of Ex: 4UL, 2LL, } 1 \mathrm{Tr} \\
\text { Equipment: variable resistance machines (Polaris) } \\
\text { Intensity: } 80 \% \text { 1RM } \\
\text { Frequency: Ex3 } \\
\text { Reps/Sets: } 8-10 / 3 \\
\text { Program Duration: } 24 \text { weeks } \\
\text { Setting: gym } \\
\text { Adherence: } 87 \% \text { of sessions } \\
\text { Supervision: full } \\
\text { 2. Control Group: maintain current routine }\end{array}$} \\
\hline Outcomes & \multicolumn{2}{|c|}{$\begin{array}{l}\text { Strength (1RM) } \\
\text { Activity performance } \\
\text { Blair Seven Day Recall } \\
\text { Comments on adverse events: yes (safety a priority objective) }\end{array}$} \\
\hline \multicolumn{3}{|l|}{ Notes } \\
\hline \multicolumn{3}{|l|}{ Risk of bias } \\
\hline Item & Authors' judgement & Description \\
\hline Allocation concealment? & Unclear & B - Unclear \\
\hline
\end{tabular}


Ouellette 2004

\begin{tabular}{|c|c|c|}
\hline Methods & \multicolumn{2}{|c|}{$\begin{array}{l}\text { RCT } \\
\text { Method of randomisation: not reported } \\
\text { Assessor blinding: not reported } \\
\text { Participant blinding: not reported } \\
\text { Loss to Follow-up: 0 } \\
\text { Intention-to-treat analysis: yes } \\
\text { Post-program follow up: no }\end{array}$} \\
\hline Participants & \multicolumn{2}{|c|}{$\begin{array}{l}\text { Location: USA } \\
\mathrm{N}=42 \text { ( } 21 \text { in each group) } \\
\text { Sample: single mild to moderate stroke } \\
\text { Age: mean } 65.8 \text { years ( } \mathrm{SD}=2.5 \text { ) } \\
\text { Inclusion criteria: subjects aged at least } 50 \text { years, } 6 \text { months to } 6 \text { years following a single } \\
\text { unilateral mild to moderate stroke with residual lower extremity hemiparesis, community } \\
\text { dwelling, independent ambulation with or without an assistive device, report of } 2 \text { or more } \\
\text { limitations on the physical function subscale (PF } 10 \text { ) of the Medical Outcomes Survey } \\
\text { Short-Form, ability to travel to the exercise laboratory, and willingness to be randomized. } \\
\text { Stroke was diagnosed by history and clinical examination, and confirmed via medical } \\
\text { records review. } \\
\text { Exclusion criteria: myocardial infarction within the past } 6 \text { months, symptomatic coronary } \\
\text { artery disease or congestive heart failure, uncontrolled hypertension, fracture within the } \\
\text { past } 6 \text { months, acute or terminal illness, score less than } 20 \text { on the MMSE, inability to } \\
\text { follow a } 3 \text {-step command, current participation in regular strength training or supervised } \\
\text { physical therapy, or pain during exercise }\end{array}$} \\
\hline Interventions & \multicolumn{2}{|c|}{$\begin{array}{l}\text { PRT versus control } \\
\text { 1. PRT } \\
\text { Type of Ex: } 4 \text { LLs } \\
\text { Equipment: Pneumatic resistance training equipment (Keiser Sports Health Equipment) and } \\
\text { modified } \\
\text { stack-pulley system (Therapy Systems) } \\
\text { Intensity: } 70 \% \text { of } 1 \text { RM } \\
\text { Frequency: Ex3 } \\
\text { Reps/Sets: } 8-10 / 3 \\
\text { Duration: } 12 \text { weeks } \\
\text { Setting: gym } \\
\text { Supervision: full } \\
\text { Adherence: } 85.4 \% \text {-PRT; } 79.9 \% \text {-controls } \\
\text { 2. Control group: bilateral range of motion ex and upper body flexibility exercise }\end{array}$} \\
\hline Outcomes & \multicolumn{2}{|c|}{$\begin{array}{l}\text { Primary: Late-Life Function and Disability Instrument, sickness impact profile } \\
\text { Secondary: strength measure (1 RM), 6-minutes walk, gait speed, stair climb, chair rise } \\
\text { Comments on adverse events: yes }\end{array}$} \\
\hline Notes & \multicolumn{2}{|c|}{ SD is obtained from SE for LLFD 1} \\
\hline \multicolumn{3}{|l|}{ Risk of bias } \\
\hline Item & Authors' judgement & Description \\
\hline Allocation concealment? & Unclear & B - Unclear \\
\hline
\end{tabular}

Parkhouse 2000

\begin{tabular}{l|l}
\hline Methods & RCT \\
& Method of randomisation: not reported \\
& Assessor blinding: no \\
& Participant blinding: no \\
& Loss to Follow-up: not reported \\
& Intention-to-treat analysis: no \\
& Post-program follow up: no \\
\hline Participants & Location: Canada \\
& N=22 \\
& Sample: sedentary older women with low bone mineral density \\
& Age: mean 68.1 years \\
& Inclusion criteria: community-dwelling, sedentary, post-menopausal women, aged 60-80 \\
& years, low bone mineral density
\end{tabular}




\begin{tabular}{l|l|l} 
& $\begin{array}{l}\text { Exclusion criteria: medical or orthopaedic problems that would interfere with their ability } \\
\text { to participate in physical activity, on hormone replacement }\end{array}$ \\
\hline Interventions & $\begin{array}{l}\text { PRT versus control } \\
\text { 1. PRT } \\
\text { Type of Ex: 9 LL } \\
\text { Equipment: not reported } \\
\text { Intensity: 75-80\% of 1RM } \\
\text { Frequency: Ex3 } \\
\text { Reps/Sets: 8-10/3 } \\
\text { Program Duration: 8 months } \\
\text { Setting: gym } \\
\text { Supervision: not reported } \\
\text { Adherence: not reported } \\
\text { 2. Control Group: not reported }\end{array}$ \\
\hline Outcomes & $\begin{array}{l}\text { Strength (1RM) } \\
\text { Comments on adverse events: no }\end{array}$ \\
\hline Notes & \multicolumn{2}{|l}{} \\
\hline Risk of bias & Authors' judgement & Description \\
\hline Item & Unclear & B - Unclear \\
\hline Allocation concealment? &
\end{tabular}

Pollock 1991

\begin{tabular}{|c|c|}
\hline Methods & $\begin{array}{l}\text { RCT with } 3 \text { groups: PRT, control, and aerobic training group } \\
\text { Method of randomisation: rank ordered then randomly stratified into } 3 \text { groups, with the } \\
\text { restriction that more would be assigned to training groups } \\
\text { Assessor blinding: no } \\
\text { Participant blinding: no } \\
\text { Loss to Follow-up: } 8 \text { total ( } 4 \text { in PRT and control) } \\
\text { Intention-to-treat analysis: no } \\
\text { Post-program follow up: no, but } 6 \text { month exercise program }\end{array}$ \\
\hline Participants & $\begin{array}{l}\text { Location: USA } \\
\mathrm{N}=57 \text { in total ( } 36 \text { in PRT and control) } \\
\text { Sample: sedentary men and women } \\
\text { Age: mean } 72 \text { years } \\
\text { Inclusion criteria: free from overt evidence of coronary heart disease or any other } \\
\text { conditions that would limit their participation in vigorous exercise; aged } 70-79 \text {, sedentary } \\
\text { for one year } \\
\text { Exclusion criteria: blood pressure > 160/100; ECG changes or cardiac symptoms during } \\
\text { exercise testing }\end{array}$ \\
\hline Interventions & $\begin{array}{l}\text { PRT versus control and versus aerobic } \\
\text { 1. PRT } \\
\text { Type of Ex: 5UL, 2LL, } 3 \mathrm{Tr} \\
\text { Equipment: variable resistance machines (Nautilus) } \\
\text { Intensity: initially light to moderate, by week } 14 \text { encouraged to train to fatigue } \\
\text { Frequency: Ex3 } \\
\text { Reps/Sets: } 8-12 / 1 \\
\text { Program Duration: } 26 \text { weeks } \\
\text { Setting: gym } \\
\text { Supervision: not reported } \\
\text { Adherence: } 97.8 \% \text { sessions attended (excluding drop-outs), } 87 \% \text { stayed with program } \\
\text { 2. Control Group: not reported } \\
\text { 3. Aerobic Training Group: } 3 \text { sessions per week of walk/jog program for } 26 \text { weeks, aimed } \\
\text { for duration of } 35-45 \text { min minutes at } 75-85 \% \text { VO2 max by week } 26\end{array}$ \\
\hline Outcomes & $\begin{array}{l}\text { Strength } \\
\text { VO2 max } \\
\text { Adverse events } \\
\text { Reaction time } \\
\text { Comments on adverse events: yes (a priority outcome, well- defined) }\end{array}$ \\
\hline Notes & Data from PRT and aerobic training group were compared \\
\hline
\end{tabular}




\begin{tabular}{l|l|l} 
Item & Authors' judgement & Description \\
\hline Allocation concealment? & Unclear & B - Unclear \\
\hline
\end{tabular}

Pu 2001

\begin{tabular}{|c|c|c|}
\hline Methods & \multicolumn{2}{|c|}{$\begin{array}{l}\text { RCT } \\
\text { Method of randomisation: matched by age then randomised } \\
\text { Assessor blinding: no } \\
\text { Participant blinding: no } \\
\text { Loss to follow-up: } 2 \\
\text { Intention-to-treat analysis: yes } \\
\text { Post-program follow up: no }\end{array}$} \\
\hline Participants & \multicolumn{2}{|c|}{$\begin{array}{l}\text { Location: USA } \\
\mathrm{N}=16 \\
\text { Sample: older women with CHF } \\
\text { Age: mean } 77 \text { years (SE } 6 \text { ) } \\
\text { Inclusion criteria: community-dwelling women; } 65 \text { years or older; mild to moderate } \\
\text { systolic heart failure New York Heart Association (NYHA) class I to III; resting ejection } \\
\text { fraction less than or equal to } 45 \% \text {, } \\
\text { Exclusion criteria: NYHA class IV heart failure; myocardial infarction within } 6 \text { months of } \\
\text { randomisation, hospitalization for CHF within } 2 \text { months, change of CHF therapy within } 1 \\
\text { MO; unstable angina pectoris, fixed ventricular rate pacemaker, abdominal aortic aneurysm } \\
>4 \mathrm{~cm}, \text { major limb amputation, symptomatic abdominal or inguinal hernias, MMSE }<23 \text {, } \\
\text { signification abnormalities on treadmill or strength testing, any unstable medical conditions }\end{array}$} \\
\hline Interventions & \multicolumn{2}{|c|}{$\begin{array}{l}\text { PRT versus control } \\
\text { 1. PRT } \\
\text { Type of Ex: } 2 \text { UL, } 2 \mathrm{LL} \\
\text { Equipment: pneumatic resistance equipment (Keiser) } \\
\text { Intensity: } 80 \% \text { of } 1 \text { RM } \\
\text { Frequency: Ex3 } \\
\text { Reps/Sets: } 8 / 3 \\
\text { Program Duration: } 10 \text { weeks } \\
\text { Setting: Gym } \\
\text { Adherence: } 98 \% \\
\text { Supervision: Full } \\
\text { 2. Control Group: sham exercise group } 2 \text { time per week of supervised, low-intensity } \\
\text { stretches for } 10 \text { weeks }\end{array}$} \\
\hline Outcomes & \multicolumn{2}{|c|}{$\begin{array}{l}\text { Exercise capacity (6-minute walk) } \\
\text { Maximal oxygen consumption } \\
\text { Comments on adverse events: yes }\end{array}$} \\
\hline \multicolumn{3}{|l|}{ Notes } \\
\hline \multicolumn{3}{|l|}{ Risk of bias } \\
\hline Item & Authors' judgement & Description \\
\hline Allocation concealment? & Unclear & B - Unclear \\
\hline
\end{tabular}

\section{Rall 1996}

\begin{tabular}{l|l}
\hline Methods & RCT: (groups of healthy young people and middle-aged people with RA not included in \\
this review) & Method of randomisation: not reported \\
& Assessor blinding: no \\
& Participant blinding: no \\
& Loss to follow-up: 0 \\
& Intention-to-treat analysis: no drop-outs, not stated \\
& Post-program follow up: no \\
\hline Participants & Location: USA \\
& N = 14 \\
& Sample: healthy elderly \\
& Age: mean 70.3 years (SD 5)
\end{tabular}

Cochrane Database Syst Rev. Author manuscript; available in PMC 2015 February 11. 


\begin{tabular}{|c|c|c|}
\hline & \multicolumn{2}{|c|}{$\begin{array}{l}\text { Inclusion criteria: healthy older people (ages } 65-80 \text { ) } \\
\text { Exclusion criteria: obese (BMI }>30 \text { ), diabetes, cancer, renal disease, liver disease, cardiac } \\
\text { artery disease, endocrine disorder, autoimmune disease }\end{array}$} \\
\hline Interventions & \multicolumn{2}{|c|}{$\begin{array}{l}\text { PRT versus control } \\
\text { 1. PRT } \\
\text { Type of Ex: 1UL, 2LL, } 2 \mathrm{Tr} \\
\text { Equipment: pneumatic resistance machines (Keiser) } \\
\text { Intensity: } 80 \% \text { of } 1 \mathrm{RM} \\
\text { Frequency: Ex2 } \\
\text { Reps/Sets: } 8 / 3 \\
\text { Program Duration: } 12 \text { weeks } \\
\text { Setting: gym } \\
\text { Supervision: full } \\
\text { Adherence: } 92 \% \\
\text { 2. Control Group: } 15 \text { minutes of water exercises }\end{array}$} \\
\hline Outcomes & \multicolumn{2}{|c|}{$\begin{array}{l}\text { Strength (1RM) } \\
\text { Aerobic capacity - VO2 max } \\
\text { Comments on adverse events: yes }\end{array}$} \\
\hline \multicolumn{3}{|l|}{ Notes } \\
\hline \multicolumn{3}{|l|}{ Risk of bias } \\
\hline Item & Authors' judgement & Description \\
\hline Allocation concealment? & Unclear & B - Unclear \\
\hline
\end{tabular}

\section{Reeves 2004}

\begin{tabular}{|c|c|c|}
\hline Methods & \multicolumn{2}{|c|}{$\begin{array}{l}\text { RCT } \\
\text { Method of randomisation: not reported } \\
\text { Assessor blinding: not reported } \\
\text { Participant blinding: not reported } \\
\text { Loss to follow-up: } 0 \\
\text { Intention-to-treat analysis: not reported } \\
\text { Post-program follow up: no }\end{array}$} \\
\hline Participants & \multicolumn{2}{|c|}{$\begin{array}{l}\text { Location: } \mathrm{UK} \\
\mathrm{N}=18 \text { ( } 9 \text { in each group) } \\
\text { Sample: physically active volunteers } \\
\text { Age: mean } 74.3 \text { years }(\mathrm{SD}=3.5) \\
\text { Inclusion criteria: no neurological or musculoskeletal disorder that might prevent } \\
\text { participation } \\
\text { Exclusion criteria: not reported }\end{array}$} \\
\hline Interventions & \multicolumn{2}{|c|}{$\begin{array}{l}\text { PRT versus control } \\
\text { 1. PRT } \\
\text { Type of Ex: } 2 \text { UL/2LL } \\
\text { Equipment: Technogym machines } \\
\text { Intensity: } 80 \% \text { of } 5 \text { RM } \\
\text { Frequency: Ex3 } \\
\text { Reps/Sets: } 10 / 2 \\
\text { Duration: } 14 \text { weeks } \\
\text { Setting: not reported } \\
\text { Supervision: full } \\
\text { Adherence: } 93 \% \\
\text { 2. Control Group: to keep normal activity level }\end{array}$} \\
\hline Outcomes & \multicolumn{2}{|c|}{$\begin{array}{l}\text { Muscle strength } \\
\text { Comments on adverse events: no }\end{array}$} \\
\hline \multicolumn{3}{|l|}{ Notes } \\
\hline \multicolumn{3}{|l|}{ Risk of bias } \\
\hline Item & Authors' judgement & Description \\
\hline Allocation concealment? & Unclear & B - Unclear \\
\hline
\end{tabular}


Rhodes 2000

\begin{tabular}{|c|c|c|}
\hline Methods & \multicolumn{2}{|c|}{$\begin{array}{l}\text { RCT } \\
\text { Method of randomisation: not reported } \\
\text { Assessor blinding: no } \\
\text { Participant blinding: no } \\
\text { Loss to follow-up: } 6 \\
\text { Intention-to-treat analysis: no } \\
\text { Post-program follow up: no, but exercise program } 1 \text { year duration }\end{array}$} \\
\hline Participants & \multicolumn{2}{|c|}{$\begin{array}{l}\text { Location: Canada } \\
\mathrm{N}=44 \\
\text { Sample: healthy, community-dwelling sedentary women } \\
\text { Age: mean } 68.8 \text { years } \\
\text { Inclusion criteria: aged } 65-75 \text {, not actively engaged in an organised activity program, had } \\
\text { independent community dwelling status, passed medical screening by doctor } \\
\text { Exclusion criteria: recent hospital stay, blind, severe hearing impairment, uncontrolled } \\
\text { hypertension and diabetes, symptomatic cardiorespiratory disease, severe renal or hepatic } \\
\text { disease, uncontrolled epilepsy, progressive neurological disease, chronic disabling arthritis, } \\
\text { MMSE<25/30, anaemia, marked obesity with the inability to exercise, regular exercise at } \\
\text { the time of screening more than } 3 \text { times } 30 \text { minutes per week, current use of Beta-blockers, } \\
\text { oral anti-coagulants or central nervous system stimulants }\end{array}$} \\
\hline Interventions & \multicolumn{2}{|c|}{$\begin{array}{l}\text { PRT versus control } \\
\text { 1. PRT } \\
\text { Type of Ex: 3UL, 3LL } \\
\text { Equipment: weight-lifting equipment (Universal Gym) } \\
\text { Intensity: } 75 \% \text { RMM } \\
\text { Frequency: Ex3 } \\
\text { Reps/Sets: } 8 / 3 \\
\text { Program duration: } 1 \text { year } \\
\text { Setting: first } 3 \text { months in supervised gym, last } 9 \text { months at a recreation facility close to } \\
\text { participants' home } \\
\text { Supervision: supervised for first } 3 \text { months, last } 9 \text { months had occasional visits from study } \\
\text { staff } \\
\text { Adherence: } 86 \% \text { (attendance) } \\
\text { 2. Control Group: asked to maintain normal lifestyle, could participate in exercises at the } \\
\text { end of the trial }\end{array}$} \\
\hline Outcomes & \multicolumn{2}{|c|}{$\begin{array}{l}\text { Muscle strength (1RM, hand grip) Flexibility (trunk flexion test) Comments on adverse } \\
\text { events: no }\end{array}$} \\
\hline \multicolumn{3}{|l|}{ Notes } \\
\hline \multicolumn{3}{|l|}{ Risk of bias } \\
\hline Item & Authors' judgement & Description \\
\hline Allocation concealment? & Unclear & B - Unclear \\
\hline
\end{tabular}

\section{Schilke 1996}

\begin{tabular}{l|l}
\hline Methods & RCT \\
& Method of randomisation: table of random numbers used \\
Assessor blinding: no \\
Participant blinding: no \\
Loss to follow-up: no \\
Intention-to-treat analysis: No dropouts, not stated ITT \\
Post-program follow up: no
\end{tabular}

Cochrane Database Syst Rev. Author manuscript; available in PMC 2015 February 11. 


\begin{tabular}{l|l|l} 
& $\begin{array}{l}\text { Intensity: high - maximal contractions } \\
\text { Frequency: Ex3 } \\
\text { Reps/Sets: 5/ 6 by session 6 (the end of week 2) } \\
\text { Program duration: 8 weeks } \\
\text { Setting: gym } \\
\text { Supervision: full } \\
\text { Adherence: not reported } \\
\text { 2. Control Group: usual activities }\end{array}$ \\
\hline Outcomes & $\begin{array}{l}\text { Strength (isokinetic dynamometer) } \\
\text { Timed walk } \\
\text { Range of motion } \\
\text { Health status (Arthritis Impact Measurement Scales; higher score = poor health status) } \\
\text { Osteoarthritis Screening Index (OASI; modified from RheumatoidArthritis Disease } \\
\text { Activity Index; higher score = worse health) } \\
\text { Comments on adverse events: no }\end{array}$ \\
\hline Notes & \multicolumn{2}{|l}{} \\
\hline Risk of bias & Authors' judgement & Description \\
\hline Item & Unclear & B - Unclear \\
\hline Allocation concealment? &
\end{tabular}

\section{Schlicht 1999}

\begin{tabular}{|c|c|c|}
\hline Methods & \multicolumn{2}{|c|}{$\begin{array}{l}\text { RCT } \\
\text { Method of randomisation: not reported } \\
\text { Assessor blinding: no } \\
\text { Participant blinding: no } \\
\text { Loss to follow-up: } 2 \\
\text { Intention-to-treat analysis: no } \\
\text { Post-program follow up: no }\end{array}$} \\
\hline Participants & \multicolumn{2}{|c|}{$\begin{array}{l}\text { Location: USA } \\
\text { N=24 } \\
\text { Sample: moderately active, community-dwelling men and women } \\
\text { Age: mean } 72 \text { years (SD 6.3) } \\
\text { Inclusion criteria: } 60 \text { years and older, community-dwelling, physician consent to participate } \\
\text { Exclusion criteria: dependent living status, current involvement in a strength training } \\
\text { program, physiological disorders that precluded strenuous exercise or affected vestibular } \\
\text { function }\end{array}$} \\
\hline Interventions & \multicolumn{2}{|c|}{$\begin{array}{l}\text { PRT versus control } \\
\text { 1. PRT } \\
\text { Type of Ex: } 6 \mathrm{LL} \\
\text { Equipment: resistance training machines (Universal, Cybex and Paramount equipment) } \\
\text { Intensity: } 75 \% \text { of } 1 \mathrm{RM} \\
\text { Frequency: Ex3 } \\
\text { Reps/Sets: } 10 / 2 \\
\text { Program Duration: } 8 \text { weeks } \\
\text { Setting: gym } \\
\text { Supervision: not reported } \\
\text { Adherence: } 99 \% \text { (excluding drop outs) } \\
\text { 2. Control Group: not reported }\end{array}$} \\
\hline Outcomes & \multicolumn{2}{|c|}{$\begin{array}{l}\text { Muscle strength (1 RM) } \\
\text { Maximum walking speed } \\
\text { 5-rep sit-to-stand } \\
\text { Balance (1-leg stance with eyes shut) } \\
\text { Comments on adverse events: yes }\end{array}$} \\
\hline \multicolumn{3}{|l|}{ Notes } \\
\hline \multicolumn{3}{|l|}{ Risk of bias } \\
\hline Item & Authors' judgement & Description \\
\hline Allocation concealment? & Unclear & B - Unclear \\
\hline
\end{tabular}


Segal 2003

\begin{tabular}{|c|c|c|}
\hline Methods & \multicolumn{2}{|c|}{$\begin{array}{l}\text { RCT } \\
\text { Method of randomisation: using a table of random numbers, which was stratified by study } \\
\text { centers and intent of treatment (curative or palliative) } \\
\text { Assessor blinding: yes } \\
\text { Participant blinding: no } \\
\text { Loss to follow-up: } 8 / 82 \text { in the PRT group; } 12 / 73 \text { in the control group } \\
\text { Intention-to-treat analysis: yes } \\
\text { Post-program follow up: no }\end{array}$} \\
\hline Participants & \multicolumn{2}{|c|}{$\begin{array}{l}\text { Location: Canada } \\
\mathrm{N}=155(82 \text { in PRT) } \\
\text { Sample: men with prostate cancer } \\
\text { Age: mean } 68.2 \text { years }(\mathrm{SD}=7.9) \\
\text { Inclusion criteria: had prostate cancer, would received androgen deprivation therapy for at } \\
\text { least } 3 \text { months after recruitment, and the treating oncologist provided consent } \\
\text { Exclusion criteria: severe cardiac disease, uncontrolled hypertension, pain, unstable bone } \\
\text { lesions, and residence more than } 1 \mathrm{hr} \text { from the study center }\end{array}$} \\
\hline Interventions & \multicolumn{2}{|c|}{$\begin{array}{l}\text { PRT versus control } \\
\text { 1. PRT } \\
\text { Type of Ex: } 6 \mathrm{UL} / 3 \mathrm{LL} \\
\text { Equipment: not reported } \\
\text { Intensity: } 60-70 \% \text { of } 1 \mathrm{RM} \text {, increased } 5 \mathrm{lb} \text { after } 12 \text { successful repetitions } \\
\text { Frequency: Ex3 } \\
\text { Reps/Sets: } 8-12 / 2 \\
\text { Duration: } 12 \text { weeks } \\
\text { Setting: fitness center } \\
\text { Supervision: full } \\
\text { Adherence: } 79 \% \\
\text { 2. Control Group: on a waiting list, offered the identical exercise advice and guideline as } \\
\text { the exercisegroup after the study period }\end{array}$} \\
\hline Outcomes & \multicolumn{2}{|c|}{$\begin{array}{l}\text { Primary: Health-related quality of life } \\
\text { Secondary: Muscle fatigue (Number of repetition) } \\
\text { Comments of adverse events: no }\end{array}$} \\
\hline \multicolumn{3}{|l|}{ Notes } \\
\hline \multicolumn{3}{|l|}{ Risk of bias } \\
\hline Item & Authors' judgement & Description \\
\hline Allocation concealment? & Unclear & B - Unclear \\
\hline
\end{tabular}

Selig 2004

\begin{tabular}{l|l}
\hline Methods & RCT \\
& Method of randomisation: not reported \\
Assessor blinding: not reported & Participant blinding: not reported \\
& Loss to follow-up: 3/19 in the PRT group \\
Intention-to-treat analysis: no & Post-program follow up: no \\
\hline \multirow{2}{*}{ Participants } & Location: Australia \\
& N=33 (14 in PRT) \\
& Sample: with chronic heart failure \\
& Age: mean 65 years (SD = 13) \\
& Inclusion criteria: left ventricular systolic failure except aortic stenosis, left ventricular \\
& ejection fraction below 40\%, and stable pharmacologic therapy \\
& Exclusion criteria: New York Heart Association Class I or IV, mayocardiact infarction in \\
& the previous 6 months, cardiac arrest, symptomatic, sustained ventricular tachycardia, \\
& current angina, conditions that constraindicate exercise, did not pass baseline assessment \\
\hline \multirow{2}{*}{ Interventions } & PRT versus control \\
& 1. PRT \\
& Type of Ex : 5 UL/4 LL \\
& Equipment: multistation hydraulic resistance training system \\
& Intensity: by increasing resistance or the number of sets
\end{tabular}

Cochrane Database Syst Rev. Author manuscript; available in PMC 2015 February 11. 


\begin{tabular}{|c|c|c|}
\hline & $\begin{array}{l}\text { Frequency: Ex3 } \\
\text { Reps/Sets: not reported } \\
\text { Duration: } 12 \text { weeks } \\
\text { Setting: hospital rehabilitation gym } \\
\text { Supervision: not reported } \\
\text { Adherence: not reported } \\
\text { 2. Control Group: usual care }\end{array}$ & \\
\hline Outcomes & $\begin{array}{l}\text { Muscle strength } \\
\text { VO2 max } \\
\text { Comments on adverse events: yes }\end{array}$ & \\
\hline \multicolumn{3}{|l|}{ Notes } \\
\hline \multicolumn{3}{|l|}{ Risk of bias } \\
\hline Item & Authors' judgement & Description \\
\hline Allocation concealment? & Unclear & B - Unclear \\
\hline
\end{tabular}

\section{Seynnes 2004}

\begin{tabular}{|c|c|c|}
\hline Methods & \multicolumn{2}{|c|}{$\begin{array}{l}\text { RCT with } 3 \text { groups: high intensity, low intensity, and control } \\
\text { Method of randomisation: not reported } \\
\text { Assessor blinding: no } \\
\text { Participant blinding: yes } \\
\text { Loss to follow-up: } 5 / 27 \text { drop out } \\
\text { Intention-to-treat analysis: no } \\
\text { Post-program follow up: no }\end{array}$} \\
\hline Participants & \multicolumn{2}{|c|}{$\begin{array}{l}\text { Location: France } \\
\mathrm{N}=8 \text {-HI; N = 6-LI; } \mathrm{N}=8 \text {-control Sample: institutionalized elders } \\
\text { Age: HI-mean } 83.3 \text { years }(\mathrm{SD}=2.8 \text { ); LI-mean } 80.7 \text { years }(\mathrm{SD}=2.3 \text { ) } \\
\text { Inclusion criteria: at least } 70 \text { years of age, ambulatory, and understand simple instructions } \\
\text { Exclusion criteria: (a) cognitive impairment precluding understanding of the written } \\
\text { informed consent; (b) practice of regular exercise outside of the research activities; (c) } \\
\text { unstable cardiovascular disease, hypertension, diabetes, or any other unstable medical } \\
\text { condition; (d) amputations; (e) hernias; (f) symptomatic known unrepaired aortic aneurysm; } \\
\text { (g) recent (within } 6 \text { months) hospitalization for myocardial infarction, stroke, fracture, eye } \\
\text { surgery, or laser treatment; (h) skin disease precluding placement of ankle weights; (i) } \\
\text { musculoskeletal deformity; (j) neuromuscular disease; and (k) symptomatic rheumatoid or } \\
\text { osteoarthritis precluding planned exercises }\end{array}$} \\
\hline Interventions & \multicolumn{2}{|c|}{$\begin{array}{l}\text { PRT (high intensity and low intensity) versus control } \\
\text { 1. PRT } \\
\text { Type of Ex: 1LL } \\
\text { Equipment: ankle cuff } \\
\text { Intensity: HI- } 80 \% \text { of } 1 \mathrm{RM} \text {; LI- } 40 \% \text { of } 1 \mathrm{RM} \\
\text { Frequency: Ex } 3 \\
\text { Reps/Sets: } 8 / 3 \\
\text { Duration: } 10 \text { weeks } \\
\text { Setting: not reported-gym? } \\
\text { Supervision: full } \\
\text { Adherence: } 99 \% \\
\text { 2. Control Group: wearing empty ankle cuff and did the same exercise as the Ex group but } \\
\text { without weights }\end{array}$} \\
\hline Outcomes & \multicolumn{2}{|c|}{$\begin{array}{l}\text { Primary: self-reported disability } \\
\text { Secondary: muscle strength (1RM), muscle endurance, 6-minute walking, chair rising, stair } \\
\text { climbing } \\
\text { Comments on adverse events: yes }\end{array}$} \\
\hline Notes & \multicolumn{2}{|c|}{$\begin{array}{l}\text { SD was calculated from SEM } \\
\text { Date from high intensity PRT and low intensity PRT were compared }\end{array}$} \\
\hline \multicolumn{3}{|l|}{ Risk of bias } \\
\hline Item & Authors' judgement & Description \\
\hline Allocation concealment? & Unclear & B - Unclear \\
\hline
\end{tabular}


Simoneau 2006

\begin{tabular}{|c|c|c|}
\hline Methods & \multicolumn{2}{|c|}{$\begin{array}{l}\text { RCT } \\
\text { Method of randomisation: not reported } \\
\text { Assessor blinding: not reported } \\
\text { Participant blinding: not reported } \\
\text { Loss to follow-up: not reported } \\
\text { Intention-to-treat analysis: no } \\
\text { Post-program follow up: no }\end{array}$} \\
\hline Participants & \multicolumn{2}{|c|}{$\begin{array}{l}\text { Location: France } \\
\mathrm{N}=20 \text { (11 in PRT) } \\
\text { Sample: healthy and community dwelling people } \\
\text { Age: mean } 78.1 \text { years }(\mathrm{SD}=3.1) \\
\text { Inclusion criteria: no muscular, neurological, cardiovascular, metabolic, and inflammatory } \\
\text { disease' moderately active individuals } \\
\text { Exclusion criteria: not reported }\end{array}$} \\
\hline Interventions & \multicolumn{2}{|c|}{$\begin{array}{l}\text { PRT versus control } \\
\text { 1. PRT } \\
\text { Type of Ex : } 1 \text { LL-ankle joint } \\
\text { Equipment: elastic bands-home } \\
\text { Intensity: increased progressively from } 50 \%-55 \% \text { of } 3 \mathrm{RM} \text { to } 70 \% \text { of } 3 \mathrm{RM} \\
\text { Frequency: Ex3 (2 supervised and } 1 \text { at home) } \\
\text { Reps/Sets: } 8 / 3 \\
\text { Duration: } 24 \text { weeks } \\
\text { Setting: gym and home } \\
\text { Supervision: } 2 \text { sessions were supervised } \\
\text { Adherence: not reported } \\
\text { 2. Control Group: maintain usual activities }\end{array}$} \\
\hline Outcomes & \multicolumn{2}{|c|}{$\begin{array}{l}\text { Muscle strength (Torques) } \\
\text { Comments on adverse events: no }\end{array}$} \\
\hline Notes & \multicolumn{2}{|l|}{ Training at ankle joints } \\
\hline \multicolumn{3}{|l|}{ Risk of bias } \\
\hline Item & Authors' judgement & Description \\
\hline Allocation concealment? & Unclear & B - Unclear \\
\hline
\end{tabular}

\section{Simons 2006}

\begin{tabular}{l|l}
\hline Methods & RCT \\
& Method of randomisation: not reported \\
Assessor blinding: not reported \\
Participant blinding: not reported \\
Loss to follow-up: $2 / 21$ in the PRT group; $1 / 21$ in the control group \\
Intention-to-treat analysis: no \\
Post-program follow up: no \\
\hline \multirow{2}{*}{ Participants } & Location: USA \\
& N=42 21 in each group) \\
& Sample: older adults from independent living facility \\
& Age: mean 84.6 years (SD = 4.5) \\
& Inclusion criteria: had clearance by the primary physician, lack of regular exercise more \\
& than 1 year, and at least 65 years of age \\
& Exclusion criteria: not reported \\
\hline \multirow{2}{*}{ Interventions } & PRT versus control \\
& 1. PRT \\
& Type of Ex: 3 UL/3LL \\
& Equipment: Keiser machines \\
& Intensity: $75 \%$ of 1 RM, increased the load of $5 \%$ \\
& Frequency: Ex2 \\
& Reps/Sets: $10 / 1$ \\
& Duration: 16 weeks \\
Setting: fitness center & Supervision: full, by trained instructors \\
& Adherence: not reported \\
\end{tabular}


2. Control Group: controls and Ex group all had 6 one-hour health lectures at 3-week intervals

\begin{tabular}{l|l|l} 
& $\begin{array}{l}\text { 2. Control Group: controls and Ex group all had 6 one-hour health lectures at 3-week } \\
\text { intervals }\end{array}$ \\
\hline Outcomes & $\begin{array}{l}\text { Muscle strength (1RM) } \\
\text { Flexibility } \\
\text { Balance and agibility } \\
\text { Eye-hand coordination } \\
\text { Comments on adverse events: yes }\end{array}$ \\
\hline Notes & Authors' judgement & Description \\
\hline Risk of bias & Unclear & B - Unclear \\
\hline Item &
\end{tabular}

Simpson 1992

\begin{tabular}{|c|c|c|}
\hline Methods & \multicolumn{2}{|c|}{$\begin{array}{l}\text { RCT } \\
\text { Method of randomisation: stratified (don't know how) and randomly assigned } \\
\text { Participant blinding: no } \\
\text { Assessor blinding: no } \\
\text { Loss to follow-up: } 6 \\
\text { Intention-to-treat analysis: no } \\
\text { Post-program follow up: no }\end{array}$} \\
\hline Participants & \multicolumn{2}{|c|}{$\begin{array}{l}\text { Location: Canada } \\
\mathrm{N}=34 \\
\text { Sample: people with chronic airflow obstruction } \\
\text { Age: mean } 73 \text { years (SD 4.8) in PRT group } \\
\text { Inclusion criteria: aged 58-80, attending a respiratory outpatient clinic, in a clinically stable } \\
\text { state, no recent infective exacerbation, drug management was considered to be optimal, FE } \\
\text { to VC ratio of less than } 0.7 \text {, body weight within } 30 \% \text { of ideal weight, absence of disorders } \\
\text { likely to affect exercise, capacity to take part in the training program, } \\
\text { Exclusion criteria: not reported }\end{array}$} \\
\hline Interventions & \multicolumn{2}{|c|}{$\begin{array}{l}\text { PRT versus control } \\
\text { 1. PRT } \\
\text { Type of Ex: 1UL, 2LL } \\
\text { Equipment: weight-lifting machines } \\
\text { Intensity: } 50-85 \% \text { of } 1 \mathrm{RM} \\
\text { Frequency: Ex3 } \\
\text { Reps/Sets: } 10 / 3 \\
\text { Program Duration: } 8 \text { weeks } \\
\text { Setting: gym } \\
\text { Supervision: not reported } \\
\text { Adherence: } 90 \% \\
\text { 2. Control Group: only attended testing sessions }\end{array}$} \\
\hline Outcomes & \multicolumn{2}{|c|}{$\begin{array}{l}\text { Strength (1RM) } \\
\text { Spirometry } \\
\text { Aerobic capacity (VO2 max) } \\
\text { 6-minute walk test } \\
\text { Likert scale rating of discomfort during four daily activities ( } 1=\text { extreme disability, } 7=\text { none } \\
\text { assessed for fatigue, dyspnoea, emotion and mastery } \\
\text { Comments on adverse events: no }\end{array}$} \\
\hline \multicolumn{3}{|l|}{ Notes } \\
\hline \multicolumn{3}{|l|}{ Risk of bias } \\
\hline Item & Authors' judgement & Description \\
\hline Allocation concealment? & Unclear & B - Unclear \\
\hline
\end{tabular}

Sims 2006

\begin{tabular}{l|l}
\hline Methods & RCT
\end{tabular}

Cochrane Database Syst Rev. Author manuscript; available in PMC 2015 February 11. 


\begin{tabular}{|c|c|c|}
\hline & \multicolumn{2}{|c|}{$\begin{array}{l}\text { Method of randomisation: by an independent person with a previously block randomised } \\
\text { list } \\
\text { Assessor blinding: yes } \\
\text { Participant blinding: no } \\
\text { Loss to follow-up: } 6 \\
\text { Intention-to-treat analysis: yes } \\
\text { Post-program follow up: yes, at the 6th month }\end{array}$} \\
\hline Participants & \multicolumn{2}{|c|}{$\begin{array}{l}\text { Location: Australia } \\
\mathrm{N}=32 \text { (14 in PRT) } \\
\text { Sample: older adults with depression symptoms } \\
\text { Age: mean } 74.28 \text { years( } \mathrm{SD}=5.87) \\
\text { Inclusion criteria: at least } 65 \text { years old; GDS score > } 11 \\
\text { Exclusion criteria: unsuitable to exercise according to the score of the Physical Activity } \\
\text { Readiness Questionnaire. Alcohol or drug related depression; depression with psychotic } \\
\text { features; schizophrenia; bipolar disorder; other psychiatric diagnoses; suicidal ideation; } \\
\text { dementia; terminally ill; uncontrolled hypertension, unstable insulin dependent diabetes, } \\
\text { and unstable angina. They excluded those currently receiving antidepressants in order to } \\
\text { determine the independent impact of PRT }\end{array}$} \\
\hline Interventions & \multicolumn{2}{|c|}{$\begin{array}{l}\text { PRT versus control } \\
\text { 1. PRT } \\
\text { Type of Ex: major UL and LL muscles } \\
\text { Equipment: weights } \\
\text { Intensity: } 80 \% \text { of } 1 \text { RM \& Borg's perceived exertion scale } \\
\text { Frequency: Ex3 } \\
\text { Reps/Sets: } 8-10 / 3 \\
\text { Duration: } 10 \text { weeks } \\
\text { Setting: gym } \\
\text { Supervision: full } \\
\text { Adherence: } 5 \text { attended } 2-15 \text { sessions, } 7 \text { attended } 18-30 \text { sessions } 58 \% \text { meet the adherence } \\
\text { criterion of } 60 \% \text { of sessions completed } \\
\text { 2. Control group: received ex information (Ex group received it too) }\end{array}$} \\
\hline Outcomes & \multicolumn{2}{|c|}{$\begin{array}{l}\text { Human Activity Profile WHO-QOL } \\
\text { PASE-functional health status } \\
\text { PGMS-well being } \\
\text { Comments on adverse events: no }\end{array}$} \\
\hline \multicolumn{3}{|l|}{ Notes } \\
\hline \multicolumn{3}{|l|}{ Risk of bias } \\
\hline Item & Authors' judgement & Description \\
\hline Allocation concealment? & Yes & A - Adequate \\
\hline
\end{tabular}

\section{Singh 1997}

\begin{tabular}{l|l}
\hline Methods & RCT \\
Method of randomisation: computer-generated list in blocks of five \\
Assessor blinding: all outcomes except strength \\
Participant blinding: no, but attention control group \\
Loss to follow-up: 0 \\
Intention-to-treat analysis: no drop-outs but not stated \\
Post-program follow up: no
\end{tabular}




\begin{tabular}{|c|c|c|}
\hline & \multicolumn{2}{|c|}{$\begin{array}{l}\text { Frequency: Ex3 } \\
\text { Reps/Sets: } 8 / 3 \\
\text { Program Duration: } 10 \text { weeks } \\
\text { Setting: gym } \\
\text { Supervision: full } \\
\text { Adherence: median } 93 \% \\
\text { 2. Control Group: health education program, } 2 \text { times per week for } 1 \text { hour }\end{array}$} \\
\hline Outcomes & \multicolumn{2}{|c|}{$\begin{array}{l}\text { Sickness Impact Profile } \\
\text { Katz ADL scale } \\
\text { Lawton Brody IADL scale } \\
\text { SF-36 } \\
\text { Strength (1RM) } \\
\text { Adverse events (chest pain, musculoskeletal pain, medication change, intercurrent illness, } \\
\text { hospitalisation, visits to a health professional, worsening of suicidality } \\
\text { Comments on adverse events: yes (a priority outcome) }\end{array}$} \\
\hline \multicolumn{3}{|l|}{ Notes } \\
\hline \multicolumn{3}{|l|}{ Risk of bias } \\
\hline Item & Authors' judgement & Description \\
\hline Allocation concealment? & Unclear & B - Unclear \\
\hline
\end{tabular}

\section{Singh 2005}

\begin{tabular}{|c|c|}
\hline Methods & $\begin{array}{l}\text { RCT with } 3 \text { groups: high intensity, low intensity, and control } \\
\text { Method of randomisation: by a computer generated random number program in blocks of } \\
15 \\
\text { Assessor blinding: yes } \\
\text { Participant blinding: yes } \\
\text { Loss to follow-up: } 2 / 20 \text { in the high intensity group; } 3 / 20 \text { in the low intensity group; } 1 / 20 \text { in } \\
\text { the control group } \\
\text { Intention-to-treat analysis: no } \\
\text { Post-program follow up: no }\end{array}$ \\
\hline Participants & $\begin{array}{l}\text { Location: Australia } \\
\mathrm{N}=20 \text { in each group } \\
\text { Sample: major or minor depression } \\
\text { Age: HI-mean } 69 \text { years }(\mathrm{SD}=5) \text {; } \mathrm{LI}-\text { mean } 70 \text { years }(\mathrm{SD}=7) \\
\text { Inclusion criteria: aged } 60 \text { years; major depression, minor depression, or dysthymia; and } \\
\text { had a GDS score at least } 14 \text {. } \\
\text { Exclusion criteria: if demented clinically according to DSM-IV criteria or if their MMSE } \\
\text { score was less than } 23 \text {, if they were suffering from unstable medical disease which would } \\
\text { preclude resistance training, had bipolar disorder or active psychosis, or were determined } \\
\text { by the study physician to be actively suicidal. They were also excluded if they were } \\
\text { currently seeing a psychiatrist, prescribed antidepressant drugs within the last } 3 \text { months, or } \\
\text { were currently participating in any exercise training more than twice a week }\end{array}$ \\
\hline Interventions & $\begin{array}{l}\text { PRT (high intensity versus low intensity) versus control } \\
\text { 1. PRT } \\
\text { Type of Ex: 3UL/3LL } \\
\text { Equipment: Keiser Sports Health Equipment } \\
\text { intensity: high intensity group- } 80 \% \text { of 1RM; low intensity group- } 20 \% \text { of } 1 \text { RM } \\
\text { Frequency: Ex3 } \\
\text { Reps/Sets: } 8 / 3 \\
\text { Duration: } 8 \text { weeks } \\
\text { Setting: outpatient gym in a hospital } \\
\text { Supervision: full } \\
\text { Adherence: high intensity group: } 95-100 \% \text {; low intensity group: } 99-100 \% \\
\text { 2. Control Group: usual care }\end{array}$ \\
\hline Outcomes & $\begin{array}{l}\text { Primary: SF-36 } \\
\text { Secondary: muscle strength (1RM) } \\
\text { Comments on adverse events: yes }\end{array}$ \\
\hline \multicolumn{2}{|l|}{ Notes } \\
\hline \multicolumn{2}{|l|}{ Risk of bias } \\
\hline Item & Authors' judgement \\
\hline
\end{tabular}




\begin{tabular}{|c|c|c|}
\hline Allocation concealment? & Unclear & B - Unclear \\
\hline \multicolumn{3}{|c|}{ Sipila 1996} \\
\hline Methods & \multicolumn{2}{|c|}{$\begin{array}{l}\text { RCT with } 3 \text { groups: PRT, control and aerobic training group } \\
\text { Method of randomisation: not reported } \\
\text { Assessor blinding: no } \\
\text { Participant blinding: no } \\
\text { Loss to follow-up: } 4 \text { in PRT/controls ( } 8 \text { total) } \\
\text { Intention-to-treat analysis: no } \\
\text { Post-program follow up: no }\end{array}$} \\
\hline Participants & \multicolumn{2}{|c|}{$\begin{array}{l}\text { Location: Finland } \\
\mathrm{N}=42 \text { total ( } 27 \text { in PRT and control) } \\
\text { Sample: healthy older women } \\
\text { Age: } 76-78 \text { years } \\
\text { Inclusion criteria: born between } 1915-17 \text { (aged } 76-78) \text {, no severe diseases or functional } \\
\text { impairments, no indications against intensive physical exercise (medical exam and exercise } \\
\text { test screening) } \\
\text { Exclusion criteria: not reported }\end{array}$} \\
\hline Interventions & \multicolumn{2}{|c|}{$\begin{array}{l}\text { PRT versus control and versus endurance (aerobic) } \\
\text { 1. PRT } \\
\text { Type of Ex: 4LL } \\
\text { Equipment: variable resistance machines (HUR equipment) } \\
\text { Intensity: } 60-75 \% \text { of } 1 \text { RM } \\
\text { Frequency: Ex3 } \\
\text { Reps/Sets: } 8-10 / 3-4 \\
\text { Program duration: } 18 \text { weeks } \\
\text { Setting: gym } \\
\text { Supervision: full } \\
\text { Adherence: } 71-86 \% \text { (varied depending upon muscle group/exercise type) } \\
\text { 2. Control Group: instructed to continue daily routines and not change their physical } \\
\text { activity levels } \\
\text { 3. Endurance exercise group: } 18 \text { weeks of track walking ( } 2 \text { times per week) and step } \\
\text { aerobics (once perweek) at } 50 \%-80 \% \text { of initial maximum heart rate reserve }\end{array}$} \\
\hline Outcomes & \multicolumn{2}{|c|}{$\begin{array}{l}\text { Strength } \\
\text { Walking speed } \\
\text { Comments on adverse events: no }\end{array}$} \\
\hline Notes & \multicolumn{2}{|c|}{ Data from PRT and aerobic training group were compared } \\
\hline \multicolumn{3}{|l|}{ Risk of bias } \\
\hline Item & Authors' judgement & Description \\
\hline Allocation concealment? & Unclear & B - Unclear \\
\hline
\end{tabular}

\section{Skelton 1995}

\begin{tabular}{l|l}
\hline Methods & RCT \\
& Method of randomisation: a random numbers table \\
& Assessor blinding: no \\
& Participant blinding: no \\
Loss to follow-up: 7 & Intention-to-treat analysis: no \\
& Post-program follow up: no \\
\hline Participants & Location: UK \\
& N=47 \\
& Sample:healthy, independent women \\
& Age: median 79.5 years (range 76-93) in PRT group \\
& Inclusion criteria: healthy; medically stable; no recent history of cardiovascular, \\
& cerebrovascular, respiratory, systemic or muscular disease; any impairment that interfered \\
& with mobility, live independently, require not help with ADLs \\
& Exclusion criteria: not reported \\
\hline
\end{tabular}




\begin{tabular}{|c|c|c|}
\hline Interventions & \multicolumn{2}{|c|}{$\begin{array}{l}\text { PRT versus control } \\
\text { 1. PRT } \\
\text { Type of Ex: } 3 \mathrm{UL}, 6 \mathrm{LL} \\
\text { Equipment: rice bags and elastic tubing } \\
\text { Intensity: resistance increased as soon as participant could complete } 3 \text { sets of } 8 \text { reps } \\
\text { Frequency: Ex3 } \\
\text { Reps/Sets: } 4-8 / 3 \\
\text { Program duration: } 12 \text { weeks } \\
\text { Setting: group exercise class } 1 \text { day per week, home } 2 \text { days } \\
\text { Supervision: not reported } \\
\text { Adherence: no one attended fewer than } 6 \text { classes or } 11 \text { home sessions } \\
\text { 2. Control Group: asked not to change their activities }\end{array}$} \\
\hline Outcomes & \multicolumn{2}{|c|}{$\begin{array}{l}\text { Human Activity Profile } \\
\text { Anthropometry } \\
\text { Strength (isometric strength and handgrip): such as extensor power } \\
\text { Functional reach } \\
\text { Chair rise } \\
\text { Timed walk } \\
\text { Stair walking } \\
\text { Comments on adverse events: yes }\end{array}$} \\
\hline \multicolumn{3}{|l|}{ Notes } \\
\hline \multicolumn{3}{|l|}{ Risk of bias } \\
\hline Item & Authors' judgement & Description \\
\hline Allocation concealment? & Unclear & B - Unclear \\
\hline
\end{tabular}

\section{Skelton 1996}

\begin{tabular}{|c|c|}
\hline Methods & $\begin{array}{l}\text { RCT } \\
\text { Method of randomisation: matched by age then randomised } \\
\text { Assessor blinding: no } \\
\text { Participant blinding: no } \\
\text { Loss to follow-up: } 2 \\
\text { Intention-to-treat analysis: no } \\
\text { Post-program follow up: no }\end{array}$ \\
\hline Participants & $\begin{array}{l}\text { Location: UK } \\
\mathrm{N}=20 \\
\text { Sample: women with functional limitations } \\
\text { Age: median } 81 \text { years } \\
\text { Inclusion criteria: age: } 75+\text {, from GP practice, have minor or major functional/mobility } \\
\text { laminations } \\
\text { Exclusion criteria: any disease / condition adversely affected by exercise }\end{array}$ \\
\hline Interventions & $\begin{array}{l}\text { PRT versus control } \\
\text { 1. PRT } \\
\text { Type of Ex: 2UL, 6LL } \\
\text { Equipment: theraband, cuff-weights } \\
\text { Intensity: resistance increased as soon as participant could complete } 3 \text { sets of } 8 \text { reps } \\
\text { Frequency: Ex3 } \\
\text { Reps/Sets: } 4-8 / 3 \\
\text { Program Duration: } 8 \text { weeks } \\
\text { Setting: } 1 \text { class per week, } 2 \text { home sessions per week } \\
\text { Supervision: class supervised, home exercises unsupervised } \\
\text { Adherence: no subject performed fewer than } 30 \text { complete sessions } \\
\text { 2. Control Group: asked not to change activities }\end{array}$ \\
\hline Outcomes & $\begin{array}{l}\text { Human Activity Profile } \\
\text { Strength (isometric strength and handgrip) } \\
1 \text {-legged balance } \\
\text { Chair rise } \\
\text { Timed walk } \\
\text { Timed up-and-go } \\
\text { Comments on adverse events: yes }\end{array}$ \\
\hline \multicolumn{2}{|l|}{ Notes } \\
\hline Risk of bias & \\
\hline
\end{tabular}




\begin{tabular}{l|l|l} 
Item & Authors' judgement & Description \\
\hline Allocation concealment? & Unclear & B - Unclear \\
\hline
\end{tabular}

\section{Sousa 2005}

\begin{tabular}{|c|c|c|}
\hline Methods & \multicolumn{2}{|c|}{$\begin{array}{l}\text { RCT } \\
\text { Method of randomisation: not reported } \\
\text { Assessor blinding: not reported } \\
\text { Participant blinding: not reported } \\
\text { Loss to follow-up: } 0 \\
\text { Intention-to-treat analysis: NA } \\
\text { Post-program follow up: no }\end{array}$} \\
\hline Participants & \multicolumn{2}{|c|}{$\begin{array}{l}\text { Location: Portugal } \\
\mathrm{N}=20 \text { (10 in each group) } \\
\text { Sample: healthy men } \\
\text { Age: mean } 73 \text { years }(\mathrm{SD}=6) \\
\text { Inclusion criteria: family physician's approval } \\
\text { Exclusion criteria: taking medications that could affect balance, smokers, history of falls, } \\
\text { and orthopedic, neurological, cardiac, or pulmonary problems }\end{array}$} \\
\hline Interventions & \multicolumn{2}{|c|}{$\begin{array}{l}\text { PRT versus control } \\
\text { 1. PRT } \\
\text { Type of Ex: 4UL/3LL } \\
\text { Equipment: Image Sport Machines } \\
\text { Intensity: increased progressively from } 50 \% \text { to } 80 \% \text { of } 1 \text { RM over the program } \\
\text { Frequency: Ex3 } \\
\text { Reps/Sets: first } 8 \text { weeks: } 8-12 / 2-3 \text {; then } 6-10 / 2-3 \\
\text { Duration: } 14 \text { weeks } \\
\text { Setting: not reported-gym? } \\
\text { Supervision: not reported } \\
\text { Adherence: } 95 \% \\
\text { 2. Control Group: not reported }\end{array}$} \\
\hline Outcomes & \multicolumn{2}{|c|}{$\begin{array}{l}\text { Primary: self-reported disability } \\
\text { Secondary: Muscle strength ( } 1 \mathrm{RM}) \text {, TUAG, functional reach test } \\
\text { Comment on adverse events: no }\end{array}$} \\
\hline \multicolumn{3}{|l|}{ Notes } \\
\hline \multicolumn{3}{|l|}{ Risk of bias } \\
\hline Item & Authors' judgement & Description \\
\hline Allocation concealment? & Unclear & B - Unclear \\
\hline
\end{tabular}

\section{Suetta 2004}

\begin{tabular}{l|l}
\hline Methods & RCT \\
& Method of randomisation: by a computer program \\
Assessor blinding: On measuring muscle cross-sectional area & Participant blinding: not reported \\
& Loss to follow-up: 2/13-PRT group, 3/12-Control \\
& Intention-to-treat analysis: no \\
& Post-program follow up: no \\
\hline Participants & Location: Denmark \\
& N=25 (13 in PRT) \\
& Sample: unilateral hip replacement due to OA \\
& Age: Mean 71 years \\
& Inclusion criteria: age at least of 60 years, and unilateral primary hip replacement due to \\
& OA \\
& Exclusion criteria: cardiopulmonary, neurological, or cognitive problems \\
\hline Interventions & PRT versus control \\
& 1. PRT \\
& Type of Ex: 2 LL and standard care
\end{tabular}




\begin{tabular}{l|l|l} 
& $\begin{array}{l}\text { Equipment: sandbags strapped to the ankle of the operated leg during hospitalization, after } \\
\text { day 7, Technogym International machines } \\
\text { Intensity: week 0-6, 20 to 12 RM; the last 6 weeks, 8 RM } \\
\text { Frequency: daily during hospitalization, Ex3 after day 7 } \\
\text { Reps/Sets: week 0-6, 10/ 3-5; the last 6 weeks, 8/3-5 } \\
\text { Duration: 12 weeks } \\
\text { Setting: not reported } \\
\text { Supervision: physical therapist } \\
\text { Adherence: not reported } \\
\text { 2. Control Group: home-based standard care }\end{array}$ \\
\hline Outcomes & $\begin{array}{l}\text { Muscle strength } \\
\text { Gait speed } \\
\text { Stair climbing } \\
\text { Sit-to-stand } \\
\text { Comments on adverse events: yes }\end{array}$ \\
\hline Notes & SD was calculated from SE & Description \\
\hline Risk of bias & \multicolumn{2}{|l}{} \\
\hline Item & Authors' judgement & B - Unclear \\
\hline Allocation concealment? & Unclear &
\end{tabular}

\section{Sullivan 2005}

\begin{tabular}{|c|c|c|}
\hline Methods & \multicolumn{2}{|c|}{$\begin{array}{l}\text { RCT } \\
\text { Method of randomisation: done by a biostatistician } \\
\text { Assessor blinding: yes } \\
\text { Participant blinding: yes for the testosterone } \\
\text { Loss to follow-up: } 2 / 17 \text { in low resistance group with placebo, } 4 / 17 \text { in high resistance group } \\
\text { with placebo } \\
\text { Intention-to-treat analysis: no } \\
\text { Post-program follow up: no }\end{array}$} \\
\hline Participants & \multicolumn{2}{|c|}{$\begin{array}{l}\text { Location: USA } \\
\mathrm{N}=17-\mathrm{HI} ; \mathrm{N}=17-\mathrm{LI} \\
\text { Sample: recent functional decline } \\
\text { Age: mean } 78.2 \text { years }(\mathrm{SD}=6.4) \\
\text { Inclusion criteria: recent functional decline, at least } 65 \text { years old, serum total testosterone } \\
\text { less than } 480 \mathrm{ngd} / \mathrm{L} \text {, and can give informed consent } \\
\text { Exclusion criteria: near terminal medical disorder, unresolved malignancy, prostate specific } \\
\text { antigen }>10 \mathrm{ngm} / \mathrm{L} \text {, possibility of prostate cancer, history of prostate cancer, disabling } \\
\text { arthritis, neurological diseases or unstable cardiovascular disease }\end{array}$} \\
\hline Interventions & \multicolumn{2}{|c|}{$\begin{array}{l}\text { PRT (High intensity versus low intensity) } \\
\text { Type of Ex: } 2 \text { LL } \\
\text { Equipment: Keiser Sport Health Equipment } \\
\text { Intensity: low intensity: } 20 \% \text { 1RM; high intensity: } 80 \% \text { of } 1 \mathrm{RM} \\
\text { Frequency: Ex3 } \\
\text { Reps/Sets: } 8 / 3 \\
\text { Duration: } 12 \text { weeks } \\
\text { Setting: not reported } \\
\text { Supervision: not reported } \\
\text { Adherence: } 99 \%\end{array}$} \\
\hline Outcomes & \multicolumn{2}{|c|}{$\begin{array}{l}\text { Muscle strength } \\
\text { Sit-to-stand } \\
\text { Gait speed } \\
\text { Stair climb } \\
\text { Comments on adverse events: yes }\end{array}$} \\
\hline Notes & \multicolumn{2}{|c|}{$\begin{array}{l}\text { Reported absolute change. High-intensity leg exercise led to greater leg strength, No } \\
\text { significance in aggregate physical performance score change between any intervention } \\
\text { groups. Final score = baseline + change score. Final SD = baseline SD } \\
\text { Date from high intensity PRT and low intensity PRT were compared }\end{array}$} \\
\hline \multicolumn{3}{|l|}{ Risk of bias } \\
\hline Item & Authors' judgement & Description \\
\hline Allocation concealment? & Yes & A - Adequate \\
\hline
\end{tabular}




\section{Symons 2005}

\begin{tabular}{l|l}
\hline Methods & RCT \\
Method of randomisation: random selection with continuing replacement method \\
Assessor blinding: no \\
Participant blinding: not reported \\
Loss to follow-up: 5/14 in isokinetic eccentric group \\
Intention-to-treat analysis: no \\
Post-program follow up: no
\end{tabular}

Taaffe 1996

\begin{tabular}{l|l}
\hline Methods & RCT with 3 groups: high intensity training, low intensity and control (high-intensity only \\
used for main comparisons) \\
Method of randomisation: not reported \\
Participant blinding: no \\
Assessor blinding: no \\
Loss to follow-up: 11 total (5 from HI PRT and control) \\
Intention-to-treat analysis: no \\
Post-program follow up: no \\
\hline \multirow{2}{*}{ Participants } & Location: USA \\
& N = 36 total (23 in control and main PRT group) \\
& Sample: healthy older women \\
& Age: mean 67 years (SE 0.2) in HI-PRT group \\
& Inclusion criteria: female, did not participate in a strength-training program; not taking \\
& HRT or on HRT for more than one year \\
& Exclusion criteria: evidence of acute or uncontrolled chronic illness or condition that would \\
prevent participation in a resistance training program; presence of vertebral compression & fracture; evidence of any disorder that would affect bone metabolism \\
\hline Interventions & PRT (high intensity and low intensity) versus control \\
& 1. PRT \\
& Type of Ex: 3LL \\
& Equipment: weight machines (Universal Gym, and Marcy equipment) \\
& Intensity: HI-80\% of 1RM; LI-40\% of 1RM \\
& Frequency: Ex3
\end{tabular}




\begin{tabular}{l|l|l}
\multicolumn{1}{l|}{} & $\begin{array}{l}\text { Reps/Sets: HI= first set at 40\% 1RM for 14 reps, last } 2 \text { had } 7 \text { reps; LI=14/3 } \\
\text { Program Duration: 52 weeks } \\
\text { Setting: gym } \\
\text { Supervision: full } \\
\text { Adherence: } 79 \% \\
\text { 2. Control Group: maintain customary dietary and activity patterns }\end{array}$ \\
\hline Outcomes & $\begin{array}{l}\text { Strength (1RM), } \\
\text { Habitual activity (4 day activity records) } \\
\text { Comments on adverse events: no }\end{array}$ \\
\hline Notes & Date from high intensity PRT and low intensity PRT were compared \\
\hline Risk of bias & Authors' judgement & Description \\
\hline Item & Unclear & B - Unclear \\
\hline Allocation concealment? &
\end{tabular}

Taaffe 1999

\begin{tabular}{|c|c|c|}
\hline Methods & \multicolumn{2}{|c|}{$\begin{array}{l}\text { RCT with } 4 \text { groups, PRT once per week, twice per week, } 3 \text { times per week and control } \\
\text { (main analyses with } 3 \text { times per week and control) } \\
\text { Method of randomisation: not reported } \\
\text { Assessor blinding: no } \\
\text { Participant blinding: no } \\
\text { Loss to follow-up: } 7 \text { total ( } 2 \text { in control and Ex } 3 \text { ) } \\
\text { Intention-to-treat analysis: no } \\
\text { Post-program follow up: no, but } 24 \text { weeks duration }\end{array}$} \\
\hline Participants & \multicolumn{2}{|c|}{$\begin{array}{l}\text { Location: USA } \\
\mathrm{N}=46 \text { total ( } 25 \text { in Ex } 3 \text { and control) } \\
\text { Sample: community-dwelling, healthy men and women } \\
\text { Age: mean } 71.0 \text { years (SD 4.1) in Ex } 3 \text { group } \\
\text { Inclusion criteria: aged } 65-79 \text { years, apparently healthy, BMI<30, no musculoskeletal } \\
\text { disorder that could inhibit them from exercising, no weight training in previous } 12 \text { months, } \\
\text { passed medical screening (including maximum exercise stress test) }\end{array}$} \\
\hline Interventions & \multicolumn{2}{|c|}{$\begin{array}{l}\text { PRT (at different frequencies) versus control } \\
\text { 1. PRT } \\
\text { Type of Ex: 6UL, 6LL } \\
\text { Equipment: Universal Gym, Marcy and Nautilus equipment } \\
\text { Intensity: } 80 \% 1 \mathrm{RM} \\
\text { Frequency: Ex1, Ex2, Ex3 } \\
\text { Reps/Sets: } 8 / 3 \\
\text { Program Duration: } 24 \text { weeks } \\
\text { Setting: gym } \\
\text { Supervision: full } \\
\text { Adherence: } 97-99 \% \\
\text { 2. Control Group: maintain customary dietary and activity patterns }\end{array}$} \\
\hline Outcomes & \multicolumn{2}{|c|}{$\begin{array}{l}\text { Strength (1RM) } \\
\text { Timed backward tandem walk } \\
\text { Chair rise } \\
\text { Comments on adverse events: no }\end{array}$} \\
\hline Notes & \multicolumn{2}{|c|}{ Data from 3 times per week and one time per week group were compared } \\
\hline \multicolumn{3}{|l|}{ Risk of bias } \\
\hline Item & Authors' judgement & Description \\
\hline Allocation concealment? & Unclear & B - Unclear \\
\hline
\end{tabular}

Topp 1993

\begin{tabular}{l|l}
\hline Methods & RCT (note: results extrapolated from graph) \\
& Method of randomisation: not reported \\
Assessor blinding: no
\end{tabular}

Cochrane Database Syst Rev. Author manuscript; available in PMC 2015 February 11. 


\begin{tabular}{|c|c|c|}
\hline & \multicolumn{2}{|c|}{$\begin{array}{l}\text { Participant blinding: no but attention control group } \\
\text { Loss to follow-up: } 7 \\
\text { Intention-to-treat analysis: no } \\
\text { Post-program follow up: no }\end{array}$} \\
\hline Participants & \multicolumn{2}{|c|}{$\begin{array}{l}\text { Location: USA } \\
\mathrm{N}=63 \\
\text { Sample: community-dwelling men and women } \\
\text { Age: mean } 69.2 \text { years (SE 0.8) in the PRT group } \\
\text { Inclusion criteria: community-dwelling, } 65+\text {, } \\
\text { Exclusion criteria: cardiopulmonary/ cardiovascular disease, intolerance to exercise, } \\
\text { functional disabilities that would contraindicate strength training, unable to commit to a 12- } \\
\text { week program, currently involved in strength training more than } 1 \text { hour per week }\end{array}$} \\
\hline Interventions & \multicolumn{2}{|c|}{$\begin{array}{l}\text { PRT versus control } \\
\text { 1. PRT } \\
\text { Type of Ex: 6UL, 6LL } \\
\text { Equipment: surgical tubing } \\
\text { Intensity: low-moderate - increased tubing thickness when they could perform } 12 \text { reps of an } \\
\text { exercise } \\
\text { Frequency: Ex3 } \\
\text { Reps/Sets: upper body 10/ 2; lower body } 10 / 3 \\
\text { Program Duration: } 12 \text { weeks } \\
\text { Setting: exercise class for at least one session per week, home for other session(s) } \\
\text { Supervision: full in exercise class, low at home } \\
\text { Adherence: } 90 \% \\
\text { 2. Control Group: attended two 3-hour driver education classes, continue usual activities, } \\
\text { could have 4weeks of exercise at the end of the trial }\end{array}$} \\
\hline Outcomes & \multicolumn{2}{|c|}{$\begin{array}{l}\text { Gait speed } \\
\text { Balance (modified Romberg protocol) } \\
\text { Comments on adverse events: no }\end{array}$} \\
\hline \multicolumn{3}{|l|}{ Notes } \\
\hline \multicolumn{3}{|l|}{ Risk of bias } \\
\hline Item & Authors' judgement & Description \\
\hline Allocation concealment? & Unclear & B - Unclear \\
\hline
\end{tabular}

\section{Topp 1996}

\begin{tabular}{|c|c|}
\hline Methods & $\begin{array}{l}\text { RCT } \\
\text { Method of randomisation: not reported } \\
\text { Assessor blinding: no } \\
\text { Participant blinding: no, but attention control group } \\
\text { Loss to follow-up: } 19 \\
\text { Intention-to-treat analysis: no - excluded people who completed }<70 \% \text { of prescribed } \\
\text { sessions } \\
\text { Post-program follow up: no }\end{array}$ \\
\hline Participants & $\begin{array}{l}\text { Location: USA } \\
\mathrm{N}=61 \\
\text { Sample: community-dwelling, sedentary } \\
\text { Age: mean } 70.8 \text { years (SE 1.03) in exercise group } \\
\text { Inclusion criteria: community dwelling older adults } \\
\text { Exclusion criteria: any contraindications to participating in regular exercise including a } \\
\text { history of coronary artery disease, more than one major coronary risk factor or major } \\
\text { symptoms or signs of cardiopulmonary or metabolic disease evident during a medically } \\
\text { supervised history and physical; already participating in a program of regular resistance } \\
\text { training, unable to make a } 14 \text {-week commitment to the project }\end{array}$ \\
\hline Interventions & $\begin{array}{l}\text { PRT versus control } \\
\text { 1. PRT } \\
\text { Type of Ex: } 11 \text { exercises (UL, LL, Tr) } \\
\text { Equipment: theraband } \\
\text { Intensity: low-moderate - used theraband of a thickness sufficient to produce moderate } \\
\text { fatigue during the final } 2 \text { reps of an exercise } \\
\text { Frequency: Ex3 } \\
\text { Reps/Sets: by end of study, } 2 / 10 \text { for UL, } 3 / 10 \text { for LL } \\
\text { Program duration: } 14 \text { weeks } \\
\text { Setting: exercise class at least once per week, home for other session(s) }\end{array}$ \\
\hline
\end{tabular}

Cochrane Database Syst Rev. Author manuscript; available in PMC 2015 February 11. 
Supervision: full for exercise class, none for home

Adherence: $93 \%$ (excluding drop-outs)

2. Control Group: two 3-hour supervised driver-education classes

\begin{tabular}{l|l|l}
\hline Outcomes & $\begin{array}{l}\text { Strength } \\
\text { Postural control (measured using a force plate) } \\
\text { Gait speed } \\
\text { Comments on adverse events: no }\end{array}$ \\
\hline Notes & Authors' judgement & Description \\
\hline Risk of bias & Unclear & B - Unclear \\
\hline Item & &
\end{tabular}

Topp 2002

\begin{tabular}{|c|c|c|}
\hline Methods & \multicolumn{2}{|c|}{$\begin{array}{l}\text { RCT } \\
\text { Method of randomisation: not reported } \\
\text { Assessor blinding: not reported } \\
\text { Participant blinding: not reported } \\
\text { Loss to follow-up: } 0 \\
\text { Intention-to-treat analysis: N/A } \\
\text { Post-program follow up: no }\end{array}$} \\
\hline Participants & \multicolumn{2}{|c|}{$\begin{array}{l}\text { Location: USA } \\
\mathrm{N}=35 \\
\text { Sample: adults with knee OA } \\
\text { Age: mean = } 65.57 \text { years (SD }=1.82 \text { ) estimated } \\
\text { Inclusion criteria: knee pain due to OA (based on WOMAC); physician validated the knee } \\
\text { pain and the } \\
\text { diagnosis of OA } \\
\text { Exclusion criteria: had any contraindications for exercise, including a history of } \\
\text { uncontrolled angina, cardiomyopathy severe enough to compromise cardiac functioning, } \\
\text { electrolyte or metabolic disturbances, disabilities that prohibited resistance training of the } \\
\text { lower extremities, or if they were currently taking nitrates, digitalis, or phenothiazine. } \\
\text { Individuals were also excluded if they were currently participating in an organized exercise } \\
\text { program or exercised more than } 1 \text { hour per week }\end{array}$} \\
\hline Interventions & \multicolumn{2}{|c|}{$\begin{array}{l}\text { PRT versus control } \\
\text { 1. PRT } \\
\text { Type of Ex: } 6 \text { LL for } 30 \text { minutes } \\
\text { Equipment: Thera-Band elastic bands } \\
\text { Intensity: self exertion of mild fatigue after } 8 \mathrm{RM} \\
\text { Frequency: Ex3 ( } 2 \text { at home } 1 \text { at gym) } \\
\text { Reps/Sets: increasing reps and sets every week and then reached } 12 \text { reps/3sets at week } 9 \text { to } \\
16 \\
\text { Duration: } 16 \text { weeks } \\
\text { Setting: home and gym } \\
\text { Supervision: provided in the gym } \\
\text { Adherence: each participant had exercise log, but results were not reported } \\
\text { 2. Control Group: no intervention }\end{array}$} \\
\hline Outcomes & \multicolumn{2}{|c|}{$\begin{array}{l}\text { WOMAC } \\
\text { Knee pain } \\
\text { Stair climbing } \\
\text { Down and up off the floor } \\
\text { Comments on adverse events: no }\end{array}$} \\
\hline Notes & \multicolumn{2}{|c|}{ Calculated SDs from reported SEMs } \\
\hline \multicolumn{3}{|l|}{ Risk of bias } \\
\hline Item & Authors' judgement & Description \\
\hline Allocation concealment? & Unclear & B - Unclear \\
\hline
\end{tabular}




\section{Topp 2005}

\begin{tabular}{|c|c|c|}
\hline Methods & \multicolumn{2}{|c|}{$\begin{array}{l}\text { RCT with } 3 \text { groups: PRT, control, and aerobic groups } \\
\text { Method of randomisation: two-coin-flip methodology } \\
\text { Assessor blinding: not reported } \\
\text { Participant blinding: not reported } \\
\text { Loss to follow-up: yes, but the number was not reported } \\
\text { Intention-to-treat analysis: not reported } \\
\text { Post-program follow up: no }\end{array}$} \\
\hline Participants & \multicolumn{2}{|c|}{$\begin{array}{l}\text { Location: USA } \\
\mathrm{N}=66 \text { ( } 31 \text { in each group) } \\
\text { Sample: older adults with limited functional ability, community dwelling } \\
\text { Age: mean } 74.1 \text { years }(\mathrm{SD}=6.2) \\
\text { Inclusion criteria: score lower then } 24 \text { in physical function domain of SF-36 } \\
\text { Exclusion criteria: could not climb } 26 \text { stairs in } 126 \text { seconds; had contraindications to } \\
\text { exercise }\end{array}$} \\
\hline Interventions & \multicolumn{2}{|c|}{$\begin{array}{l}\text { PRT versus control and versus aerobic } \\
\text { 1. PRT } \\
\text { Type of Ex: } 12 \text { exercises } \\
\text { Equipment: Thera-Band elastic bands } \\
\text { Intensity: self exertion of mild fatigue after } 8 \mathrm{RM} \\
\text { Frequency: Ex3 ( } 2 \text { at home } 1 \text { at gym) } \\
\text { Reps/Sets: started with } 10 / 1-2 \text {, mild fatigue; then increased to } 10 / 3 \text { moderate fatigue at } \\
\text { week } 8 \text { to week } 16 \\
\text { Duration: } 16 \text { weeks } \\
\text { Setting: home and gym } \\
\text { Supervision: provided in the gym } \\
\text { Adherence: each participant had exercise log, but results were not reported Participants in } \\
\text { the final analysis had } 70 \% \text { compliance rate. } \\
\text { 2. Control Group: no intervention, maintain usual activities } \\
\text { 3. Aerobic walking group: N=33, } 3 \text { times/week; between } 50 \% \text { METs to } 75 \% \text { METs; } \\
\text { endurance increasedfrom } 10 \text { minutes to } 35 \text { minutes }\end{array}$} \\
\hline Outcomes & \multicolumn{2}{|l|}{$\begin{array}{l}\text { Arm curls (repetitions) } \\
\text { Chair rise (repetitions) } \\
\text { Stair ascend/descend } \\
\text { Down and up off the floor } \\
\text { Comments on adverse events: no }\end{array}$} \\
\hline Notes & \multicolumn{2}{|c|}{ Numerical results of SDs were not reported. Data were not pooled } \\
\hline \multicolumn{3}{|l|}{ Risk of bias } \\
\hline Item & Authors' judgement & Description \\
\hline Allocation concealment? & Unclear & B - Unclear \\
\hline
\end{tabular}

Tracy 2004

\begin{tabular}{l|l}
\hline Methods & RCT \\
& Method of randomisation: not reported \\
Assessor blinding: not reported & Participant blinding: not reported \\
& Loss to follow-up: 0 (?) \\
& Intention-to-treat analysis: N/A \\
& Post-program follow up: no \\
\hline Participants & Location: USA \\
& N=20 (11 in PRT) \\
& Sample: healthy older adults \\
& Age: mean 73.1 years (SD = 4.9) \\
& Inclusion criteria: no neurological disease, free of medications known to affect the outcome \\
& measures; less than 3 hours a week of low to moderate intensity endurance exercise \\
& Exclusion criteria: not reported \\
\hline Interventions & PRT versus control \\
& 1. PRT \\
& Type of Ex : knee extension, each leg trained separately \\
& Equipment: weight-stack machine (Icarian)
\end{tabular}

Cochrane Database Syst Rev. Author manuscript; available in PMC 2015 February 11. 


\begin{tabular}{|c|c|c|}
\hline & \multicolumn{2}{|l|}{$\begin{array}{l}\text { Intensity: } 80 \% \text { of } 1 \mathrm{RM} \\
\text { Frequency: Ex3 } \\
\text { Reps/Sets: } 10 / 3 \\
\text { Duration: } 16 \text { weeks } \\
\text { Setting: lab } \\
\text { Supervision: full } \\
\text { Adherence: not reported } \\
\text { 2. Control Group: no training involved }\end{array}$} \\
\hline Outcomes & \multicolumn{2}{|c|}{$\begin{array}{l}\text { Primary: physical function tests (including gait speed, chair rise, stair ascent/descent) } \\
\text { Secondary: muscle strength (1RM) } \\
\text { Comments on adverse events: no }\end{array}$} \\
\hline \multicolumn{3}{|l|}{ Notes } \\
\hline \multicolumn{3}{|l|}{ Risk of bias } \\
\hline Item & Authors' judgement & Description \\
\hline Allocation concealment? & Unclear & B - Unclear \\
\hline
\end{tabular}

Tsutsumi 1997

\begin{tabular}{|c|c|c|}
\hline Methods & \multicolumn{2}{|c|}{$\begin{array}{l}\text { RCT with } 3 \text { groups: High-intensity PRT, low-intensity PRT, and control } \\
\text { Method of randomisation: not reported } \\
\text { Assessor blinding: no } \\
\text { Participant blinding: no } \\
\text { Loss to follow-up: } 1 \\
\text { Intention-to-treat analysis: no } \\
\text { Post-program follow up: no }\end{array}$} \\
\hline Participants & \multicolumn{2}{|c|}{$\begin{array}{l}\text { Location: USA } \\
\mathrm{N}=42 \text { total ( } 28 \text { in HI and control) } \\
\text { Sample: sedentary, healthy } \\
\text { Age: mean } 68.9 \text { years (SD } 5.7 \text { years) } \\
\text { Inclusion criteria: aged } 60+\text {, medically healthy, sedentary (no involvement in regular } \\
\text { exercise for the previous } 6 \text { months) } \\
\text { Exclusion criteria: not reported }\end{array}$} \\
\hline Interventions & \multicolumn{2}{|c|}{$\begin{array}{l}\text { PRT versus control } \\
\text { 1. PRT } \\
\text { Type of Ex: 7UL/2LL, 2Tr } \\
\text { Equipment: dynamic variable resistance weight machines } \\
\text { Intensity: HI-75-85\% 1RM; LI-55-65\% 1RM } \\
\text { Frequency: Ex3 } \\
\text { Reps/Sets: HI 8-12/2; LI 12-16/2 } \\
\text { Program duration: 12 weeks } \\
\text { Setting: gym } \\
\text { Supervision: full } \\
\text { Adherence: not reported } \\
\text { 2. Control Group: not reported }\end{array}$} \\
\hline Outcomes & \multicolumn{2}{|c|}{$\begin{array}{l}\text { Strength (1RM) } \\
\text { Aerobic capacity (VO2 max; bicycle ergometer testing) } \\
\text { SF-36 } \\
\text { Physical self-efficacy } \\
\text { Comments on adverse events: no }\end{array}$} \\
\hline Notes & \multicolumn{2}{|c|}{ Date from high intensity PRT and low intensity PRT were compared } \\
\hline \multicolumn{3}{|l|}{ Risk of bias } \\
\hline Item & Authors' judgement & Description \\
\hline Allocation concealment? & Unclear & B - Unclear \\
\hline
\end{tabular}

Tyni-Lenne 2001

\begin{tabular}{l|l}
\hline Methods & RCT
\end{tabular}




\begin{tabular}{l|l|l} 
& $\begin{array}{l}\text { Method of randomisation: not reported } \\
\text { Assessor blinding: no } \\
\text { Participant blinding: no } \\
\text { Loss to follow-up: 0 } \\
\text { Intention-to-treat analysis: no } \\
\text { Post-program follow up: no }\end{array}$ \\
\hline Participants & $\begin{array}{l}\text { Location: Sweden } \\
\text { N }=24 \\
\text { Sample: people with moderate to severe CHF } \\
\text { Age: mean 63 years (SD 9) in PRT group }\end{array}$ \\
& $\begin{array}{l}\text { Inclusion criteria: diagnosed with CHF; medically stable CHF in New York Heart } \\
\text { Association Class II or III } \\
\text { Exclusion criteria: angina pectoris, valvular heart disease determined by Doppler, co- } \\
\text { morbidity such as intermittent claudication, diabetes mellitus, chronic obstructive } \\
\text { pulmonary disease or any other disorder limiting physical performance other than heart } \\
\text { failure }\end{array}$ \\
\hline Interventions & $\begin{array}{l}\text { PRT versus control } \\
\text { 1. PRT } \\
\text { Type of Ex: many UL and LL exercises }\end{array}$ \\
\hline Equipment: theraband \\
Intensity: low-moderate, used Borg rating scale and increased resistance when people rated \\
peripheral resistance <13 \\
Frequency: Ex3 \\
Reps/Sets: 25/2 \\
Program Duration: 8 weeks \\
Setting: group activity \\
Supervision: full \\
Adherence: 95\% \\
2. Control Group: not reported
\end{tabular}

Vincent 2002

\begin{tabular}{l|l}
\hline Methods & RCT with 3 groups: High-intensity PRT, low-intensity PRT and control \\
Method of randomisation: stratified by strength, randomised using a random numbers table \\
Assessor blinding: not reported \\
Participant blinding: not reported \\
Loss to follow-up: 22 \\
Intention-to-treat analysis: no \\
Post-program follow up: no \\
\hline \multirow{2}{*}{ Participants } & Location: USA \\
& N=38 (in HI group and control); N=36-LL \\
& Sample: healthy men and women \\
& Age: mean 67 years (SD 7) \\
& Inclusion criteria: free from cardiovascular or orthopedic problems that would limit \\
& exercise (assessment included physical exam), had not participated in resistance exercise \\
& for at least one year \\
& Excludion Criteria: not reported \\
& PRT versus control \\
& 1. PRT \\
& Type of Ex: 5 UL/ 6LL \\
& Equipment: resistance machines (MedX) \\
& Intensity: high intensity: (80\% of 1RM); low Intensity: (50\% of 1RM) \\
& Frequency: Ex3 \\
& Reps/Sets: high Intensity: $8 / 1 ;$ low Intensity: 13/1 \\
& Program Duration: 6 months \\
& Setting: gym \\
& Supervision: full \\
&
\end{tabular}




\begin{tabular}{l|l|l} 
& $\begin{array}{l}\text { Adherence: excluded those who completed less than 85\% of sessions } \\
\text { 2. Control Group: instructed not to make any changes in their lifestyle during the study }\end{array}$ \\
\hline Outcomes & $\begin{array}{l}\text { Strength (1RM) } \\
\text { Peak VO2 (update) } \\
\text { Stair climb (update) } \\
\text { Comments on adverse events: yes }\end{array}$ \\
\hline Notes & $\begin{array}{l}\text { Added results from more recent publications } \\
\text { Date from high intensity PRT and low intensity PRT were compared }\end{array}$ \\
\hline Risk of bias & \multicolumn{2}{|l}{} \\
\hline Item & Authors' judgement & Description \\
\hline Allocation concealment? & Unclear & B- Unclear \\
\hline
\end{tabular}

\section{Westhoff 2000}

\begin{tabular}{|c|c|c|}
\hline Methods & \multicolumn{2}{|c|}{$\begin{array}{l}\text { RCT } \\
\text { Method of randomisation: not reported } \\
\text { Assessor blinding: yes } \\
\text { Participant blinding: no } \\
\text { Loss to follow-up: } 5 \\
\text { Intention-to-treat analysis: no } \\
\text { Post-program follow up: no }\end{array}$} \\
\hline Participants & \multicolumn{2}{|c|}{$\begin{array}{l}\text { Location: The Netherlands } \\
\mathrm{N}=26 \\
\text { Sample: low knee-extensor muscle strength } \\
\text { Age: mean } 75.9 \text { years (SD 6.8) in the exercise group } \\
\text { Inclusion criteria: local residents } 65 \text { years and over } \\
\text { Exclusion criteria: maximum knee extensor torque for both legs }>87.5 \mathrm{Nm} \text {, self-reported } \\
\text { disease or condition such as uncontrolled heart failure or a neurological disease that would } \\
\text { be adversely affected by the exercises in the program }\end{array}$} \\
\hline Interventions & \multicolumn{2}{|c|}{$\begin{array}{l}\text { PRT versus control and versus aerobic } \\
\text { 1. PRT } \\
\text { Type of Ex: } 5 \mathrm{UL}, 3 \mathrm{LL} \\
\text { Equipment: resistance training machines } \\
\text { Intensity: } 75 \% \text { of } 5 \mathrm{RM} \text { at first, progressed to } 8-12 \mathrm{RM} \\
\text { Frequency: Ex3 } \\
\text { Reps/Sets } 8-12 / 1-2 \\
\text { Program Duration: } 12 \text { weeks } \\
\text { Setting: gym } \\
\text { Supervision: not reported } \\
\text { Adherence: excluded those who did not have } 80 \% \text { or more attendance } \\
\text { 2. Control Group: asked not to make significant changes in their physical activity and } \\
\text { nutrition habitsover a } 12 \text {-week period } \\
\text { 3. Aerobic Training: trained on treadmills and cycle ergometers } 3 \text { times per week at } \\
\text { 60-70\% estimatedHR reserve, for } 21-45 \text { minutes per session }\end{array}$} \\
\hline Outcomes & \multicolumn{2}{|c|}{$\begin{array}{l}\text { Strength (maximum torque measured by the Quadriso-tester) } \\
\text { Gronigen Activity Restriction Scale, an ADL/IADL Index with scores from } 18 \text { (no } \\
\text { limitations) to } 72 \text { (fully dependent) } \\
\text { Timed walking test } \\
\text { Timed up-and-go } \\
\text { Balance (FICSIT balance test, graded from 1-6) } \\
\text { Comments on adverse events: yes (asked about complaints during exercise) }\end{array}$} \\
\hline \multicolumn{3}{|l|}{ Notes } \\
\hline \multicolumn{3}{|l|}{ Risk of bias } \\
\hline Item & Authors' judgement & Description \\
\hline Allocation concealment? & Unclear & B - Unclear \\
\hline
\end{tabular}


Wieser 2007

\begin{tabular}{|c|c|c|}
\hline Methods & \multicolumn{2}{|c|}{$\begin{array}{l}\text { RCT } \\
\text { Method of randomisation: used www.randomization.com } \\
\text { Assessor blinding: not reported } \\
\text { Participant blinding: not reported } \\
\text { Loss to follow-up: } 0 \text { in PRT group, } 4 / 14 \text { in the control group } \\
\text { Intention-to-treat analysis: no } \\
\text { Post-program follow up: no }\end{array}$} \\
\hline Participants & \multicolumn{2}{|c|}{$\begin{array}{l}\text { Location: Austria } \\
\mathrm{N}=28 \text { ( } 14 \text { in each group) } \\
\text { Sample: healthy older adults } \\
\text { Age: mean } 76.2 \text { years }(\mathrm{SD}=3.2) \\
\text { Inclusion criteria: older than } 70 \text { years, healthy cardio-pulmonary system, untrained } \\
\text { Exclusion criteria: participated in a resistance training program; or cardiac arrhythmia, } \\
\text { recent myocardial infarct, stroke, cancer, or an ill-treated hypertonia }\end{array}$} \\
\hline Interventions & \multicolumn{2}{|c|}{$\begin{array}{l}\text { PRT versus control } \\
\text { 1. PRT } \\
\text { Type of Ex: 4UL/1LL } \\
\text { Equipment: machines } \\
\text { Intensity: increase weight after } 10 \text { th repetitions } \\
\text { Frequency: Ex2 } \\
\text { Reps/sets week 1-4: } 8 / 1 \text { week 5-8: 8/3; week 9-12: 8/4 } \\
\text { Duration: } 12 \text { weeks } \\
\text { Setting: not reported } \\
\text { Supervision: not reported } \\
\text { Adherence: not reported, provided make-up sessions } \\
\text { 2. Control Group: not reported }\end{array}$} \\
\hline Outcomes & \multicolumn{2}{|c|}{$\begin{array}{l}\text { VO2max } \\
\text { Muscle strength } \\
\text { Comments on adverse events: no }\end{array}$} \\
\hline Notes & \multicolumn{2}{|c|}{ Numerical results of muscle strength were not reported } \\
\hline \multicolumn{3}{|l|}{ Risk of bias } \\
\hline Item & Authors' judgement & Description \\
\hline Allocation concealment? & Unclear & B - Unclear \\
\hline
\end{tabular}

\section{Wood 2001}

\begin{tabular}{l|l}
\hline Methods & RCT with 4 groups: PRT alone, aerobic training alone, combined PRT and aerobic training \\
and control & Method of randomisation: not reported \\
Assessor blinding: no & Participant blinding: no \\
Loss to follow-up: 9 in four groups - drop outs not reported by group & Intention-to-treat analysis: no \\
& Post-program follow up: no
\end{tabular}




\begin{tabular}{|c|c|c|}
\hline & \multicolumn{2}{|c|}{$\begin{array}{l}\text { Frequency: Ex3 } \\
\text { Reps/Sets } 8-12 \text { from progressed from } 1 \text { set to } 2 \text { sets } \\
\text { Program Duration: } 12 \text { weeks } \\
\text { Setting: gym } \\
\text { Supervision: not reported } \\
\text { Adherence: excluded those who did not have } 80 \% \text { or more attendance } \\
\text { 2. Control Group: asked not to make significant changes in their physical activity and } \\
\text { nutrition habitsover a } 12 \text {-week period } \\
\text { 3. Aerobic Training: trained on treadmills and cycle ergometers } 3 \text { times per week at } \\
\text { 60-70\% estimatedHR reserve, for } 21-45 \text { minutes per session }\end{array}$} \\
\hline Outcomes & \multicolumn{2}{|c|}{$\begin{array}{l}\text { Strength }(5 \mathrm{RM}) \\
\text { Submaximal aerobic capacity } \\
\text { Co-ordination } \\
\text { Comments on adverse events: no }\end{array}$} \\
\hline Notes & \multicolumn{2}{|c|}{ Data from PRT and aerobic training group were compared } \\
\hline \multicolumn{3}{|l|}{ Risk of bias } \\
\hline Item & Authors' judgement & Description \\
\hline Allocation concealment? & Unclear & B - Unclear \\
\hline
\end{tabular}

ADL: activities of daily living

Age: overall age of all groups. If this is not available age for progressive resistance training group alone is reported

CHF: congestive heart failure

CHD:coronary heart disease

COPD: chronic obstructive pulmonary disease

Ex: exercise

Ex1: exercise once per week

Ex2: exercise twice per week

Ex3: exercise three times per week

HI: high intensity

MI: Medium intensity

LI: low intensity

LL: lower limb

METs: maximum metabolic equivalents

MMSE: the Mini-Mental State Examination

$\mathrm{N}$ : number of participants allocated to strength training group and control group; or number of participants allocated to additional intervention group

NA: not applicable

OA: osteoarthritis

PAD: peripheral arterial disease RCT: Randomised controlled trial

PRT: progressive resistance strength training Reps: repetitions

RM: repetition maximum

SF-36: Medical Outcome Studies 36 Item Short Form questionnaire

Tr: trunk

TUAG: timed "up-and-go" test

UL: upper limb

WOMAC: Western Ontario/McMaster Universities Arthritis Index 
Characteristics of excluded studies [ordered by study ID]

\begin{tabular}{|c|c|}
\hline Study & Reason for exclusion \\
\hline Adami 1999 & Not a RCT \\
\hline Adams 2001 & Participants too young (mean age $<60$ ) \\
\hline Agre 1988 & Not a RCT \\
\hline Alexander 2003 & Combined program - not PRT alone \\
\hline Aniansson 1981 & Not a RCT \\
\hline Annesi 2004 & Combined intervention program - not PRT alone \\
\hline Ardman 1998 & Not a RCT \\
\hline Ballard 2004 & Combined program - not PRT alone \\
\hline Barbosa 2002 & Not a RCT \\
\hline Baum 2003b & Does not meet criteria for PRT \\
\hline Bean 2002 & Does not meet criteria for PRT \\
\hline Bellew 2003 & Not a RCT \\
\hline Beniamini 1997 & Participants too young (mean age $<60$ ) \\
\hline Beniamini 1999 & Participants too young (mean age $<60$ ) \\
\hline Berg 1998 & Not a RCT \\
\hline Bernard 1999 & Combined program - not PRT alone \\
\hline Bilodeau 2000 & Participants too young (mean age $<60$ ) \\
\hline Binda 2003 & Does not meet for criteria for PRT \\
\hline Binder 2002 & Combined program - not PRT alone \\
\hline Boardley 2007 & No relevant outcomes to the review \\
\hline Braith 2005 & No relevant outcomes to the review \\
\hline Brandon 2003b & Does not meet the criteria for PRT - not progressive \\
\hline Brandon 2004 & Combined program - not PRT alone \\
\hline Brill 1998 & Not a RCT \\
\hline Brose 2003 & Combined program - not PRT alone \\
\hline Brown 1990 & Not a RCT \\
\hline Brown 1991 & Combined program - not PRT alone \\
\hline Brown 2000 & Combined program - not PRT alone \\
\hline Bunout 2001 & Combined program - not PRT alone \\
\hline Campbell 2002 & No relevant outcomes to the review \\
\hline Campbell 2004 & No relevant outcomes to the review \\
\hline Cancela 2003 & Article cannot be located. \\
\hline Candow 2004 & Combined program (with supplement) - not PRT alone \\
\hline Capodaglio 2002 & Not a RCT \\
\hline Carter 2002 & Does not meet the criteria for PRT \\
\hline Carter 2005 & Included participants younger than 60 \\
\hline Carvalho 2002 & No relevant outcomes to the review \\
\hline Cauza 2005 & No relevant outcomes to the review \\
\hline
\end{tabular}




\begin{tabular}{|c|c|}
\hline Study & Reason for exclusion \\
\hline Cauza 2005b & Included young participants (younger than 60 years old) \\
\hline Chaloupka 2000 & Participants too young (mean age $<60$ ) \\
\hline Chetlin 2004 & Included young participants (younger than 60 years old) \\
\hline Chiba 2006 & Not a RCT \\
\hline Chien 2005 & Does not meet the criteria for PRT \\
\hline Connelly 1995 & Not a RCT \\
\hline Connelly 2000 & Not a RCT \\
\hline Cramp 2006 & Not a RCT \\
\hline Cress 1991 & Not a RCT \\
\hline Cress 1999 & Combined program - not PRT alone \\
\hline Daepp 2006 & Does not meet the criteria for PRT \\
\hline Daly 2005 & Combined program - not PRT alone \\
\hline de Bruin 2007 & No relevant comparisons to the review \\
\hline de Vito 1999 & Combined program - not PRT alone \\
\hline DeBolt 2004 & Included young participants (younger than 60 years old) \\
\hline Delagardelle 2002 & Combined program - not PRT alone \\
\hline Delecluse 2004 & Combined program - not PRT alone \\
\hline DeVito 2003 & Combined program - not PRT alone \\
\hline Dibble 2006 & Not a RCT \\
\hline Dibble $2006 \mathrm{~b}$ & Not a RCT \\
\hline Dunstan 2002 & Not PRT alone - with eating plan \\
\hline Dunstan 2005 & Not PRT alone - with eating plan \\
\hline Dupler 1993 & Not a RCT \\
\hline Fernandez Ramirez 99 & Combined program - not PRT alone \\
\hline Ferrara 2006 & Not a RCT \\
\hline Ferri 2003 & Not a RCT \\
\hline Fiatarone 1990 & Not a RCT \\
\hline Fisher 1991 & Not a RCT \\
\hline Forte 2003 & Not a RCT \\
\hline Frontera 1988 Frontera 1990 & Not a RCT Not a RCT \\
\hline Frontera 1990 & Not a RCT \\
\hline Galvao 2006 & Not a RCT \\
\hline Grimby 1992 & Not a RCT \\
\hline Gur 2002 & Included young participants (younger than 60 years old) \\
\hline Hageman 2002 & Not a RCT \\
\hline Hakkinen 1999 & Participants too young (mean age $<60$ ) \\
\hline Hameed 2004 & Combined program (with hormone intervention) \\
\hline Hartard 1996 & Not a RCT \\
\hline Haub 2002 & Combined program (protein) - not PRT alone \\
\hline
\end{tabular}

Cochrane Database Syst Rev. Author manuscript; available in PMC 2015 February 11. 


\begin{tabular}{|c|c|}
\hline Study & Reason for exclusion \\
\hline Heiwe 2005 & No relevant outcomes to the review \\
\hline Henwood 2006 & Does not meet the criteria for PRT - not progressive \\
\hline Hess 2005 & Not a RCT \\
\hline Hess 2006 & Not a RCT \\
\hline Hirsch 2003 & Combined program - not PRT alone \\
\hline Host 2007 & Combined program - not PRT alone \\
\hline Huggett 2004 & No relevant outcomes to the review \\
\hline Hughes 2004 & Combined program - not PRT alone \\
\hline Humphries 2000 & Participants too young (mean age $<60$ ) \\
\hline Hung 2004 & Does not meet the criteria for PRT \\
\hline Hunter 1995 & Not a RCT \\
\hline Hunter 2002 & Not a RCT \\
\hline Ibanez 2005 & Not a RCT \\
\hline Ivey 2000 & Not a RCT \\
\hline Johansen 2006 & Included young participants (younger than 60 years old) \\
\hline Jones 1987 & Participants too young (mean age $<60$ ) \\
\hline Judge 2005 & No relevant outcomes to the review \\
\hline Katula 2006 & Not a RCT \\
\hline Kerr 2001 & No relevant outcomes to the review \\
\hline Kolbe-Alexander 2006 & Not a RCT \\
\hline Komatireddy 1997 & Participants too young (mean age $<60$ ) \\
\hline La Forge 2002 & No relevant outcomes to the review \\
\hline Labarque 2002 & Training did not meet criteria for PRT \\
\hline Lambert 2002 & No relevant outcomes to the review \\
\hline Lambert 2003 & Combined program (with hormone) - not PRT alone \\
\hline Lamotte 2005 & No relevant outcomes to the review \\
\hline Levinger 2005 & Included young participants (younger than 60 years old) \\
\hline Lexell 1992 & Not a RCT \\
\hline Lexell 1995 & $\begin{array}{l}\text { Not a RCT (not clearly stated that patients were } \\
\text { randomised) }\end{array}$ \\
\hline Littbrand 2006 & Combined program - not PRT alone \\
\hline Liu 2004 & Training did not meet criteria for PRT - not progressive \\
\hline Liu-Ambrose 2004 & No relevant outcomes to the review \\
\hline Loeppky 2005 & Does not meet the criteria for PRT \\
\hline Lohman 1995 & Participants too young (mean age $<60$ ) \\
\hline Maddalozzo 2000 & Participants too young (mean age <60) \\
\hline Magnusson 1996 & Participants too young (mean age $<60$ ) \\
\hline Marcora 2005 & Not a RCT \\
\hline Martin Ginis 2006 & No relevant comparisons to the review \\
\hline McCool 1991 & Not a RCT \\
\hline
\end{tabular}




\begin{tabular}{|c|c|}
\hline Study & Reason for exclusion \\
\hline McMurdo 1994 & Training did not meet criteria for PRT \\
\hline Mobily 2004 & Not a RCT \\
\hline Morey 1989 & Combined program - not PRT alone \\
\hline Morey 1991 & Combined program - not PRT alone \\
\hline Morse 2005 & Combined program - not PRT alone \\
\hline Narici 1989 & Participants too young (mean age $<60$ ) \\
\hline Nelson 1997 & Combined program - not PRT alone \\
\hline Ochala 2005 & Training did not meet criteria for PRT \\
\hline Ohira 2006 & $\begin{array}{l}\text { Training did not meet criteria for PRT - not } \\
\text { progressive/included young participants }\end{array}$ \\
\hline Oka 2000 & Combined program - not PRT alone \\
\hline Okawa 2004 & Included younger participants (middle age) \\
\hline Okumiya 1996 & Combined program - not PRT alone \\
\hline Panton 2004 & Combined program - not PRT alone \\
\hline Parsons 1992 & Not a RCT \\
\hline Perhonen 1992 & Training did not meet criteria for PRT \\
\hline Perkins 1961 & Training did not meet criteria for PRT \\
\hline Perrig-Chiello 1998 & No relevant outcomes to the review \\
\hline Petrella 2000 & Training did not meet criteria for PRT \\
\hline Phillips 2004 & Not a RCT \\
\hline Pyka 1994 & $\begin{array}{l}\text { Serious threats to internal validity - participants } \\
\text { allowed to move from exercise to control group-Not a } \\
\text { RCT }\end{array}$ \\
\hline Rabelo 2004 & Training did not meet criteria for PRT - not progressive \\
\hline Ramsbottom 2004 & Combined program - not PRT alone \\
\hline Reeves 2004b & Does not meet the criteria for PRT \\
\hline Reeves 2005 & Not a RCT \\
\hline Reeves 2006 & Does not meet the criteria for PRT \\
\hline Richards 1996 & Not a RCT \\
\hline Roman 1993 & Not a RCT \\
\hline Rooks 1997 & Training did not meet criteria for PRT \\
\hline Salli 2006 & Training did not meet criteria for PRT - not progressive \\
\hline Sallinen 2006 & Combined program (with diet) - not PRT alone \\
\hline Sanders 1998 & Not a RCT \\
\hline Sartorio 2001 & No relevant outcomes to the review \\
\hline Sauvage 1992 & Combined program - not PRT alone \\
\hline Sayers 2003 & Not a RCT \\
\hline Schott 2006 & Combined program (with supplement) - not PRT alone \\
\hline Sharp 1997 & Not a RCT \\
\hline Shaw 1998 & Not a RCT \\
\hline Sherrington 1997 & Training did not meet criteria for PRT \\
\hline
\end{tabular}




\begin{tabular}{|c|c|}
\hline Study & Reason for exclusion \\
\hline Signorile 2005 & Does not meet the criteria for PRT - not progressive \\
\hline Sinaki 1996 & Participants too young (mean age <60) \\
\hline Sipila 1994 & Not a RCT \\
\hline Spruit 2002 & Combined program - not PRT alone \\
\hline Sullivan 2001 & Not a RCT \\
\hline Taaffe 1997 & Not a RCT \\
\hline Teixeira 2002 & Does not meet the criteria for PRT \\
\hline Teixeira 2003 & Included young participants (younger than 60 years old) \\
\hline Teixeira-Salm. 2005 & Combined program - not PRT alone \\
\hline Thielman 2004 & No relevant outcomes to the review \\
\hline Thomas 2004 & Combined program - not PRT alone \\
\hline Thomas 2005 & $\begin{array}{l}\text { Training did not meet criteria for PRT - The resistance } \\
\text { was not progressively increased }\end{array}$ \\
\hline Thompson 1988 & Combined program - not PRT alone \\
\hline Timonen 2002 & Combined program - not PRT alone \\
\hline Timonen 2006 & Combined program - not PRT alone \\
\hline Timonen $2006 b$ & Combined program - not PRT alone \\
\hline Treuth 1994 & Not a RCT \\
\hline Trudelle-Jack. 2004 & Combined program - not PRT alone \\
\hline Tsuji 2000 & Combined program - not PRT alone \\
\hline Vad 2002 & Combined program - not PRT alone \\
\hline Vale 2003 & Article cannot be identified \\
\hline Valkeinen 2005 & $\begin{array}{l}\text { Participants too young (mean age of the control group }< \\
60 \text { ) }\end{array}$ \\
\hline Van den Ende 2000 & Combined program - not PRT alone \\
\hline Vanbiervliet 2003 & Included young participants (younger than 60 years old) \\
\hline Veloso 2003 & Does not meet the criteria for PRT \\
\hline Verfaillie 1997 & Combined program - not PRT alone \\
\hline Villareal 2003 & Combined program (with hormone)-not PRT alone \\
\hline Villareal 2006b & Combined program - not PRT alone \\
\hline Vincent $2002 b$ & No relevant outcomes to the review \\
\hline Vincent 2003 & No relevant outcomes to the review \\
\hline Vincent 2006 & Included young participants (younger than 60 years old) \\
\hline Woo 2007 & $\begin{array}{l}\text { Training did not meet criteria for PRT. The resistance } \\
\text { was not progressively increased }\end{array}$ \\
\hline Yang 2006 & Does not meet the criteria for PRT \\
\hline Zion 2003 & Not a RCT \\
\hline
\end{tabular}

$\mathrm{RCT}=$ randomised controlled trial $; \mathrm{PRT}=$ progressive resistance strength training 
Data and Analyses

\section{Comparison 1. PRT versus control}

\begin{tabular}{|c|c|c|c|c|}
\hline Outcome or subgroup title & No. of studies & No. of participants & Statistical method & Effect size \\
\hline $\begin{array}{l}1 \text { Main function measure } \\
\text { (higher score = better } \\
\text { function) }\end{array}$ & 33 & 2172 & $\begin{array}{l}\text { Std. Mean Difference } \\
\text { (IV, Fixed, 95\% CI) }\end{array}$ & $0.14[0.05,0.22]$ \\
\hline $\begin{array}{l}2 \text { Physical function domain } \\
\text { of SF-36/SF-12 (Higher score } \\
=\text { better function) }\end{array}$ & 14 & 778 & $\begin{array}{l}\text { Std. Mean Difference } \\
\text { (IV, Fixed, 95\% CI) }\end{array}$ & $0.07[-0.08,0.21]$ \\
\hline $\begin{array}{l}3 \text { Activities of daily living } \\
\text { measure (higher score }= \\
\text { better function) }\end{array}$ & 3 & 330 & $\begin{array}{l}\text { Std. Mean Difference } \\
\text { (IV, Fixed, 95\% CI) }\end{array}$ & $0.04[-0.18,0.26]$ \\
\hline $\begin{array}{l}4 \text { Activity level measure (kJ/ } \\
\text { week) }\end{array}$ & 2 & & $\begin{array}{l}\text { Mean Difference (IV, } \\
\text { Fixed, 95\% CI) }\end{array}$ & Subtotals only \\
\hline $\begin{array}{l}5 \text { Main lower limb (LL) } \\
\text { strength measure }\end{array}$ & 73 & 3059 & $\begin{array}{l}\text { Std. Mean Difference } \\
\text { (IV, Random, 95\% } \\
\text { CI) }\end{array}$ & $0.84[0.67,1.00]$ \\
\hline $\begin{array}{l}6 \text { Main measure of aerobic } \\
\text { function }\end{array}$ & 29 & 1138 & $\begin{array}{l}\text { Std. Mean Difference } \\
\text { (IV, Random, 95\% } \\
\text { CI) }\end{array}$ & $0.31[0.09,0.53]$ \\
\hline $7 \mathrm{VO} 2$ or peak oxygen uptake & 19 & & $\begin{array}{l}\text { Mean Difference (IV, } \\
\text { Random, 95\% CI) }\end{array}$ & Subtotals only \\
\hline 7.1 VO2max-ml/kg.min & 18 & 710 & $\begin{array}{l}\text { Mean Difference (IV, } \\
\text { Random, 95\% CI) }\end{array}$ & $1.50[0.49,2.51]$ \\
\hline $\begin{array}{l}\text { 7.2 Peak oxygen uptake- } \\
\text { L/min }\end{array}$ & 2 & 47 & $\begin{array}{l}\text { Mean Difference (IV, } \\
\text { Random, 95\% CI) }\end{array}$ & $0.10[-0.04,0.24]$ \\
\hline $\begin{array}{l}8 \text { Six-minute walk test } \\
\text { (meters) }\end{array}$ & 11 & 325 & $\begin{array}{l}\text { Mean Difference (IV, } \\
\text { Random, 95\% CI) }\end{array}$ & $52.37[17.38,87.37]$ \\
\hline $\begin{array}{l}9 \text { Balance measures (higher }= \\
\text { better balance) }\end{array}$ & 17 & 996 & $\begin{array}{l}\text { Std. Mean Difference } \\
\text { (IV, Fixed, 95\% CI) }\end{array}$ & $0.12[-0.00,0.25]$ \\
\hline $\begin{array}{l}10 \text { Balance measures }(\text { Low }= \\
\text { better balance) }\end{array}$ & 1 & & $\begin{array}{l}\text { Mean Difference (IV, } \\
\text { Fixed, 95\% CI) }\end{array}$ & Totals not selected \\
\hline $\begin{array}{l}10.1 \text { PRT (high intensity) } \\
\text { versus control }\end{array}$ & 1 & & $\begin{array}{l}\text { Mean Difference (IV, } \\
\text { Fixed, 95\% CI) }\end{array}$ & Not estimable \\
\hline $\begin{array}{l}10.2 \text { PRT (low intensity) } \\
\text { versus control }\end{array}$ & 1 & & $\begin{array}{l}\text { Mean Difference (IV, } \\
\text { Fixed, 95\% CI) }\end{array}$ & Not estimable \\
\hline 11 Gait speed $(\mathrm{m} / \mathrm{s})$ & 24 & 1179 & $\begin{array}{l}\text { Mean Difference (IV, } \\
\text { Random, 95\% CI) }\end{array}$ & $0.08[0.04,0.12]$ \\
\hline 12 Timed walk (seconds) & 8 & 204 & $\begin{array}{l}\text { Mean Difference (IV, } \\
\text { Fixed, 95\% CI) }\end{array}$ & $-0.23[-1.07,0.62]$ \\
\hline $\begin{array}{l}13 \text { Timed "Up-and-Go" } \\
\text { (seconds) }\end{array}$ & 12 & 691 & $\begin{array}{l}\text { Mean Difference (IV, } \\
\text { Fixed, 95\% CI) }\end{array}$ & $-0.69[-1.11,-0.27]$ \\
\hline 14 Time to stand from a chair & 11 & 384 & $\begin{array}{l}\text { Std. Mean Difference } \\
\text { (IV, Random, 95\% } \\
\text { CI) }\end{array}$ & $-0.94[-1.49,-0.38]$ \\
\hline 15 Stair climbing (seconds) & 8 & 268 & $\begin{array}{l}\text { Mean Difference (IV, } \\
\text { Random, 95\% CI) }\end{array}$ & $-1.44[-2.51,-0.37]$ \\
\hline $\begin{array}{l}16 \text { Chair stand within time } \\
\text { limit (number of times) }\end{array}$ & 1 & & $\begin{array}{l}\text { Mean Difference (IV, } \\
\text { Fixed, 95\% CI) }\end{array}$ & Totals not selected \\
\hline $\begin{array}{l}17 \text { Vitality (SF-36/Vitality } \\
\text { plus scale, higher = more } \\
\text { vitality) }\end{array}$ & 10 & 611 & $\begin{array}{l}\text { Mean Difference (IV, } \\
\text { Fixed, 95\% CI) }\end{array}$ & $1.33[-0.89,3.55]$ \\
\hline $\begin{array}{l}18 \text { Pain (higher }=\text { less pain, } \\
\text { Bodily pain on SF-36) }\end{array}$ & 10 & 587 & $\begin{array}{l}\text { Mean Difference (IV, } \\
\text { Fixed, 95\% CI) }\end{array}$ & $0.34[-3.44,4.12]$ \\
\hline
\end{tabular}

Cochrane Database Syst Rev. Author manuscript; available in PMC 2015 February 11. 


\begin{tabular}{lccll}
\hline Outcome or subgroup title & No. of studies & No. of participants & Statistical method & Effect size \\
\hline $\begin{array}{l}\text { 19 Pain (lower score = less } \\
\text { pain) }\end{array}$ & 6 & 503 & $\begin{array}{l}\text { Std. Mean Difference } \\
\text { (IV, Fixed, 95\% CI) }\end{array}$ & $-0.30[-0.48,-0.13]$ \\
& \multirow{2}{*}{13} & 1125 & $\begin{array}{l}\text { Risk Ratio (M-H, } \\
\text { Fixed, 95\% CI) }\end{array}$ & $0.89[0.52,1.54]$ \\
\hline
\end{tabular}

\section{Comparison 2. High versus low intensity PRT}

\begin{tabular}{|c|c|c|c|c|}
\hline $\begin{array}{l}\text { Outcome or subgroup } \\
\text { title }\end{array}$ & No. of studies & No. of participants & Statistical method & Effect size \\
\hline $\begin{array}{l}1 \text { Main function } \\
\text { measure (higher score } \\
=\text { better function) }\end{array}$ & 2 & 62 & $\begin{array}{l}\text { Std. Mean Difference (IV, } \\
\text { Fixed, } 95 \% \text { CI) }\end{array}$ & $-0.17[-0.67,0.33]$ \\
\hline $\begin{array}{l}2 \text { Main lower limb } \\
\text { (LL) strength measure }\end{array}$ & 9 & 219 & $\begin{array}{l}\text { Std. Mean Difference (IV, } \\
\text { Random, 95\% CI) }\end{array}$ & $0.48[0.03,0.93]$ \\
\hline $\begin{array}{l}3 \mathrm{VO} 2 \mathrm{Max}(\mathrm{ml} / \mathrm{kg} / \\
\min )\end{array}$ & 3 & 101 & $\begin{array}{l}\text { Mean Difference (IV, } \\
\text { Random, 95\% CI) }\end{array}$ & $1.82[-0.79,4.43]$ \\
\hline $\begin{array}{l}4 \text { Pain (higher score }= \\
\text { less pain) }\end{array}$ & 2 & 62 & $\begin{array}{l}\text { Std. Mean Difference (IV, } \\
\text { Fixed, 95\% CI) }\end{array}$ & $-0.05[-0.55,0.45]$ \\
\hline $\begin{array}{l}5 \text { Vitality }(\mathrm{SF}-36, \\
\text { higher score }=\text { more } \\
\text { vitality) }\end{array}$ & 2 & 62 & $\begin{array}{l}\text { Mean Difference (IV, Fixed, } \\
95 \% \text { CI) }\end{array}$ & $6.54[0.69,12.39]$ \\
\hline
\end{tabular}

Comparison 3. High versus variable intensity PRT

\begin{tabular}{lclll}
\hline $\begin{array}{l}\text { Outcome or subgroup } \\
\text { title }\end{array}$ & No. of studies & No. of participants & Statistical method & Effect size \\
\hline $\begin{array}{l}\text { 1 Main lower limb } \\
\text { (LL) strength measure }\end{array}$ & 1 & $\begin{array}{l}\text { Std. Mean Difference (IV, } \\
\text { Random, 95\% CI) }\end{array}$ & Totals not selected \\
$\begin{array}{l}\text { 2 VO2 Max (ml/kg/ } \\
\text { min) }\end{array}$ & 1 & $\begin{array}{l}\text { Mean Difference (IV, Fixed, } \\
95 \% \text { CI) }\end{array}$ & Totals not selected \\
\hline
\end{tabular}

\section{Comparison 4. PRT frequency}

\begin{tabular}{|c|c|c|c|c|}
\hline $\begin{array}{l}\text { Outcome or } \\
\text { subgroup title }\end{array}$ & No. of studies & No. of participants & Statistical method & Effect size \\
\hline $\begin{array}{l}1 \text { Main LL strength } \\
\text { measure }\end{array}$ & 2 & & $\begin{array}{l}\text { Std. Mean Difference (IV, } \\
\text { Fixed, 95\% CI) }\end{array}$ & Totals not selected \\
\hline $\begin{array}{l}1.1 \text { Three times } \\
\text { versus once per week }\end{array}$ & 1 & & $\begin{array}{l}\text { Std. Mean Difference (IV, } \\
\text { Fixed, 95\% CI) }\end{array}$ & Not estimable \\
\hline $\begin{array}{l}1.2 \text { Twice versus } \\
\text { once per week }\end{array}$ & 1 & & $\begin{array}{l}\text { Std. Mean Difference (IV, } \\
\text { Fixed, 95\% CI) }\end{array}$ & Not estimable \\
\hline
\end{tabular}

Cochrane Database Syst Rev. Author manuscript; available in PMC 2015 February 11. 
Comparison 5. PRT: 3-sets versus 1-sets

\begin{tabular}{|c|c|c|c|c|}
\hline $\begin{array}{l}\text { Outcome or } \\
\text { subgroup title }\end{array}$ & No. of studies & No. of participants & Statistical method & Effect size \\
\hline $\begin{array}{l}1 \text { Main lower limb } \\
\text { (LL) strength measure }\end{array}$ & 1 & & $\begin{array}{l}\text { Std. Mean Difference (IV, } \\
\text { Fixed, 95\% CI) }\end{array}$ & Totals not selected \\
\hline $\begin{array}{l}2 \text { Six-minute walk test } \\
\text { (meters) }\end{array}$ & 1 & & $\begin{array}{l}\text { Mean Difference (IV, Fixed, } \\
95 \% \text { CI) }\end{array}$ & Totals not selected \\
\hline $\begin{array}{l}3 \text { Timed walk } \\
\text { (seconds) }\end{array}$ & 1 & & $\begin{array}{l}\text { Mean Difference (IV, Fixed, } \\
95 \% \text { CI) }\end{array}$ & Totals not selected \\
\hline $\begin{array}{l}4 \text { Time to stand from } \\
\text { a chair (seconds) }\end{array}$ & 1 & & $\begin{array}{l}\text { Mean Difference (IV, Fixed, } \\
\text { 95\% CI) }\end{array}$ & Totals not selected \\
\hline $\begin{array}{l}5 \text { Stair climbing } \\
\text { (seconds) }\end{array}$ & 1 & & $\begin{array}{l}\text { Mean Difference (IV, Fixed, } \\
95 \% \text { CI) }\end{array}$ & Totals not selected \\
\hline
\end{tabular}

\section{Comparison 6. PRT versus aerobic training}

\begin{tabular}{|c|c|c|c|c|}
\hline $\begin{array}{l}\text { Outcome or } \\
\text { subgroup title }\end{array}$ & No. of studies & No. of participants & Statistical method & Effect size \\
\hline $\begin{array}{l}1 \text { Main function } \\
\text { measure (higher } \\
\text { score = better } \\
\text { function) }\end{array}$ & 4 & 125 & $\begin{array}{l}\text { Std. Mean Difference (IV, } \\
\text { Fixed, 95\% CI) }\end{array}$ & $-0.21[-0.56,0.15]$ \\
\hline $\begin{array}{l}2 \text { Main function } \\
\text { measure (lower score } \\
=\text { better function) }\end{array}$ & 1 & & $\begin{array}{l}\text { Std. Mean Difference (IV, } \\
\text { Fixed, 95\% CI) }\end{array}$ & Totals not selected \\
\hline $\begin{array}{l}3 \text { Main lower limb } \\
\text { strength measure }\end{array}$ & 10 & 487 & $\begin{array}{l}\text { Std. Mean Difference (IV, } \\
\text { Random, 95\% CI) }\end{array}$ & $0.44[0.08,0.80]$ \\
\hline $\begin{array}{l}4 \mathrm{VO} 2 \max (\mathrm{ml} / \\
\text { kg.min) }\end{array}$ & 8 & 423 & $\begin{array}{l}\text { Mean Difference (IV, } \\
\text { Random, 95\% CI) }\end{array}$ & $-1.13[-2.63,0.38]$ \\
\hline $\begin{array}{l}5 \text { Six minute walk } \\
\text { test (meters) }\end{array}$ & 2 & 63 & $\begin{array}{l}\text { Mean Difference (IV, Fixed, } \\
95 \% \text { CI) }\end{array}$ & $\begin{array}{l}-4.28[-48.24,39 . \\
67]\end{array}$ \\
\hline 6 Gait speed $(\mathrm{m} / \mathrm{s})$ & 1 & & $\begin{array}{l}\text { Mean Difference (IV, Fixed, } \\
95 \% \text { CI) }\end{array}$ & Totals not selected \\
\hline $\begin{array}{l}7 \text { Pain (lower score }= \\
\text { less pain) }\end{array}$ & 1 & & $\begin{array}{l}\text { Std. Mean Difference (IV, } \\
\text { Fixed, 95\% CI) }\end{array}$ & Totals not selected \\
\hline
\end{tabular}

\section{Comparison 7. PRT versus functional exercise}

\begin{tabular}{lccll}
\hline $\begin{array}{l}\text { Outcome or subgroup } \\
\text { title }\end{array}$ & No. of studies & No. of participants & Statistical method & Effect size \\
\hline $\begin{array}{l}\text { 1 Main function } \\
\text { measure (higher score } \\
\text { better function) }\end{array}$ & 1 & & $\begin{array}{l}\text { Mean Difference (IV, Fixed, } \\
95 \% \text { CI) }\end{array}$ & Totals not selected \\
$\begin{array}{l}\text { 2 Main lower limb } \\
\text { strength measure }\end{array}$ & 3 & & $\begin{array}{l}\text { Mean Difference (IV, Fixed, } \\
95 \% \text { CI) }\end{array}$ & $-6.51[-21.05,8.04]$ \\
$\begin{array}{l}3 \text { Timed "Up-and-Go" } \\
\text { (seconds) }\end{array}$ & 1 & 158 & $\begin{array}{l}\text { Mean Difference (IV, Fixed, } \\
95 \% \text { CI) }\end{array}$ & Totals not selected \\
$\begin{array}{l}4 \text { Vitality (SF-36/ } \\
\begin{array}{l}\text { Vitality plus scale, } \\
\text { higher = more vitality) }\end{array}\end{array}$ & 2 & & $\begin{array}{l}\text { Mean Difference (IV, Fixed, } \\
95 \% \text { CI) }\end{array}$ & -0.07 [-2.68, 2.54]
\end{tabular}




\begin{tabular}{|c|c|c|c|c|}
\hline $\begin{array}{l}\text { Outcome or subgroup } \\
\text { title }\end{array}$ & No. of studies & No. of participants & Statistical method & Effect size \\
\hline $\begin{array}{l}5 \text { Pain (higher }=\text { less } \\
\text { pain, Bodily pain on } \\
\text { SF-36) }\end{array}$ & 1 & & $\begin{array}{l}\text { Mean Difference (IV, Fixed, } \\
95 \% \text { CI) }\end{array}$ & Totals not selected \\
\hline
\end{tabular}

\section{Comparison 8. PRT versus flexibility training}

\begin{tabular}{|c|c|c|c|c|}
\hline $\begin{array}{l}\text { Outcome or subgroup } \\
\text { title }\end{array}$ & No. of studies & No. of participants & Statistical method & Effect size \\
\hline $\begin{array}{l}1 \mathrm{SF} 36 \text { (higher score }= \\
\text { better function) }\end{array}$ & 1 & & $\begin{array}{l}\text { Mean Difference (IV, Fixed, } \\
95 \% \text { CI) }\end{array}$ & Totals not selected \\
\hline $\begin{array}{l}2 \text { Main lower limb (LL) } \\
\text { strength measure }\end{array}$ & 1 & & $\begin{array}{l}\text { Mean Difference (IV, Fixed, } \\
95 \% \text { CI) }\end{array}$ & Totals not selected \\
\hline 3 Timed walk (seconds) & 1 & & $\begin{array}{l}\text { Mean Difference (IV, Fixed, } \\
95 \% \text { CI) }\end{array}$ & Totals not selected \\
\hline $\begin{array}{l}4 \text { Time to stand from a } \\
\text { chair (seconds) }\end{array}$ & 1 & & $\begin{array}{l}\text { Mean Difference (IV, Fixed, } \\
95 \% \text { CI) }\end{array}$ & Totals not selected \\
\hline $\begin{array}{l}5 \text { Vitality (SF-36/ } \\
\text { Vitality plus scale, } \\
\text { higher = more vitality) }\end{array}$ & 1 & & $\begin{array}{l}\text { Mean Difference (IV, Fixed, } \\
95 \% \text { CI) }\end{array}$ & Totals not selected \\
\hline $\begin{array}{l}6 \text { Pain (higher }=\text { less } \\
\text { pain, Bodily pain on } \\
\text { SF- 36) }\end{array}$ & 1 & & $\begin{array}{l}\text { Mean Difference (IV, Fixed, } \\
95 \% \text { CI) }\end{array}$ & Totals not selected \\
\hline
\end{tabular}

\section{Comparison 9. Power training}

\begin{tabular}{|c|c|c|c|c|}
\hline $\begin{array}{l}\text { Outcome or subgroup } \\
\text { title }\end{array}$ & No. of studies & No. of participants & Statistical method & Effect size \\
\hline $\begin{array}{l}1 \text { Main lower limb } \\
\text { strength measure }\end{array}$ & 3 & & $\begin{array}{l}\text { Std. Mean Difference (IV, } \\
\text { Fixed, 95\% CI) }\end{array}$ & Totals not selected \\
\hline $\begin{array}{l}1.1 \text { High intensity } \\
\text { (power treatment) } \\
\text { versus control (control) }\end{array}$ & 2 & & $\begin{array}{l}\text { Std. Mean Difference (IV, } \\
\text { Fixed, 95\% CI) }\end{array}$ & Not estimable \\
\hline $\begin{array}{l}1.2 \text { High intensity } \\
\text { (treatment) versus low } \\
\text { intensity (control) }\end{array}$ & 2 & & $\begin{array}{l}\text { Std. Mean Difference (IV, } \\
\text { Fixed, 95\% CI) }\end{array}$ & Not estimable \\
\hline
\end{tabular}

\section{Comparison 10. PRT versus control supplementary analyses}

\begin{tabular}{|c|c|c|c|c|}
\hline Outcome or subgroup title & No. of studies & No. of participants & Statistical method & Effect size \\
\hline $\begin{array}{l}1 \text { Strength (grouped by allocation } \\
\text { concealment) }\end{array}$ & 73 & & $\begin{array}{l}\text { Std. Mean } \\
\text { Difference (IV, } \\
\text { Fixed, } 95 \% \mathrm{CI})\end{array}$ & Subtotals only \\
\hline 1.1 Allocation concealed & 6 & 607 & $\begin{array}{l}\text { Std. Mean } \\
\text { Difference (IV, } \\
\text { Fixed, } 95 \% \text { CI) }\end{array}$ & $0.12[-0.04,0.28]$ \\
\hline 1.2 Concealment unknown & 67 & 2452 & $\begin{array}{l}\text { Std. Mean } \\
\text { Difference (IV, } \\
\text { Fixed, } 95 \% \mathrm{CI})\end{array}$ & $0.65[0.56,0.73]$ \\
\hline
\end{tabular}




\begin{tabular}{|c|c|c|c|c|}
\hline Outcome or subgroup title & No. of studies & No. of participants & Statistical method & Effect size \\
\hline $\begin{array}{l}2 \text { Strength (grouped by assessor } \\
\text { blinding) }\end{array}$ & 73 & & $\begin{array}{l}\text { Std. Mean } \\
\text { Difference (IV, } \\
\text { Fixed, 95\% CI) }\end{array}$ & Subtotals only \\
\hline 2.1 Blinded assessors & 19 & 1523 & $\begin{array}{l}\text { Std. Mean } \\
\text { Difference (IV, } \\
\text { Fixed, } 95 \% \text { CI) }\end{array}$ & $0.23[0.13,0.34]$ \\
\hline 2.2 Assessors were not blinded & 54 & 1536 & $\begin{array}{l}\text { Std. Mean } \\
\text { Difference (IV, } \\
\text { Fixed, } 95 \% \text { CI) }\end{array}$ & $0.88[0.77,0.99]$ \\
\hline $\begin{array}{l}3 \text { Strength (grouped by intention- } \\
\text { to-treat) }\end{array}$ & 73 & 3059 & $\begin{array}{l}\text { Std. Mean } \\
\text { Difference (IV, } \\
\text { Fixed, } 95 \% \mathrm{CI})\end{array}$ & $0.53[0.46,0.61]$ \\
\hline 3.1 Intention-to-treat was used & 12 & 1041 & $\begin{array}{l}\text { Std. Mean } \\
\text { Difference (IV, } \\
\text { Fixed, } 95 \% \mathrm{CI})\end{array}$ & $0.18[0.06,0.30]$ \\
\hline $\begin{array}{l}3.2 \text { Intention-to-treat was not } \\
\text { used }\end{array}$ & 61 & 2018 & $\begin{array}{l}\text { Std. Mean } \\
\text { Difference (IV, } \\
\text { Fixed, 95\% CI) }\end{array}$ & $0.74[0.64,0.83]$ \\
\hline $\begin{array}{l}4 \text { Strength (grouped by attention } \\
\text { control) }\end{array}$ & 73 & 3059 & $\begin{array}{l}\text { Std. Mean } \\
\text { Difference (IV, } \\
\text { Fixed, 95\% CI) }\end{array}$ & $0.53[0.46,0.61]$ \\
\hline 4.1 Attention control & 24 & 1408 & $\begin{array}{l}\text { Std. Mean } \\
\text { Difference (IV, } \\
\text { Fixed, 95\% CI) }\end{array}$ & $0.34[0.23,0.44]$ \\
\hline 4.2 No attention control & 49 & 1651 & $\begin{array}{l}\text { Std. Mean } \\
\text { Difference (IV, } \\
\text { Fixed, 95\% CI) }\end{array}$ & $0.72[0.61,0.82]$ \\
\hline $\begin{array}{l}5 \text { Strength (grouped by exercise } \\
\text { intensity) }\end{array}$ & 72 & 3052 & $\begin{array}{l}\text { Std. Mean } \\
\text { Difference (IV, } \\
\text { Fixed, 95\% CI) }\end{array}$ & $0.53[0.45,0.60]$ \\
\hline 5.1 High intensity & 54 & 2026 & $\begin{array}{l}\text { Std. Mean } \\
\text { Difference (IV, } \\
\text { Fixed, } 95 \% \text { CI) }\end{array}$ & $0.60[0.51,0.70]$ \\
\hline 5.2 Low-to-moderate intensity & 19 & 1026 & $\begin{array}{l}\text { Std. Mean } \\
\text { Difference (IV, } \\
\text { Fixed, } 95 \% \text { CI) }\end{array}$ & $0.39[0.26,0.51]$ \\
\hline $\begin{array}{l}6 \text { Strength (grouped by exercise } \\
\text { duration) }\end{array}$ & 56 & 2564 & $\begin{array}{l}\text { Std. Mean } \\
\text { Difference (IV, } \\
\text { Fixed, } 95 \% \text { CI) }\end{array}$ & $0.53[0.45,0.61]$ \\
\hline 6.1 Less than 12 weeks & 20 & 828 & $\begin{array}{l}\text { Std. Mean } \\
\text { Difference (IV, } \\
\text { Fixed, } 95 \% \text { CI) }\end{array}$ & $0.52[0.37,0.66]$ \\
\hline 6.2 Longer than 12 weeks & 36 & 1736 & $\begin{array}{l}\text { Std. Mean } \\
\text { Difference (IV, } \\
\text { Fixed, } 95 \% \text { CI) }\end{array}$ & $0.53[0.43,0.63]$ \\
\hline $\begin{array}{l}7 \text { Strength (grouped by health } \\
\text { status) }\end{array}$ & 65 & 2428 & $\begin{array}{l}\text { Std. Mean } \\
\text { Difference (IV, } \\
\text { Fixed, } 95 \% \text { CI) }\end{array}$ & $0.60[0.52,0.69]$ \\
\hline 7.1 Healthy participants & 46 & 1502 & $\begin{array}{l}\text { Std. Mean } \\
\text { Difference (IV, } \\
\text { Fixed, } 95 \% \text { CI) }\end{array}$ & $0.77[0.66,0.88]$ \\
\hline $\begin{array}{l}7.2 \text { Older adults with a specific } \\
\text { health problem }\end{array}$ & 19 & 926 & $\begin{array}{l}\text { Std. Mean } \\
\text { Difference (IV, } \\
\text { Fixed, } 95 \% \text { CI) }\end{array}$ & $0.37[0.24,0.51]$ \\
\hline $\begin{array}{l}8 \text { Strength (grouped by functional } \\
\text { limitations) }\end{array}$ & 54 & 2133 & $\begin{array}{l}\text { Std. Mean } \\
\text { Difference (IV, } \\
\text { Fixed, } 95 \% \mathrm{CI})\end{array}$ & $0.60[0.51,0.70]$ \\
\hline
\end{tabular}




\begin{tabular}{lcclc}
\hline Outcome or subgroup title & No. of studies & No. of participants & Statistical method & Effect size \\
\hline & & & Std. Mean & \\
& & & Difference (IV, & \\
8.1 No functional limitations & 41 & 1349 & Fixed, 95\% CI) & $0.81[0.69,0.93]$ \\
& & & Std. Mean & \\
8.2 With functional limitations & 13 & 784 & Difference (IV, & $0.30[0.16,0.44]$ \\
\hline
\end{tabular}

\section{Analysis 1.1. Comparison 1 PRT versus control, Outcome 1 Main function measure (higher score = better function)}

Review, Progressive resistance strength training for improving physical function in older adults

Comparison: I PRT versus control

Outcome: 1 Main function measure (higher score $=$ better function)

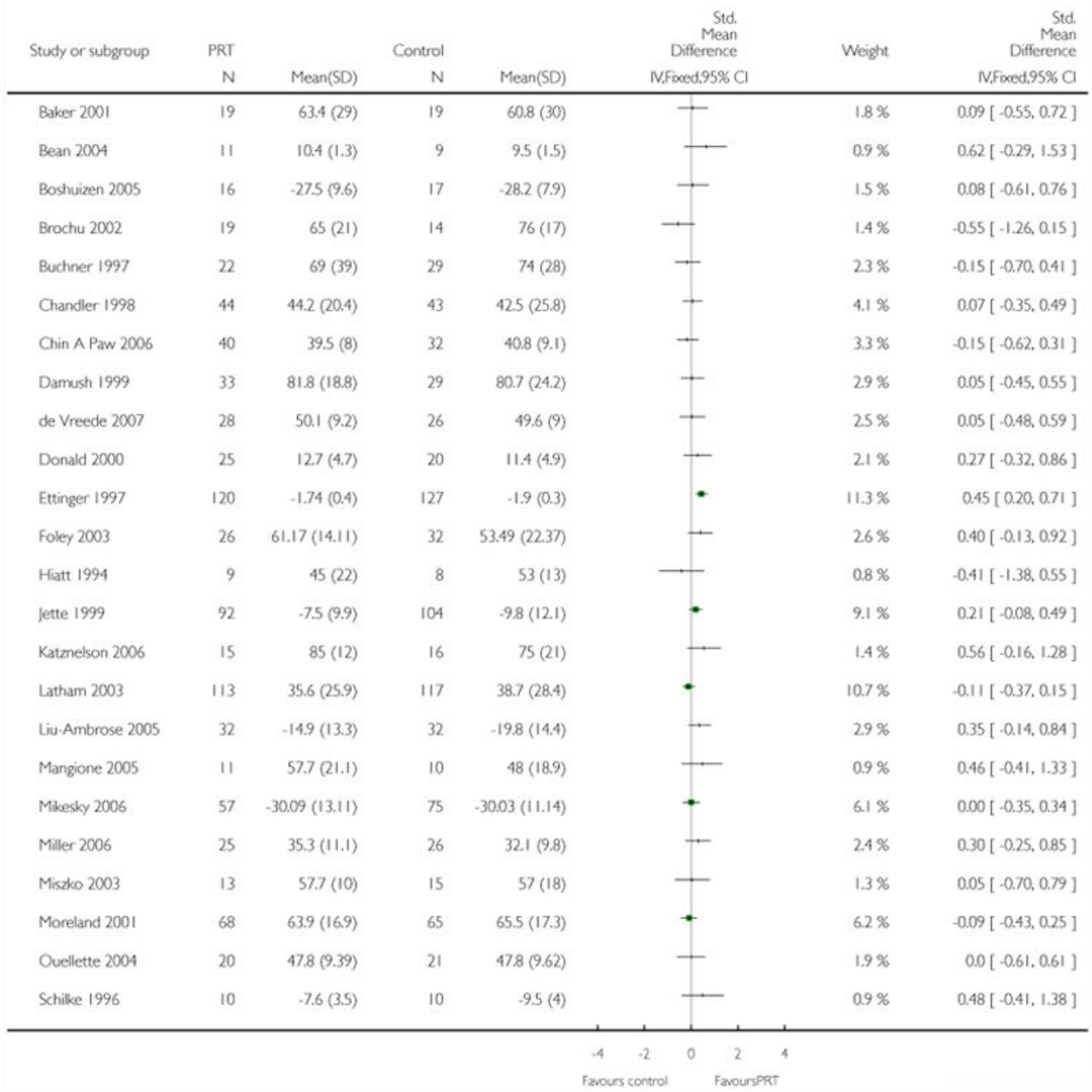

Cochrane Database Syst Rev. Author manuscript; available in PMC 2015 February 11. 


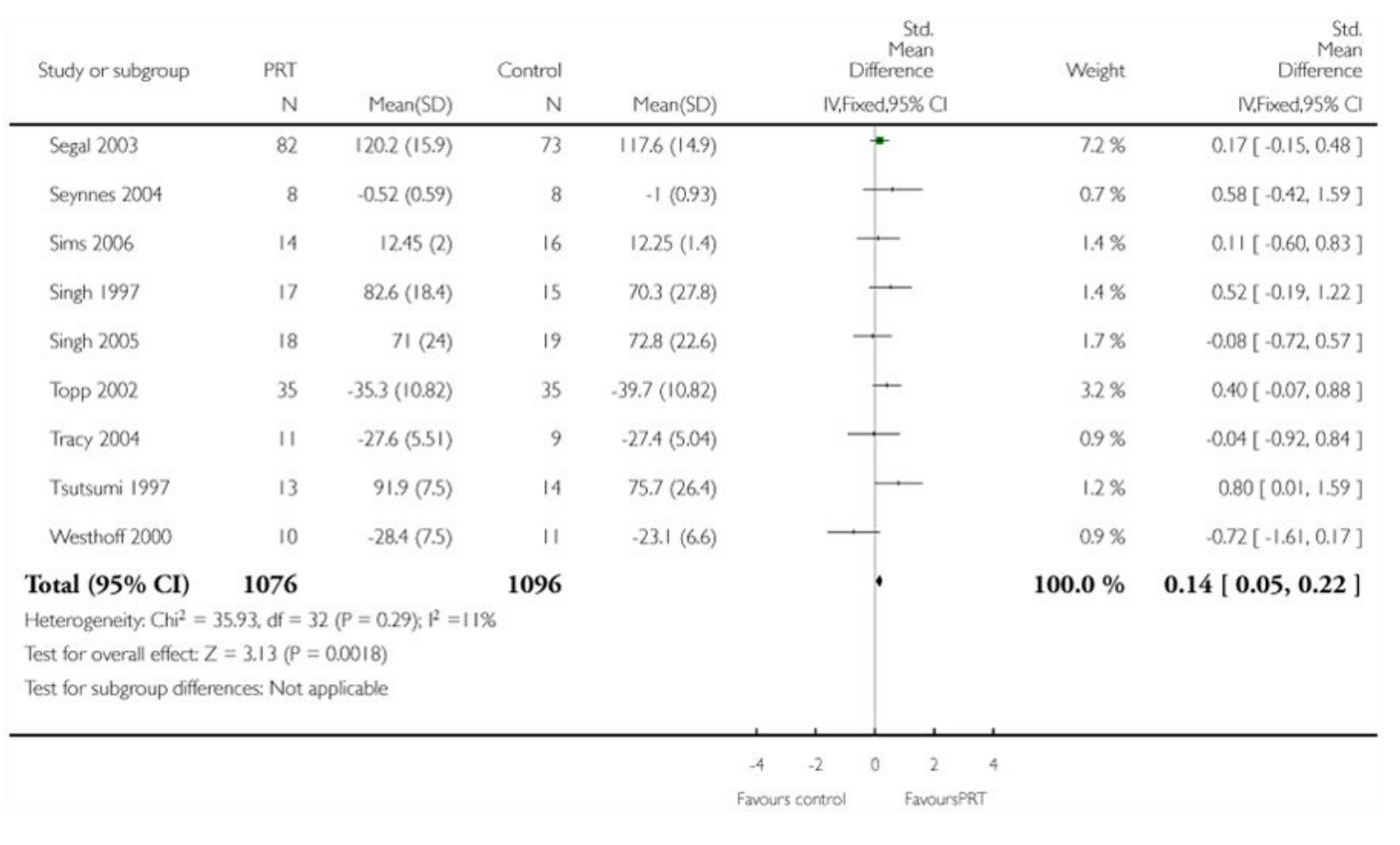




\section{Analysis 1.3. Comparison 1 PRT versus control, Outcome 3 Activities of daily living measure (higher score $=$ better function)}

\begin{tabular}{|c|c|c|c|c|c|c|c|}
\hline \multicolumn{8}{|c|}{$\begin{array}{l}\text { Review: Progressive resistance strength training for improving physical function in older adults } \\
\text { Comparison: I PRT versus control }\end{array}$} \\
\hline \multicolumn{8}{|c|}{ Outcome: 3 Activities of daily living measure (higher score $=$ better function) } \\
\hline \multirow[t]{2}{*}{ Study or subgroup } & \multirow{2}{*}{$\begin{array}{r}\text { PRT } \\
\mathrm{N}\end{array}$} & \multicolumn{3}{|c|}{ Control } & \multirow{2}{*}{$\begin{array}{r}\text { Std. } \\
\text { Mean } \\
\text { Difference } \\
\text { IV,Fixed,95\% Cl } \\
\end{array}$} & \multirow[t]{2}{*}{ Weight } & $\begin{array}{r}\text { Std. } \\
\text { Mean } \\
\text { Difference }\end{array}$ \\
\hline & & Mean(SD) & N & Mean(SD) & & & IV,Fixed,95\% Cl \\
\hline Chin A Paw 2006 & 40 & $39.5(8)$ & 32 & $40.8(9.1)$ & 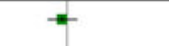 & $21.6 \%$ & $-0.15[-0.62,0.31]$ \\
\hline Latham 2003 & 111 & $18.5(2.4)$ & 116 & $18.2(3.2)$ & E & $69.0 \%$ & $0.11[-0.16,0.37]$ \\
\hline Singh 1997 & 17 & $6.1(0.8)$ & 14 & $6.1(0.9)$ & $\rightarrow$ & $9.4 \%$ & $0.0[-0.71,0.71]$ \\
\hline Total $(95 \% \mathrm{CI})$ & 168 & & 162 & & $\bullet$ & $100.0 \%$ & $0.04[-0.18,0.26]$ \\
\hline \multicolumn{8}{|c|}{ Heterogeneity: $\mathrm{Chi}^{2}=0.90, \mathrm{df}=2(\mathrm{P}=0.64) ; \mathrm{I}^{2}=0.0 \%$} \\
\hline \multicolumn{8}{|c|}{ Test for overall effect: $Z=0.36(P=0.72)$} \\
\hline \multicolumn{8}{|c|}{ Test for subgroup differences: Not applicable } \\
\hline
\end{tabular}

\section{Analysis 1.4. Comparison 1 PRT versus control, Outcome 4 Activity level measure (kJ/week)}

\begin{tabular}{|c|c|c|c|c|c|c|c|c|c|}
\hline \multicolumn{10}{|c|}{ Review: Progressive resistance strength training for improving physical function in older adults } \\
\hline \multicolumn{10}{|c|}{ Comparison: I PRT versus control } \\
\hline \multicolumn{10}{|c|}{ Outcome: 4 Activity level measure (k/week) } \\
\hline \multirow[t]{2}{*}{ Study or subgroup } & \multirow{2}{*}{$\begin{array}{r}\text { PRT } \\
\mathrm{N}\end{array}$} & \multicolumn{3}{|c|}{ Control } & \multirow{2}{*}{\multicolumn{3}{|c|}{$\begin{array}{c}\text { Mean } \\
\text { Difference } \\
\text { IV.Fixed,95\% Cl }\end{array}$}} & \multirow[t]{2}{*}{ Weight } & \multirow{2}{*}{$\begin{array}{r}\text { Mean } \\
\text { Difference } \\
\text { IV,Fixed,95\% Cl }\end{array}$} \\
\hline & & $\operatorname{Mean}(\mathrm{SD})$ & $\mathrm{N}$ & $\operatorname{Mean}(\mathrm{SD})$ & & & & & \\
\hline Nelson 1994 & 20 & $8610(1109)$ & 19 & $5393(697)$ & & & & $\cdot$ & $3217.00[2638.68 .3795 .32]$ \\
\hline Taaffe 1996 & 8 & $1870(221.9)$ & 11 & $1937.2(201.4)$ & & & - & & $-67.20[-261.65,127.25]$ \\
\hline Subtotal $(95 \% \mathrm{CI})$ & $\mathbf{0}$ & & $\mathbf{0}$ & & & & & & $0.0[0.0,0.0]$ \\
\hline \multicolumn{10}{|c|}{ Heterogeneity: $\mathrm{Chi}^{2}=0.0, \mathrm{df}=0(\mathrm{P}<0.0000 \mathrm{I}) ; \mathrm{I}^{2}=0.0 \%$} \\
\hline \multicolumn{10}{|c|}{ Test for overall effect: $Z=0.0(P<0.0000 I)$} \\
\hline \multicolumn{10}{|c|}{ Test for subgroup differences: Not applicable } \\
\hline & & & & & -500 & -250 & 250 & 500 & \\
\hline \multicolumn{10}{|c|}{ Favours control } \\
\hline
\end{tabular}

Cochrane Database Syst Rev. Author manuscript; available in PMC 2015 February 11. 


\section{Analysis 1.5. Comparison 1 PRT versus control, Outcome 5 Main lower limb (LL) strength measure}

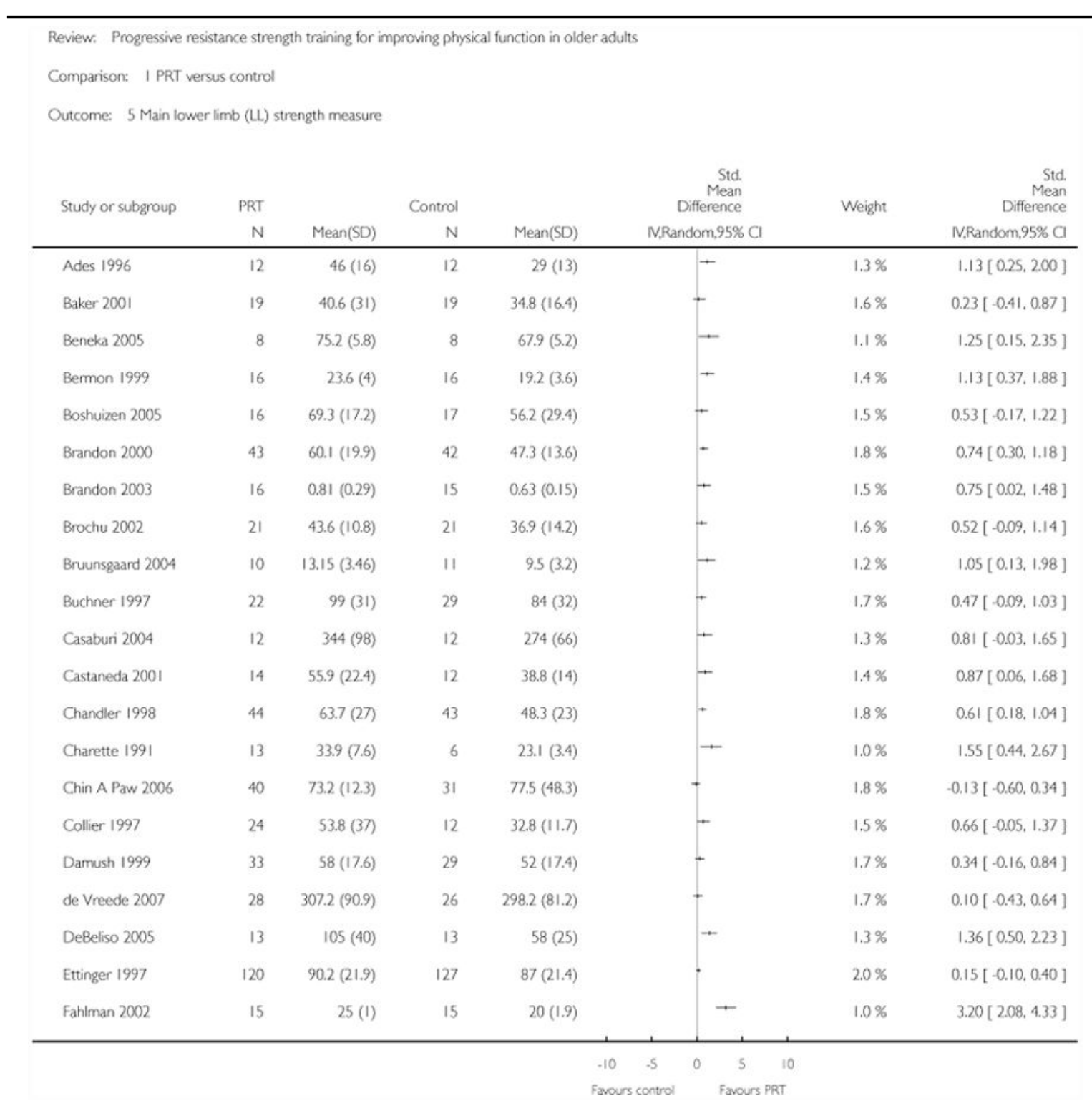

Cochrane Database Syst Rev. Author manuscript; available in PMC 2015 February 11. 


\begin{tabular}{|c|c|c|c|c|c|c|c|}
\hline \multirow[t]{2}{*}{ Study or subgroup } & \multirow{2}{*}{$\begin{array}{r}\text { PRT } \\
\text { N }\end{array}$} & \multicolumn{3}{|c|}{ Control } & \multirow{2}{*}{$\begin{array}{c}\text { Sid. } \\
\text { Mean } \\
\text { Difference } \\
\text { N.Random,95\% a }\end{array}$} & \multirow[t]{2}{*}{ Weight } & \multirow{2}{*}{$\begin{array}{r}\text { Std } \\
\text { Mean } \\
\text { Difference } \\
\text { N.Random } 95 \% \text { a }\end{array}$} \\
\hline & & $\operatorname{Mean}(\mathrm{SD})$ & $\mathrm{N}$ & $\operatorname{Mean}(\mathrm{SO})$ & & & \\
\hline Fatouros 2002 & 8 & $116.1(9.8)$ & 8 & $665(8.5)$ & 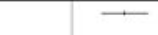 & $0.4 \%$ & $5.11[285,7.38]$ \\
\hline Fatouros 2005 & 20 & $91.7(8.2)$ & 14 & $56.4(8.5)$ & - & $0.9 \%$ & $4.14[289,5.39]$ \\
\hline Foley 2003 & 26 & $26.73(14.2)$ & 32 & $21.94(11.61)$ & - & $1.7 \%$ & $0.37[-0.15,0.89]$ \\
\hline Frontera 2003 & 7 & $18.4(5.1)$ & 7 & $125(28)$ & - & $1.0 \%$ & $1.34[0.14 .2 .54]$ \\
\hline Haykowsky 2000 & 10 & $367(47)$ & 8 & $290(53)$ & $\rightarrow$ & $1.1 \%$ & $1.48[0.40 .255]$ \\
\hline Hortobagi 2001 & 18 & $1122(340)$ & 9 & $877(203)$ & - & $1.3 \%$ & $0.78[-0.05,1.62]$ \\
\hline Hruda 2003 & 18 & $81.9(23.3)$ & 7 & $71(222)$ & - & $1.3 \%$ & $0.46[-0.43,1.34]$ \\
\hline Jette 1996 & 35 & $52(18.5)$ & 38 & $45.8(19.1)$ & 5 & $18 \%$ & $0.33[-0.14,0.79]$ \\
\hline Jette 1999 & 96 & $15(5.3)$ & 104 & $13.7(5.5)$ & - & $1.9 \%$ & $0.24[-0.04,0.52]$ \\
\hline Jones 1994 & 30 & $55.17(15.87)$ & 12 & 50.17 (8.75) & t & $1.5 \%$ & $0.34[-0.33,1.02]$ \\
\hline Judge 1994 & 22 & $8(3.8)$ & 23 & $69(1.9)$ & - & $1.6 \%$ & $0.36[-0.23,0.95]$ \\
\hline Kalapotharakos 2005 & 11 & 43.12 (11.49) & 10 & $20.75(7.16)$ & - & $1.0 \%$ & $222[1.08 .3 .35]$ \\
\hline Kongrgaard 2004 & 6 & $61(7)$ & 7 & S1 (6.5) & - & $0.9 \%$ & $1.38[0.12 .2 .64]$ \\
\hline Lamoureux 2003 & 29 & $31.8(1.7)$ & 15 & $21.2(24)$ & - & $0.9 \%$ & $5.31[3.99 .6 .63]$ \\
\hline Latham 2001 & 10 & $123(7.1)$ & 10 & $7.4(3.5)$ & - & $1.2 \%$ & $0.84[-0.08,1.76]$ \\
\hline Latham 2003 & 108 & $126(5.4)$ & 112 & $129(5.3)$ & t & $20 \%$ & $.0 .06[-0.320 .21]$ \\
\hline Liu-Ambrose 2005 & 32 & $189(8.6)$ & 32. & $17.5(6.4)$ & t & $1.7 \%$ & $0.18[-0.31,0.67)$ \\
\hline Maiorana 1997 & 12 & $1324(26.4)$ & 14 & $116.6(26.6)$ & - & $1.4 \%$ & $0.58[-0.21,1.37]$ \\
\hline Malliou 2003 & 15 & $79.2(6.8)$ & 10 & $65.9(5.2)$ & - & $1.1 \%$ & $207[1.05,3.08]$ \\
\hline McCartney 1995 & 20 & $124(22)$ & 23 & $98(18)$ & - & $1.5 \%$ & $1.28[0.62 .1 .94]$ \\
\hline Mikesky 2006 & 59 & 109.4 (35.85) & 78 & 103.89 (35.86) & - & $1.9 \%$ & $0.15[-0.19,0.49]$ \\
\hline Miller 2006 & 25 & $5.9(4.1)$ & 26 & $5.4(3.4)$ & t & $1.7 \%$ & $0.13[-0.42,0.68]$ \\
\hline Miscbo 2003 & 13 & $105.27(53.1)$ & 15 & $79.71(37.5)$ & - & $1.4 \%$ & $0.55[-0.21,1.31]$ \\
\hline Nelson 1994 & 20 & $37.8(10.4)$ & 19 & $25.8(8.5)$ & - & $1.5 \%$ & $1.23[0.54,1.93]$ \\
\hline Newnham 1995 & 12 & $15.3(7.5)$ & 12 & $7.4(3.6)$ & - & $1.3 \%$ & $1.30[0.40 .2 .19]$ \\
\hline Nichols 1993 & 15 & $39.2(12.7)$ & 15 & $322(9.7)$ & - & $1.5 \%$ & $0.60[-0.13,1.34]$ \\
\hline Parkhouse 2000 & 13 & $98(21.6)$ & 9 & $54(18)$ & - & $1.1 \%$ & $209[1.00,3.18]$ \\
\hline Pollock 1991 & 20 & $53.4(21.1)$ & 12 & $43.2(20.3)$ & 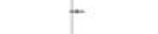 & $15 \%$ & $0.48[-0.25,1.20]$ \\
\hline Pu 2001 & 9 & $1630(516)$ & 7 & $1292(352)$ & - & $1.1 \%$ & $0.71[-0.32,1.73]$ \\
\hline Rall 1996 & 8 & $47.3(14.8)$ & 6 & $322(11.2)$ & - & $1.0 \%$ & $1.05[-0.10 .221]$ \\
\hline Reeres 2004 & 9 & $115(51.7)$ & 9 & $98.5(29.9)$ & + & $1.2 \%$ & $0.37[-0.56,1.31]$ \\
\hline
\end{tabular}

Cochrane Database Syst Rev. Author manuscript; available in PMC 2015 February 11. 


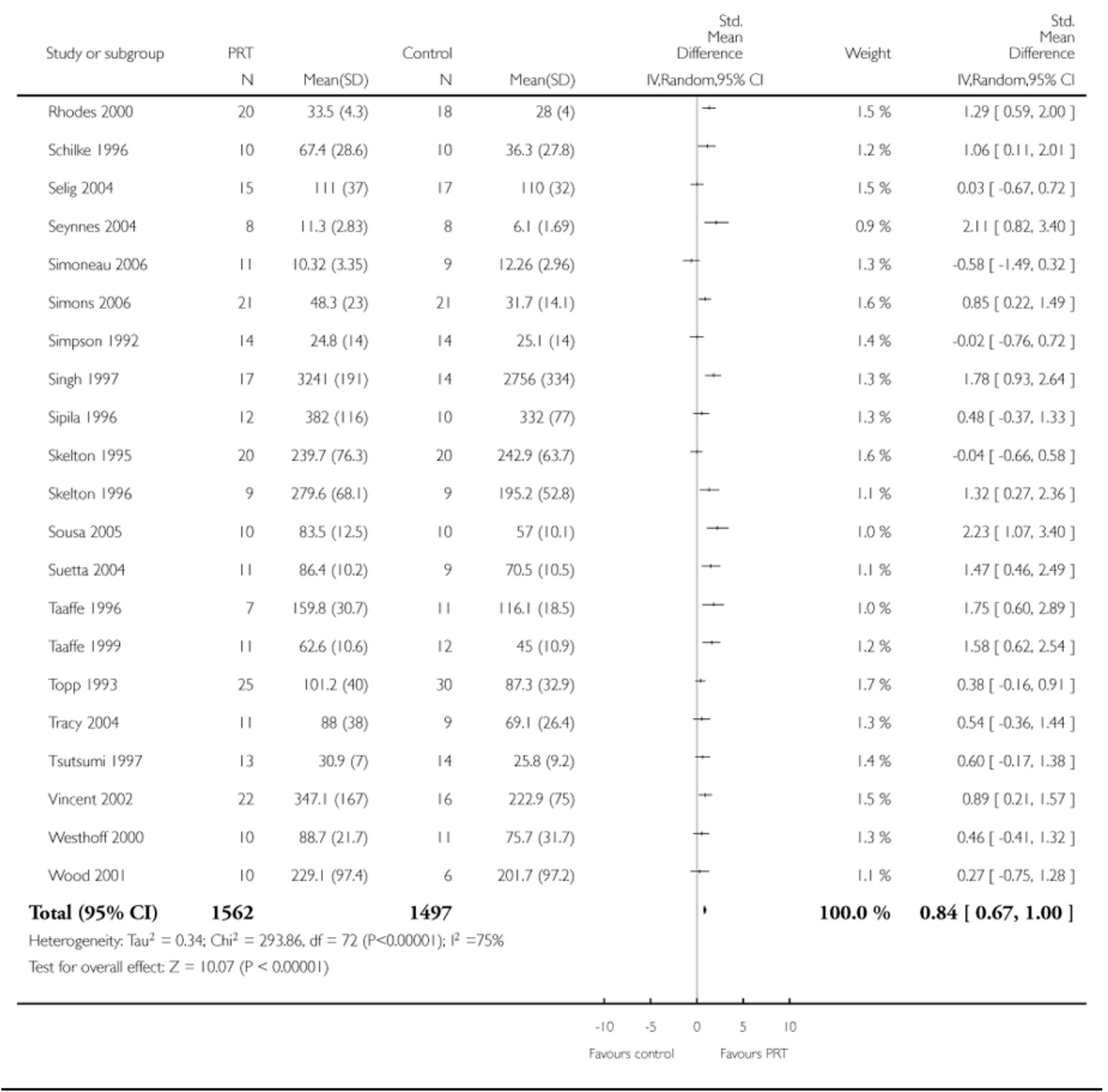

Cochrane Database Syst Rev. Author manuscript; available in PMC 2015 February 11. 


\section{Analysis 1.6. Comparison 1 PRT versus control, Outcome 6 Main measure of aerobic function}

Review: Progressive resistance strength training for improving physical function in older adults

Comparison: I PRT versus control

Outcome: 6 Main measure of aerobic function

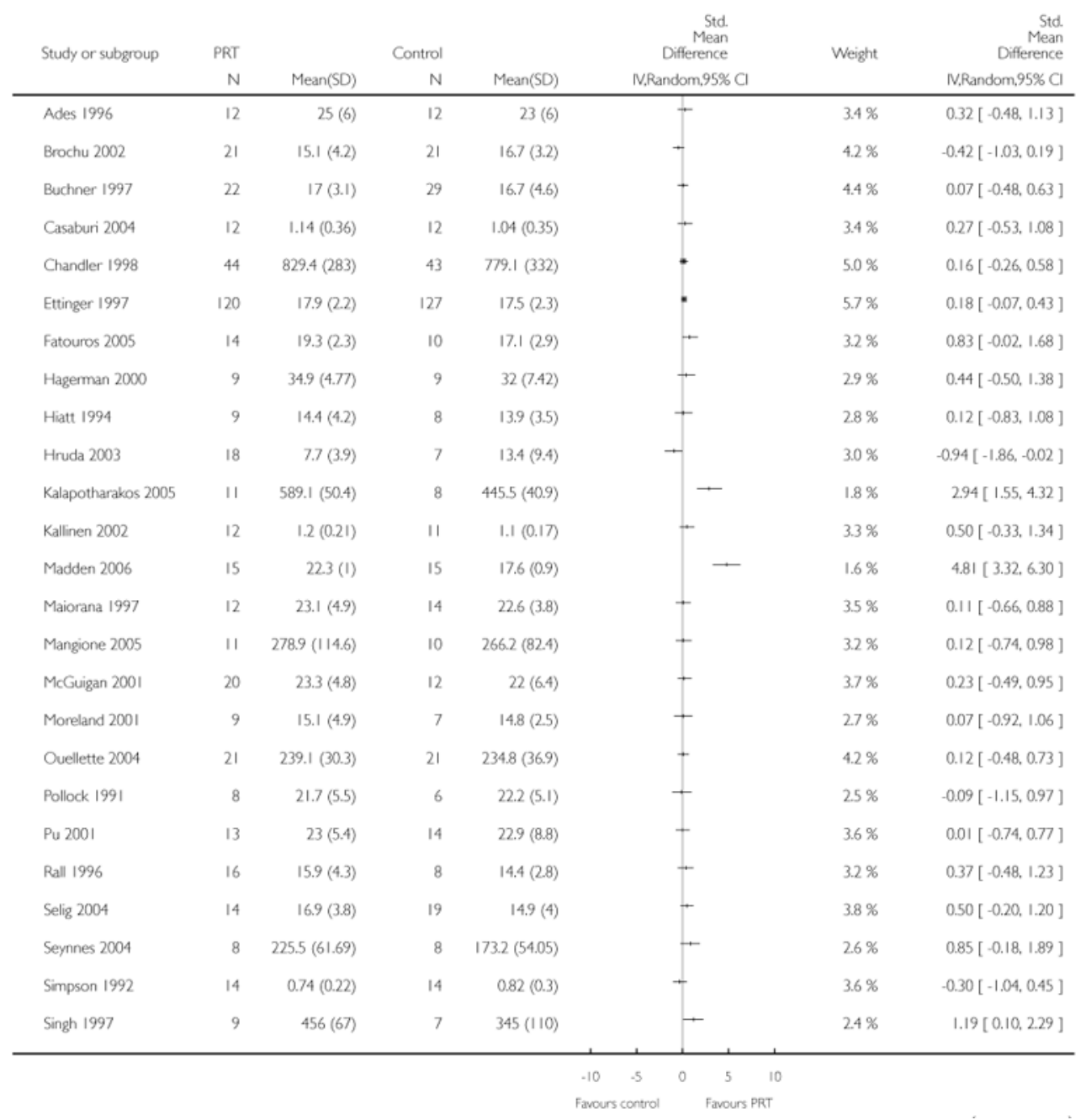

Cochrane Database Syst Rev. Author manuscript; available in PMC 2015 February 11. 


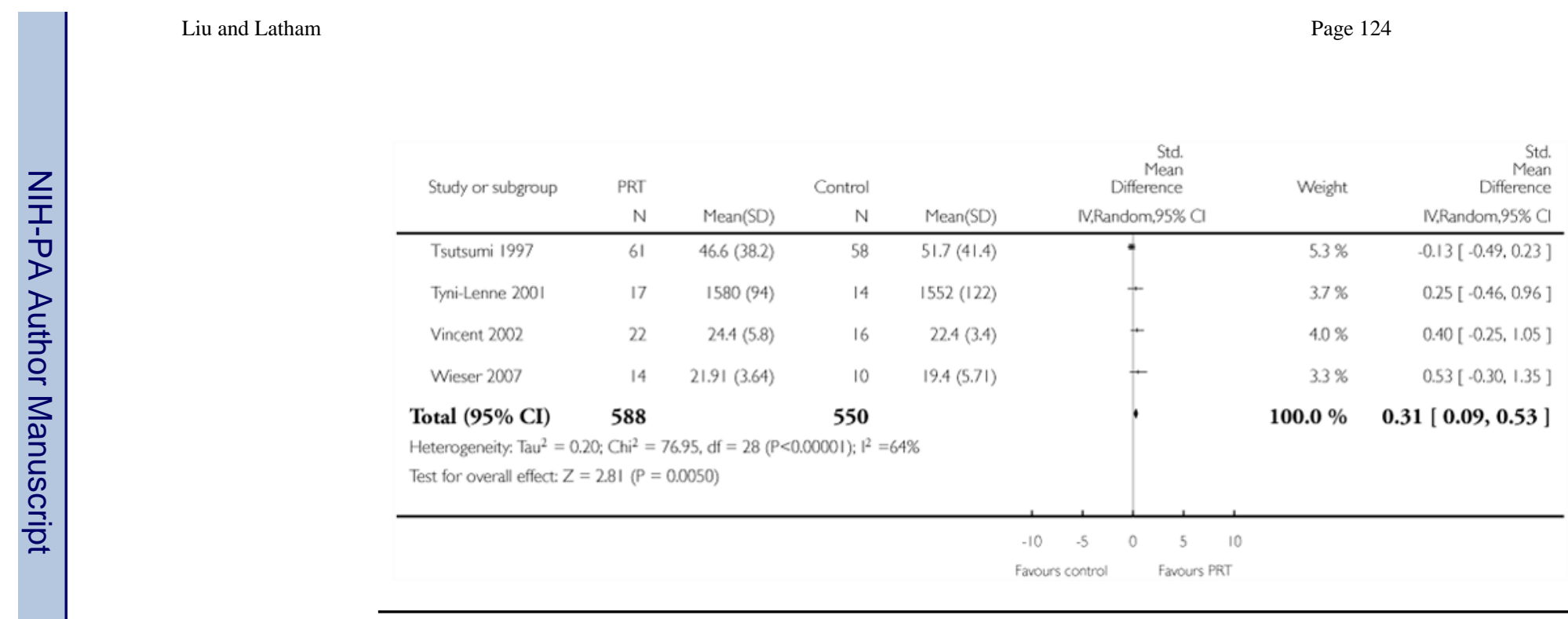

\section{Analysis 1.7. Comparison 1 PRT versus control, Outcome 7 VO2 or peak oxygen uptake}

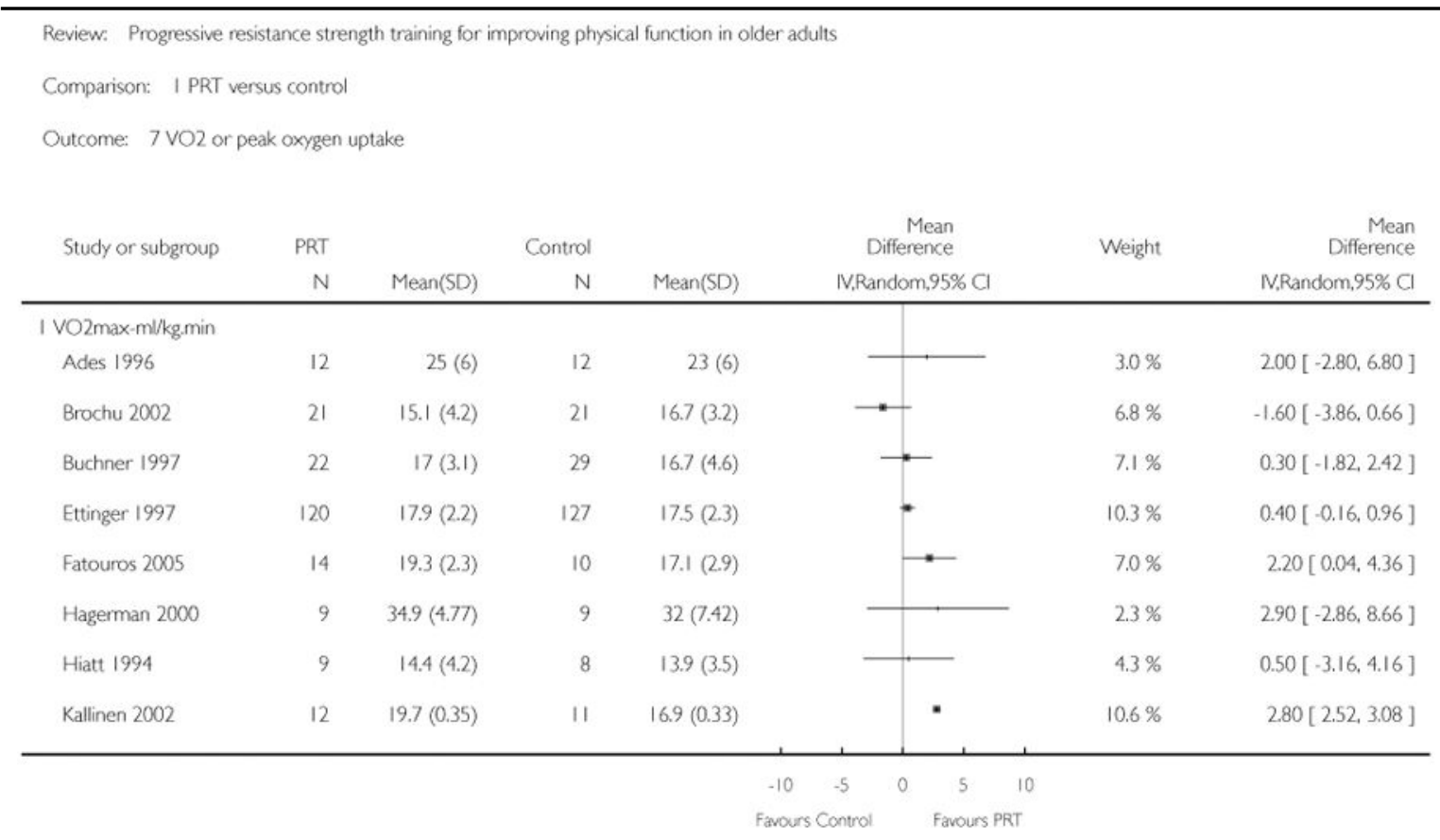

Cochrane Database Syst Rev. Author manuscript; available in PMC 2015 February 11. 


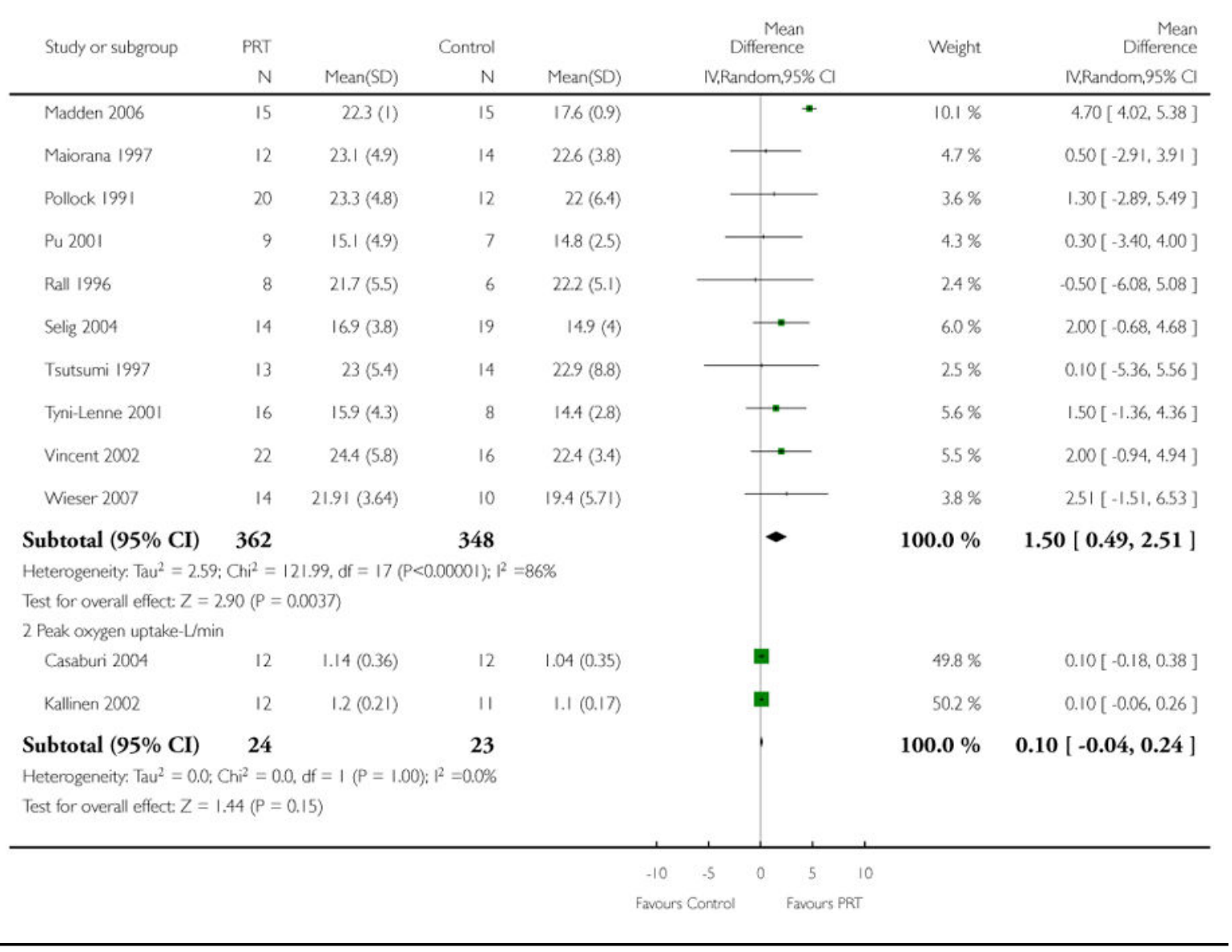




\section{Analysis 1.8. Comparison 1 PRT versus control, Outcome 8 Six-minute walk test (meters)}

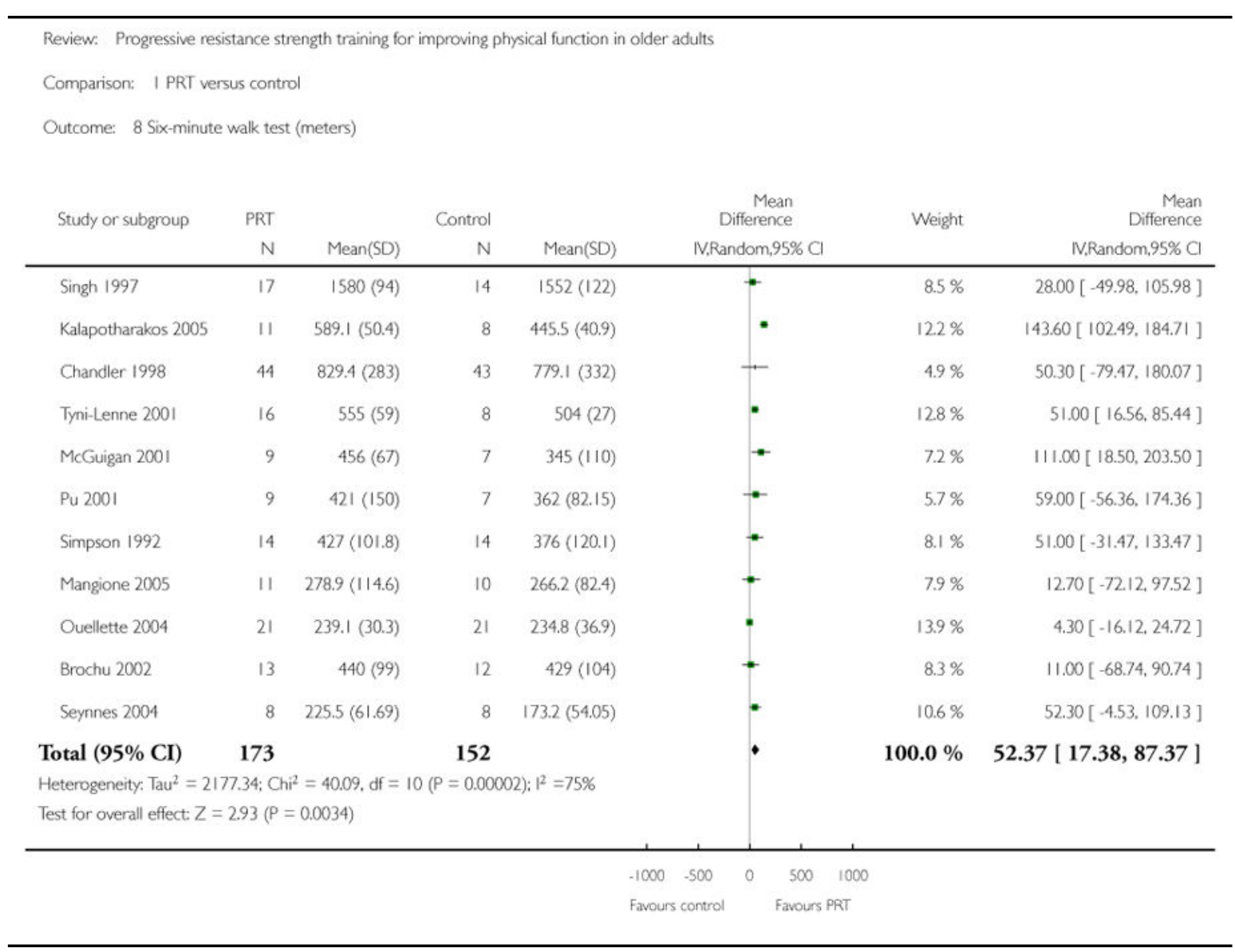

Cochrane Database Syst Rev. Author manuscript; available in PMC 2015 February 11. 


\section{Analysis 1.9. Comparison 1 PRT versus control, Outcome 9 Balance measures (higher $=$ better balance)}

\begin{tabular}{|c|c|c|c|c|c|c|c|}
\hline \multicolumn{8}{|c|}{ Review: Progressive resistance strength training for improving physical function in older adults } \\
\hline \multicolumn{8}{|c|}{ Comparison: I PRT versus control } \\
\hline \multicolumn{8}{|c|}{ Outcome: 9 Balance measures (higher $=$ better balance) } \\
\hline \multirow[t]{2}{*}{ Study or subgroup } & \multirow{2}{*}{$\begin{array}{r}\text { PRT } \\
N\end{array}$} & \multicolumn{3}{|c|}{ Control } & $\begin{array}{r}\text { Std. } \\
\text { Mean } \\
\text { Difference }\end{array}$ & \multirow[t]{2}{*}{ Weight } & \multirow{2}{*}{$\begin{array}{r}\text { Std } \\
\text { Mear } \\
\text { Difference } \\
\text { IV.Fixed,95\% C }\end{array}$} \\
\hline & & Mean(SD) & $\mathrm{N}$ & Mean(SD) & IV,Fixed,95\% Cl & & \\
\hline Kalapotharakos 2005 & 11 & $85.4(42.7)$ & 8 & $55(20)$ & -1 & $1.7 \%$ & $0.83[-0.13,1.78]$ \\
\hline Newnham 1995 & 12 & $44.3(10.2)$ & 12 & $32.3(18.7)$ & - & $2.3 \%$ & $0.77[-0.07,1.60]$ \\
\hline Buchner 1997 & 22 & $9.5(2.2)$ & 29 & $8.4(3.1)$ & $\rightarrow$ & $5.0 \%$ & $0.39[-0.17,0.95]$ \\
\hline Topp 1993 & 25 & $25.1(20)$ & 30 & $21.3(16.4)$ & $\rightarrow$ & $5.5 \%$ & $0.21[-0.33,0.74]$ \\
\hline Chandler 1998 & 44 & $9.92(2.8)$ & 43 & $9.82(3.3)$ & $\rightarrow$ & $8.9 \%$ & $0.03[-0.39,0.45]$ \\
\hline Latham 2001 & 9 & $44.9(10.5)$ & 10 & $40.5(10.4)$ & - & $1.9 \%$ & $0.40[-0.51,1.31]$ \\
\hline Schlicht 1999 & 11 & $5.08(3.5)$ & 11 & $4.7(4.2)$ & - & $2.2 \%$ & $0.09[-0.74,0.93]$ \\
\hline Judge 1994 & 19 & $10(10.9)$ & 19 & $9.4(8.7)$ & - & $3.9 \%$ & $0.06[-0.58,0.70]$ \\
\hline Westhoff 2000 & 10 & $5.3(0.8)$ & 11 & $5.5(0.8)$ & $\div$ & $2.1 \%$ & $-0.24[-1,10,0.62]$ \\
\hline Boshuizen 2005 & 16 & $4.8(1)$ & 17 & $4.5(1.5)$ & 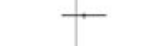 & $3.3 \%$ & $0.23[-0.46,0.91]$ \\
\hline Latham 2003 & 110 & $39.9(10.6)$ & 110 & $41.9(11.1)$ & * & $22.4 \%$ & $-0.18[-0.45,0.08]$ \\
\hline Jette 1999 & 92 & $10.9(2.7)$ & 104 & $10.1(3.5)$ & * & $19.8 \%$ & $0.25[-0.03,0.53]$ \\
\hline Bean 2004 & 11 & $6.76(5.4)$ & 9 & $6.31(5.9)$ & 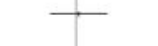 & $20 \%$ & $0.08[-0.80,0.96]$ \\
\hline Skelton 1995 & 20 & $96(5)$ & 20 & $95(8.1)$ & + & $4.1 \%$ & $0.15[-0.48,0.77]$ \\
\hline Liu-Ambrose 2005 & 32 & $51.2(21.9)$ & 32 & $45(17.4)$ & $\rightarrow$ & $6.5 \%$ & $0.31[-0.18,0.80]$ \\
\hline Chin A Paw 2006 & 40 & $16.6(12.8)$ & 31 & $18.5(12.4)$ & $\rightarrow$ & $7.1 \%$ & $-0.15[-0.62,0.32]$ \\
\hline Skelton 1996 & 8 & $3.1(1.3)$ & 8 & $1.7(0.9)$ & $\longrightarrow$ & $1.3 \%$ & $1.18[0.10,2.27]$ \\
\hline Total $(95 \%$ CI) & 492 & & 504 & & - & $100.0 \%$ & $0.12[0.00,0.25]$ \\
\hline \multicolumn{8}{|c|}{ Heterogeneity: $C$ hi ${ }^{2}=18.19, d f=16(P=0.31): 1^{2}=12 \%$} \\
\hline \multicolumn{8}{|c|}{ Test for overall effect: $Z=1.90(P=0.057)$} \\
\hline \multicolumn{8}{|c|}{ Test for subgroup differences: Not applicable } \\
\hline & & & & & 2 & & \\
\hline \multicolumn{8}{|c|}{ Favours control Favours PRT } \\
\hline
\end{tabular}

Cochrane Database Syst Rev. Author manuscript; available in PMC 2015 February 11. 


\section{Analysis 1.10. Comparison 1 PRT versus control, Outcome 10 Balance measures (Low $=$ better balance)}

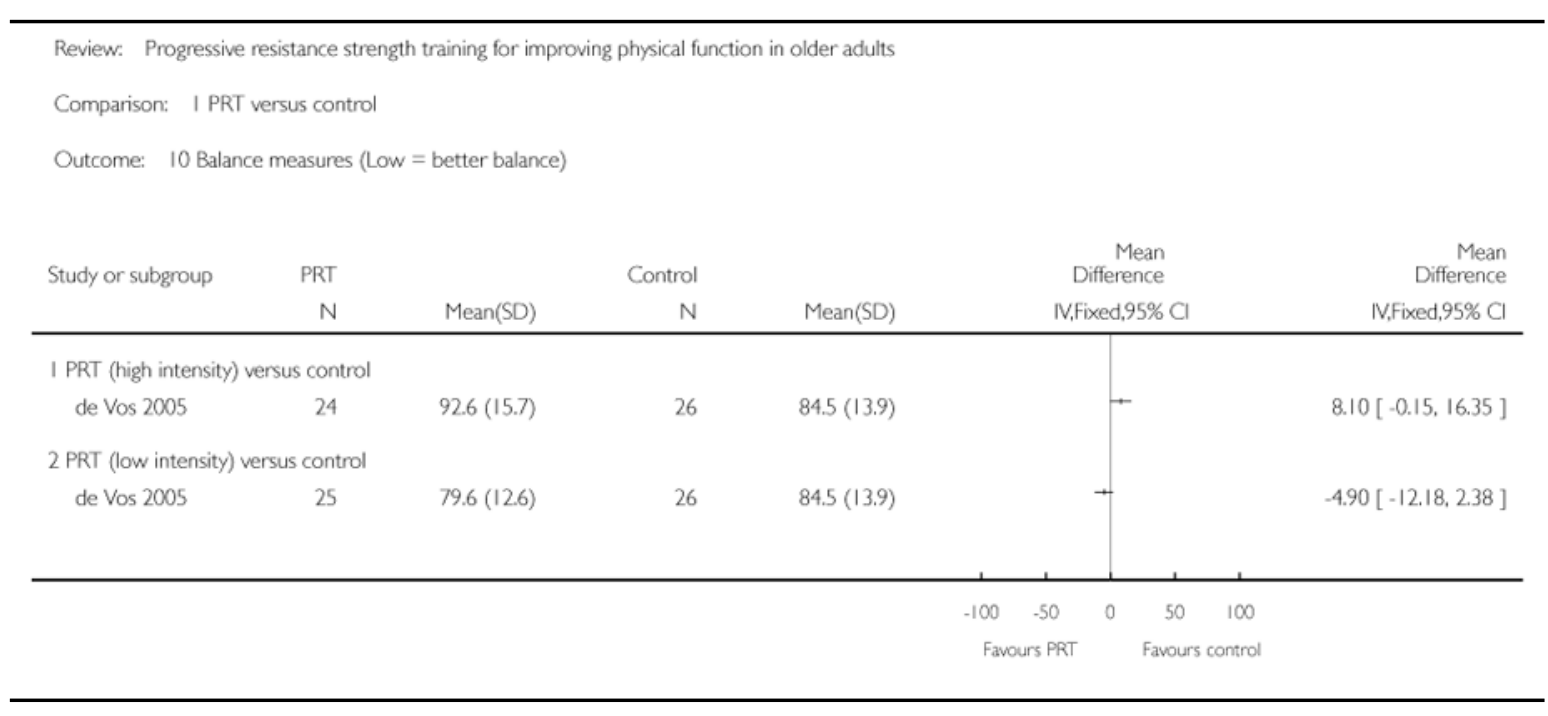




\section{Analysis 1.11. Comparison 1 PRT versus control, Outcome 11 Gait speed $(\mathrm{m} / \mathrm{s})$}

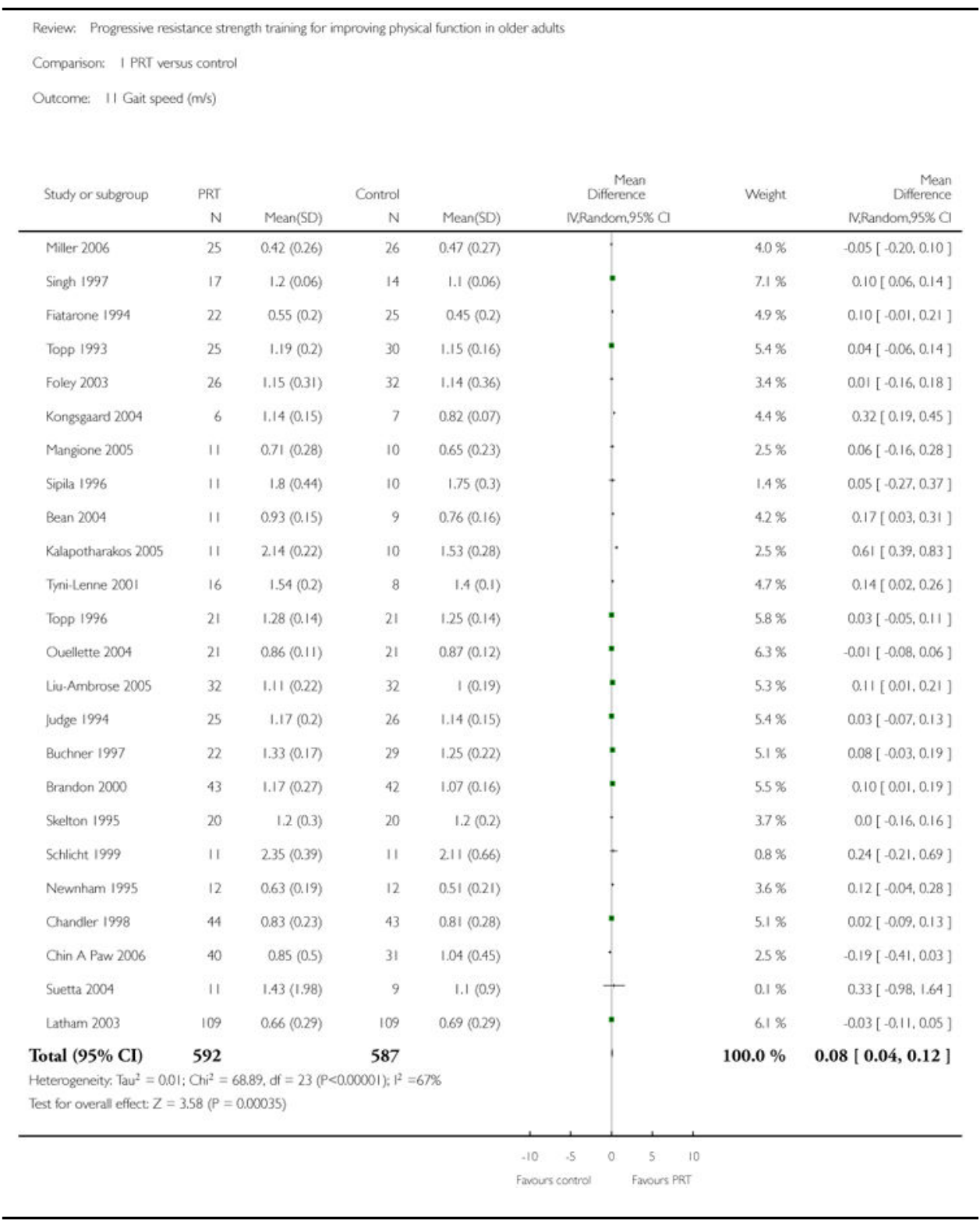

Cochrane Database Syst Rev. Author manuscript; available in PMC 2015 February 11. 


\section{Analysis 1.12. Comparison 1 PRT versus control, Outcome 12 Timed walk (seconds)}

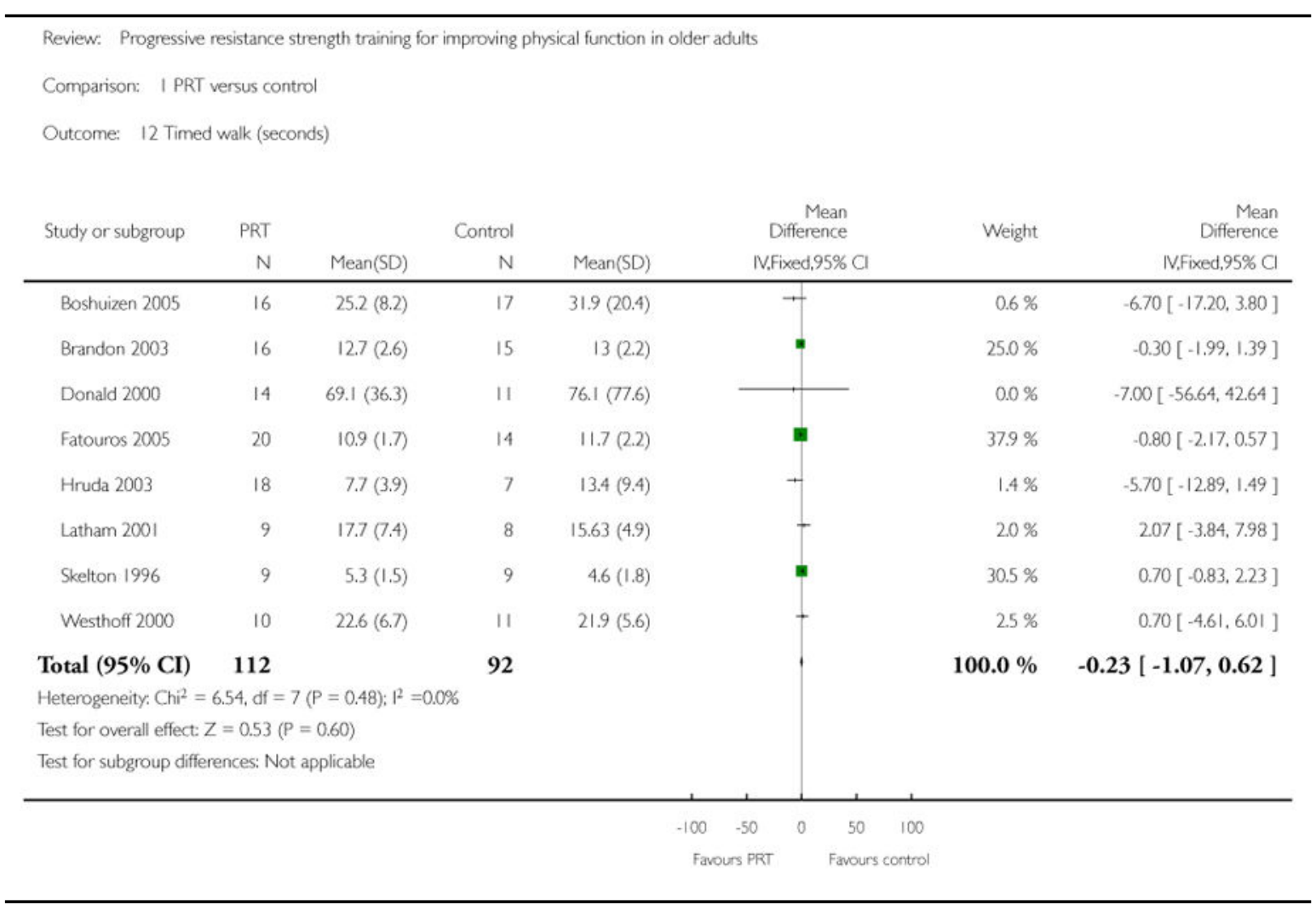




\section{Analysis 1.13. Comparison 1 PRT versus control, Outcome 13 Timed "Up-and-Go" (seconds)}

\begin{tabular}{|c|c|c|c|c|c|c|c|c|c|}
\hline \multicolumn{10}{|c|}{ Review: Progressive resistance strength training for improving physical function in older adults } \\
\hline \multicolumn{10}{|c|}{ Comparison: I PRT versus control } \\
\hline \multicolumn{10}{|c|}{ Outcome: 13 Timed "Up-and-Go" (seconds) } \\
\hline \multirow{2}{*}{ Study or subgroup } & \multirow{2}{*}{$\begin{array}{r}\text { PRT } \\
\mathrm{N}\end{array}$} & \multicolumn{3}{|c|}{ Control } & \multirow{2}{*}{\multicolumn{3}{|c|}{$\begin{array}{c}\text { Mean } \\
\text { Difference } \\
\text { IV.Fixed,95\% CI }\end{array}$}} & \multirow[t]{2}{*}{ Weight } & \multirow{2}{*}{$\begin{array}{r}\text { Mean } \\
\text { Difference } \\
\text { IV.Fixed,95\% Cl }\end{array}$} \\
\hline & & Mean(SD) & $\mathrm{N}$ & $\operatorname{Mean}(\mathrm{SD})$ & & & & & \\
\hline Boshuizen 2005 & 16 & $14(7.5)$ & 17 & $17.5(10.5)$ & & + & & $0.5 \%$ & $-3.50[-9.70,2.70]$ \\
\hline Brandon 2003 & 16 & $7.6(1.8)$ & 15 & $8.3(1)$ & & 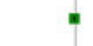 & & $17.0 \%$ & $-0.70[-1.72,0.32]$ \\
\hline de Vreede 2007 & 28 & $5.3(1.1)$ & 26 & $5.2(1.3)$ & & 1 & & $42.3 \%$ & $0.10[-0.54,0.74]$ \\
\hline Fatouros 2005 & 20 & $7.1(1.1)$ & 14 & $7.9(26)$ & & $\cdot$ & & $8.4 \%$ & $-0.80[-2.24,0.64]$ \\
\hline Hruda 2003 & 18 & $14.4(6.5)$ & 7 & $16.3(18.9)$ & & $\rightarrow$ & & $0.1 \%$ & $-1.90[-16.22,12.42]$ \\
\hline Jette 1999 & 92 & $11.6(5)$ & 103 & $13.3(10)$ & & . & & $3.7 \%$ & $-1.70[-3.88,0.48]$ \\
\hline Latham 2001 & 9 & $17.4(8.1)$ & 6 & $20.3(8.9)$ & & + & & $0.2 \%$ & $-2.90[-11.77,5.97]$ \\
\hline Latham 2003 & 111 & $28.1(29.7)$ & 110 & $22.6(20.6)$ & & - & & $0.4 \%$ & $5.50[-1.23,12.23]$ \\
\hline Newnham 1995 & 12 & $21.5(8.9)$ & 12 & $43.5(52.5)$ & & - & & $0.0 \%$ & $-22.00[-52.13,8.13]$ \\
\hline Skelton 1996 & 9 & $10.3(6.6)$ & 9 & $12.8(4.9)$ & & + & & $0.6 \%$ & $-2.50[-7.87,2.87]$ \\
\hline Sousa 2005 & 10 & $5.1(0.4)$ & 10 & $6.9(1.3)$ & & 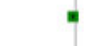 & & $24.7 \%$ & $-1.80[-2.64,-0.96]$ \\
\hline Westhoff 2000 & 10 & $10.8(3.1)$ & 11 & $11.7(3.6)$ & & t & & $2.1 \%$ & $-0.90[-3.77,1.97]$ \\
\hline Total $(95 \% \mathrm{CI})$ & 351 & & 340 & & & 1 & & $100.0 \%$ & $-0.69[-1.11,-0.27]$ \\
\hline \multicolumn{10}{|c|}{ Heterogeneity: $C \mathrm{Ch}^{2}=19.95, \mathrm{df}=11 \quad(\mathrm{P}=0.05) ; \mathrm{I}^{2}=45 \%$} \\
\hline \multicolumn{10}{|c|}{ Test for overall effect: $Z=3.24(P=0.0012)$} \\
\hline \multicolumn{10}{|c|}{ Test for subgroup differences: Not applicable } \\
\hline \multirow{2}{*}{\multicolumn{10}{|c|}{$\begin{array}{ccccc}-100 & -50 & 0 & 50 & 100 \\
- \text { Favours } & P R T & & & \text { Favours control }\end{array}$}} \\
\hline & & & & & Favou & & & & \\
\hline
\end{tabular}




\section{Analysis 1.14. Comparison 1 PRT versus control, Outcome 14 Time to stand from a chair}

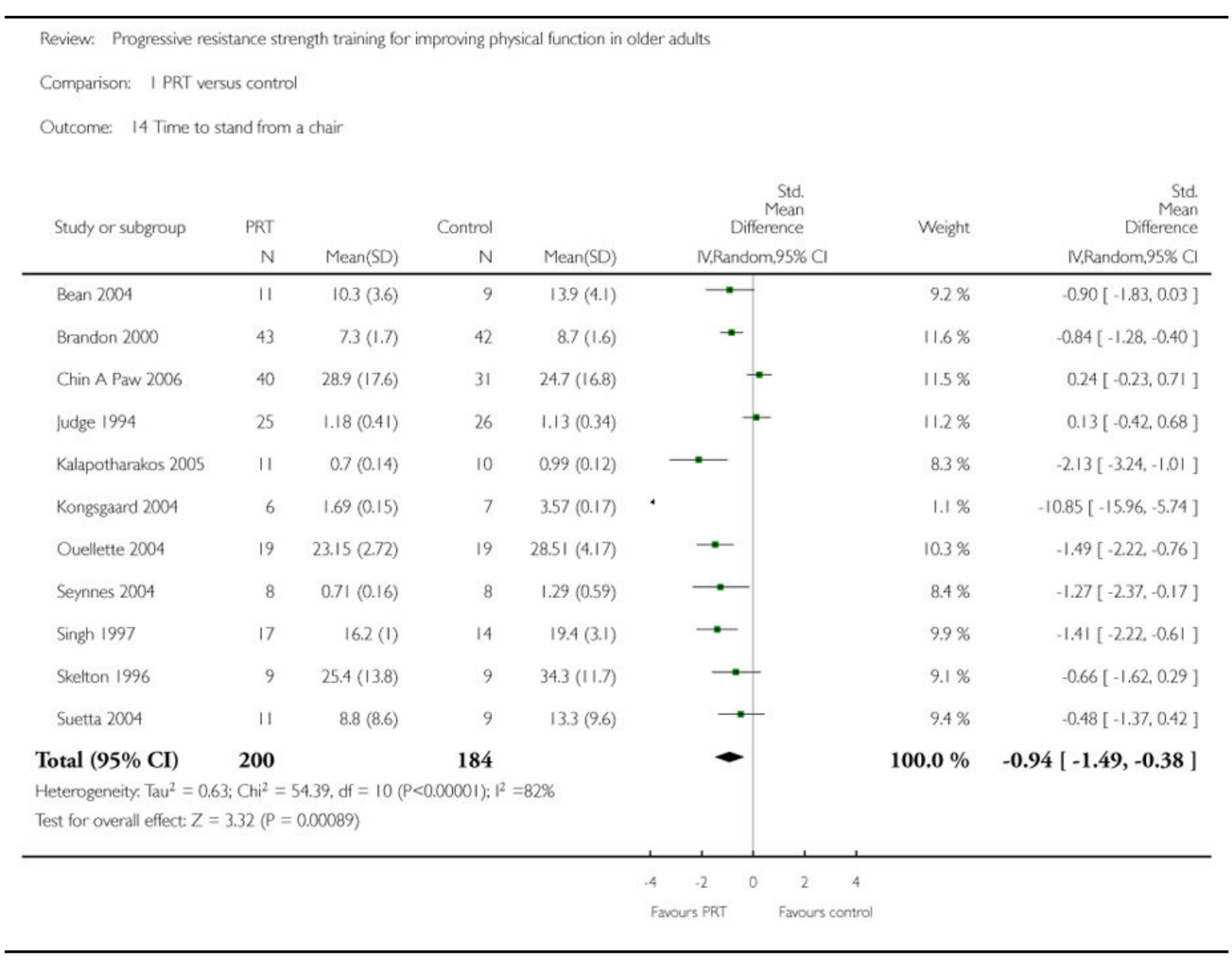




\section{Analysis 1.15. Comparison 1 PRT versus control, Outcome 15 Stair climbing (seconds)}

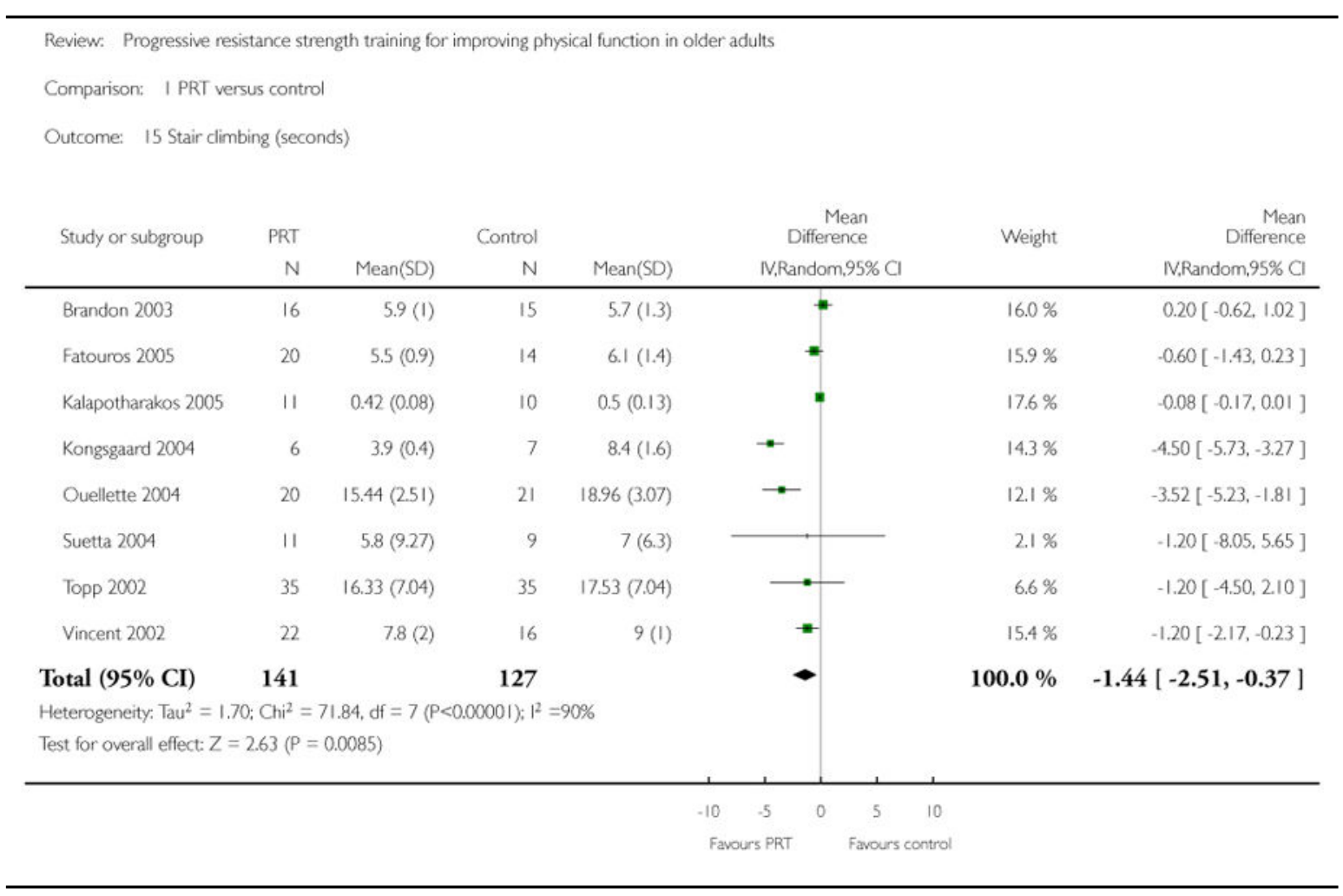

\section{Analysis 1.16. Comparison 1 PRT versus control, Outcome 16 Chair stand within time limit (number of times)}

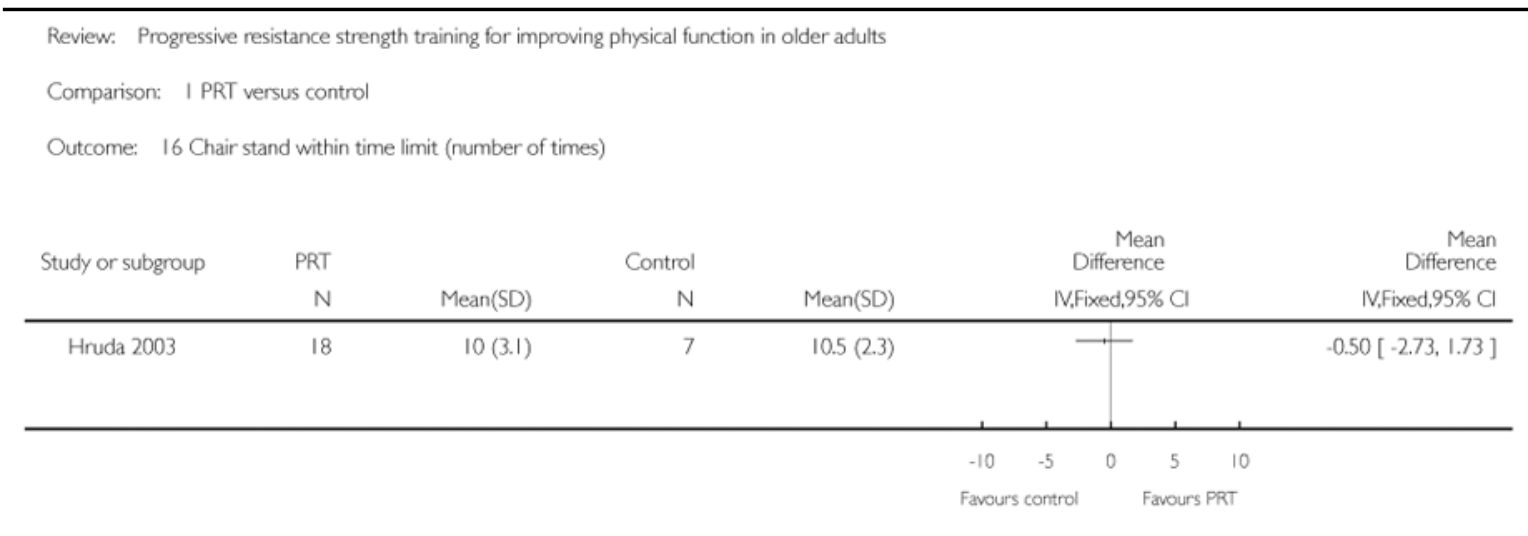




\section{Analysis 1.17. Comparison 1 PRT versus control, Outcome 17 Vitality (SF-36/Vitality plus scale, higher = more vitality)}

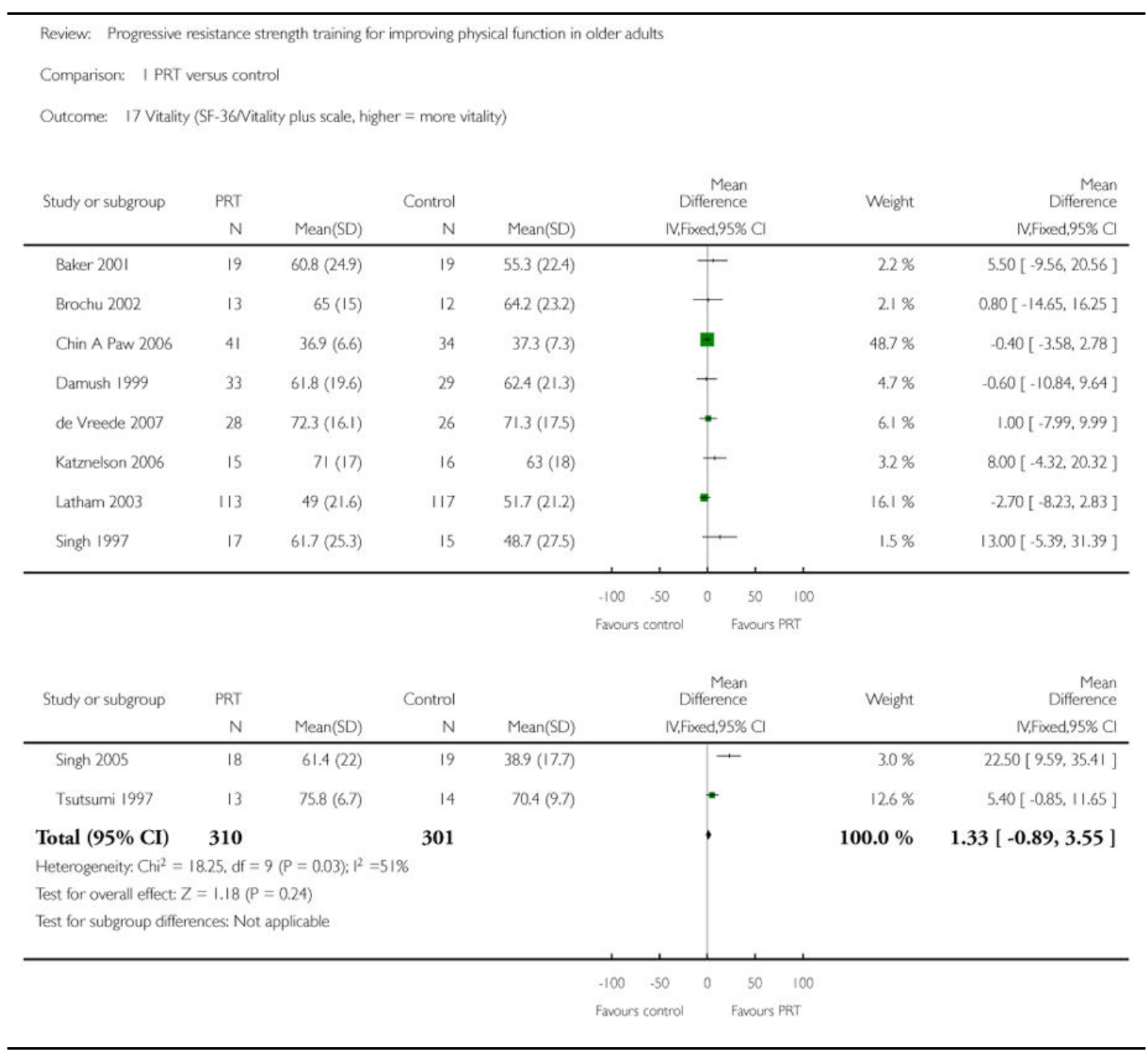

Cochrane Database Syst Rev. Author manuscript; available in PMC 2015 February 11. 


\section{Analysis 1.18. Comparison 1 PRT versus control, Outcome 18 Pain (higher = less pain, Bodily pain on SF-36)}

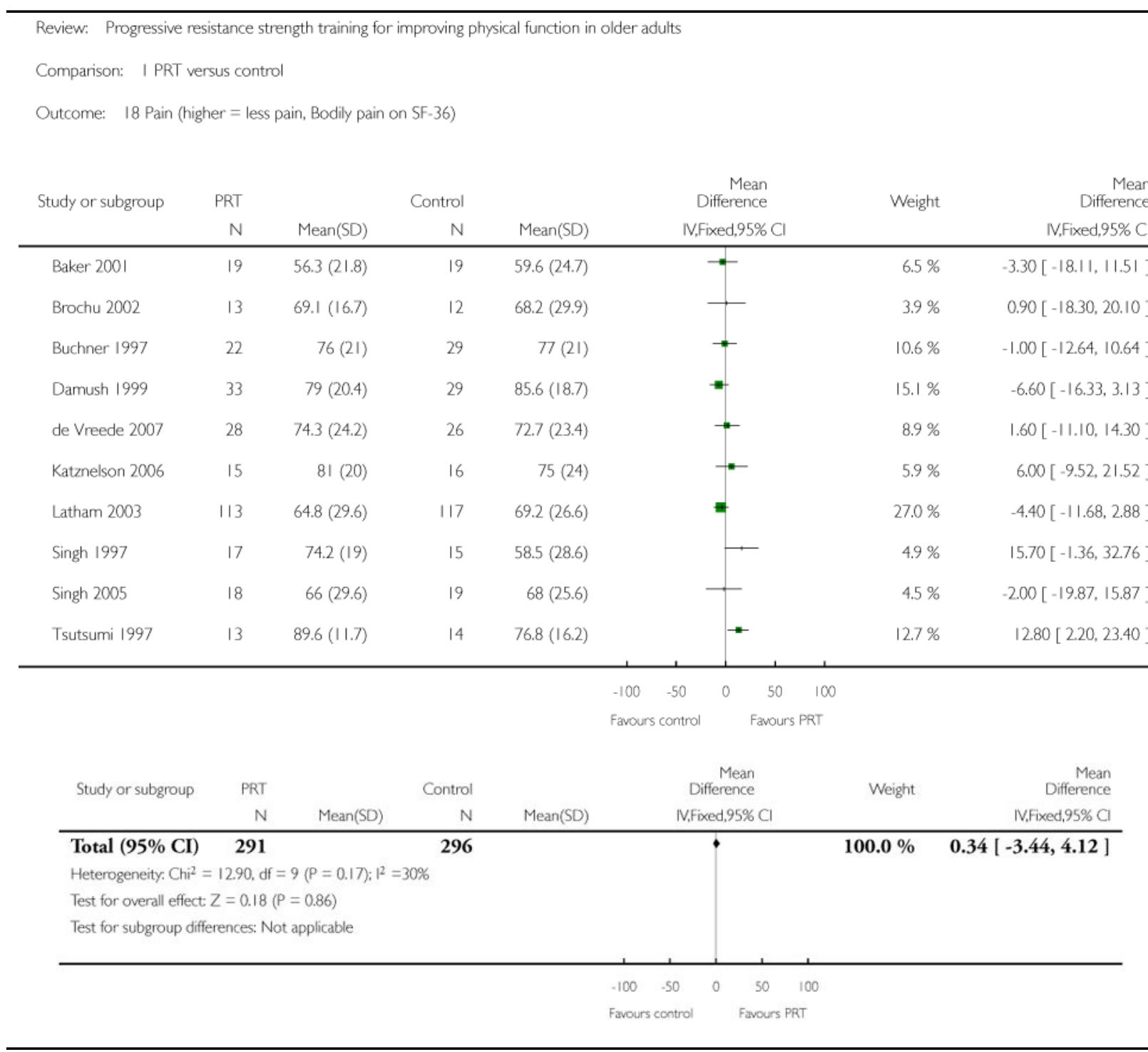




\section{Analysis 1.19. Comparison 1 PRT versus control, Outcome 19 Pain (lower score = less pain)}

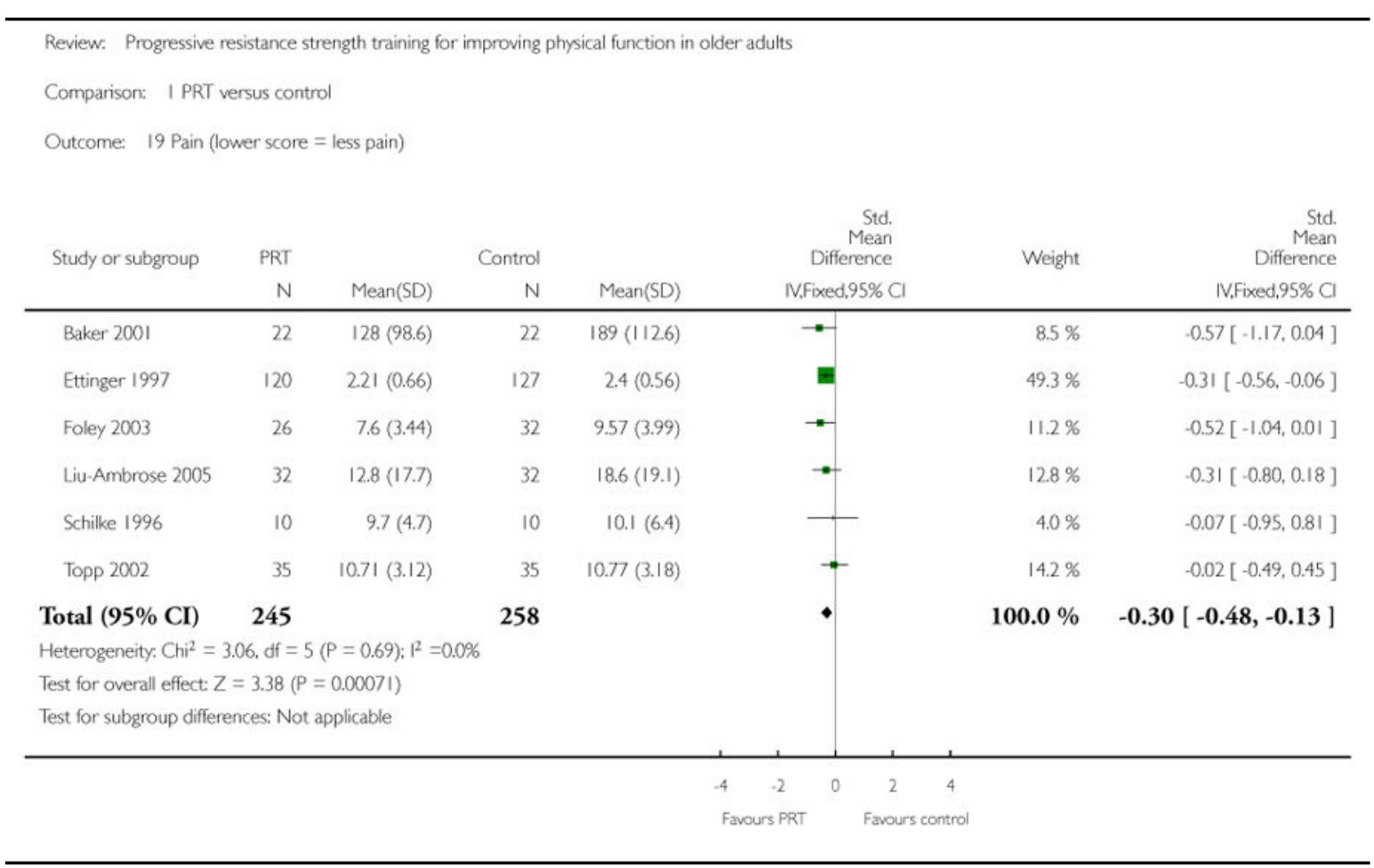




\section{Analysis 1.20. Comparison 1 PRT versus control, Outcome 20 Death}

\begin{tabular}{|c|c|c|c|c|c|}
\hline \multicolumn{6}{|c|}{ Review: Progressive resistance strength training for improving physical function in older adults } \\
\hline \multicolumn{6}{|c|}{ Comparison: I PRT versus control } \\
\hline Outcome: 20 Death & & & & & \\
\hline \multirow[t]{2}{*}{ Study or subgroup } & PRT & Control & Risk Ratio & Weight & Risk Ratio \\
\hline & $n / N$ & $n / N$ & \multicolumn{2}{|l|}{ M-H,Fixed,95\% Cl } & M-H,Fixed, $95 \% \mathrm{Cl}$ \\
\hline Baum 2003 & $1 / 11$ & 0/9 & ? & $2.1 \%$ & $2.50[0.11 .54 .87]$ \\
\hline Boshuizen 2005 & $0 / 24$ & $1 / 22$ & - & $6.1 \%$ & $0.31[0.01,7.16]$ \\
\hline Chin A Paw 2006 & 1/57 & $2 / 51$ & $\bullet$ & $8.2 \%$ & $0.45[0.04,4.79]$ \\
\hline Donald 2000 & $3 / 30$ & $3 / 24$ & 一 & $13.0 \%$ & $0.80[0.18,3.61]$ \\
\hline Ettinger 1997 & $0 / 146$ & $1 / 149$ & 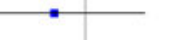 & $5.8 \%$ & $0.34[0.01,8.28]$ \\
\hline Fiatarone 1994 & $0 / 25$ & 1/26 & 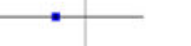 & $5.7 \%$ & $0.35[0.01 .8 .12]$ \\
\hline Kallinen 2002 & $1 / 16$ & $0 / 11$ & . & $2.3 \%$ & $2.12[0.09,47.68]$ \\
\hline Latham 2003 & $6 / 120$ & $8 / 123$ & $\rightarrow$ & $30.9 \%$ & $0.77[0.27,2.15]$ \\
\hline Mangione 2005 & $2 / 17$ & $0 / 11$ & & $2.3 \%$ & $3.33[0.17,63.51]$ \\
\hline Miller 2006 & $2 / 25$ & $1 / 26$ & & $3.8 \%$ & $208[0.20,21.52]$ \\
\hline Moreland 2001 & $0 / 68$ & $1 / 65$ & - & $6.0 \%$ & $0.32[0.01,7.69]$ \\
\hline Newnham 1995 & $3 / 15$ & $3 / 15$ & 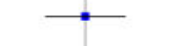 & $11.7 \%$ & $1.00[0.24,4.18]$ \\
\hline Selig 2004 & $1 / 19$ & $0 / 20$ & & $1.9 \%$ & $3.15[0.14,72.88]$ \\
\hline Total $(95 \% \mathrm{CI})$ & 573 & 552 & - & $100.0 \%$ & $0.89[0.52,1.54]$ \\
\hline \multicolumn{6}{|c|}{ Total events: 20 (PRT). 21 (Control) } \\
\hline \multicolumn{6}{|c|}{ Heterogeneity: $\mathrm{Chi}^{2}=4.61, \mathrm{df}=12(P=0.97) ; 1^{2}=0.0 \%$} \\
\hline \multicolumn{6}{|c|}{ Test for overall effect: $Z=0.41(P=0.68)$} \\
\hline & & & 10 & 100 & \\
\hline \multicolumn{6}{|c|}{ Favours treatment Favours control } \\
\hline
\end{tabular}




\section{Analysis 2.1. Comparison 2 High versus low intensity PRT, Outcome 1 Main function measure (higher score = better function)}

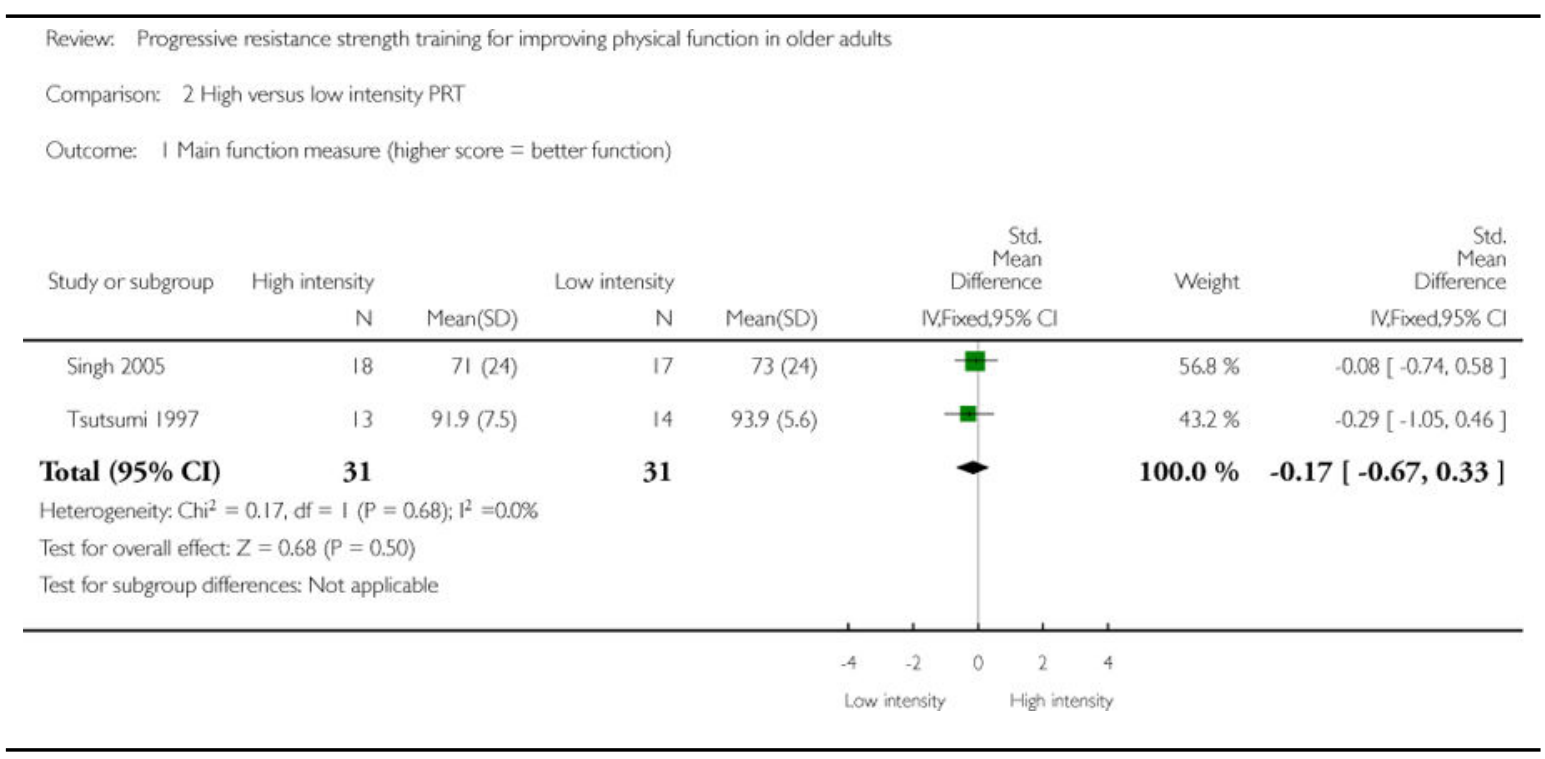




\section{Analysis 2.2. Comparison 2 High versus low intensity PRT, Outcome 2 Main lower limb (LL) strength measure}

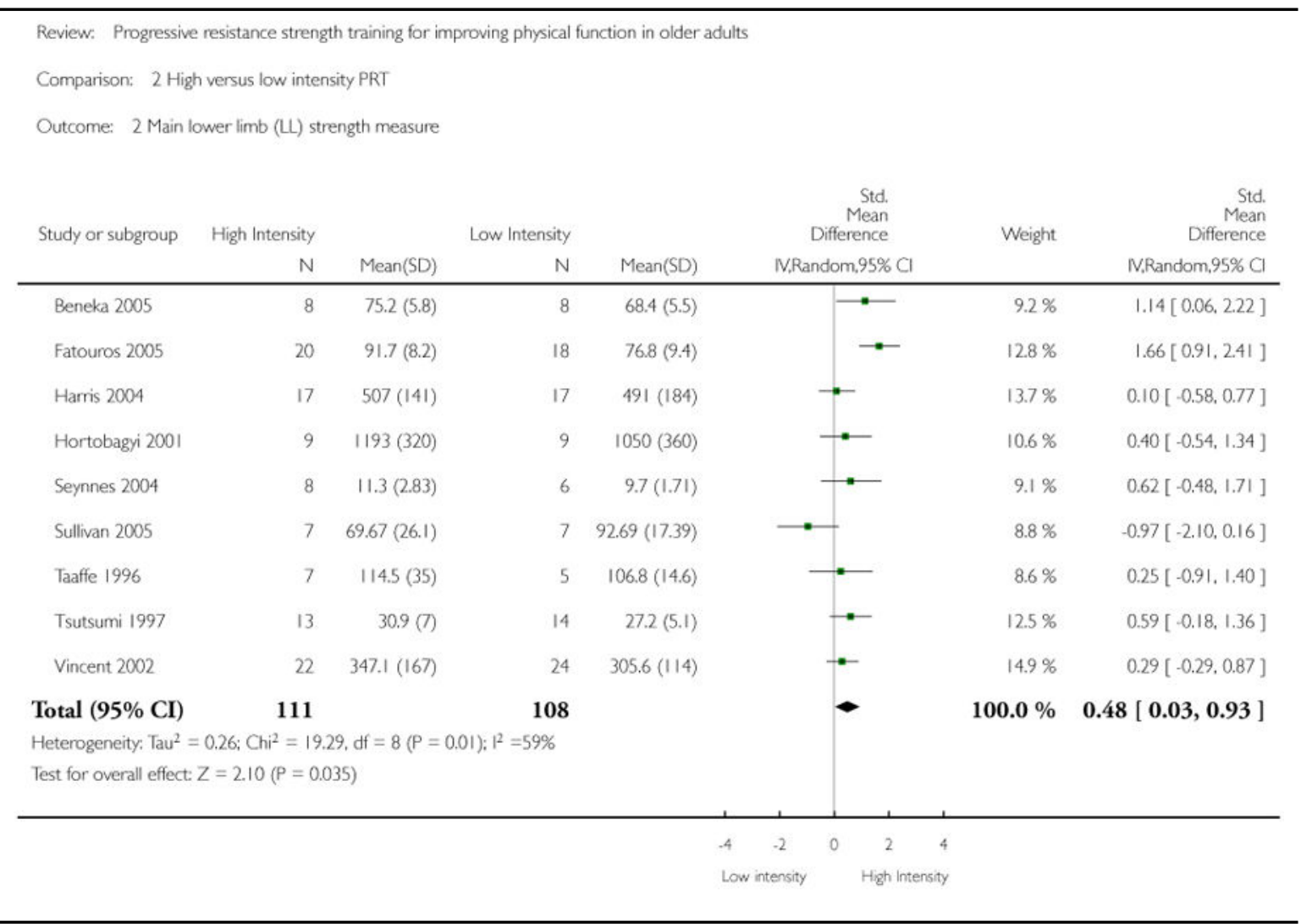




\section{Analysis 2.3. Comparison 2 High versus low intensity PRT, Outcome 3 VO2 Max (ml/kg/min)}

\begin{tabular}{|c|c|c|c|c|c|c|c|c|}
\hline \multicolumn{9}{|c|}{ Review: Progressive resistance strength training for improving physical function in older adults } \\
\hline \multicolumn{9}{|c|}{ Comparison: 2 High versus low intensity PRT } \\
\hline \multicolumn{9}{|c|}{ Outcome: $3 \mathrm{VO} 2 \mathrm{Max}(\mathrm{m} / \mathrm{kg} / \mathrm{min})$} \\
\hline \multirow[t]{2}{*}{ Study or subgroup } & High intensity & \multicolumn{3}{|c|}{ Low intensity } & \multirow{2}{*}{\multicolumn{2}{|c|}{$\begin{array}{l}\text { Mean } \\
\text { Difference } \\
\text { IV.Random,95\% Cl }\end{array}$}} & \multirow[t]{2}{*}{ Weight } & \multirow{2}{*}{$\begin{array}{r}\text { Mear } \\
\text { Difference } \\
\text { IV,Random,95\% C }\end{array}$} \\
\hline & $\mathrm{N}$ & Mean(SD) & $\mathrm{N}$ & $\operatorname{Mean}(\mathrm{SD})$ & & & & \\
\hline Fatouros 2005 & 14 & $19.3(2.3)$ & 14 & $17.9(3.1)$ & & $-=$ & $42.9 \%$ & $1.40[-0.62,3.42]$ \\
\hline Tsutsumi 1997 & 13 & $23(5.4)$ & 14 & $17.8(4.9)$ & & 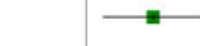 & $25.2 \%$ & $5.20[1.30,9.10]$ \\
\hline Vincent 2002 & 22 & $24.4(5.8)$ & 24 & $24.7(4.8)$ & & $\longrightarrow$ & $31.9 \%$ & $-0.30[-3.39,2.79]$ \\
\hline Total $(95 \% \mathrm{CI})$ & 49 & & 52 & & & - & $100.0 \%$ & $1.82[-0.79,4.43]$ \\
\hline \multirow{2}{*}{\multicolumn{9}{|c|}{$\begin{array}{l}\text { Heterogeneity: } \mathrm{Tau}^{2}=3.07 ; \mathrm{Chi}^{2}=4.76, \mathrm{df}=2(\mathrm{P}=0.09) ; 1^{2}=58 \% \\
\text { Test for overall effect: } Z=1.36(P=0.17)\end{array}$}} \\
\hline & & & & & & & & \\
\hline \multicolumn{9}{|c|}{0} \\
\hline \multicolumn{9}{|c|}{ Low intensity High intensity } \\
\hline
\end{tabular}

\section{Analysis 2.4. Comparison 2 High versus low intensity PRT, Outcome 4 Pain (higher score = less pain)}

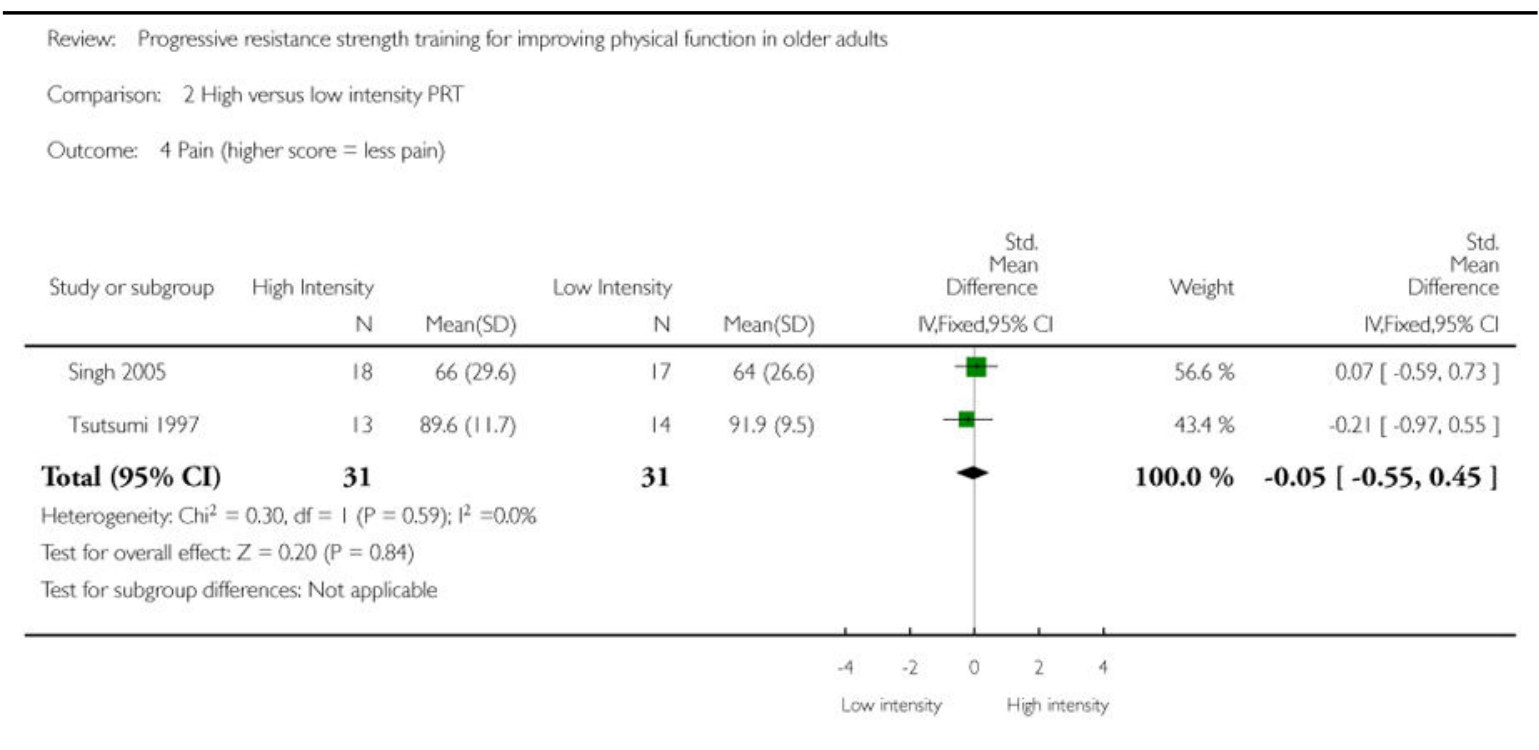

Cochrane Database Syst Rev. Author manuscript; available in PMC 2015 February 11. 


\title{
Analysis 2.5. Comparison 2 High versus low intensity PRT, Outcome 5 Vitality $($ SF-36, higher score $=$ more vitality)
}

\begin{tabular}{|c|c|c|c|c|c|c|c|}
\hline \multicolumn{8}{|c|}{ Review: Progressive resistance strength training for improving physical function in older adults } \\
\hline \multicolumn{8}{|c|}{ Comparison: 2 High versus low intensity PRT } \\
\hline \multicolumn{8}{|c|}{ Outcome: 5 vitality $(\mathrm{SF}-36$, higher score $=$ more vitality $)$} \\
\hline \multirow[t]{2}{*}{ Study or subgroup } & \multirow{2}{*}{$\begin{array}{r}\text { High Intensity } \\
\mathrm{N}\end{array}$} & \multicolumn{2}{|r|}{ Low Intensity } & \multirow[b]{2}{*}{$\operatorname{Mean}(\mathrm{SD})$} & \multirow{2}{*}{$\begin{array}{c}\text { Mean } \\
\text { Difference } \\
\text { IV.Fixed,95\% Cl }\end{array}$} & \multirow[t]{2}{*}{ Weight } & \multirow{2}{*}{$\begin{array}{r}\text { Mean } \\
\text { Difference } \\
\text { IV.Fixed,95\% Cl }\end{array}$} \\
\hline & & Mean(SD) & N & & & & \\
\hline Singh 2005 & 18 & $61.4(22)$ & 17 & $46.8(27.7)$ & $\bullet-$ & $124 \%$ & $14.60[-2.03,31.23]$ \\
\hline Tsutsumi 1997 & 13 & $75.8(6.7)$ & 14 & $70.4(9.7)$ & ت & $87.6 \%$ & $5.40[-0.85,11.65]$ \\
\hline Total $(95 \% \mathrm{CI})$ & 31 & & 31 & & $\bullet$ & $100.0 \%$ & $6.54[0.69,12.39]$ \\
\hline \multicolumn{8}{|c|}{ Heterogeneity: $\mathrm{Ch}^{2}=1.03, \mathrm{df}=1(\mathrm{P}=0.31) ; \mathrm{I}^{2}=3 \%$} \\
\hline \multicolumn{8}{|c|}{ Test for overall effect: $Z=2.19(P=0.029)$} \\
\hline \multicolumn{8}{|c|}{ Test for subgroup differences: Not applicable } \\
\hline & & & & .100 & -50 & 100 & \\
\hline \multicolumn{8}{|c|}{ Low intensity } \\
\hline
\end{tabular}

\begin{abstract}
Analysis 3.1. Comparison 3 High versus variable intensity PRT, Outcome 1 Main lower limb (LL) strength measure
\end{abstract}

Review: Progressive resistance strength training for improving physical function in older adults

Comparison: 3 High versus variable intensity PRT

Outcome: I Main lower limb (LL) strength measure

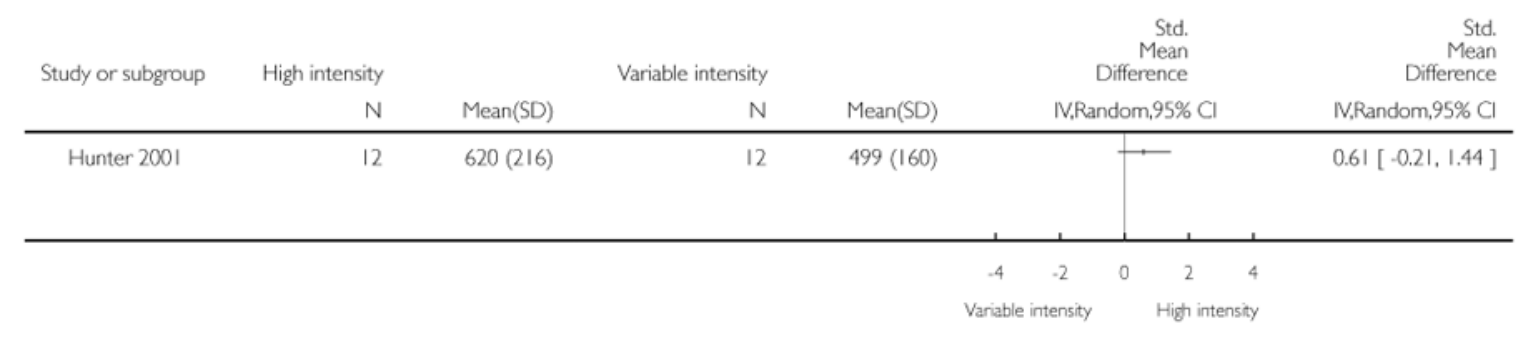




\section{Analysis 3.2. Comparison 3 High versus variable intensity PRT, Outcome 2 VO2 Max (ml/kg/min)}

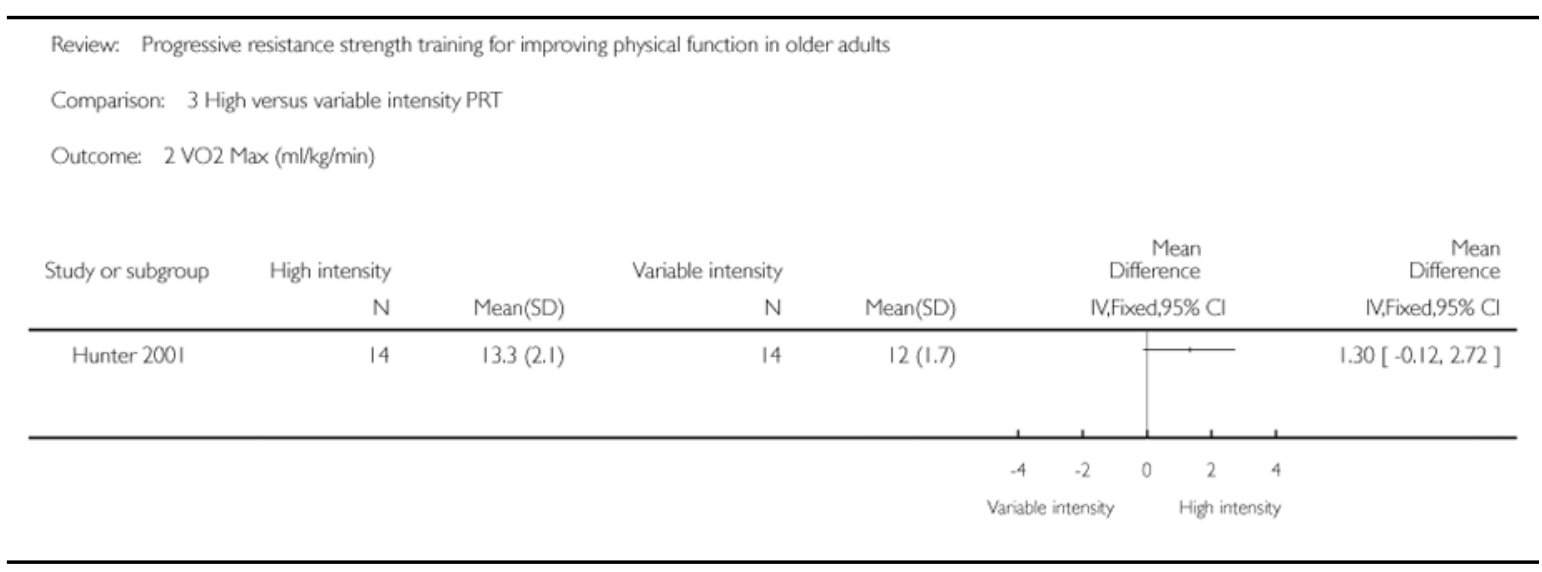

\section{Analysis 4.1. Comparison 4 PRT frequency, Outcome 1}

Main LL strength measure

\section{Review: Progressive resistance strength training for improving physical function in older adults}

Comparison: 4 PRT frequency

Outcome: I Main LL strength measure

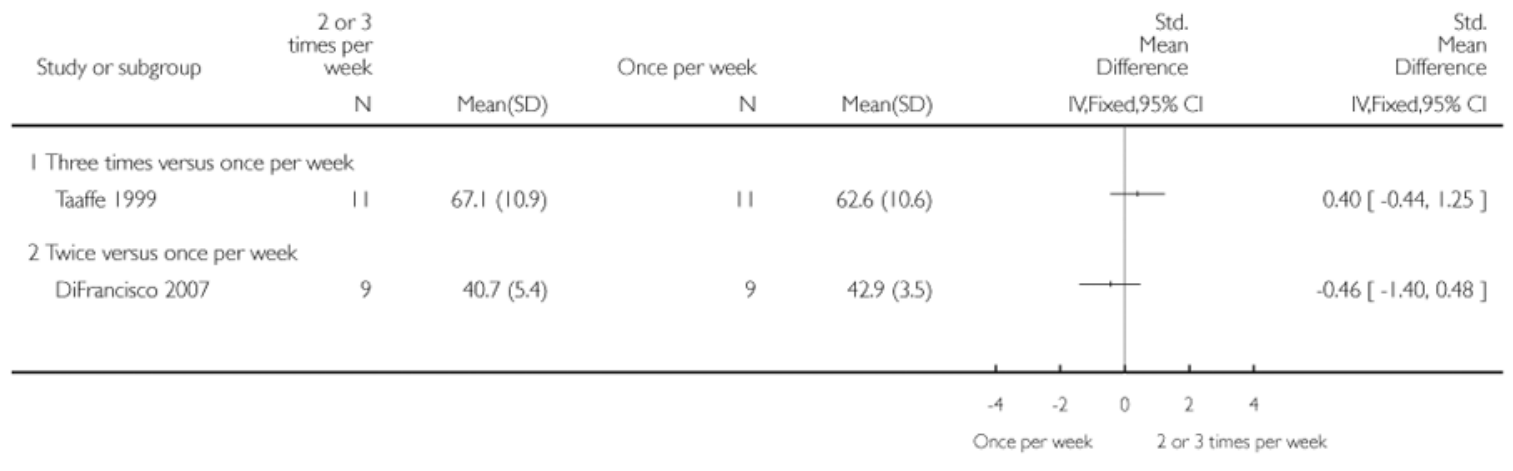




\section{Analysis 5.1. Comparison 5 PRT: 3-sets versus 1-sets, Outcome 1 Main lower limb (LL) strength measure}

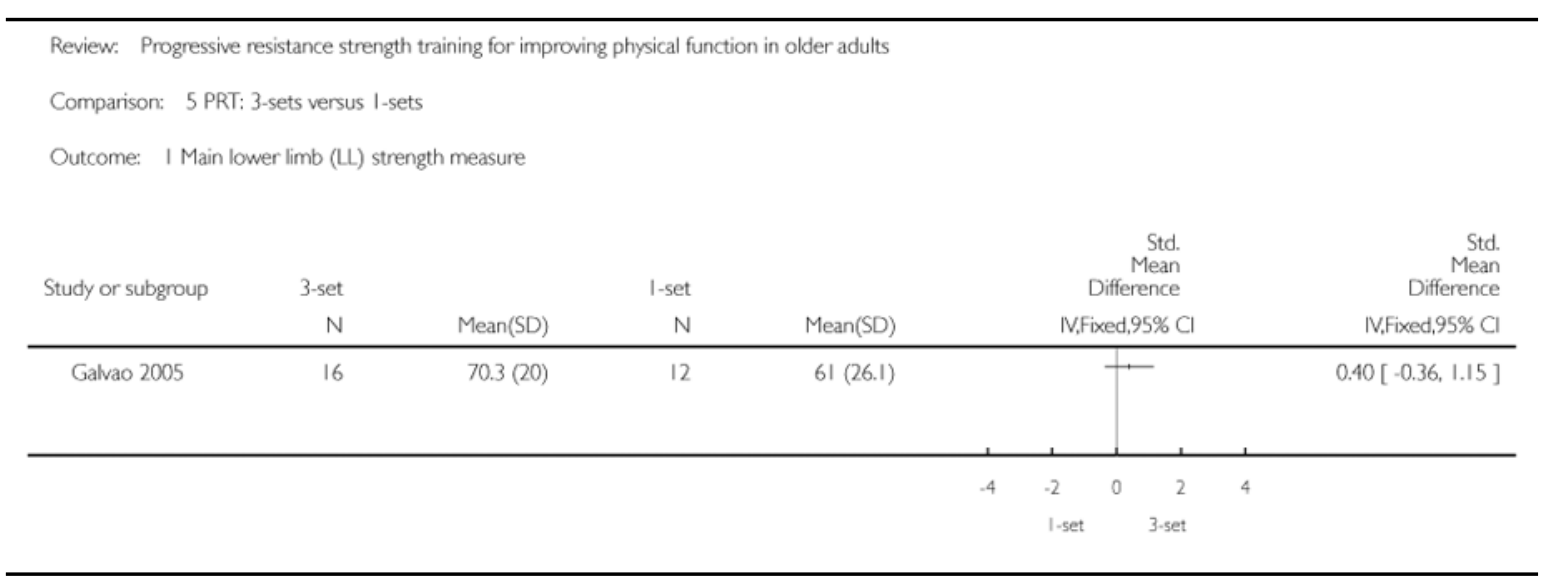

Analysis 5.2. Comparison 5 PRT: 3-sets versus 1-sets, Outcome 2 Six-minute walk test (meters)

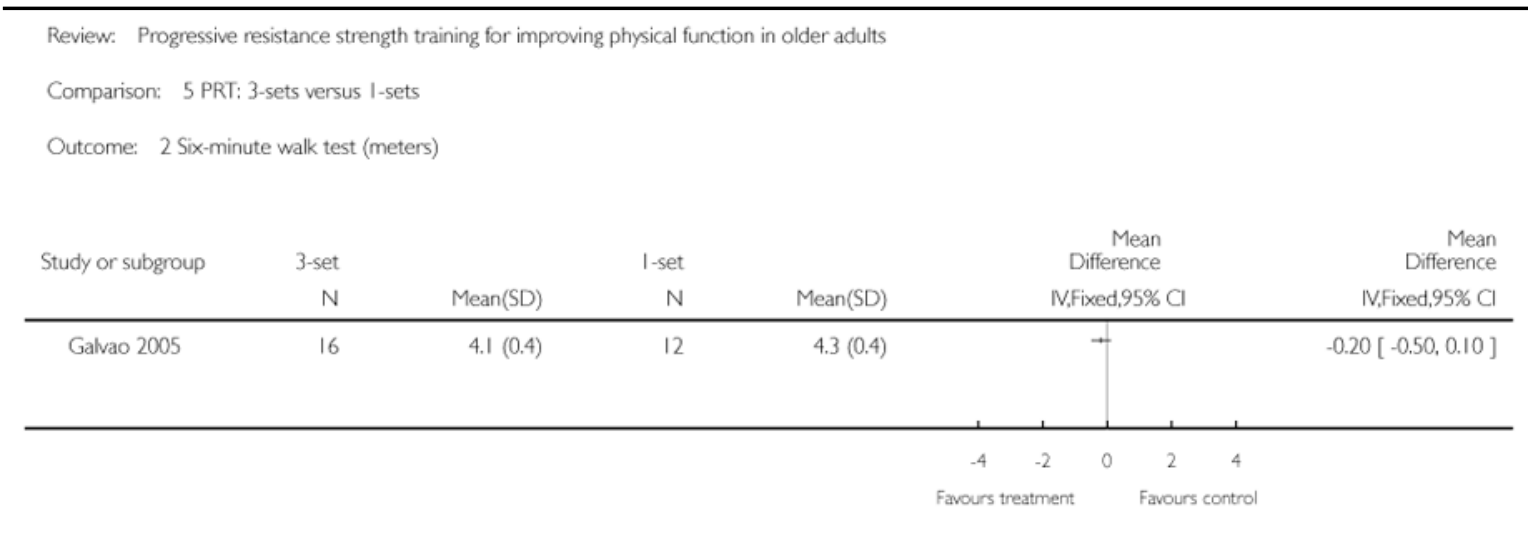


Analysis 5.3. Comparison 5 PRT: 3-sets versus 1-sets, Outcome 3 Timed walk (seconds)

\begin{tabular}{|c|c|c|c|c|c|c|c|c|c|}
\hline \multicolumn{10}{|c|}{ Review: Progressive resistance strength training for improving physical function in older adults } \\
\hline \multicolumn{10}{|c|}{ Comparison: 5 PRT: 3 -sets versus I-sets } \\
\hline \multicolumn{10}{|c|}{ Outcome: 3 Timed walk (seconds) } \\
\hline \multirow[t]{2}{*}{ Study or subgroup } & 3-set & \multicolumn{3}{|c|}{ 1-set } & \multicolumn{4}{|c|}{$\begin{array}{r}\text { Mean } \\
\text { Difference }\end{array}$} & $\begin{array}{r}\text { Mean } \\
\text { Difference }\end{array}$ \\
\hline & $\mathrm{N}$ & $\operatorname{Mean}(\mathrm{SD})$ & $\mathrm{N}$ & Mean(SD) & \multicolumn{4}{|c|}{ N,Fixed,95\% Cl } & IV,Fixed,95\% CI \\
\hline \multirow[t]{3}{*}{ Galvao 2005} & 16 & $213.3(26.1)$ & 12 & $242.9(37.2)$ & & $\longrightarrow$ & & & $-29.60[-54.23,-4.97]$ \\
\hline & & & & & -100 & $.50 \quad 0$ & 50 & 100 & \\
\hline & & & & & & 3-set & 1-set & & \\
\hline
\end{tabular}

\section{Analysis 5.4. Comparison 5 PRT: 3 -sets versus 1-sets,} Outcome 4 Time to stand from a chair (seconds)

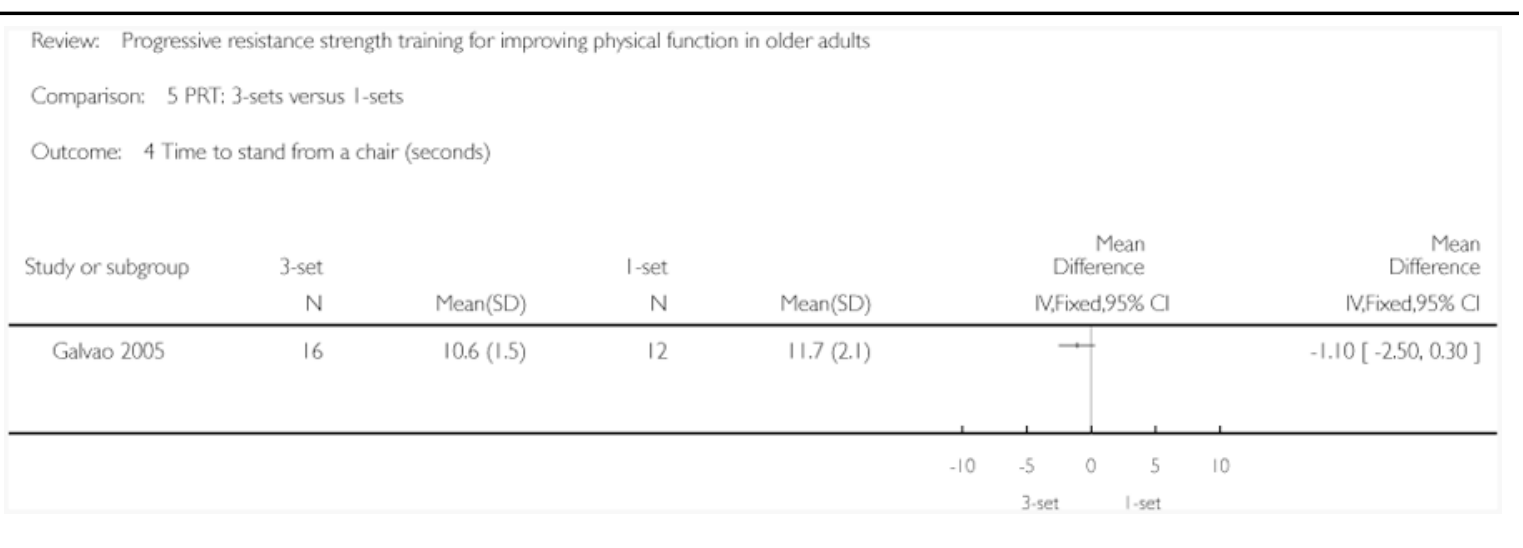




\section{Analysis 5.5. Comparison 5 PRT: 3-sets versus 1-sets, Outcome 5 Stair climbing (seconds)}

\begin{tabular}{|c|c|c|c|c|c|c|c|c|c|}
\hline \multicolumn{10}{|c|}{$\begin{array}{l}\text { Review: Progressive resistance strength training for improving physical function in older adults } \\
\text { Comparison: } 5 \text { PRT: 3-sets versus I-sets }\end{array}$} \\
\hline \multirow[t]{2}{*}{ Study or subgroup } & 3-set & \multicolumn{3}{|c|}{ 1-set } & \multicolumn{4}{|c|}{$\begin{array}{r}\text { Mean } \\
\text { Difference }\end{array}$} & $\begin{array}{r}\text { Mean } \\
\text { Difference }\end{array}$ \\
\hline & $\mathrm{N}$ & Mean(SD) & $\mathrm{N}$ & Mean(SD) & \multicolumn{4}{|c|}{ IV,Fixed,95\% Cl } & IV,Fixed,95\% Cl \\
\hline \multirow[t]{2}{*}{ Galvao 2005} & 16 & $4.2(0.5)$ & 12 & $4.8(1.1)$ & & + & & & $-0.60[-1.27,0.07]$ \\
\hline & & & & & -10 & $\begin{array}{ll}-5 & 0 \\
3-\text { set } & \end{array}$ & $\begin{array}{r}5 \\
\text { 1.set }\end{array}$ & 10 & \\
\hline
\end{tabular}

\section{Analysis 6.1. Comparison 6 PRT versus aerobic training, Outcome 1 Main function measure (higher score $=$ better function)}

Review: Progressive resistance strength training for improving physical function in older adults

Comparison: 6PRT versus aerobic training

Outcome: I Main function measure (higher score = better function)

\begin{tabular}{|c|c|c|c|c|c|c|c|}
\hline \multirow[t]{2}{*}{ Study or subgroup } & \multirow{2}{*}{$\begin{array}{r}\text { PRT } \\
\mathrm{N}\end{array}$} & \multicolumn{3}{|c|}{ Aerobic } & \multirow{2}{*}{$\begin{array}{r}\text { Std. } \\
\text { Mean } \\
\text { Difference } \\
\text { IV,Fixed,95\% Cl }\end{array}$} & \multirow[t]{2}{*}{ Weight } & \multirow{2}{*}{$\begin{array}{r}\text { Std. } \\
\text { Mean } \\
\text { Difference } \\
\text { IV.Fixed,95\% Cl }\end{array}$} \\
\hline & & Mean(SD) & $\mathrm{N}$ & $\operatorname{Mean}(\mathrm{SD})$ & & & \\
\hline Mangione 2005 & 11 & $57.7(21.1)$ & 12 & $57.5(24.3)$ & $\rightarrow-$ & $18.9 \%$ & $0.01[-0.81,0.83]$ \\
\hline Buchner 1997 & 22 & $69(39)$ & 21 & $83(31)$ & $\rightarrow$ & $34.7 \%$ & $-0.39[-0.99,0.22]$ \\
\hline Earles 2001 & 18 & $48(6)$ & 22 & $47(6)$ & $\mp$ & $32.5 \%$ & $0.16[-0.46,0.79]$ \\
\hline Hiatt 1994 & 9 & $45(22)$ & 10 & $66(22)$ & $\longrightarrow$ & $13.8 \%$ & $-0.91[-1.87,0.05]$ \\
\hline Total $(95 \%$ CI) & 60 & & 65 & & $\bullet$ & $100.0 \%$ & $1[-0.56,0.15]$ \\
\hline
\end{tabular}

Heterogeneity: $\mathrm{Chi}^{2}=4.05, \mathrm{df}=3(\mathrm{P}=0.26) ; \mathrm{I}^{2}=26 \%$

Test for overall effect: $Z=1.14(P=0.26)$

Test for subgroup differences: Not applicable 


\section{Analysis 6.2. Comparison 6 PRT versus aerobic training, Outcome 2 Main function measure (lower score $=$ better function)}

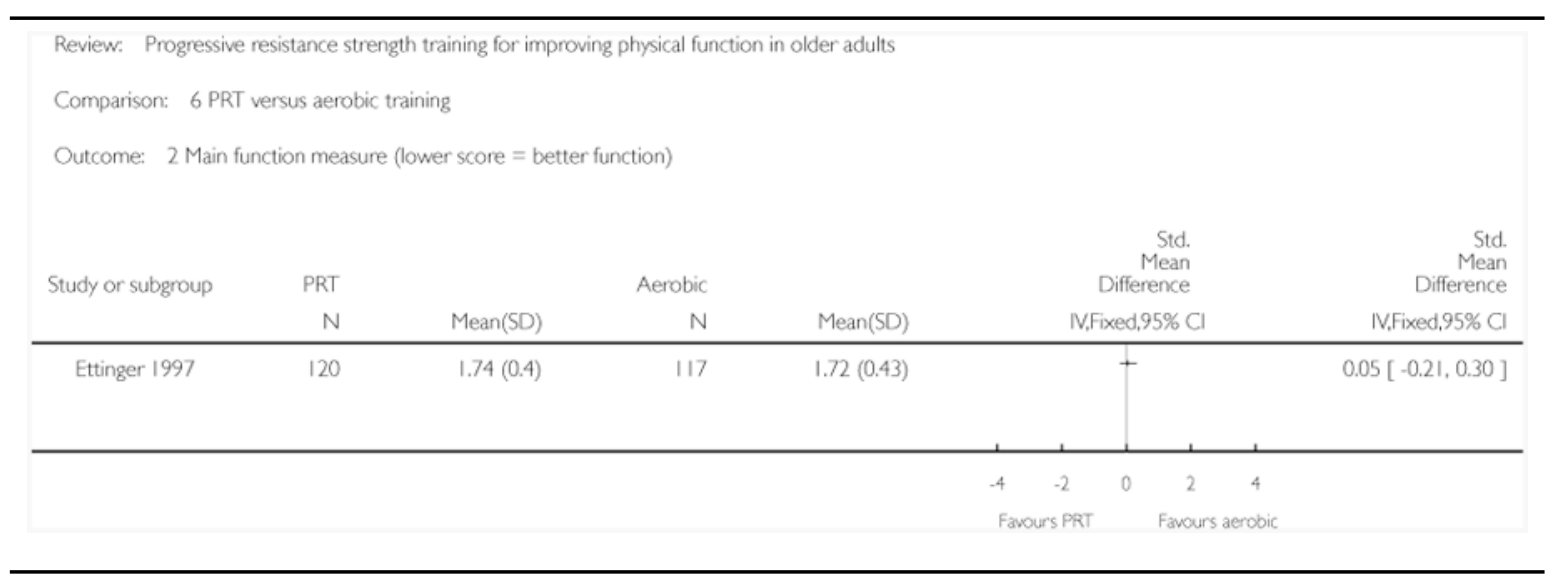

\section{Analysis 6.3. Comparison 6 PRT versus aerobic training, Outcome 3 Main lower limb strength measure}

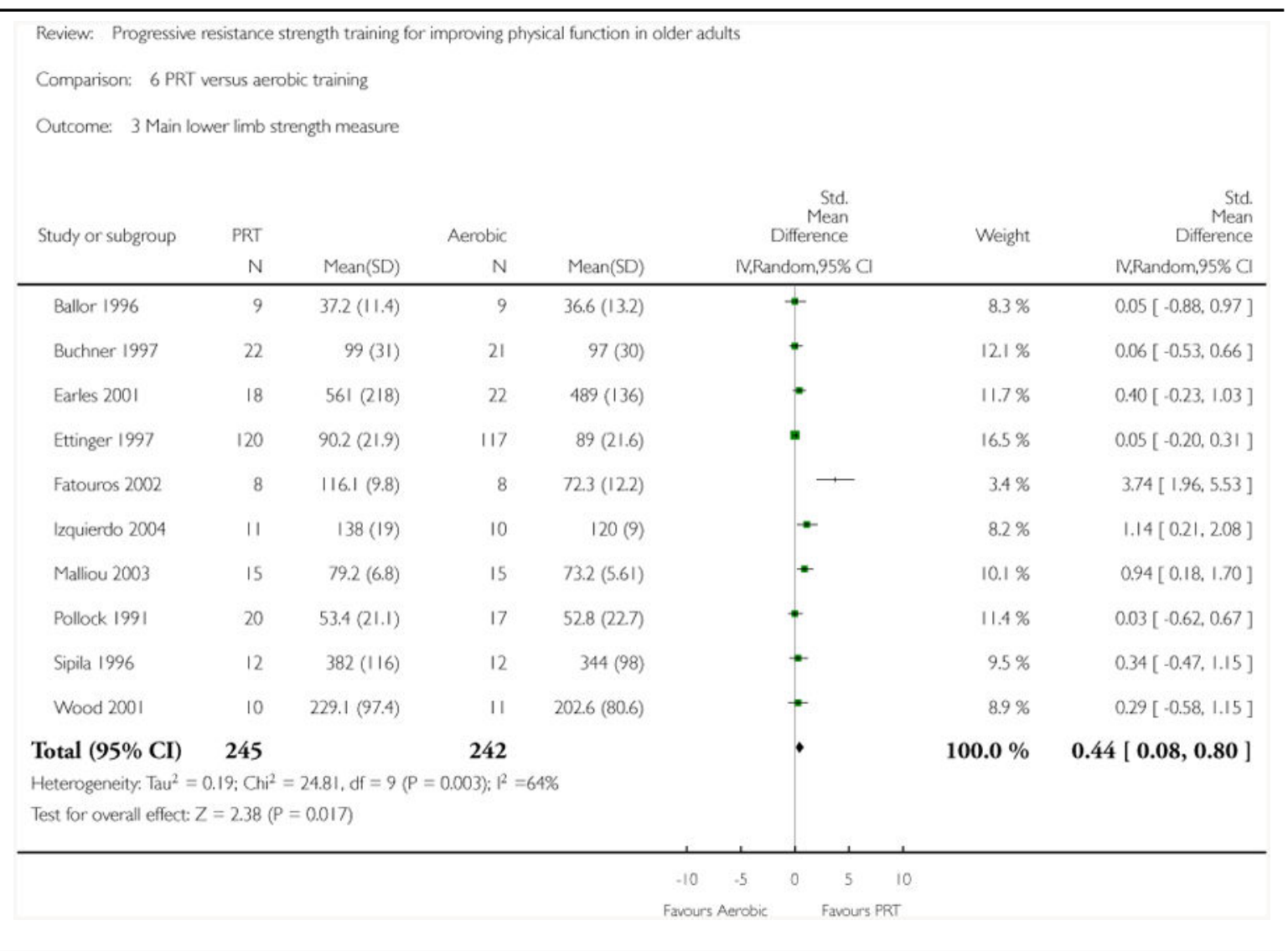




\section{Analysis 6.4. Comparison 6 PRT versus aerobic training, Outcome $4 \mathrm{VO2}$ max (ml/kg.min)}

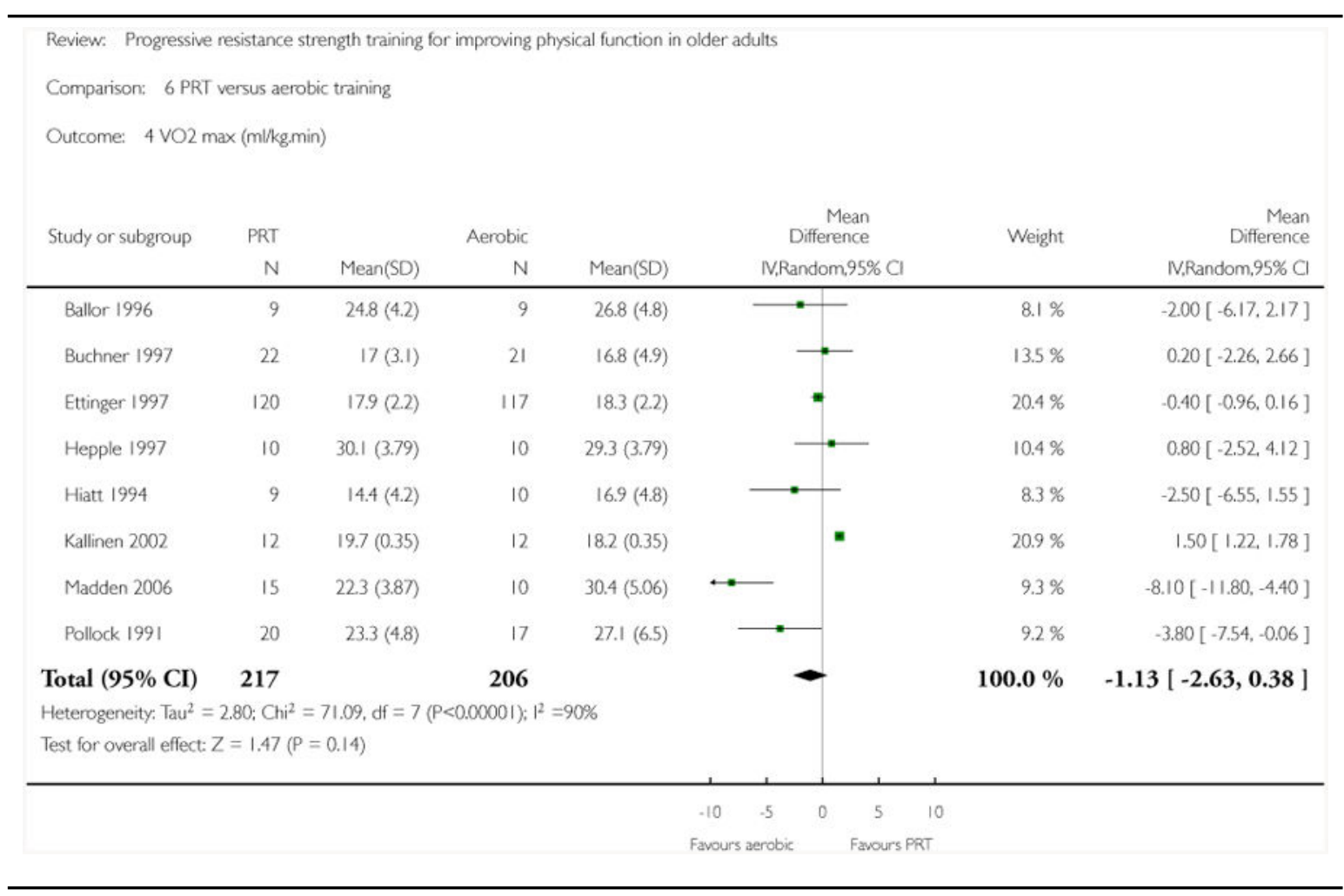

\section{Analysis 6.5. Comparison 6 PRT versus aerobic training, Outcome 5 Six minute walk test (meters)}

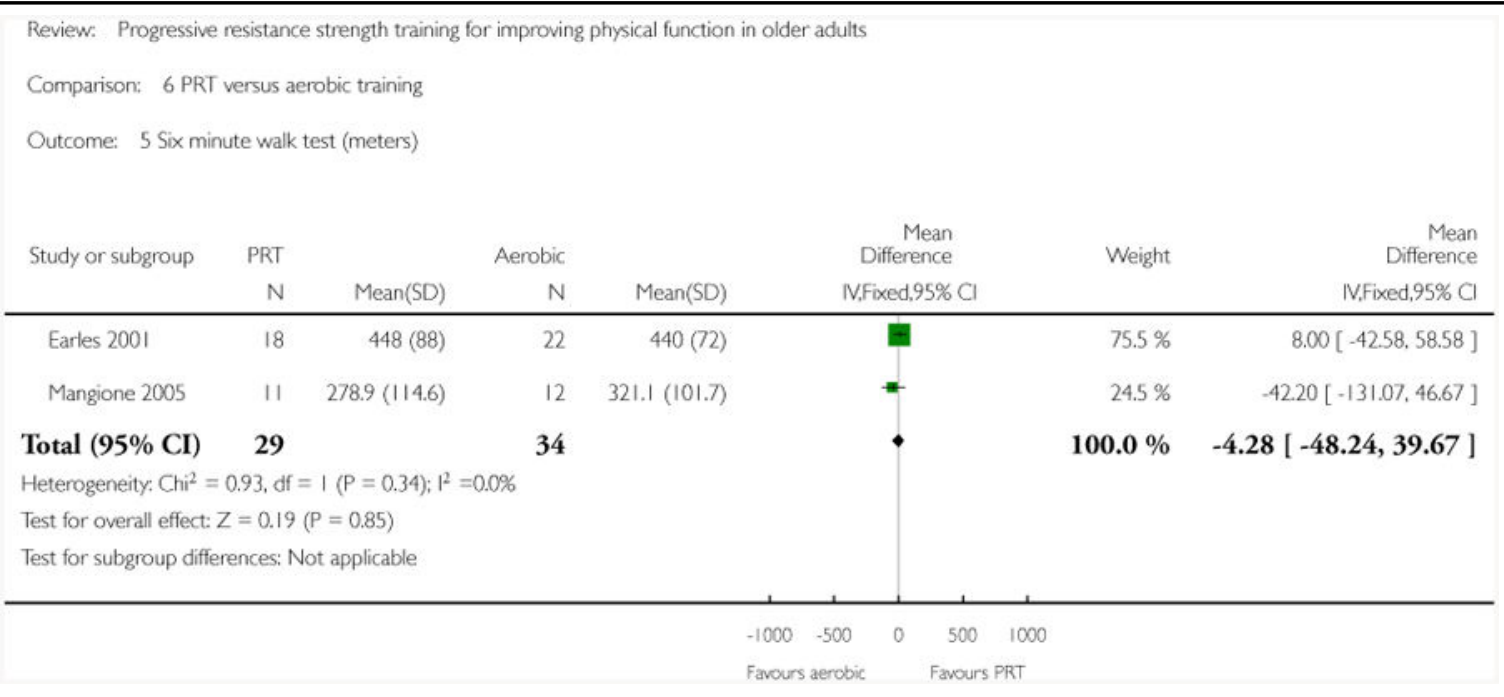




\section{Analysis 6.6. Comparison 6 PRT versus aerobic training, Outcome 6 Gait speed $(\mathrm{m} / \mathrm{s})$}

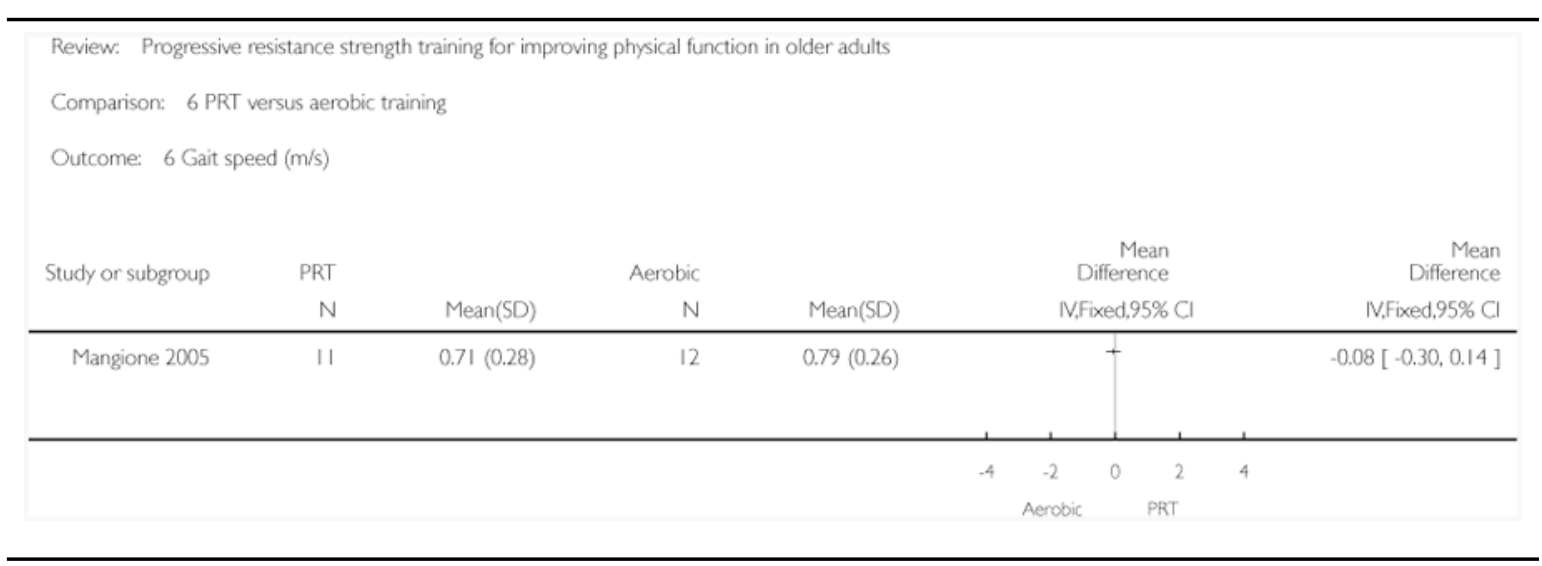

\section{Analysis 6.7. Comparison 6 PRT versus aerobic} training, Outcome 7 Pain (lower score = less pain)

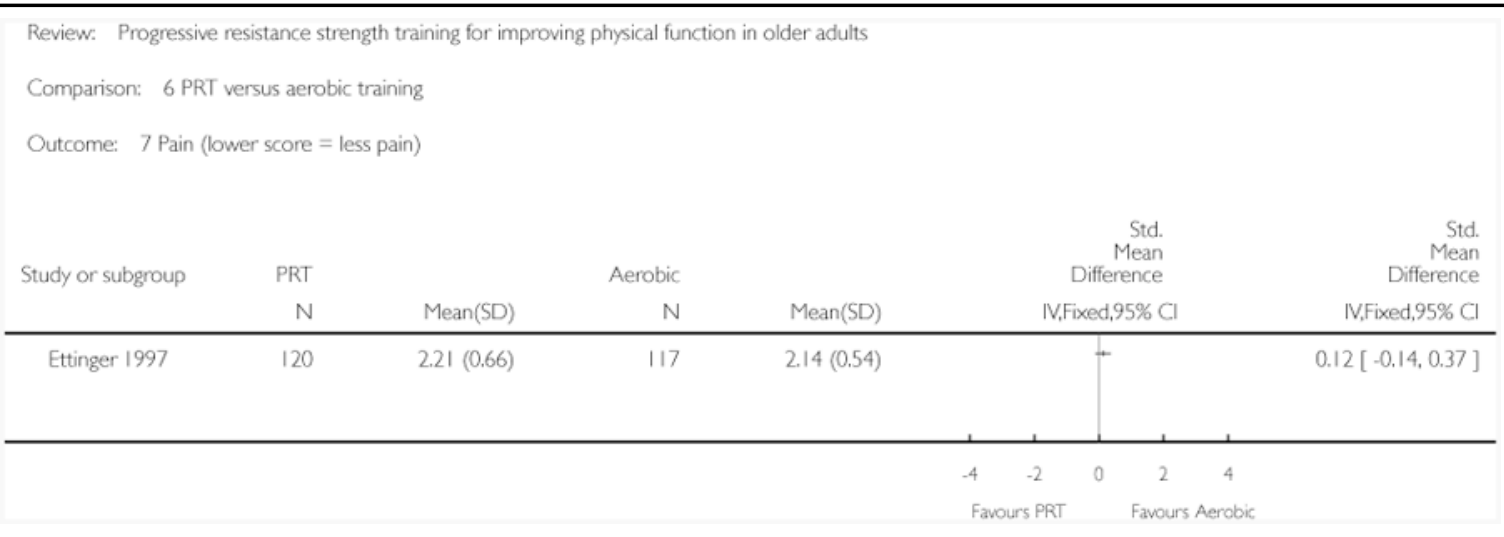




\section{Analysis 7.1. Comparison 7 PRT versus functional exercise, Outcome 1 Main function measure (higher score $=$ better function)}

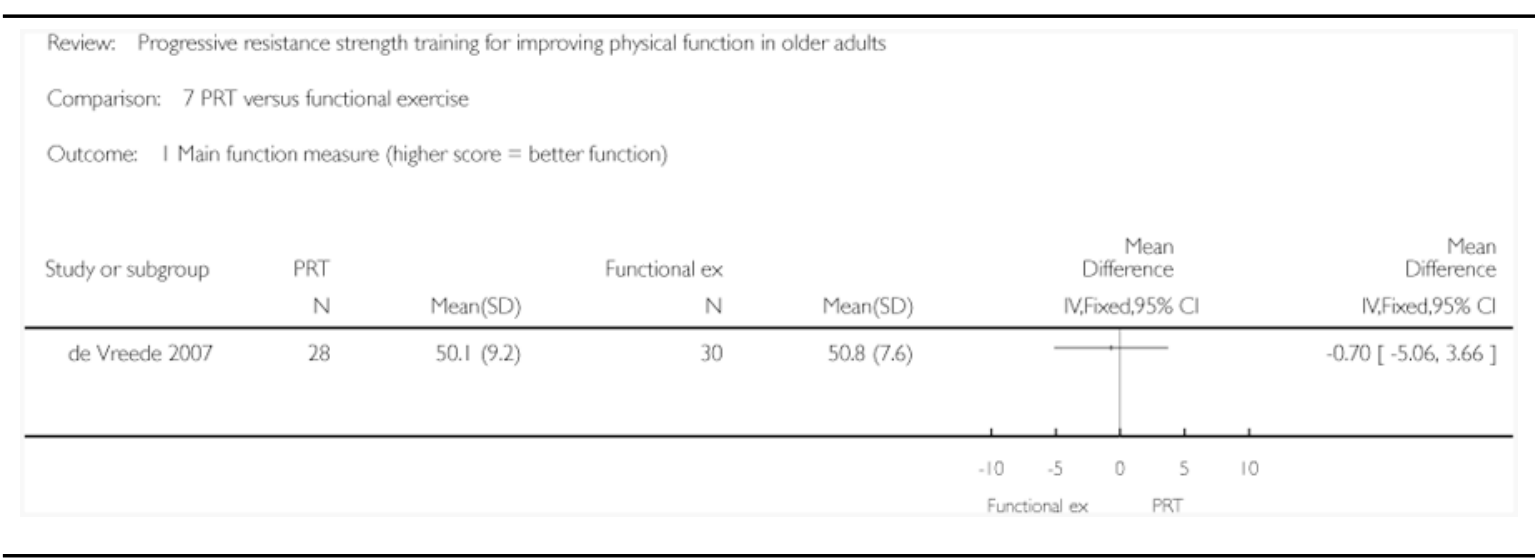

Analysis 7.2. Comparison 7 PRT versus functional exercise, Outcome 2 Main lower limb strength measure

Review: Progressive resistance strength training for improving physical function in older adults

Comparison: 7 PRT versus functional exercise

Outcome: 2 Main lower limb strength measure

\begin{tabular}{|c|c|c|c|c|c|c|c|}
\hline \multirow[t]{2}{*}{ Study or subgroup } & \multirow{2}{*}{$\begin{array}{l}\text { PRT } \\
\mathrm{N}\end{array}$} & \multicolumn{3}{|c|}{ Functional ex } & \multirow{2}{*}{$\begin{array}{c}\text { Mean } \\
\text { Difference } \\
\text { IV,Fixed,95\% Cl }\end{array}$} & \multirow[t]{2}{*}{ Weight } & \multirow{2}{*}{$\begin{array}{r}\text { Mean } \\
\text { Difference } \\
\text { IV,Fixed,95\% Cl }\end{array}$} \\
\hline & & Mean(SD) & $\mathrm{N}$ & Mean(SD) & & & \\
\hline Chin A Paw 2006 & 40 & $73.2(12.3)$ & 44 & $82.1(52.7)$ & $-t$ & $82.4 \%$ & $-8.90[-24.93,7.13]$ \\
\hline de Vreede 2007 & 28 & $307.2(90.9)$ & 30 & $300.3(85.7)$ & & $10.2 \%$ & $6.90[-38.64,52.44]$ \\
\hline Manini 2005 & 9 & $166.2(60.4)$ & 7 & $164.6(48.4)$ & & $7.4 \%$ & $1.60[-51.72,54.92]$ \\
\hline Total $(95 \%$ CI) & 77 & & 81 & & & $100.0 \%$ & $-6.51[-21.05,8.04]$ \\
\hline
\end{tabular}

Heterogeneity; $\mathrm{Chi}^{2}=0.51$, df $=2(P=0.78) ; 1^{2}=0.0 \%$

Test for overall effect: $Z=0.88(P=0.38)$

Test for subgroup differences: Not applicable 


\section{Analysis 7.3. Comparison 7 PRT versus functional exercise, Outcome 3 Timed "Up-and-Go" (seconds)}

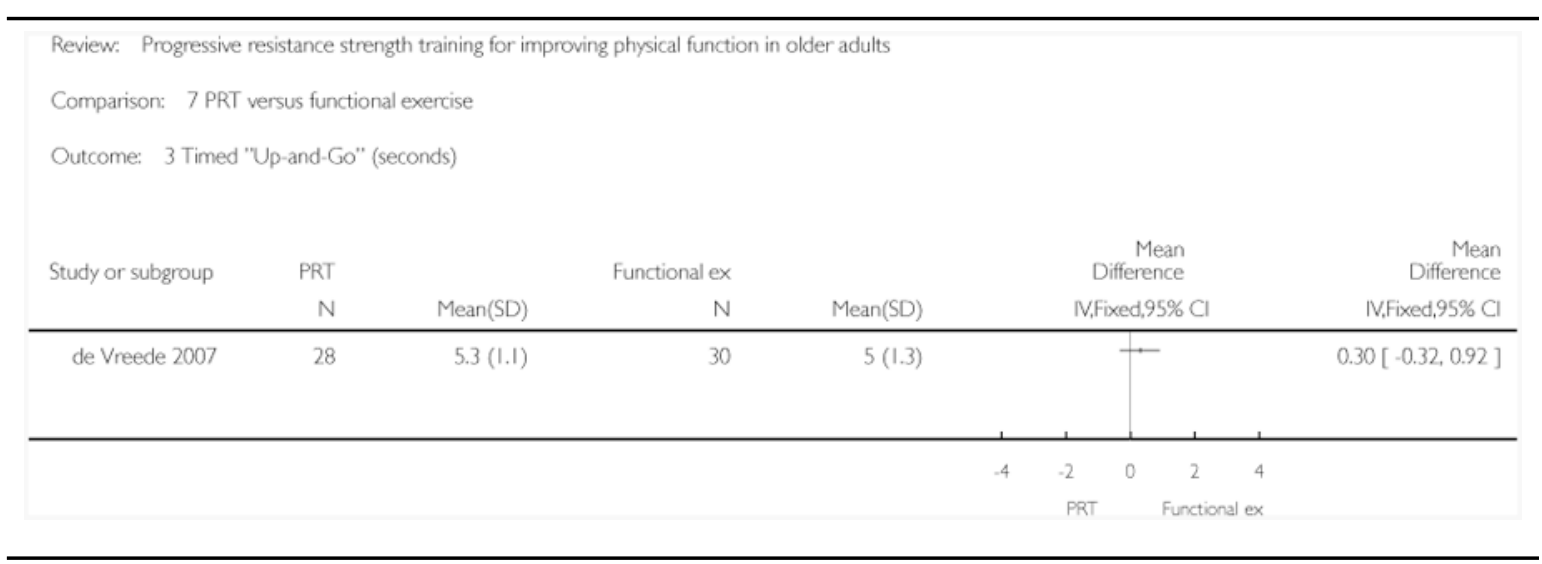

\section{Analysis 7.4. Comparison 7 PRT versus functional exercise, Outcome 4 Vitality (SF-36/Vitality plus scale, higher $=$ more vitality)}

Review: Progressive resistance strength training for improving physical function in older adults

Comparison: 7 PRT versus functional exercise

Outcome: 4 Vitality (SF-36Nitality plus scale, higher $=$ more vitality)

\begin{tabular}{|c|c|c|c|c|c|c|c|}
\hline \multirow[t]{2}{*}{ Study or subgroup } & \multirow{2}{*}{$\begin{array}{r}\text { PRT } \\
\mathrm{N}\end{array}$} & \multicolumn{3}{|c|}{ Functional ex } & \multirow{2}{*}{$\begin{array}{c}\text { Mean } \\
\text { Difference } \\
\text { Nv,Fixed, } 95 \% \mathrm{Cl}\end{array}$} & \multirow[t]{2}{*}{ Weight } & \multirow{2}{*}{$\begin{array}{r}\text { Mean } \\
\text { Difference } \\
\text { IV,Fixed, } 95 \% \mathrm{Cl}\end{array}$} \\
\hline & & $\operatorname{Mean}(\mathrm{SD})$ & $\mathrm{N}$ & $\operatorname{Mean}(\mathrm{SD})$ & & & \\
\hline Chin A Paw 2006 & 41 & $36.9(6.6)$ & 48 & $36.5(6.6)$ & & $89.8 \%$ & $0.40[-2.35,3.15]$ \\
\hline de Vreede 2007 & 28 & $72.3(16.1)$ & 30 & $76.5(15.6)$ & - & $10.2 \%$ & $-4.20[-12.37,3.97]$ \\
\hline
\end{tabular}

Total (95\% CI) 69

78

Heterogeneity: Chi $^{2}=1.09, \mathrm{df}=1(\mathrm{P}=0.30) ; \mathrm{I}^{2}=9 \%$

Test for overall effect: $Z=0.05(P=0.96)$

Test for subgroup differences: Not applicable 


\section{Analysis 7.5. Comparison 7 PRT versus functional exercise, Outcome 5 Pain (higher = less pain, Bodily pain on SF-36)}

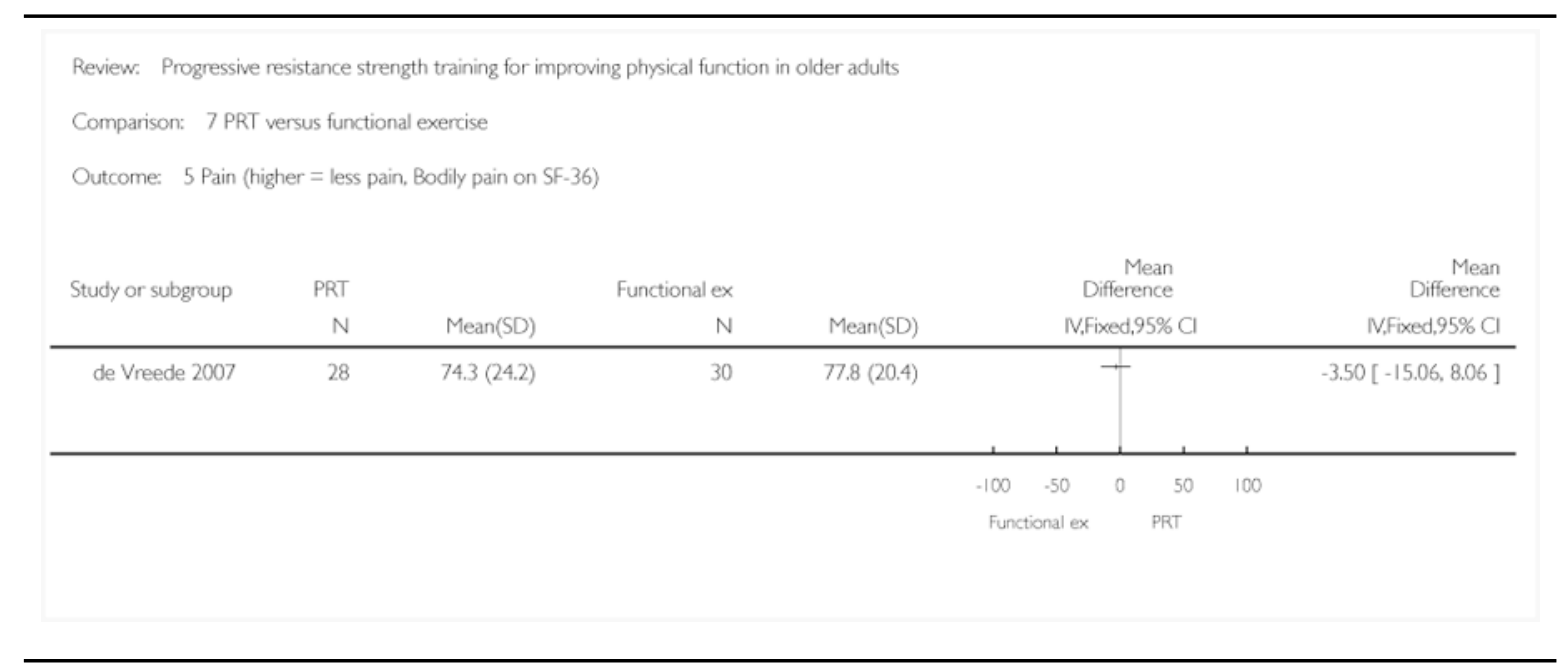

Analysis 8.1. Comparison 8 PRT versus flexibility training, Outcome 1 SF36 (higher score $=$ better function)

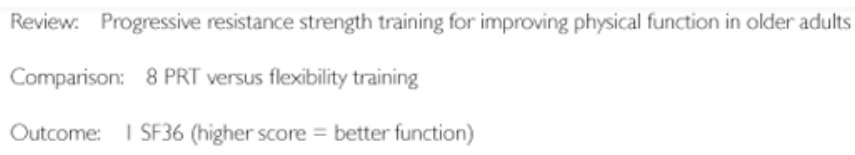

\begin{tabular}{|c|c|c|c|c|c|c|c|c|c|c|}
\hline \multirow[t]{2}{*}{ Study or subgroup } & \multirow{2}{*}{$\begin{array}{l}\text { PRT } \\
N\end{array}$} & \multicolumn{3}{|c|}{ Flexibility } & \multicolumn{5}{|c|}{$\begin{array}{l}\text { Mean } \\
\text { Difference }\end{array}$} & \multirow{2}{*}{$\begin{array}{r}\text { Mean } \\
\text { Difference } \\
\text { IV.Fixed,95\% Cl }\end{array}$} \\
\hline & & Mean(SD) & $\mathrm{N}$ & Mean(SD) & \multicolumn{5}{|c|}{ IV,Fixed,95\% Cl } & \\
\hline \multirow[t]{3}{*}{ Barrett 2002} & 22 & $73.8(19)$ & 22 & $78.5(16.9)$ & & & $\rightarrow$ & & & $-4.70[-15.33,5.93]$ \\
\hline & & & & & -100 & -50 & 0 & so & 100 & \\
\hline & & & & & Flexibilit & training & & RT & & \\
\hline
\end{tabular}




\section{Analysis 8.2. Comparison 8 PRT versus flexibility training, Outcome 2 Main lower limb (LL) strength measure}

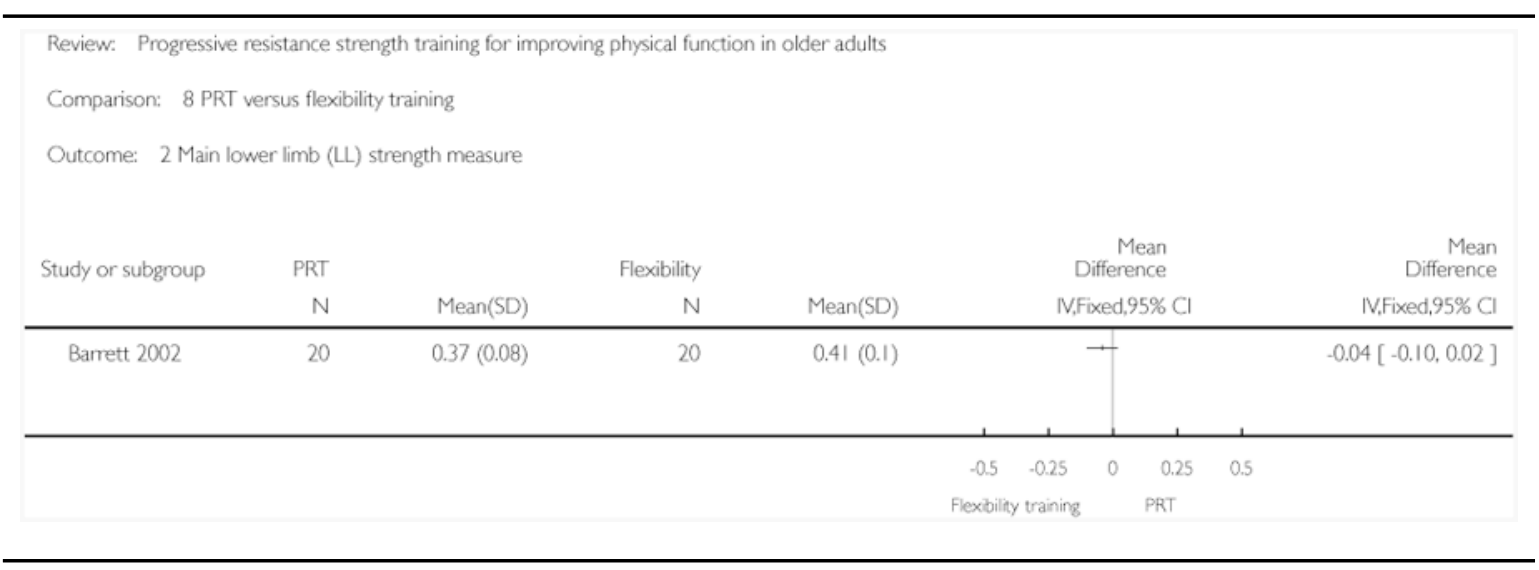

\section{Analysis 8.3. Comparison 8 PRT versus flexibility training, Outcome 3 Timed walk (seconds)}

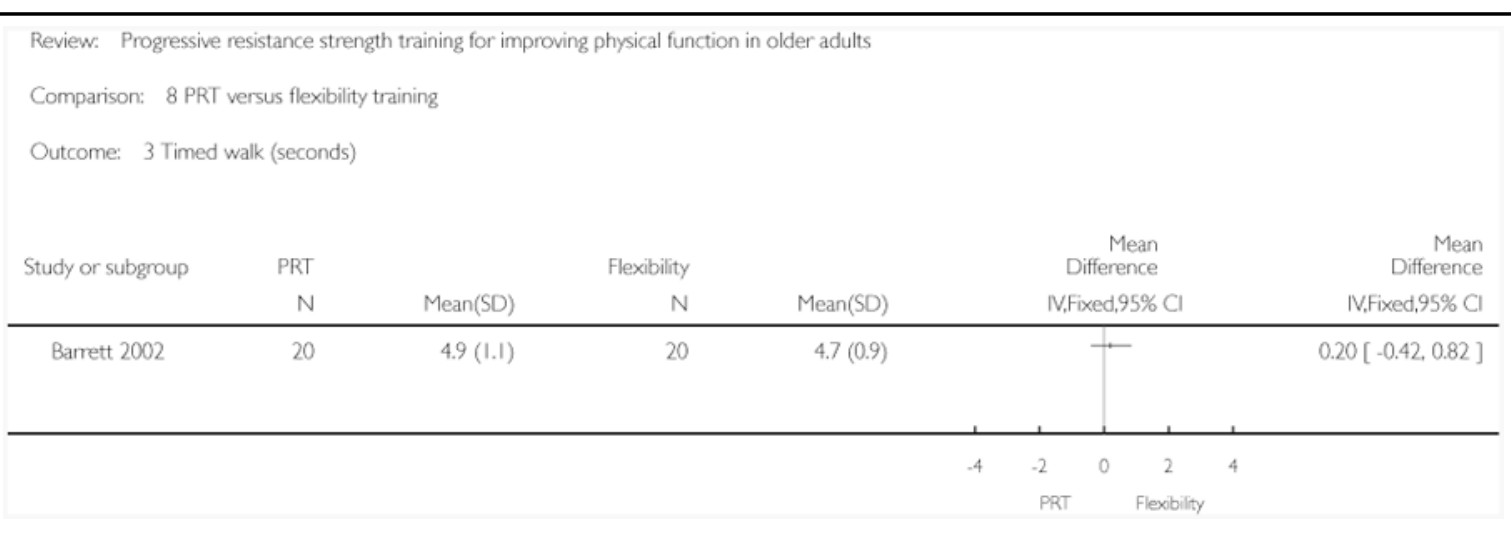




\section{Analysis 8.4. Comparison 8 PRT versus flexibility training, Outcome 4 Time to stand from a chair (seconds)}

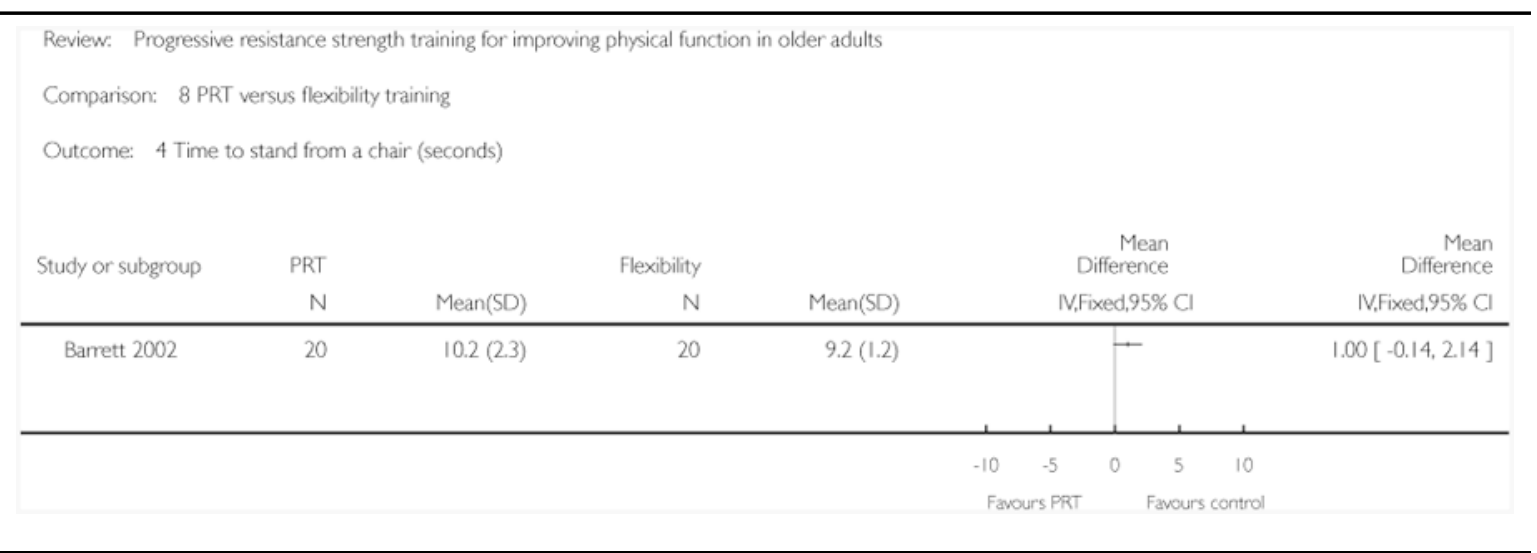

\section{Analysis 8.5. Comparison 8 PRT versus flexibility training, Outcome 5 Vitality (SF-36/Vitality plus scale, higher $=$ more vitality)}

Review: Progressive resistance strength training for improving physical function in older adults

Comparison: 8 PRT versus flexibility training

Outcome: 5 Vitality (SF-36/Vitality plus scale, higher = more vitality)

\begin{tabular}{|c|c|c|c|c|c|c|c|c|c|}
\hline \multirow[t]{2}{*}{ Study or subgroup } & \multirow{2}{*}{$\begin{array}{r}\text { PRT } \\
\mathrm{N}\end{array}$} & \multicolumn{3}{|c|}{ Flexibility } & \multicolumn{4}{|c|}{$\begin{array}{l}\text { Mean } \\
\text { Difference }\end{array}$} & \multirow{2}{*}{$\begin{array}{r}\text { Mean } \\
\text { Difference } \\
\text { IV,Fixed,95\% Cl }\end{array}$} \\
\hline & & Mean(SD) & $\mathrm{N}$ & Mean(SD) & & & Fixed,95\% Cl & & \\
\hline \multirow[t]{3}{*}{ Barrett 2002} & 22 & $53.5(21.7)$ & 22 & $63.5(13.1)$ & & & 4 & & $-10.00[-20.59,0.59]$ \\
\hline & & & & & -100 & -50 & 50 & 100 & \\
\hline & & & & & & ility ex & PRT & & \\
\hline
\end{tabular}




\section{Analysis 8.6. Comparison 8 PRT versus flexibility training, Outcome 6 Pain (higher = less pain, Bodily pain on SF- 36)}

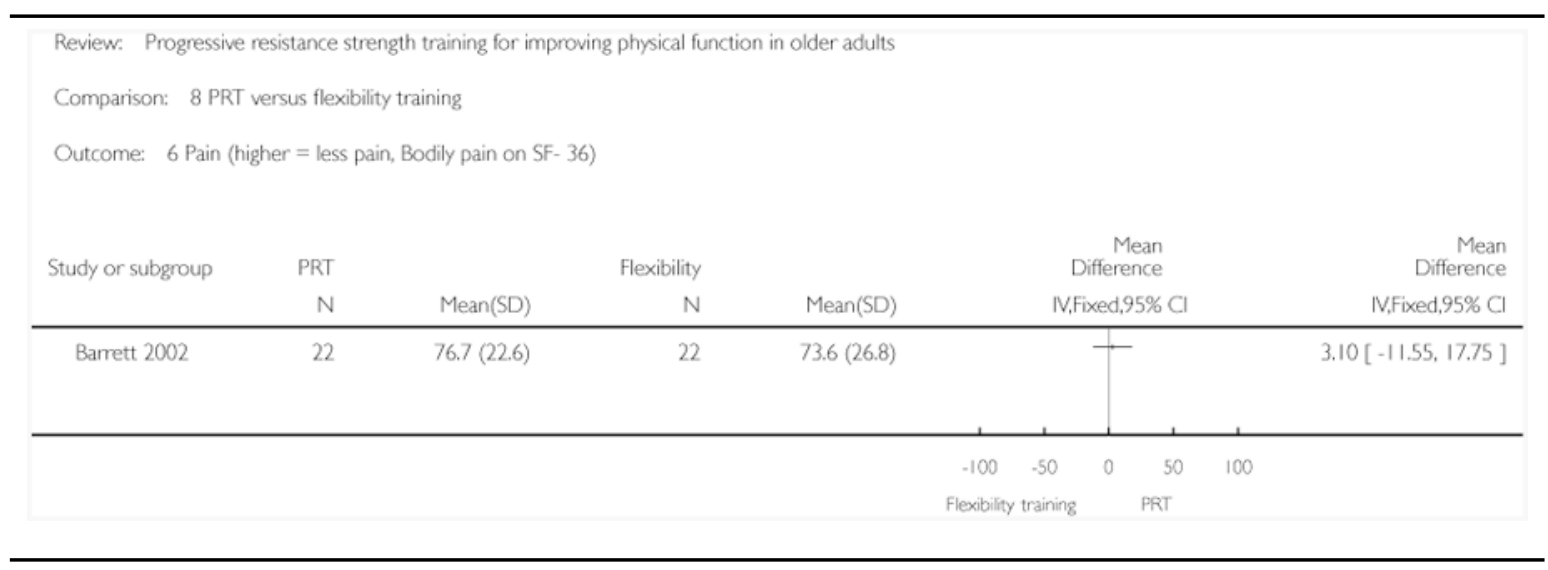

\section{Analysis 9.1. Comparison 9 Power training, Outcome 1 Main lower limb strength measure}

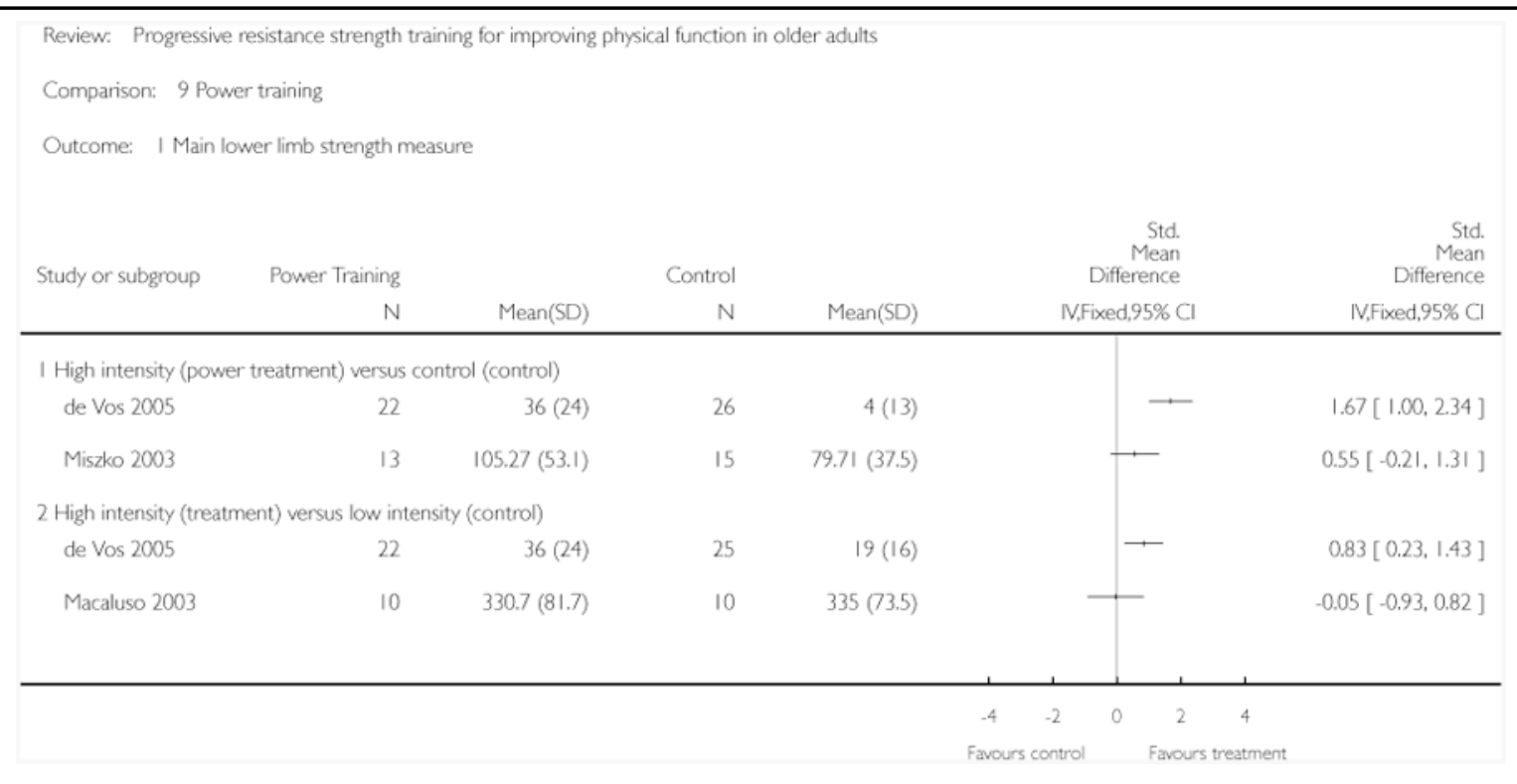




\section{Analysis 10.1. Comparison 10 PRT versus control supplementary analyses, Outcome 1 Strength (grouped by allocation concealment)}

Review. Progressive resistance strength training for improving physical function in older adults

Comparison: 10 PRT versus control supplementary analyses

Outcome: I Strength (grouped by allocation concealment)

\begin{tabular}{|c|c|c|c|c|c|c|c|}
\hline \multirow[t]{2}{*}{ Study or subgroup } & \multirow{2}{*}{$\begin{array}{r}\text { PRT } \\
\mathrm{N}\end{array}$} & \multicolumn{3}{|c|}{ Control } & \multirow{2}{*}{$\begin{array}{r}\text { Std. } \\
\text { Mean } \\
\text { Difference } \\
\text { N.Fixed,95\% Cl }\end{array}$} & \multirow[t]{2}{*}{ Weight } & \multirow{2}{*}{$\begin{array}{r}\text { Std. } \\
\text { Mean } \\
\text { Difference } \\
\text { IV.Fixed,95\% Cl }\end{array}$} \\
\hline & & Mean(SD) & N & $\operatorname{Mean}(\mathrm{SD})$ & & & \\
\hline \multicolumn{8}{|l|}{ I Allocation concealed } \\
\hline Baker 2001 & 19 & $40.6(31)$ & 19 & $34.8(16.4)$ & $\rightarrow-$ & $6.3 \%$ & $0.23[-0.41,0.87]$ \\
\hline Chin A Paw 2006 & 40 & $73.2(12.3)$ & 31 & $77.5(48.3)$ & $\rightarrow$ & $11.6 \%$ & $-0.13[-0.60,0.34]$ \\
\hline Foley 2003 & 26 & $26.73(14.2)$ & 32 & $21.94(11.61)$ & $\rightarrow$ & $9.4 \%$ & $0.37[-0.15,0.89]$ \\
\hline Jette 1999 & 96 & $15(5.3)$ & 104 & $13.7(5.5)$ & $\equiv$ & $33.0 \%$ & $0.24[-0.04,0.52]$ \\
\hline Latham 2001 & 10 & $12.3(7.1)$ & 10 & $7.4(3.5)$ & - & $3.0 \%$ & $0.84[-0.08,1.76]$ \\
\hline Latham 2003 & 108 & $12.6(5.4)$ & 112 & $12.9(5.3)$ & $\equiv$ & $36.6 \%$ & $-0.06[-0.32,0.21]$ \\
\hline Subtotal $(95 \% \mathrm{CI})$ & 299 & & 308 & & - & $100.0 \%$ & $0.12[-0.04,0.28]$ \\
\hline \multicolumn{8}{|c|}{ Heterogeneity: $\mathrm{Ch}^{2}=6.79 . \mathrm{df}=5(\mathrm{P}=0.24): \mathrm{I}^{2}=26 \%$} \\
\hline \multicolumn{8}{|c|}{ Test for overall effect: $Z=1.44(P=0.15)$} \\
\hline \multicolumn{8}{|l|}{2 Concealment unknown } \\
\hline Ades 1996 & 12 & $46(16)$ & 12 & $29(13)$ & $\longrightarrow$ & $0.9 \%$ & $1.13[0.25 .2 .00]$ \\
\hline Beneka 2005 & 8 & $75.2(5.8)$ & 8 & $67.9(5.2)$ & & $0.6 \%$ & $1.25[0.15,2.35]$ \\
\hline Bermon 1999 & 16 & $23.6(4)$ & 16 & $19.2(3.6)$ & $\longrightarrow$ & $1.3 \%$ & $1.13[0.37,1.88]$ \\
\hline Boshuizen 2005 & 16 & $69.3(17.2)$ & 17 & $56.2(29.4)$ & - & $1.5 \%$ & $0.53[-0.17,1.22]$ \\
\hline Brandon 2000 & 43 & $60.1(19.9)$ & 42 & $47.3(13.6)$ & $\leftarrow$ & $3.7 \%$ & $0.74[0.30,1.18]$ \\
\hline Brandon 2003 & 16 & $0.81(0.29)$ & 15 & $0.63(0.15)$ & $\longrightarrow$ & $1.3 \%$ & $0.75[0.02,1.48]$ \\
\hline Brochu 2002 & 21 & $43.6(10.8)$ & 21 & $36.9(14.2)$ & + & $1.9 \%$ & $0.52[-0.09,1.14]$ \\
\hline Bruunsgaard 2004 & 10 & $13.15(3.46)$ & 11 & $9.5(3.2)$ & $\longrightarrow$ & $0.8 \%$ & $1.05[0.13,1.98]$ \\
\hline Buchner 1997 & 22 & $99(31)$ & 29 & $84(32)$ & 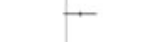 & $2.3 \%$ & $0.47[-0.09,1.03]$ \\
\hline Casaburi 2004 & 12 & $344(98)$ & 12 & $274(66)$ & $\rightarrow$ & $1.0 \%$ & $0.81[-0.03,1.65]$ \\
\hline Castaneda 2001 & 14 & $55.9(22.4)$ & 12 & $38.8(14)$ & $\longrightarrow$ & $1.1 \%$ & $0.87[0.06,1.68]$ \\
\hline Chandler 1998 & 44 & $63.7(27)$ & 43 & $48.3(23)$ & + & $3.9 \%$ & $0.61[0.18,1.04]$ \\
\hline Charette 1991 & 13 & $33.9(7.6)$ & 6 & $23.1(3.4)$ & - & $0.6 \%$ & $1.55[0.44,267]$ \\
\hline \multirow[t]{3}{*}{ Collier 1997} & 24 & $53.8(37)$ & 12 & $328(11.7)$ & - & $1.4 \%$ & $0.66[-0.05,1.37]$ \\
\hline & & & & & 2 & & \\
\hline & & & & & Favou & & \\
\hline
\end{tabular}

Cochrane Database Syst Rev. Author manuscript; available in PMC 2015 February 11. 


\begin{tabular}{|c|c|c|c|c|c|c|c|}
\hline \multirow[t]{2}{*}{ Study or subgroup } & \multirow{2}{*}{$\begin{array}{r}\text { PRT } \\
\text { N }\end{array}$} & \multicolumn{3}{|c|}{ Control } & \multirow{2}{*}{$\begin{array}{r}\text { Std. } \\
\text { Mean } \\
\text { Difference } \\
\text { MVFixed, } 95 \% \mathrm{Cl} \\
\end{array}$} & \multirow[t]{2}{*}{ Weight } & \multirow{2}{*}{$\begin{array}{r}\text { Std. } \\
\text { Mean } \\
\text { Difference } \\
\text { NV.ixed,95\% C }\end{array}$} \\
\hline & & $\operatorname{Mean}(S D)$ & $\mathrm{N}$ & $\operatorname{Mean}(S D)$ & & & \\
\hline Damush 1999 & 33 & $58(17.6)$ & 29 & $52(17,4)$ & $\leftarrow$ & $28 \%$ & $0.34[-0.16,0.84]$ \\
\hline de Vreede 2007 & 28 & $307.2(90.9)$ & 26 & $298.2(81.2)$ & + & $25 \%$ & $0.10[-0.43,0.64]$ \\
\hline DeBeliso 2005 & 13 & $105(40)$ & 13 & $58(25)$ & $\rightarrow$ & $1.0 \%$ & $1.36[0.50 .223]$ \\
\hline Ettinger 1997 & 120 & $90.2(21.9)$ & 127 & $87(21.4)$ & * & $11.5 \%$ & $0.15[-0.10,0.40]$ \\
\hline Fahlman 2002 & 15 & $25(1)$ & 15 & $20(1.9)$ & $\longrightarrow$ & $0.6 \%$ & $3.20[208,4.33]$ \\
\hline Fatouros 2002 & 8 & $116.1(9.8)$ & 8 & $66.5(8.5)$ & - & $0.1 \%$ & $5.11[2.85,7.38]$ \\
\hline Fatouros 2005 & 20 & $91.7(8.2)$ & 14 & $56.4(8.5)$ & - & $0.5 \%$ & $4.14[2.89,5.39]$ \\
\hline Frontera 2003 & 7 & $18.4(5.1)$ & 7 & $12.5(2.8)$ & & $0.5 \%$ & $1.34[0.14,2.54]$ \\
\hline Haykowsky 2000 & 10 & $367(47)$ & 8 & $290(53)$ & $\longrightarrow$ & $0.6 \%$ & $1.48[0.40,2.55]$ \\
\hline Hortobagyi 2001 & 18 & $1122(340)$ & 9 & $877(203)$ & - & $1.0 \%$ & $0.78[-0.05,1.62]$ \\
\hline Hruda 2003 & 18 & $81.9(23.3)$ & 7 & $71(22.2)$ & - & $0.9 \%$ & $0.46[-0.43,1.34]$ \\
\hline Jette 1996 & 35 & $52(18.5)$ & 38 & $45.8(19.1)$ & + & $3.4 \%$ & $0.33[-0.14,0.79]$ \\
\hline Jones 1994 & 30 & $55.17(15.87)$ & 12 & $50.17(8.75)$ & - & $1.6 \%$ & $0.34[-0.33,1.02]$ \\
\hline Judge 1994 & 22 & $8(3.8)$ & 23 & $6.9(1.9)$ & - & $21 \%$ & $0.36[-0.23,0.95]$ \\
\hline Kalapotharakos 2005 & 11 & $43.12(11.49)$ & 10 & $20.75(.16)$ & - & $0.6 \%$ & $2.22[1.08,3.35]$ \\
\hline Kongsgaard 2004 & 6 & $61(7)$ & 7 & $51(6.5)$ & ?. & $0.5 \%$ & $1.38[0.12 .2 .64]$ \\
\hline Lamoureux 2003 & 29 & $31.8(1.7)$ & is & $21.2(2.4)$ & & $0.4 \%$ & $5.31[3.99,6.63]$ \\
\hline Liu-Ambrose 2005 & 32 & $18.9(8.6)$ & 32 & $17.5(6.4)$ & - & $3.0 \%$ & $0.18[-0.31,0.67]$ \\
\hline Maiorana 1997 & 12 & $132.4(26.4)$ & 14 & $116.6(26.6)$ & - & $1.2 \%$ & $0.58[-0.21,1.37]$ \\
\hline Malliou 2003 & 15 & $79.2(6.8)$ & 10 & $65.9(5.2)$ & 一 & $0.7 \%$ & $2.07[1.05,3.08]$ \\
\hline McCartney 1995 & 20 & $124(22)$ & 23 & $98(18)$ & - & $1.6 \%$ & $1.28[0.62,1.94]$ \\
\hline Mkesky 2006 & 59 & $109.4(35.85)$ & 78 & $103.89(35.86)$ & - & $63 \%$ & $0.15[-0.19,0.49]$ \\
\hline Miler 2006 & 25 & $5.9(4.1)$ & 26 & $5.4(3.4)$ & - & $24 \%$ & $0.13[-0.42,0.68]$ \\
\hline Missko 2003 & 13 & $105.27(53.1)$ & 15 & $79.71(37.5)$ & - & $1.2 \%$ & $0.55[-0.21,1.31]$ \\
\hline Neson 1994 & 20 & $37.8(10.4)$ & 19 & $25.8(8.5)$ & $\leftarrow$ & $1.5 \%$ & $1.23[0.54,1.93]$ \\
\hline Newnham 1995 & 12 & $15.3(7.5)$ & 12 & $7.4(3.6)$ & $\rightarrow$ & $0.9 \%$ & $1.30[0.40,2.19]$ \\
\hline Nichols 1993 & 15 & $39.2(127)$ & 15 & $322(9.7)$ & - & $1.3 \%$ & $0.60[-0.13,1.34]$ \\
\hline Parkhouse 2000 & 13 & $98(21.6)$ & 9 & 54 (18) & 一 & $0.6 \%$ & $2.09[1.00,3.18]$ \\
\hline Pollock 1991 & 20 & $53.4(21.1)$ & 12 & $43.2(20.3)$ & - & $1.4 \%$ & $0.48[-0.25,1.20]$ \\
\hline Pu 2001 & 9 & $1630(516)$ & 7 & $1292(352)$ & - & $0.7 \%$ & $0.71[-0.32,1.73]$ \\
\hline Rall 1996 & 8 & $47.3(14.8)$ & 6 & $32.2(11.2)$ & - & $0.5 \%$ & $1.05[-0.10 .221]$ \\
\hline
\end{tabular}

Cochrane Database Syst Rev. Author manuscript; available in PMC 2015 February 11. 


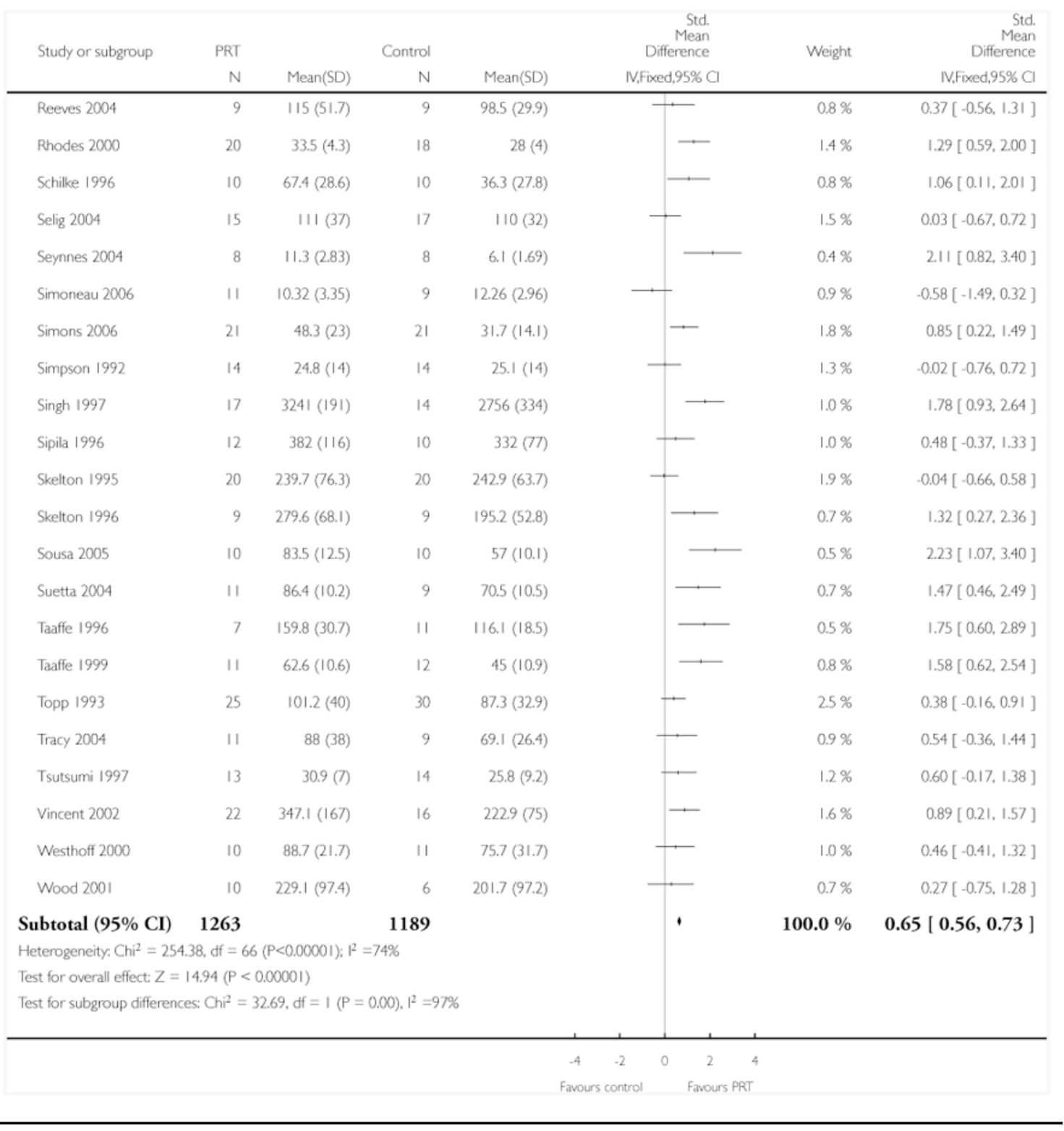

Cochrane Database Syst Rev. Author manuscript; available in PMC 2015 February 11. 


\section{Analysis 10.2. Comparison 10 PRT versus control supplementary analyses, Outcome 2 Strength (grouped by assessor blinding)}

Review. Progressive resistance strength training for improving physical function in older adults

Comparison: 10 PRT versus control supplementary analyses

Outcome: 2 Strength (grouped by assessor blinding)

\begin{tabular}{|c|c|c|c|c|c|c|c|}
\hline \multirow[t]{2}{*}{ Study or subgroup } & \multirow{2}{*}{$\begin{array}{r}\text { PRT } \\
\mathrm{N}\end{array}$} & \multicolumn{3}{|c|}{ Control } & \multirow{2}{*}{$\begin{array}{r}\text { Std. } \\
\text { Mean } \\
\text { Difference } \\
\text { N.Fixed, } 95 \% \mathrm{Cl}\end{array}$} & \multirow[t]{2}{*}{ Weight } & \multirow{2}{*}{$\begin{array}{r}\text { Std. } \\
\text { Mean } \\
\text { Difference } \\
\text { IV.Fixed, } 95 \% \mathrm{Cl}\end{array}$} \\
\hline & & $\operatorname{Mean}(\mathrm{SD})$ & $N$ & $\operatorname{Mean}(\mathrm{SD})$ & & & \\
\hline \multicolumn{8}{|l|}{ I Blinded assessors } \\
\hline Boshuizen 2005 & 16 & $69.3(17.2)$ & 17 & $56.2(29.4)$ & 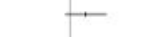 & $2.1 \%$ & $0.53[-0.17,1.22]$ \\
\hline Buchner 1997 & 22 & $99(31)$ & 29 & $84(32)$ & 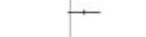 & $3.3 \%$ & $0.47[-0.09,1.03]$ \\
\hline Casaburi 2004 & 12 & 344 (98) & 12 & $274(66)$ & + & $1.5 \%$ & $0.81[-0.03,1.65]$ \\
\hline Chandler 1998 & 44 & $63.7(27)$ & 43 & $48.3(23)$ & $\bullet$ & $5.6 \%$ & $0.61[0.18,1.04]$ \\
\hline Chin A Paw 2006 & 40 & $73.2(123)$ & 31 & $77.5(48.3)$ & - & $4.7 \%$ & $-0.13[-0.60,0.34]$ \\
\hline de Vreede 2007 & 28 & $307.2(90.9)$ & 26 & $298.2(81.2)$ & + & $3.6 \%$ & $0.10[-0.43,0.64]$ \\
\hline Ettinger 1997 & 120 & $90.2(21.9)$ & 127 & $87(21.4)$ & 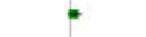 & $16.6 \%$ & $0.15[-0.10,0.40]$ \\
\hline Foley 2003 & 26 & $26.73(14.2)$ & 32 & $21.94(11.61)$ & $\leftarrow$ & $3.8 \%$ & $0.37[-0.15,0.89]$ \\
\hline Jette 1996 & 35 & $52(18.5)$ & 38 & $45.8(19.1)$ & $\leftarrow$ & $4.9 \%$ & $0.33[-0.14,0.79]$ \\
\hline Jette 1999 & 96 & $15(5.3)$ & 104 & $13.7(5.5)$ & $*$ & $13.4 \%$ & $0.24[-0.04,0.52]$ \\
\hline Jones 1994 & 30 & $55.17(15.87)$ & 12 & $50.17(8.75)$ & 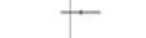 & $2.3 \%$ & $0.34[-0.33,1.02]$ \\
\hline Judge 1994 & 22 & $8(3.8)$ & 23 & $6.9(1.9)$ & 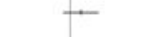 & $3.0 \%$ & $0.36[-0.23,0.95]$ \\
\hline Kalapotharakos 2005 & 11 & $43.12(11.49)$ & 10 & $20.75(7.16)$ & & $0.8 \%$ & $2.22[1.08,3.35]$ \\
\hline Latham 2003 & 108 & $12.6(5.4)$ & 112 & $12.9(5.3)$ & $*$ & $14.9 \%$ & $-0.06[-0.32,0.21]$ \\
\hline Liu-Ambrose 2005 & 32 & $18.9(8.6)$ & 32 & $17.5(6.4)$ & $\leftarrow$ & $4.3 \%$ & $0.18[-0.31,0.67]$ \\
\hline Mikesky 2006 & 59 & $109.4(35.85)$ & 78 & $103.89(35.86)$ & $\rightarrow$ & $9.1 \%$ & $0.15[-0.19,0.49]$ \\
\hline Miller 2006 & 25 & $5.9(4.1)$ & 26 & $5.4(3.4)$ & 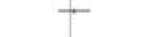 & $3.4 \%$ & $0.13[-0.42,0.68]$ \\
\hline Newnham 1995 & 12 & $15.3(7.5)$ & 12 & $7.4(3.6)$ & $\longrightarrow$ & $1.3 \%$ & $1.30[0.40 .2 .19]$ \\
\hline Westhoff 2000 & 10 & $88.7(21.7)$ & 11 & $75.7(31.7)$ & + & $1.4 \%$ & $0.46[-0.41,1.32]$ \\
\hline Subtotal $(95 \% \mathrm{CI})$ & 748 & & 775 & & - & $100.0 \%$ & $0.23[0.13,0.34]$ \\
\hline \multicolumn{8}{|c|}{ Heterogeneity: $\mathrm{Chi}^{2}=32.15 . \mathrm{df}=18(\mathrm{P}=0.02): \mathrm{I}^{2}=44 \%$} \\
\hline \multicolumn{8}{|c|}{ Test for overall effect: $Z=4.51(P<0.00001)$} \\
\hline \multicolumn{8}{|c|}{2 Assessors were not blinded } \\
\hline \multirow[t]{3}{*}{ Ades 1996} & 12 & $46(16)$ & 12 & $29(13)$ & $\longrightarrow$ & $1.6 \%$ & $1.13[0.25 .200]$ \\
\hline & & & & & -2 & 4 & \\
\hline & & & & & ontrol & & \\
\hline
\end{tabular}

Cochrane Database Syst Rev. Author manuscript; available in PMC 2015 February 11. 


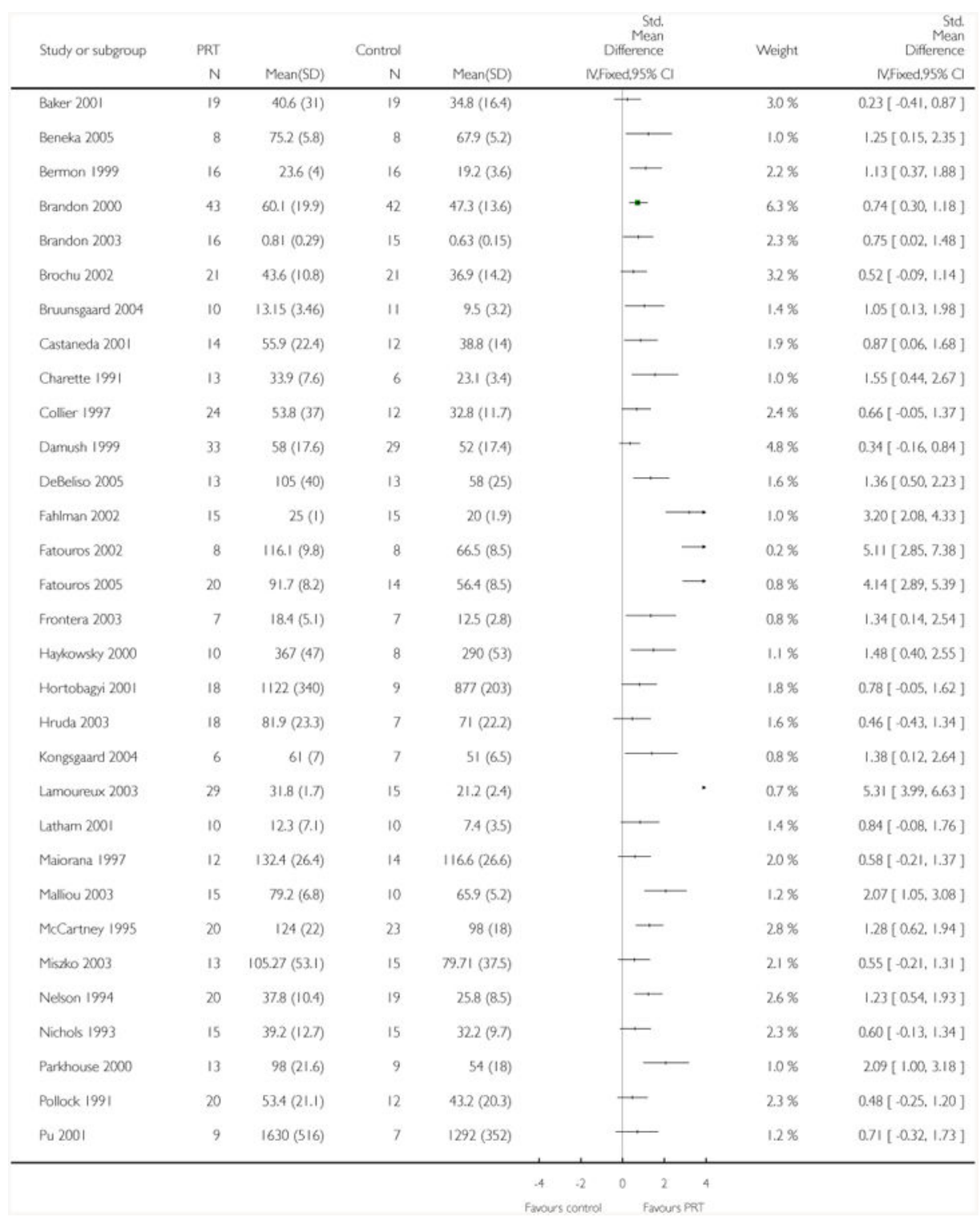

Cochrane Database Syst Rev. Author manuscript; available in PMC 2015 February 11. 


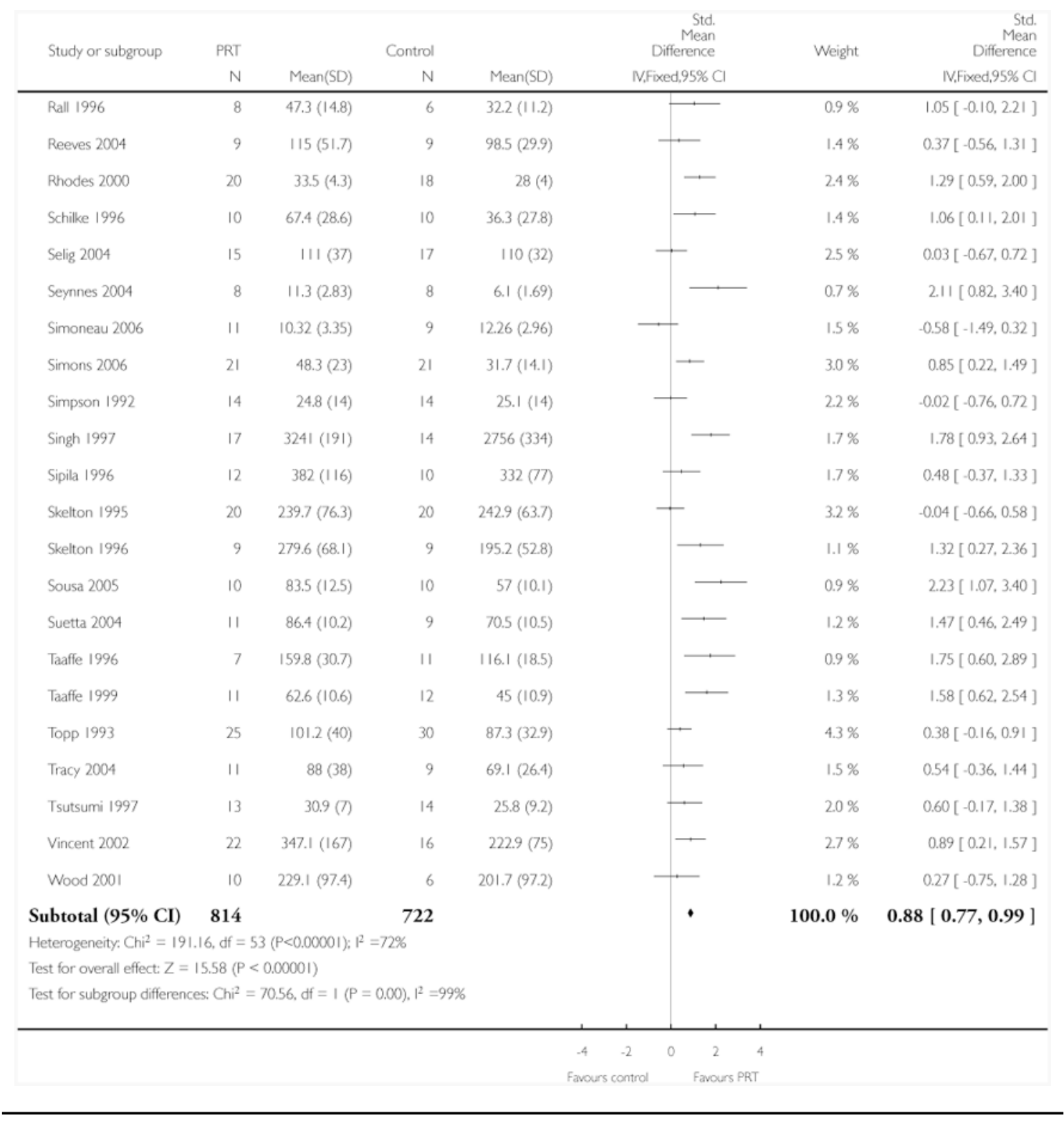

Cochrane Database Syst Rev. Author manuscript; available in PMC 2015 February 11. 


\section{Analysis 10.3. Comparison 10 PRT versus control supplementary analyses, Outcome 3 Strength (grouped by intention-to-treat)}

\begin{tabular}{|c|c|c|c|c|c|c|c|}
\hline \multirow{3}{*}{\multicolumn{4}{|c|}{$\begin{array}{l}\text { Review. Progressive resistance strength training for improving phys } \\
\text { Comparison: 10 PRT versus control supplementary analyses } \\
\text { Outcome: 3 Strength (grouped by intention-to-treat) } \\
\text { Study or subgroup } \quad \text { PRT }\end{array}$}} & \multirow{3}{*}{ Mean(SD) } & \multirow{3}{*}{$\begin{array}{l}\text { Std. } \\
\text { Mean } \\
\text { Difference } \\
\text { IV.Fixed,95\% a }\end{array}$} & \multirow{3}{*}{ Weight } & \multirow{3}{*}{$\begin{array}{r}\text { Std. } \\
\text { Mean } \\
\text { Difference } \\
\text { IV.Fixed,95\% C }\end{array}$} \\
\hline & & & & & & & \\
\hline & & & & & & & \\
\hline I Intention-to-treat was us & & & & & & & \\
\hline Baker 2001 & 19 & $40.6(31)$ & 19 & $34.8(16.4)$ & - & $1.4 \%$ & $0.23[-0.41,0.87]$ \\
\hline Buchner 1997 & 22 & $99(31)$ & 29 & $84(32)$ & + & $1.8 \%$ & $0.47[-0.09,1.03]$ \\
\hline Chin A Paw 2006 & 40 & $73.2(12.3)$ & 31 & $77.5(48.3)$ & $T$ & $2.5 \%$ & $-0.13[-0.60,0.34]$ \\
\hline Ettinger 1997 & 120 & $90.2(21.9)$ & 127 & $87(21.4)$ & * & $9.0 \%$ & $0.15[-0.10,0.40]$ \\
\hline Foley 2003 & 26 & $26.73(14.2)$ & 32 & $21.94(11.61)$ & $\rightarrow$ & $2.1 \%$ & $0.37[-0.15,0.89]$ \\
\hline Judge 1994 & 22 & $8(3.8)$ & 23 & $6.9(1.9)$ & + & $1.6 \%$ & $0.36[-0.23,0.95]$ \\
\hline Latham 2003 & 108 & $12.6(5.4)$ & 112 & $12.9(5.3)$ & 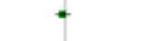 & $8.0 \%$ & $-0.06[-0.32,0.21]$ \\
\hline Liu-Ambrose 2005 & 32 & $18.9(8.6)$ & 32 & $17.5(6.4)$ & + & $23 \%$ & $0.18[-0.31,0.67]$ \\
\hline Mikesky 2006 & 59 & $109.4(35.85)$ & 78 & $103.89(35.86)$ & + & $4.9 \%$ & $0.15[-0.19,0.49]$ \\
\hline Miller 2006 & 25 & $5.9(4.1)$ & 26 & $5.4(3.4)$ & - & $1.9 \%$ & $0.13[-0.42,0.68]$ \\
\hline Nelson 1994 & 20 & $37.8(10.4)$ & 19 & $25.8(8.5)$ & $\rightarrow$ & $1.2 \%$ & $1.23[0.54,1.93]$ \\
\hline Suetta 2004 & 11 & $86.4(10.2)$ & 9 & $70.5(10.5)$ & $\longrightarrow$ & $0.5 \%$ & $1.47[0.46,2.49]$ \\
\hline Subtotal $(95 \% \mathrm{CI})$ & 504 & & 537 & & - & $37.2 \%$ & $0.18[0.06,0.30]$ \\
\hline Heterogeneity, $\mathrm{Chi}^{2}=21.8$ & $d f=11$ & $=0.03): 1^{2}=50$ & & & & & \\
\hline Test for overall effect: $Z$ = & $8(P=0$ & 040) & & & & & \\
\hline 2 Intention-to-treat was no & sed & & & & & & \\
\hline Ades 1996 & 12 & $46(16)$ & 12 & $29(13)$ & $\longrightarrow$ & $0.7 \%$ & $1.13[0.25 .2 .00]$ \\
\hline Beneka 2005 & 8 & $75.2(5.8)$ & 8 & $67.9(5.2)$ & & $0.5 \%$ & $1.25[0.15 .2 .35]$ \\
\hline Bermon 1999 & 16 & $23.6(4)$ & 16 & $19.2(3.6)$ & $\rightarrow$ & $1.0 \%$ & $1.13[0.37,1.88]$ \\
\hline Boshuizen 2005 & 16 & $69.3(17.2)$ & 17 & $56.2(29.4)$ & - & $1.2 \%$ & $0.53[-0.17,1.22]$ \\
\hline Brandon 2000 & 43 & $60.1(19.9)$ & 42 & $47.3(13.6)$ & $\leftarrow$ & $29 \%$ & $0.74[0.30,1.18]$ \\
\hline Brandon 2003 & 16 & $0.81(0.29)$ & 15 & $0.63(0.15)$ & $\rightarrow$ & $1.0 \%$ & $0.75[0.02,1.48]$ \\
\hline Brochu 2002 & 21 & $43.6(10.8)$ & 21 & $36.9(14.2)$ & - & $1.5 \%$ & $0.52[-0.09,1.14]$ \\
\hline Bruunsgaard 2004 & 10 & $13.15(3.46)$ & 11 & $9.5(3.2)$ & $\rightarrow$ & $0.7 \%$ & $1.05[0.13,1.98]$ \\
\hline & & & & & 2 & & \\
\hline & & & & & Favour & & \\
\hline
\end{tabular}

Cochrane Database Syst Rev. Author manuscript; available in PMC 2015 February 11. 


\begin{tabular}{|c|c|c|c|c|c|c|c|}
\hline \multirow[t]{2}{*}{ Study or subgroup } & \multirow{2}{*}{$\begin{array}{r}\text { PRT } \\
\mathrm{N}\end{array}$} & \multicolumn{3}{|c|}{ Control } & \multirow{2}{*}{$\begin{array}{r}\text { Std. } \\
\text { Mean } \\
\text { Difference } \\
\text { IV.fixed,95\% CI } \\
\end{array}$} & \multirow[t]{2}{*}{ Weight } & \multirow{2}{*}{ 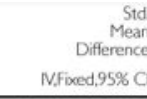 } \\
\hline & & $\operatorname{Mean}(\mathrm{SD})$ & $\mathrm{N}$ & $\operatorname{Mean}(\mathrm{SD})$ & & & \\
\hline Cassaburi 2004 & 12 & $344(98)$ & 12 & $274(66)$ & 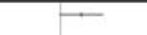 & $0.8 \%$ & $0.81[-0.03,1.65]$ \\
\hline Castaneda 2001 & 14 & $55.9(22.4)$ & 12 & $38.8(14)$ & - & $0.9 \%$ & $0.87[0.06,1.68]$ \\
\hline Chander 1998 & 44 & $63.7(27)$ & 43 & $48.3(23)$ & - & $3.0 \%$ & $0.61[0.18,1.04]$ \\
\hline Charette 1991 & 13 & $33.9(7.6)$ & 6 & $23.1(3.4)$ & $\longrightarrow$ & $0.5 \%$ & $1.55[0.44 .267]$ \\
\hline Collier 1997 & 24 & $538(37)$ & 12 & $32.8(11.7)$ & - & $1.1 \%$ & $0.66[-0.05,1.37]$ \\
\hline Damush 1999 & 33 & $58(17.6)$ & 29 & $52(17.4)$ & - & $22 \%$ & $0.34[-0.16,0.84]$ \\
\hline de Vreede 2007 & 28 & $307.2(90.9)$ & 26 & $298.2(81.2)$ & - & $20 \%$ & $0.10[-0.43,0.64]$ \\
\hline DeBeliso 2005 & 13 & $105(40)$ & 13 & $58(25)$ & $\rightarrow$ & $0.7 \%$ & $1.36[0.50 .2 .23]$ \\
\hline Fatiman 2002 & 15 & $25(1)$ & 15 & $20(1.9)$ & - & $0.4 \%$ & $3.20[208,4.33]$ \\
\hline Fatouros 2002 & 8 & $\mid 16.1(9.8)$ & 8 & $66.5(85)$ & & $0.1 \%$ & $5.11[285,7.38]$ \\
\hline Fatouros 2005 & 20 & $91.7(8.2)$ & 14 & $56.4(8.5)$ & & $0.4 \%$ & $4.14[289,5.39]$ \\
\hline Frontera 2003 & 7 & $18.4(5.1)$ & 7 & $125(28)$ & 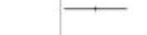 & $0.4 \%$ & $1.34[0.14,2.54]$ \\
\hline Haykowsky 2000 & 10 & $367(47)$ & 8 & $290(53)$ & $\longrightarrow$ & $0.5 \%$ & $1.48[0.40,2.55]$ \\
\hline Hortobaggi 2001 & 18 & $1122(340)$ & 9 & $877(203)$ & - & $0.8 \%$ & $0.78[-0.05,1.62]$ \\
\hline Hruda 2003 & 18 & $81.9(23.3)$ & 7 & $71(22.2)$ & - & $0.7 \%$ & $0.46[-0.43,1.34]$ \\
\hline Jette 1996 & 35 & $52(18.5)$ & 38 & 45.8 (19.1) & - & $26 \%$ & $0.33[-0.14,0.79]$ \\
\hline Jette 1999 & 96 & $15(5.3)$ & 104 & $13.7(5.5)$ & - & $7.2 \%$ & $0.24[-0.04,0.52]$ \\
\hline Jones 1994 & 30 & $55.17(15.87)$ & 12 & $50.17(8.75)$ & - & $1.2 \%$ & $0.34[-0.33,1.02]$ \\
\hline Kalapotharakos 2005 & 11 & $43.12(11.49)$ & 10 & $20.75(7.16)$ & - & $0.4 \%$ & $2.22[1.08,3.35]$ \\
\hline Kongsgaard 2004 & 6 & $61(7)$ & 7 & $51(6.5)$ & - & $0.4 \%$ & $1.38[0.12 .2 .64]$ \\
\hline Lamoureux 2003 & 29 & $31.8(1.7)$ & 15 & $21.2(24)$ & & $0.3 \%$ & $5.31[3.99,6.63]$ \\
\hline Latham 2001 & 10 & $12.3(7.1)$ & 10 & $7.4(3.5)$ & - & $0.7 \%$ & $0.84[-0.08,1.76]$ \\
\hline Maiorana 1997 & 12 & $1324(26.4)$ & 14 & $116.6(26.6)$ & - & $0.9 \%$ & $0.58[-0.21,1.37]$ \\
\hline Malliou 2003 & 15 & $79.2(6.8)$ & 10 & $65.9(5.2)$ & $\rightarrow$ & $0.5 \%$ & $2.07[1.05 .3 .08]$ \\
\hline McCartney 1995 & 20 & $124(22)$ & 23 & $98(18)$ & - & $1.3 \%$ & $1.28[0.62 .1 .94]$ \\
\hline Miszko 2003 & 13 & $105.27(53.1)$ & 15 & 79.71 (37.5) & $\leftarrow$ & $1.0 \%$ & $0.55[-0.21,1.31]$ \\
\hline Newnham 1995 & 12 & $15.3(7.5)$ & 12 & $7.4(3.6)$ & - & $0.7 \%$ & $1.30[0.40,2.19]$ \\
\hline Nichols 1993 & 15 & $39.2(12.7)$ & 15 & $32.2(9.7)$ & - & $1.0 \%$ & $0.60[-0.13,1.34]$ \\
\hline Parkhouse 2000 & 13 & $98(21.6)$ & 9 & 54 (18) & $\longrightarrow$ & $0.5 \%$ & $2.09[1.00,3.18]$ \\
\hline Pollock 1991 & 20 & $53.4(21.1)$ & 12 & $43.2(20.3)$ & - & $1.1 \%$ & $0.48[-0.25,1.20]$ \\
\hline Pu 2001 & 9 & $1630(516)$ & 7 & $1292(352)$ & - & $0.5 \%$ & $0.71[-0.32,1.73]$ \\
\hline
\end{tabular}

Cochrane Database Syst Rev. Author manuscript; available in PMC 2015 February 11. 


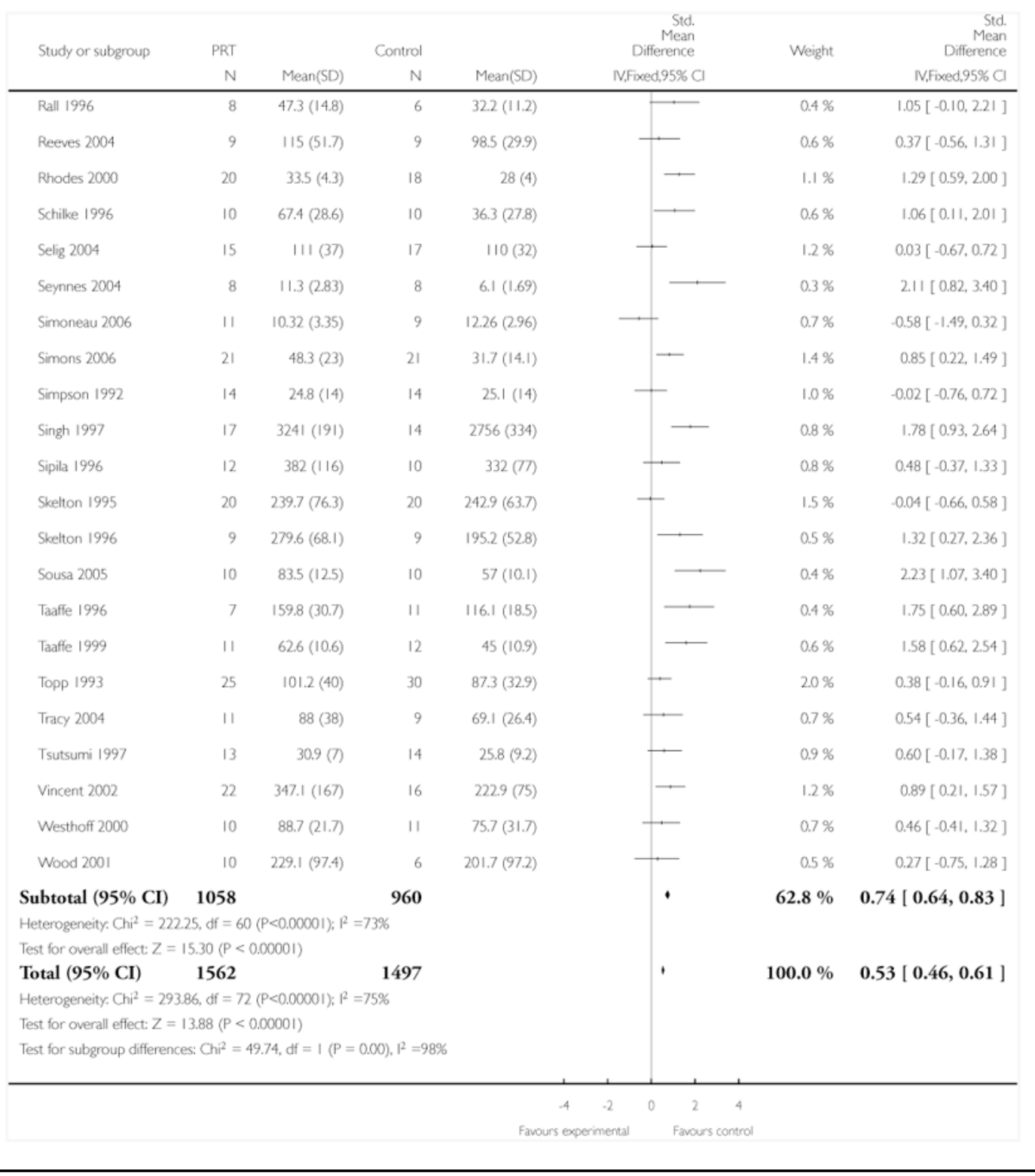

Cochrane Database Syst Rev. Author manuscript; available in PMC 2015 February 11. 


\section{Analysis 10.4. Comparison 10 PRT versus control supplementary analyses, Outcome 4 Strength (grouped by attention control)}

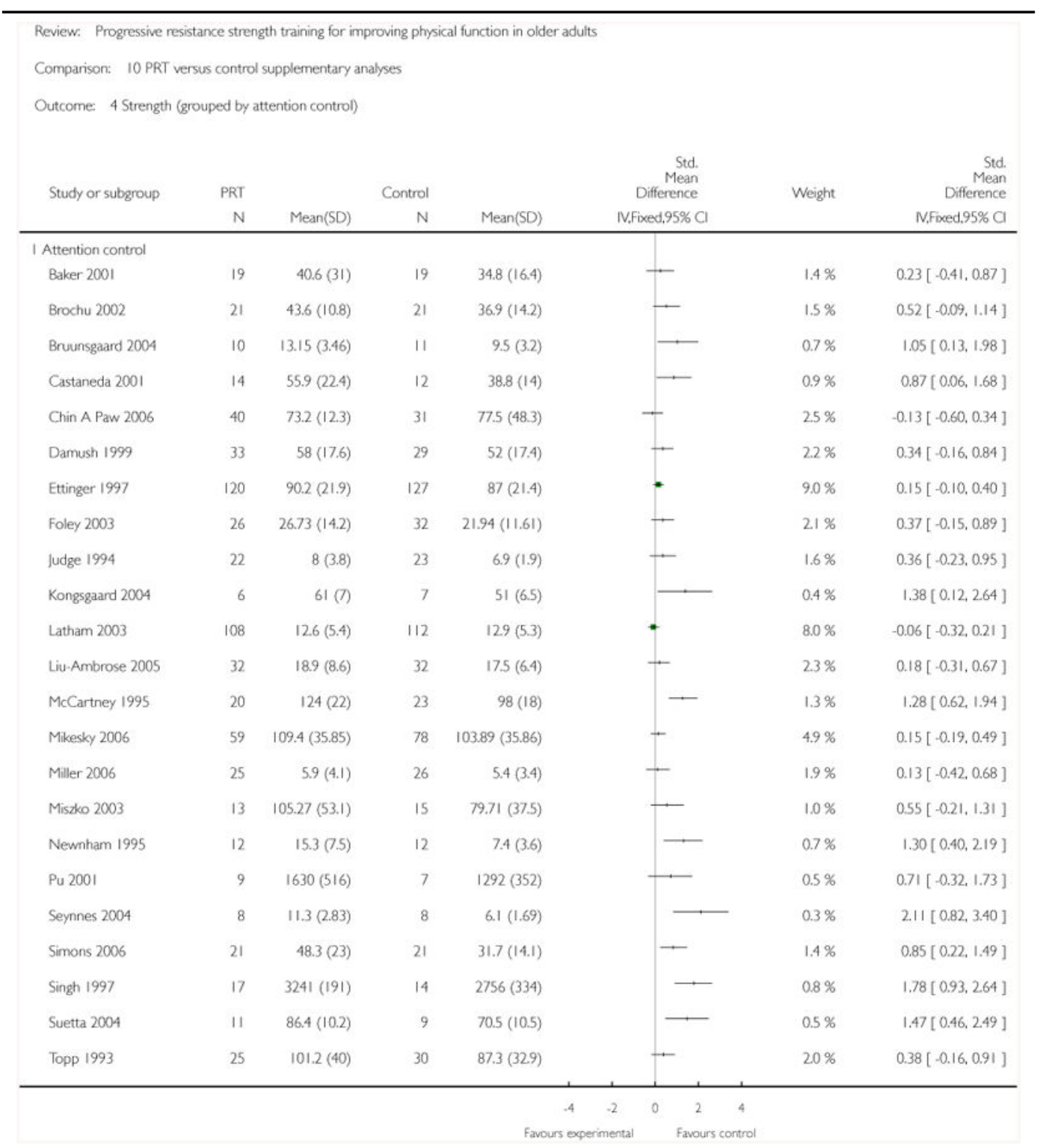

Cochrane Database Syst Rev. Author manuscript; available in PMC 2015 February 11. 


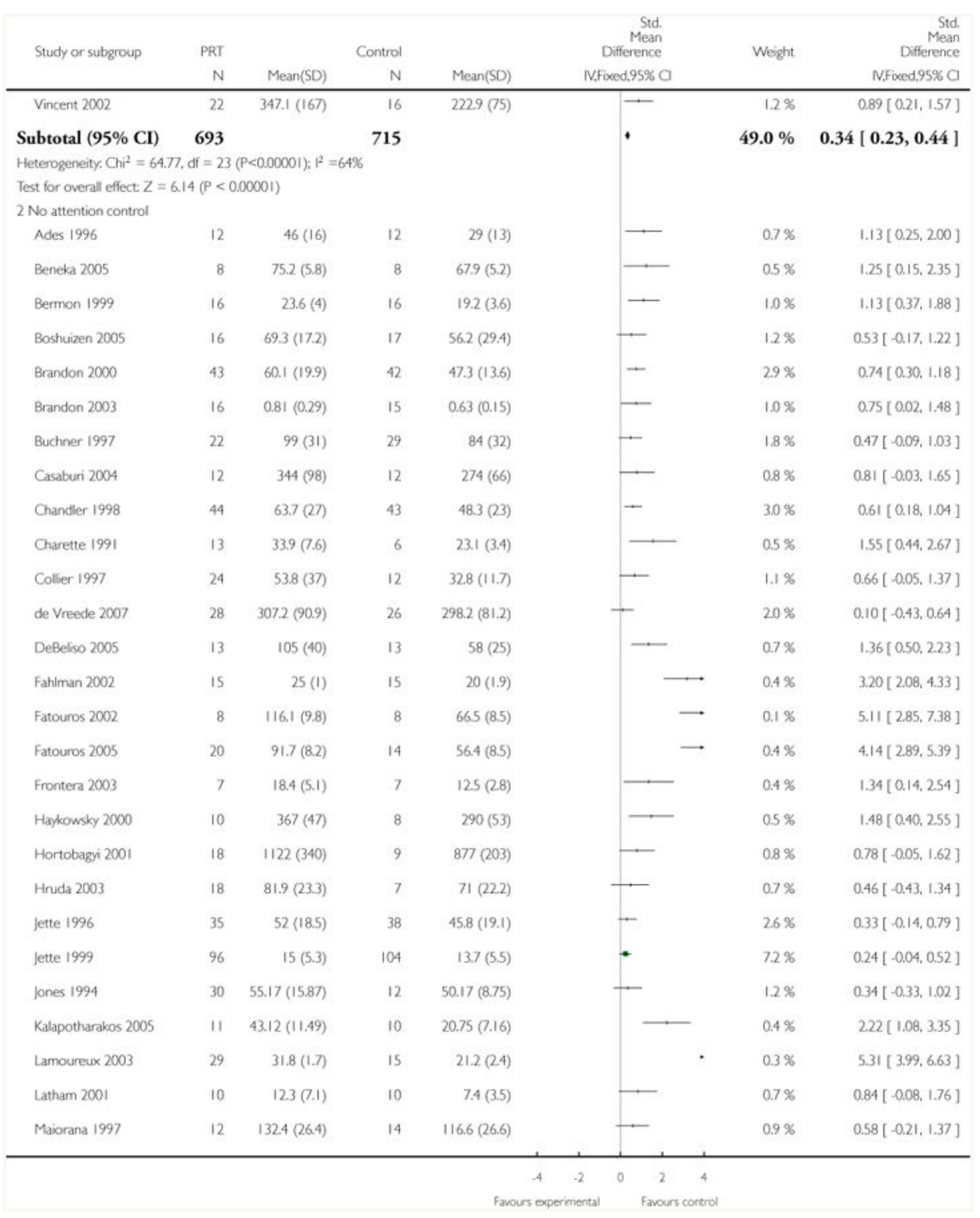

Cochrane Database Syst Rev. Author manuscript; available in PMC 2015 February 11. 


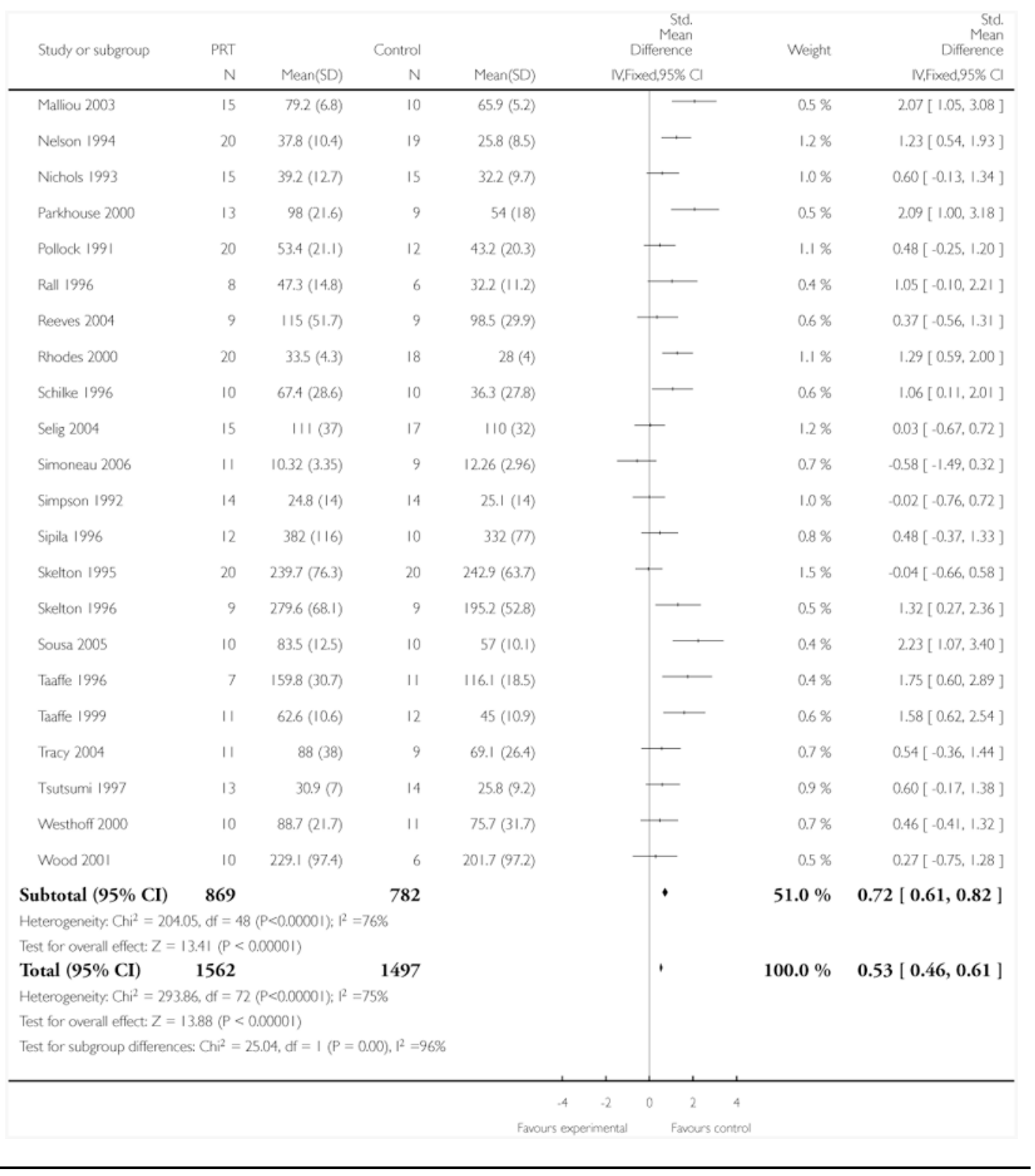

Cochrane Database Syst Rev. Author manuscript; available in PMC 2015 February 11. 


\section{Analysis 10.5. Comparison 10 PRT versus control supplementary analyses, Outcome 5 Strength (grouped by exercise intensity)}

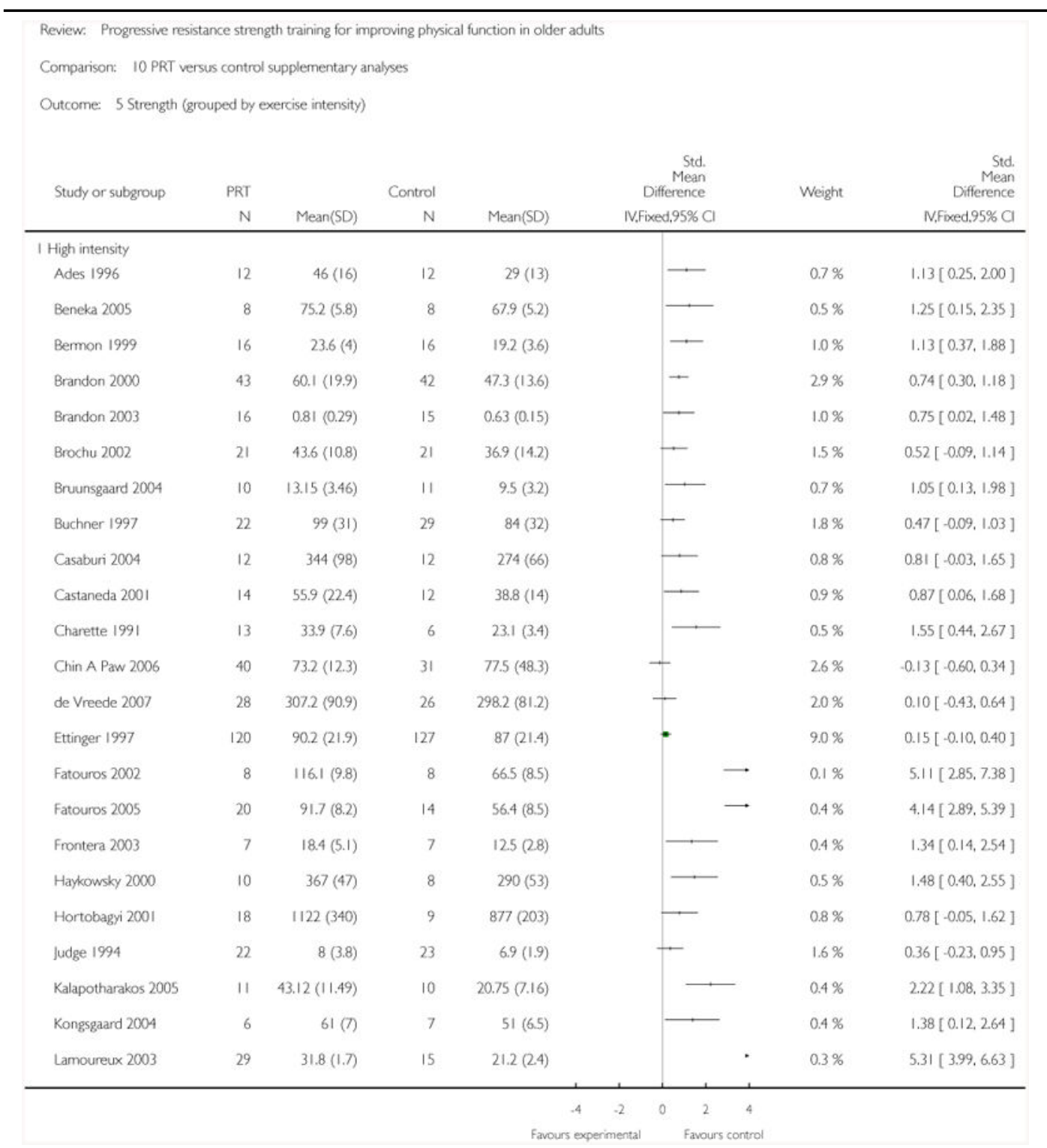

Cochrane Database Syst Rev. Author manuscript; available in PMC 2015 February 11. 


\begin{tabular}{|c|c|c|c|c|c|c|c|}
\hline \multirow[t]{2}{*}{ Study or subgroup } & \multirow{2}{*}{$\begin{array}{l}\text { PRT } \\
\text { N }\end{array}$} & \multicolumn{3}{|c|}{ Control } & \multirow{2}{*}{$\begin{array}{r}\text { Std. } \\
\text { Mean } \\
\text { Difference } \\
\text { NV.fixed,95\% C }\end{array}$} & \multirow[t]{2}{*}{ Weight } & \multirow{2}{*}{$\begin{array}{r}\text { Std. } \\
\text { Mean } \\
\text { Difference } \\
\text { IV.fixed,95\% C } \\
\end{array}$} \\
\hline & & $\operatorname{Mean}(\mathrm{SD})$ & $\mathrm{N}$ & $\operatorname{Mean}(\mathrm{SD})$ & & & \\
\hline Latham 2001 & 10 & $12.3(7.1)$ & 10 & $7.4(3.5)$ & 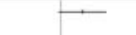 & $0.7 \%$ & $0.84[-0.08,1.76]$ \\
\hline Latham 2003 & 108 & $12.6(5.4)$ & 112 & $12.9(5.3)$ & $\bullet$ & $8.1 \%$ & $-0.06[-0.32,0.21]$ \\
\hline Liu-Ambrose 2005 & 32 & $189(8.6)$ & 32 & $17.5(6.4)$ & 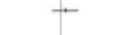 & $2.3 \%$ & $0.18[-0.31,0.67]$ \\
\hline Maiorana 1997 & 12 & $132.4(26.4)$ & 14 & $116.6(26.6)$ & - & $0.9 \%$ & $0.58[-0.21,1.37]$ \\
\hline Malliou 2003 & 15 & $79.2(6.8)$ & 10 & $65.9(5.2)$ & $\longrightarrow$ & $0.5 \%$ & $2.07[1.05,3.08]$ \\
\hline McCartney 1995 & 20 & $124(22)$ & 23 & 98 (18) & $一$ & $1.3 \%$ & $1.28[0.62 .1 .94]$ \\
\hline Miller 2006 & 25 & $5.9(4.1)$ & 26 & $5.4(3.4)$ & 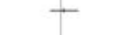 & $1.9 \%$ & $0.13[-0.42 .0 .68]$ \\
\hline Misdko 2003 & 13 & $105.27(53.1)$ & 15 & $79.71(37.5)$ & - & $1.0 \%$ & $0.55[-0.21,1.31]$ \\
\hline Neson 1994 & 20 & $37.8(10.4)$ & 19 & $25.8(8.5)$ & 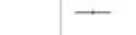 & $1.2 \%$ & $1.23[0.54,1.93]$ \\
\hline Newnham 1995 & 12 & $15.3(7.5)$ & 12 & $7.4(3.6)$ & $\rightarrow$ & $0.7 \%$ & $1.30[0.40 .2 .19]$ \\
\hline Nichols 1993 & 15 & $39.2(127)$ & 15 & $322(9.7)$ & - & $1.0 \%$ & $0.60[-0.13,1.34]$ \\
\hline Parkhouse 2000 & 13 & $98(21.6)$ & 9 & $54(18)$ & $\longrightarrow$ & $0.5 \%$ & $2.09[1.00,3.18]$ \\
\hline Pollock 1991 & 20 & $53.4(21.1)$ & 12 & $43.2(20.3)$ & - & $1.1 \%$ & $0.48[-0.25,1.20]$ \\
\hline Pu 2001 & 9 & $1630(516)$ & 7 & $1292(352)$ & - & $0.5 \%$ & $0.71[-0.32,1.73]$ \\
\hline Rall 1996 & 8 & $47.3(14.8)$ & 6 & $32.2(11.2)$ & 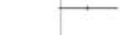 & $0.4 \%$ & $1.05[-0.10 .2 .21]$ \\
\hline Reeves 2004 & 9 & $115(51.7)$ & 9 & $98.5(29.9)$ & - & $0.6 \%$ & $0.37[-0.56,1.31]$ \\
\hline Rhodes 2000 & 20 & $33.5(4.3)$ & 18 & $28(4)$ & 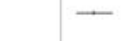 & $1.1 \%$ & $1.29[0.59 .200]$ \\
\hline Schilke 1996 & 10 & $67.4(28.6)$ & 10 & $36.3(27.8)$ & {[} & $0.6 \%$ & $1.06[0.11,2.01]$ \\
\hline Seynnes 2004 & 8 & $11.3(283)$ & 8 & $6.1(1.69)$ & - & $0.3 \%$ & $2.11[0.82 .3 .40]$ \\
\hline Simoneau 2006 & 11 & $10.32(3.35)$ & 9 & $12.26(2.96)$ & 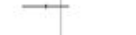 & $0.7 \%$ & $-0.58[-1.49,0.32]$ \\
\hline Simons 2006 & 21 & $48.3(23)$ & 21 & $31.7(14.1)$ & - & $1.4 \%$ & $0.85[0.22,1.49]$ \\
\hline Simpson 1992 & 14 & $24.8(14)$ & 14 & $25.1(14)$ & - & $1.0 \%$ & $-0.02[-0.76,0.72]$ \\
\hline Singh 1997 & 17 & 3241 (191) & 14 & 2756 (334) & - & $0.8 \%$ & $1.78[0.93 .2 .64]$ \\
\hline Sipila 1996 & 12 & 382 (116) & 10 & $332(77)$ & $\leftarrow$ & $0.8 \%$ & $0.48[-0.37,1.33]$ \\
\hline Sousa 2005 & 10 & $83.5(12.5)$ & 10 & $57(10.1)$ & - & $0.4 \%$ & $2.23[1.07,3.40]$ \\
\hline Taaffe 1996 & 7 & $159.8(30.7)$ & 11 & 116.1 (18.5) & $\longrightarrow$ & $0.4 \%$ & $1.75[0.60 .2 .89]$ \\
\hline Taaffe 1999 & 11 & $62.6(10.6)$ & 12 & $45(10.9)$ & $\longrightarrow$ & $0.6 \%$ & $1.58[0.62 .2 .54]$ \\
\hline Tracy 2004 & 11 & $88(38)$ & 9 & $69.1(26.4)$ & - & $0.7 \%$ & $0.54[-0.36,1.44]$ \\
\hline Tsutsumi 1997 & 13 & $30.9(7)$ & 14 & $25.8(9.2)$ & 5 & $0.9 \%$ & $0.60[-0.17,1.38]$ \\
\hline Vincent 2002 & 22 & $347.1(167)$ & 16 & $222.9(75)$ & - & $1.2 \%$ & $0.89[0.21,1.57]$ \\
\hline Wood 2001 & 10 & $229.1(97.4)$ & 6 & $201.7(97.2)$ & - & $0.5 \%$ & $0.27[-0.75,1.28]$ \\
\hline
\end{tabular}

Cochrane Database Syst Rev. Author manuscript; available in PMC 2015 February 11. 


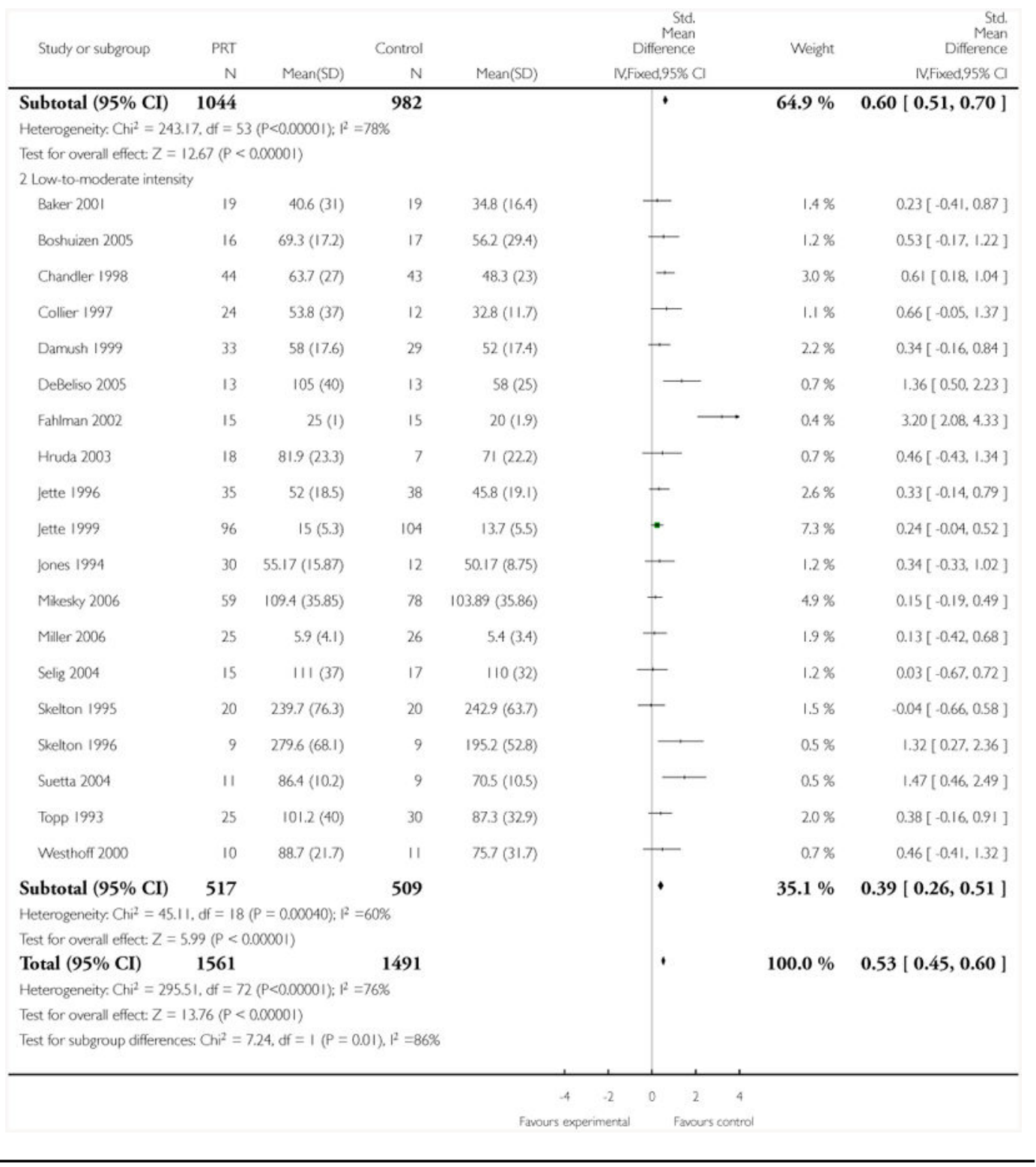

Cochrane Database Syst Rev. Author manuscript; available in PMC 2015 February 11. 


\section{Analysis 10.6. Comparison 10 PRT versus control supplementary analyses, Outcome 6 Strength (grouped by exercise duration)}

Review. Progressive resistance strength training for improving physical function in older adults

Comparison: 10 PRT versus control supplementary analyses

Outcome: 6 Strength (grouped by exercise duration)

\begin{tabular}{|c|c|c|c|c|c|c|c|}
\hline \multirow[t]{2}{*}{ Study or subgroup } & \multirow{2}{*}{$\begin{array}{r}\text { PRT } \\
\mathrm{N} \\
\end{array}$} & \multicolumn{3}{|c|}{ Control } & \multirow{2}{*}{$\begin{array}{r}\text { Std. } \\
\text { Mean } \\
\text { Difference } \\
\text { IV.Fixed,95\% C }\end{array}$} & \multirow[t]{2}{*}{ Weight } & \multirow{2}{*}{$\begin{array}{r}\text { Std. } \\
\text { Mean } \\
\text { Difference } \\
\text { IV.Fixed, } 95 \% \text { C }\end{array}$} \\
\hline & & $\operatorname{Mean}(\mathrm{SD})$ & $\mathrm{N}$ & $\operatorname{Mean}(S D)$ & & & \\
\hline \multicolumn{8}{|l|}{1 Less than 12 weeks } \\
\hline Bermon 1999 & 16 & $23,6(4)$ & 16 & $19.2(3.6)$ & $\longrightarrow$ & $1.2 \%$ & $1.13[0.37,1.88]$ \\
\hline Boshuizen 2005 & 16 & $69.3(17.2)$ & 17 & $56.2(29.4)$ & - & $1.4 \%$ & $0.53[-0.17,1.22]$ \\
\hline Casaburi 2004 & 12 & 344 (98) & 12 & $274(66)$ & $\rightarrow$ & $1.0 \%$ & $0.81[-0.03,1.65]$ \\
\hline Chandler 1998 & 44 & $63.7(27)$ & 43 & $48.3(23)$ & $\rightarrow$ & $3.6 \%$ & $0.61[0.18,1.04]$ \\
\hline Collier 1997 & 24 & $53.8(37)$ & 12 & $32.8(11.7)$ & 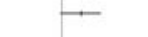 & $1.3 \%$ & $0.66[-0.05,1.37]$ \\
\hline Damush 1999 & 33 & $58(17.6)$ & 29 & $52(17.4)$ & $\leftarrow$ & $27 \%$ & $0.34[-0.16,0.84]$ \\
\hline Fahlman 2002 & is & $25(1)$ & is & $20(1.9)$ & & $0.5 \%$ & $3.20[2.08,4.33]$ \\
\hline Foley 2003 & 26 & $26.73(14.2)$ & 32 & $21.94(11.61)$ & + & $2.5 \%$ & $0.37[-0.15,0.89]$ \\
\hline Hruda 2003 & 18 & $81.9(23.3)$ & 7 & $71(22.2)$ & - & $0.9 \%$ & $0.46[-0.43,1.34]$ \\
\hline Latham 200I & 10 & $12.3(7.1)$ & 10 & $7.4(3.5)$ & - & $0.8 \%$ & $0.84[-0.08,1.76]$ \\
\hline Latham 2003 & 108 & $12.6(5.4)$ & 112 & $12.9(5.3)$ & $*$ & $9.6 \%$ & $-0.06[-0.32,0.21]$ \\
\hline Maiorana 1997 & 12 & $132.4(26.4)$ & 14 & $116.6(26.6)$ & - & $1.1 \%$ & $0.58[-0.21,1.37]$ \\
\hline Malliou 2003 & 15 & $79.2(6.8)$ & 10 & $65.9(5.2)$ & - & $0.7 \%$ & $207[1.05,3.08]$ \\
\hline Pu 2001 & 9 & $1630(516)$ & 7 & $1292(352)$ & & $0.6 \%$ & $0.71[-0.32,1.73]$ \\
\hline Schilke 1996 & 10 & $67.4(28.6)$ & 10 & $36.3(27.8)$ & & $0.7 \%$ & $1.06[0.11 .201]$ \\
\hline Seynnes 2004 & 8 & $11.3(283)$ & 8 & $6.1(1.69)$ & & $0.4 \%$ & $2.11[0.82,3.40]$ \\
\hline Simpson 1992 & 14 & $24.8(14)$ & 14 & $25.1(14)$ & 1 & $1.2 \%$ & $-0.02[-0.76,0.72]$ \\
\hline Singh 1997 & 17 & $3241(191)$ & 14 & $2756(334)$ & & $0.9 \%$ & $1.78[0.93 .2 .64]$ \\
\hline Skelton 1996 & 9 & $279.6(68.1)$ & 9 & $195.2(52.8)$ & & $0.6 \%$ & $1.32[0.27,2.36]$ \\
\hline Westhoff 2000 & 10 & $88.7(21.7)$ & 11 & $75.7(31.7)$ & - & $0.9 \%$ & $0.46[-0.41,1.32]$ \\
\hline Subtotal $(95 \% \mathrm{CI})$ & 426 & & 402 & & - & $32.6 \%$ & $0.52[0.37,0.66]$ \\
\hline \multicolumn{8}{|c|}{ Heterogeneity. $C i^{2}=73.47, d f=19(P<0.00001) ; 1^{2}=74 \%$} \\
\hline \multicolumn{8}{|c|}{ Test for overall effect: $Z=7.06(P<0.00001)$} \\
\hline \multicolumn{8}{|l|}{2 Longer than 12 weeks } \\
\hline & & & & & 2 & & \\
\hline & & & & & al & & \\
\hline
\end{tabular}

Cochrane Database Syst Rev. Author manuscript; available in PMC 2015 February 11. 


\begin{tabular}{|c|c|c|c|c|c|c|c|c|}
\hline \multirow[t]{2}{*}{ Study or subgroup } & \multirow{2}{*}{$\begin{array}{l}\text { PRT } \\
\text { N }\end{array}$} & \multicolumn{3}{|c|}{ Control } & \multirow{2}{*}{\multicolumn{2}{|c|}{$\begin{array}{r}\text { Std. } \\
\text { Mean } \\
\text { Difference } \\
\text { N. Fixed,95\% a }\end{array}$}} & \multirow[t]{2}{*}{ Weight } & \multirow{2}{*}{ 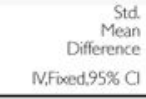 } \\
\hline & & $\operatorname{Mean}(S D)$ & $\mathrm{N}$ & $\operatorname{Mean}(S D)$ & & & & \\
\hline Baker 2001 & 19 & $40.6(31)$ & 19 & 34.8 (16.4) & $=$ & & $1.6 \%$ & $0.23[-0.41,0.87]$ \\
\hline Beneks 2005 & 8 & $75.2(5.8)$ & 8 & $67.9(5.2)$ & 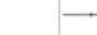 & & $0.6 \%$ & $1.25[0.15,2.35]$ \\
\hline Brandon 2000 & 43 & $60.1(19.9)$ & 42 & $47.3(13.6)$ & - & & $3.5 \%$ & $0.74[0.30 .1 .18]$ \\
\hline Brandon 2003 & 16 & $0.81(0.29)$ & 15 & $0.63(0.15)$ & - & & $13 \%$ & $0.75\left[\begin{array}{ll}0.02 & 1.48\end{array}\right]$ \\
\hline Brochu 2002 & 21 & $43.6(10.8)$ & 21 & $36.9(14.2)$ & - & & $1.8 \%$ & $0.52[-0.09,1.14]$ \\
\hline Brvunsgard 2004 & 10 & $13.15(3.46)$ & 11 & $9.5(3.2)$ & $\longrightarrow$ & & $0.8 \%$ & $1.05[0.13,1.98]$ \\
\hline Buchner 1997 & 22 & 99 (31) & 29 & $84(32)$ & - & & $21 \%$ & $0.47[-0.09,1.03]$ \\
\hline Chin A Paw 2006 & 40 & $73.2(12.3)$ & 31 & $77.5(48.3)$ & - & & $3.0 \%$ & $-0.13[-0.60,0.34]$ \\
\hline DeBeliso 2005 & 13 & $105(40)$ & 13 & $58(25)$ & $\longrightarrow$ & & $0.9 \%$ & $1.36[0.50 .2 .23]$ \\
\hline Ettinger 1997 & 120 & $90.2(21.9)$ & 127 & $87(21.4)$ & - & & $10.8 \%$ & $0.15[-0.10,0.40]$ \\
\hline Fatouros 2002 & 8 & $116.1(9.8)$ & 8 & $665(8.5)$ & & 一 & $0.1 \%$ & $5.11[2.85,7.38]$ \\
\hline Fatouros 2005 & 20 & $91.7(8.2)$ & 14 & $564(8.5)$ & & $\longrightarrow$ & $0.4 \%$ & $4.14[2.89 .5 .39]$ \\
\hline Haykowsky 2000 & 10 & $367(47)$ & 8 & 290 (53) & 一 & & $0.6 \%$ & $1.48[0.40,2.55]$ \\
\hline Hortobagyi 2001 & 18 & $1122(340)$ & 9 & $877(203)$ & - & & $1.0 \%$ & $0.78[-0.05,1.62]$ \\
\hline Jette 1996 & 35 & $52(18.5)$ & 38 & $45.8(19.1)$ & - & & $3.1 \%$ & $0.33[-0.14,0.79]$ \\
\hline Jette 1999 & 96 & $15(5.3)$ & 104 & $13.7(5.5)$ & - & & $8.7 \%$ & $0.24[-0.04,0.52]$ \\
\hline Jones 1994 & 30 & $55.17(15.87)$ & 12 & $50.17(8.75)$ & - & & $15 \%$ & $0.34[-0.33,1.02]$ \\
\hline Lamoureux 2003 & 29 & $31.8(1.7)$ & 15 & $21.2(2.4)$ & & - & $0.4 \%$ & $5.31[3.99 .6 .63]$ \\
\hline Liu-Ambrose 2005 & 32 & $18.9(8.6)$ & 32 & $17.5(6.4)$ & - & & $28 \%$ & $0.18[-0.31,0.67]$ \\
\hline McCartney 1995 & 20 & $124(22)$ & 23 & $98(18)$ & - & & $1.5 \%$ & $1.28[0.62 .1 .94]$ \\
\hline Mikesky 2006 & 59 & $109.4(35.85)$ & 78 & $103.89(35.86)$ & - & & $5.9 \%$ & $0.15[-0.19,0.49]$ \\
\hline Miszko 2003 & 13 & $105.27(53.1)$ & 15 & $79.71(37.5)$ & - & & $1.2 \%$ & $0.55[-0.21,1.31]$ \\
\hline Nelson 1994 & 20 & $37.8(10.4)$ & 19 & $25.8(8.5)$ & $\rightarrow$ & & $1.4 \%$ & $1.23[0.54,1.93]$ \\
\hline Nichols 1993 & 15 & $39.2(12.7)$ & 15 & $32.2(9.7)$ & - & & $1.2 \%$ & $0.60[-0.13,1.34]$ \\
\hline Parkhouse 2000 & 13 & $98(21.6)$ & 9 & $54(18)$ & - & & $0.6 \%$ & $209[1.00,3.18]$ \\
\hline Pollock 1991 & 20 & $53.4(21.1)$ & 12 & $43.2(20.3)$ & - & & $13 \%$ & $0.48[-0.25,1.20]$ \\
\hline Reeves 2004 & 9 & $115(51.7)$ & 9 & $98.5(29.9)$ & - & & $0.8 \%$ & $0.37[-0.56,1.31]$ \\
\hline Rhodes 2000 & 20 & $33.5(4.3)$ & 18 & $28(4)$ & - & & $13 \%$ & $1.29[0.59 .200]$ \\
\hline Simoneau 2006 & 11 & $10.32(3.35)$ & 9 & $12.26(2.96)$ & 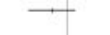 & & $0.8 \%$ & $-0.58[-1.49,0.32]$ \\
\hline Simons 2006 & 21 & $48.3(23)$ & 21 & $31.7(14.1)$ & - & & $1.7 \%$ & $0.85[0.22,1.49]$ \\
\hline Sipila 1996 & 12 & $382(116)$ & 10 & $332(77)$ & - & & $0.9 \%$ & $0.48[-0.37,1.33]$ \\
\hline
\end{tabular}

Cochrane Database Syst Rev. Author manuscript; available in PMC 2015 February 11. 


\begin{tabular}{|c|c|c|c|c|c|c|c|c|}
\hline \multirow[t]{2}{*}{ Study or subgroup } & \multirow{2}{*}{$\begin{array}{r}\text { PRT } \\
\mathrm{N}\end{array}$} & \multicolumn{3}{|c|}{ Control } & \multirow{2}{*}{\multicolumn{2}{|c|}{$\begin{array}{r}\text { Std. } \\
\text { Mean } \\
\text { Difference } \\
\text { IV,Fixed,95\% CI }\end{array}$}} & \multirow[t]{2}{*}{ Weight } & \multirow{2}{*}{$\begin{array}{r}\text { Std. } \\
\text { Mean } \\
\text { Difference } \\
\text { IV.Fixed,95\% Cl }\end{array}$} \\
\hline & & $\operatorname{Mean}(\mathrm{SD})$ & N & $\operatorname{Mean}(\mathrm{SD})$ & & & & \\
\hline Sousa 2005 & 10 & $83.5(12.5)$ & 10 & $57(10.1)$ & & $\longrightarrow$ & $0.5 \%$ & $2.23[1.07,3.40]$ \\
\hline Taaffe 1996 & 7 & $159.8(30.7)$ & 11 & $116.1(18.5)$ & & $\longrightarrow$ & $0.5 \%$ & $1.75[0.60,2.89]$ \\
\hline Taaffe 1999 & 11 & $62.6(10.6)$ & 12 & $45(10.9)$ & & 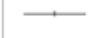 & $0.7 \%$ & $1.58[0.62 .2 .54]$ \\
\hline Tracy 2004 & 11 & $88(38)$ & 9 & $69.1(26.4)$ & & 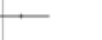 & $0.8 \%$ & $0.54[-0.36,1.44]$ \\
\hline Vincent 2002 & 22 & $347.1(167)$ & 16 & $222.9(75)$ & & 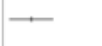 & $1.5 \%$ & $0.89[0.21,1.57]$ \\
\hline Subtotal $(95 \% \mathrm{CI})$ & 884 & & 852 & & & - & $67.4 \%$ & $0.53[0.43,0.63]$ \\
\hline \multicolumn{9}{|c|}{ Heterogeneity: $\mathrm{Chi}^{2}=185.04, \mathrm{df}=35(\mathrm{P}<0.00001) ; 1^{2}=81 \%$} \\
\hline \multicolumn{9}{|c|}{ Test for overall effect: $Z=10.50(P<0.00001)$} \\
\hline Total $(95 \% \mathrm{CI})$ & 1310 & & 1254 & & & $\cdot$ & $100.0 \%$ & $0.53[0.45,0.61]$ \\
\hline \multicolumn{9}{|c|}{ Heterogeneity: $\mathrm{Chi}^{2}=258.55, \mathrm{df}=55(\mathrm{P}<0.0000 \mathrm{I}): \mathrm{I}^{2}=79 \%$} \\
\hline \multicolumn{9}{|c|}{ Test for overall effect: $Z=12.64(P<0.00001)$} \\
\hline \multicolumn{9}{|c|}{ Test for subgroup differences: $\mathrm{Chi}^{2}=0.04, \mathrm{df}=\mathrm{I}(\mathrm{P}=0.85), \mathrm{I}^{2}=0.0 \%$} \\
\hline & & & & & -2 & 2 & & \\
\hline & & & & Favou & mental & Favours & & \\
\hline
\end{tabular}




\section{Analysis 10.7. Comparison 10 PRT versus control supplementary analyses, Outcome 7 Strength (grouped by health status)}

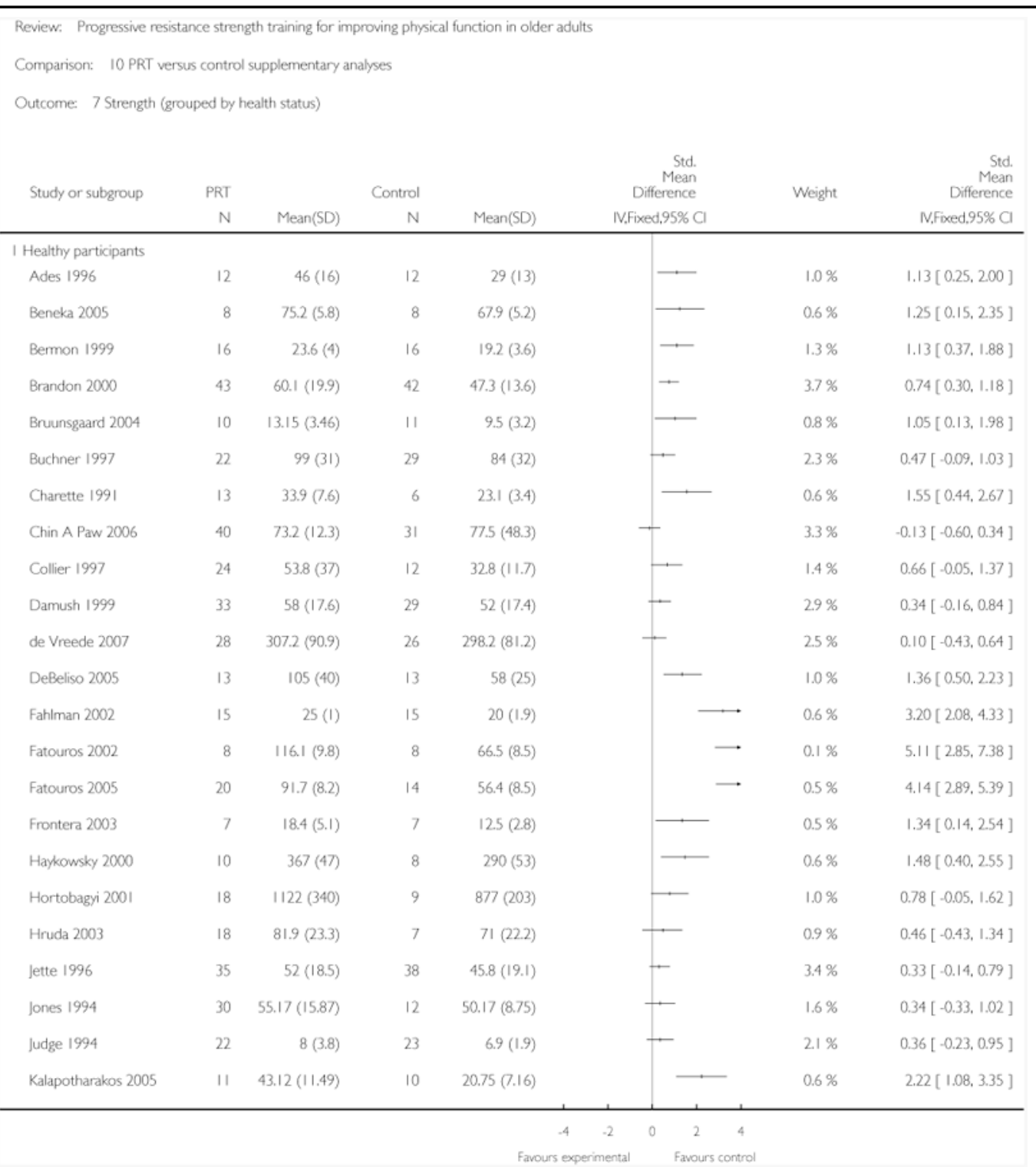

Cochrane Database Syst Rev. Author manuscript; available in PMC 2015 February 11. 


\begin{tabular}{|c|c|c|c|c|c|c|c|}
\hline \multirow[t]{2}{*}{ Study or subgroup } & \multirow{2}{*}{$\begin{array}{r}\text { PRT } \\
\mathrm{N}\end{array}$} & \multicolumn{3}{|c|}{ Control } & \multirow{2}{*}{$\begin{array}{r}\text { Std. } \\
\text { Mean } \\
\text { Difference } \\
\text { IV.Fixed.95\% CI }\end{array}$} & \multirow[t]{2}{*}{ Weight } & \multirow{2}{*}{$\begin{array}{r}\text { Std. } \\
\text { Mean } \\
\text { Difference } \\
\text { N.Fixed,95\% CI }\end{array}$} \\
\hline & & $\operatorname{Mean}(\mathrm{SD})$ & $\mathrm{N}$ & $\operatorname{Mean}(S D)$ & & & \\
\hline Lamoureux 2003 & 29 & $31.8(1.7)$ & 15 & $21.2(24)$ & & $0.4 \%$ & $5.31[3.99,6.63]$ \\
\hline Latham 2001 & 10 & $12.3(7.1)$ & 10 & $7.4(3.5)$ & - & $0.8 \%$ & $0.84[-0.08,1.76]$ \\
\hline Malliou 2003 & 15 & $79.2(6.8)$ & 10 & $65.9(5.2)$ & - & $0.7 \%$ & $2.07[1.05,3.08]$ \\
\hline McCartney 1995 & 20 & $124(22)$ & 23 & 98 (18) & - & $1.6 \%$ & $1.28[0.62,1.94]$ \\
\hline Nelson 1994 & 20 & $37.8(10.4)$ & 19 & $25.8(8.5)$ & - & $1.5 \%$ & $1.23[0.54,1.93]$ \\
\hline Nichols 1993 & 15 & $39.2(127)$ & 15 & $322(9.7)$ & - & $1.3 \%$ & $0.60[-0.13,1.34]$ \\
\hline Pollock 1991 & 20 & $53.4(21.1)$ & 12 & $43.2(20.3)$ & - & $1.4 \%$ & $0.48[-0.25,1.20]$ \\
\hline Rall 1996 & 8 & $47.3(14.8)$ & 6 & $32.2(11.2)$ & 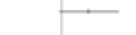 & $0.5 \%$ & $1.05[-0.10,221]$ \\
\hline Reeves 2004 & 9 & $115(51.7)$ & 9 & $98.5(29.9)$ & - & $0.8 \%$ & $0.37[-0.56,1.31]$ \\
\hline Rhodes 2000 & 20 & $33.5(4.3)$ & 18 & 28 (4) & $一$ & $1.4 \%$ & $1.29[0.59,200]$ \\
\hline Seynnes 2004 & 8 & $11.3(2.83)$ & 8 & 6.1 (1.69) & - & $0.4 \%$ & $2.11[0.82,3.40]$ \\
\hline Simoneau 2006 & 11 & $10.32(3.35)$ & 9 & $1226(296)$ & 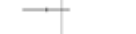 & $0.9 \%$ & $-0.58[-1.49,0.32]$ \\
\hline Simons 2006 & 21 & $48.3(23)$ & 21 & $31.7(14.1)$ & - & $1.8 \%$ & $0.85[0.22,1.49]$ \\
\hline Sipila 1996 & 12 & 382 (116) & 10 & $332(77)$ & - & $1.0 \%$ & $0.48[-0.37,1.33]$ \\
\hline Skelton 1995 & 20 & $239.7(76.3)$ & 20 & $242.9(63.7)$ & - & $1.9 \%$ & $-0.04[-0.66,0.58]$ \\
\hline Sousa 2005 & 10 & $83.5(12.5)$ & 10 & $57(10.1)$ & - & $0.5 \%$ & $2.23[1.07,3.40]$ \\
\hline Taaffe 1996 & 7 & $159.8(30.7)$ & 11 & $116.1(18.5)$ & $\longrightarrow$ & $0.6 \%$ & $1.75[0.60,289]$ \\
\hline Taaffe 1999 & 11 & $62.6(10.6)$ & 12 & $45(10.9)$ & $\longrightarrow$ & $0.8 \%$ & $1.58[0.62 .254]$ \\
\hline Topp 1993 & 25 & $101.2(40)$ & 30 & $87.3(32.9)$ & - & $25 \%$ & $0.38[-0.16,0.91]$ \\
\hline Tracy 2004 & 11 & $88(38)$ & 9 & $69.1(26.4)$ & - & $0.9 \%$ & $0.54[-0.36,1.44]$ \\
\hline Tsutsumi 1997 & 13 & $30.9(7)$ & 14 & $25.8(9.2)$ & - & $1.2 \%$ & $0.60[-0.17,1.38]$ \\
\hline Vincent 2002 & 22 & $347.1(167)$ & 16 & $222.9(75)$ & $\rightarrow$ & $1.6 \%$ & $0.89[0.21,1.57]$ \\
\hline Wood 2001 & 10 & $229.1(97.4)$ & 6 & $201.7(97.2)$ & - & $0.7 \%$ & $0.27[-0.75,1.28]$ \\
\hline Subtotal $(95 \% \mathrm{CI})$ & 803 & & 699 & & - & $58.7 \%$ & $0.77[0.66,0.88]$ \\
\hline \multicolumn{8}{|c|}{ Heterogeneity: $\mathrm{Chi}^{2}=201.20$, df $=45(P<0.00001) ; R^{2}=78 \%$} \\
\hline \multirow{2}{*}{\multicolumn{8}{|c|}{$\begin{array}{l}\text { Test for overall effect: } Z=13.52 \text { ( } P<0.00001) \\
2 \text { Older adults with a specific health problem }\end{array}$}} \\
\hline & & & & & & & \\
\hline Baker 2001 & 19 & $40.6(31)$ & 19 & $34.8(16.4)$ & T & $1.8 \%$ & $0.23[-0.41,0.87]$ \\
\hline Brandon 2003 & 16 & $0.81(0.29)$ & 15 & $0.63(0.15)$ & +5 & $1.3 \%$ & $0.75[0.02,1.48]$ \\
\hline Brochu 2002 & 21 & $43.6(10.8)$ & 21 & $36.9(14.2)$ & - & $1.9 \%$ & $0.52[-0.09,1.14]$ \\
\hline Casaburin 2004 & 12 & $344(98)$ & 12 & $274(66)$ & - & $1.0 \%$ & $0.81[-0.03,1.65]$ \\
\hline Castareda 2001 & 14 & $55.9(224)$ & 12 & $38.8(14)$ & - & $1.1 \%$ & $0.87[0.06,1.68]$ \\
\hline
\end{tabular}

Cochrane Database Syst Rev. Author manuscript; available in PMC 2015 February 11. 


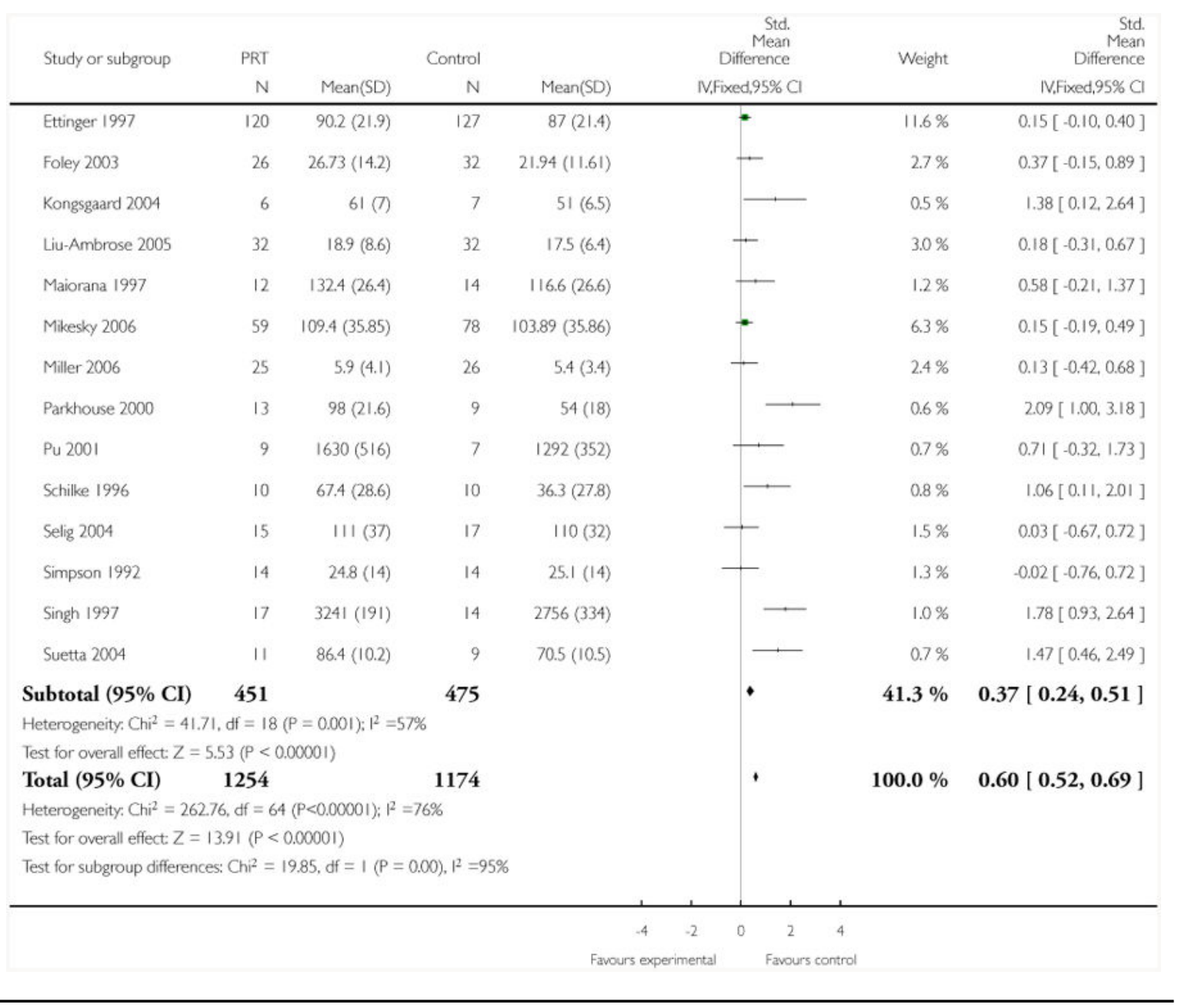

Cochrane Database Syst Rev. Author manuscript; available in PMC 2015 February 11. 


\section{Analysis 10.8. Comparison 10 PRT versus control supplementary analyses, Outcome 8 Strength (grouped by functional limitations)}

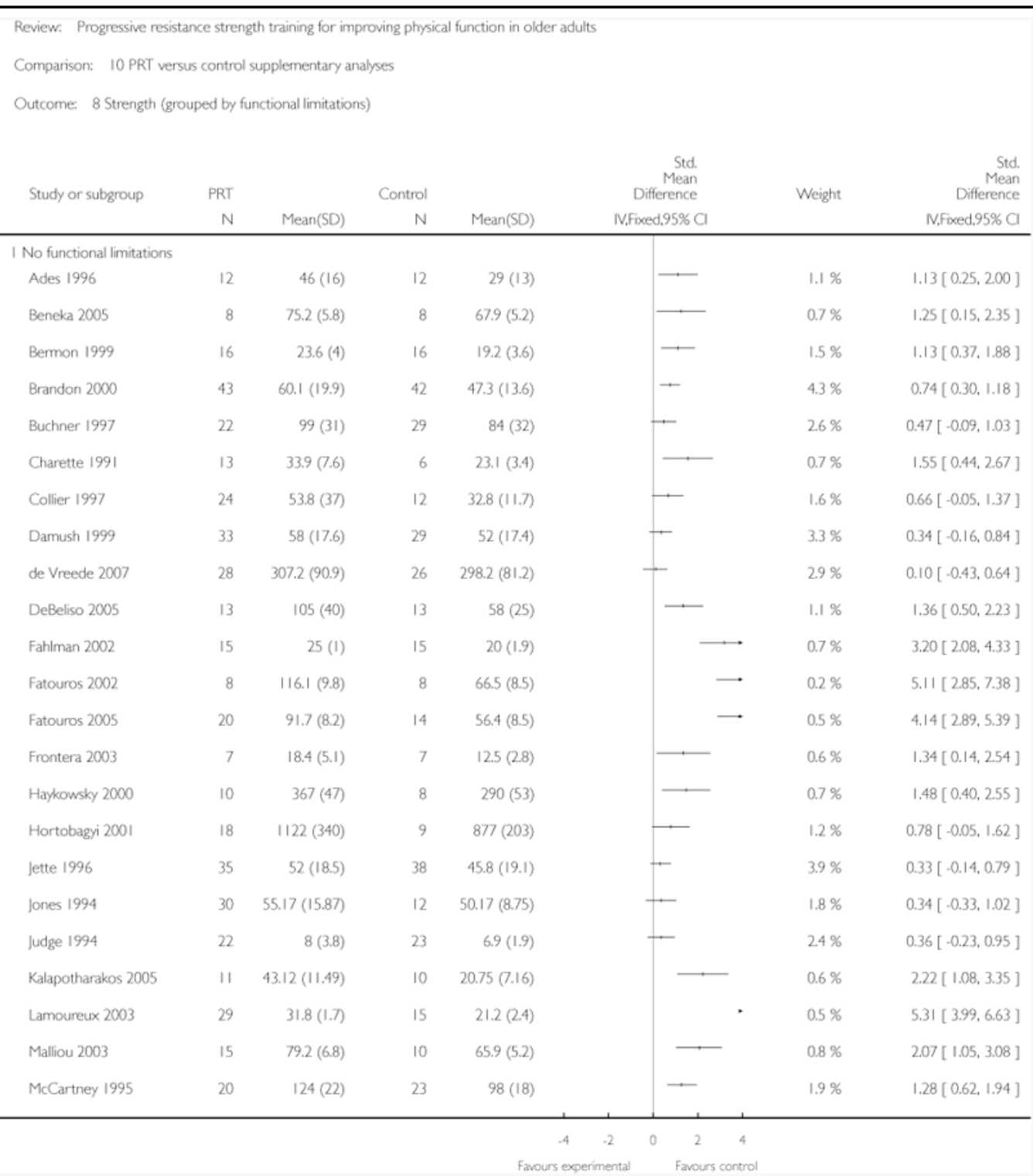




\begin{tabular}{|c|c|c|c|c|c|c|c|}
\hline \multirow[t]{2}{*}{ Study or subgroup } & \multirow{2}{*}{$\begin{array}{r}\text { PRT } \\
\mathrm{N}\end{array}$} & \multicolumn{3}{|c|}{ Control } & \multirow{2}{*}{$\begin{array}{c}\text { Std. } \\
\text { Difference } \\
\text { IV.Fixed,95\% CI }\end{array}$} & \multirow[t]{2}{*}{ Weight } & \multirow{2}{*}{$\begin{array}{r}\text { Mear } \\
\text { Difference } \\
\text { N.,Fixed,95\% } \\
\end{array}$} \\
\hline & & $\operatorname{Mean}(\mathrm{SD})$ & $\mathrm{N}$ & $\operatorname{Mean}(\mathrm{SD})$ & & & \\
\hline Netson 1994 & 20 & $37.8(10.4)$ & 19 & $25.8(8.5)$ & $\div$ & $1.7 \%$ & $1.23[0.54,1.93]$ \\
\hline Nichols 1993 & 15 & $39.2(127)$ & 15 & $32.2(9.7)$ & - & $1.5 \%$ & $0.60[-0.13,1.34]$ \\
\hline Pollock 1991 & 20 & $53.4(21.1)$ & 12 & $43.2(20.3)$ & - & $1.6 \%$ & $0.48[-0.25,1.20]$ \\
\hline Rall 1996 & 8 & $47.3(14.8)$ & 6 & $322(11.2)$ & $\longrightarrow$ & $0.6 \%$ & $1.05[-0.10,221]$ \\
\hline Reeves 2004 & 9 & $115(51.7)$ & 9 & $98.5(29.9)$ & - & $0.9 \%$ & $0.37[-0.56,1.31]$ \\
\hline Rhodes 2000 & 20 & $33.5(4.3)$ & 18 & 28 (4) & - & $1.7 \%$ & $1.29[0.59 .200]$ \\
\hline Simoneau 2006 & 11 & $10.32(3.35)$ & 9 & $12.26(296)$ & 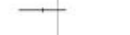 & $1.0 \%$ & $-0.58[-1.49,0.32]$ \\
\hline Simons 2006 & 21 & $48.3(23)$ & 21 & $31.7(14.1)$ & $\leftarrow$ & $2.1 \%$ & $0.85[0.22,1.49]$ \\
\hline Sipila 1996 & 12 & $382(116)$ & 10 & $332(77)$ & - & $1.1 \%$ & $0.48[-0.37,1.33]$ \\
\hline Skelton 1995 & 20 & $239.7(76.3)$ & 20 & $2429(63.7)$ & - & $22 \%$ & $-0.04[-0.66,0.58]$ \\
\hline Sousa 2005 & 10 & $83.5(125)$ & 10 & $57(10.1)$ & 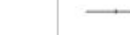 & $0.6 \%$ & $2.23[1,07,3,40]$ \\
\hline Taaffe 1996 & 7 & $159.8(30.7)$ & 11 & $116.1(18.5)$ & $\longrightarrow$ & $0.6 \%$ & $1.75[0.60,289]$ \\
\hline Taaffe 1999 & 11 & $62.6(10.6)$ & 12 & $45(10.9)$ & $\longrightarrow$ & $0.9 \%$ & $1.58[0.62,2.54]$ \\
\hline Topp 1993 & 25 & $101.2(40)$ & 30 & $87.3(329)$ & - & $29 \%$ & $0.38[-0.16,0.91]$ \\
\hline Tracy 2004 & 11 & $88(38)$ & 9 & $69.1(26.4)$ & - & $1.0 \%$ & $0.54[-0.36,1.44]$ \\
\hline Tsutsumi 1997 & 13 & $30.9(7)$ & 14 & $25.8(9.2)$ & - & $1.4 \%$ & $0.60[-0.17,1.38]$ \\
\hline Vincent 2002 & 22 & $347.1(167)$ & 16 & $222.9(75)$ & - & $1.8 \%$ & $0.89[0.21,1.57]$ \\
\hline Wood 2001 & 10 & $229.1(97.4)$ & 6 & $201.7(97.2)$ & - & $0.8 \%$ & $0.27[-0.75,1.28]$ \\
\hline Subtotal $(95 \% \mathrm{CI})$ & 717 & & 632 & & - & $59.8 \%$ & $0.81[0.69,0.93]$ \\
\hline \multicolumn{8}{|c|}{ Heterogeneity. $C \mathrm{i}^{2}=181.69 . \mathrm{df}=40(\mathrm{P}<0.00001): \mathrm{R}^{2}=78 \%$} \\
\hline \multicolumn{8}{|c|}{ Test for overall effect: $Z=13.51(P<0.00001)$} \\
\hline 2 With functional limitatio & & & & & & & \\
\hline Boshuizen 2005 & 16 & $69.3(17.2)$ & 17 & $56.2(29.4)$ & - & $1.7 \%$ & $0.53[-0.17,1.22]$ \\
\hline Bruunsgaard 2004 & 10 & $13.15(3.46)$ & 11 & $9.5(3.2)$ & - - & $1.0 \%$ & $1.05[0.13,1.98]$ \\
\hline Chandler 1998 & 44 & $63.7(27)$ & 43 & $48.3(23)$ & - & $4.5 \%$ & $0.61[0.18,1.04]$ \\
\hline Chin A Paw 2006 & 40 & $73.2(12.3)$ & 31 & $77.5(48.3)$ & - & $38 \%$ & $-0.13[-0.60,0.34]$ \\
\hline Hruda 2003 & 18 & $81.9(23.3)$ & 7 & $71(22.2)$ & $\rightarrow$ & $1.1 \%$ & $0.46[-0.43,1.34]$ \\
\hline Jette 1999 & 96 & $15(5.3)$ & 104 & $13.7(5.5)$ & - & $10.7 \%$ & $0.24[-0.04,0.52]$ \\
\hline Latham 2001 & 10 & $12.3(7.1)$ & 10 & $7.4(3.5)$ & & $1.0 \%$ & $0.84[-0.08,1.76]$ \\
\hline Latham 2003 & 108 & $12.6(5.4)$ & 112 & $12.9(5.3)$ & * & $11.8 \%$ & $-0.06[-0.32,0.21]$ \\
\hline Miszko 2003 & 13 & 105.27 (53.1) & 15 & 79.71 (37.5) & 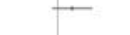 & $1.4 \%$ & $0.55[-0.21,1.31]$ \\
\hline Newnham 1995 & 12 & $15.3(7.5)$ & 12 & $7.4(3.6)$ & $\rightarrow$ & $1.0 \%$ & $1.30[0.40,2.19]$ \\
\hline
\end{tabular}

Cochrane Database Syst Rev. Author manuscript; available in PMC 2015 February 11. 


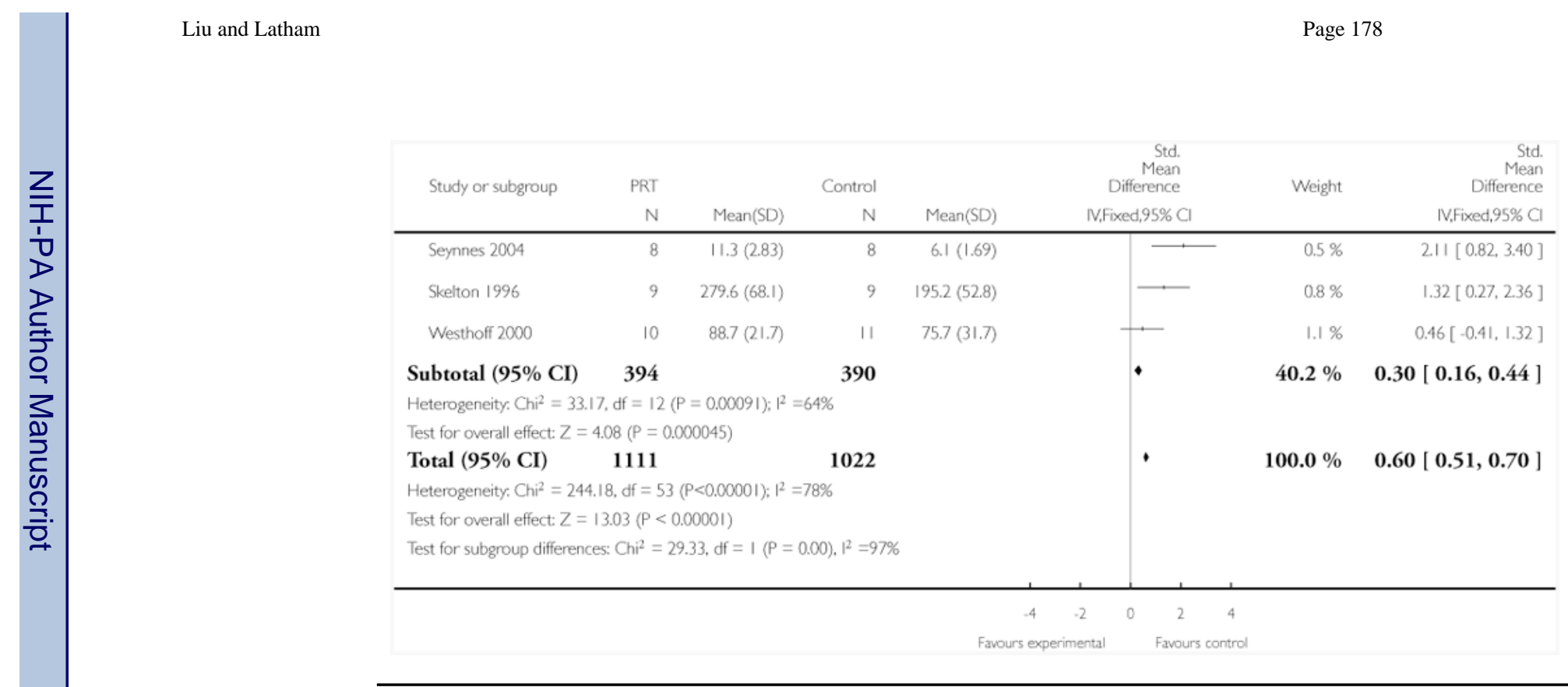

\section{Appendix 1. Search strategies}

\section{Medline (OVID WEB)}

1. ((strength $\$$ or resist $\$$ or weight $\$)$ adj 3 training).tw.

2. progressive resist $\$$.tw.

3. or $/ 1-2$

4. Exercise/

5. Exercise Therapy/

6. exercise\$.tw.

7. or $/ 4-6$

8. (Resist\$ training or strength\$).tw.

9. and $/ 7-8$

10. or $/ 3,9$

11. limit 10 to ("all aged (65 and over)" or "aged ( 80 and over)")

12. (elderly or senior\$).tw.

13. and $/ 10,12$

14. or $/ 11,13$

15. randomized controlled trial.pt.

16. controlled clinical trial.pt.

17. Randomized Controlled Trials/ 

18. Random Allocation/
19. Double Blind Method/
20. Single Blind Method/
21. or $/ 15-20$
22. Animals/not Humans/
23. 21 not 22
24. clinical trial.pt.
25. exp Clinical Trials as topic/
26. (clinic $\$$ adj25 trial\$).tw.
27. (( $\operatorname{sing} 1 \$$ or doubl\$ or trebl $\$$ or tripl\$) adj25 (blind $\$$ or mask $\$)$ ).tw.
28. Placebos/
29. placebo\$.tw.
30. random $\$$.tw.
31. Research Design/
32. or $/ 24-31$
33. 32 not 22
34. 33 not 23
35. or $/ 23,34$
36. and $/ 14,35$

\section{Embase (OVID WEB)}

1. ((strength $\$$ or resist $\$$ or weight $\$)$ adj3 training).tw.

2. progressive resist $\$$.tw.

3. or $/ 1-2$

4. Exercise/

5. Kinesiotherapy/or Therapy Resistance/

6. exercise $\$ . t w$.

7. or $/ 4-6$

8. (resist\$ or strength\$).tw.

9. and $/ 7-8$

10. or $/ 3,9$

11. limit 10 to aged $\langle 65+$ years $>$ 
12. (elderly or senior\$).tw.

13. and $/ 10,12$

14. or $/ 11,13$

15. Clinical trial/

16. Randomized controlled trial/

17. Randomization/

18. Single blind procedure/

19. Double blind procedure/

20. Crossover procedure/

21. Placebo/

22. Randomi?ed controlled trial\$.tw.

23. Rct.tw.

24. Random allocation.tw.

25. Randomly allocated.tw.

26. Allocated randomly.tw.

27. (allocated adj2 random).tw.

28. Single blind $\$$.tw.

29. Double blind\$.tw.

30. ((treble or triple) adj blind\$).tw.

31. Placebo\$.tw.

32. Prospective study/

33. or $/ 15-32$

34. Case study/

35. Case report.tw.

36. Abstract report/or letter/

37. or $/ 34-36$

38. 33 not 37

39. limit 38 to human

40. and $/ 14,39$

\section{The Cochrane Library (Wiley)}

\#1 ((strength* or resist* or weight*) NEAR/3 training):ti,ab,kw 


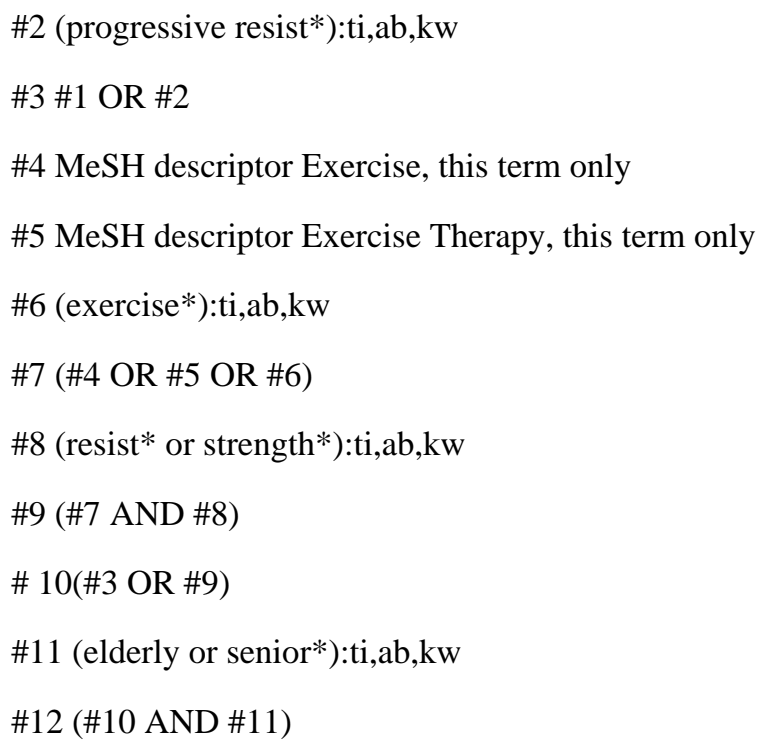

\section{Cinahl (OVID WEB)}

1. ((strength $\$$ or resist $\$$ or weight $\$)$ adj3 training).tw.

2. progressive resist $\$ . t w$.

3. or/1-2

4. Exercise/

5. Therapeutic Exercise/

6. "Exercise therapy: ambulation (iowa nic)"/or "Exercise therapy: balance (iowa nic)"/or "Exercise therapy: joint mobility (iowa nic)"/or "Exercise therapy: muscle control (iowa nic)"/or "Teaching: prescribed activity/exercise (iowa nic)"/

7. exercise\$.tw.

8. or $/ 4-7$

9. (resist\$ or strength\$).tw.

10. and $/ 8-9$

11. or $/ 3,10$

12. limit 11 to (aged $<65$ to 79 years $>$ or "aged $<80$ and over $>$ ")

13. (elderly or senior\$).tw.

14. and $/ 11,13$

15. or $/ 12,14$

16. exp Clinical Trials/

17. $\exp$ Evaluation Research/

18. exp Comparative Studies/ 
19. exp Crossover Design/

20. clinical trial.pt.

21. or $/ 16-20$

22. ((clinical or controlled or comparative or placebo or prospective or randomi\#ted) adj3 (trial or study)).tw.

23. (random $\$$ adj7 (allocat $\$$ or allot $\$$ or assign $\$$ or basis $\$$ or divid $\$$ or order $\$$ )).tw.

24. ((singl\$ or doubl\$ or trebl\$ or tripl\$) adj7 (blind\$ or mask\$)).tw.

25. (cross?over\$ or (cross adj1 over\$)).tw.

26. ( (allocat $\$$ or allot $\$$ or assign $\$$ or divid $\$$ ) adj3 (condition $\$$ or experiment $\$$ or intervention $\$$ or treatment $\$$ or therap $\$$ or control $\$$ or group $\$)$ ).tw.

27. or $/ 22-26$

28. or $/ 21,27$

29. and $/ 15,28$

\section{SPORTDiscus (OVID WEB)}

1. ((strength $\$$ or resist $\$$ or weight $\$)$ adj 3 training).tw.

2. progressive resist $\$ . t w$.

3. or $/ 1-2$

4. Exercise/

5. Exercise therapy/

6. exercise\$.tw.

7. or $/ 4-6$

8. (resist\$ or strength\$).tw.

9. and $/ 7-8$

10. or $/ 3,9$

11. (elderly or senior\$).tw.

12. and $/ 10-11$

13. exp Clinical trial/

14. exp Randomized controlled trial/

15. Placebo/

16. ((clinical or controlled or comparative or placebo or prospective or randomi\#ed) $\operatorname{adj} 3$ (trial or study)).tw.

17. (random $\$$ adj7 (allocat $\$$ or allot $\$$ or assign $\$$ or basis $\$$ or divid $\$$ or order $\$$ )).tw. 
18. ((singl\$ or doubl\$ or trebl\$ or tripl\$) adj7 (blind $\$$ or mask $\$))$.tw.

19. (cross?over\$ or (cross adj1 over\$)).tw.

20. ( (allocat\$ or allot\$ or assign $\$$ or divid\$) adj3 (condition\$ or experiment\$ or intervention\$ or treatment\$ or therap\$ or control\$ or group\$)).tw.

21. or $/ 13-20$

22. and $/ 12,21$

\section{References to studies included in this review}

Ades PA, Ballor DL, Ashikaga T, Utton JL, Nair KS. Weight training improves walking endurance in healthy elderly persons. Annals of Internal Medicine. 1996; 124(6):568-72. MEDLINE: 96175655. [PubMed: 8597320]

Baker KR, Nelson ME, Felson DT, Layne JE, Sarno R, Roubenoff R. The efficacy of home based progressive strength training in older adults with knee osteoarthritis: A randomized controlled trial. Journal of Rheumatology. 2001; 28:1655-65. [PubMed: 11469475]

Balagopal P, Schimke JC, Ades P, Adey D, Nair KS. Age effect on transcript levels and synthesis rate of muscle MHC and response to resistance exercise. American Journal of Physiology Endocrinology and Metabolism. 2001; 280(2):E203-8. [PubMed: 11158921]

Ballor DL, Harvey-Berino JR, Ades PA, Cryan J, Calles-Escandon J. Contrasting effects of resistance and aerobic training on body composition and metabolism after diet-induced weight loss. Metabolism: Clinical \& Experimental. 1996; 45(2):179-83. [PubMed: 8596486]

Barrett CJ, Smerdely P. A comparison of community-based resistance exercise and flexibility exercise for seniors. Australian Journal of Physiotherapy. 2002; 48(3):215-9. [PubMed: 12217071]

Baum EE, Jarjoura D, Polen AE, Faur D, Rutechi G. Effectiveness of a group exercise program in a long-term care facility: a randomized pilot trial. Journal of the American Medical Directors Association. 2003; 4(2):74-80. [PubMed: 12807578]

Bean JF, Herman S, Kiely DK, Frey IC, Leveille SG, Fielding RA, et al. Increased Velocity Exercise Specific to Task (InVEST) training: a pilot study exploring effects on leg power, balance, and mobility in community-dwelling older women. Journal of the American Geriatrics Society. 2004; 52(5):799-804. [PubMed: 15086665]

Beneka A, Malliou P, Fatouros I, Jamurtas A, Gioftsidou A, Godolias G, et al. Resistance training effects on muscular strength of elderly are related to intensity and gender. Journal of Science \& Medicine in Sport. 2005; 8(3):274-83. [PubMed: 16248468]

Bermon S, Philip P, Ferrari P, Candito M, Dolisi C. Effects of a short-term strength training programme on lymphocyte subsets at rest in elderly humans. European Journal of Applied Physiology and Occupational Physiology. 1999; 79(4):336-40. [PubMed: 10090633]

Boshuizen HC, Stemmerik L, Westhoff MH, Hopman-Rock M. The effects of physical therapists' guidance on improvement in a strength-training program for the frail elderly. Journal of Aging \& Physical Activity. 2005; 13(1):5-22. [PubMed: 15677832]

Brandon LJ, Boyetter LW, Gaasch DA, Lloyd DG. Effects of lower extremity strength training on functional mobility in older adults. Journal of Aging \& Physical Activity. 2000; 8(3):214-27.

Brandon LJ, Gaasch DA, Boyette LW, Lloyd AM. Effects of long-term resistive training on mobility and strength in older adults with diabetes. Journals of Gerontology Series A-Biological Sciences \& Medical Sciences. 2003; 58(8):740-5.

Ades PA, Savage PD, Brochu M, Tischler MD, Lee NM, Poehlman ET. Resistance training increases total daily energy expenditure in disabled older women with coronary heart disease. Journal of Applied Physiology. 2005; 98(4):1280-5. [PubMed: 15772059]

Ades PA, Savage PD, Cress ME, Brochu M, Lee NM, Poehlman ET. Resistance training on physical performance in disabled older female cardiac patients. Medicine \& Science in Sports \& Exercise. 2003; 35(8):1265-70. [PubMed: 12900677] 
*. Brochu M, Savage P, Lee M, Dee J, Cress ME, Poehlman ET, et al. Effects of resistance training on physical function in older disabled women with coronary heart disease. Journal of Applied Physiology. 2002; 92(2):672-8. [PubMed: 11796680]

Bruunsgaard H, Bjerregaard E, Schroll M, Pedersen BK. Muscle strength after resistance training is inversely correlated with baseline levels of soluble tumor necrosis factor receptors in the oldest old. Journal of the American Geriatrics Society. 2004; 52(2):237-41. [PubMed: 14728633]

*. Buchner DM, Cress ME, de Lateur BJ. The effect of strength and endurance training on gait, balance, fall risk and health services use in community-living older adults. Journals of Gerontology Series A-Biological Sciences \& Medical Sciences. 1997; 52(4):M218-24.

Buchner DM, Cress ME, de Lateur BJ, Wagner EH. Variability in the effect of strength training on skeletal muscle in older adults. Facts and Research in Gerontology. 1993; 7:143-53.

Coleman EA, Buchner DM, Cress ME, Chan BKS, de Lateur BJ. The relationship of joint symptoms with exercise performance in older adults. Journal of the American Geriatrics Society. 1996; 44(1):14-21. [PubMed: 8537585]

Casaburi R, Bhasin S, Cosentino L, Porszasz J, Somfay A, Lewis MI, et al. Effects of testosterone and resistance training in men with chronic obstructive pulmonary disease. American Journal of Respiratory \& Critical Care Medicine. 2004; 170(8):870-8. [PubMed: 15271690]

Castaneda C, Gordon PL, Uhlin KL, Levery AS, Kehayias JJ, Dwyer JT, et al. Resistance training to counteract the catabolism of a low-protein diet in patients with chronic renal insufficiency. Annals of Internal Medicine. 2001; 135(11):965-76. [PubMed: 11730397]

Castaneda C, Gordon PL, Parker RC, Uhlin KL, Roubenoff R, Levey AS. Resistance training to reduce the malnutrition-inflammation complex syndrome of chronic kidney disease. American Journal of Kidney Diseases. 2004; 43(4):607-16. [PubMed: 15042537]

Chandler JM, Duncan PW, Kochersberger G, Studenski S. Is lower extremity strength gain associated with improvement in physical performance and disability in frail, community-dwelling elders? Archives of Physical Medicine and Rehabilitation. 1998; 79(1):24-30. [PubMed: 9440412]

Charette S, McEvoy L, Pyka G. Muscle hypertrophy response to resistance training in older women. Journal of Applied Physiology. 1991; 70(5):1912-6. [PubMed: 1864770]

Chin A Paw MJM, Van Poppel MNM, Twisk JWR, Van Mechelen W. Effects of resistance and allround, functional training on quality of life, vitality and depression of older adults living in longterm care facilities: A 'randomized' controlled trial. BMC Geriatrics. 2004; 4:1-9. ISRCTN87177281. [PubMed: 15050032]

*. Chin A Paw MJM, Van Poppel MNM, Twisk JWR, Van Mechelen W. Once a week not enough, twice a week not feasible? A randomised controlled exercise trial in long-term care facilities. Patient Education \& Counseling. 2006; 63(1-2):205-14. ISRCTN87177281. [PubMed: 16426800]

Chin A Paw MJM, Van Poppel MNM, Van Mechelen W. Effects of resistance and functional-skills training on habitual activity and constipation among older adults living in long-term care facilities: A randomized controlled trial. BMC Geriatrics. 2006; 6:9. [PubMed: 16875507]

Collier, CD. Isotonic resistance training related functional fitness, physical self-efficacy and depression in adults ages 65-85 (thesis). Stillwater (OK): Oklahoma State University; 1997.

Damush TM, Damush JG Jr. The effects of strength training on strength and health-related quality of life in older adult women. Gerontologist. 1999; 39(6):705-10. [PubMed: 10650680]

*. de Vos NJ, Singh NA, Ross DA, Stavrinos TM, Orr R, Fiatarone-Singh MA. Optimal load for increasing muscle power during explosive resistance training in older adults. Journals of Gerontology Series A-Biological Sciences \& Medical Sciences. 2005; 60(5):638-47.

Orr R, de Vos NJ, Singh NA, Ross DA, Stavrinos TM, Fiatarone-Singh MA. Power training improves balance in healthy older adults. Journals of Gerontology Series A-Biological Sciences \& Medical Sciences. 2006; 61(1):78-85.

de Vreede PL, Samson MM, Van Meeteren NL, Van der Bom JG, Duursma SA, Verhaar HJ. Functional tasks exercise versus resistance exercise to improve daily function in older women: a feasibility study. Archives of Physical Medicine \& Rehabilitation. 2004; 85(12):1952-61. [PubMed: 15605332] 
de Vreede PL, Samson MM, Van Meeteren NLU, Duursma SA, Verhaar HJJ. Functional-task exercise versus resistance strength exercise to improve daily function in older women: A randomized, controlled trial. Journal of the American Geriatrics Society. 2005; 53(1):2-10. [PubMed: 15667369]

*. de Vreede PL, Van Meeteren NL, Samson MM, Wittink HM, Duursma SA, Verhaar HJ. The effect of functional tasks exercise and resistance exercise on health-related quality of life and physical activity: A randomized controlled trial. Gerontology. 2007; 53(1):12-20. [PubMed: 16940735]

DeBeliso M, Harris C, Spitzer-Gibson T, Adams KJ. A comparison of periodised and fixed repetition training protocol on strength in older adults. Journal of Science \& Medicine in Sport. 2005; 8(2): 190-9. [PubMed: 16075779]

DiFrancisco-Donoghue J, Werner W, Douris P. Comparison of once-weekly and twice-weekly strength training in older adults. British Journal of Sports Medicine. 2007; 41(1):19-22. [PubMed: 17062657]

Donald IP, Pitt K, Armstrong E, Shuttleworth H. Preventing falls on an elderly care rehabilitation ward. Clinical Rehabilitation. 2000; 14(2):178-85. [PubMed: 10763795]

Earles DR, Judge JO, Gunnarsson OT. Velocity training induces power-specific adaptations in highly functioning older adults. Archives of Physical Medicine and Rehabilitation. 2001; 82(7):872-8. [PubMed: 11441371]

*. Ettinger WH Jr, Burns R, Messier SP, Applegate W, Rejeski WJ, Morgan T, et al. A randomized trial comparing aerobic exercise and resistance exercise with a health education program in older adults with knee osteoarthritis. The Fitness Arthritis and Seniors Trial (FAST). JAMA. 1997; 277(1):25-31. [PubMed: 8980206]

Mangani I, Cesari M, Kritchevsky SB, Maraldi C, Carter CS, Atkinson HH, et al. Physical exercise and comorbidity. Results from the Fitness and Arthritis in Seniors Trial (FAST). Aging-Clinical \& Experimental Research. 2006; 18(5):374-80. [PubMed: 17167301]

Messier SP, Royer TD, Craven TE, O'Toole ML, Burns R, Ettinger WH Jr. Long-term exercise and its effect on balance in older, osteoarthritic adults: results from the Fitness, Arthritis and Seniors Trial (FAST). Journal of the American Geriatrics Society. 2000; 48(2):131-8. [PubMed: 10682941]

Penninx B, Rejeski WJ, Pandya J, Miller ME, Di Bari M, Applegate WB, et al. Exercise and depressive symptoms: A comparison of aerobic and resistance exercise effects on emotional and physical function in older persons with high and low depressive symptomatology. Journals of Gerontology Series B-Psychological Sciences \& Social Sciences. 2002; 57(2):124-32.

Rejeski WJ, Ettinger WH Jr, Martin K, Morgan T. Treating disability in knee osteoarthritis with exercise therapy: a central role for self-efficacy and pain. Arthritis Care \& Research. 1998; 11(2): 94-101. [PubMed: 9668732]

Sevick MA, Bradham DD, Muender M, Chen GJ, Enarson C, Dailey M, et al. Cost-effectiveness of aerobic and resistance exercise in seniors with knee osteoarthritis. Medicine \& Science in Sports \& Exercise. 2000; 32(9):1534-40. [PubMed: 10994901]

Fahlman MM, Boardley D, Lambert CP, Flynn MG. Effects of endurance training and resistance training on plasma lipoprotein profiles in elderly women. Journals of Gerontology Series ABiological Sciences \& Medical Sciences. 2002; 57(2):B54-60.

Fatouros I, Taxildaris K, Tokmakidis S, Kalapotharakos VI, Aggelousis N, Athanasopoulos S, et al. The effects of strength training, cardiovascular training and their combination on flexibility of inactive older adults. International Journal of Sports Medicine. 2002; 23(2):112-9. [PubMed: 11842358]

Fatouros IG, Kambas A, Katrabasas I, Leontsini D, Chatzinikolaou A, Jamurtas AZ, et al. Resistance training and detraining effects on flexibility performance in the elderly are intensity-dependent. Journal of Strength \& Conditioning Research. 2006; 20(3):634-42. [PubMed: 16937978]

*. Fatouros IG, Kambas A, Katrabasas I, Nikolaidis K, Chatzinikolaou A, Leontsini D, et al. Strength training and detraining effects on muscular strength, anaerobic power, and mobility of inactive older men are intensity dependent. British Journal of Sports Medicine. 2005; 39(10):776-80. [PubMed: 16183776]

Fatouros IG, Tournis S, Leontsini D, Jamurtas AZ, Sxina M, Thomakos P, et al. Leptin and adiponectin responses in overweight inactive elderly following resistance training and detraining 
are intensity related. Journal of Clinical Endocrinology \& Metabolism. 2005; 90(11):5970-7. [PubMed: 16091494]

*. Fiatarone MA, O'Neill EF, Ryan ND, Clements KM, Solares GR, Nelson ME, et al. Exercise training and nutritional supplementation for physical frailty in very elderly people. New England Journal of Medicine. 1994; 330(25):1769-75. [PubMed: 8190152]

Singh MA, Ding W, Manfredi TJ, Solares GS, O'Neill EF, Clements KM. Insulin-like growth factor I in skeletal muscle after weight-lifting exercise in frail elders. American Journal of Physiology Endocrinology and Metabolism. 1999; 277(40):E135-43.

Fiatarone, MA.; O'Neill, EF.; Doyle, RN.; Clements, K. Efficacy of home-based resistance training in frail elders; Abstracts of the 16th Congress of the International Association of Gerontology; Bedford Park, South Australia. 1997 World Congress of Gerontology Inc; 1997. Abstract 985

*. Fielding RA, LeBrasseur NK, Cuoco A, Bean J, Mizer K, Fiatarone Singh MA. High-velocity resistance training increases skeletal muscle peak power in older women. Journal of the American Geriatrics Society. 2002; 50(4):655-62. see comment. [PubMed: 11982665]

Sayers SP, Bean J, Cuoco A, LeBrasseur NK, Jette A, Fielding RA. Changes in function and disability after resistance training: does velocity matter? A pilot study. American Journal of Physical Medicine \& Rehabilitation. 2003; 82(8):605-13. [PubMed: 12872017]

Flynn MG, Fahlman M, Braun WA, Lambert CP, Bouillon LE, Brolinson PG, et al. Effects of resistance training on selected indexes of immune function in elderly women. Journal of Applied Physiology. 1999; 86(6):1905-13. [PubMed: 10368355]

Foley A, Halbert J, Hewitt T, Crotty M. Does hydrotherapy improve strength and physical function in patients with osteoarthritis - A randomised controlled trial comparing a gym based and a hydrotherapy based strengthening programme. Annals of the Rheumatic Diseases. 2003; 62(12): 1162-7. [PubMed: 14644853]

Frontera WR, Hughes VA, Krivickas LS, Kim SK, Foldvari M, Roubenoff R. Strength training in older women: early and late changes in whole muscle and single cells. Muscle \& Nerve. 2003; 28(5):601-8. [PubMed: 14571463]

Galvao DA, Taaffe DR. Resistance exercise dosage in older adults: Single- versus multiset effects on physical performance and body composition. Journal of the American Geriatrics Society. 2005; 53(12):2090-7. [PubMed: 16398892]

Hagerman FC, Walsh SJ, Staron RS, Hikida RS, Gilders RM, Murray TF, et al. Effects of highintensity resistance strength training on untrained older men 1. Strength, cardiovascular and metabolic responses. Journals of Gerontology Series A-Biological Sciences \& Medical Sciences. 2000; 55A(7):B336-46.

Harris C, DeBeliso MA, Spitzer-Gibson TA, Adams KJ. The effect of resistance-training intensity on strength-gain response in the older adult. Journal of Strength \& Conditioning Research. 2004; 18(4):833-8. [PubMed: 15574091]

Haykowsky M, Humen D, Teo K, Quinney A, Souster M, Bell G, et al. Effects of 16 weeks of resistance training on left ventricular morphology and systolic function in healthy men $>60$ years of age. American Journal of Cardiology. 2000; 85(8):1002-6. [PubMed: 10760343]

Haykowsky M, McGavock J, Vonder Muhll I, Koller M, Mandic S, Welsh R, et al. Effect of exercise training on peak aerobic power, left ventricular morphology, and muscle strength in healthy older women. Journals of Gerontology Series A-Biological Sciences \& Medical Sciences. 2005; 60(3): $307-11$.

Hennessey JV, Chromiak JA, DellaVentura S, Reinert SE, Puhl J, Kiel DP, et al. Growth hormone administration and exercise effects on muscle fibre type and diameter in moderately frail older people. Journal of the American Geriatrics Society. 2001; 49(7):852-8. [PubMed: 11527474]

Hepple RT, Mackinnon SL, Goodman MJ, Thomas SG, Plyley MJ. Resistance and aerobic training in older men: effects on VO2 peak and the capillary supply to skeletal muscle. Journal of Applied Physiology. 1997; 82(4):1305-10. [PubMed: 9104869]

*. Hiatt WR, Wolfel EE, Meier RH, Regensteiner JG. Superiority of treadmill walking exercise versus strength training for patients with peripheral arterial disease. Circulation. 1994; 90(4):1866-74. [PubMed: 7923674] 
Regensteiner JG, Steiner JF, Hiatt WR. Exercise training improves functional status in patients with peripheral arterial disease. Journal of Vascular Surgery. 1996; 23(1):104-15. [PubMed: 8558725]

Hortobagyi T, Tunnel D, Moody J, Beam S, DeVita P. Low- or high-intensity strength training partially restores impaired quadriceps force accuracy and steadiness in aged adults. Journals of Gerontology Series A-Biological Sciences \& Medical Sciences. 2001; 56(1):B38-47.

Hruda KV, Hicks AL, McCartney N. Training for muscle power in older adults: effects on functional abilities. Canadian Journal of Applied Physiology. 2003; 28(2):178-89. [PubMed: 12825328]

Hunter GR, Wetzstein CA, McLafferty CL Jr, Zuckerman PA, Landers KA, Bamman MM. Highresistance versus variable-resistance training in older adults. Medicine \& Science in Sports \& Exercise. 2001; 33(10):1759-64. [PubMed: 11581563]

Izquierdo M, Ibanez J, Hakkinen K, Kraemer WJ, Larrion JL, Gorostiaga EM. Once weekly combined resistance and cardiovascular training in healthy older men. Medicine \& Science in Sports \& Exercise. 2004; 36(3):435-43. [PubMed: 15076785]

Jette AM, Harris BA, Sleeper L, Lachman ME, Heislein D, Giorgetti M, et al. A home-based exercise program for nondisabled older adults. Journal of the American Geriatrics Society. 1996; 44(6): 644-9. [PubMed: 8642153]

Dancewicz TM, Krebs DE, McGibbon CA. Lower-limb extensor power and lifting characteristics in disabled elders. Journal of Rehabilitation Research and Development. 2003; 40(4):337-47. [PubMed: 15074445]

*. Jette AM, Lachman M, Giorgetti MM, Assman SF, Harris BA, Levenson C, et al. Exercise - it's never too late: The strong-for-life program. American Journal of Public Health. 1999; 89(1):6672. [PubMed: 9987467]

Krebs DE, Jette AM, Assmann SF. Moderate exercise improves gait stability in disabled elders. Archives of Physical Medicine \& Rehabilitation. 1998; 79(12):1489-95. [PubMed: 9862288]

Jones CJ, Rikli RE, Benedict J, Williamson P. Effects of a resistance training program on leg strength and muscular endurance in older women. Scandinavian Journal of Medicine and Science in Sports. 1995; 5(6):329-41. [PubMed: 8775717]

Jubrias SA, Esselman PC, Price LB, Cress ME, Conley KE. Large energetic adaptations of elderly muscle to resistance and endurance training. Journal of Applied Physiology. 2001; 90(5):1663-70. [PubMed: 11299253]

*. Judge JO, Whipple RH, Wolfson LI. Effects of resistance and balance exercises on isokinetic strength in older persons. Journal of the American Geriatrics Society. 1994; 42(9):937-46. [PubMed: 8064101]

Wolfson L, Whipple R, Derby C, Judge J, King M, Amerman P, et al. Balance and strength training in older adults: intervention gains and tai chi maintenance. Journal of the American Geriatrics Society. 1996; 44(5):498-506. [PubMed: 8617896]

*. Kalapotharakos VI, Michalopoulos M, Tokmakidis SP, Godolias G, Gourgoulis V. Effects of a heavy and a moderate resistance training on functional performance in older adults. Journal of Strength \& Conditioning Research. 2005; 19(3):652-7. [PubMed: 16095421]

Kalapotharakos VI, Michalopoulou M, Godolias G, Tokmakidis SP, Malliou PV, Gourgoulis V. The effects of high- and moderate-resistance training on muscle function in the elderly. Journal of Aging \& Physical Activity. 2004; 12(2):131-43. [PubMed: 15223882]

Kalapotharakos VI, Michalopoulou M, Tokmakidis S, Godolias G, Strimpakos N, Karteroliotis K. Effects of a resistance exercise programme on the performance of inactive older adults. International Journal of Therapy and Rehabilitation. 2004; 11(7):318-23.

Kalapotharakos VI, Tokmakidis SP, Smilios I, Michalopoulos M, Gliatis J, Godolias G. Resistance training in older women: effect on vertical jump and functional performance. Journal of Sports Medicine \& Physical Fitness. 2005; 45(4):570-5. [PubMed: 16446692]

Kallinen M, Sipila S, Alen M, Suominen H. Improving cardiovascular fitness by strength or endurance training in women aged 76-78 years. A population-based, randomized controlled trial. Age and Ageing. 2002; 31(4):247-54. [PubMed: 12147561]

Katznelson L, Robinson MW, Coyle CL, Lee H, Farrell CE. Effects of modest testosterone supplementation and exercise for 12 weeks on body composition and quality of life in elderly men. European Journal of Endocrinology. 2006; 155(6):867-75. [PubMed: 17132757] 
Kongsgaard M, Backer V, Jorgensen K, Kjaer M, Beyer N. Heavy resistance training increases muscle size, strength and physical function in elderly male COPD-patients - a pilot study. Respiratory Medicine. 2004; 98(10):1000-7. [PubMed: 15481277]

Krebs DE, Scarborough DM, McGibbon CA. Functional vs. strength training in disabled elderly outpatients. American Journal of Physical Medicine \& Rehabilitation. 2007; 86(2):93-103. [PubMed: 17251692]

*. Lamoureux E, Sparrow WA, Murphy A, Newton RU. The effects of improved strength on obstacle negotiation in community-living older adults. Gait \& Posture. 2003; 17(3):273-83. [PubMed: 12770641]

Lamoureux EL, Murphy A, Sparrow A, Newton RU. The effects of progressive resistance training on obstructed-gait tasks in community-living older adults. Journal of Aging and Physical Activity. 2003; 11(1):98-110.

Latham NK, Stretton CS, Ronald M. Progressive resistance strength training in hospitalised older people: a preliminary investigation. New Zealand Journal of Physiotherapy. 2001; 29(2):41-8.

*. Latham NK, Anderson CS, Lee A, Bennett D, Moseley AM, Cameron ID. A randomized, controlled trial of quadriceps resistance exercise and vitamin D in frail older people: the Frailty Interventions Trial in Elderly Subjects (FITNESS). Journal of the American Geriatrics Society. 2003; 51(3):291-9. [PubMed: 12588571]

Liu-Ambrose T, Khan KM, Eng JJ, Janssen PA, Lord SR, McKay HA. Resistance and agility training reduce fall risk in women aged 75 to 85 with low bone mass: a 6-month randomized, controlled trial. Journal of the American Geriatrics Society. 2004; 52(5):657-65. [PubMed: 15086643]

Liu-Ambrose T, Khan KM, Eng JJ, Lord SR, McKay HA. Balance confidence improves with resistance or agility training: Increase is not correlated with objective changes in fall risk and physical abilities. Gerontology. 2004; 50(6):373-82. [PubMed: 15477698]

*. Liu-Ambrose TYL, Khan KM, Eng JJ, Lord SR, Lentle B, McKay HA. Both resistance and agility training reduce back pain and improve health-related quality of life in older women with low bone mass. Osteoporosis International. 2005; 16(11):1321-9. [PubMed: 15702262]

Liu-Ambrose, TYL. Studies of fall risk and bone morphology in older women with low bone mass. British Columbia (Canada): The University of British Columbia; 2004. dissertation

Macaluso A, Young A, Gibb KS, Rowe DA, De Vito G. Cycling as a novel approach to resistance training increases muscle strength, power, and selected functional abilities in healthy older women. Journal of Applied Physiology. 2003; 95(6):2544-53. [PubMed: 12937026]

Madden KM, Levy WC, Stratton JK. Exercise training and heart rate variability in older adult female subjects. Clinical \& Investigative Medicine - Medecine Clinique et Experimentale. 2006; 29(1): 20-8. [PubMed: 16553360]

Maiorana AJ, Briffa TG, Goodman C, Hung J. A controlled trial of circuit weight training on aerobic capacity and myocardial oxygen demand in men after coronary artery bypass surgery. Journal of Cardiopulmonary Rehabilitation. 1997; 17(4):239-47. [PubMed: 9271767]

Malliou P, Fatouros I, Beneka A, Gioftsidou A, Zissi V, Godolias G, et al. Different training programs for improving muscular performance in healthy inactive elderly. Isokinetics and Exercise Science. 2003; 11(4):189-95.

Mangione KK, Craik RL, Tomlinson SS, Palombaro KM. Can elderly patients who have had a hip fracture perform moderate- to high-intensity exercise at home? Physical Therapy. 2005; 85(8): 727-39. [PubMed: 16048421]

Manini TM, Clark BC, Tracy BL, Burke J, Ploutz-Snyder L. Resistance and functional training reduces knee extensor position fluctuations in functionally limited older adults. European Journal of Applied Physiology. 2005; 95(5-6):436-46. [PubMed: 16193338]

Maurer BT, Stern AG, Kinossian B, Cook KD, Schumacher HR. Osteoarthritis of the knee: isokinetic quadriceps exercise versus an educational intervention. Archives of Physical Medicine and Rehabilitation. 1999; 80(10):1293-9. [PubMed: 10527090]

McCartney N, Hicks A, Martin J, Webber C. A longitudinal trial of weight training in the elderly: continued improvements in year 2. Journals of Gerontology Series A-Biological Sciences \& Medical Sciences. 1996; 51(6):B425-33. 
*. McCartney N, Hicks AL, Martin J, Webber CE. Long-term resistance training in elderly: Effects on dynamic strength, exercise capacity, muscle and bone. Journals of Gerontology Series ABiological Sciences \& Medical Sciences. 1995; 50(2):B97-104.

McGuigan MR, Bronks R, Newton RU, Sharman MJ, Graham JC, Cody DV, et al. Resistance training in patients with peripheral arterial disease: effects on myosin isoforms, fiber type distribution and capillary supply to skeletal muscle. Journals of Gerontology Series A-Biological Sciences \& Medical Sciences. 2001; 56(7):B302-10.

McMurdo ME, Johnstone R. A randomized controlled trial of a home exercise programme for elderly people with poor mobility. Age and Ageing. 1995; 24(5):425-8. [PubMed: 8669348]

Mihalko SL, McAuley E. Strength training effects on subjective well-being and physical function in the elderly. Journal of Aging and Physical Activity. 1996; 4(1):56-68.

Mikesky AE, Mazzuca SA, Brandt KD, Perkins SM, Damush T, Lane KA. Effects of strength training on the incidence and progression of knee osteoarthritis. Arthritis and Rheumatism - Arthritis Care and Research. 2006; 55(5):690-9. [PubMed: 17013851]

Miller MD, Crotty M, Whitehead C, Bannerman E, Daniels LA. Nutritional supplementation and resistance training in nutritionally at risk older adults following lower limb fracture: a randomized controlled trial. Clinical Rehabilitation. 2006; 20(4):311-23. [PubMed: 16719029]

Miszko TA, Cress ME, Slade JM, Covey CJ, Agrawal SK, Doerr CE. Effect of strength and power training on physical function in community-dwelling older adults. Journals of Gerontology Series A-Biological Sciences \& Medical Sciences. 2003; 58(2):171-5.

*. Moreland J. Personal Communication. 2001

Moreland JD, Goldsmith CH, Huijbregts MP, Anderson RE, Prentice DM, Brunton KB, et al. Progressive resistance strengthening exercises after stroke: a single-blind randomized controlled trial. Archives of Physical Medicine and Rehabilitation. 2003; 84(10):1433-40. [PubMed: 14586909]

Morganti CM, Nelson ME, Fiatarone MA, Dallal GE, Economos CD, Crawford BM, et al. Strength improvements with $1 \mathrm{yr}$ of progressive resistance training in older women. Medicine \& Science in Sports \& Exercise. 1995; 27(6):906-12. [PubMed: 7658954]

*. Nelson ME, Fiatarone MA, Morganti CM, Trice I, Greenberg RA, Evans WJ. Effects of highintensity strength training on multiple risk factors for osteoporotic fractures. JAMA. 1994; 272(24):1909-14. [PubMed: 7990242]

Newnham, J. The effects of a strengthening program on muscle function and mobility skills in an elderly instituionalised population [thesis]. Montreal (Quebec): McGill University; 1995.

Nichols JF, Omizo DK, Peterson KK, Nelson KP. Efficacy of heavy-resistance training for active women over sixty: Muscular strength, body composition and program adherence. Journal of the American Geriatrics Society. 1993; 41(3):205-10. [PubMed: 8440838]

Ouellette MM, LeBrasseur NK, Bean JF, Phillips E, Stein J, Frontera WR, et al. High-intensity resistance training improves muscle strength, self-reported function, and disability in long-term stroke survivors. Stroke. 2004; 35(6):1404-9. [PubMed: 15105515]

Parkhouse WS, Coupland DC, Li C, Vanderhoek KJ. IGF-1 bioavailabililty is increased by resistance training in older women with low bone mineral density. Mechanisms of Ageing and Development. 2000; 113(2):75-83. [PubMed: 10708256]

Hagberg JM, Graves JE, Limacher M, Woods DR, Leggett SH, Cononie C, et al. Cardiovascular responses of 70- to 79-year-old mean and women to exercise training. Journal of Applied Physiology. 1989; 66(6):2589-94. [PubMed: 2745320]

Panton LB, Graves JE, Pollock ML, Hagberg JM, Chen W. Effect of aerobic and resistance training on fractionated reaction time and speed of movement. Journal of Gerontology. 1990; 45(1):M26-31. [PubMed: 2295775]

*. Pollock ML, Carroll JF, Graves JE, Leggett SH, Braith RW, Limacher M, et al. Injuries and adherence to walk/jog and resistance training programs in the elderly. Medicine \& Science in Sports \& Exercise. 1991; 23(10):1194-200. [PubMed: 1758297]

Pu CT, Johnson MT, Forman DE, Hausdorff JM, Roubenoff R, Foldvari M, et al. Randomized trial of progressive resistance training to counteract the myopathy of chronic heart failure. Journal of Applied Physiology. 2001; 90(6):2341-50. [PubMed: 11356801] 
Rall LC, Meydani SN, Kehayias JJ, Dawson Hughes B, Roubenoff R. The effect of progressive resistance training in rheumatoid arthritis. Increased strength without changes in energy balance or body composition. Arthritis and Rheumatism. 1996; 39(3):415-26. [PubMed: 8607890]

Rall LC, Rosen CJ, Dolnikowski G, Hartman WJ, Lundgren N, Abad LW, et al. Protein metabolism in rheumatoid arthritis and aging. Effects of muscle strength training and tumor necrosis factor alpha. Arthritis and Rheumatism. 1996; 39(7):1115-24. [PubMed: 8670319]

*. Rall LC, Roubenoff R, Cannon JG, Abad LW, Dinarello CA, Meydani SN. Effects of progressive resistance training on immune response in aging and chronic inflammation. Medicine \& Science in Sports \& Exercise. 1996; 28(11):1356-65. [PubMed: 8933485]

*. Reeves ND, Narici MV, Maganaris CN. In vivo human muscle structure and function: adaptations to resistance training in old age. Experimental Physiology. 2004; 89(6):675-89. [PubMed: 15328305]

Reeves ND, Narici MV, Maganaris CN. Strength training alters the viscoelastic properties of tendons in elderly humans. Muscle \& Nerve. 2003; 28(1):74-81. [PubMed: 12811776]

Rhodes EC, Martin AD, Taunton JE, Donnely M, Warren J, Elliot J. Effects of one year of resistance training on the relation between muscular strength and bone density in elderly women. British Journal of Sports Medicine. 2000; 34(1):18-22. [PubMed: 10690445]

Schilke JM, Johnson GO, Housh TJ, O'Dell JR. Effects of muscle-strength training on the functional status of patients with osteoarthritis of the knee joint. Nursing Research. 1996; 45(2):68-72. [PubMed: 8604366]

*. Schlicht, J. Effect of intense strength training on walking speed, standing balance and sit-to-stand performance in older adults [thesis]. Storrs (CT): Univ. of Connecticut; 1999.

Schlicht J, Camaione DN, Owen SV. Effect of intense strength training on standing balance, walking speed and sit-to-stand performance in older adults. Journals of Gerontology Series A-Biological Sciences \& Medical Sciences. 2001; 56(5):M281-6.

Segal RJ, Reid RD, Courneya KS, Malone SC, Parliament MB, Scott CG, et al. Resistance exercise in men receiving androgen deprivation therapy for prostate cancer. Journal of Clinical Oncology. 2003; 21(9):1653-9. [PubMed: 12721238]

Selig SE, Carey MF, Menzies DG, Patterson J, Geerling RH, Williams AD, et al. Moderate-intensity resistance exercise training in patients with chronic heart failure improves strength, endurance, heart rate variability, and forearm blood flow. Journal of Cardiac Failure. 2004; 10(1):21-30. [PubMed: 14966771]

Seynnes O, Fiatarone Singh MA, Hue O, Pras P, Legros P, Bernard PL. Physiological and functional responses to low-moderate versus high-intensity progressive resistance training in frail elders. Journals of Gerontology Series A-Biological Sciences \& Medical Sciences. 2004; 59(5):503-9.

Simoneau E, Martin A, Porter MM, Van Hoecke J. Strength training in old age: adaptation of antagonist muscles at the ankle joint. Muscle \& Nerve. 2006; 33(4):546-55. [PubMed: 16372326]

Simons R, Andel R. The effects of resistance training and walking on functional fitness in advanced old age. Journal of Aging \& Health. 2006; 18(1):91-105. [PubMed: 16470977]

Simpson K, Killian K, McCartney N, Lloyd DG, Jones NL. Randomised controlled trial of weightlifting exercise in patients with chronic airflow limitation. Thorax. 1992; 47(2):70-5. [PubMed: 1549826]

Sims J, Hill K, Davidson S, Gunn J, Huang N. Exploring the feasibility of a community-based strength training program for older people with depressive symptoms and its impact on depressive symptoms. BMC Geriatrics. 2006; 6:18. [PubMed: 17134517]

*. Singh NA, Clements KM, Fiatarone MA. A randomised trial of progressive resistance training in depressed elders. Journals of Gerontology Series A-Biological Sciences \& Medical Sciences. 1997; 52(1):M27-35.

Singh NA, Clements KM, Fiatarone Singh MA. The efficacy of exercise as a long-term antidepressant in elderly subjects: a randomized controlled trial. Journals of Gerontology Series A-Biological Sciences and Medical Sciences. 2001; 56(8):497-504.

Singh NA, Stavrinos TM, Scarbek Y, Galambos G, Liber C, Fiatarone Singh MA. A randomized controlled trial of high versus low intensity weight training versus general practitioner care for 
clinical depression in older adults. Journals of Gerontology Series A-Biological Sciences \& Medical Sciences. 2005; 60(6):768-76.

Siplia S, Multanen J, Kallinen M, Era P, Suominen H. Effects of strength and endurance training on isometric muscle strength and walking speed in elderly women. Acta Physiologica Scandinavica. 1996; 156(4):457-64. [PubMed: 8732251]

Skelton DA, Young A, Greig CA, Malbut KE. Effects of resistance training on strength, power, and functional abilities of women aged 75 and older. Journal of the American Geriatrics Society. 1995; 43(10):1081-7. [PubMed: 7560695]

Skelton DA, McLaughlin AW. Training functional ability in old age. Physiotherapy. 1996; 82(3):159_ 67.

Sousa N, Sampaio J. Effects of progressive strength training on the performance of the Functional Reach Test and the Timed Get-Up-and-Go Test in an elderly population from the rural north of Portugal. American Journal of Human Biology. 2005; 17(6):746-51. [PubMed: 16254902]

Suetta C, Aagaard P, Rosted A, Jakobsen AK, Duus B, Kjaer M, et al. Training-induced changes in muscle CSA, muscle strength, EMG, and rate of force development in elderly subjects after longterm unilateral disuse. Journal of Applied Physiology. 2004; 97(5):1954-61. [PubMed: 15247162]

Suetta C, Magnusson SP, Rosted A, Aagaard P, Jakobsen AK, Larsen LH, et al. Resistance training in the early postoperative phase reduces hospitalization and leads to muscle hypertrophy in elderly hip surgery patients - A controlled, randomized study. Journal of the American Geriatrics Society. 2004; 52(12):2016-22. [PubMed: 15571536]

*. Sullivan DH, Roberson PK, Johnson LE, Bishara O, Evans WJ, Smith ES, et al. Effects of muscle strength training and testosterone in frail elderly males. Medicine \& Science in Sports \& Exercise. 2005; 37(10):1664-72. [PubMed: 16260965]

Sullivan DH, Roberson PK, Smith ES, Price JA, Bopp MM. Effects of muscle strength training and megestrol acetate on strength, muscle mass, and function in frail older people. Journal of the American Geriatrics Society. 2007; 55(1):20-8. [PubMed: 17233681]

Symons TB, Vandervoort AA, Rice CL, Overend TJ, Marsh GD. Effects of maximal isometric and isokinetic resistance training on strength and functional mobility in older adults. Journals of Gerontology Series A-Biological Sciences \& Medical Sciences. 2005; 60(6):777-81.

Pruitt LA, Taaffe DR, Marcus R. Effects of a one-year high-intensity versus low-intensity resistance training program on bone mineral density in older women. Journal of Bone \& Mineral Research. 1995; 10(11):1788-95. [PubMed: 8592957]

*. Taaffe DR, Pruitt L, Pyka G, Guido D, Marcus R. Comparative effects of high- and low- intensity resistance training on thigh muscle strength, fiber area, and tissue composition in elderly women. Clinical Physiology. 1996; 16(4):381-92. [PubMed: 8842574]

Taaffe DR, Pruitt L, Reim J, Butterfield G, Marcus R. Effect of sustained resistance training on basal metabolic rate in older women. Journal of the American Geriatrics Society. 1995; 43(5):465-71. [PubMed: 7730525]

Taaffe DR, Duret C, Wheeler S, Marcus R. Once-weekly resistance exercise improves strength and neuromuscular performance in older adults. Journal of the American Geriatrics Society. 1999; 47(10):1208-14. [PubMed: 10522954]

Mikesky AE, Topp R, Wigglesworth JK, Harsha DM, Edwards JE. Efficacy of a home-based training program for older adults using elastic tubing. European Journal of Applied Physiology and Occupational Physiology. 1994; 69(4):316-20. [PubMed: 7851367]

*. Topp R, Mikesky A, Wigglesworth J, Holt W, Edwards JE. The effect of a 12-week dynamic resistance strength training on gait velocity and balance in older adults. Gerontologist. 1993; 33(4):501-6. [PubMed: 8375679]

Topp R, Mikesky A, Dayhoff NE, Holt W. Effect of resistance training on strength, postural control and gait velocity in older adults. Clinical Nursing Research. 1996; 5(4):407-21. [PubMed: 8970279]

Topp R, Woolley S, Hornyak J 3rd, Khuder S, Kahaleh B. The effect of dynamic versus isometric resistance training on pain and functioning among adults with osteoarthritis of the knee. Archives of Physical Medicine \& Rehabilitation. 2002; 83(9):1187-95. [PubMed: 12235596] 
Topp R, Boardley D, Morgan AL, Fahlman M, McNevin N. Exercise and functional tasks among adults who are functionally limited. Western Journal of Nursing Research. 2005; 27(3):252-70. [PubMed: 15781902]

Tracy BL, Byrnes WC, Enoka RM. Strength training reduces force fluctuations during anisometric contractions of the quadriceps femoris muscles in old adults. Journal of Applied Physiology. 2004; 96(4):1530-40. [PubMed: 14565966]

*. Tsutsumi, T. The effects of strength training on mood, self-efficacy, cardiovascular reactivity and quality of life in older adults [thesis]. Boston (MA): Boston University; 1997.

Tsutsumi T, Don BM, Zaichkowsky LD, Delizonna LL. Physical fitness and psychological benefits of strength training in community dwelling older adults. Applied Human Science. 1997; 16(6):25766. [PubMed: 9545677]

Tsutsumi T, Don BM, Zaichkowsky LD, Takenaka K, Oka K, Ohno T. Comparison of high and moderate intensity of strength training on mood and anxiety in older adults. Perceptual and Motor Skills. 1998; 87(3 Pt 1):1003-11. [PubMed: 9885072]

Tyni-Lenne R, Dencker K, Gordon A, Jansson E, Sylven C. Comprehensive local muscle training increases aerobic working capacity and quality of life and decreases neurohormonal activation in patients with chronic heart failure. European Journal of Heart Failure. 2001; 3(1):47-52. [PubMed: 11163735]

*. Vincent KR, Braith BW. Resistance exercise and bone turnover in elderly men and women. Medicine \& Science in Sports \& Exercise. 2002; 34(1):17-23. [PubMed: 11782642]

Vincent KR, Braith RW, Bottiglieri T, Vincent HK, Lowenthal DT. Homocysteine and lipoprotein levels following resistance training in older adults. Preventive Cardiology. 2003; 6(4):197-203. [PubMed: 14605513]

Vincent KR, Braith RW, Feldman RA, Kallas HE, Lowenthal DT. Improved cardiorespiratory endurance following 6 months of resistance exercise in elderly men and women. Archives of Internal Medicine. 2002; 162(6):673-8. [PubMed: 11911721]

Vincent KR, Braith RW, Feldman RA, Magyari PM, Cutler RB, Persin SA, et al. Resistance exercise and physical performance in adults aged 60 to 83. Journal of the American Geriatrics Society. 2002; 50(6):1100-7. [PubMed: 12110072]

Westhoff MH, Stemmerik L, Boshuizen HC. Effects of a low-intensity strength-training program on knee-extensor strength and functional ability of frail older people. Journal of Aging and Physical Activity. 2000; 8(4):325-42.

Wieser M, Haber P. The effects of systematic resistance training in the elderly. International Journal of Sports Medicine. 2007; 28(1):59-65. [PubMed: 16761216]

Wood RH, Reyes R, Welsch MA, Favaloro-Sabatier J, Sabatier M, Lee CM, et al. Concurrent cardiovascular and resistance training in healthy older adults. Medicine \& Science in Sports \& Exercise. 2001; 33(10):1751-8. [PubMed: 11581562]

\section{References to studies excluded from this review}

Adami S, Gattie D, Braga V, Bianchini D, Rossini M. Site-specific effects of strength training on bone structure and geometry of ultradistal radius in postmenopausal women. Journal of Bone and Mineral Research. 1999; 14(1):120-4. [PubMed: 9893073]

Adams KJ, Swank AM, Berning JM, Sevene-Adams PG, Barnard KL, et al. Progressive strength training in sedentary older African American women. Medicine \& Science in Sports \& Exercise. 2001; 33(9):1567-76. [PubMed: 11528347]

*. Agre JC, Pierce LE, Raab DM, McAdams M, Smith EL. Light resistance and stretching exercise in elderly women: effect upon strength. Archives of Physical Medicine and Rehabilitation. 1988; 69(4):273-6. [PubMed: 3355357]

Raab DM, Agre JC, McAdam M, Smith EL. Light resistance and stretching exercise in elderly women: effect upon flexibility. Archives of Physical Medicine and Rehabilitation. 1988; 69(4):268-72. [PubMed: 3355356]

Alexander, JL. Effect of strength training on functional fitness in older chronic obstructive pulmonary disease patients. Arizona State University; 2003. dissertation 
Aniansson A, Gustafsson E. Physical training in elderly men with special reference to quadriceps muscle strength and morphology. Clinical Physiology. 1981; 1(1):87-98.

Annesi JJ, Gann S, Westcott WW, Annesi JJ, Gann S, Westcott WW. Preliminary evaluation of a 10wk. resistance and cardiovascular exercise protocol on physiological and psychological measures for a sample of older women. Perceptual \& Motor Skills. 2004; 98(1):163-70. [PubMed: 15058879]

Ardman, O. The effects of strength training on strength, mobility and balance in two groups of institutionalized elderly subjects. Montreal (Quebec): McGill University; 1998. dissertation

Ballard JE, McFarland C, Wallace LS, Holiday DB, Roberson G. The effect of 15 weeks of exercise on balance, leg strength, and reduction in falls in 40 women aged 65 to 89 years. Journal of the American Medical Womens Association. 2004; 59(4):255-61.

Barbosa AR, Santarem JM, Filho WJ, Marucci MFN. Effects of resistance training on the sit-and-reach test in elderly women. Journal of Strength \& Conditioning Research. 2002; 16(1):14-8. [PubMed: 11834101]

Baum K, Ruether T, Essfeld D. Reduction of blood pressure response during strength training through intermittent muscle relaxations. International Journal of Sports Medicine. 2003; 24(6):441-5. [PubMed: 12905093]

Bean J, Herman S, Kiely DK, Callahan D, Mizer K, Frontera WR, et al. Weighted stair climbing in mobility-limited older people: a pilot study. Journal of the American Geriatrics Society. 2002; 50(4):663-70. [PubMed: 11982666]

Bellew JW, Yates JW, Gater DR. The initial effects of low-volume strength training on balance in untrained older men and women. Journal of Strength \& Conditioning Research. 2003; 17(1):1218. [PubMed: 12580667]

Beniamini Y, Rubenstein JJ, Zaichkowsky LD, Leonard D, Crim MC. Effects of high-intensity strength training on quality-of-life parameters in cardiac rehabilitation patients. American Journal of Cardiology. 1997; 80(7):841-6. [PubMed: 9381995]

Beniamini Y, Rubenstein JJ, Faigehbaum AD, Lichenstein AH, Crim MC. High-intensity strength training of patients enrolled in an outpatient cardiac rehabilitation program. Journal of Cardiopulmonary Rehabilitation. 1999; 19(1):8-17. [PubMed: 10079415]

Berg WP, Lapp BA. The effect of a "practical" resistance training intervention on mobility in independent, community-dwelling older adults. Journal of Aging and Physical Activity. 1998; 6(1):18-35.

Bernard S, Whittom F, Leblanc P, Jobin J, Belleau R, Berube C, et al. Aerobic and strength training in patients with chronic obstructive pulmonary disease. American Journal of Respiratory Care and Critical Care Medicine. 1999; 159(3):896-901.

Bilodeau M, Keen DA, Sweeney PJ, Shields RW, Enoka RM. Strength training can improve steadiness in persons with essential tremor. Muscle and Nerve. 2000; 23:771-8. [PubMed: 10797401]

Binda SM, Culham EG, Brouwer B, Binda SM, Culham EG, Brouwer B. Balance, muscle strength, and fear of falling in older adults. Experimental Aging Research. 2003; 29(2):205-19. [PubMed: 12623729]

Binder EF, Schechtman KB, Ehsani AA, Steger-May K, Brown M, Sinacore DR, et al. Effects of exercise training on frailty in community-dwelling older adults: results of a randomized, controlled trial. Journal of the American Geriatrics Society. 2002; 50(12):1921-8. [PubMed: 12473001]

Binder EF, Yarasheski KE, Steger-May K, Sinacore DR, Brown M, Schechtman KB, et al. Effects of progressive resistance training on body composition in frail older adults: Results of a randomized, controlled trial. Journals of Gerontology Series A-Biological Sciences \& Medical Sciences. 2005; 60(11):1425-31.

Boardley D, Fahlman M, Topp R, Morgan AL, McNevin N. The impact of exercise training on blood lipids in older adults. American Journal of Geriatric Cardiology. 2007; 16(1):30-5. [PubMed: 17215640]

Braith RW, Magyari PM, Pierce GL, Edwards DG, Hill JA, White LJ, et al. Effect of resistance exercise on skeletal muscle myopathy in heart transplant recipients. American Journal of Cardiology. 2005; 95(10):1192-8. [PubMed: 15877992] 
Brandon LJ, Gaasch DA, Boyette LW, Lloyd AM. Effects of long-term resistive training on mobility and strength in older adults with diabetes. Journals of Gerontology Series A-Biological Sciences \& Medical Sciences. 2003; 58(8):740-5.

Brandon LJ, Boyette LW, Lloyd A, Gaasch DA. Resistive training and long-term function in older adults. Journal of Aging and Physical Activity. 2004; 12(1):10-28. [PubMed: 15211018]

*. Brill PA, Matthews M, Mason J, Davis D, Mustafa T, Macera C. Improving functional performance through a group-based free weight strength training program in residents of two assisted living communities. Physical \& Occupational Therapy in Geriatrics. 1998; 15(3):57-69.

Brill PA, Probst JC, Greenhouse DL, Schell B, Macera CA. Clinical feasibility of a free-weight strength-training program for older adults. Journal of the American Board of Family Practice. 1998; 11(6):445-51. [PubMed: 9875999]

Brose A, Parise G, Tarnopolsky MA. Creatine supplementation enhances isometric strength and body composition improvements following strength exercise training in older adults. Journals of Gerontology Series A-Biological Sciences \& Medical Sciences. 2003; 58(1):11-9.

Brown AB, McCartney N, Sale DG. Positive adaptations to weight-lifting in the elderly. Journal of Applied Physiology. 1990; 69(5):1725-33. [PubMed: 2272965]

Brown M, Holloszy JO. Effects of a low intensity exercise program on selected physical performance characteristics of 60- to 70- year olds. Aging (Milan, Italy). 1991; 3(2):129-39.

Brown M, Sinacore DR, Ehsani AA, Binder EF, Holloszy JO, Kohrt WM. Low-intensity exercise as a modifier of physical frailty in older adults. Archives of Physical Medicine and Rehabilitation. 2000; 81(7):960-5. [PubMed: 10896013]

Bunout B, Barrera G, de la Maza P, Avendano M, Gattas V, Petermann M, et al. Effects of nutritional supplementation and resistance training on muscle strength in free living elders. Results of one year follow. Journal of Nutrition, Health \& Aging. 2004; 8(2):68-75.

Bunout D, Barrera G, Avendano M, de la Maza P, Gattas V, Leiva L, et al. Results of a communitybased weight-bearing resistance training programme for healthy Chilean elderly subjects. Age and Ageing. 2005; 34(1):80-3. [PubMed: 15591487]

Bunout D, Barrera G, de la Maza P, Avendano M, Gattas V, Petermann M, et al. The impact of nutritional supplementation and resistance training on the health and function of free-living Chilean elders: results of 18 months of follow-up. Journal of Nutrition. 2001; 131(9):2441S-6S. [PubMed: 11533291]

Bunout D, Barrera G, Leiva L, Gattas V, de la Maza MP, Avendano M, et al. Effects of vitamin D supplementation and exercise training on physical performance in Chilean vitamin D deficient elderly subjects. Experimental Gerontology. 2006; 41(8):746-52. [PubMed: 16797903]

Campbell WW, Joseph LJO, Anderson RA, Davey SL, Hinton J, Evans WJ. Effects of resistive training and chromium picolinate on body composition and skeletal muscle size in older women. International Journal of Sport Nutrition and Exercise Metabolism. 2002; 12(2):125-35. MEDLINE: Wrong outcomes and involved medication, not RCT. [PubMed: 12187613]

Campbell WW, Joseph LJO, Ostlund RE Jr, Anderson RA, Farrell PA, Evans WJ. Resistive training and chromium picolinate: Effects on inositols and liver and kidney functions in older adults. International Journal of Sport Nutrition \& Exercise Metabolism. 2004; 14(4):430-42. [PubMed: 15467101]

Cancela Carral JM, Romo Perez V, Camina Fernandez F. Effect of strength program on a 65 years old elderly woman [Spanish]. Gerokomos. 2003; 14(2):80-9.

Candow DG, Chilibeck PD, Chad KE, Chrusch MJ, Shawn Davison K, Burke DG. Effect of ceasing creatine supplementation while maintaining resistance training in older men. Journal of Aging \& Physical Activity. 2004; 12(3):219-31. [PubMed: 15263100]

Capodaglio P, Facioli M, Burroni E, Giordano A, Ferri A, Scaglioni G. Effectiveness of a home-based strengthening program for elderly males in Italy. A preliminary study. Aging-Clinical \& Experimental Research. 2002; 14(1):28-34. [PubMed: 12027149]

Carter ND, Khan KM, McKay HA, Petit MA, Waterman C, Heinonen A, et al. Community-based exercise program reduces risk factors for falls in 65- to 75-year-old women with osteoporosis: randomized controlled trial. CMAJ. 2002; 167(9):997-1004. [PubMed: 12403738] 
Carter JM, Bemben DA, Knehans AW, Bemben MG, Witten MS. Does nutritional supplementation influence adaptability of muscle to resistance training in men aged 48 to 72 years. Journal of Geriatric Physical Therapy. 2005; 28(2):40-7. [PubMed: 16236227]

Carvalho, J.; Oliveira, J.; Magalhaes, J.; Ascensao, A.; Cabri, J.; Soares, JMC. The influence of initial strength levels on isokinetic torque after training in elderly adults; European College of Sport Science, Proceedings of the 7th annual congress of the European College of Sport Science; 2002 July 24-28; Athens Greece. Pashalidis Medical Publisher; p. 513

Cauza E, Hanusch-Enserer U, Strasser B, Kostner K, Dunky A, Haber P. Strength and endurance training lead to different post exercise glucose profiles in diabetic participants using a continuous subcutaneous glucose monitoring system. European Journal of Clinical Investigation. 2005; 35(12):745-51. [PubMed: 16313250]

Cauza E, Hanusch-Enserer U, Strasser B, Ludvik B, Metz-Schimmerl S, Pacini G, et al. The relative benefits of endurance and strength training on the metabolic factors and muscle function of people with type 2 diabetes mellitus. Archives of Physical Medicine and Rehabilitation. 2005; 86(8):1527-33. [PubMed: 16084803]

Chaloupka V, Elbl L, Nehyba S. Strength training in patients after myocardial infarct. Vnitrni Lekarstvi. 2000; 46(12):829-34. Silovy trenink u nemocnych po infarktu myokardu. [PubMed: 11214360]

Chetlin RD, Gutmann L, Tarnopolsky M, Ullrich IH, Yeater RA. Resistance training effectiveness in patients with Charcot-Marie-Tooth disease: recommendations for exercise prescription. Archives of Physical Medicine \& Rehabilitation. 2004; 85(8):1217-23. [PubMed: 15295743]

Chiba A. Positive effects of resistance training on QOL in the frail elderly. Japanese Journal of Public Health. 2006; 53(11):851-8. [PubMed: 17228754]

Chien MY, Yang RS, Tsauo JY. Home-based trunk-strengthening exercise for osteoporotic and osteopenic postmenopausal women without fracture - A pilot study. Clinical Rehabilitation. 2005; 19(1):28-36. [PubMed: 15704506]

Connelly DM, Vandervoort AA. Effects of detraining on knee extensor strength and functional mobility in a group of elderly women. Journal of Orthopaedic \& Sports Physical Therapy. 1997; 26(6):340-6. [PubMed: 9402571]

*. Connelly DM, Vandervoort AA. Improvement in knee extensor strength of institutionalized elderly women after exercise with ankle weights. Physiotherapy Canada. 1995; 41(1):15-23.

Connelly DM, Vandervoort AA. Effects of isokinetic strength training on concentric and eccentric torque development in the ankle dorsiflexors of older adults. The Journals of Gerontology Series A, Biological Sciences and Medical Sciences. 2000; 55:B465-72.

Cramp MC, Greenwood RJ, Gill M, Rothwell JC, Scott OM. Low intensity strength training for ambulatory stroke patients. Disability \& Rehabilitation. 2006; 28(13-14):883-9. [PubMed: 16777776]

Cress ME, Thomas DP, Johnson J, Kasch FW, Cassens RG, Smith EL, et al. Effect of training on VO2 max, thigh strength and muscle morphology in septuagenarian women. Medicine and Science in Sports and Exercise. 1991; 23:752-8. [PubMed: 1886486]

Cress ME, Buchner DM, Questad KA, Esselman PC, de Lateur BJ, Schwartz RS. Exercise: effects on physical functional performance in older adults. The Journals of Gerontology Series A, Biological Sciences and Medical Sciences. 1999; 54(5):M242-8.

Daepp C, Vogt M, Klossner S, Popp A, Lippuner K, Hoppeler H. Slimmer and stronger - the effect of eccentric exercise in the elderly. Schweizerische Zeitschrift Fuer Sportmedizin Und Sporttraumatologie/Revue Suisse De Medecine Et De Traumatologie Du Sport/Rivista Svizzera Di Medicina e Traumatologia Dello Sport. 2006; 54(2):71. 1422-0644.

Daly RM, Dunstan DW, Owen N, Jolley D, Shaw JE, Zimmet PZ. Does high-intensity resistance training maintain bone mass during moderate weight loss in older overweight adults with type 2 diabetes? Osteoporosis International. 2005; 16(12):1703-12. [PubMed: 15937634]

de Bruin ED, Menzi C, Waelle R, Murer K. Strength training and balance performance compared to combined strength and agility training in elderly over 80 years: a three months RCT. Isokinetics and Exercise Science. 2004; 12(1):33-4. 
*. de Bruin ED, Murer K. Effect of additional functional exercises on balance in elderly people. Clinical Rehabilitation. 2007; 21(2):112-21. [PubMed: 17264105]

de Vito G, Bernardi M, Forte R, Pulejo C, Figura F. Effects of a low-intensity programme on VO2 max and maximal instantaneous peak power in elderly women. European Journal of Applied Physiology and Occupational Physiology. 1999; 80(3):227-32. [PubMed: 10453925]

DeBolt LS, McCubbin JA, DeBolt LS, McCubbin JA. The effects of home-based resistance exercise on balance, power, and mobility in adults with multiple sclerosis. Archives of Physical Medicine \& Rehabilitation. 2004; 85(2):290-7. [PubMed: 14966716]

Delagardelle C, Feiereisen P, Autier P, Shita R, Krecke R, Beissel J. Strength/endurance training versus endurance training in congestive heart failure. Medicine \& Science in Sports \& Exercise. 2002; 34(12):1868-72. [PubMed: 12471289]

Delecluse C, Colman V, Roelants M, Verschueren S, Derave W, Ceux T, et al. Exercise programs for older men: mode and intensity to induce the highest possible health-related benefits. Preventive Medicine. 2004; 39(4):823-33. [PubMed: 15351552]

De Vito CA, Morgan RO, Duque M, Abdel-Moty E, Virnig BA. Physical performance effects of lowintensity exercise among clinically defined high-risk elders. Gerontology. 2003; 49(3):146-54. [PubMed: 12679604]

Dibble LE, Hale T, Marcus RL, Gerber JP, Lastayo PC. The safety and feasibility of high-force eccentric resistance exercise in persons with Parkinson's disease. Archives of Physical Medicine \& Rehabilitation. 2006; 87(9):1280-2. [PubMed: 16935068]

Dibble LE, Hale TF, Marcus RL, Droge J, Gerber JP, LaStayo PC. High-intensity resistance training amplifies muscle hypertrophy and functional gains in persons with Parkinson's disease. Movement Disorders. 2006; 21(9):1444-52. [PubMed: 16773643]

Dunstan DW, Daly RM, Owen N, Jolley D, De Courten M, Shaw J, et al. High-intensity resistance training improves glycemic control in older patients with type 2 diabetes. Diabetes Care. 2002; 25(10):1729-36. [PubMed: 12351469]

Dunstan DW, Daly RM, Owen N, Jolley D, Vulikh E, Shaw J, et al. Home-based resistance training is not sufficient to maintain improved glycemic control following supervised training in older individuals with type 2 diabetes. Diabetes Care. 2005; 28(1):3-9. [PubMed: 15616225]

Dupler TL, Cortes C. Effects of a whole-body resistive training regimen in the elderly. Gerontology. 1993; 39(6):314-9. [PubMed: 8144045]

Fernandez Ramirez AI, Fernandez Ramirez AS. Effect of an exercise program on physical fitness of institutionalized elderly men. Archivos de Medicina del Deporte. 1999; 16(72):325-32.

Ferrara CM, Goldberg AP, Ortmeyer HK, Ryan AS. Effects of aerobic and resistive exercise training on glucose disposal and skeletal muscle metabolism in older men. Journals of Gerontology Series A-Biological Sciences \& Medical Sciences. 2006; 61(5):480-7.

Ferri A, Scaglioni G, Pousson M, Capodaglio P, Van Hoecke J, Narici MV. Strength and power changes of the human plantar flexors and knee extensors in response to resistance training in old age. Acta Physiologica Scandinavica. 2003; 177(1):69-78. [PubMed: 12492780]

Fiatarone MA, Marks ED, Ryan ND, Meredith CN, Lipsitz LA, Evans WJ. High-intensity strength training in nonagenarians. Effects on skeletal muscle. JAMA. 1990; 263(22):3029-34. [PubMed: 2342214]

Fisher NM, Pendergast DR, Calkins E. Muscle rehabilitation in impaired elderly nursing home residents. Archives of Physical Medicine and Rehabilitation. 1991; 72:181-5. [PubMed: 1998450]

*. Fisher NM, Pendergast DR, Gresham GE, Calkins E. Muscle rehabilitation: its effect on muscular and functional performance of patients with knee osteoarthritis. Archives of Physical Medicine and Rehabilitation. 1991; 72(6):367-74. [PubMed: 2059102]

Forte R, De Vito G, Figura F. Effects of dynamic resistance training on heart rate variability in healthy older women. European Journal of Applied Physiology. 2003; 89(1):85-9. [PubMed: 12627310]

Frontera WR, Meredith C, O'Reilly KP, Knuttgen HG, Evans W. Strength conditioning in older men: skeletal muscle hypertrophy and improved function. Journal of Applied Physiology. 1988; 64(3): 1038-44. [PubMed: 3366726] 
Frontera WR, Meredith CN, O'Reilly KP, Evans WJ. Strength training and determinants of VO2 Max in older men. Journal of Applied Physiology. 1990; 68(1):329-33. [PubMed: 2312474]

Galvao DA, Nosaka K, Taaffe DR, Spry N, Kristjanson LJ, McGuigan MR, et al. Resistance training and reduction of treatment side effects in prostate cancer patients. Medicine \& Science in Sports \& Exercise. 2006; 38(12):2045-52. [PubMed: 17146309]

Grimby G, Aniansson A, Hedberg M, Henning GB, Grangard U, Kvist H. Training can improve muscle strength and endurance in 78- to 84-year-old men. Journal of Applied Physiology. 1992; 73(6):2517-23. [PubMed: 1490965]

Gur H, Cakin N, Akova B, Okay E, Kucukoglu S. Concentric versus combined concentric-eccentric isokinetic training: effects on functional capacity and symptoms in patients with osteoarthritis of the knee. Archives of Physical Medicine and Rehabilitation. 2002; 83(3):308-16. [PubMed: 11887109]

Hageman PA, Thomas VS. Gait performance in dementia: the effects of a 6-week resistance training program in an adult day-care setting. International Journal of Geriatric Psychiatry. 2002; 17(4): 329-34. [PubMed: 11994886]

Hakkinen A, Sokka T, Kotaniemi A, Kautiainen H, Jappinen I, Laitinen L, et al. Dynamic strength training in patients with early rheumatoid arthritis increases muscle strength but not bone mineral density. Journal of Rheumatology. 1999; 26(6):1257-63. [PubMed: 10381039]

Hameed M, Lange KHW, Andersen JL, Schjerling P, Kjaer M, Harridge SDR, et al. The effect of recombinant human growth hormone and resistance training on IGF-I mRNA expression in the muscles of elderly men. Journal of Physiology. 2004; 555(1):231-40. [PubMed: 14565994]

Hartard M, Haber P, Ilieva D, Preisinger E, Seidl G, Huber J. Systematic strength training as a model of therapeutic intervention. A controlled trial in postmenopausal women with osteopenia. American Journal of Physical Medicine \& Rehabilitation. 1996; 75(1):21-8. [PubMed: 8645434]

Haub MD, Wells AM, Tarnopolsky MA, Campbell WW. Effect of protein source on resistive-traininginduced changes in body composition and muscle size in older men. American Journal of Clinical Nutrition. 2002; 76(3):511-7. [PubMed: 12197993]

Heiwe S, Clyne N, Tollback A, Borg K. Effects of regular resistance training on muscle histopathology and morphometry in elderly patients with chronic kidney disease. American Journal of Physical Medicine \& Rehabilitation. 2005; 84(11):865-74. [PubMed: 16244524]

Henwood TR, Taaffe DR. Short-term resistance training and the older adult: The effect of varied programmes for the enhancement of muscle strength and functional performance. Clinical Physiology \& Functional Imaging. 2006; 26(5):305-13. [PubMed: 16939509]

Hess JA, Woollacott M. Effect of high-intensity strength-training on functional measures of balance ability in balance-impaired older adults. Journal of Manipulative \& Physiological Therapeutics. 2005; 28(8):582-90. [PubMed: 16226626]

Hess JA, Woollacott M, Shivitz N. Ankle force and rate of force production increase following high intensity strength training in frail older adults. Aging-Clinical \& Experimental Research. 2006; 18(2):107-115. [PubMed: 16702779]

Hirsch MA, Toole T, Maitland CG, Rider RA. The effects of balance training and high-intensity resistance training on persons with idiopathic Parkinson's disease. Archives of Physical Medicine and Rehabilitation. 2003; 84(8):1109-17. [PubMed: 12917847]

Host HH, Sinacore DR, Bohnert KL, Steger-May K, Brown M, Binder EF. Training-induced strength and functional adaptations after hip fracture. Physical Therapy. 2007; 87:292-303. [PubMed: 17284548]

Huggett DL, Elliott ID, Overend TJ, Vandervoort AA. Comparison of heart-rate and blood-pressure increases during isokinetic eccentric versus isometric exercise in older adults. Journal of Aging \& Physical Activity. 2004; 12(2):157-69. [PubMed: 15223884]

Hughes SL, Seymour RB, Campbell R, Pollak N, Huber G, Sharma L. Impact of the fit and strong intervention on older adults with osteoarthritis. Gerontologist. 2004; 44(2):217-28. [PubMed: 15075418]

Hughes SL, Seymour RB, Campbell RT, Huber G, Pollak N, Sharma L, et al. Long-term impact of fit and strong! On older adults with osteoarthritis. Gerontologist. 2006; 46(6):801-14. [PubMed: 17169935] 
Humphries B, Newton RU, Bronks R, Marshall S, McBride J, Triplett-McBride T, et al. Effect of exercise intensity on bone density, strength, and calcium turnover in older women. Medicine \& Science in Sports \& Exercise. 2000; 32(6):1043-50. [PubMed: 10862528]

Hung C, Daub B, Black B, Welsh R, Quinney A, Haykowsky M. Exercise training improves overall physical fitness and quality of life in older women with coronary artery disease. Chest. 2004; 126(4):1026-31. see comment. [PubMed: 15486358]

Hunter GR, Treuth MS, Weinsier RL, Kekes-Szabo T, Kell SH, Roth DL, et al. The effects of strength conditioning on older women's ability to perform daily tasks. Journal of the American Geriatrics Society. 1995; 43(7):756-60. [PubMed: 7602026]

Hunter GR, Bryan DR, Wetzstein CJ, Zuckerman PA, Bamman MM. Resistance training and intraabdominal adipose tissue in older men and women. Medicine \& Science in Sports \& Exercise. 2002; 34(6):1023-8. [PubMed: 12048332]

Ibanez J, Izquierdo M, Arguelles I, Forga L, Larrion JL, Garcia-Unciti M, et al. Twice-weekly progressive resistance training decreases abdominal fat and improves insulin sensitivity in older men with type 2 diabetes. Diabetes Care. 2005; 28(3):662-7. [PubMed: 15735205]

Ivey FM, Tracy BL, Lemmer JT, NessAiver M, Metter EJ, Fozard JL, et al. Effects of strength training and detraining on muscle quality: age and gender comparisons. Journals of Gerontology Series A, Biological Sciences and Medical Sciences. 2000; 55(3):B152-7.

Johansen KL, Painter PL, Sakkas GK, Gordon P, Doyle J, Shubert T. Effects of resistance exercise training and nandrolone decanoate on body composition and muscle function among patients who receive hemodialysis: A randomized, controlled trial. Journal of the American Society of Nephrology. 2006; 17(8):2307-14. [PubMed: 16825332]

Jones D, Rutherford O. Human muscle strength training: The effects of three different regimes and the nature of the resultant changes. Journal of Physiology. 1987; 391:1-11. [PubMed: 3443943]

Judge JO, Kleppinger A, Kenny A, Smith JA, Biskup B, Marcella G. Home-based resistance training improves femoral bone mineral density in women on hormone therapy. Osteoporosis International. 2005; 16(9):1096-108. [PubMed: 15754082]

Katula JA, Sipe M, Rejeski WJ, Focht BC. Strength training in older adults: an empowering intervention. Medicine and Science in Sports and Exercise. 2006; 38(1):106-11. [PubMed: 16394961]

Kerr D, Ackland T, Maslen B, Morton A, Prince R. Resistance training over 2 years increases bone mass in calcium-replete postmenopausal women. Journal of Bone \& Mineral Research. 2001; 16(1):175-81. [PubMed: 11149482]

Kolbe-Alexander TL, Charlton KE, Lambert EV. Effectiveness of a community based low intensity exercise program for older adults. Journal of Nutrition, Health \& Aging. 2006; 10(1):21-9.

Komatireddy GR, Leitch RW, Cella K, Browning G, Minor M. Efficacy of low load resistive muscle training in patients with rheumatoid arthritis functional class II and III. Journal of Rheumatology. 1997; 24(8):1531-9. [PubMed: 9263147]

La Forge R. Effect of high-intensity resistance exercise on elderly bones. IDEA Health \& Fitness Source. 2002; 20(5):17. 1096-8156.

Labarque V, 'T Eijnde BO, Van Leemputte M. Resistance training alters torque-velocity relation of elbow flexors in elderly men. Medicine \& Science in Sports \& Exercise. 2002; 34(5):851-56. L ' entrainement de force altere la relation torsion-velocite des muscles flechisseurs du coude chez les hommes ages. 0195-9131. [PubMed: 11984305]

Lambert CP, Sullivan DH, Freeling SA, Lindquist DM, Evans WJ. Effects of testosterone replacement and/or resistance exercise on the composition of megestrol acetate stimulated weight gain in elderly men: A randomized controlled trial. Journal of Clinical Endocrinology \& Metabolism. 2002; 87(5):2100-6. [PubMed: 11994348]

Lambert CP, Sullivan DH, Evans WJ. Megestrol acetate-induced weight gain does not negatively affect blood lipids in elderly men: Effects of resistance training and testosterone replacement. Journals of Gerontology Series A-Biological Sciences \& Medical Sciences. 2003; 58(7):644647. 
Lamotte M, Niset G, Van de Borne P. The effect of different intensity modalities of resistance training on beat-to-beat blood pressure in cardiac patients. European Journal of Cardiovascular Prevention \& Rehabilitation. 2005; 12(1):12-7. [PubMed: 15703501]

Levinger I, Bronks R, Cody DV, Linton I, Davie A. Resistance training for chronic heart failure patients on beta blocker medications. International Journal of Cardiology. 2005; 102(3):493-9. [PubMed: 16004896]

Lexell J, Robertsson E, Stenstrom E. Effects of strength training in elderly women. Journal of the American Geriatrics Society. 1992; 40(2):190-1. letter. [PubMed: 1740607]

Lexell J, Downham DY, Larsson Y, Bruhn E, Morsing B. Heavy-resistance training in older Scandinavian men and women: short- and long-term effects on arm and leg muscles. Scandinavian Journal of Medicine \& Science in Sports. 1995; 5(6):329-41. [PubMed: 8775717]

Littbrand H, Rosendahl E, Lindelof N, Lundin-Olsson L, Gustafson Y, Nyberg L. A high-intensity functional weight-bearing exercise program for older people dependent in activities of daily living and living in residential care facilities: evaluation of the applicability with focus on cognitive function. Physical Therapy. 2006; 86(4):489-98. [PubMed: 16579666]

Liu SW, Liu HB, Tang D. Strengthened training of knee joint isolated movement for ambulation ability in patients with stroke. Zhongguo Linchuang Kangfu. 2004; 8(25):5214-5. Chinese.

Liu-Ambrose TYL, Khan KM, Eng JJ, Heinonen A, McKay HA. Both resistance and agility training increase cortical bone density in 75- to 85-year-old women with low bone mass: A 6-month randomized controlled trial. Journal of Clinical Densitometry. 2004; 7(4):390-8. [PubMed: 15618599]

Loeppky JA, Gurney B, Kobayashi Y, Icenogle MV. Effects of ischemic training on leg exercise endurance. Journal of Rehabilitation Research and Development. 2005; 42(4):511-21. [PubMed: 16320146]

Lohman T, Going S, Pamenter R, Hall M, Boyden T, Houtkooper L, et al. Effects of resistance training on regional and total bone mineral density in premenopausal women: a randomized prospective study. Journal of Bone \& Mineral Research. 1995; 10(7):1015-24. [PubMed: 7484276]

Maddalozzo GF, Snow CM. High intensity resistance training: effects on bone in older men and women. Calcified Tissue International. 2000; 66(6):394-404.

Magnusson G, Gordon A, Kaijser L, Sylven C, Isberg B, Karpakka J, et al. High intensity knee extensor training in patients with chronic heart failure. European Heart Journal. 1996; 17(7): 1048-55. [PubMed: 8809523]

Marcora SM, Lemmey AB, Maddison PJ. Can progressive resistance training reverse cachexia in patients with rheumatoid arthritis? Results of a pilot study. Journal of Rheumatology. 2005; 32(6):1031-9. [PubMed: 15940763]

Martin Ginis KA, Latimer AE, Brawley LR, Jung ME, Hicks AL. Weight training to activities of daily living: Helping older adults make a connection. Medicine \& Science in Sports \& Exercise. 2006; 38(1):116-21. [PubMed: 16394963]

McCool J, Schneider J. Home-based leg strengthening for older adults initiated through private practice. Preventative Medicine. 1991; 28:105-10.

McMurdo M, Rennie L. Improvements in quadriceps strength with regular seated exercise in the institutionalised elderly. Archives of Physical Medicine and Rehabilitation. 1994; 75:600-3. [PubMed: 8185457]

Mobily KE, Mobily PR, Raimondi RM, Walter KL, Rubenstein LM. Strength training and falls among older adults: a community-based TR intervention. Annual in Therapeutic Recreation. 2004; 13:111. 109-18.

Morey MC, Cowper PA, Feussner JR, DiPasquale RC, Crowley GM, Kitzman DW, et al. Evaluation of a supervised exercise program in a geriatric population. Journal of the American Geriatrics Society. 1989; 37:348-54. [PubMed: 2921457]

Morey MC, Cowper PA, Feussner JR, DiPasquale RC, Crowley GM, Sullivan RJ Jr. Two-year trends in physical performance following supervised exercise among community-dwelling older veterans. Journal of the American Geriatrics Society. 1991; 39(6):549-54. [PubMed: 2037744] 
Morse CI, Thom JM, Mian OS, Muirhead A, Birch KM, Narici MV. Muscle strength, volume and activation following 12-month resistance training in 70-year-old males. European Journal of Applied Physiology. 2005; 95(2-3):197-204. [PubMed: 16003538]

Narici MV, Roi GS, Landoni L, Minetti AE, Cerretelli P. Changes in force, cross-sectional area and neural activation during strength training and detraining of the human quadriceps. European Journal of Applied Physiology and Occupational Physiology. 1989; 59(4):310-9. [PubMed: 2583179]

Hausdorff JM, Nelson ME, Kaliton D, Layne JE, Bernstein MJ, Nuernberger A, et al. Etiology and modification of gait instability in older adults: A randomized controlled trial of exercise. Journal of Applied Physiology. 2001; 90:2117-29. [PubMed: 11356774]

*. Nelson ME, Layne JE, Nuernberger A, Allen MJ, Judge J, Kailiton D, et al. Home-based exercise training in the frail elderly: Effects on physical performance. Medicine and Science in Sports and Exercise. 1997; 29(Suppl 5):S110.

Ochala J, Lambertz D, Van Hoecke J, Pousson M, Ochala J, Lambertz D, et al. Effect of strength training on musculotendinous stiffness in elderly individuals. European Journal of Applied Physiology. 2005; 94(1-2):126-33. [PubMed: 15702341]

Ohira T, Schmitz KH, Ahmed RL, Yee D. Effects of weight training on quality of life in recent breast cancer survivors: the Weight Training for Breast Cancer Survivors (WTBS) study. Cancer. 2006; 106(9):2076-83. [PubMed: 16568409]

Oka RK, De Marco T, Haskell WL, Botvinick E, Dae MW, Bolen K, et al. Impact of a home-based walking and resistance training program on quality of life in patients with heart failure. American Journal of Cardiology. 2000; 85(3):365-9. [PubMed: 11078308]

Okawa T, Sato T, Koike T. Effect of exercises on bone mineral density and physical strength in elderly women. Nippon Rinsho - Japanese Journal of Clinical Medicine. 2004; 62(Suppl 2):510-4.

Okumiya K, Matsubayashi K, Wada T, Kimura S, Doi Y, Ozawa T. Effects of exercise on neurobehavioral function in community-dwelling older people more than 75 years of age. Journal of the American Geriatrics Society. 1996; 44(5):569-72. [PubMed: 8617907]

Panton LB, Golden J, Broeder CE, Browder KD, Cestaro-Seifer DJ, Seifer FD. The effects of resistance training on functional outcomes in patients with chronic obstructive pulmonary disease. European Journal of Applied Physiology. 2004; 91(4):443-9. [PubMed: 14639479]

Parsons D, Foster V, Harman F, Dickinson A, Olivia P, Westerlind K. Balance and strength changes in elderly subjects after heavy resistance strength training. Medicine and Science in Sports and Exercise. 1992; 24(Suppl 5):S21. abstract.

Perhonen M, Komi P, Hakkinen K, Von Bonsdorff H, Partio E. Strength training and neuromuscular function in elderly people with total knee endoprosthesis. Scandinavian Journal of Medicine \& Science in Sports. 1992; 2:234-43.

Perkins L, Kaiser H. Results of short-term isotonic and isometric exercise programs in persons over sixty. Physical Therapy Review. 1961; 41:633-5. [PubMed: 13734405]

Perrig-Chiello P, Perrig WJ, Ehrsam R, Staehelin HB, Krings F. The effects of resistance training on well-being and memory in elderly volunteers. Age and Ageing. 1998; 27(4):469-76. [PubMed: 9884004]

Petrella R, Bartha C. Home based exercise therapy for older patients with knee osteoarthritis: a randomized clinical trial. Journal of Rheumatology. 2000; 27(9):2215-21. [PubMed: 10990236]

Phillips WT, Ziuraitis JR. Energy cost of single-set resistance training in older adults. Journal of Strength and Conditioning Research. 2004; 18(3):606-9. [PubMed: 15320686]

Pyka G, Lindenberger E, Charette S, Marcux R. Muscle strength and fiber adaptations to a year-long resistance training program. Journal of Gerontology. 1994; 49(1):M22-7. [PubMed: 8282977]

Rabelo HT, Oliveira RJ, Bottaro M. Effects of resistance training on activities of daily living in older women. Biology of Sport. 2004; 21(4):325-36. 0860-021X.

Ramsbottom R, Ambler A, Potter J, Jordan B, Nevill A, Williams C. The effect of 6 months training on leg power, balance, and functional mobility of independently living adults over 70 years old. Journal of Aging \& Physical Activity. 2004; 12(4):497-510. [PubMed: 15851822] 
Reeves ND, Narici MV, Maganaris CN. Effect of resistance training on skeletal muscle-specific force in elderly humans. Scandinavian Journal of Medicine \& Science in Sports. 2004; 14(2):134-5. Abstract. 0905-7188.

Reeves ND, Maganaris CN, Narici MV. Plasticity of dynamic muscle performance with strength training in elderly humans. Muscle \& Nerve. 2005; 31(3):355-64. [PubMed: 15654690]

Reeves ND, Narici MV, Maganaris CN. Musculoskeletal adaptations to resistance training in old age. Manual Therapy. 2006; 11(3):192-6. [PubMed: 16782393]

Richards, D. Efficacy of upper extremity strength training on upper extremity functional performance among elderly long-term care residents [thesis]. Pittsburgh (PA): Univ. of Pittsburgh; 1996.

Roman WJ, Fleckenstein J, Stray-Gundersen J, Alway SE, Peshock R, Gonyea WJ. Adaptations in the elbow flexors of elderly males after heavy-resistance training. Journal of Applied Physiology. 1993; 74(2):750-4. [PubMed: 8458791]

Rooks D, Kiel D, Parsons C, Hayes W. Self-paced resistance training and walking exercise in community-dwelling older adults: effects on neuromotor performance. Journals of Gerontology Series A, Biological Sciences and Medical Sciences. 1997; 52(3):M161-8.

Salli A, Ugurlu H, Emlik D. Comparison of the effectiveness of concentric, combined concentriceccentric and isometric exercises on symptoms and functional capacity in patients with knee osteoarthritis. Turkiye Fiziksel Tip ve Rehabilitasyon Dergisi. 2006; 52(2):61-7. Diz Osteoartritinde Konsantrik, Kombine Konsantrik-Eksantrik ve Izometrik Egzersizlerin Semptomlar ve Fonksiyonel Kapasite Uzerine Etkinliginin Karsilastirilmasi.

Sallinen J, Pakarinen A, Fogelholm M, Sillanpaa E, Alen M, Volek JS, et al. Serum basal hormone concentrations and muscle mass in aging women: effects of strength training and diet. International Journal of Sport Nutrition \& Exercise Metabolism. 2006; 16(3):316-31. [PubMed: 16948487]

Sanders, S. The effects of two modes of strength training on elderly men [thesis]. Minneapolis (MN): Walden University; 1998.

Sartorio A, Lafortuna C, Capodaglio P, Vangeli V, Narici MV, Faglia G. Effects of a 16-week progressive high-intensity strength training (HIST) on indexes of bone turnover in men over 65 years: A randomized controlled study. Journal of Endocrinological Investigation. 2001; 24(11): 882-6. [PubMed: 11817713]

Sauvage LJ, Myklebust B, Crow-Pan J, Novak S, Millington P, Hoffman MD, et al. A clinical trial of strengthening and aerobic exercise to improve gait and balance in elderly male nursing home residents. American Journal of Physical Medicine and Rehabilitation. 1992; 71:333-42. [PubMed: 1466871]

Sayers SP, Bean J, Cuoco A, LeBrasseur NK, Jette A, Fielding RA. Changes in function and disability after resistance training: does velocity matter? A pilot study. American Journal of Physical Medicine \& Rehabilitation. 2003; 82(8):605-13. [PubMed: 12872017]

Schott N, Konietzny S, Raschka C. Red ginseng enhances the effectiveness of strength training in elderly: A randomized placebo-controlled double-blind trial. Schweizerische Zeitschrift fur Ganzheitsmedizin. 2006; 18(7-8):376-83. Einfluss von Rotem Ginseng auf ein Krafttraining bei alteren Erwachsenen: Eine randomisierte placebokontrollierte Doppelblindstudie.

Sharp S, Brouwer B. Isokinetic strength training of the hemiparetic knee: effects on function and spasticity. Archives of Physical Medicine and Rehabilitation. 1997; 78:1231-6. [PubMed: 9365354]

Shaw JM, Snow CM. Weighted vest exercise improves indices of fall risk in older women. Journals of Gerontology Series A, Biological Sciences and Medical Sciences. 1998; 53(1):M53-8.

Sherrington C, Lord SR. Home exercise to improve strength and walking velocity after hip fracture: a randomized controlled trial. Archives of Physical Medicine and Rehabilitation. 1997; 78:208-12. [PubMed: 9041904]

Signorile JF, Carmel MP, Lai S, Roos BA. Early plateaus of power and torque gains during high- and low-speed resistance training of older women. Journal of Applied Physiology. 2005; 98(4):121320. [PubMed: 15772058]

Sinaki M, Wahner H, Bergstralh E, Hodgson SF, Offord KP, Squires RW, et al. Three-year controlled, randomized trial of the effect of dose-specified loading and strengthening exercises on bone 
mineral density of spine and femur in nonalthletic, physically active women. Bone. 1996; 19(3): 233-44. [PubMed: 8873964]

Sipila S, Suominen H. Knee extension strength and walking speed in relation to quadriceps muscle composition and training in elderly women. Clinical Physiology. 1994; 14(4):433-42. [PubMed: 7955941]

Spruit MA, Gosselink R, Troosters T, De Paepe K, Decramer M. Resistance versus endurance training in patients with COPD and peripheral muscle weakness. European Respiratory Journal. 2002; 19(6):1072-8. [PubMed: 12108859]

Sullivan DH, Wall PT, Bariola JR, Bopp MM, Frost YM. Progressive resistance muscle strength training of hospitalized frail elderly. American Journal of Physical Medicine \& Rehabilitation. 2001; 80:503-9. [PubMed: 11421518]

Taaffe DR, Marcus R. Dynamic muscle strength alterations to detraining and retraining in elderly men. Clinical Physiology. 1997; 17(3):311-24. [PubMed: 9171971]

Teixeira R, Guerra S, Esculcas C, Ribeiro JC, Carvalho J, Mota J. Influence of age on physical fitness improvements after training on elderly women. Koskolou. 2002; 514 Abstract.

Teixeira PJ, Going SB, Houtkooper LB, Metcalfe LL, Blew RM, Flint-Wagner HG, et al. Resistance training in postmenopausal women with and without hormone therapy. Medicine and Science in Sports and Exercise. 2003; 35(4):555-62. [PubMed: 12673136]

Teixeira-Salmela LF, Santiago L, Lima RC, Lana DM, Camargos FF, Cassiano JG. Functional performance and quality of life related to training and detraining of community-dwelling elderly. Disability \& Rehabilitation. 2005; 27(17):1007-12. [PubMed: 16096254]

Thielman GT, Dean CM, Gentile AM. Rehabilitation of reaching after stroke: Task-related training versus progressive resistive exercise. Archives of Physical Medicine \& Rehabilitation. 2004; 85(10):1613-8. [PubMed: 15468020]

Thomas KJ, Tomsic JB, Martin MS. Does participation in light to moderate strength and endurance exercise result in measurable physical benefits for older adults? Journal of Geriatric Physical Therapy. 2004; 27(2):53-8.

Thomas GN, Hong AW, Tomlinson B, Lau E, Lam CW, Sanderson JE, et al. Effects of Tai Chi and resistance training on cardiovascular risk factors in elderly Chinese subjects: a 12-month longitudinal, randomized, controlled intervention study. Clinical Endocrinology. 2005; 63(6): 663-9. [PubMed: 16343101]

Thompson RF, Crist DM, Marsh M, Rosenthal M. Effects of physical exercise for elderly patients with physical impairments. Journal of the American Geriatrics Society. 1988; 36(2):130-5. [PubMed: 3339217]

Timonen L, Rantanen T, Ryynanen OP, Taimela S, Timonen TE, Sulkava R. A randomized controlled trial of rehabilitation after hospitalization in frail older women: effects on strength, balance and mobility. Scandinavian Journal of Medicine \& Science in Sports. 2002; 12(3):186-92. [PubMed: 12135452]

Timonen L, Rantanen T, Makinen E, Timonen TE, Tormakangas T, Sulkava R. Effects of group-based exercise program on functional abilities in frail older women after hospital discharge. AgingClinical \& Experimental Research. 2006; 18(1):50-6. [PubMed: 16608136]

Timonen L, Rantanen T, Timonen TE, Sulkava R. Effects of a group-based exercise program on the mood state of frail older women after discharge from hospital. International Journal of Geriatric Psychiatry. 2006; 17(12):1106-11. [PubMed: 12461758]

Treuth MS, Ryan AS, Pratley RE, Rubin MA, Miller JP, Nicklas BJ, et al. Effects of strength training on total and regional body composition in older men. Journal of Applied Physiology. 1994; 77(2):614-20. [PubMed: 8002507]

Trudelle-Jackson E, Smith SS. Effects of a late-phase exercise program after total hip arthroplasty: a randomized controlled trial. Archives of Physical Medicine and Rehabilitation. 2004; 85(7): 1056-62. [PubMed: 15241750]

Tsuji I, Tamagawa A, Nagatomi R, Irie N, Ohkubo T, Saito M, et al. Randomised controlled trial of exercise training for older people: study design and primary outcome. Journal of Epidemiology. 2000; 10(1):55-64. [PubMed: 10695262] 
Vad E, Worm C, Lauritsen JM, Poulsen PB, Puggaard L, Stovring H, et al. Physical training as treatment of reduced functional ability in frail $75+$ year-olds living at home. A randomized intervention study in general practice with technological assessment elements. Ugeskrift for Laeger. 2002; 164(44):5140-4. Fysisk traening som behandling af nedsat funktionsevne hos svage, hjemmeboende 75+-arige. Et randomiseret interventionsstudie i almen praksis omfattende elementer til en teknologivurdering. [PubMed: 12448161]

Vale RGS, Damasceno V, Cordeiro LS, Baptista MR, Pernambuco CS, Motta T, et al. Effects of supine resistance strength training in independent elderly women. Fitness \& Performance Journal. 2003; 2(4):255. Efeitos de um treinamento resistido de forca no supino reto em idosas independentes [Abstract]. 1519-9088.

Valkeinen H, Hakkinen K, Pakarinen A, Hannonen P, Hakkinen A, Airaksinen O, et al. Muscle hypertrophy, strength development, and serum hormones during strength training in elderly women with fibromyalgia. Scandinavian Journal of Rheumatology. 2005; 34(4):309-14. [PubMed: 16195165]

Van den Ende CH, Breedveld FC, le Cessie S, Dijkmans BA, de Mug AW, Hazes JM. Effect of intensive exercise on patients with active rheumatoid arthritis: a randomised clinical trial. Annals of the Rheumatic Diseases. 2000; 59(8):615-21. [PubMed: 10913058]

Vanbiervliet W, Pelissier J, Ledermann B, Kotzki N, Benaim C, Herisson C, et al. Strength training with elastic bands: measure of its effects in cardiac rehabilitation after coronary diseases. Annales de Readaptation et de Medecine Physique. 2003; 46(8):545-52. Le renforcement musculaire par bandes elastiques: evaluation de ses effets dans le reentrainement a l'effort du coronarien. [PubMed: 14585532]

Veloso U, Monteiro W, Farinatti P. Do continuous and intermittent exercises sets induce similar cardiovascular responses in the elderly women? Revista Brasileira de Medicina do Esporte. 2003; 9(2):85-90. 1517-8692.

Verfaillie D, Nichols J, Turkel E, Hovell M. Effects of resistance, balance and gait training on reduction of risk factors leading to falls in elders. Journal of Aging and Physical Activity. 1997; 5:213-28.

Villareal DT, Binder EF, Yarasheski KE, Williams DB, Brown M, Sinacore DR, et al. Effects of exercise training added to ongoing hormone replacement therapy on bone mineral density in frail elderly women. Journal of the American Geriatrics Society. 2003; 51(7):985-90. [PubMed: 12834519]

Villareal DT, Holloszy JO. DHEA enhances effects of weight training on muscle mass and strength in elderly women and men. American Journal of Physiology -Endocrinology \& Metabolism. 2006; 291(5):E1003-8. [PubMed: 16787962]

Vincent KR, Vincent HK, Braith RW, Lennon SL, Lowenthal DT. Resistance exercise training attenuates exercise-induced lipid peroxidation in the elderly. European Journal of Applied Physiology. 2002; 87(4/5):416-23. 1439-6327. [PubMed: 12172882]

Vincent KR, Vincent HK, Braith RW, Bhatnagar V, Lowenthal DT. Strength training and hemodynamic responses to exercise. American Journal of Geriatric Cardiology. 2003; 12(2):97106. [PubMed: 12624579]

Vincent KR, Braith RW, Vincent HK. Influence of resistance exercise on lumbar strength in older, overweight adults. Archives of Physical Medicine and Rehabilitation. 2006; 87(3):383-9. 00039993. [PubMed: 16500173]

Woo J, Hong A, Lau E, Lynn H. A randomised controlled trials of Tai Chi and resistance exercise on bone health, muscle strength and balance in community-living elderly people. Age and Ageing. 2007; 36:262-8. [PubMed: 17356003]

Yang Y, Wang R, Lin K, Chu M, Chan R. Task-oriented progressive resistance strength training improves muscle strength and functional performance in individuals with stroke. Clinical Rehabilitation. 2006; 20(10):860-70. [PubMed: 17008338]

Zion AS, De Meersman R, Diamond BE, Bloomfield DM, Zion AS, De Meersman R, et al. A homebased resistance-training program using elastic bands for elderly patients with orthostatic hypotension. Clinical Autonomic Research. 2003; 13(4):286-92. [PubMed: 12955554] 


\section{References to studies awaiting assessment}

Bennell KL, Hunt MA, Wrigley TV, Hunter DJ. The effects of hip muscle strengthening on knee load, pain, and function in people with knee osteoarthritis: a protocol for a randomised, single-blind controlled trial. BMC Musculoskeletal Disorders. 2007; 8:121. [PubMed: 18067658]

Cheema B, Abas H, Smith B, O'Sullivan A, Chan M, Patwardhan A, et al. Progressive exercise for anabolism in kidney disease (PEAK): a randomized, controlled trial of resistance training during hemodialysis. Journal of the American Society of Nephrology. 2007; 18(5):1594-601. [PubMed: 17409306]

Fahlman M, Morgan A, McNevin N, Topp R, Boardley D. Combination training and resistance training as effective interventions to improve functioning in elders. Journal of Aging \& Physical Activity. 2007; 15(2):195-205. [PubMed: 17556785]

*. Henwood TR, Riek S, Taaffe DR. Strength versus muscle power-specific resistance training in community-dwelling older adults. Journals of Gerontology Series A-Biological Sciences \& Medical Sciences. 2008; 63(1):83-91.

Karinkanta S, Heinonen A, Sievanen H, Uusi-Rasi K, Pasanen M, Ojala K, et al. A multi-component exercise regimen to prevent functional decline and bone fragility in home-dwelling elderly women: randomized, controlled trial. Osteoporosis International. 2007; 18(4):453-62. MEDLINE: Yes. [PubMed: 17103296]

Lin DH, Lin YF, Chai HM, Han YC, Jan MH. Comparison of proprioceptive functions between computerized proprioception facilitation exercise and closed kinetic chain exercise in patients with knee osteoarthritis. Clinical Rheumatology. 2007; 26(4):520-8. erratum appears in Clin Rheumatol. 2007 Apr;26(4):617 Note: Chai, Hei-Min [corrected to Chai, Huei-Ming]. MEDLINE: yes. [PubMed: 16786252]

Mitchell SL, Stott DJ, Martin BJ, Grant SJ. Randomized controlled trial of quadriceps training after proximal femoral fracture. Clinical Rehabilitation. 2001; 15(3):282-290. [PubMed: 11386398]

O'Shea SD, Taylor NF, Paratz JD. A predominantly home-based progressive resistance exercise program increases knee extensor strength in the short-term in people with chronic obstructive pulmonary disease: a randomised controlled trial. Australian Journal of Physiotherapy. 2007; 53(4):229-37. [PubMed: 18047457]

Raso V, Benard G, Da Silva Duarte AJ, Natale VM. Effect of resistance training on immunological parameters of healthy elderly women. Medicine \& Science in Sports \& Exercise. 2007; 39(12): 2152-9. [PubMed: 18046186]

Williams AD, Carey MF, Selig S, Hayes A, Krum H, Patterson J, et al. Circuit resistance training in chronic heart failure improves skeletal muscle mitochondrial ATP production rate - a randomized controlled trial. Journal of Cardiac Failure. 2007; 13(2):79-85. [PubMed: 17395046]

\section{Additional references}

Guideline for the prevention of falls in older persons. American Geriatrics Society, British Geriatrics Society, and American Academy of Orthopaedic Surgeons Panel on Falls Prevention. Journal of the American Geriatrics Society. 2001; 49(5):664-72. [PubMed: 11380764]

Brosseau L, MacLeay L, Welch V, Tugwell P, Wells G. Intensity of exercise for the treatment of osteoarthritis. Cochrane Database of Systematic Reviews. 2003; (2)10.1002/14651858CD004259

Buchner DM. Understanding variability in studies of strength training in older adults: a meta-analytic perspective. Topics in Geriatric Rehabilitation. 1993; 8(3):1-21.

Buchner D, Larson E, Wagner E, Koepsell T, De Lateur B. Evidence for a non-linear relationship between leg strength and gait speed. Age and Ageing. 1996; 25:386-91. [PubMed: 8921145]

Chandler JM, Hadley EC. Exercise to improve physiologic and functional performance in old age. Clinics in Geriatric Medicine. 1996; 12(4):761-84. [PubMed: 8890115]

Doherty TJ, Vandervoort AA, Brown WF. Effects of ageing on the motor unit: A brief review. Canadian Journal of Applied Physiology. 1993; 18(4):331-58. [PubMed: 8275048]

Fiatarone M, Evans W. The etiology and reversibility of muscle dysfunction in the aged. Journals of Gerontology. 1993; 48(special):77-83. [PubMed: 8409245] 
Frontera W, Meredith CN, O'Reilly K, Knuttgen H, Evans W. Strength conditioning in older men: skeletal muscle hypertrophy and improved function. Journal of Applied Physiology. 1988; 64(3): 1038-44. [PubMed: 3366726]

Gillespie LD, Gillespie WJ, Robertson MC, Lamb SE, Cumming RG, Rowe BH. Interventions for preventing falls in elderly people. Cochrane Database of Systematic Reviews. 2009; (2)10.1002/14651858CD000340.pub2

Guralnik J, Ferrucci L, Simonsick E, Salive M, Wallace R. Lower-extremity function in persons over the age of 70 years as a predictor of subsequent disability. The New England Journal of Medicine. 1995; 332:556-61. [PubMed: 7838189]

Higgins, JPT.; Green, S. [accessed 01 May 2007] Highly sensitive search strategies for identifying reports of randomized controlled trials in MEDLINE. Cochrane Handbook for Systematic Reviews of Interventions 42 6. updated September 2006; Appendix 5b. www.cochrane.org/ resources/handbook/hbook.htm

Howe TE, Rochester L, Jackson A, Banks PMH, Blair VA. Exercise for improving balance in older people. Cochrane Database of Systematic Reviews. 2007; (4)10.1002/14651858.CD004963.pub2

Keysor JJ, Jette AM. Have we oversold the benefit of late-life exercise? Journals of Gerontology: Medical Sciences. 2001; 56A(7):M412-M23.

King AC, Rejeski WJ, Buchner DM. Physical activity interventions targeting older adults: a critical review and recommendations. American Journal of Preventative Medicine. 1998; 15(4):316-33.

King AC. Interventions to promote physical activity by older adults. Journals of Gerontology Series ABiological Sciences \& Medical Sciences. 2001; 56(Spec No 2):36-46.

Mazzeo RS, Cavanagh P, Evans WJ, Fiatarone M, Hagberg J, McAuley E, et al. American College of Sports Medicine position stand: exercise and physical activity for older adults. Medicine and Science in Sports and Exercise. 1998; 30(6):992-1008. [PubMed: 9624662]

Nagi, SZ. Disability concepts revisited: Implications for prevention. In: Pope, AM.; Tarlov, AR., editors. Disability in America: Toward a national agenda for prevention. Washington, D.C: National Academy Press; 1991. p. 309-327.

Province MA, Hadley EC, Hornbrook MC, Lipsitz LA, Philip-Miller J, Mulrow CD, et al. The effects of exercise on falls in elderly patients. A preplanned meta-analysis of the FICSIT trials. Journal of the American Geriatrics Society. 1995; 273(17):1341-7.

Singh MA. Exercise comes of age: rationale and recommendations for a geriatric exercise prescription. Journals of Gerontology: Medical Sciences. 2002; 57(5):M262-82.

Tinetti M, Williams T, Mayewski R. Fall risk index for elderly patients based on number of chronic disabilities. The American Journal of Medicine. 1986; 80:429-34. [PubMed: 3953620]

World Health Organization. ICIDH-2: International classification of functioning, disability and health (final draft). Geneva: World Health Organization; 2001.

\section{References to other published versions of this review}

Latham NK, Anderson CS, Bennett DA, Stretton C. Progressive resistance strength training for physical disability in older people. Cochrane Database of Systematic Reviews. 2003a; (2)10.1002/1465.1858.CD002759

Latham NK, Bennett DA, Stretton CM, Anderson CS. Systematic review of progressive resistance strength training in older adults. Journals of Gerontology Series A-Biological Sciences \& Medical Sciences. 2004; 59(1):48-61. 


\section{Plain Language Summary}

Progressive resistance strength training for improving physical function in older adults

Older people generally lose muscle strength as they age. This reduction in muscle strength and associated weakness means that older people are more likely to have problems carrying out their daily activities and to fall. Progressive resistance training (PRT) is a type of exercise where participants exercise their muscles against some type of resistance that is progressively increased as their strength improves. The exercise is usually conducted two to three times a week at moderate to high intensity by using exercise machines, free weights, or elastic bands. This review sets out to examine if PRT can help to improve physical function and muscle strength in older people.

Evidence from 121 randomised controlled trials (6,700 participants) shows that older people who exercise their muscles against a force or resistance become stronger. They also improve their performance of simple activities such as walking, climbing steps, or standing up from a chair more quickly. The improvement in activities such as getting out of a chair or stair climbing is generally greater than walking speed. Moreover, these strength training exercises also improved older people's physical abilities, including more complex daily activities such as bathing or preparing a meal. PRT also reduced pain in people with osteoarthritis. There was insufficient evidence to comment on the risks of PRT or long term effects. 


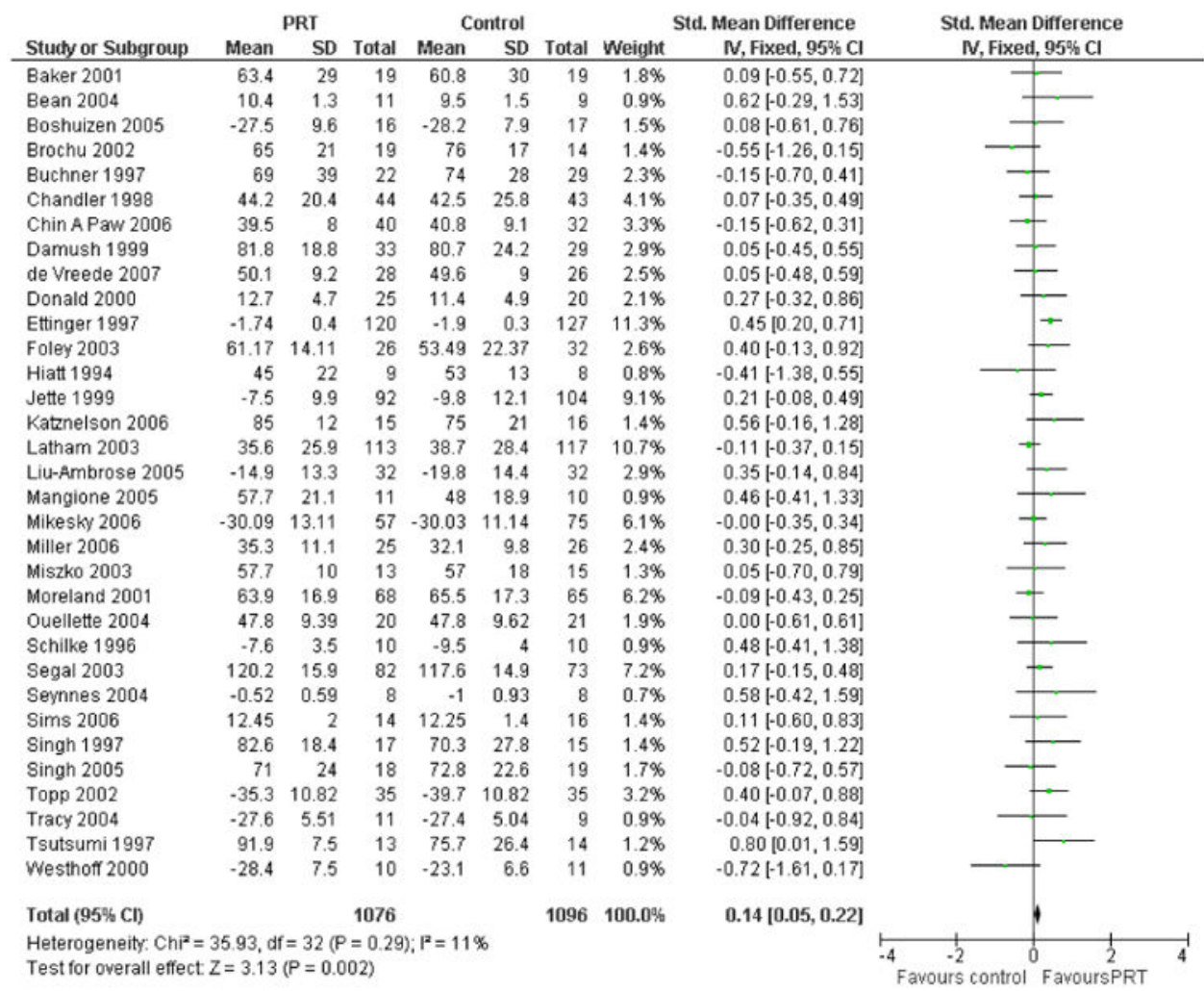

Figure 1. Forest plot of comparison: 1 PRT versus control, outcome: 1.1 Main function measure (higher score $=$ better function) 
Table 1

Assessment of methodological quality scheme

\begin{tabular}{|c|c|c|}
\hline Items & Scores & Notes \\
\hline $\begin{array}{l}\text { A. Was the assigned treatment } \\
\text { adequately concealed prior to allocation? }\end{array}$ & $\begin{array}{l}2=\text { method did not allow disclosure of assignment. } \\
1=\text { small but possible chance of disclosure of assignment or } \\
\text { unclear. } \\
0=\text { quasi-randomised or open list/tables. }\end{array}$ & \\
\hline $\begin{array}{l}\text { B. Were the outcomes of patients/ } \\
\text { participants who withdrew described and } \\
\text { included in the analysis (intention-to- } \\
\text { treat)? }\end{array}$ & $\begin{array}{l}2=\text { withdrawals well described and accounted for in analysis. } \\
1=\text { withdrawals described and analysis not possible. } \\
0=\text { no mention, inadequate mention, or obvious differences } \\
\text { and no adjustment }\end{array}$ & \\
\hline $\begin{array}{l}\text { C. Were the outcome assessors blind to } \\
\text { treatment status? }\end{array}$ & $\begin{array}{l}2=\text { effective action taken to blind assessors. } \\
1=\text { small or moderate chance of un blinding of assessors. } \\
0=\text { not mentioned }, \text { or not possible. }\end{array}$ & \\
\hline $\begin{array}{l}\text { D. Were the participants blinded to the } \\
\text { treatment status? }\end{array}$ & $\begin{array}{l}2=\text { effective action taken to blind assessors. } \\
1=\text { small or moderate chance of un blinding of assessors. } \\
0=\text { not mentioned }, \text { or not possible. }\end{array}$ & \\
\hline $\begin{array}{l}\text { E. Were the treatment and control group } \\
\text { comparable at entry? Specifically, were } \\
\text { the groups comparable with respect to } \\
\text { age, medical co-morbidities (one or more } \\
\text { of history of coronary artery disease, } \\
\text { stroke, hypertension, diabetes, chronic } \\
\text { lung disease), pre-entry physical } \\
\text { dependency (independent vs dependent } \\
\text { in self-care ADL) and mental status } \\
\text { (clinical evidence of cognitive } \\
\text { impairment, yes or no)? }\end{array}$ & $\begin{array}{l}2=\text { good comparability of groups, or confounding adjusted for } \\
\text { in analysis. } \\
1=\text { confounding small; mentioned but not adjusted for. } \\
0=\text { large potential for confounding, or not discussed. }\end{array}$ & \\
\hline $\begin{array}{l}\text { F. Were care programmes, other than the } \\
\text { trial options, identical? }\end{array}$ & $\begin{array}{l}2=\text { care programmes clearly identical } \\
1=\text { clear but trivial differences. } \\
0=\text { not mentioned or clear and important differences in care } \\
\text { programmes }\end{array}$ & \\
\hline $\begin{array}{l}\text { G. Were the inclusion and exclusion } \\
\text { criteria clearly defined? }\end{array}$ & $\begin{array}{l}2=\text { clearly defined } \\
1=\text { inadequately defined } \\
0=\text { not defined. }\end{array}$ & \\
\hline $\begin{array}{l}\text { H. Were the interventions clearly } \\
\text { defined? }\end{array}$ & $\begin{array}{l}2=\text { clearly defined interventions are applied with a } \\
\text { standardised protocol. } \\
1=\text { clearly defined interventions are applied but the } \\
\text { application protocol is not standardised. } \\
0=\text { intervention and/or application protocol are poorly or not } \\
\text { defined }\end{array}$ & \\
\hline $\begin{array}{l}\text { I. Were the outcome measures used } \\
\text { clearly defined? }\end{array}$ & $\begin{array}{l}2=\text { clearly defined measures and the method of data collection } \\
\text { and scoring are clearly described } \\
1=\text { inadequately defined measures } \\
0=\text { not defined. }\end{array}$ & $\begin{array}{l}\text { For our primary outcome, } \\
\text { physical disability in terms of } \\
\text { self-report measures of physical } \\
\text { function, we considered the } \\
\text { outcome clearly defined if a } \\
\text { validated and standardised scale } \\
\text { was used and the method of data } \\
\text { collection was clearly described. } \\
\text { Our secondary outcome } \\
\text { measures included gait speed, } \\
\text { muscle strength (e.g. one } \\
\text { repetition maximum test, } \\
\text { isokinetic and isometric } \\
\text { dynamometry), balance (e.g. } \\
\text { Berg Balance Scale, Functional } \\
\text { Reach Test), aerobic capacity, } \\
\text { and chair rise. These secondary } \\
\text { outcomes were considered well } \\
\text { defined if validated and } \\
\text { standardised measures were } \\
\text { used, and the method of data } \\
\text { collection and scoring of any } \\
\text { scales was clearly described }\end{array}$ \\
\hline
\end{tabular}




\begin{tabular}{lll}
\hline Items & Scores & Notes \\
\hline J. Was the surveillance active and of & $2=$ active and appropriate duration (three months follow-up or \\
clinically appropriate duration (i.e. at & greater). \\
least 3 months)? & $1=$ active but inadequate duration (less than three months \\
& follow-up). \\
& $0=$ not active or surveillance period not defined. \\
\hline
\end{tabular}



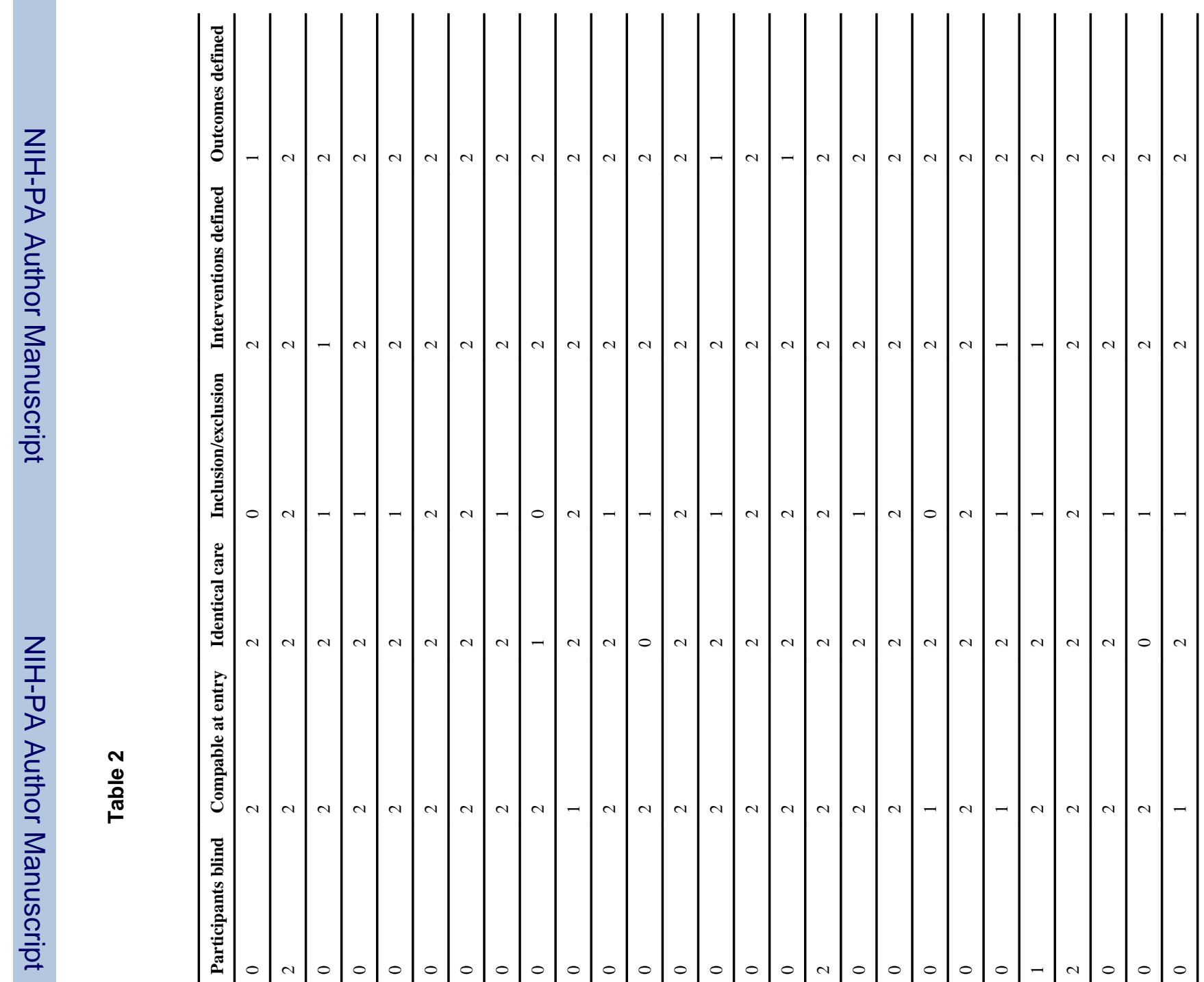

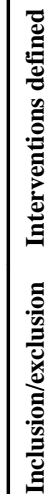

(1)

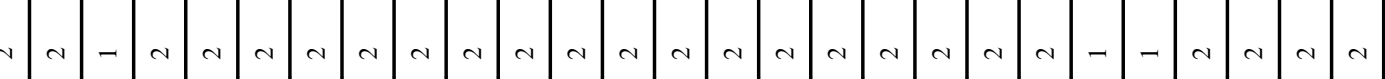

ป气

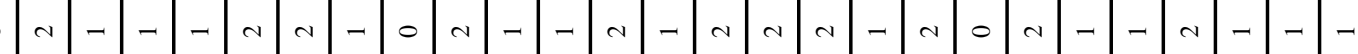

焉

竞

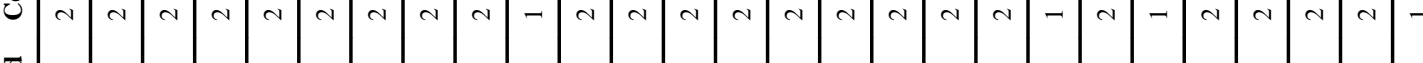

产

产

$\int_{0}^{\frac{\pi}{0}}$

를

要

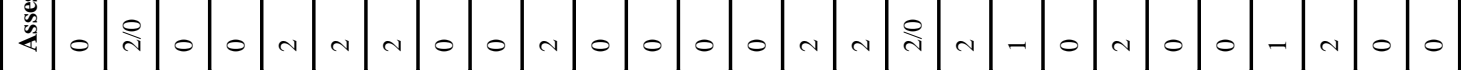

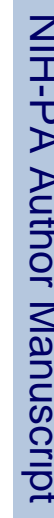

$\sum_{0}$
$\circ$

(ี) 


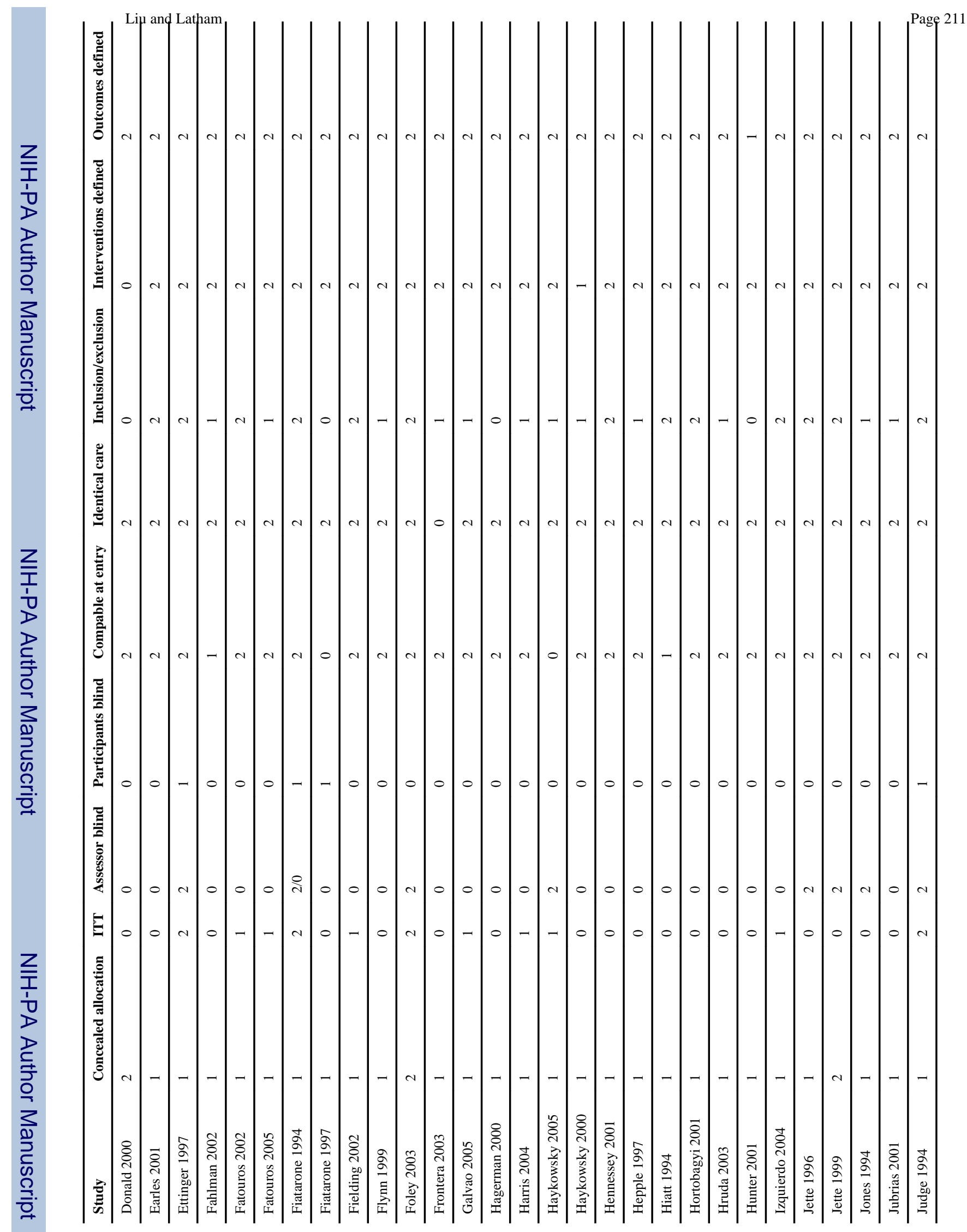




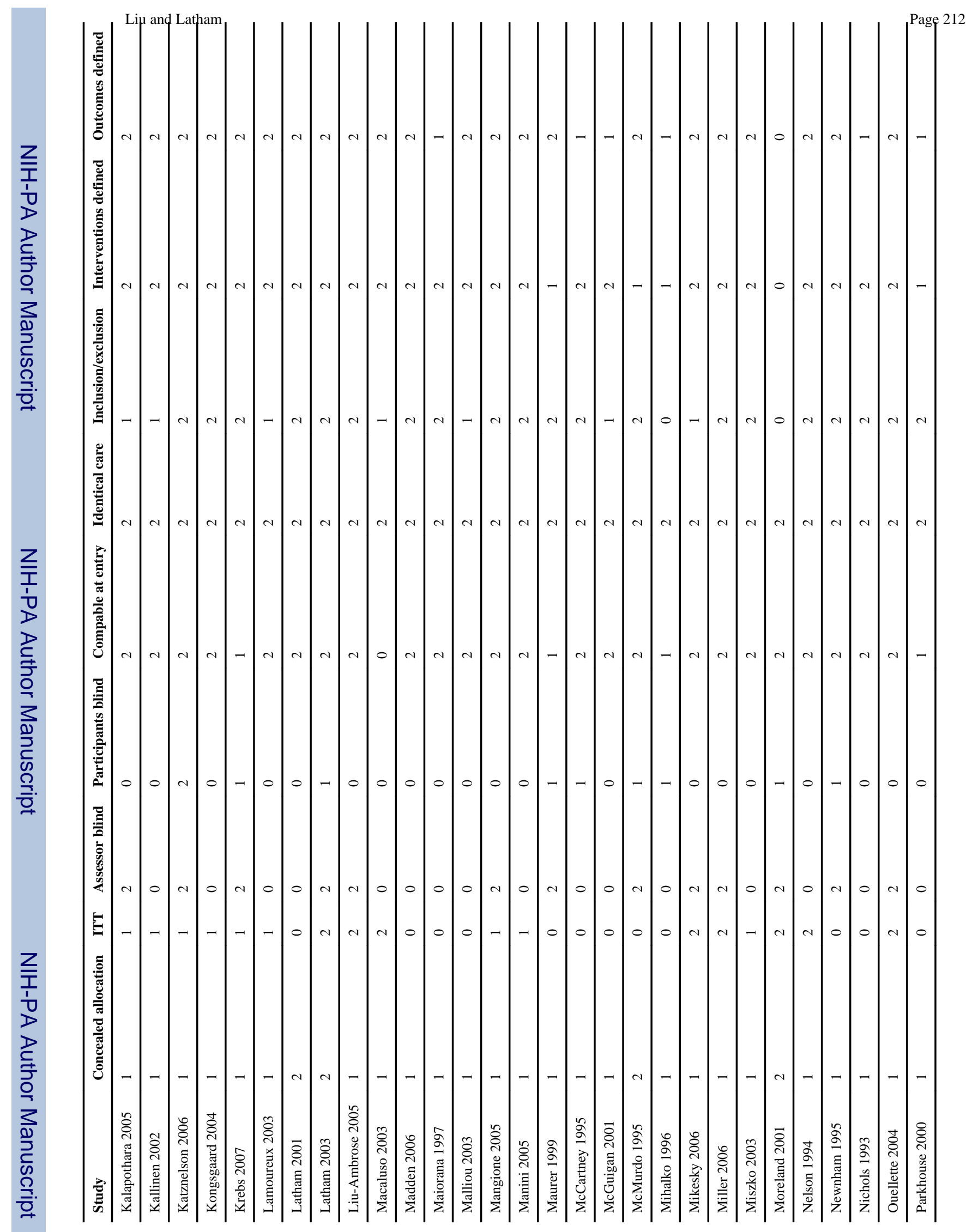




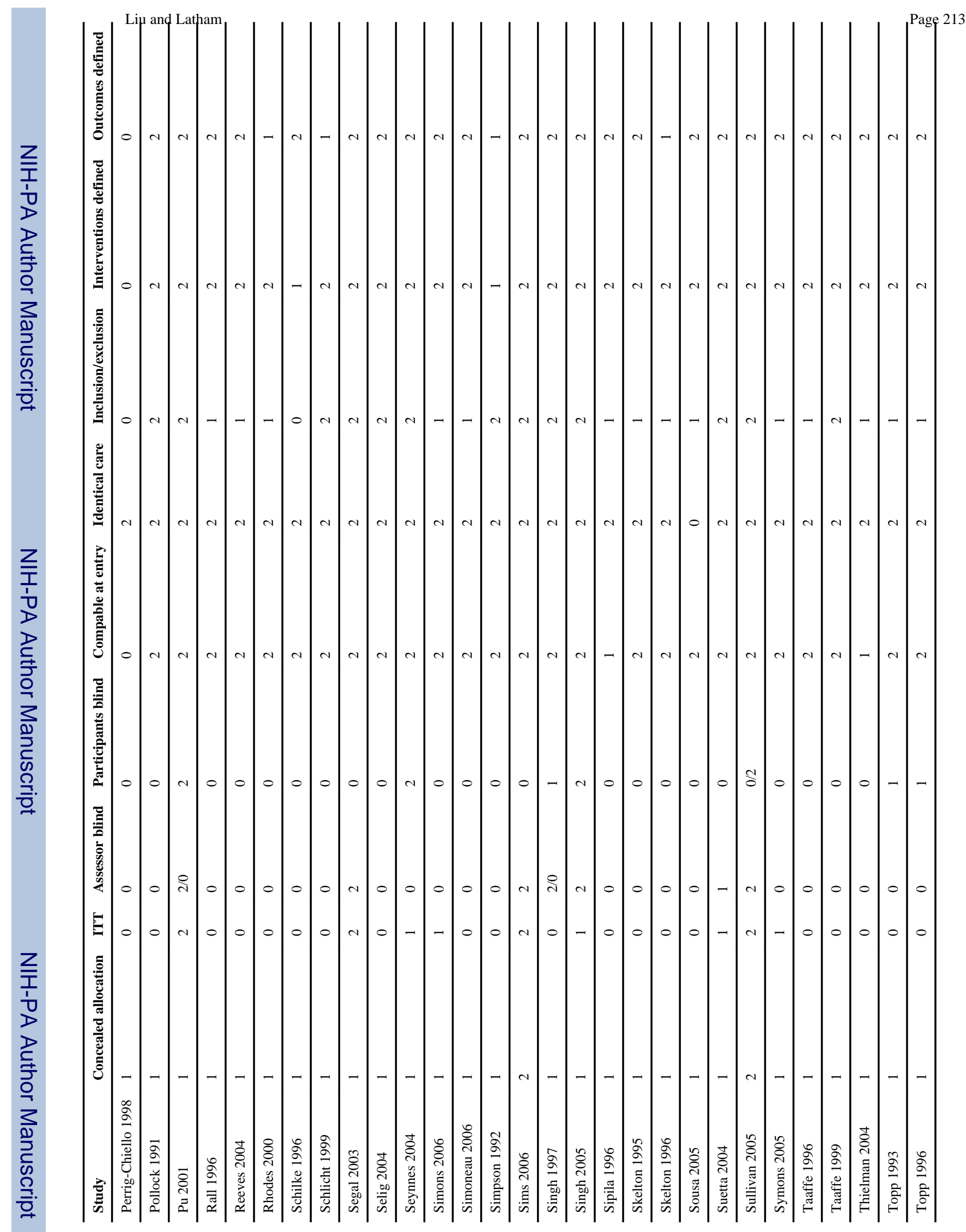




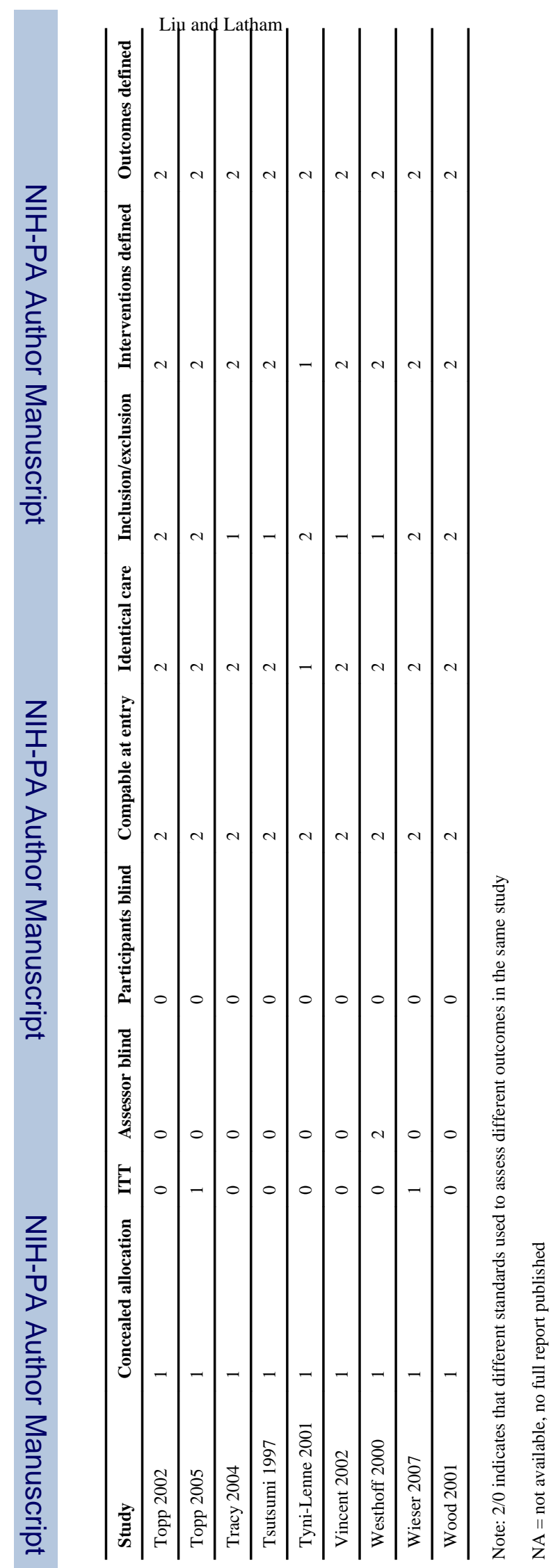

Page 214 
Table 3

Functional or quality of life measures that could not be pooled

\begin{tabular}{|c|c|c|c|}
\hline Study & Outcome Measure & Treatment Group & Control Group \\
\hline Baum 2003 & $\begin{array}{l}\text { Physical performance test at } 6 \text { month. Mean }= \\
\text { baseline score }+ \text { change score. SD was not } \\
\text { reported }\end{array}$ & 9.2 & 8.1 \\
\hline Buchner 1997 & mean change in number of independent IADL's & mean $0.1(\mathrm{SD} 0.7)$ & mean $0.2(\mathrm{SD} 0.7)$ \\
\hline Donald 2000 & Barthel Index (actual data not in paper) & no significant difference & \\
\hline Fiatarone 1994 & ankle activity monitors (counts/day) & mean change 3412 (SD 1700) & $\begin{array}{l}\text { mean change }-1230(\mathrm{SD} \\
1670)\end{array}$ \\
\hline Fiatarone 1997 & $\begin{array}{l}\text { overall self-reported activity level (measure not } \\
\text { specified) }\end{array}$ & $\begin{array}{l}\text { significant improvement }(\mathrm{p}<0.05) \text { in } \\
\text { exercise group }\end{array}$ & NR \\
\hline Fielding 2002 & SF-36-PF & $\begin{array}{l}\text { No significant differences between } \\
\text { high intensity and low intensity } \\
\text { groups }\end{array}$ & \\
\hline Jette 1996 & SF-36 - PF (actual data not reported) & $\begin{array}{l}\text { no significant difference between } \\
\text { groups (data not reported) }\end{array}$ & \\
\hline Kongsgaard 2004 & $\begin{array}{l}\text { three ADLs of a questionnaire developed by the } \\
\text { Danish Institute of Clinical Epidemiology }\end{array}$ & $\begin{array}{l}\text { Actual data not reported. The author } \\
\text { stated that the self-reported ADL } \\
\text { level was significantly higher in the } \\
\text { Ex group than in the control group }\end{array}$ & \\
\hline Krebs 2007 & $\begin{array}{l}\text { SF-36. } 7 \text { people ( } 2 \text { in PRT, } 5 \text { in Functional } \\
\text { training) reported improvement in the SF-36 } \\
\text { items }\end{array}$ & & \\
\hline Maiorana 1997 & $\begin{array}{l}\text { Physical Activity Questionnaire (no reference) } \\
\text { self report }\end{array}$ & mean $209.8(\mathrm{SD} 142.9) \mathrm{kJ} / \mathrm{kg}$ & mean $250.1(\mathrm{SD} 225) \mathrm{kJ} / \mathrm{kg}$ \\
\hline Maurer 1999 & SF-36 PF (no SD/SE reported) & mean 50.3 & mean 49.2 \\
\hline Maurer 1999 & WOMAC section C (no SD/SE reported) & 464.4 & 606.6 \\
\hline Maurer 1999 & Aims Mobility (no SD/SE reported) & 1.28 & 1.21 \\
\hline McMurdo 1995 & Barthel Index (medians reported) & median change 0 (range -1 to 2 ) & $\begin{array}{l}\text { median change control } 0 \\
\text { (range }-1 \text { to } 1 \text { ) }\end{array}$ \\
\hline Mihalko 1996 & $\begin{array}{l}\text { adapted version of Lawton and Brody's IADL } \\
\text { scale (higher = better, not pooled because study } \\
\text { was cluster randomised) }\end{array}$ & mean $105(\mathrm{SD} 12)$ & mean 68 (SD 25) \\
\hline Mikesky 2006 & $\begin{array}{l}\text { SF-36 physical function at } 30 \text { month (the } \\
\text { intervention was } 1 \text { - year) }\end{array}$ & $\mathrm{n}=81$, mean $=65.37(\mathrm{SD}=25.05)$ & $\begin{array}{l}\mathrm{n}=79, \text { mean }=63.88(\mathrm{SD}= \\
25.48)\end{array}$ \\
\hline Nichols 1993 & $\begin{array}{l}\text { Blair Seven-day recall Caloric Expenditure } \\
\text { (KCalories) }\end{array}$ & not significantly altered & not significantly altered \\
\hline Schilke 1996 & AIMS mobility score (actual data not reported) & $\begin{array}{l}\text { "no significant differences between } \\
\text { or within groups" }\end{array}$ & \\
\hline Singh 1997 & IADL (Lawton Brody Scale) & mean $23.4(\mathrm{SD} 0.4)$ & mean $23.9($ SD 0.1$)$ \\
\hline Skelton 1996 & $\begin{array}{l}\text { Human Activity Profile - (only reported training } \\
\text { groups \% change and the P-value of the change) }\end{array}$ & $3.9 \%$ change $3.9 \%$ change & NR \\
\hline Skelton 1996 & Human Activity Profile - Max Activity Score & $0 \%$ change & NR \\
\hline Skelton 1995 & Human Activity Profile & no difference from baseline & no difference from baseline \\
\hline Thielman 2004 & Rivermead Motor Assessment & $\begin{array}{l}\text { Significant improvement was found } \\
\text { for people in the control group with } \\
\text { low-level function }\end{array}$ & \\
\hline Tyni-Lenne 2001 & $\begin{array}{l}\text { Minnesota Living with Heart Failure } \\
\text { Questionnaire (lower score = better QOL, } \\
\text { medians reported) }\end{array}$ & median 19 (range 0-61) & median 44 (range $3-103$ ) \\
\hline
\end{tabular}




\section{Table 4}

\section{Falls}

\begin{tabular}{|c|c|c|c|}
\hline Study & Fall Statistic & PRT & Control \\
\hline \multirow[t]{3}{*}{ Buchner 1997} & $\begin{array}{l}\text { 1) Cox regression analysis, time to first fall, } 0.53,95 \% \mathrm{CI} \\
0.3-0.91 \text { for exercise group (including endurance exercise } \\
\text { groups) }\end{array}$ & & \\
\hline & 2) proportion of people who fell in one year & all exercise groups: $42 \%$ & $60 \%$ \\
\hline & 3) fall rate (falls/year) & all exercise: 0.81 falls/year & 0.49 falls/year \\
\hline \multirow[t]{2}{*}{ Donald 2000} & 1) number of falls & $7(n=32)$ & $4(n=27)$ \\
\hline & 2) number of people who fell & $6(n=32)$ & $2(\mathrm{n}=27)$ \\
\hline \multirow[t]{2}{*}{ * Fiatarone 1994} & 1) average falls/subject & 2.32 & 2.77 \\
\hline & $\begin{array}{l}\text { 2) covariance adjusted treatment incidence ratio (PRT vs } \\
\text { control) }\end{array}$ & $0.95(95 \%$ CI $0.64,1.41)$ & \\
\hline Fiatarone 1997 & Falls & $\begin{array}{l}\text { no difference between groups (no } \\
\text { data provided) }\end{array}$ & \\
\hline \multirow[t]{2}{*}{ * Judge 1994} & 1) Average falls/subject & 0.82 & 1.22 \\
\hline & $\begin{array}{l}\text { 2) Co-variate adjusted treatment incidence ratio (PRT vs } \\
\text { control) }\end{array}$ & 0.61 (95\%CI $0.34,1.09)$ & \\
\hline \multirow[t]{2}{*}{ * Buchner 1997} & 1) Average falls/subject & 0.68 & 1.6 \\
\hline & $\begin{array}{l}\text { 2) Co-variate adjusted treatment incidence ratio (PRT vs } \\
\text { control) }\end{array}$ & $0.91(95 \%$ CI $0.48,1.74)$ & \\
\hline Krebs 2007 & $\begin{array}{l}1 \text { in the PRT group sustained an unrelated fall halfway through } \\
\text { the } 6 \text {-week intervention, resulting in injury of her dominate } \\
\text { shoulder. Exercise was modified for her }\end{array}$ & 1 & 0 \\
\hline Latham 2001 & total falls & 164 & 149 \\
\hline \multirow[t]{2}{*}{ Latham 2003} & 1) number of people who fell & 60 & 64 \\
\hline & 2) fall-rate, person years & 1.02 & 1.07 \\
\hline Liu-Ambrose 2005 & $\begin{array}{l}\text { the frequency of falls (excluded falls occurred in exercise } \\
\text { classes) }\end{array}$ & 18 (1 subject fell 7 times) & 0 \\
\hline Mangione 2005 & $\begin{array}{l}\text { Reported the number of participants fell during post-training } \\
\text { examination ( } \mathrm{n}=1 \text { - group was not reported) }\end{array}$ & & \\
\hline Miszko 2003 & Report number of people & 5 & 1 \\
\hline Singh 2005 & Numbers per person, no statistical difference between groups & $.15(.37)$ & 0 \\
\hline
\end{tabular}

Note: Data marked with * were obtained from Province 1995 


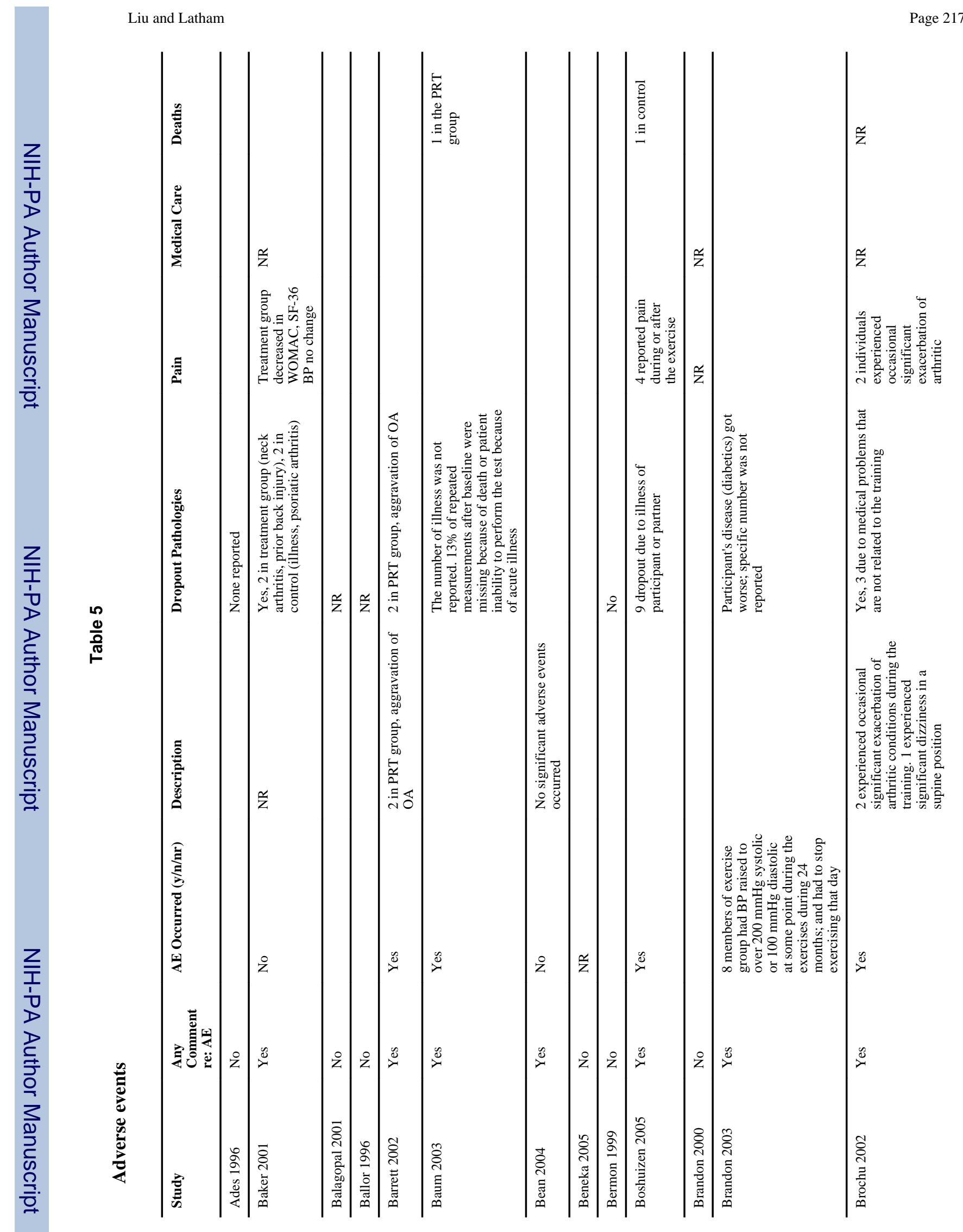




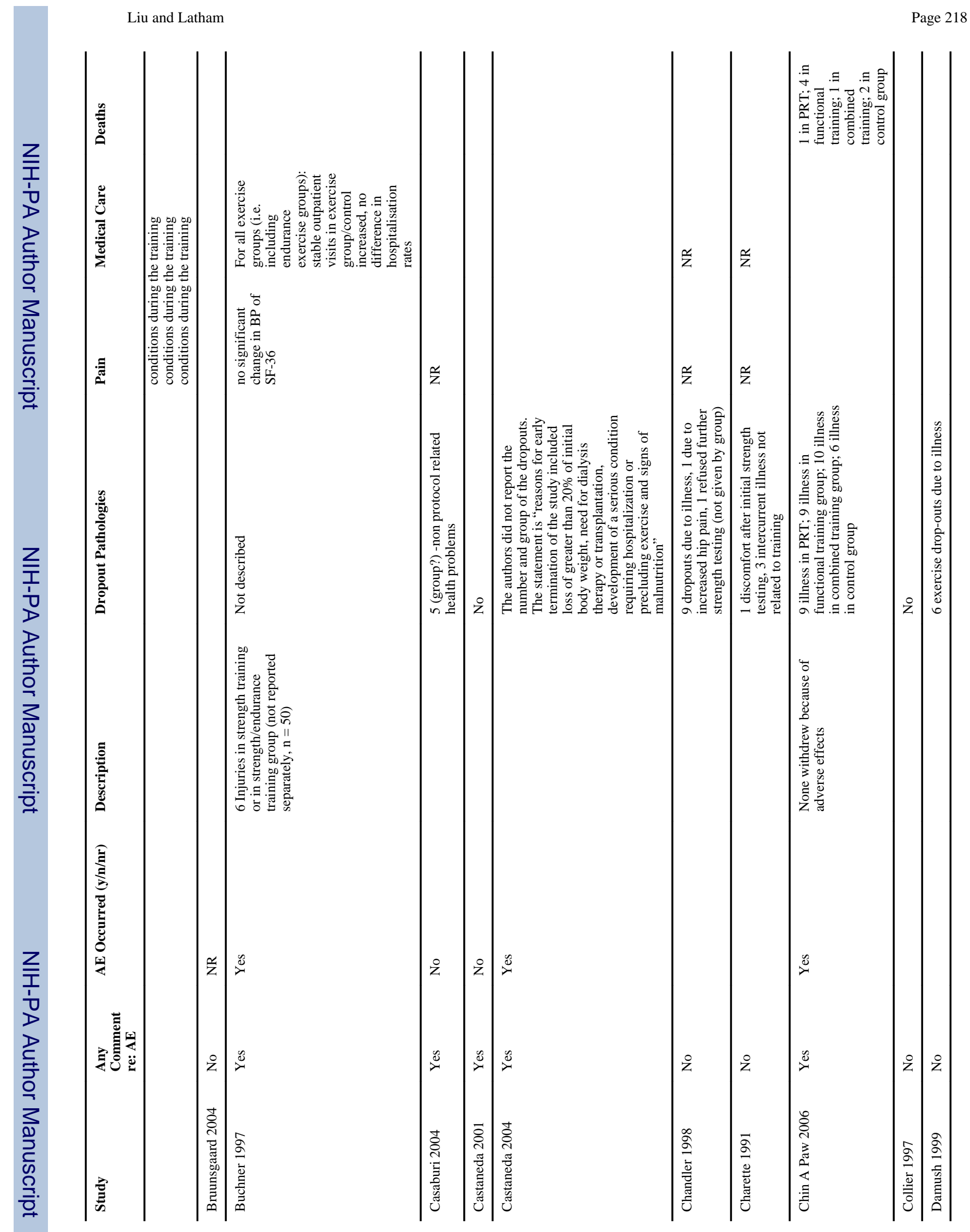

Cochrane Database Syst Rev. Author manuscript; available in PMC 2015 February 11. 


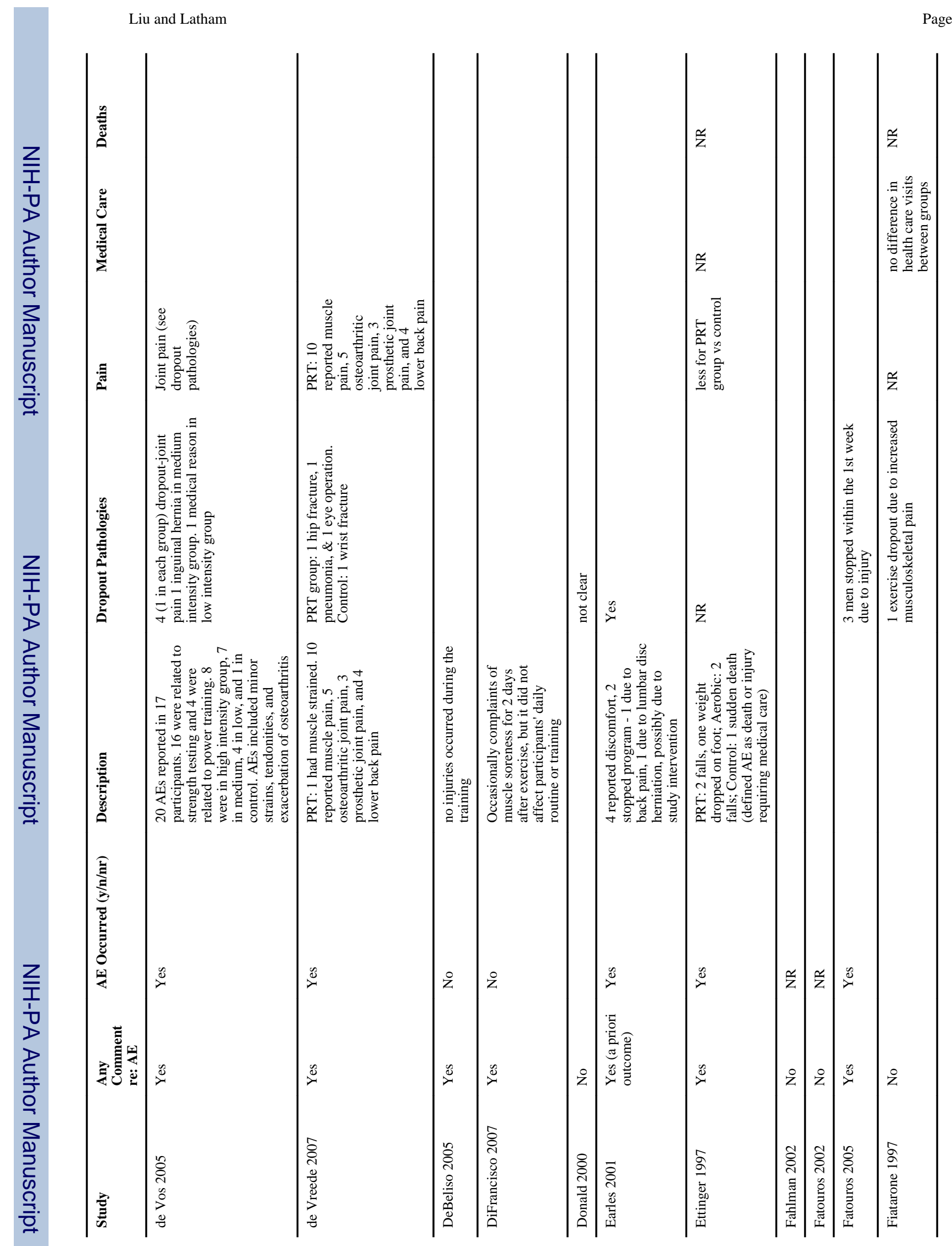

Cochrane Database Syst Rev. Author manuscript; available in PMC 2015 February 11. 


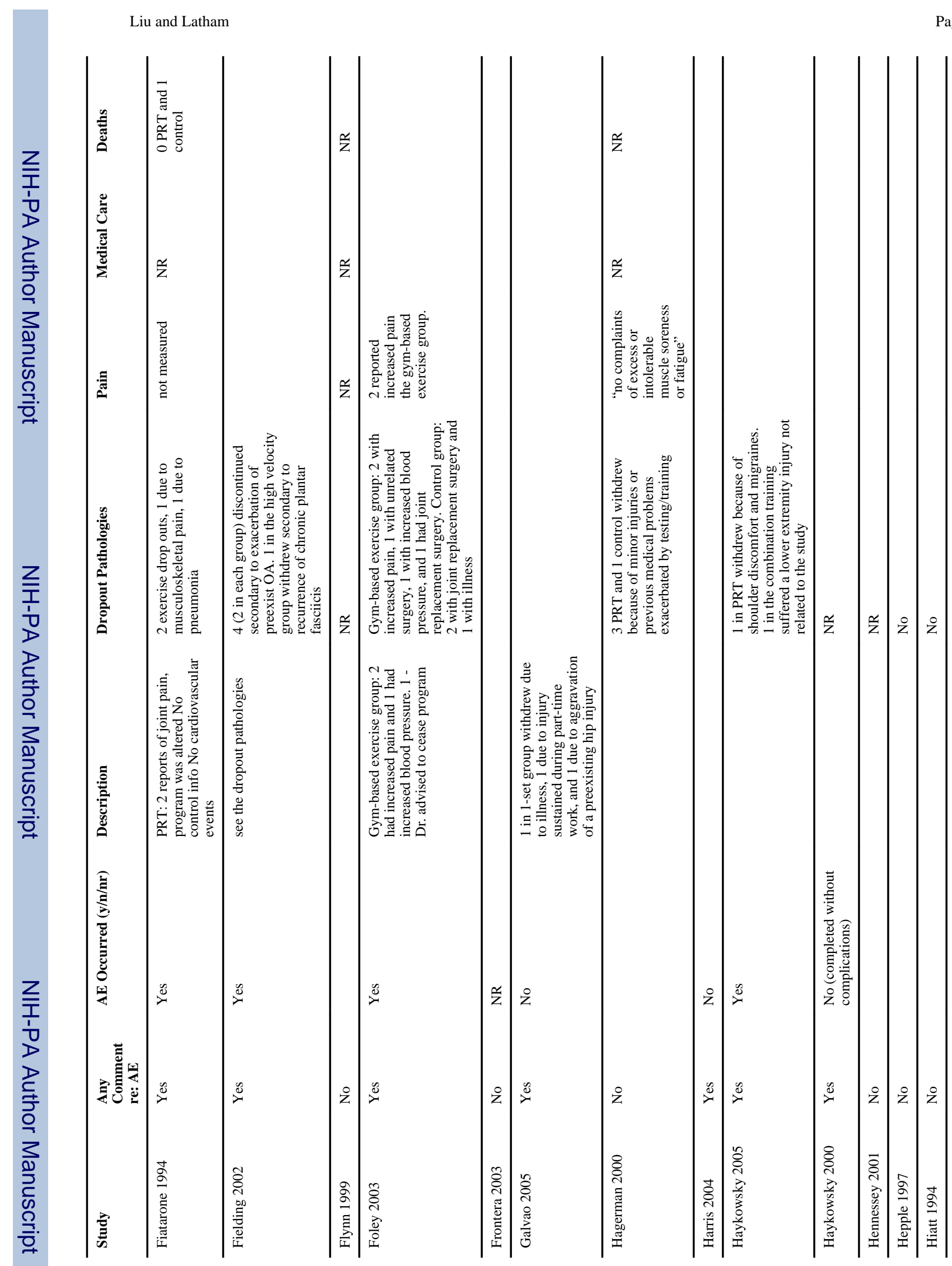




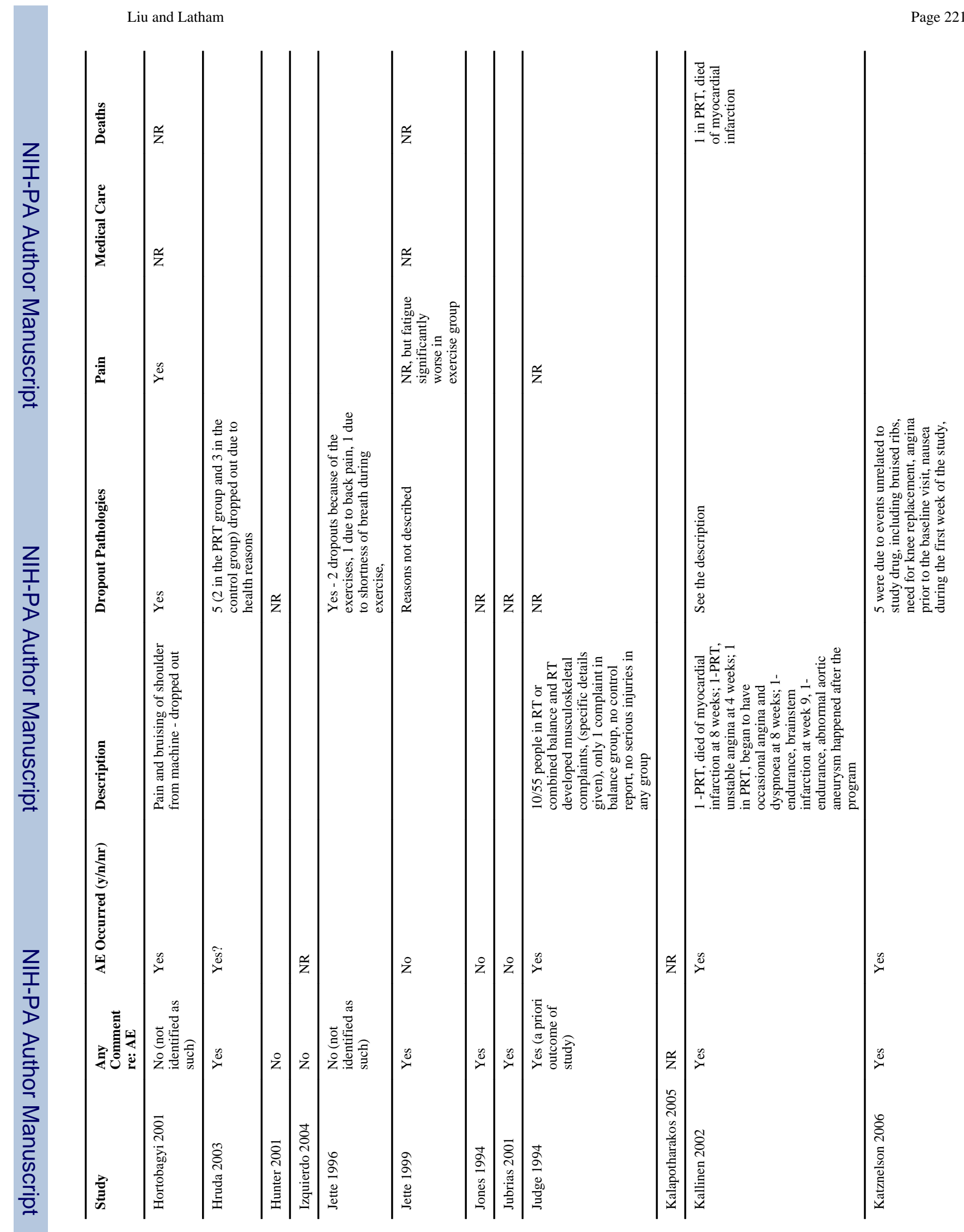




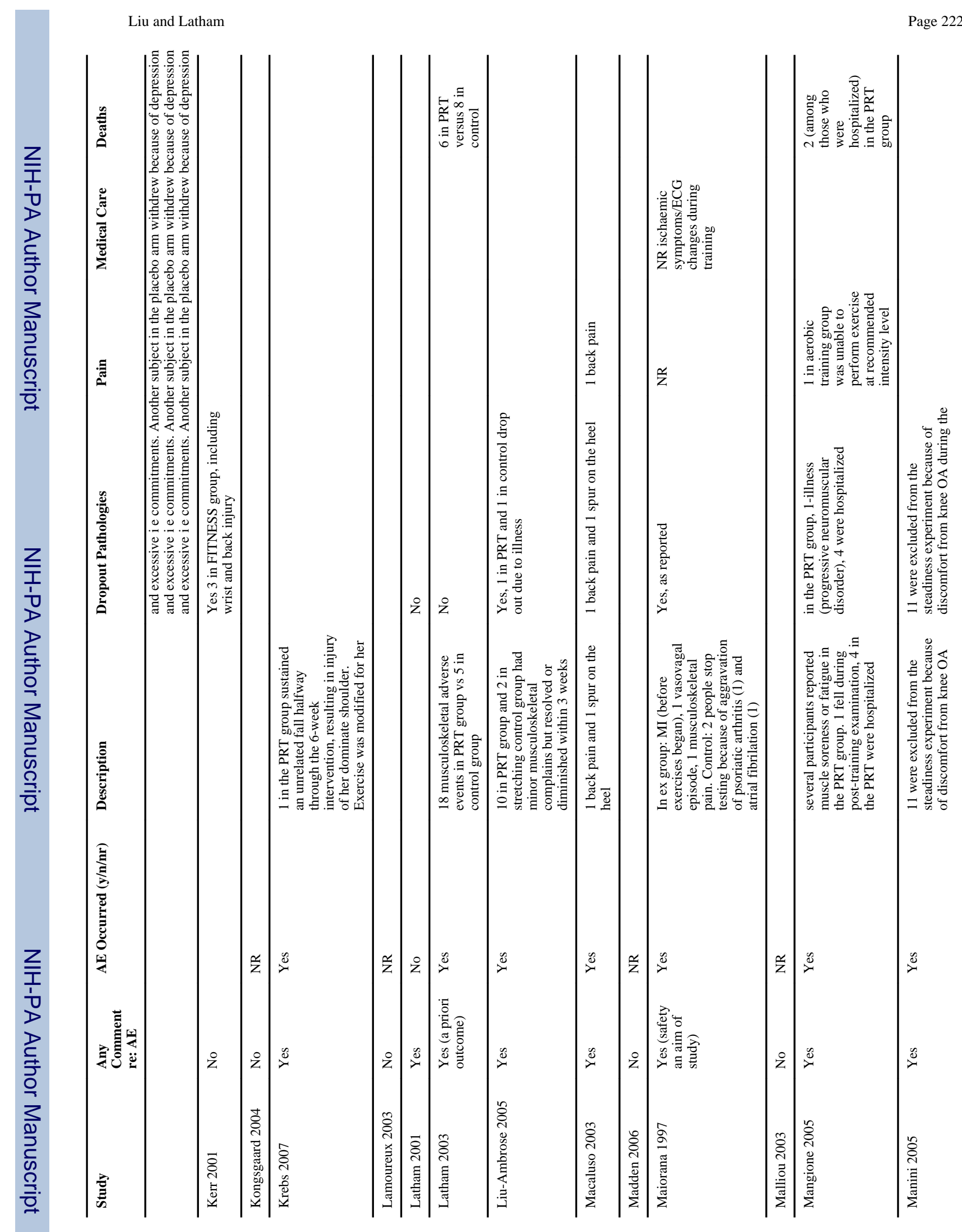




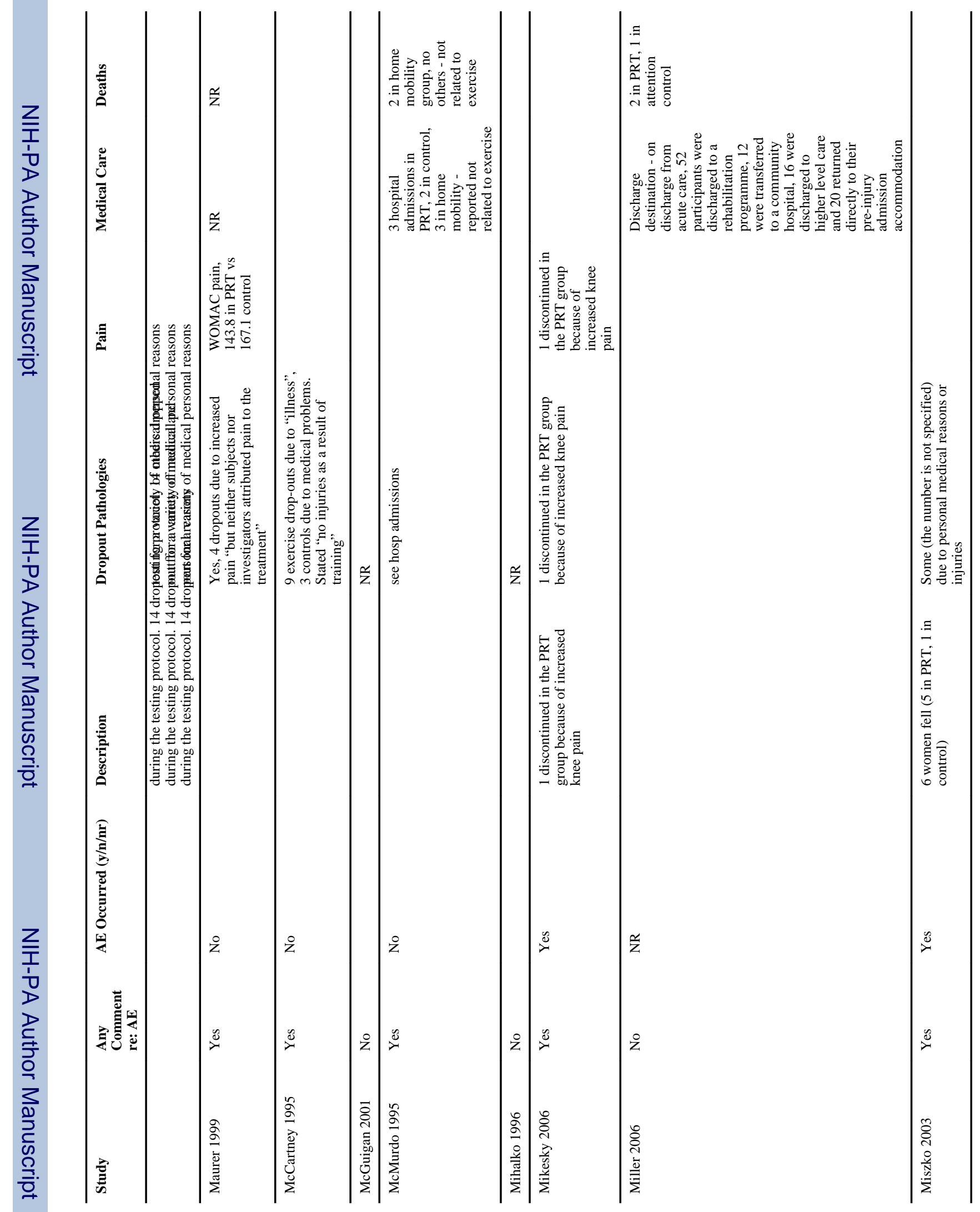




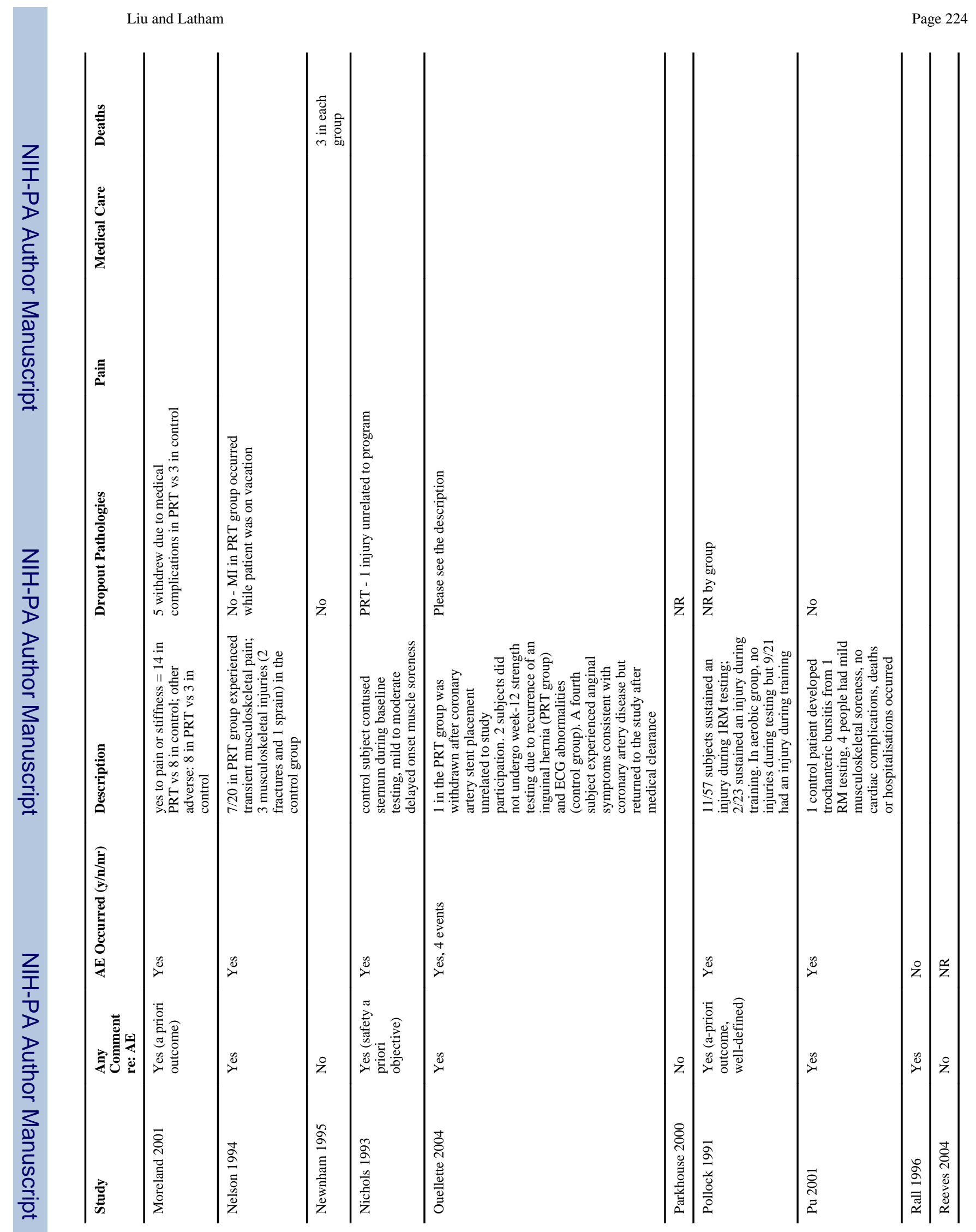

Cochrane Database Syst Rev. Author manuscript; available in PMC 2015 February 11. 


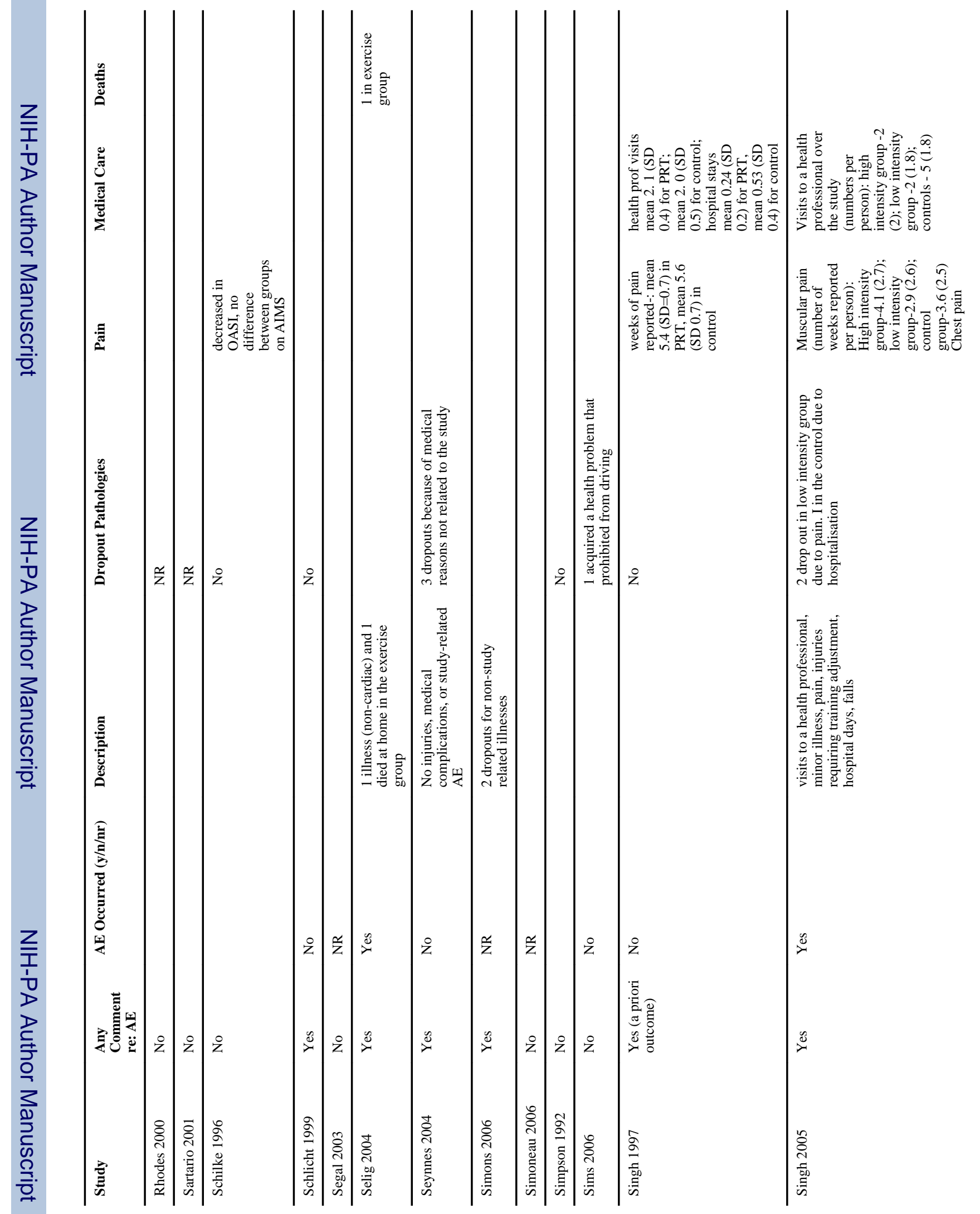




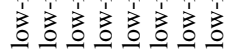

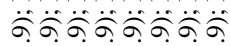

Liuarn Daphamb a

Page 226
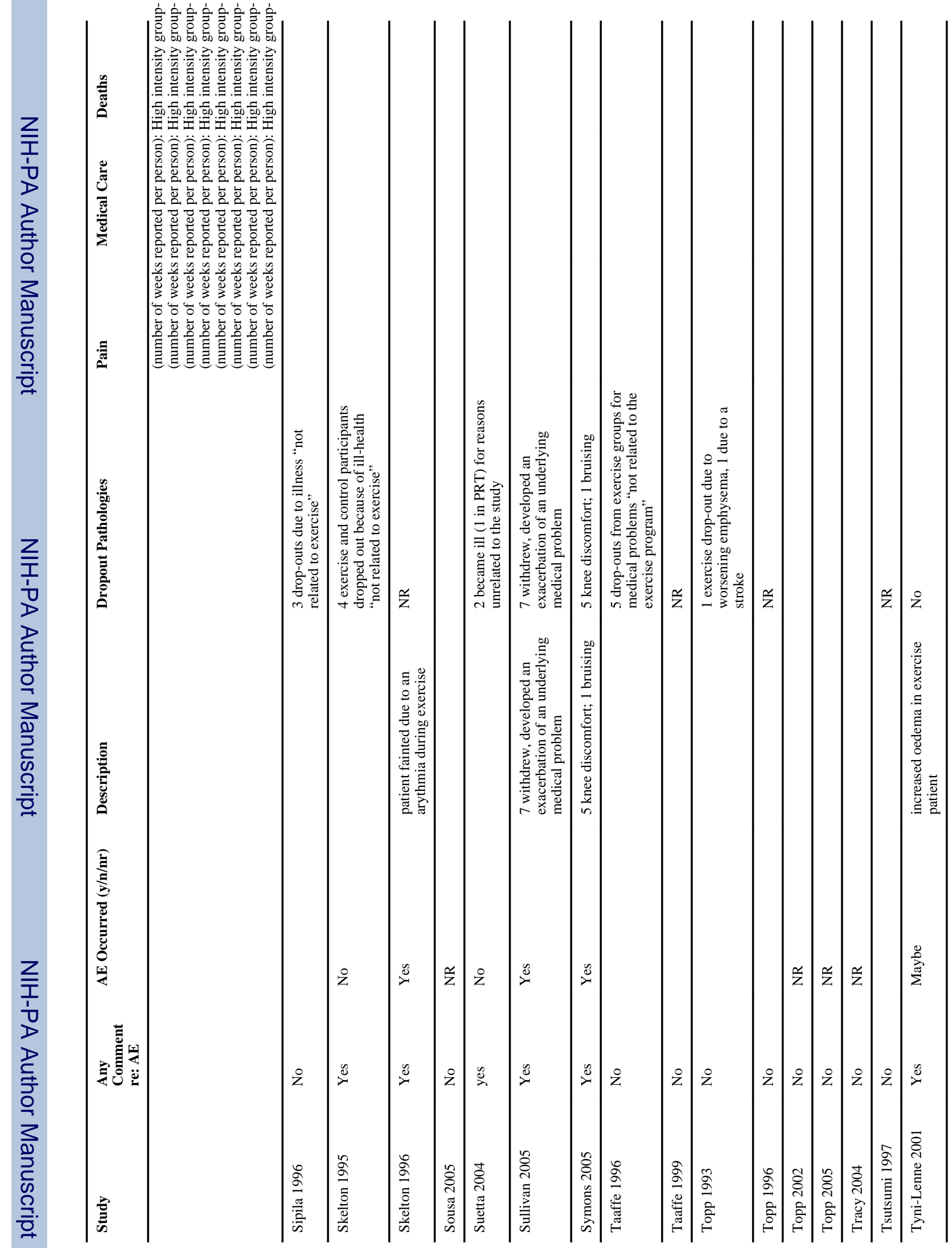


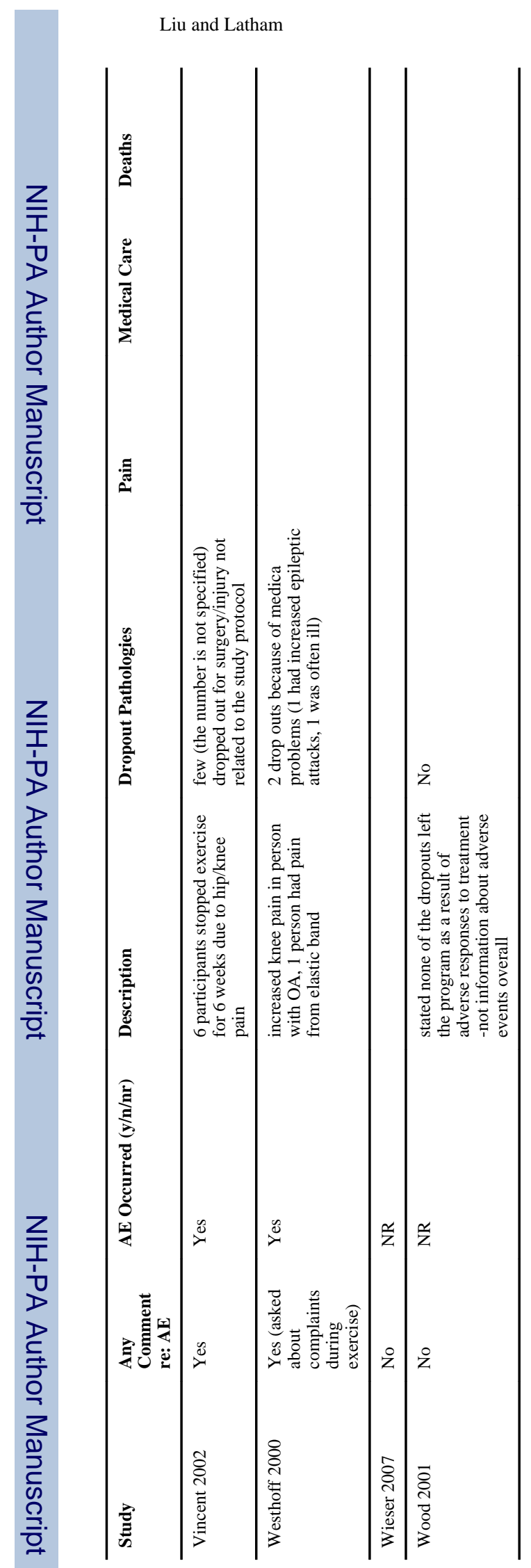

Page 227 